\title{
MÉTODO DOS ELEMENTOS DE CONTORNO COM A RECIPROCIDADE DUAL PARA A ANÁLISE TRANSIENTE TRIDIMENSIONAL DA MECÂNICA DO FRATURAMENTO
}

JOÃO CARLOS CORDEIRO BARBIRATO

Tese apresentada à Escola de Engenharia de São Carlos, da Universidade de São Paulo, como parte dos requisitos para obtenção do Título de Doutor em Engenharia de Estruturas.

ORIENTADOR: Prof. Dr. Wilson Sérgio Venturini

São Carlos

1999 
Barbirato, João Carlos Cordeiro

B237m

Método dos elementos de contorno com a reciprocidade dual para a análise transiente tridimensional da mecânica do fraturamento / João Carlos Cordeiro Barbirato. -- São Carlos, 1999.

Tese (Doutorado) -- Escola de Engenharia de São Carlos-Universidade de São Paulo, 1999.

Área: Engenharia de Estruturas.

Orientador: Prof. Dr. Wilson Sérgio Venturini.

1. Método dos elementos de contorno. 2. Método da reciprocidade dual. 3. Fratura dinâmica. I. Título. 
FOLHA DE APROVAÇ̃̃O

Candidato: Engenheiro JOÃO CARLOS CORDEIRO BARBIRATO

Tese defendida e aprovada em 24-09-1999 pela Comissão Julgadora:

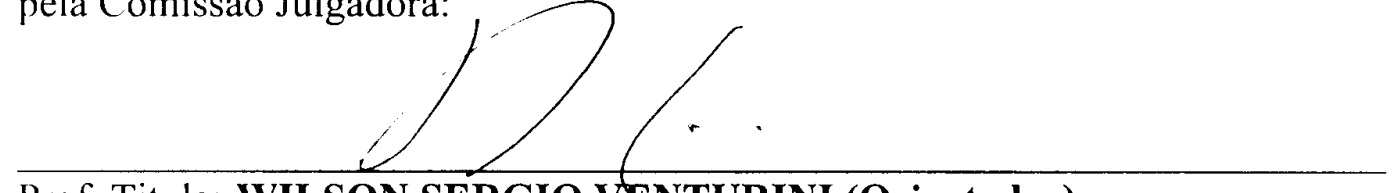

Prof. Titular WILSQN SERGIO VENTURINI (Orientador)

(Escola de Engenharia de São Carlos - Universidade de São Paulo)

Prof. Titular JOSÉ ELIAS LAIER

(Escola de Engenharia de São Carlos - Universidade de São Paulo)

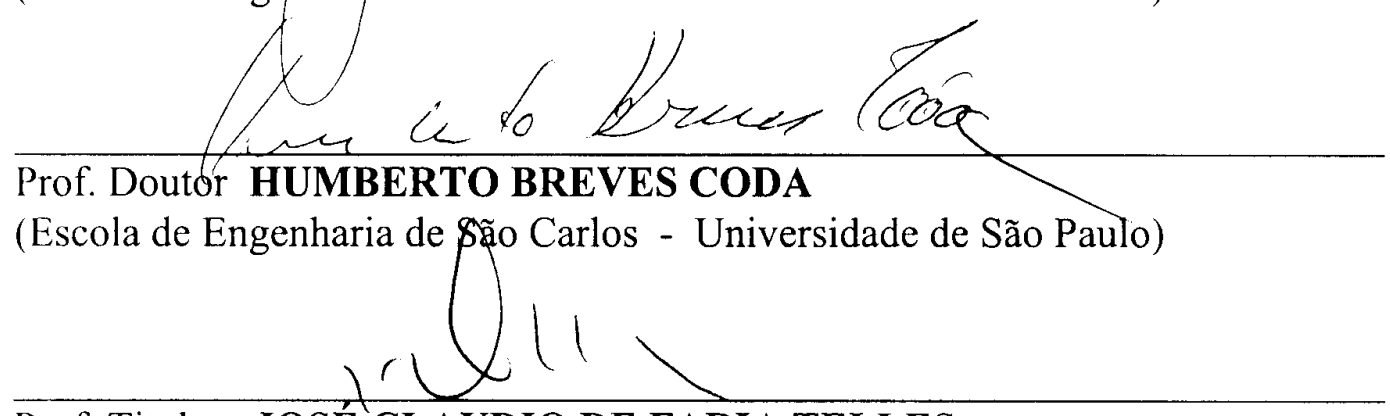

Prof. Titular JOSÉ CLAUDIO DE FARIA TELLES

(COPPE - Universidade Federal do Rio de Janeiro)

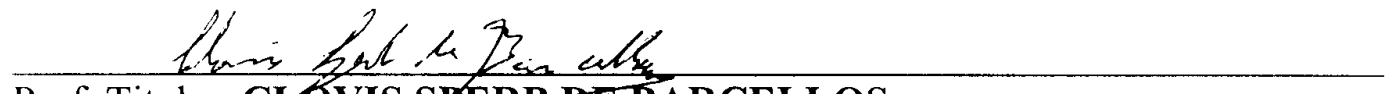

Prof. Titular CLOVIS SPERB DE BARCELLOS

(Universidade Feđeral de Santa Catarina - UFSC)

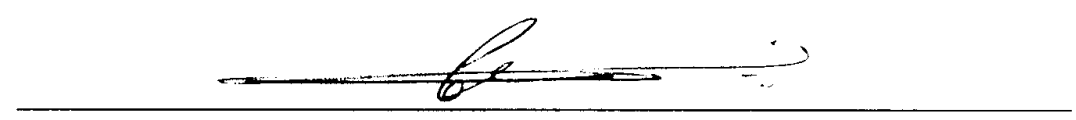

Prof. Titular CARLITO CALIL JUNIOR

Coordenador da Área de Engenharia de Estruturas

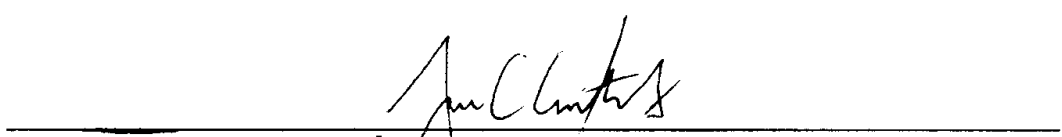

JOSÉ CARLOS A. CINTRA

Presidente dà Comissão de Pós-Graduação 
À minha esposa Gianna, e ao meu filho João Gabriel.

À memória de meu irmão Francisco. 


\section{AGRADECIMENTOS}

Ao Professor Dr. Wilson Sérgio Venturini, pela orientação sempre presente, amizade e compreensão. É, também, exemplo de estímulo à pesquisa e à docência.

À Gianna, pela paciência em revisar os capítulos, mesmo não sendo da sua área, o que me trouxe uma ajuda adicional.

A todos os amigos da pós-graduação de estruturas da EESC/USP pela convivência agradável e enriquecedora; em especial ao amigo Mário César e Yansheng Jiang pela troca de informações e discussões sobre fraturamento.

Aos Professores e Funcionários do SET/EESC/USP que direta ou indiretamente colaboraram para a realização deste trabalho.

Ao Departamento de Engenharia Estrutural do Centro de Tecnologia da UFAL que, ao implementar um programa de capacitação docente, propiciou meu afastamento para a realização deste trabalho, além do apoio recebido ao retornar.

Aos grandes amigos de ontem e de sempre, Aline, Valéria, Edna, Solange, Léa, Marcio, Flávio, Jefferson, Sérgio e Nelson, pelo constante apoio e amizade.

Ao povo brasileiro que paga impostos para manter, entre outros, o programa CAPES-PICD do qual fui beneficiado com uma bolsa de doutoramento. 


\section{SUMÁRIO}

LISTA DE FIGURAS $\ldots$ i

LISTA DE TABELAS

LISTA DE ABREVIATURAS E SIGLAS $\ldots$ viii

LISTA DE SÍMBOLOS _.. ix

RESUMO

ABSTRACT _... xiv

CAPÍTULO 1

CONSIDERAÇÕES INICIAIS

1.1 - INTRODUÇÃO $\ldots 1$

1.2 - ESTRUTURAÇÃO DO TRABALHO $\ldots 2$

1.3 - ÁREAS DE ABORDAGEM $\ldots 4$

1.3.1 - O Método dos Elementos de Contorno $\quad \ldots 4$

1.3.2 - Elastodinâmica $\quad \ldots 5$

1.3.3 - Mecânica da Fratura $\quad \ldots 5$

1.4 - REVISÃO BIBLIOGRÁRICA $\quad \ldots 7$

CAPÍTULO 2

FORMULAÇÃO ELASTOSTÁTICA TRIDIMENSIONAL DO

MEC

2.1 - INTRODUÇÃO $\quad \ldots 16$

2.2 - NOTAÇÃO INDICIAL $\quad \ldots 17$

2.3 - RELAÇÕES DA ELASTOSTÁTICA $\quad \ldots 18$ 
2.4 - SOLUÇÕES FUNDAMENTAIS

2.4.1 - Solução Fundamental de Kelvin …24

2.4.2 - Solução Fundamental de Mindlin …26

2.5 - EQUAÇÕES INTEGRAIS DE CONTORNO _.. 32

2.5.1 - Para Pontos do Domínio … 32

2.5.2 - Para Pontos do Contorno … 34

2.6 - MÉTODO DOS ELEMENTOS DE CONTORNO _... 38

2.6.1 - Discretizações $\quad \ldots 38$

2.6.2 - Elementos de Contorno $\quad \ldots 42$

2.6.2.1 - Elemento Constante $\quad \ldots 44$

2.6.2.2 - Elemento Linear $\quad \ldots 45$

2.6.3 - Integrações Numéricas — ... 49

2.6.3.1 - Integração Singular $\quad \ldots 50$

2.6.3.2 - Integração Numérica $\quad \ldots 56$

2.6.4 - Deslocamentos e Tensões em Pontos do Domínio _.. 58

2.6.5 - Tensões em Pontos do Contorno _.. 59

2.7 - APLICAÇÕES $\quad \ldots 63$

2.7.1 - Aplicação 1 - Sólido elástico paralelepipédico, contido

lateralmente $\quad \ldots 63$

2.7.2 - Aplicação 2: Viga elástica solicitada à flexão _..66

2.7.3 - Aplicação 3: Sólido cruciforme $\quad \ldots 68$

2.7.4 - Aplicação 4: Superfície retangular carregada no meio semi-

infinito $\quad \ldots 71$

2.7.5 - Aplicação 5: Cavidade próxima à superfície do semi-infinito

\section{CAPÍTULO 3}

FORMULAÇÃO ELASTODINÂMICA TRIDIMENSIONAL DO

MEC - MATRIZ DE MASSA

3.1 - INTRODUÇÃO $\quad \ldots 81$

3.2 - RELAÇÕES DA ELASTODINÂMICA $\quad \ldots 82$ 
3.3 - REPRESENTAÇÕES INTEGRAIS

3.3.1 - Equações Básicas

3.3.2 - Para Pontos do Contorno

3.4 - MÉTODO DA RECIPROCIDADE DUAL

3.4.1 - Formulação Integral

3.4.2 - Discretizações

... 92

3.5 - TÉCNICA DA INTEGRAÇÃO DIRETA

... 96

3.5.1 - Transformação do Termo de Domínio para o Contorno Forças de Inércia

3.5.2 - Discretizações

3.6 - ALGORITMOS PARA INTEGRAÇÃO NO TEMPO

3.6.1 - Algoritmo de Newmark

3.6.2 - Algoritmo de Houbolt

3.7 - APLICAÇÕES

... 105

3.7.1 - Aplicação 1: Sólido contido lateralmente

... 105

3.7.2 - Aplicação 2: Sólido à flexão

... 108

\section{CAPÍTULO 4}

MECÂNICA DO FRATURAMENTO - MODELO COESIVO

4.1 - INTRODUÇÃO

4.2 - MECÂNICA DA FRATURA ELÁSTICA E LINEAR

4.2.1 - Generalidades

4.2.2 - Fatores de Intensidade de Tensão

\section{CAPÍTULO 5}

FORMULAÇÃO DO MEC PARA PROBLEMAS DE FRATURAMENTO - TEORIA DE TENSÕES INICIAIS

5.1 - INTRODUÇÃO

5.2 - EQUAÇÕES GOVERNANTES 
5.4 - SOBRE O INTEGRANDO CORRESPONDENTE AOS DIPOLOS

5.5 - CONTRIBUIÇÃO DOS DIPOLOS PARA A ABERTURA DA TRINCA

5.6 - CONTRIBUIÇÃO DOS DIPOLOS NAS TENSÕES EM PONTOS INTERNOS

5.7 - DISCRETIZAÇÕES

5.8 - ASPECTOS COMPUTACIONAIS

5.9 - APLICAÇÕES

5.9.1 - Aplicação 1: Sólido paralelepipédico elástico solicitado axialmente por tensões uniformemente distribuídas

5.9.2 - Aplicação 2: Sólido paralelepipédico elástico solicitado axialmente por tensões triangularmente distribuídas

\section{CAPÍTULO 6}

FRATURAMENTO DINÂMICO - TEORIA DE TENSÕES INICIAIS E MATRIZ DE MASSA

6.1 - INTRODUÇÃO

6.2 - EQUAÇÕES GOVERNANTES

... 164

6.3 - EQUAÇÕES INTEGRAIS

... 165

6.4 - EMPREGO DO MÉTODO DA RECIPROCIDADE DUAL

6.5 - DISCRETIZAÇÕES

... 168

6.6 - SOLUÇÃO DAS EQUAÇÕES

... 170

6.6.1 - Algoritmo de Newmark

... 170

6.6.2 - Algoritmo de Houbolt

... 173

6.7 - APLICAÇÕES

... 176

6.7.1 - Aplicação 1: Problema de Chen

... 176

6.7.2 - Aplicação 2: Sólido paralelepipédico elástico solicitado axialmente por tensões dinâmicas 
CAPÍTULO 7

IMPLEMENTAÇÕES COMPUTACIONAIS

7.1 - INTRODUÇÃO

... 182

7.2 - ALGORITMO ELASTOSTÁTICO

... 182

7.2.1 - Para a Solução Fundamental de Kelvin

... 192

7.2.2 - Para a Solução Fundamental de Mindlin

... 194

7.3 - ALGORITMO ELASTODINÂMICO

... 196

7.3.1 - Método da Reciprocidade Dual

... 197

7.3.2 - Técnica da Integração Direta

... 199

7.4 - ALGORITMO PARA FRATURA ELÁSTICA

... 200

7.5 - ALGORITMO PARA FRATURA DINÂMICA

... 208

\section{CAPÍTULO 8}

CONCLUSÕES

$\underline{\text { ANEXOS }}$

ANEXO A: DELTA DE DIRAC

ANEXO B: DERIVADAS DO TENSOR DAS TENSÕES PARA A SOLUÇÃO FUNDAMENTAL DE MINDLIN

ANEXO C: DEDUÇÃO DO TENSOR D̂

ANEXO D: DETERMINAÇÃO DE UMA PRIMITIVA

... 222

ANEXO E: DETERMINAÇÃO DE TENSORES PARA M'

ANEXO F: DESENVOLVIMENTO DAS DERIVADAS PARA SE OBTER O TENSOR $\mathrm{G}_{\mathrm{ij}}^{\ell}$, SUAS COMPONENTES E A MATRIZ K

ANEXO G: COMPONENTES DA MATRIZ DE CONTRIBUIÇÃO DOS DIPOLOS ÀS TENSÕES EM PONTOS INTERNOS 


\section{LISTA DE FIGURAS}

Figura 2.3.1 - Definição do objeto de estudo: sólido tridimensional de domínio $\Omega$ e contorno $\Gamma$

Figura 2.3.2 - Tetraedro de Cauchy

Figura 2.3.3 - Definição das condições de contorno

Figura 2.4.1 - Definição do problema fundamental e a interdepen-dência do problema que se deseja analisar

Figura 2.4.2 - Efeitos das forças unitárias aplicadas em $\Omega^{*}$ : solução fundamental

Figura 2.4.3 - Definição do problema fundamental de Kelvin

Figura 2.4.4 - Definição do problema fundamental de Mindlin

.... 26

Figura 2.5.1 - Transformação de um ponto do contorno para o domínio

Figura 2.5.2 - Região infinita - espaço de Kelvin

Figura 2.5.3 - Região semi-infinita - espaço de Mindlin

Figura 2.6.1 - Sólidos discretizados por elementos de contorno: (a) constante; (b) linear; e (c) quadrático

Figura 2.6.2 - Geometria do elemento triangular plano a partir dos diversos sistemas de coordenadas

Figura 2.6.3 - Variação das coordenadas homogêneas .... 43

Figura 2.6.4 - Elemento triangular constante .... 44

Figura 2.6.5 - Variações do elemento linear .... 45

Figura 2.6.6 - Elemento triangular isoparamétrico linear .... 46

Figura 2.6.7 - Elemento triangular descontínuo .... 48

Figura 2.6.8 - Definição de parâmetros para a integração singular 
Figura 2.6.9 - (a) integração no contorno fictício do elemento triangular;

(b) elemento unidimensional utilizado .... 54

Figura 2.6.10 - Proximidade dos pontos fonte S e de campo Q .... 57

Figura 2.6.11 - Divisão do elemento triangular em 25 subelementos .... 57

Figura 2.6.12 - Ponto do contorno para análise de tensões

Figura 2.7.1 - Definição do sólido e suas condições de contorno: (a) contorno contido lateralmente e pontos para avaliação; e (b) sólido, carga e condições de contorno parciais

Figura 2.7.2 - Discretizações do contorno por elementos triangulares planos descontínuos: (a) 12 elementos e (b) 40 elementos

Figura 2.7.3 - Viga engastada com carregamento transversal na extremidade livre

Figura 2.7.4 - Discretizações utilizadas: (a) 40 elementos triangulares planos; e (b) 72 elementos

Figura 2.7.5 - Linha elástica da viga obtida das três formas ... 68

Figura 2.7.6 - Sólido elástico em forma de cruz

Figura 2.7.7 - Discretizações em 152 elementos triangulares planos com aproximação linear (456 pontos de colocação)

Figura 2.7.8 - Direções principais das tensões em pontos internos ... 70

Figura 2.7.9 - Direções principais das tensões em pontos internos

Figura 2.7.10 - Área retangular na superfície livre do semi-infinito, uniformemente carregada

Figura 2.7.11 - Discretizações utilizadas: (a) 16 elementos; e (b) 64 elementos

Figura 2.7.12 - Deslocamentos em $\mathrm{X}_{3}$ ao longo de $\mathrm{X}_{1}(\mathrm{em} \mathrm{cm})$ comparação entre as soluções fundamentais de Mindlin e Kelvin (discretização (a))

Figura 2.7.13 - Deslocamentos em $\mathrm{X}_{3}$ ao longo de $\mathrm{X}_{2}(\mathrm{em} \mathrm{cm})$ comparação entre as soluções fundamentais de Mindlin e Kelvin (discretização (a)) 
Figura 2.7.14 - Deslocamentos em $X_{3}$ ao longo de $X_{1}($ em cm) comparação entre as discretizações (a) e (b) para a solução $\ldots 75$ fundamental de Mindlin

Figura 2.7.15 - Deslocamentos em $X_{3}$ ao longo de $X_{2}(e m c m)-$ comparação entre as discretizações (a) e (b) para a solução fundamental de Mindlin

Figura 2.7.16 - Tensões $\sigma_{33}$ ao longo do eixo $\mathrm{X}_{3}$ (em Pa) - comparação entre as discretizações (a) e (b) para a solução fundamental de Mindlin

Figura 2.7.17 - Definição do problema: cavidade no meio semi-infinito, próxima da superfície, com carga distribuída agindo na superfície

Figura 2.7.18 - Discretização utilizada: 152 elementos triangulares planos

Figura 2.7.19 - Deslocamentos horizontais na face mais próxima da carga, em $X_{3}=2 \mathrm{~m}$

Figura 2.7.20 - Deslocamentos horizontais na face mais próxima da carga, no eixo $\mathrm{X}_{3}$

Figura 2.7.21 - Superfície de deslocamentos da face vertical da escavação mais próxima da carga: curvas de nível (valores em $\mathrm{cm}$ )

Figura 3.2.1 - Definição do objeto de estudo: sólido tridimensional de domínio $\Omega$ e contorno $\Gamma$ (nos instantes 0 e t)

Figura 3.7.1 - Características geométricas do sólido, condições de contorno e comportamento temporal da força $f(t)$

Figura 3.7.2 - Discretizações do contorno por elementos triangulares planos: (a) 40 elementos e (b) 80 elementos

Figura 3.7.3 - Resultados para a discretização (a) ... 106

Figura 3.7.4 - Resultados para a discretização (b) 
Figura 3.7.5 - Resultados de comparação entre os algoritmos de Newmark e Houbolt

Figura 3.7.6 - Resultados do processamento utilizando-se Newmark para İ $=0,0$

Figura 3.7.7 - Características geométricas do sólido, condições de contorno e comportamento temporal da força $f(t)$

Figura 3.7.8 - Discretização do contorno do sólido - 80 elementos triangulares planos

Figura 3.7.9 - Deslocamentos verticais no ponto A ao longo do tempo

Figura 3.7.10 - Deslocamentos horizontais no ponto A ao longo do tempo

Figura 3.7.11 - Suavização da resposta à medida que se aumenta o valor de $\Delta \mathrm{t}$

Figura 3.7.12 - Oscilação da resposta à medida que se aumenta o valor de $\Delta \mathrm{t}$

Figura 4.2.1 - Modelo experimentado por Inglis - furo elíptico numa placa solicitada por tensões constantes

Figura 4.2.2 - Modos de fraturamento: (a) modo I - abertura; (b) modo II, de deslizamento; (c) modo III, de rasgamento

Figura 4.2.3 - Frente da fratura - sistemas de coordenadas

Figura 4.3.1 - Definição do modelo idealizado - distribuição de tensões na zona coesiva

Figura 4.3.2 - Leis constitutivas do material: (a) tensão x deformação; (b) tensão x abertura da trinca - modelo ideal; (c) tensão x abertura da trinca - modelo simplificado

Figura 5.2.1 - Modelo Elastoplástico - parcelas de deformação ... 124

Figura 5.3.1 - Definição da descontinuidade no meio (fratura)

Figura 5.3.2 - Coordenadas globais e locais para a superfície de fraturamento

Figura 5.4.1 - Elemento do contorno da fratura com o seu sistema local de coordenadas e vetor normal 
Figura 5.4.2 - Elemento infinitesimal no contorno da fratura com as componentes dos dipolos

Figura 5.5.1 - Esquema de dois pontos próximos da fratura - na esquerda e na direita

Figura 5.9.1 - Sólido para análise: (a) geometria e deslocamentos prescritos; (b) discretização do contorno; (c) discretização da superfície da trinca

Figura 5.9.2 - Resultados: (a) tensão em um nó da fratura $\mathrm{x}$ abertura da fratura; (b) força de superfície na extremidade do sólido $\mathrm{x}$ deslocamento no nó 2'

Figura 5.9.3 - Configuração final do corpo fraturado

Figura 5.9.4 - Geometria e deslocamentos prescritos

Figura 5.9.5 - Discretização do contorno do sólido: 40 elementos triangulares planos

Figura 5.9.6 - Discretizações da superfície da fratura: (a) 32 elementos constantes, (b) 64 elementos e (c) 64 elementos

Figura 5.9.7 - Abertura da fratura para as discretizações adotadas

Figura 5.9.8 - Deslocamentos na direção do eixo $\mathrm{X}_{3}$ para os três pontos previamente definidos

Figura 5.9.9 - Redução gradativa da capacidade de resistência da zona de fraturamento - modelo coesivo

Figura 6.2.1 - Sólido em movimento com uma região de tensões iniciais $\Omega_{\mathrm{c}}$

Figura 6.7.1 - Definição do objeto de estudo: geometria do sólido, condições de contorno e comportamento temporal da força

Figura 6.7.2 - Discretização do contorno do sólido por elementos lineares

Figura 6.7.3 - Discretização da superfície da trinca na seção central do sólido 
Figura 6.7.4 - Valores de $K_{I}$ (normalizado por $\sigma \sqrt{\pi a}$ ) ao longo do ... 178 tempo

Figura 6.7.5 - Definição do objeto de estudo: geometria do sólido, condições de contorno e comportamento temporal da força

Figura 6.7.6 - Discretizações: (a) contorno do sólido e (b) superfície da fratura

Figura 6.7.7 - Deslocamento no ponto 1 na direção $X_{3}$, ao longo do tempo

Figura 6.7.8 - Abertura da fratura $\left(\mathrm{w}_{\mathrm{c}} / \mathrm{w}_{\mathrm{c}_{-} \text {ad }}\right)$ ao longo do tempo

Figura 6.7.9 - Deslocamentos (corrigidos) dos pontos 2' e 2',

... 181

Figura 7.2.1 - Desenvolvimento em módulos do algoritmo elastostático

Figura 7.2.2 - Rotina principal do módulo I do programa elastostático

... 184

Figura 7.2.3 - Fluxograma da sub-rotina "montagem de H e G"

... 182

Figura 7.3.1 - Rotina principal do algoritmo elastodinâmico

Figura 7.3.2 - Fluxograma da sub-rotina de montagem da matriz de massa

Figura 7.4.1 - Desenvolvimento em módulos do algoritmo para análise de fratura mecânica

Figura 7.4.2 - Fluxograma da sub-rotina que calcula a matriz K dos dipolos

Figura 7.4.3 - Fluxograma da sub-rotina que calcula a matriz KS dos dipolos

Figura 7.4.4 - Rotina principal do Módulo III do programa de fratura mecânica

Figura 7.4.5 - Fluxograma do procedimento incremental

Figura 7.4.6 - Fluxograma do procedimento iterativo de fratura mecânica

Figura 7.5.1 - Desenvolvimento em módulos do algoritmo para análise de fratura dinâmica 
Tabela 2.7.1 - Deslocamentos na direção axial, $X_{3},\left(\times 10^{-5} \mathrm{~m}\right)$ .... 65

Tabela 2.7.2 - Tensões na direção axial, $\mathrm{X}_{3},(\mathrm{~Pa})$ ... 65

Tabela 2.7.3 - Linha elástica da viga analisada (valores em $\mathrm{cm}$ )

Tabela 2.7.4 - Deslocamentos axiais máximos nas direções $\mathrm{X}_{2}$ e $\mathrm{X}_{3}$ ... 70

Tabela 2.7.5 - Deslocamentos axiais máximos nas direções $\mathrm{X}_{2}$ e $\mathrm{X}_{3}$ $\ldots 71$

Tabela 2.7.6 - Deslocamentos em $\mathrm{X}_{3}$ ao longo de $\mathrm{X}_{1}(\mathrm{em} \mathrm{cm})$ $\ldots 73$

Tabela 2.7.7 - Deslocamentos em $\mathrm{X}_{3}$ ao longo de $\mathrm{X}_{2}(\mathrm{em} \mathrm{cm})$ $\ldots 74$

Tabela 2.7.8 - Deslocamentos em $\mathrm{X}_{3}$ ao longo de $\mathrm{X}_{1}(\mathrm{em} \mathrm{cm})$ ... 75

Tabela 2.7.9 - Deslocamentos em $\mathrm{X}_{3}$ ao longo de $\mathrm{X}_{2}(\mathrm{em} \mathrm{cm})$ ... 75

Tabela 2.7.10 - Tensões $\sigma_{33}$ ao longo do eixo $\mathrm{X}_{3}(\mathrm{~Pa})$ ... 76

Tabela 3.7.1 - Casos processados .... 106

Tabela 5.9.1 - Deslocamento durante o processo de carregamento

Tabela 5.9.2 - Valores de $\mathrm{w}_{\mathrm{c}}$ (abertura da fratura) ao longo de $\mathrm{X}_{1}$, para $\Delta \mathrm{u}_{3}=0,00008$ 


\section{LISTA DE ABREVIATURAS E SIGLAS}

$\begin{array}{lll}\text { MEC } & - & \text { Método dos Elementos de Contorno } \\ \text { MEF } & - & \text { Método dos Elementos Finitos } \\ \text { MFLE } & \text { - } & \text { Mecânica da Fratura Linear Elástica } \\ \text { MRD } & - & \text { Método da Reciprocidade Dual } \\ \text { FIT } & \text { - } & \text { Fatores de Intensidade de Tensão }\end{array}$




\section{LISTA DE SÍMBOLOS}

$\begin{array}{ll}\delta_{\mathrm{ij}} & \text { delta de Kronecker } \\ \delta(\mathrm{s}, \mathrm{q}) & \text { delta de Dirac } \\ \Gamma & \text { contorno, } \\ & \text { trabalho necessário para a formação de uma nova trinca } \\ \beta & \text { parâmetro do algoritmo de Newmark } \\ \Omega & \text { domínio } \\ \sigma_{\mathrm{ij}} & \text { componentes do tensor das tensões } \\ \sigma_{\mathrm{ij}}^{\mathrm{o}} & \text { tensor das tensões iniciais } \\ \sigma^{*} & \text { tensor das tensões do problema fundamental } \\ \chi & \text { ponto material de referência } \\ \varepsilon_{\mathrm{ij}} & \text { componentes do tensor das deformações } \\ \varepsilon_{\mathrm{ij}}^{\mathrm{e}} & \text { tensor das deformações elásticas } \\ \varepsilon_{\mathrm{ij}}^{\mathrm{o}} & \text { tensor das deformações iniciais } \\ \nu & \text { coeficiente de Poisson } \\ \lambda & \text { constante de Lamé } \\ \alpha_{\ell}, \beta_{\ell}, \gamma_{\ell} & \text { aunções de interpolação } \\ \xi_{\mathrm{i}} & \begin{array}{l}\text { coeficientes aproximadoras } \\ \varphi\end{array}\end{array}$




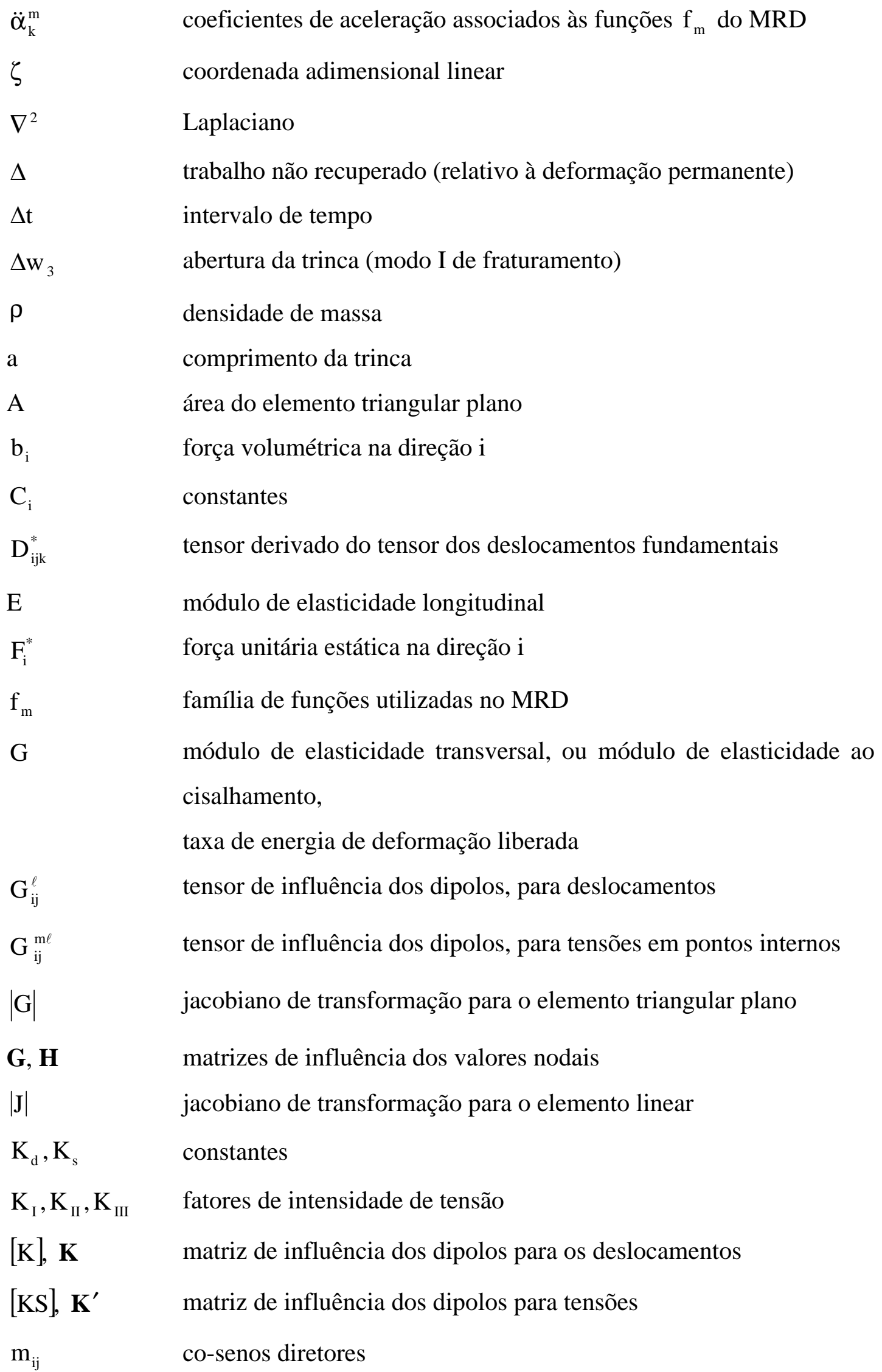




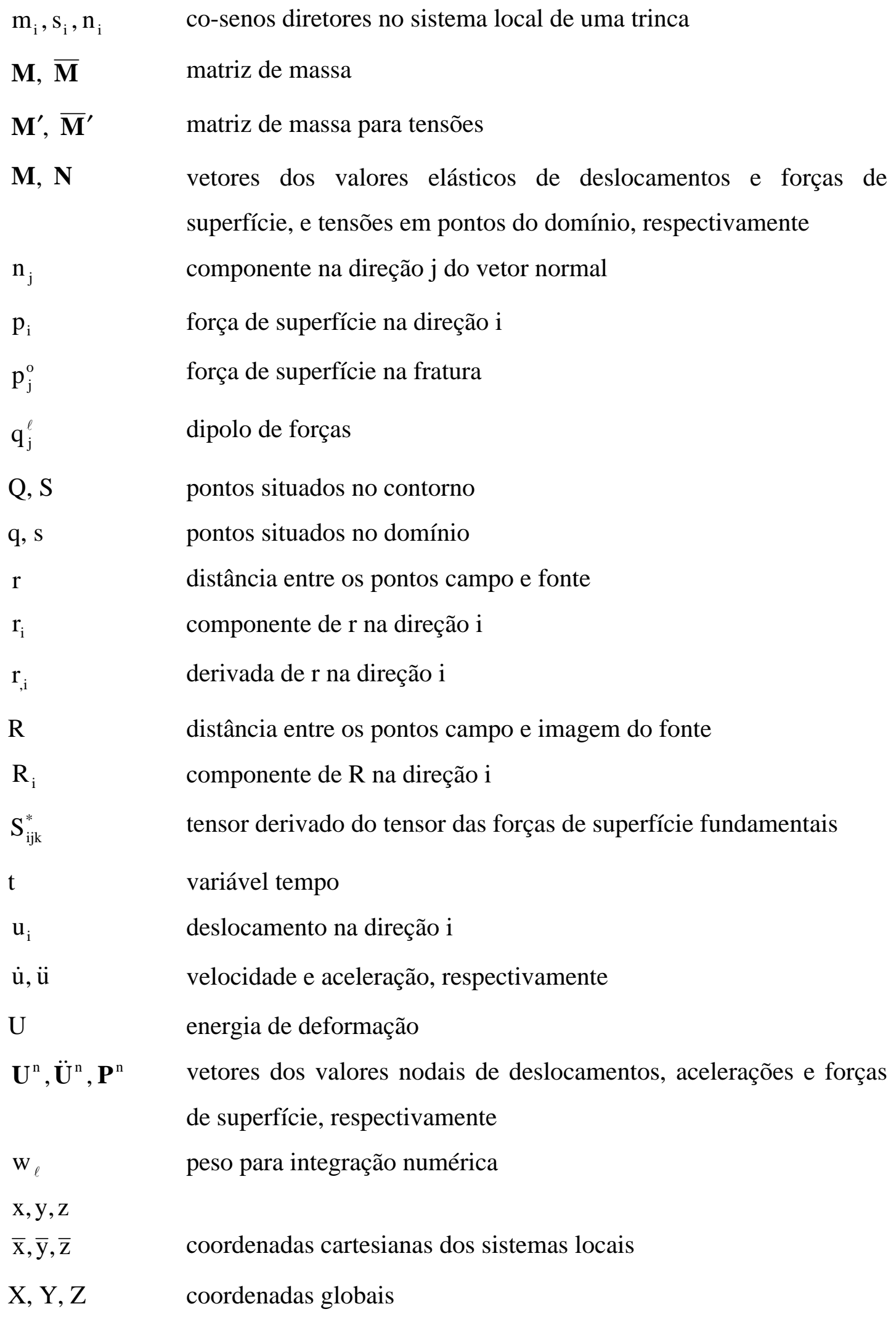




\section{RESUMO}

BARBIRATO, J.C.C. Método dos elementos de contorno com a reciprocidade dual para a análise transiente tridimensional da mecânica do fraturamento. São Carlos, 1999. 246p. Tese (Doutorado) - Escola de Engenharia de São Carlos, Universidade de São Paulo.

O presente trabalho desenvolve uma formulação do Método dos Elementos de Contorno para análise de problemas tridimensionais de fraturamento no regime transiente. Utilizam-se as soluções fundamentais da elastostática para obter a matriz de massa, empregando-se o Método da Reciprocidade Dual e a discretização do domínio por células tridimensionais. Para a integração no tempo são utilizados os algoritmos de Newmark e Houbolt. O fenômeno do fraturamento é abordado através da consideração de um campo de tensões iniciais, introduzindo-se o conceito de dipolos de tensão. Os tensores desenvolvidos que se relacionam aos dipolos, derivados das soluções fundamentais, são também apresentados. É utilizado o modelo de fratura coesiva. O contorno é discretizado utilizando-se elementos triangulares planos com aproximação linear, e elementos constantes para a superfície fictícia de fraturamento. São feitas várias aplicações cujos resultados obtidos confirmam a importância e a adequação da formulação apresentada para os problemas propostos.

Palavras-chave: Método dos Elementos de Contorno; Método da Reciprocidade Dual; Fratura Dinâmica. 


\section{ABSTRACT}

BARBIRATO, J.C.C. Boundary Element Method for Three-dimensional Transient Analysis of Fracture Mechanics using Dual Reciprocity Method. São Carlos, 1999. 246p. Tese (Doutorado) - Escola de Engenharia de São Carlos, Universidade de São Paulo.

This work presents a Boundary Element Method (BEM) formulation for analysis of three-dimensional fracture mechanics transient problems. Elastostatics fundamental solutions are considered in order to obtain the mass matrix, using both Dual Reciprocity Method and three-dimensional cell discretization. Newmark and Houbolt algorithms are employed to evaluate the time integrals. The fracture effects are captured by using dipoles of stresses, derived from an initial stress field. The tensors related to those dipoles, developed in the present work, are presented. The cohesive crack is the adopted model. Body boundary is discretized though linear flat triangular elements and the fracture surfaces are approximated by constant flat triangular elements. Some applications are processed to show the efficiency of presented BEM formulations.

Key words: Boundary Element Method, Dual Reciprocity Method, Dynamic Fracture. 


\section{CAPÍTULO 1}

\section{CONSIDERAÇÕES INICIAIS}

\section{1 - INTRODUÇÃO}

O Método dos Elementos de Contorno (MEC) tem se destacado no campo da engenharia como um importante método de simulação numérica, com boa precisão em seus resultados para vários problemas da mecânica do contínuo. Sobretudo nas últimas duas décadas, o MEC teve grande desenvolvimento. Para problemas de elastodinâmica e fratura mecânica em particular, o método tem-se mostrado eficiente e confiável. Em casos de fratura mecânica, o acompanhamento da propagação desta requer pouco esforço computacional, evitando-se refazer a rede de elementos (VENTURINI, 1994b; 1995).

O estudo com problemas da elastodinâmica justifica-se por esta ser a teoria mais genérica e próxima das leis da natureza, quando comparada à elastostática. Cada vez mais os efeitos dinâmicos fazem parte da análise estrutural, determinandose seu comportamento frente às condições de serviço e segurança (em prédios, pontes, plataformas "offshore", veículos, etc.) sob a ação de solicitações dinâmicas (ações sísmicas, vento, ondas, tráfego, impacto mecânico, dentre outras).

Também é preocupação da engenharia avaliar o comportamento estrutural na presença de trincas, inerentes ao material empregado ou provocadas por carregamentos. Observando-se as publicações sobre o assunto (BROEK, 1989; ALIABADI et al., 1994, entre outras), nota-se a tendência cada vez maior do estudo 
da Mecânica da Fratura. Vê-se, por exemplo, que algumas lacunas ainda existem na análise transiente tridimensional da mecânica da fratura, conforme constata DOMINGUEZ (1993).

Portanto, a abordagem transiente tridimensional da mecânica da fratura, objeto deste trabalho, está presente nas discussões sobre o desenvolvimento tecnológico atual.

O presente trabalho apresenta-se, portanto, no contexto da Mecânica da Fratura para problemas elastodinâmicos. Seu objetivo principal é desenvolver uma formulação do Método dos Elementos de Contorno capaz de analisar problemas transientes tridimensionais da Mecânica da Fratura, empregando, para tanto, o Método da Reciprocidade Dual, para a obtenção da "matriz de massa", juntamente com a inclusão de um campo de tensões iniciais.

A partir do objetivo principal surgem os objetivos específicos, que subsidiam o primeiro com suas formulações. São eles: o aprofundamento nos conhecimentos sobre a formulação do método dos elementos de contorno para problemas elastostáticos tridimensionais, utilizando as soluções fundamentais de Kelvin e Mindlin; as formulações do MEC para problemas elastodinâmicos, sobretudo as relacionadas à matriz de massa, obtidas através da discretização do domínio em células e pelo Método da Reciprocidade Dual; estudo da Mecânica da Fratura, inclinando-se para o modelo de fratura coesiva; formulações do MEC para análise de fratura mecânica, escolhendo aquela onde uma superfície fictícia carregada por tensões particulares, dipolos, simula a presença da trinca.

\section{2 - ESTRUTURAÇÃO DO TRABALHO}

O trabalho está estruturado de forma a permitir a abordagem isolada de cada assunto, com suas características particulares, formando uma seqüência de formulações que vão se interligando para compor a formulação do MEC para problemas tridimensionais de fratura dinâmica. Assim, após as considerações iniciais do presente capítulo, é apresentada no capítulo 2 a formulação tridimensional elástica do Método dos Elementos de Contorno, bem como as equações básicas da 
elasticidade linear, representações integrais e as correspondentes equações algébricas, utilizando-se elementos triangulares planos com aproximação linear nas discretizações. São abordados, ainda, alguns procedimentos de integração (semianalítico e numérico), encerrando-se o capítulo com algumas aplicações.

Em seguida, no capítulo 3, é apresentada uma formulação do MEC para a elastodinâmica, utilizando-se o Método da Reciprocidade Dual e a Técnica da Integração Direta para a definição da "matriz de massa". Nesta formulação, as soluções fundamentais requeridas são as mesmas da análise estática, o que facilita o seu desenvolvimento teórico. São apresentados exemplos processados para testar a formulação apresentada.

O capítulo 4 é dedicado à teoria da mecânica da fratura. Em uma abordagem sucinta, definem-se as bases sobre o assunto e introduz-se o modelo de fratura coesiva utilizado.

A mecânica da fratura é objeto de estudo no capítulo 5. Tomam-se as equações da elastostática apresentadas no capítulo 2 e introduz-se um campo de tensões iniciais a fim de caracterizar uma zona de descontinuidade (a fratura). Define-se o ente "dipolo" capaz de modelar a presença da fratura, que vai se formando obedecendo ao modelo coesivo apresentado no capítulo 4. É apresentada a formulação do MEC para analisar este problema, finalizando-se com aplicações que evidenciam a importância do método.

No capítulo 6 é apresentada uma formulação do Método dos Elementos de Contorno para análise da mecânica da fratura, com os procedimentos vistos no capítulo 5, porém no regime elastodinâmico, baseando-se na formulação apresentada no capítulo 3. Também aí estão incluídas aplicações para avaliar a formulação apresentada.

O capítulo 7 trata dos algoritmos computacionais utilizados, apresentando os fluxogramas das rotinas mais importantes.

Por fim, são apresentadas algumas considerações finais sobre os assuntos abordados, as conclusões gerais e as possibilidades de extensão deste trabalho.

Seguem-se os anexos, com o desenvolvimento e apresentação de equações importantes para o completo entendimento dos diversos assuntos abordados. Em seguida, é apresentada a bibliografia utilizada. 


\section{3 - ÁREAS DE ABORDAGEM}

A seguir são apresentadas algumas características e justificativas a respeito das áreas de abordagem escolhidas para o presente trabalho.

\subsection{1 - O Método dos Elementos de Contorno}

O Método dos Elementos de Contorno (MEC) é o método numérico mais recente do ponto de vista de aplicações computacionais, dentre os mais utilizados. Ganhou esta denominação a partir do trabalho de BREBBIA (1978). Resumidamente, o MEC consiste em obter a solução das equações diferenciais que descrevem o comportamento de um corpo no seu domínio, através da solução de equações integrais sobre o contorno. Isso reduz de uma unidade as dimensões de problemas lineares analisados, o que leva a menores quantidades de dados de entrada e, conseqüentemente, menor sistema de equações algébricas. Por outro lado, a matriz do sistema é geralmente cheia e não simétrica.

Para obter-se a equação integral de contorno que possibilite a análise do problema, o MEC necessita de uma solução fundamental. Esta representa a resposta em um ponto do domínio infinito devido à aplicação de força unitária em outro ponto do mesmo domínio. A utilização de uma solução fundamental, que genericamente pode ser classificada como uma desvantagem, na verdade proporciona versatilidade e precisão ao método (segundo BECKER, 1992).

O MEC tem emergido como uma força alternativa, principalmente nos problemas cujos domínios são estendidos ao espaço infinito (ou semi-infinito). Nestes casos, a rede de elementos utilizada pelo MEC na discretização do contorno necessita modelar apenas parte deste, uma vez que a solução fundamental utilizada no método já contempla a influência do infinito (ou semi-infinito).

Outros métodos, como por exemplo o Método dos Elementos Finitos (MEF) COOK et al. (1989), utilizam contornos fictícios para representar o infinito. Isto pode causar sérios erros nos resultados numéricos, sobretudo na elastodinâmica, onde ondas podem ser refletidas por tais contornos. 


\subsection{2 - Elastodinâmica}

Vários problemas governados pela teoria da Elastodinâmica têm sido estudados nos diversos centros de pesquisa do mundo (ações dinâmicas em edifícios, em pontes, em torres, análise dinâmica da mecânica da fratura, análise dinâmica da interação solo - estrutura, dentre outras), conforme BESKOS (1987) e MOAN et al. (1993).

A formulação elastodinâmica no domínio do tempo permite melhor aproximação para resolver alguns problemas da engenharia e, em alguns destes, trata-se da única formulação capaz de fornecer resultados aproximados, segundo DOMÍNGUEZ (1993).

A formulação no domínio do tempo é adequada para problemas transientes, já que uma solução, a mais precisa quanto possível, é necessária para o início da análise. Outra aplicação para esta formulação, com dependência geométrica no tempo, refere-se a problemas de propagação dinâmica de fraturas.

O Método dos Elementos de Contorno tem aplicação destacada na análise de problemas da elastodinâmica. Suas características permitem analisar problemas da elastodinâmica no espaço tridimensional (infinito ou semi-infinito) com consideráveis avanços em comparação a outros métodos numéricos, utilizando para tanto o conceito de "matriz de massa".

$\mathrm{Na}$ análise elastodinâmica com o MEC, surge um termo de domínio em sua equação integral. Dentre as várias técnicas para tratamento desta parcela está a da Reciprocidade Dual, cuja utilização tem trazido ótimos resultados (PARTRIDGE et al., 1992). Pode-se obter melhores resultados com esta técnica utilizando-se uma função alternativa para a obtenção da "matriz de massa", como utilizado em CODA (1990) e CALDERÓN \& VENTURINI (1991).

\subsection{3 - Mecânica da Fratura}

Os sólidos sempre contêm alguma fissura em seu interior, resultado do processo de fabricação ou simplesmente devido às cargas atuantes. Essas fissuras 
agem como pontos de elevação de tensões e a sua propagação pode levar à degeneração da resistência da estrutura.

É interessante para a engenharia poder analisar estruturas onde existem trincas, conhecendo sua intensidade e seu modo de propagação. Neste contexto se insere a Ciência da Mecânica da Fratura, cujo significado prático na engenharia é importante, pois define a base racional para o cálculo da resistência de estruturas com trincas e permite a determinação das taxas de propagação das mesmas na fadiga.

Soluções analíticas para problemas da mecânica da fratura são limitadas a um pequeno número de situações idealizadas, nas quais o domínio é bem definido e o carregamento relativamente simples. Na prática, os problemas de mecânica da fratura têm características geométricas e carregamentos complexos.

As trincas relacionadas a problemas elásticos lineares são analisadas pela formulação da Mecânica da Fratura Elástica Linear (MFEL). A MFEL é aplicável a uma série de casos práticos e por isso o fator de intensidade de tensão (parâmetro que quantifica a magnitude do campo de tensão na ponta da trinca) é tabelado para uma quantidade expressiva de configurações de carregamento e geometria. Entretanto, a engenharia se depara freqüentemente com casos cujas soluções não são conhecidas, surgindo, assim, a necessidade da investigação própria utilizando métodos numéricos.

O MEC é um dos métodos numéricos utilizados na determinação do fator de intensidade de tensão em uma trinca e a propagação da mesma (como em INGRAFFEA \& MANU, 1980; CRUSE, 1988; LUCHI \& RIZZUTI, 1987; GUIMARÃES, 1992; NISITANI \& CHEN, 1993; PORTELA et al., 1993; entre outros).

Para o problema de trincas no espaço tridimensional elástico, o MEC está sendo utilizado e, segundo BAINS et al. (1993) e DOMINGUEZ \& SÁEZ (1998), com ganhos computacionais relevantes frente a outros métodos numéricos. 


\section{4 - REVISÃO BIBLIOGRÁFICA}

A técnica de transformação da equação diferencial de um determinado problema em uma equação integral constitui a base matemática do Método dos Elementos de Contorno. A literatura especializada remonta a 1823 o registro da utilização por Abel de uma equação integral para resolver o problema chamado “pêndulo isócrono", segundo Elliot ${ }^{1}$ apud SILVA (1996).

A representação integral para a elastostática é estabelecida por SOMIGLIANA $^{2}$ (1886) apud DOMINGUEZ (1993), conhecida como Identidade Somigliana.

Desde esta época até os anos cinqüenta, alguns trabalhos utilizando equações integrais são desenvolvidos, principalmente no campo da mecânica dos fluidos e potencial, tornando-as mais conhecidas. Nesse contexto, pode-se citar os trabalhos de FREDHOLM (1903), MUSKHELISHVILI (1953), VOLTERRA (1956), MIKHLIN (1957).

As equações integrais são até então conhecidas como um método indireto de análise, isto é, a solução de um problema é obtida por fontes fictícias aplicadas ao contorno em que, após a determinação de seus valores, calculam-se as variáveis físicas do problema, como em KUPRADZE (1965).

Outra formulação, denominada direta, utilizando as próprias variáveis físicas do problema, é apresentada em RIZZO (1967). Com aplicações na elasticidade bidimensional, este trabalho apresenta a discretização do contorno feita por segmentos de reta, sendo os deslocamentos e forças de superfície constantes em cada um deles com a integração realizada analiticamente. Constitui-se em um dos primeiros registros onde o Método das Equações Integrais toma forma de técnica numérica, similar a de outros métodos - Método das Diferenças Finitas e Método dos Elementos Finitos.

Vários trabalhos seguiram-se ao de RIZZO (1967), divulgando o que se denomina Método das Equações Integrais de Contorno. Dentre estes, podem ser

\footnotetext{
${ }^{1}$ ANDERSEN,R.S. et al. (1980). The application and numerical solution of integral equations. Alphen aan den Rijn, The Netherlands, Sijthoff \& Noordhoff.

${ }^{2}$ SOMIGLIANA, C. (1886). Sopra l'equilibrio di un corpo elastico isotropo.Il Nuovo cimento. Ser. 3, v. 17-20.
} 
citados CRUSE \& RIZZO (1968), CRUSE (1968) e RIZZO \& SHIPPY (1968). Os trabalhos de CRUSE (1969), CRUSE \& VAN BUREN (1971) e CRUSE (1973) utilizam o método para a análise de problemas tridimensionais.

Mas foi a partir dos trabalhos realizados por LACHAT (1975), DOMINGUEZ ${ }^{3}$ (1977) apud DOMÍNGUEZ (1993), BANERJEE \& BUTTERFIELD (1977), BREBBIA \& DOMINGUEZ (1977) e BREBBIA (1978) que o Método das Equações Integrais de Contorno ganha forma consistente de método numérico, utilizando-se a técnica dos resíduos ponderados e funções de forma tão utilizadas no Método dos Elementos Finitos. Passa, então, a ser denominado de Método dos Elementos de Contorno (MEC).

A aplicação do MEC para o estudo de problemas tridimensionais tem como precursores os trabalhos CRUSE (1968) e LACHAT (1975), já citados. Este tema também é abordado em NAKAGUMA (1979), CUROTTO (1981), SÁ \& TELLES (1986), SILVA (1989), BARBIRATO (1991), CODA (1993), entre outros. Em NAKAGUMA (1979) e BARBIRATO (1991) são utilizadas formulações do MEC para análise tridimensional com as soluções fundamentais de Kelvin e Mindlin.

A utilização na elastodinâmica do MEC tem início nos trabalhos de FRIEDMAN \& SHAW $^{4}$ (1962) e BANAUGH \& GOLDSMITH ${ }^{5}$ (1963), apud DOMINGUEZ (1993). Mas é nos trabalhos de CRUSE \& RIZZO (1968) e CRUSE (1968) que a formulação direta do MEC para a resolução de problemas da elastodinâmica transiente apresenta-se pela primeira vez. Seguem-se os trabalhos de MANOLIS \& BESKOS (1981), NARDINI \& BREBBIA (1983; 1985), MANSUR \& BREBBIA (1982; 1985), CODA (1990), CODA \& VENTURINI (1990a; b), DOMINGUEZ \& GALLEGO (1991), ARAÚJO (1994), CHIRINO et. al. (1994), entre outros.

A análise tridimensional transiente através do MEC é também abordada nos trabalhos de CODA (1993), onde foi apresentada uma formulação mista entre o MEC e o Método dos Elementos Finitos (MEF), e CODA \& VENTURINI (1995a; b).

\footnotetext{
3 DOMÍNGUEZ, J. (1977) Computation of stresses near anchor plates: an application of the boundary element method. Ph.D. Thesis, Univ. of Seville, Spanish

${ }^{4}$ FRIEDMAN, M.B. and SHAW, R.P. (1962). Diffraction of pulses by cylindrical obstacles of arbitrary cross section. J. Appl. Mech., Vol. 29, pp. 40-46.

${ }^{5}$ BANAUGH, R.P. and GOLDSMITH, W. (1962). Diffraction of steady elastic waves by surfaces of arbitrary shape. J. Appl. Mech., Vol. 30, pp. 589-597.
} 
Outro trabalho relevante neste contexto é o de DOMINGUEZ (1993), que discorre sobre vários campos dentro da elastodinâmica.

CODA \& VENTURINI (1996) estudam o MEC para análise elastodinâmica transiente tridimensional para solução de problemas práticos, utilizando aspectos importantes das soluções fundamentais no domínio do tempo - clássica e alternativa (CODA; 1993). Constatam que, apesar de similares, as duas formulações mostram resultados completamente diferentes quando aplicadas a alguns problemas particulares. Para problemas com razões grandes entre contorno e volume, a formulação clássica produz resultados instáveis. Para razões pequenas, como no caso do domínio finito (e com adoção de colocações singulares), essa formulação mostra bons resultados. Por outro lado, a solução fundamental mais recente, proporciona representação integral muito mais conveniente, e, consequentemente, relações algébricas mais apropriadas e resultados mais precisos e estáveis.

Em LEITÃO (1998), tem-se a descrição da aplicação do método de Trefftz com multi-regiões, na análise de problemas de fratura mecânica elástica linear bidimensional.

Diferentes técnicas têm sido desenvolvidas para tratar o termo de domínio, segundo PARTRIDGE et al. (1992). Dentre elas, pode-se destacar: integração analítica das integrais de domínio; expansões em série de Fourier; vetor de Galerkin; Reciprocidade Múltipla e Reciprocidade Dual.

A técnica da Reciprocidade Dual, propósito deste trabalho, é apresentada primeiramente por NARDINI \& BREBBIA ${ }^{6}$ (1982) apud PARTRIDGE et al. (1992), que procuram tratar o problema dinâmico como sendo estático, considerando as forças de inércia como forças de domínio. Baseada nesta idéia, a Reciprocidade Dual obtém um conjunto de funções que transformam a integral de domínio em uma série de integrais de contorno.

BREBBIA \& NARDINI (1983) propõem um procedimento alternativo para análise dinâmica transiente na mecânica dos sólidos utilizando a abordagem integral de contorno. Nesse trabalho obtém-se a "matriz de massa" usando valores de contorno com a aplicação de uma classe especial de funções coordenadas. Essa

6 NARDINI, D. and BREBBIA, C.A. (1982). A new approach to free vibration analysis using boudary elements. in: Computational Mechanics Publications, Southampton. 
técnica permite a aplicação do MEC em elastodinâmica, sem necessitar da implementação computacional de integrais de domínio. Entretanto, são necessários alguns pontos base localizados no domínio, denominados pólos, para garantir respostas mais precisas.

CODA (1990) e CODA \& VENTURINI (1990a,b) apresentam uma formulação alternativa que transforma a integral de domínio em uma soma de integrais de contorno. Em VENTURINI (1994a) é apresentada uma análise crítica das várias formas de o tratamento das integrais de domínio. Propõe uma família de funções para uso da técnica da Reciprocidade Dual, mostrando sua utilidade para análise de problemas não-lineares. Em PARTRIDGE (1995), tem-se, dentro da Reciprocidade Dual no MEC, a comparação de funções locais e globais de aproximação em problemas de convecção, de difusão e similares, sendo que tais soluções dependem, entre outros fatores, da função escolhida.

FEDELINSKI et al. (1996) utilizam o DBEM (Método dos Elementos de Contorno Dual) em três abordagens (domínio tempo, transformada de Laplace e Método da Reciprocidade Dual) no comportamento dinâmico de fraturas estacionárias no domínio finito e em duas dimensões, e aplicam essas mesmas abordagens em problemas de fratura de modo misto. Concluem, entre outros aspectos, que a implementação computacional realizada pelo domínio do tempo e pela transformada de Laplace são mais rápidas, embora a cada intervalo de tempo, duas novas matrizes grandes são calculadas. Por outro lado, o Método da Reciprocidade Dual consome mais tempo de processamento, mas a solução em cada passo de tempo é mais rápida (já que não são criadas novas matrizes). Constatam, ainda, que o domínio do tempo e a transformada de Laplace são particularmente eficientes para análise de domínios infinitos, e que o Método da Reciprocidade Dual pode ser facilmente aplicado para condições iniciais não homogêneas e forças de volume.

BARBIRATO \& VENTURINI (1997) apresentam uma formulação do Método dos Elementos de Contorno para a análise de problemas transientes de sólidos tridimensionais, utilizando o conceito de matriz de massa obtida a partir do emprego do Método da Reciprocidade Dual. Concluem que a formulação é adequada ao problema, tendo em vista o exemplo numérico mostrado, comparando os 
resultados com os obtidos através do procedimento que discretiza o domínio em células.

ALIABADI \& ROOKE (1991) mostram uma formulação utilizando o Método dos Elementos de Contorno Dual na resolução de problemas de fratura dinâmica em material elástico-linear. Mostram exemplos de implementações computacionais dos fatores de intensidade de tensão dinâmicos usando as aberturas da trinca que demostram a eficiência da estratégia apresentada. Da mesma forma, CISILINO \& ALIABADI (1998) utilizam o mesmo método na análise elastoplástica tridimensional de corpos fraturados, encontrando resultados precisos para problemas onde o fronte da trinca é curvado.

CHEN \& CHEN (1998) mostram a extensão da técnica dos elementos de contorno dual para análise de contato friccional em superfícies de fraturas para formas e distribuições arbitrárias.

As aplicações do Método dos Elementos de Contorno na Mecânica da Fratura em três dimensões são introduzidas por CRUSE \& Van BUREN (1971). Seguem-se os trabalhos de CRUSE (1974) e o de SNYDER \& CRUSE (1975), onde é apresentada uma solução fundamental - uma função de Green - que embute uma trinca linear sem cargas no meio infinito. Em CRUSE \& MEYERS (1977) são calculadas distribuições do fator de intensidade de tensão através do MEC, utilizando-se uma variação da taxa de energia de deformação, devido Griffith.

ROCHA \& VENTURINI (1998) formulam, no contexto da mecânica do contínuo aplicada à problemas de fratura, um novo critério para iniciação de trincas em sólidos deformáveis, baseado no critério de Griffith propondo uma forma teórica estendida a problemas dinâmicos e processos não isotérmicos.

TELLES \& GUIMARÃES (1998) obtêm a solução da função de Green para problemas gerais de fraturas mecânicas, como em simulações de multi-fratura curva, estática e harmônica em 2-D e 3-D. Mostram, portanto, que a função de Green numérica pode ser implementada em um programa computacional de elementos de contorno, na forma de solução fundamental, produzindo resultados eficientes e precisos. 
Os trabalhos de CARTWRIGHT \& ROOKE (1985) e ALIABADI et al. (1987) mostram bons resultados na utilização do "Método da Função Peso", baseado na interpretação da integral J de $\operatorname{RICE}^{7}$ (1968) apud ALIABADI \& ROOKE (1991).

RIGBY \& ALIABADI (1993) aplicam a técnica da integral J de Rice para obtenção de fatores de intensidade de tensão em problemas com modos de fratura combinados.

WEARING \& AHMADI-BROOGHANI (1999) discutem a aplicação do MEC para a determinação dos fatores de intensidade de tensão em problemas de placas, utilizando o método da integral J, o método da extrapolação do deslocamento, o elemento "quarter-point" e o método da extrapolação da tensão. Os resultados, em comparação com os analíticos ou utilizando o método dos elementos finitos, foram considerados satisfatórios.

Outra vertente na aplicação do MEC à Mecânica da Fratura é a que utiliza a “Técnica dos Domínios Múltiplos”, conforme LACHAT \& WATSON (1976). CROUCH \& STARFIELD (1983) utilizam o Método da Descontinuidade de Deslocamento, apresentando uma discussão extensiva sobre o assunto.

Em BRADY \& BRAY (1978) são analisadas inclusões finas e descontinuidades em escavações de minas, utilizando-se a formulação indireta do MEC juntamente com forças fictícias chamadas "quadripolos". Em ROCHA (1988) são analisados problemas com inclusão de descontinuidades, utilizando-se a formulação direta do MEC juntamente com os dipolos. O assunto pode ser visto ainda em VENTURINI (1994b), VENTURINI (1995), SALEH \& ALIABADI (1994), entre outros. Em VENTURINI (1994a e b; 1995), LOPES JR (1996), as formulações referem-se à análise de problemas bidimensionais.

BARBIRATO \& VENTURINI (1998) apresentam uma formulação do MEC para análise de fratura mecânica em sólidos tridimensionais, utilizando o conceito de dipolos. Apresentam os tensores derivados da solução fundamental de Kelvin (para 3D) e verificam o potencial da formulação ao mostrar os resultados obtidos da aplicação processada.

\footnotetext{
${ }^{7}$ RICE, J.R. (1968). A path independent integral and the approximate analysis of strain concentration by notches and cracks. Trans. ASME, J.Appl.Mech., 35, 379-386.
} 
CARPINTERI (1989); ALLIABADI \& ROOKE (1991); ALIABADI \& BREBBIA (1993) e ALIABADI et al. (1994) trazem formulações completas sobre aplicações do MEC à mecânica da fratura nas análises estática e dinâmica.

O modelo de fratura coesiva tem sido empregado com eficiência para representar o comportamento do material do tipo "quasi-brittle" frente ao processo de fraturamento. Em HILLERBORG \& MODÉER (1976), o modelo é testado e modificado utilizando-se corpo de prova homogêneo e de área constante, solicitado até a ruptura, em ensaios laboratoriais. É definida uma zona de fratura (ou zona de processo) com largura limitada na direção da tensão, formada em algum lugar do modelo de prova. Esta zona de fratura perde gradualmente suas propriedades mecânicas à medida que o dano causado pelo surgimento de microfissuras aumenta, e, portanto, tem-se um comportamento de material coesivo. Da mesma forma, em CARPINTERI (1989) são encontrados novos experimentos e análise através de modelagem numérica de fraturas coesivas no intuito de descrever a influência do efeito escala no processo, constituindo-se em uma referência importante sobre o assunto.

O Método dos Elementos de Contorno também é aplicado à Mecânica da Fratura Linear Elástica em GUIMARÃES (1992). Uma formulação mista para deslocamentos e forças de superfície é utilizada juntamente com o elemento "quarterpoint".

GUIMARÃES \& TELLES (1994) discutem a aplicação da equação integral de contorno hipersingular (formulação de tração) na resolução de problemas envolvendo fraturas mecânicas lineares e elásticas. Enfatizam a importância de se considerar continuidades na implementação numérica e no uso dos elementos "quarter-point".

Uma extensão na aplicabilidade da formulação do MEC com as funções de Green para fraturas únicas e múltiplas, é apresentada no trabalho de TELLES et al. (1994).

CARRER \& MANSUR (1995) apresenta uma formulação do MEC empregando o conceito de parte finita de integral nas representações integrais do método para o cálculo de deslocamentos e de tensões nos pontos internos. Constatam 
a vantagem do método no emprego de expressões mais compactas, e sugerem a extensão dessa formulação nos casos que envolvam plasticidade e visco-plasticidade.

WEN et al. (1995) desenvolvem a aplicação dos métodos da carga fictícia e de descontinuidade de deslocamento para mecânica da fratura dinâmica tridimensional, obtendo resultados substituindo as soluções fundamentais de Laplace em um programa de cálculo estático.

SILVEIRA \& TELLES (1997) propõem uma formulação do MEC para solução de problemas planos de Mecânica da Fratura Linear Elástica com trincas internas de geometria qualquer. Utilizam a solução fundamental de Kelvin para deslocamento e força de superfície e parte complementar, para obtenção da função de Green. Os resultados numéricos dos exemplos apresentados mostram a correta implementação das expressões propostas.

BARRA et al. (1997) mostram a expansão do procedimento da função Green Numérica para o cálculo de fatores de intensidade de tensão dinâmicos transientes obtidos através da formulação no domínio transformado de Laplace, obtendo bom desempenho na aplicação.

CASTOR \& TELLES (1997) enfocam a aplicação da função de Green numérica (FGN) a problemas tridimensionais, apresentando resultados numéricos para problemas de fissuras internas, introduzindo, da metodologia aplicada para problemas bidimensionais, algumas técnicas para regularização das integrais de partes finitas. Concluem que a precisão alcançada se deu a partir da aproximação geométrica da fissura e da forma de cálculo dos fatores de intensidade de tensões (a partir das aberturas da fissura).

BARRA \& TELLES (1999) mostram a extensão da abordagem da função de Green numérica para problemas de fraturas elastodinâmicas, com aplicações que demonstram boa precisão para problemas de trincas múltiplas e geometria geral.

DOMÍNGUEZ \& SÁEZ (1998) mostram uma formulação de elementos de contorno para problemas de fratura estática e dinâmica tridimensional em corpos sólidos isotrópicos e transversalmente isotrópicos. Comprova a precisão e robustez da abordagem utilizada, comparando os resultados obtidos com soluções já existentes. 
ZHAO et al. (1998) estudam o Método das Equações Integrais de Contorno de Deslocamento e Descontinuidade para um sólido elástico, isotrópico transversalmente e tridimensional, utilizando equações hipersingulares, obtendo deslocamentos e tensões próximos da borda de uma trinca.

CARRER \& MANSUR (1999) desenvolvem equações integrais para calcular as componentes de velocidade e tensões na análise elastodinâmica transiente do MEC. Os exemplos apresentados demonstram a precisão da formulação, comparada à soluções analíticas e com a utilização da formulação alternativa do MEC que emprega a solução fundamental estática.

WEN et al. (1999) desenvolvem o Método dos Elementos de Contorno com a Reciprocidade Dual no domínio de Laplace, para a análise de problemas da mecânica da fratura elastodinâmica tridimensional com modos de fratura combinados. Apresentam exemplos numéricos que demonstram boa concordância com soluções já existentes.

MUKHERJEE et al. (1999) utilizam equações integrais de contorno hipersingulares em elasticidade linear tridimensional, para a mecânica da fratura termoelástica.

Outras aplicações do MEC para problemas de propagação de fraturas podem ser vistas em LUTZ et al. (1992), MARTHA et al. (1992), MI \& ALIABADI (1994) e SOUZA et. al. (1995), entre outros. Particularmente em SOUZA et al. (1995) são mostradas diretrizes para simulação tridimensional de propagação de fissuras. Destaca-se a apresentação de um modelo para representar o fronte da fissura, que pode não ser necessariamente plana e contida em sólidos de geometria arbitrária.

Dois trabalhos trazem uma revisão de literatura sobre mecânica da fratura em seus aspectos computacionais. Em NISHIOKA (1994) é apresentado o estado da arte da mecânica computacional da fratura dinâmica. Em CRUSE (1995) encontra-se um breve histórico sobre a utilização do Método das Integrais de Contorno na Mecânica da Fratura. 


\section{CAPÍTULO 2}

\section{FORMULAÇÃO ELASTOSTÁTICA TRIDIMENSIONAL DO MEC}

\section{1 - INTRODUÇÃO}

Neste capítulo é apresentada a formulação estática do Método dos Elementos de Contorno (MEC) para sólidos elásticos tridimensionais.

Inicialmente, pretende-se indicar a notação matemática que deve ser empregada em várias expressões ao longo do texto da tese. Passa-se, então, para uma breve revisão da elastostática, apresentando suas relações e constantes mais importantes. Mostram-se os problemas fundamentais e suas soluções, que serão utilizadas para deduzir as representações integrais para pontos do domínio e especificamente para o contorno. Na seqüência, são apresentados os elementos de contorno usados na discretização do contorno do corpo, determinando-se as equações matriciais do método dos elementos de contorno propriamente dito, bem como os procedimentos utilizados para a integração numérica e semi-analítica necessárias à solução do método. São ainda apresentadas as expressões para o cálculo de deslocamentos e tensões em pontos do domínio e tensões em pontos do contorno. Por fim, apresentam-se alguns exemplos para mostrar a eficiência da formulação. 


\section{2 - NOTAÇÃO INDICIAL}

Muitas das expressões que são apresentadas neste trabalho estão escritas utilizando-se a notação indicial. O resultado desta escolha é a forma sucinta e elegante de escrevê-las.

Por exemplo, o sistema de coordenadas cartesianas, geralmente representado pelos eixos $\mathrm{x}, \mathrm{y}$ e $\mathrm{z}$, passa a ser $\mathrm{x}_{1}, \mathrm{x}_{2}$ e $\mathrm{x}_{3}$, respectivamente. Portanto, as direções cartesianas são definidas pelos índices 1,2 e 3, ou, de maneira genérica, por $x_{i}$. Outras variáveis que aparecem ao longo do texto, referidas às direções cartesianas, têm o mesmo tratamento indicial (deslocamentos, $\mathrm{u}_{\mathrm{i}}$; forças de superfície, $\mathrm{p}_{\mathrm{i}}$; forças de volume, $\mathrm{b}_{\mathrm{i}}$; acelerações, $\ddot{\mathrm{u}}_{\mathrm{i}}$; tensões, $\sigma_{\mathrm{ij}}$; deformações, $\varepsilon_{\mathrm{ij}}$; dentre outras).

A convenção implícita de somatório também é utilizada neste trabalho. $\mathrm{O}$ surgimento de um índice repetido em uma expressão representa um somatório. Por exemplo,

$$
c_{j}=a_{1 j} b_{1}+a_{2 j} b_{2}+a_{3 j} b_{3}=\sum_{i=1}^{3} a_{i j} b_{i}=a_{i j} b_{i}
$$

$\mathrm{e}$

$$
\mathrm{c}=\sum_{\mathrm{j}=1}^{\mathrm{NPG}} \mathrm{w}_{\mathrm{j}} \sum_{\mathrm{i}=1}^{3} \mathrm{a}_{\mathrm{ij}} \mathrm{b}_{\mathrm{i}}=\mathrm{w}_{\mathrm{j}}\left(\mathrm{a}_{\mathrm{ij}} \mathrm{b}_{\mathrm{i}}\right)
$$

Nesta forma de representação sucinta, as indicações tradicionais de derivadas parciais com relação ao espaço dão lugar a uma simples vírgula, conforme mostram os exemplos dados a seguir:

$$
\begin{gathered}
\frac{\partial \xi_{\mathrm{i}}}{\partial \mathrm{x}_{\ell}}=\xi_{\mathrm{i}, \ell} \\
\frac{\partial \sigma_{\mathrm{ij}}}{\partial \mathrm{X}_{\mathrm{k}}}=\sigma_{\mathrm{ij}, \mathrm{k}} \\
\frac{\partial^{2} \mathrm{G}_{\mathrm{ij}}}{\partial \mathrm{x}_{\mathrm{k}} \partial \mathrm{x}_{\ell}}=\mathrm{G}_{\mathrm{ij}, \mathrm{k} \ell}
\end{gathered}
$$


O Delta de Kronecker, utilizado ao longo do texto, é definido da seguinte forma:

$$
\delta_{i j}=\left\{\begin{array}{l}
1, \text { se } i=j \\
0, \text { se } i \neq j
\end{array}\right.
$$

Pode-se encontrar maiores detalhes matemáticos requeridos pelo método consultando-se os trabalhos de ROCHA (1988), BREBBIA \& DOMINGUEZ (1989), KANE (1994), entre outros.

\section{3 - RELAÇÕES DA ELASTOSTÁTICA}

Considere-se um sólido elástico-linear tridimensional, homogêneo e isotrópico, definido pelo domínio $\Omega$ e seu contorno $\Gamma$, conforme apresentado na figura 2.3.1.

FIGURA 2.3.1 - Definição do objeto de estudo: sólido tridimensional de domínio $\Omega$

$$
\text { e contorno } \Gamma \text {. }
$$

Admitindo-se que o corpo esteja em equilíbrio estático, pode-se afirmar que um de seus elementos infinitesimais, tomado como referência, também encontra-se equilibrado. Portanto, equacionando-se o equilíbrio das forças que agem nas faces deste elemento e as forças volumétricas, chega-se à expressão diferencial

$$
\sigma_{\mathrm{ij}, \mathrm{j}}(\chi)+\mathrm{b}_{\mathrm{i}}(\chi)=0
$$


onde,

$\sigma_{\mathrm{ij}, \mathrm{j}}(\chi)$ representa a derivada do tensor das tensões;

$\mathrm{b}_{\mathrm{i}}(\chi)$ o vetor das forças volumétricas; $\mathrm{e}$

$\chi$ representa o ponto material analisado.

Por sua vez, o equilíbrio rotacional das forças que agem sobre o elemento em questão indica a simetria do tensor das tensões, classicamente chamada de condição de Cauchy. Portanto,

$$
\sigma_{\mathrm{ij}}(\chi)=\sigma_{\mathrm{ji}}(\chi)
$$

Para a análise completa do equilíbrio de forças atuantes no corpo, deve-se tomar, ainda, um elemento infinitesimal situado em seu contorno. Surgem, então, o que se convencionou chamar de forças de superfície, $\mathrm{p}_{\mathrm{i}}$, conforme mostra a figura 2.3.2.

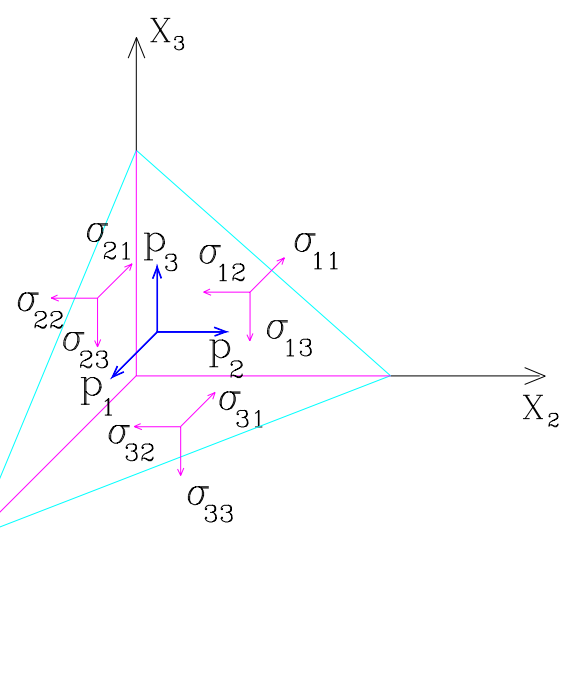

FIGURA 2.3.2 - Tetraedro de Cauchy.

As componentes das forças de superfície, representadas por $\mathrm{p}_{\mathrm{i}}$, podem ser expressas em função das componentes de tensão, considerando-se o equilíbrio nas três direções cartesianas, onde $\mathrm{n}_{\mathrm{j}}$ são os co-senos diretores dos ângulos entre a normal à face 
inclinada (superfície do contorno) e o eixo cartesiano $\mathrm{X}_{\mathrm{j}}$. Chega-se, portanto, na seguinte relação:

$$
\mathrm{p}_{\mathrm{i}}(\chi)=\sigma_{\mathrm{ij}}(\chi) \mathrm{n}_{\mathrm{j}}
$$

Quanto às deformações que se desenvolvem no corpo elástico linear, considerando-se as hipóteses de continuidade de deslocamentos, estas podem ser representadas pelo tensor

$$
\varepsilon_{\mathrm{ij}}(\chi)=\frac{1}{2}\left(\mathrm{u}_{\mathrm{i}, \mathrm{j}}(\chi)+\mathrm{u}_{\mathrm{j}, \mathrm{i}}(\chi)\right),
$$

linearizado para pequenas deformações (relaciona deformações aos deslocamentos).

A expressão diferencial conhecida como Lei de Hooke relaciona os tensores de tensão e deformação de um determinado sólido elástico-linear isotrópico, e se apresenta da seguinte forma:

$$
\sigma_{\mathrm{ij}}(\chi)=2 \mathrm{G} \frac{v}{1-2 v} \delta_{\mathrm{ij}} \varepsilon_{\mathrm{kk}}(\chi)+2 \mathrm{G} \varepsilon_{\mathrm{ij}}(\chi)
$$

ou ainda na forma inversa

$$
\varepsilon_{\mathrm{ij}}(\chi)=\frac{1}{2 \mathrm{G}}\left(\sigma_{\mathrm{ij}}(\chi)-\frac{v}{1+v} \sigma_{\mathrm{kk}}(\chi) \delta_{\mathrm{ij}}\right)
$$

Na lei de Hooke aparecem as constantes elásticas básicas, dadas a seguir:

$v$, representa o coeficiente de Poisson;

G, módulo de elasticidade transversal, ou módulo de elasticidade ao cisalhamento; e, ainda,

E, módulo de elasticidade longitudinal, ou módulo de Young; 
As relações entre as constantes elásticas são dadas a seguir:

$$
\begin{aligned}
& \mathrm{G}=\frac{\mathrm{E}}{2(1+v)} \\
& \lambda=\frac{2 \mathrm{G} v}{1-2 v}=\frac{v \mathrm{E}}{(1-2 v)(1+v)}
\end{aligned}
$$

Substituindo-se a expressão (2.3.4) em (2.3.5) e o resultado desta em (2.3.1), obtém-se a equação diferencial do problema elástico em termos de deslocamentos, conhecida como equação de Navier-Cauchy para a estática, ou seja:

$$
\mathrm{u}_{\mathrm{i}, \mathrm{j} j}(\chi)+\frac{1}{1-2 v} \mathrm{u}_{\mathrm{j}, \mathrm{j}}(\chi)+\frac{1}{\mathrm{G}} \mathrm{b}_{\mathrm{i}}(\chi)=0
$$

Para que o problema elástico-linear fique completamente definido, além das equações diferenciais de equilíbrio (2.3.1), deformação/deslocamento (2.3.4) e a Lei de Hooke (2.3.5) ou (2.3.6), é necessário conhecer as condições de contorno. Assim, considera-se o contorno do sólido, denotado por $\Gamma$, composto por duas partes: $\Gamma_{1}$ e $\Gamma_{2}$ (sendo $\Gamma=\Gamma_{1}+\Gamma_{2}$ ), conforme mostrado na figura 2.3.3.

FIGURA 2.3.3 - Definição das condições de contorno. 
Pode-se, então, definir os valores prescritos das variáveis de interesse para a análise (deslocamentos $u_{i}$ e forças de superfície $p_{i}$ ) em cada uma das partes, estabelecendo-se

$\mathrm{u}_{\mathrm{i}}(\mathrm{Q})=\overline{\mathrm{u}}_{\mathrm{i}}(\mathrm{Q}), \mathrm{Q} \in \Gamma_{1} \quad$ (condições essenciais)

$\mathrm{p}_{\mathrm{i}}(\mathrm{S})=\overline{\mathrm{p}}_{\mathrm{i}}(\mathrm{S}), \quad \mathrm{S} \in \Gamma_{2} \quad$ (condições naturais)

onde a barra sobre as variáveis representa valores prescritos.

\section{4 - SOLUÇÕES FUNDAMENTAIS}

Para que as formulações do Método dos Elementos de Contorno fiquem completamente definidas, torna-se necessário o conhecimento prévio da solução de um problema padrão da área que se deseja analisar. A este problema dá-se o nome de "problema fundamental", conforme apresentado em BREBBIA (1978). Utilizam-se no presente trabalho os problemas fundamentais de Kelvin e Mindlin com suas respectivas soluções.

Para a definição do problema fundamental, considere-se $\Omega^{*}$ um domínio infinito cujo contorno é denotado por $\Gamma^{*}$. O sólido que se deseja analisar, de domínio $\Omega$ e contorno $\Gamma$, está contido em $\Omega^{*}$. O problema particular indicado pelo asterisco é chamado de problema fundamental e encontra-se definido na figura 2.4.1.

FIGURA 2.4.1 - Definição do problema fundamental e a interdependência do problema que se deseja analisar. 
Aplica-se uma força unitária estática em um ponto $\mathrm{s}$ (ponto fonte) do domínio na direção cartesiana $\mathrm{i}, \mathrm{F}_{\mathrm{i}}{ }^{*}(\mathrm{~s})$, e avaliam-se os seus efeitos nas direções cartesianas em um outro ponto, $\mathrm{q}$ (ponto de campo), conforme mostra a figura 2.4.2.

Observe-se que nas respostas para deslocamentos e forças de superfície , $\mathrm{u}_{\mathrm{ij}}^{*} \mathrm{e}$ $\mathrm{p}_{\mathrm{ij}}^{*}$ respectivamente, o primeiro índice representa a direção cartesiana de aplicação da força e o segundo a direção do efeito medido. Estas respostas da carga unitária são chamadas de solução fundamental do problema particular analisado.

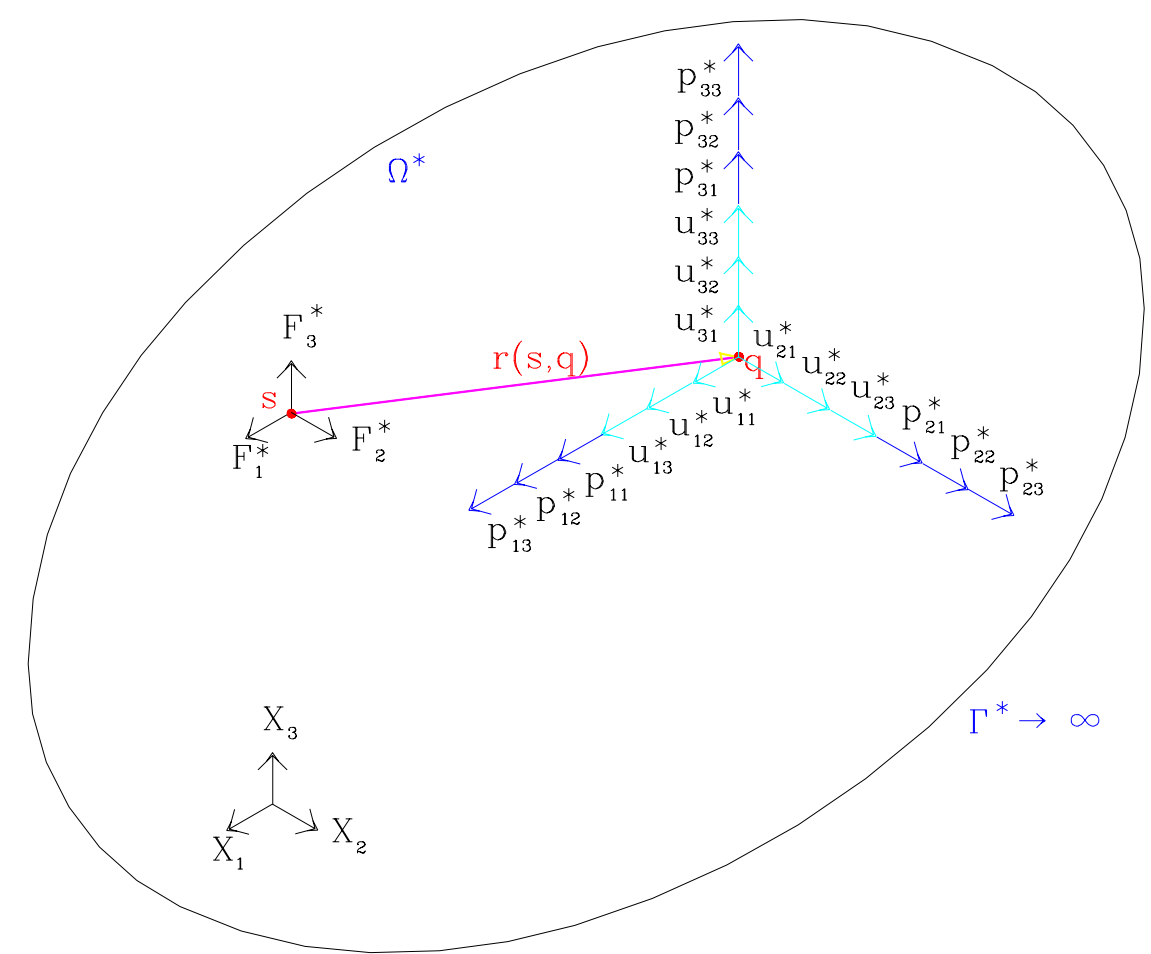

FIGURA 2.4.2 - Efeitos das forças unitárias aplicadas em $\Omega^{*}$ : solução fundamental.

As expressões analíticas da solução fundamental de deslocamentos e forças de superfície são obtidas substituindo-se o termo das forças volumétricas na equação de equilíbrio para o problema estático (2.3.1) e na equação de deslocamentos (2.3.9) pela distribuição Delta de Dirac (ver anexo A), que passa a ser um multiplicador da força unitária aplicada no ponto fonte s. Isto resulta em:

$$
\mathrm{b}_{\mathrm{i}}(\mathrm{q})=\delta(\mathrm{s}, \mathrm{q}) \delta_{\mathrm{ki}}
$$




$$
\sigma_{\mathrm{ij}, \mathrm{j}}+\delta(\mathrm{s}, \mathrm{q}) \delta_{\mathrm{ki}}=0
$$

$\mathrm{e}$

$$
\mathrm{u}_{\mathrm{ki}, \mathrm{jj}}+\frac{1}{1-2 v} \mathrm{u}_{\mathrm{kj}, \mathrm{ji}}+\frac{1}{\mathrm{G}} \delta(\mathrm{s}, \mathrm{q}) \delta_{\mathrm{ki}}=0
$$

respectivamente.

Por definição, a solução fundamental é originária de um problema conhecido e particular. Portanto, dependendo das características do problema fundamental, tais como o espaço a que seus domínio $\Omega^{*}$ e contorno $\Gamma^{*}$ pertencem (infinito ou semiinfinito, por exemplo) e, resolvendo-se as equações (2.4.2) e (2.4.3), tem-se diferentes soluções fundamentais.

Neste trabalho utilizam-se as soluções fundamentais dos problemas de Kelvin, para o domínio infinito, e de Mindlin, para o domínio semi-infinito, ambos tridimensionais.

\subsection{1 - Solução Fundamental de Kelvin}

A solução fundamental de Kelvin é, sem dúvida, a mais difundida e utilizada pelo meio técnico. Esta solução clássica foi desenvolvida por Lord Kelvin, conforme LOVE (1944), considerando sólidos tridimensionais elásticos, isotrópicos e homogêneos, cujo domínio $\Omega^{*}$ se estende ao infinito. A figura 2.4.3 mostra a definição do problema apresentando o ponto fonte $\mathrm{s}$ com suas forças unitárias, o ponto de campo q com as respostas das forças unitárias (deslocamentos fundamentais $\mathrm{u}_{\mathrm{ij}}^{*}$ e forças de superfície fundamentais $\mathrm{p}_{\mathrm{ij}}^{*}$ ), e a variável esférica $\mathrm{r}$ e suas componentes cartesianas. 


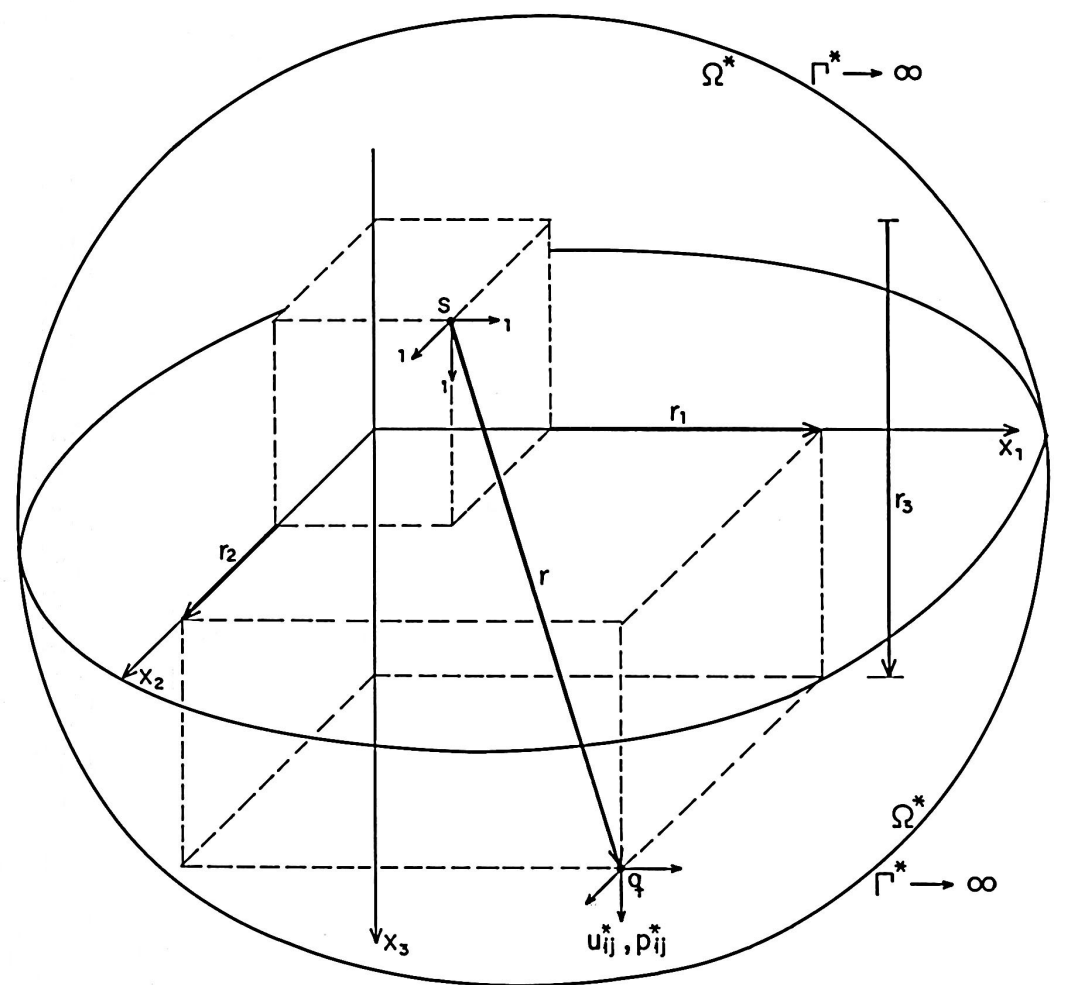

FIGURA 2.4.3 - Definição do problema fundamental de Kelvin.

As expressões de deslocamentos e forças de superfície para o problema de Kelvin são:

$$
\begin{aligned}
& \mathrm{u}_{\mathrm{ij}}^{*}(\mathrm{~s}, \mathrm{q})=\frac{1}{16 \pi(1-v) \mathrm{Gr}}\left\{(3-4 v) \delta_{\mathrm{ij}}+\mathrm{r}_{, \mathrm{i}} \mathrm{r}_{\mathrm{j}}\right\} \\
& \mathrm{p}_{\mathrm{ij}}^{*}(\mathrm{~s}, \mathrm{q})=-\frac{1}{8 \pi(1-v) \mathrm{r}^{2}}\left\{\left[(1-2 v) \delta_{\mathrm{ij}}+3 \mathrm{r}_{, \mathrm{i}} \mathrm{r}_{, \mathrm{j}}\right] \mathrm{r}_{, \mathrm{n}}-(1-2 v)\left(\mathrm{r}_{\mathrm{i}} \mathrm{n}_{\mathrm{j}}-\mathrm{r}_{, \mathrm{j}} \mathrm{n}_{\mathrm{i}}\right)\right\}
\end{aligned}
$$

onde,

$$
\begin{aligned}
& r=\sqrt{r_{i} r_{i}}=|s-q| \\
& r_{i}=X_{i}(q)-X_{i}(s) \\
& r_{, i}=\frac{r_{i}}{r}
\end{aligned}
$$




\subsection{2 - Solução Fundamental de Mindlin}

A solução fundamental de Mindlin (MINDLIN, 1936) caracteriza-se por ter seu domínio $\Omega^{*}$ um semi-espaço infinito, sólido elástico, isotrópico e homogêneo. A figura 2.4.4 apresenta o problema definindo o ponto de campo q, o ponto fonte $\mathrm{s}$ e sua imagem s' distante c do plano $\mathrm{x}_{1} \mathrm{x}_{2}$. Define, ainda, as variáveis esféricas $\mathrm{r}$ e $\mathrm{R}$ e suas componentes cartesianas. $\mathrm{O}$ plano $\mathrm{x}_{3}=0$ (ou $\bar{\Gamma}$ ) representa parte da superfície de contorno onde admite-se a ausência de trações.

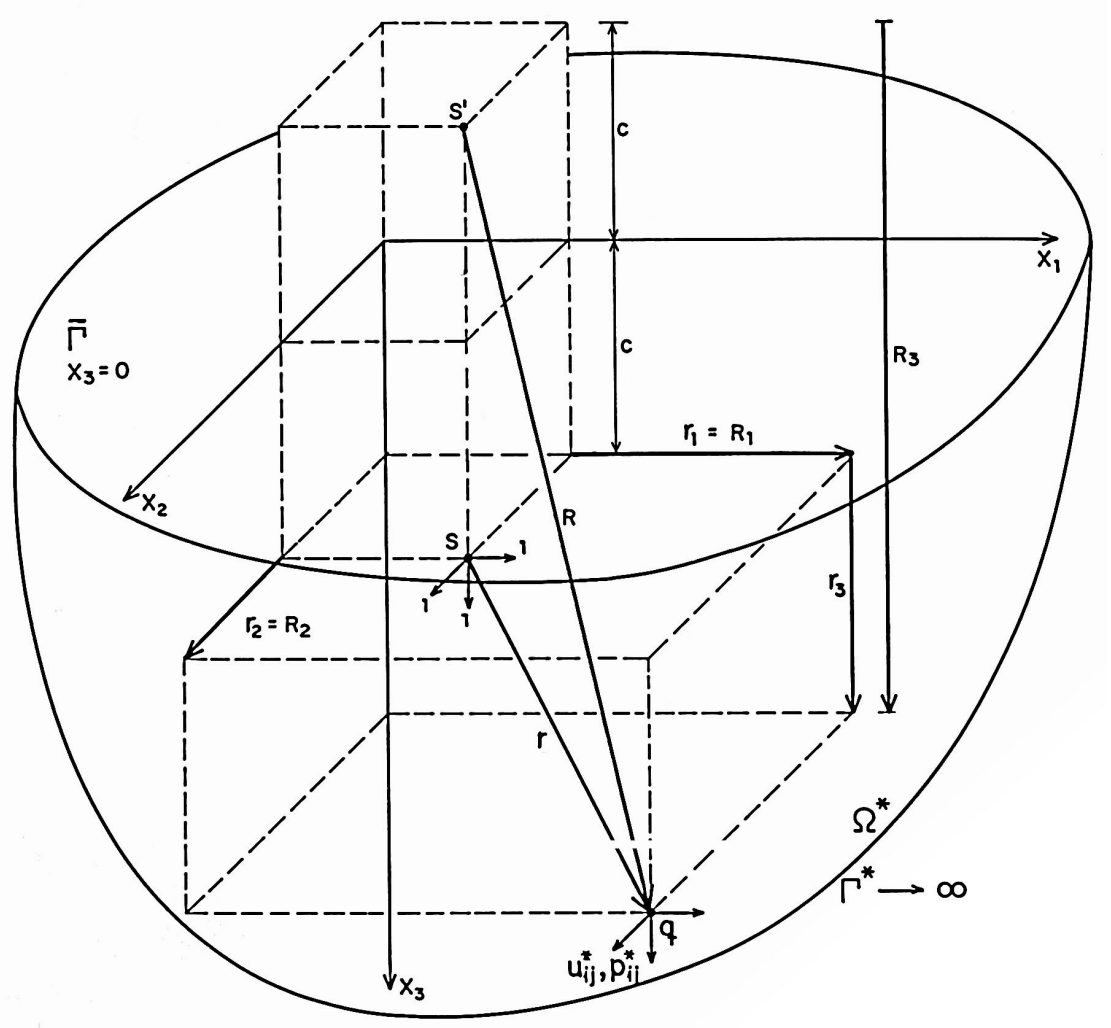

FIGURA 2.4.4 - Definição do problema fundamental de Mindlin.

As expressões fundamentais de Mindlin para deslocamentos e forças de superfície são apresentadas a seguir, após a definição de alguns parâmetros mostrados na figura 2.4 .4 e que serão utilizados nas expressões: 


$$
\begin{aligned}
& \mathrm{r}_{\mathrm{i}}=\mathrm{X}_{\mathrm{i}}(\mathrm{q})-\mathrm{X}_{\mathrm{i}}(\mathrm{s}) \\
& \mathrm{R}_{\mathrm{i}}=\mathrm{X}_{\mathrm{i}}(\mathrm{q})-\mathrm{X}_{\mathrm{i}}\left(\mathrm{s}^{\prime}\right) \\
& \mathrm{r}=\sqrt{\mathrm{r}_{\mathrm{i}} \mathrm{r}_{\mathrm{i}}} \\
& \mathrm{R}=\sqrt{\mathrm{R}_{\mathrm{i}} \mathrm{R}_{\mathrm{i}}} \\
& \mathrm{c}=\mathrm{X}_{3}(\mathrm{~s}) \geq 0 \\
& \mathrm{z}=\mathrm{X}_{3}(\mathrm{q}) \geq 0 \\
& \mathrm{~K}_{\mathrm{d}}=\frac{1}{16 \pi \mathrm{G}(1-v)} \\
& \mathrm{K}_{\mathrm{s}}=\frac{1}{8 \pi(1-v)} \\
& \mathrm{C}_{1}=1-v \\
& \mathrm{C}_{2}=1-2 v \\
& \mathrm{C}_{3}=3-4 v \\
& \mathrm{C}_{4}=3-2 v \\
& \mathrm{C}_{5}=5-4 v
\end{aligned}
$$

A seguir, tem-se as expressões para deslocamentos (2.4.8):

$$
\begin{aligned}
& \mathrm{u}_{11}^{*}=\mathrm{K}_{\mathrm{d}}\left\{\frac{\mathrm{C}_{3}}{\mathrm{r}}+\frac{\mathrm{r}_{1}^{2}}{\mathrm{r}^{3}}+\frac{1}{\mathrm{R}}+\frac{\mathrm{C}_{3} \mathrm{r}_{1}^{2}}{\mathrm{R}^{3}}+\frac{2 \mathrm{cz}}{\mathrm{R}^{3}}\left(1-\frac{3 \mathrm{r}_{1}^{2}}{\mathrm{R}^{2}}\right)+\frac{4 \mathrm{C}_{1} \mathrm{C}_{2}}{\mathrm{R}+\mathrm{R}_{3}}\left(1-\frac{\mathrm{r}_{1}^{2}}{\mathrm{R}\left(\mathrm{R}+\mathrm{R}_{3}\right)}\right)\right\} \\
& \mathrm{u}_{12}^{*}=\mathrm{K}_{\mathrm{d}} \mathrm{r}_{1} \mathrm{r}_{2}\left\{\frac{1}{\mathrm{r}^{3}}+\frac{\mathrm{C}_{3}}{\mathrm{R}^{3}}-\frac{6 \mathrm{cz}}{\mathrm{R}^{5}}-\frac{4 \mathrm{C}_{1} \mathrm{C}_{2}}{\mathrm{R}\left(\mathrm{R}+\mathrm{R}_{3}\right)^{2}}\right\} \\
& \mathrm{u}_{13}^{*}=\mathrm{K}_{\mathrm{d}} \mathrm{r}_{1}\left\{\frac{\mathrm{r}_{3}}{\mathrm{r}^{3}}+\frac{\mathrm{C}_{3} \mathrm{r}_{3}}{\mathrm{R}^{3}}-\frac{6 \mathrm{czR} \mathrm{R}_{3}}{\mathrm{R}^{5}}+\frac{4 \mathrm{C}_{1} \mathrm{C}_{2}}{\mathrm{R}\left(\mathrm{R}+\mathrm{R}_{3}\right)}\right\} \\
& \mathrm{u}_{21}^{*}=\mathrm{u}_{12}^{*}
\end{aligned}
$$




$$
\begin{aligned}
& \mathrm{u}_{22}^{*}=\mathrm{K}_{\mathrm{d}}\left\{\frac{\mathrm{C}_{3}}{\mathrm{r}}+\frac{\mathrm{r}_{2}^{2}}{\mathrm{r}^{3}}+\frac{1}{\mathrm{R}}+\frac{\mathrm{C}_{3} \mathrm{r}_{2}^{2}}{\mathrm{R}^{3}}+\frac{2 \mathrm{cz}}{\mathrm{R}^{3}}\left(1-\frac{3 \mathrm{r}_{2}^{2}}{\mathrm{R}^{2}}\right)+\frac{4 \mathrm{C}_{1} \mathrm{C}_{2}}{\mathrm{R}+\mathrm{R}_{3}}\left(1-\frac{\mathrm{r}_{2}^{2}}{\mathrm{R}\left(\mathrm{R}+\mathrm{R}_{3}\right)}\right)\right\} \\
& \mathrm{u}_{23}^{*}=\frac{\mathrm{r}_{2}}{\mathrm{r}_{1}} \mathrm{u}_{13}^{*} \\
& \mathrm{u}_{31}^{*}=\mathrm{K}_{\mathrm{d}} \mathrm{r}_{1}\left\{\frac{\mathrm{r}_{3}}{\mathrm{r}^{3}}+\frac{\mathrm{C}_{3} \mathrm{r}_{3}}{\mathrm{R}^{3}}+\frac{6 \mathrm{czR} \mathrm{R}_{3}}{\mathrm{R}^{5}}-\frac{4 \mathrm{C}_{1} \mathrm{C}_{2}}{\mathrm{R}\left(\mathrm{R}+\mathrm{R}_{3}\right)}\right\} \\
& \mathrm{u}_{32}^{*}=\frac{\mathrm{r}_{2}}{\mathrm{r}_{1}} \mathrm{u}_{31}^{*} \\
& \mathrm{u}_{33}^{*}=\mathrm{K}_{\mathrm{d}}\left\{\frac{\mathrm{C}_{3}}{\mathrm{r}}+\frac{\mathrm{r}_{3}^{2}}{\mathrm{r}^{3}}+\frac{8 \mathrm{C}_{1}^{2}-\mathrm{C}_{3}}{\mathrm{R}}+\frac{\mathrm{C}_{3} \mathrm{R}_{3}^{2}-2 \mathrm{cz}}{\mathrm{R}^{3}}+\frac{6 \mathrm{czR} \mathrm{R}_{3}^{2}}{\mathrm{R}^{5}}\right\}
\end{aligned}
$$

As expressões para as forças de superfície são obtidas em função do tensor de terceira ordem das tensões, do problema fundamental, e das componentes do vetor normal à superfície no ponto $\mathrm{q}\left(\mathrm{n}_{\mathrm{k}}\right)$, ou seja:

$$
\mathrm{p}_{\mathrm{ij}}^{*}=\sigma_{\mathrm{jk}}^{* \mathrm{i}} \mathrm{n}_{\mathrm{k}} \text {. }
$$

As expressões do tensor de terceira ordem das tensões, são (2.4.10):

$$
\begin{aligned}
\sigma_{11}^{*_{1}}= & \mathrm{K}_{\mathrm{s}} \mathrm{r}_{1}\left\{-\frac{\mathrm{C}_{2}}{\mathrm{r}^{3}}-\frac{3 \mathrm{r}_{1}^{2}}{\mathrm{r}^{5}}+\frac{\mathrm{C}_{2} \mathrm{C}_{5}}{\mathrm{R}^{3}}-\frac{3 \mathrm{C}_{3} \mathrm{r}_{1}^{2}}{\mathrm{R}^{5}}+\right. \\
& \left.-\frac{4 \mathrm{C}_{1} \mathrm{C}_{2}}{\mathrm{R}\left(\mathrm{R}+\mathrm{R}_{3}\right)^{2}}\left[3-\frac{\mathrm{r}_{1}^{2}\left(3 \mathrm{R}+\mathrm{R}_{3}\right)}{\mathrm{R}^{2}\left(\mathrm{R}+\mathrm{R}_{3}\right)}\right]+\frac{6 \mathrm{c}}{\mathrm{R}^{5}}\left[3 \mathrm{c}-\mathrm{C}_{4} \mathrm{R}_{3}+\frac{5 \mathrm{r}_{1}^{2} \mathrm{z}}{\mathrm{R}^{2}}\right]\right\}
\end{aligned}
$$




$$
\begin{aligned}
& \sigma_{12}^{* 1}=\mathrm{K}_{\mathrm{s}} \mathrm{r}_{2}\left\{-\frac{\mathrm{C}_{2}}{\mathrm{r}^{3}}-\frac{3 \mathrm{r}_{1}^{2}}{\mathrm{r}^{5}}+\frac{\mathrm{C}_{2}}{\mathrm{R}^{3}}-\frac{3 \mathrm{C}_{3} \mathrm{r}_{1}^{2}}{\mathrm{R}^{5}}+\right. \\
& \left.-\frac{4 \mathrm{C}_{1} \mathrm{C}_{2}}{\mathrm{R}\left(\mathrm{R}+\mathrm{R}_{3}\right)^{2}}\left[1-\frac{\mathrm{r}_{1}^{2}\left(3 \mathrm{R}+\mathrm{R}_{3}\right)}{\mathrm{R}^{2}\left(\mathrm{R}+\mathrm{R}_{3}\right)}\right]-\frac{6 \mathrm{cz}}{\mathrm{R}^{5}}\left[1-\frac{5 \mathrm{r}_{1}^{2}}{\mathrm{R}^{2}}\right]\right\} \\
& \sigma_{13}^{* 1}=K_{s}\left\{-\frac{C_{2} r_{3}}{r^{3}}-\frac{3 r_{1}^{2} r_{3}}{r^{5}}+\frac{C_{2} r_{3}}{R^{3}}-\frac{3 C_{3} r_{1}^{2} R_{3}}{R^{5}}+\right. \\
& \left.-\frac{6 c}{\mathrm{R}^{5}}\left[\mathrm{zR}_{3}-\mathrm{C}_{2} \mathrm{r}_{1}^{2}-\frac{5 \mathrm{r}_{1}^{2} \mathrm{zR}_{3}}{\mathrm{R}^{2}}\right]\right\} \\
& \sigma_{22}^{*_{1}}=\mathrm{K}_{\mathrm{s}} \mathrm{r}_{1}\left\{\frac{\mathrm{C}_{2}}{\mathrm{r}^{3}}-\frac{3 \mathrm{r}_{2}^{2}}{\mathrm{r}^{5}}+\frac{\mathrm{C}_{2} \mathrm{C}_{3}}{\mathrm{R}^{3}}-\frac{3 \mathrm{C}_{3} \mathrm{r}_{2}^{2}}{\mathrm{R}^{5}}+\right. \\
& \left.-\frac{4 C_{1} C_{2}}{R\left(R+R_{3}\right)^{2}}\left[1-\frac{r_{2}^{2}\left(3 R+R_{3}\right)}{R^{2}\left(R+R_{3}\right)}\right]+\frac{6 c}{R^{5}}\left[c-C_{2} R_{3}+\frac{5 r_{2}^{2} z}{R^{2}}\right]\right\} \\
& \sigma_{23}^{*_{1}}=\mathrm{K}_{\mathrm{s}} \mathrm{r}_{1} \mathrm{r}_{2}\left\{-\frac{3 \mathrm{r}_{3}}{\mathrm{r}^{5}}-\frac{3 \mathrm{C}_{3} \mathrm{R}_{3}}{\mathrm{R}^{5}}+\frac{6 \mathrm{c}}{\mathrm{R}^{5}}\left[\mathrm{C}_{2}+\frac{5 \mathrm{zR}_{3}}{\mathrm{R}^{2}}\right]\right\} \\
& \sigma_{33}^{*_{1}}=\mathrm{K}_{\mathrm{s}} \mathrm{r}_{1}\left\{\frac{\mathrm{C}_{2}}{\mathrm{r}^{3}}-\frac{3 \mathrm{r}_{3}^{2}}{\mathrm{r}^{5}}-\frac{\mathrm{C}_{2}}{\mathrm{R}^{3}}-\frac{3 \mathrm{C}_{3} \mathrm{R}_{3}^{2}}{\mathrm{R}^{5}}+\frac{6 \mathrm{c}}{\mathrm{R}^{5}}\left[\mathrm{c}+\mathrm{C}_{2} \mathrm{R}_{3}+\frac{5 \mathrm{zR}_{3}^{2}}{\mathrm{R}^{2}}\right]\right\} \\
& \sigma_{11}^{* 2}=\mathrm{K}_{\mathrm{s}} \mathrm{r}_{2}\left\{\frac{\mathrm{C}_{2}}{\mathrm{r}^{3}}-\frac{3 \mathrm{r}_{1}^{2}}{\mathrm{r}^{5}}+\frac{\mathrm{C}_{2} \mathrm{C}_{3}}{\mathrm{R}^{3}}-\frac{3 \mathrm{C}_{3} \mathrm{r}_{1}^{2}}{\mathrm{R}^{5}}+\right. \\
& \left.-\frac{4 \mathrm{C}_{1} \mathrm{C}_{2}}{\mathrm{R}\left(\mathrm{R}+\mathrm{R}_{3}\right)^{2}}\left[1-\frac{\mathrm{r}_{1}^{2}\left(3 \mathrm{R}+\mathrm{R}_{3}\right)}{\mathrm{R}^{2}\left(\mathrm{R}+\mathrm{R}_{3}\right)}\right]+\frac{6 \mathrm{c}}{\mathrm{R}^{5}}\left[\mathrm{c}-\mathrm{C}_{2} \mathrm{R}_{3}+\frac{5 \mathrm{r}_{1}^{2} \mathrm{z}}{\mathrm{R}^{2}}\right]\right\} \\
& \sigma_{12}^{* 2}=\mathrm{K}_{\mathrm{s}} \mathrm{r}_{1}\left\{-\frac{\mathrm{C}_{2}}{\mathrm{r}^{3}}-\frac{3 \mathrm{r}_{2}^{2}}{\mathrm{r}^{5}}+\frac{\mathrm{C}_{2}}{\mathrm{R}^{3}}-\frac{3 \mathrm{C}_{3} \mathrm{r}_{2}^{2}}{\mathrm{R}^{5}}+\right. \\
& \left.-\frac{4 \mathrm{C}_{1} \mathrm{C}_{2}}{\mathrm{R}\left(\mathrm{R}+\mathrm{R}_{3}\right)^{2}}\left[1-\frac{\mathrm{r}_{2}^{2}\left(3 \mathrm{R}+\mathrm{R}_{3}\right)}{\mathrm{R}^{2}\left(\mathrm{R}+\mathrm{R}_{3}\right)}\right]-\frac{6 \mathrm{cz}}{\mathrm{R}^{5}}\left[1-\frac{5 \mathrm{r}_{2}^{2}}{\mathrm{R}^{2}}\right]\right\}
\end{aligned}
$$




$$
\begin{aligned}
& \sigma_{13}^{* 2}=\sigma_{23}^{* 1} \\
& \sigma_{22}^{*_{2}}=\mathrm{K}_{\mathrm{s}} \mathrm{r}_{2}\left\{-\frac{\mathrm{C}_{2}}{\mathrm{r}^{3}}-\frac{3 \mathrm{r}_{2}^{2}}{\mathrm{r}^{5}}+\frac{\mathrm{C}_{2} \mathrm{C}_{5}}{\mathrm{R}^{3}}-\frac{3 \mathrm{C}_{3} \mathrm{r}_{2}^{2}}{\mathrm{R}^{5}}+\right. \\
& \left.-\frac{4 \mathrm{C}_{1} \mathrm{C}_{2}}{\mathrm{R}\left(\mathrm{R}+\mathrm{R}_{3}\right)^{2}}\left[3-\frac{\mathrm{r}_{2}^{2}\left(3 \mathrm{R}+\mathrm{R}_{3}\right)}{\mathrm{R}^{2}\left(\mathrm{R}+\mathrm{R}_{3}\right)}\right]+\frac{6 \mathrm{c}}{\mathrm{R}^{5}}\left[3 \mathrm{c}-\mathrm{C}_{4} \mathrm{R}_{3}+\frac{5 \mathrm{r}_{2}^{2} \mathrm{z}}{\mathrm{R}^{2}}\right]\right\} \\
& \sigma_{23}^{* 2}=K_{s}\left\{-\frac{C_{2} r_{3}}{r^{3}}-\frac{3 r_{2}^{2} r_{3}}{r^{5}}+\frac{C_{2} r_{3}}{R^{3}}-\frac{3 C_{3} r_{2}^{2} R_{3}}{R^{5}}-\frac{6 c}{R^{5}}\left[z R_{3}-C_{2} r_{2}^{2}-\frac{5 r_{2}^{2} z R_{3}}{R^{2}}\right]\right\} \\
& \sigma_{33}^{* 2}=\frac{r_{2}}{r_{1}} \sigma_{33}^{* 1} \\
& \sigma_{11}^{* 3}=K_{s}\left\{\frac{C_{2} r_{3}}{r^{3}}-\frac{3 r_{1}^{2} r_{3}}{r^{5}}+\frac{C_{2}\left(3 r_{3}-4 v R_{3}\right)}{R^{3}}-\frac{3 C_{3} r_{1}^{2} r_{3}}{R^{5}}+\right. \\
& \left.-\frac{4 \mathrm{C}_{1} \mathrm{C}_{2}}{\mathrm{R}\left(\mathrm{R}+\mathrm{R}_{3}\right)}\left[1-\frac{\mathrm{r}_{1}^{2}}{\mathrm{R}\left(\mathrm{R}+\mathrm{R}_{3}\right)}-\frac{\mathrm{r}_{1}^{2}}{\mathrm{R}^{2}}\right]+\frac{6 \mathrm{cR} \mathrm{R}_{3}}{\mathrm{R}^{5}}\left[\mathrm{C}_{2} \mathrm{z}-2 \mathrm{vc}-\frac{5 \mathrm{r}_{1}^{2} \mathrm{z}}{\mathrm{R}^{2}}\right]\right\} \\
& \sigma_{12}^{* 3}=\mathrm{K}_{\mathrm{s}} \mathrm{r}_{1} \mathrm{r}_{2}\left\{-\frac{3 \mathrm{r}_{3}}{\mathrm{r}^{5}}-\frac{3 \mathrm{C}_{3} \mathrm{r}_{3}}{\mathrm{R}^{5}}-\frac{30 \mathrm{czR} \mathrm{R}_{3}}{\mathrm{R}^{7}}-\frac{4 \mathrm{C}_{1} \mathrm{C}_{2}}{\mathrm{R}^{2}\left(\mathrm{R}+\mathrm{R}_{3}\right)}\left[\frac{1}{\mathrm{R}+\mathrm{R}_{3}}+\frac{1}{\mathrm{R}}\right]\right\} \\
& \sigma_{13}^{* 3}=K_{s} r_{1}\left\{-\frac{C_{2}}{r^{3}}-\frac{3 r_{3}^{2}}{r^{5}}+\frac{C_{2}}{R^{3}}-\frac{3 C_{3} z R_{3}}{R^{5}}+\frac{3 c(3 z+c)}{R^{5}}-\frac{30 c z R_{3}^{2}}{R^{7}}\right\} \\
& \sigma_{22}^{* 3}=K_{s}\left\{\frac{C_{2} r_{3}}{r^{3}}-\frac{3 r_{2}^{2} r_{3}}{r^{5}}+\frac{C_{2}\left(3 r_{3}-4 v R_{3}\right)}{R^{3}}-\frac{3 C_{3} r_{2}^{2} r_{3}}{R^{5}}+\right. \\
& \left.-\frac{4 \mathrm{C}_{1} \mathrm{C}_{2}}{\mathrm{R}\left(\mathrm{R}+\mathrm{R}_{3}\right)}\left[1-\frac{\mathrm{r}_{2}^{2}}{\mathrm{R}\left(\mathrm{R}+\mathrm{R}_{3}\right)}-\frac{\mathrm{r}_{2}^{2}}{\mathrm{R}^{2}}\right]+\frac{6 \mathrm{cR} \mathrm{R}_{3}}{\mathrm{R}^{5}}\left[\mathrm{C}_{2} \mathrm{z}-2 \mathrm{vc}-\frac{5 \mathrm{r}_{2}^{2} \mathrm{z}}{\mathrm{R}^{2}}\right]\right\}
\end{aligned}
$$




$$
\begin{aligned}
& \sigma_{23}^{* 3}=\mathrm{K}_{\mathrm{s}} \mathrm{r}_{2}\left\{-\frac{\mathrm{C}_{2}}{\mathrm{r}^{3}}-\frac{3 \mathrm{r}_{3}^{2}}{\mathrm{r}^{5}}+\frac{\mathrm{C}_{2}}{\mathrm{R}^{3}}-\frac{3 \mathrm{C}_{3} \mathrm{zR}_{3}}{\mathrm{R}^{5}}+\frac{3 \mathrm{c}(3 \mathrm{z}+\mathrm{c})}{\mathrm{R}^{5}}-\frac{30 \mathrm{czR} \mathrm{R}_{3}^{2}}{\mathrm{R}^{7}}\right\} \\
& \sigma_{33}^{* 3}=\mathrm{K}_{\mathrm{s}}\left\{-\frac{\mathrm{C}_{2} \mathrm{r}_{3}}{\mathrm{r}^{3}}-\frac{3 \mathrm{r}_{3}^{2}}{\mathrm{r}^{5}}+\frac{\mathrm{C}_{2} \mathrm{r}_{3}}{\mathrm{R}^{3}}-\frac{3 \mathrm{C}_{3} \mathrm{zR}_{3}^{2}}{\mathrm{R}^{5}}+\frac{3 \mathrm{cR}_{3}(5 \mathrm{z}-\mathrm{c})}{\mathrm{R}^{5}}-\frac{30 \mathrm{czR} \mathrm{R}_{3}^{3}}{\mathrm{R}^{7}}\right\}
\end{aligned}
$$

O problema determinado por Mindlin veio preencher uma lacuna entre dois problemas já devidamente conhecidos e equacionados: o problema de Kelvin (item 2.4.1) e o problema de Boussinesq-Cerruti (NAKAGUMA, 1979), de aplicação de forças somente na superfície livre do semi-espaço infinito ( $\bar{\Gamma}$ na figura 2.4.4). Portanto, à medida que o parâmetro c cresce os valores encontrados nas expressões fundamentais de Mindlin coincidem com os obtidos através de Kelvin. Por outro lado, com o parâmetro c igual a zero, as expressões de Mindlin coincidem com as de Boussinesq-Cerruti (ver NAKAGUMA, 1979 e BARBIRATO, 1991).

Pode-se, de outra forma, definir o problema de Mindlin a partir do problema de Kelvin, somando-se a este uma parcela complementar (BREBBIA et al., 1984). Isto resulta em

$$
\begin{aligned}
& \left(u_{i j}^{*}\right)^{M}=\left(u_{i j}^{*}\right)^{\mathrm{K}}+\left(u_{i j}^{*}\right)^{C}, e \\
& \left(\sigma_{i j}^{* \ell}\right)^{M}=\left(\sigma_{i j}^{* \ell}\right)^{\mathrm{K}}+\left(\sigma_{i j}^{* \ell}\right)^{\mathrm{C}} .
\end{aligned}
$$

Nas expressões (2.4.8) e (2.4.10), os primeiros termos (cujos denominadores se referem à variável $r$ ), são os mesmos obtidos com a solução fundamental de Kelvin. Os demais, relativos à variável $\mathrm{R}$, são reunidos na parcela complementar.

As expressões fundamentais de Boussinesq-Cerruti são muito simples, o que torna seu emprego mais indicado do que as de Mindlin, para forças agindo na superfície livre de trações. São elas: 


$$
\begin{aligned}
& u_{11}^{*}=\frac{1}{2 \pi \mathrm{Gr}}\left(\mathrm{C}_{1}+v \mathrm{r}_{, 1}^{2}\right) \\
& \mathrm{u}_{12}^{*}=\frac{1}{2 \pi \mathrm{Gr}} v \mathrm{r}_{, 1} \mathrm{r}_{, 2} \\
& \mathrm{u}_{13}^{*}=-\frac{1}{2 \pi \mathrm{Gr}} \mathrm{r}_{, 1}\left(\frac{1}{2}-v\right) \\
& \mathrm{u}_{21}^{*}=\mathrm{u}_{12}^{*} \\
& \mathrm{u}_{22}^{*}=\frac{1}{2 \pi \mathrm{Gr}}\left(\mathrm{C}_{1}+v \mathrm{r}_{, 2}^{2}\right) \\
& \mathrm{u}_{23}^{*}=-\frac{1}{2 \pi \mathrm{Gr}} \mathrm{r}_{, 2}\left(\frac{1}{2}-v\right) \\
& \mathrm{u}_{31}^{*}=-\mathrm{u}_{13}^{*} \\
& \mathrm{u}_{32}^{*}=-\mathrm{u}_{23}^{*} \\
& \mathrm{u}_{33}^{*}=\frac{1}{2 \pi \mathrm{Gr}} \mathrm{C}_{1} \\
& \mathrm{e}_{\mathrm{ij}}^{*}=0
\end{aligned}
$$

\section{5 - EQUAÇÕES INTEGRAIS DE CONTORNO}

As representações integrais para pontos do domínio e do contorno servem de base para o Método dos Elementos de Contorno (ver BREBBIA et al., 1984, ROCHA, 1988, dentre outros).

\subsection{1 - Para Pontos do Domínio}

A representação integral para pontos situados no domínio do sólido tridimensional pode ser obtida através da utilização da técnica dos resíduos ponderados ou do teorema de Betti, da Reciprocidade Estática. Com os resíduos 
ponderados a formulação do Método dos Elementos de Contorno ganha portabilidade para a associação com outros métodos numéricos (por exemplo, Método dos Elementos Finitos). Mas foi utilizando o teorema de Betti que Somigliana ${ }^{1}$ chegou à representação integral de deslocamento, conhecida como identidade Somigliana, apresentada da seguinte forma:

$$
\begin{aligned}
\mathrm{u}_{\mathrm{i}}(\mathrm{s}) & =-\int_{\Gamma} \mathrm{p}_{\mathrm{ij}}^{*}(\mathrm{~s}, \mathrm{Q}) \mathrm{u}_{\mathrm{j}}(\mathrm{Q}) \mathrm{d} \Gamma(\mathrm{Q})+\int_{\Gamma} \mathrm{u}_{\mathrm{ij}}^{*}(\mathrm{~s}, \mathrm{Q}) \mathrm{p}_{\mathrm{j}}(\mathrm{Q}) \mathrm{d} \Gamma(\mathrm{Q})+ \\
& +\int_{\Omega} \mathrm{u}_{\mathrm{ij}}^{*}(\mathrm{~s}, \mathrm{q}) \mathrm{b}_{\mathrm{j}}(\mathrm{q}) \mathrm{d} \Omega(\mathrm{q})
\end{aligned}
$$

A equação (2.5.1) fornece o deslocamento no ponto s do domínio na direção cartesiana i, a partir dos valores de deslocamentos e forças de superfície no ponto $\mathrm{Q}$ do contorno e, na presença de forças de volume, das componentes $b_{j}$ no ponto $q$ do domínio.

Uma vez determinada a representação integral para deslocamentos em pontos do domínio, basta utilizá-la na equação (2.3.4) e substituir o resultado na lei de Hooke (2.3.5), para que se chegue na representação integral de tensões para pontos do domínio.

$$
\begin{aligned}
\sigma_{\mathrm{ij}}(\mathrm{s}) & =-\int_{\Gamma} \mathrm{S}_{\mathrm{ijk}}^{*}(\mathrm{~s}, \mathrm{Q}) \mathrm{u}_{\mathrm{k}}(\mathrm{Q}) \mathrm{d} \Gamma(\mathrm{Q})+\int_{\Gamma} \mathrm{D}_{\mathrm{ijk}}^{*}(\mathrm{~s}, \mathrm{Q}) \mathrm{p}_{\mathrm{k}}(\mathrm{Q}) \mathrm{d} \Gamma(\mathrm{Q})+ \\
& +\int_{\Omega} \mathrm{D}_{\mathrm{ijk}}^{*}(\mathrm{~s}, \mathrm{q}) \mathrm{b}_{\mathrm{k}}(\mathrm{q}) \mathrm{d} \Omega(\mathrm{q})
\end{aligned}
$$

A expressão (2.5.2) fornece os valores das tensões no ponto interno s a partir dos valores de deslocamentos e forças de superfície do ponto Q do contorno, acrescidos da parcela relativa à forças de volume, ponto q do domínio, quando considerada. Os tensores $\mathrm{S}_{\mathrm{ijk}}^{*}$ e $\mathrm{D}_{\mathrm{ijk}}^{*}$ que nela aparecem são determinados através da derivação dos tensores de deslocamentos e forças de superfície do problema fundamental, respectivamente. Portanto, suas componentes são dadas pelas

\footnotetext{
${ }^{1}$ SOMIGLIANA, C. (1886). Sopra l'equilibrio di un corpo elastico isotropo.Il Nuovo cimento. Ser. 3 , v. 17-20.
} 
expressões inerentes a cada solução fundamental escolhida. Assim, para Kelvin, temse as seguintes expressões:

$$
\begin{aligned}
\mathrm{S}_{\mathrm{ijk}}^{*} & =\frac{\mathrm{G}}{4 \pi(1-v) \mathrm{r}^{3}}\left\{3 \mathrm{r}_{, \mathrm{n}}\left[(1-2 v) \delta_{\mathrm{ij}} \mathrm{r}_{, \mathrm{k}}+v\left(\delta_{\mathrm{ik}} \mathrm{r}_{, \mathrm{j}}+\delta_{\mathrm{jk}} \mathrm{r}_{, \mathrm{i}}\right)-5 \mathrm{r}_{, \mathrm{i}} \mathrm{r}_{, \mathrm{j}} \mathrm{r}_{, \mathrm{k}}\right]+\right. \\
& +3 v\left(\mathrm{n}_{\mathrm{i}} \mathrm{r}_{, \mathrm{j}} \mathrm{r}_{, \mathrm{k}}+\mathrm{n}_{\mathrm{j}} \mathrm{r}_{, \mathrm{i}} \mathrm{r}_{, \mathrm{k}}\right)+(1-2 v)\left(3 \mathrm{n}_{\mathrm{k}} \mathrm{r}_{, \mathrm{i}} \mathrm{r}_{, \mathrm{j}}+\mathrm{n}_{\mathrm{j}} \delta_{\mathrm{ik}}+\mathrm{n}_{\mathrm{i}} \delta_{\mathrm{jk}}\right)+ \\
& \left.-(1-4 v) \mathrm{n}_{\mathrm{k}} \delta_{\mathrm{ij}}\right\} \\
\mathrm{D}_{\mathrm{ijk}}^{*} & =\frac{1}{8 \pi(1-v) \mathrm{r}^{2}}\left\{(1-2 v)\left(\delta_{\mathrm{ki}} \mathrm{r}_{, \mathrm{j}}+\delta_{\mathrm{kj}} \mathrm{r}_{, \mathrm{i}}-\delta_{\mathrm{ij}} \mathrm{r}_{, \mathrm{k}}\right)+3 \mathrm{r}_{\mathrm{i}} \mathrm{r}_{, \mathrm{j}} \mathrm{r}_{, \mathrm{k}}\right\} .
\end{aligned}
$$

As expressões dos tensores $\mathrm{S}_{\mathrm{ijk}}^{*}$ e $\mathrm{D}_{\mathrm{ijk}}^{*}$ para a solução fundamental de Mindlin estão na forma implícita, uma vez que as expressões de deslocamentos (2.4.8) e de forças de superfície (2.4.9), tendo em vista (2.4.10), são extensas. Assim,

$$
\begin{aligned}
& \mathrm{S}_{\mathrm{ijk}}^{*}=\left[\lambda \delta_{\mathrm{ij}} \sigma_{\mathrm{k} \ell, \mathrm{j}}^{*_{\mathrm{m}}}+\mathrm{G}\left(\sigma_{\mathrm{k} \ell, \mathrm{j}}^{*_{\mathrm{i}}}+\sigma_{\mathrm{k} \ell, \mathrm{i}}^{* \mathrm{j}}\right)\right] \mathrm{n}_{\ell} \\
& \mathrm{e} \\
& \mathrm{D}_{\mathrm{ijk}}^{*}=-\sigma_{\mathrm{jk}}^{*_{\mathrm{i}}} .
\end{aligned}
$$

As derivadas do tensor das tensões, obtidas de LAETHEM et al. (1984) encontram-se apresentadas no anexo B.

\subsection{2 - Para Pontos do Contorno}

A identidade Somigliana (2.5.1) é válida apenas para pontos contidos no interior do sólido em estudo. Para o Método dos Elementos de Contorno é fundamental que se tenha a expressão correspondente para pontos que pertençam ao contorno $\Gamma$. O artifício utilizado correntemente é o de transformar o ponto de contorno em um de domínio, onde se pode aplicar a identidade Somigliana, 
acrescentando-se parte de uma esfera $\left(\Omega_{\varepsilon}\right)$ centrada no ponto do contorno e de raio $\varepsilon$ (ver figura 2.5.1). Assim, um ponto $\mathrm{S}$ do contorno passa a ser um ponto $\mathrm{s}$ do domínio.

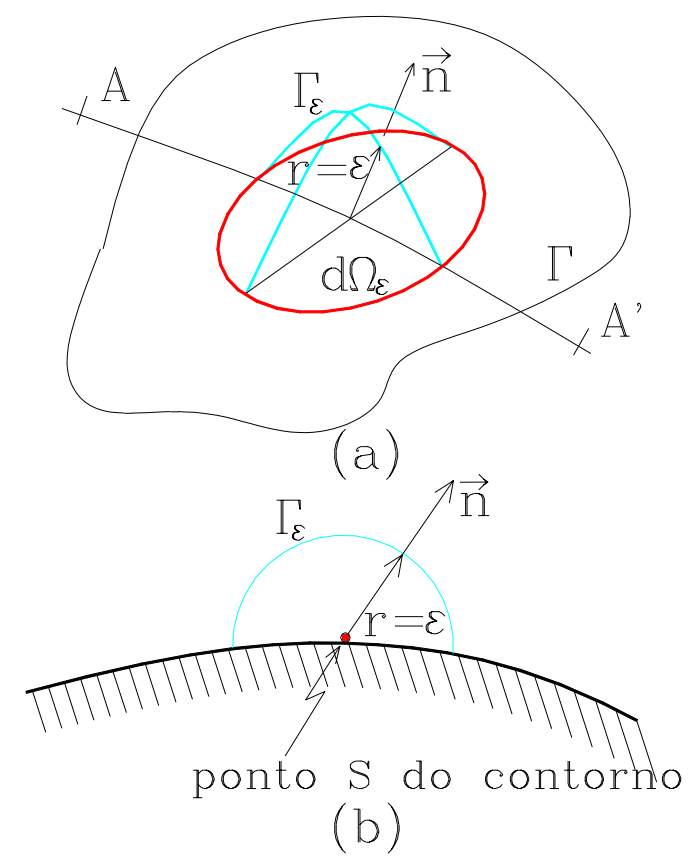

FIGURA 2.5.1 - Transformação de um ponto do contorno para o domínio: a) acréscimo no domínio em torno do ponto $\mathrm{S} \rightarrow \mathrm{s}$; e b) detalhe de $\mathrm{AA}^{\prime}$.

Com a modificação sugerida, um novo domínio fica estabelecido: $\Omega+\Omega_{\varepsilon}$ O Contorno do sólido também sofre alterações, passando a ser $\Gamma-\bar{\Gamma}+\Gamma_{\varepsilon}$. Portanto, a identidade Somigliana (2.5.1) passa a ser escrita com novos trechos:

$$
\begin{aligned}
u_{i}(s) & =-\int_{\Gamma-\bar{\Gamma}+\Gamma_{\varepsilon}} p_{i j}^{*}(s, Q) u_{j}(Q) d \Gamma(Q)+\int_{\Gamma-\bar{\Gamma}+\Gamma_{\varepsilon}} u_{i j}^{*}(s, Q) p_{j}(Q) d \Gamma(Q)+ \\
& +\int_{\Omega+\Omega_{\varepsilon}} u_{i j}^{*}(s, q) b_{j}(q) d \Omega(q)
\end{aligned}
$$

Agora, encontrada a identidade (2.5.7), deve-se efetuar o procedimento inverso, ou seja, o de levar as novas parcelas (correspondentes ao acréscimo de domínio) ao limite quando $\varepsilon \rightarrow 0, \Omega_{\varepsilon}, \bar{\Gamma}, \Gamma_{\varepsilon} \rightarrow 0$ e o ponto volta a ser de contorno, quando $s \rightarrow$ S . Em ROCHA (1988), por exemplo, são mostrados todos os detalhes destes limites. A expressão resultante de particular interesse para este trabalho é: 


$$
\begin{aligned}
\mathrm{c}_{\mathrm{ij}}(\mathrm{S}) \mathrm{u}_{\mathrm{j}}(\mathrm{S}) & =-\int_{\Gamma} \mathrm{p}_{\mathrm{ij}}^{*}(\mathrm{~S}, \mathrm{Q}) \mathrm{u}_{\mathrm{j}}(\mathrm{Q}) \mathrm{d} \Gamma(\mathrm{Q})+\int_{\Gamma} \mathrm{u}_{\mathrm{ij}}^{*}(\mathrm{~S}, \mathrm{Q}) \mathrm{p}_{\mathrm{j}}(\mathrm{Q}) \mathrm{d} \Gamma(\mathrm{Q})+ \\
& +\int_{\Omega} \mathrm{u}_{\mathrm{ij}}^{*}(\mathrm{~S}, \mathrm{q}) \mathrm{b}_{\mathrm{j}}(\mathrm{q}) \mathrm{d} \Omega(\mathrm{q}),
\end{aligned}
$$

onde $\mathrm{c}_{\mathrm{ij}}(\mathrm{S})=\frac{1}{2} \mathbf{I}$ para pontos de um contorno sem angulosidades ("smooth"); e I é a matriz identidade (aqui de ordem 3x3, para cada ponto de colocação S).

A identidade encontrada para pontos do contorno é semelhante àquela para pontos do domínio (2.5.1) e é também válida para pontos localizados fora do domínio do sólido. O coeficiente multiplicador $\mathrm{c}_{\mathrm{ij}}$ passa a caracterizar a natureza da localização do ponto, assumindo os valores:

$$
\mathrm{c}_{\mathrm{ij}}=\mathbf{I}\left\{\begin{array}{l}
0, \text { para pontos externos ao dominio } \Omega \\
\frac{1}{2}, \text { para pontos do contorno } \Gamma \\
1, \text { para pontos internos ao dominio } \Omega
\end{array}\right.
$$

A representação integral (2.5.8) é determinada considerando-se que o sólido tridimensional é definido mantendo-se a orientação do vetor normal ao seu contorno sempre para fora. Assim, os problemas relacionados a escavações e cavidades ainda não estão equacionados. Uma vez que neste trabalho utiliza-se a solução fundamental de Mindlin, e sua aplicação em escavações e cavidades próximas à superfície livre é imediata, faz-se necessário conhecer a representação integral para esses problemas.

Considere-se um sólido tridimensional, elástico-linear, homogêneo, de domínio $\bar{\Omega}$ e contorno $\bar{\Gamma}$, e que contém uma cavidade definida pelo contorno $\Gamma$ (ver figuras 2.5.2 e 2.5.3). Este sólido, definido por $\Gamma+\bar{\Omega}+\bar{\Gamma}$, em forma de uma esfera de raio $r_{o}$ centrado em um ponto $S$ do contorno $\Gamma$ da cavidade, está contido no espaço $\Omega^{*}$ infinito (ou semi-infinito). O procedimento utilizado para obter a sua representação integral passa por acrescentar à identidade (2.5.8) parcelas correspondentes ao domínio $\bar{\Omega}$ e contorno $\bar{\Gamma}$ e, depois, fazer com que o raio da esfera tenda para o infinito $\left(r_{o} \rightarrow \infty\right)$, situação limite do problema. Após efetuaremse os limites descritos, por exemplo, em ROCHA (1988), verifica-se que a expressão 
resultante é a mesma (2.5.8), ou seja, a representação integral determinada para os problemas de geometria finita também é válida para regiões infinitas, e, portanto, fica estabelecida uma formulação para análise de problemas de escavações.

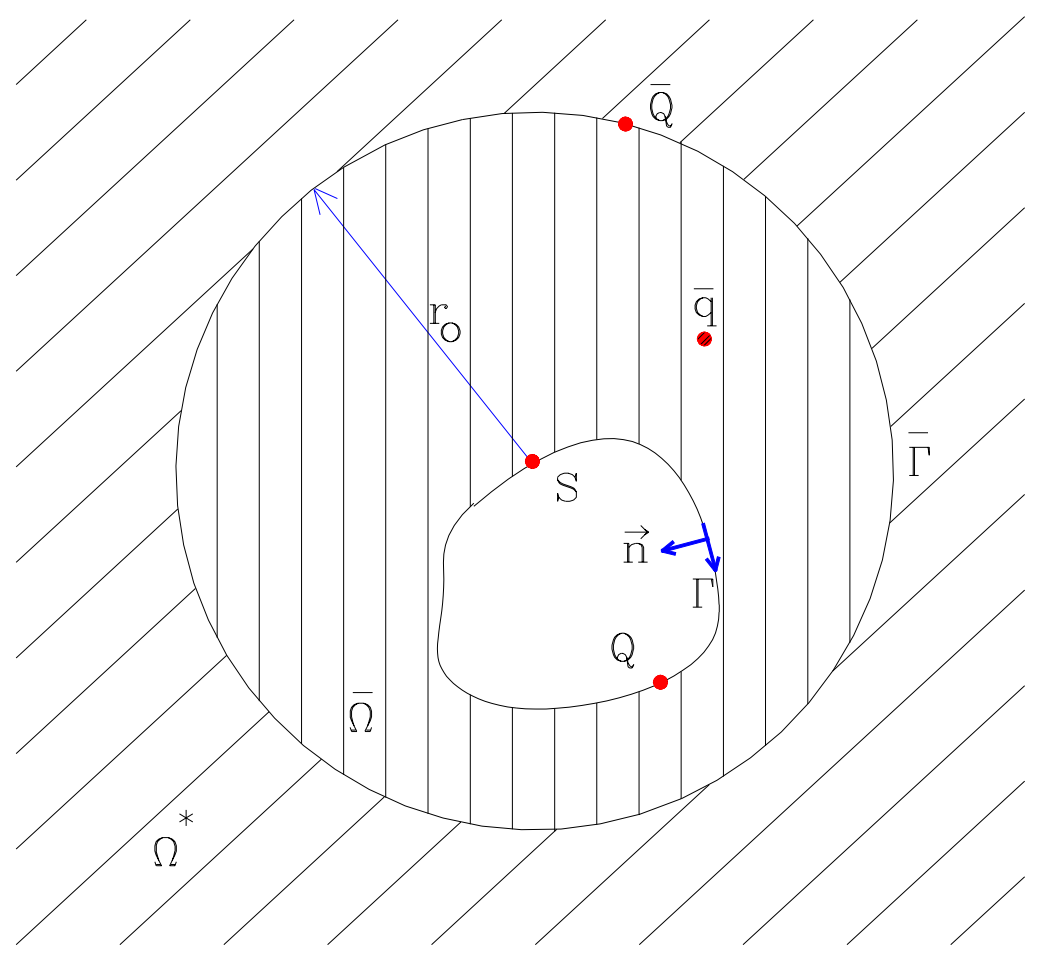

FIGURA 2.5.2 - Região infinita - espaço de Kelvin.

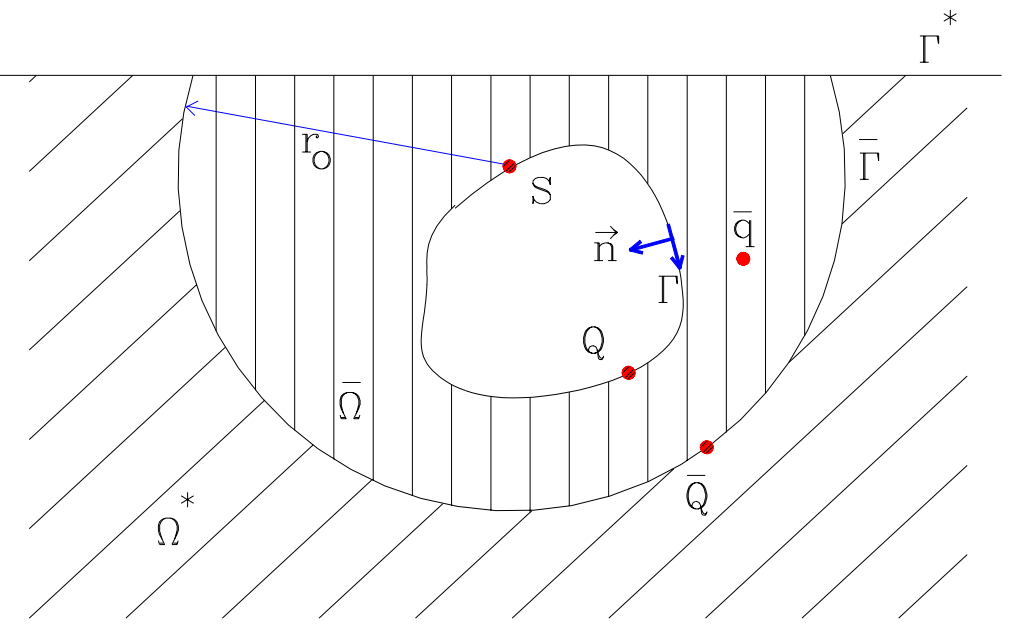

FIGURA 2.5.3 - Região semi-infinita - espaço de Mindlin. 


\section{6 - MÉTODO DOS ELEMENTOS DE CONTORNO}

Até aqui foi apresentada uma formulação, baseada nas equações integrais de contorno, que permite a análise de sólidos elásticos tridimensionais, isotrópicos e homogêneos. Entretanto, sua utilização prática se verifica com a transformação em equações algébricas. Portanto, neste item é detalhado o procedimento numérico conhecido por Método dos Elementos de Contorno (MEC). As matrizes serão representadas por caracteres em negrito.

\subsection{1 - Discretizações}

Toma-se o sólido que se deseja analisar e mapeia-se todo o seu contorno por um número finito de elementos: planos ou curvos, triangulares ou quadrangulares (conforme a figura 2.6.1).
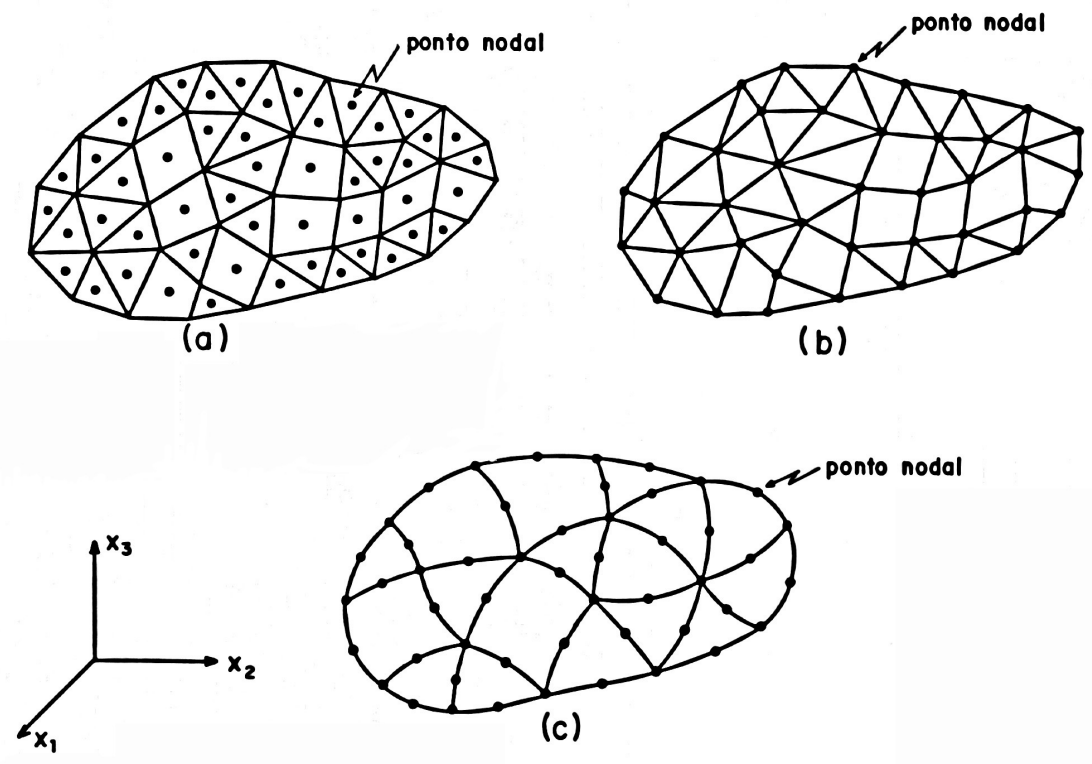

FIGURA 2.6.1 - Sólidos discretizados por elementos de contorno: (a) constante; (b) linear; e (c) quadrático. 
Um elemento qualquer é geometricamente definido pelas coordenadas cartesianas de seus pontos nodais, $\mathbf{X}^{\mathrm{n}}$. Adotando-se as funções de interpolação $\Psi$, as coordenadas de um ponto qualquer do elemento são obtidas através de:

$$
\mathbf{X}=\Psi^{\mathrm{T}} \mathbf{X}^{\mathrm{n}}
$$

As variáveis físicas do problema (deslocamentos e forças de superfícies) são aproximadas através de funções interpoladoras utilizando-se os respectivos valores nodais (valores dos nós funcionais). De acordo com a escolha das funções interpoladoras, os elementos de contorno recebem uma classificação: constantes; lineares; quadráticos; e de ordem superior, para funções polinomiais (ver figura 2.6.1).

Os valores de deslocamentos e forças de superfície (u e p), aproximados por seus valores nodais $\left(\mathbf{U}^{\mathrm{n}}\right.$ e $\left.\mathbf{P}^{\mathrm{n}}\right)$, são portanto expressos por:

$$
\begin{aligned}
& \mathbf{u}=\phi^{\mathrm{T}} \mathbf{U}^{\mathrm{n}} \\
& \mathbf{p}=\phi^{\mathrm{T}} \mathbf{P}^{\mathrm{n}},
\end{aligned}
$$

onde $\phi$ são as funções interpoladoras.

A discretização do domínio é feita, de maneira mais direta, dividindo-o em células tridimensionais, geralmente na forma de hexaedros e tetraedros. Porém, a equação básica do Método dos Elementos de Contorno (2.5.8) fica expressa por um termo de domínio, o que de certa forma não está coerente com o princípio do método. Outras técnicas são apresentadas na tentativa de utilizar somente elementos de contorno, dentre elas a Reciprocidade Dual e a Integração Direta (que serão abordadas em detalhes no próximo capítulo). Mas, para permitir a discretização geral de (2.5.8) por ora, seguem-se as relações necessárias para o uso de células. As coordenadas $\mathbf{X}_{\mathbf{c}}$ de um ponto qualquer da célula ficam definidas em termos das funções interpoladoras $\Psi_{c}$ e das coordenadas $\mathbf{X}_{\mathbf{c}}^{\mathbf{n}}$ dos pontos geométricos da mesma. 


$$
\mathbf{X}_{\mathrm{c}}=\Psi_{\mathrm{c}}^{\mathrm{T}} \mathbf{X}_{\mathrm{c}}{ }^{\mathrm{n}}
$$

As forças de volume b, por sua vez, podem ser aproximadas sobre cada célula por funções interpoladoras $\phi_{\mathrm{c}}$ e valores nodais $\mathbf{B}^{\mathbf{n}}$ (nos nós funcionais).

A fim de utilizar as expressões aproximadas mostradas neste item, é conveniente apresentar a identidade (2.5.8) na forma matricial, onde a substituição daquelas é imediata. Assim,

$$
\begin{aligned}
\mathbf{c}(\mathrm{S}) \mathbf{u}(\mathrm{S}) & =-\int_{\Gamma} \mathbf{p}^{*}(\mathrm{~S}, \mathrm{Q}) \mathbf{u}(\mathrm{Q}) \mathrm{d} \Gamma(\mathrm{Q})+\int_{\Gamma} \mathbf{u}^{*}(\mathrm{~S}, \mathrm{Q}) \mathbf{p}(\mathrm{Q}) \mathrm{d} \Gamma(\mathrm{Q})+ \\
& +\int_{\Omega} \mathbf{u}^{*}(\mathrm{~S}, \mathrm{q}) \mathbf{b}(\mathrm{q}) \mathrm{d} \Omega(\mathrm{q})
\end{aligned}
$$

Portanto, aproximando o contorno do sólido em "J" elementos, com "N" pontos nodais (nós funcionais), e o seu domínio em "M" células, a representação integral para deslocamentos, (2.6.4), passa a ser:

$$
\begin{aligned}
\mathbf{c}(\mathrm{S}) \mathbf{u}(\mathrm{S}) & =-\sum_{\mathrm{j}=1}^{\mathrm{J}}\left[\int_{\Gamma_{\mathrm{j}}} \mathbf{p}^{*}(\mathrm{~S}, \mathrm{Q}) \phi^{\mathrm{T}}(\mathrm{Q}) \mathrm{d} \Gamma(\mathrm{Q})\right] \mathbf{U}^{\mathrm{n}}+ \\
& +\sum_{\mathrm{j}=1}^{\mathrm{J}}\left[\int_{\Gamma_{\mathrm{j}}} \mathbf{u}^{*}(\mathrm{~S}, \mathrm{Q}) \phi^{\mathrm{T}}(\mathrm{Q}) \mathrm{d} \Gamma(\mathrm{Q}) \mathbf{P}^{\mathbf{n}}+\right. \\
& +\sum_{\mathrm{m}=1}^{\mathrm{M}}\left[\int_{\Omega_{\mathrm{m}}} \mathbf{u}^{*}(\mathrm{~S}, \mathrm{q}) \phi_{\mathrm{c}}^{\mathrm{T}}(\mathrm{q}) \mathrm{d} \Omega(\mathrm{q})\right] \mathbf{B}^{\mathbf{n}}
\end{aligned}
$$

Uma vez resolvidas as integrais de (2.6.5), com procedimentos que serão vistos no item 2.6.3, e escrevendo-as para cada ponto de colocação $\mathrm{S}$, tem-se a seguinte equação matricial:

$$
\overline{\mathbf{c}} \mathbf{U}+\hat{\mathbf{H}} \mathbf{U}=\mathbf{G P}+\mathbf{D B},
$$


onde as matrizes $\hat{\mathbf{H}}, \mathbf{G}$ e D vêm, respectivamente, dos somatórios das integrais sobre cada elemento j, definidos em (2.6.5).

Observando-se a equação (2.6.6), nota-se que é possível agrupar as matrizes que multiplicam o vetor dos valores nodais de deslocamentos $(\mathbf{H}=\overline{\mathbf{c}}+\hat{\mathbf{H}})$, resultando na equação:

$$
\mathbf{H U}=\mathbf{G P}+\mathbf{D B} .
$$

Um sistema de equações algébricas pode ser montado a partir da definição das matrizes $\mathbf{H}, \mathbf{G}$ e $\mathbf{D}$ e dos valores prescritos de deslocamentos $\mathbf{U}$, forças de superfície $\mathbf{P}$ e forças de volume $\mathbf{B}$. Assim,

$$
\mathbf{A} \mathbf{V}_{\mathrm{DF}}=\mathbf{F} .
$$

onde:

A é uma matriz de ordem $3 \mathrm{Nx} 3 \mathrm{~N}$ que contem elementos das matrizes $\mathbf{H}$ e G devidamente trocados (troca de colunas) para agrupar todas incógnitas do lado esquerdo da igualdade, sejam elas deslocamentos ou forças de superfície;

$\mathbf{V}_{\mathrm{DF}}$ é o vetor das incógnitas, deslocamentos e forças de superfícies, de acordo com as condições de contorno; e

F o vetor independente formado pela multiplicação dos coeficientes das matrizes $\mathbf{H}$ e $\mathbf{G}$ relativos às componentes prescritas de deslocamentos e forças de superfície, somando-se, ainda, valores da parcela das forças de volume.

A solução do sistema (2.6.8) é simples, podendo ser representada por:

$$
\mathbf{V}_{\mathrm{DF}}=\mathbf{A}^{-1} \mathbf{F}
$$

Podem ser utilizados os já consagrados métodos de resolução de sistema linear de equações, como por exemplo o algoritmo de Gauss. Entretanto, é necessário lembrar-se que a matriz A é cheia e não simétrica. 


\subsection{2 - Elementos de Contorno}

Neste trabalho utiliza-se o elemento triangular plano para a discretização da superfície de contorno do sólido tridimensional. Este elemento é bastante conhecido, uma vez que foi desenvolvido para uso no Método dos Elementos Finitos - MEF (COOK et al., 1989). Portanto, as coordenadas oblíquas (ou homogêneas) e as funções interpoladoras utilizadas no MEC são praticamente as mesmas utilizadas nas formulações do MEF. Vale ressaltar uma diferença entre as exigências feitas para ambos os métodos: para a formulação do MEF é necessário garantir a continuidade de deslocamentos, enquanto que para o MEC não.

A geometria do elemento triangular plano é determinada a partir das coordenadas cartesianas dos três nós posicionados nos vértices (nós geométricos), para o sistema global de coordenadas $\left(\mathrm{X}_{\mathrm{i}}\right)$ ou para um sistema local de coordenadas cartesianas $\left(\mathrm{x}_{\mathrm{i}}\right)$. Recomenda-se ainda a adoção de um outro sistema local utilizando as chamadas coordenadas homogêneas ou oblíquas $\left(\xi_{\mathrm{i}}\right)$. A figura 2.6.2 mostra o elemento e seus sistemas de coordenadas. No item 2.6.3, tem-se os procedimentos de integração que justificam a utilização de vários sistemas de coordenadas.

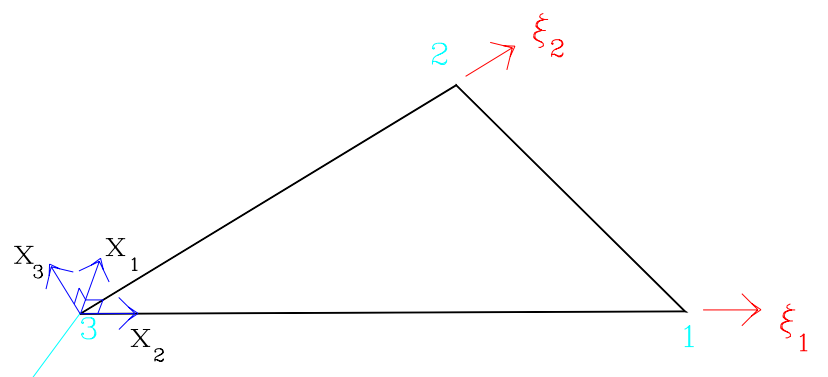

FIGURA 2.6.2 - Geometria do elemento triangular plano a partir dos diversos sistemas de coordenadas.

Tendo em vista as coordenadas homogêneas, um ponto qualquer do elemento é determinado pela expressão (2.6.1) modificada, ou seja, 


$$
\mathbf{X}=\Psi^{\mathrm{T}}\left(\xi_{\mathrm{i}}\right) \mathbf{X}^{\mathrm{n}}
$$

A variação das coordenadas homogêneas é de 0 a 1 e, por definição, a coordenada $\xi_{3}$ é função das outras duas $\left(\xi_{1}\right.$ e $\left.\xi_{2}\right)$, como mostra a figura 2.6.3.

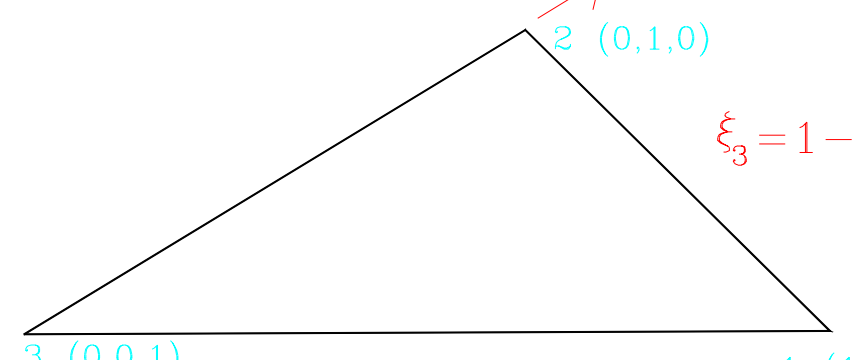

FIGURA 2.6.3 - Variação das coordenadas homogêneas.

A formulação integral até aqui apresentada diz respeito ao sistema de coordenadas cartesianas. Na mudança para o sistema de coordenadas homogêneas, aparece um valor que relaciona os dois: o Jacobiano de transformação (denotado por $|G|$ ), a partir da utilização da regra da cadeia:

$$
\frac{\partial \mathrm{r}}{\partial \xi_{\mathrm{k}}}=\frac{\partial \mathrm{X}_{\mathrm{j}}}{\partial \xi_{\mathrm{k}}} \frac{\partial \mathrm{r}}{\partial \mathrm{X}_{\mathrm{j}}}=\mathrm{G}_{\mathrm{kj}} \frac{\partial \mathrm{r}}{\partial \mathrm{X}_{\mathrm{j}}}
$$

ou, matricialmente,

$$
\left\{\begin{array}{c}
\frac{\partial \mathrm{r}}{\partial \xi_{1}} \\
\frac{\partial \mathrm{r}}{\partial \xi_{2}}
\end{array}\right\}=\left[\begin{array}{lll}
\frac{\partial \mathrm{X}_{1}}{\partial \xi_{1}} & \frac{\partial \mathrm{X}_{2}}{\partial \xi_{1}} & \frac{\partial \mathrm{X}_{3}}{\partial \xi_{1}} \\
\frac{\partial \mathrm{X}_{1}}{\partial \xi_{2}} & \frac{\partial \mathrm{X}_{2}}{\partial \xi_{2}} & \frac{\partial \mathrm{X}_{3}}{\partial \xi_{2}}
\end{array}\right]\left\{\begin{array}{c}
\frac{\partial \mathrm{r}}{\partial \mathrm{X}_{1}} \\
\frac{\partial \mathrm{r}}{\partial \mathrm{X}_{2}} \\
\frac{\partial \mathrm{r}}{\partial \mathrm{X}_{3}}
\end{array}\right\}
$$

Obtendo-se o determinante para a matriz G em (2.6.11), chega-se ao valor do Jacobiano de transformação para o elemento triangular de área A, equivalente a: 


$$
|\mathrm{G}|=2 \mathrm{~A} .
$$

Uma vez discutido o aspecto da geometria do elemento a ser empregado ao longo deste trabalho, passa-se a abordar as funções de interpolação que são utilizadas para as variáveis do problema: deslocamentos e forças de superfície.

\subsubsection{1 - Elemento Constante}

Utilizando-se a mais simples das funções aproximadoras, o elemento passa a ser denominado constante. Possui um único ponto funcional, cuja posição é no centróide de sua área. Trata-se de um elemento fácil de ser implementado de boas respostas obtidas. A precisão dos valores é conseguida a partir de uma discretização com um número relativamente grande de elementos, se comparada com outras discretizações com elementos lineares, quadráticos, etc. Uma vantagem importante no elemento constante é que as descontinuidades são perfeitamente consideradas. A figura 2.6.4 mostra o elemento constante com sua função aproximadora $\phi$ e também a definição do nó funcional.

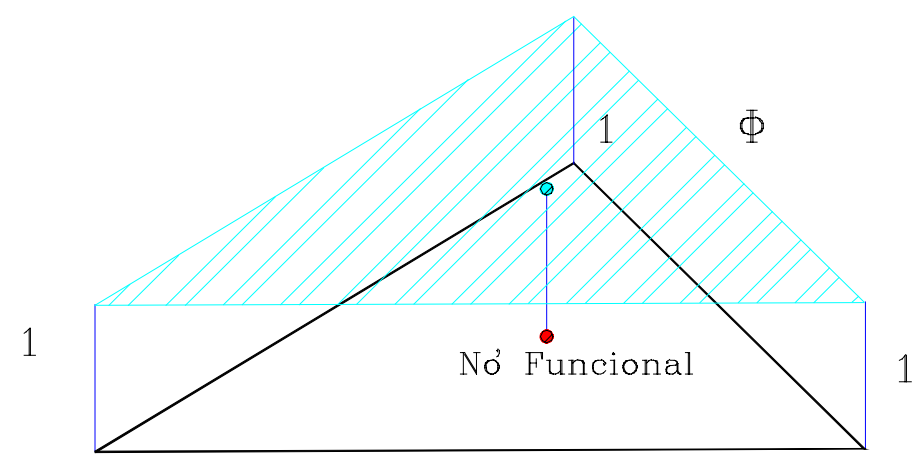

FIGURA 2.6.4 - Elemento triangular constante.

As aproximações dos valores de deslocamentos e forças de superfície (sobre cada elemento) são expressas por:

$$
\begin{aligned}
& \mathbf{u}=\phi^{\mathrm{T}}\left(\xi_{\mathrm{i}}\right) \mathbf{U}^{\mathbf{n}}=\mathbf{I U}^{\mathbf{n}} \\
& \mathbf{p}=\phi^{\mathrm{T}}\left(\xi_{\mathrm{i}}\right) \mathbf{P}^{\mathbf{n}}=\mathbf{I P}^{\mathbf{n}},
\end{aligned}
$$


onde $\mathbf{U}^{\mathrm{n}}$ e $\mathbf{P}^{\mathrm{n}}$ possuem os valores de deslocamentos e forças de superfície, respectivamente, no centróide do elemento (nó funcional).

\subsubsection{2 - Elemento Linear}

O elemento linear permite aproximações muito boas das variáveis do problema. Porém, surgem alguns problemas com relação à descontinuidade de forças de superfície, solucionados pela aplicação conveniente dos tipos de elementos lineares que existem: contínuo, de transição e descontínuo (ver figura 2.6.5).

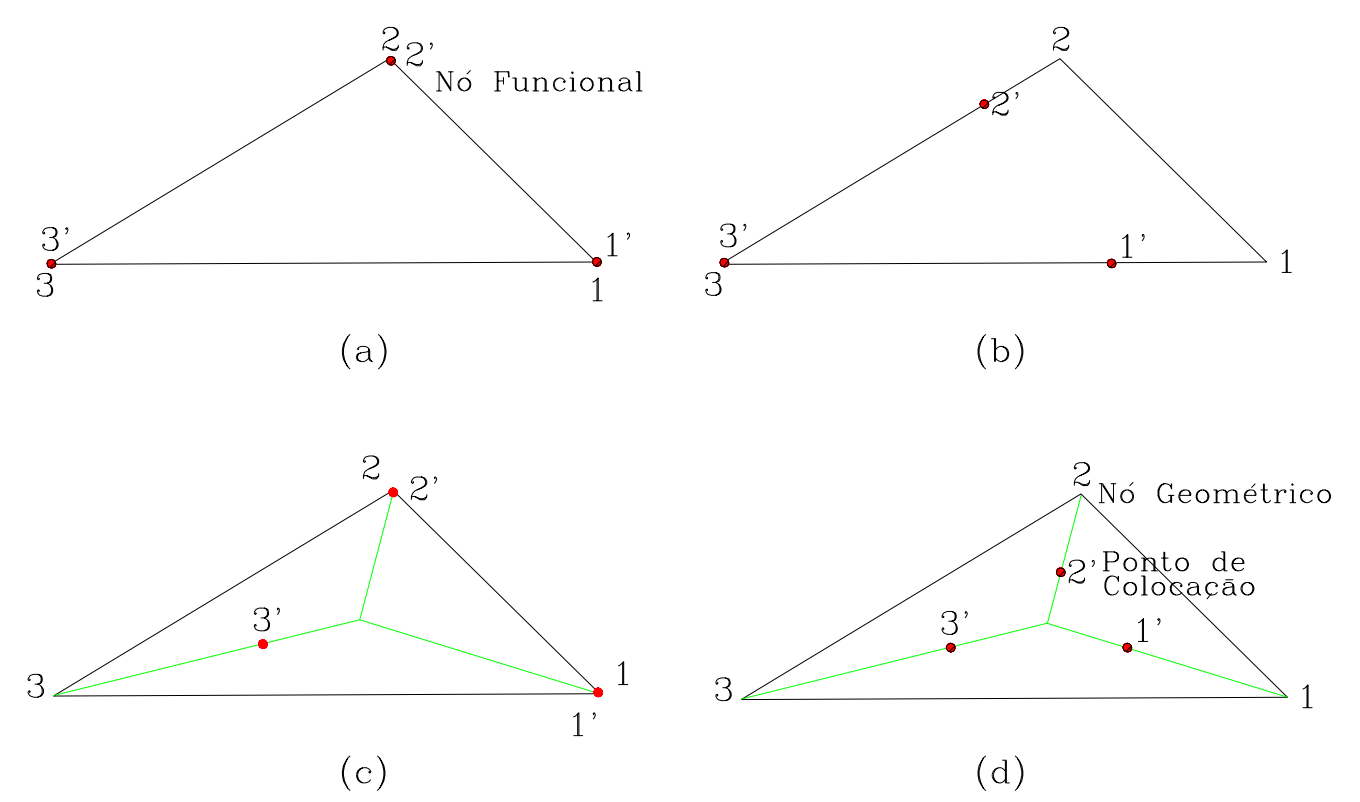

FIGURA 2.6.5 - Variações do elemento linear: (a) contínuo; (b) e (c) de transição; e (d) descontínuo.

O elemento linear contínuo, ou isoparamétrico linear, tem seus nós funcionais coincidentes com os nós geométricos (figura 2.6.5a), o que significa que a descontinuidade não pode ser modelada através de seu emprego.

O elemento linear descontínuo, não-conforme ou de colocação não nodal, tem os três nós de colocação deslocados de seus nós e estão associados a um único elemento (figura 2.6.5d). Permite, portanto, representar descontinuidades. 
O elemento linear de transição tem alguns de seus pontos de colocação coincidentes com os nós geométricos (figuras 2.6.5b-c). Como indica o próprio nome, é ele que tem a tarefa de unir os elementos descontínuos aos contínuos, permitindo uma discretização mais racional (com o número de pontos de colocação estritamente necessário). Sua formulação é uma combinação das formulações dos elementos contínuos e descontínuos.

\section{A) Elemento Triangular Linear Contínuo}

O elemento triangular isoparamétrico linear tem seus três pontos de colocação coincidentes com os nós geométricos, conforme mostra a figura 2.6.6. Note-se as funções de interpolação $\phi$ observando-se a localização de seus valores extremos, 0 e 1 .
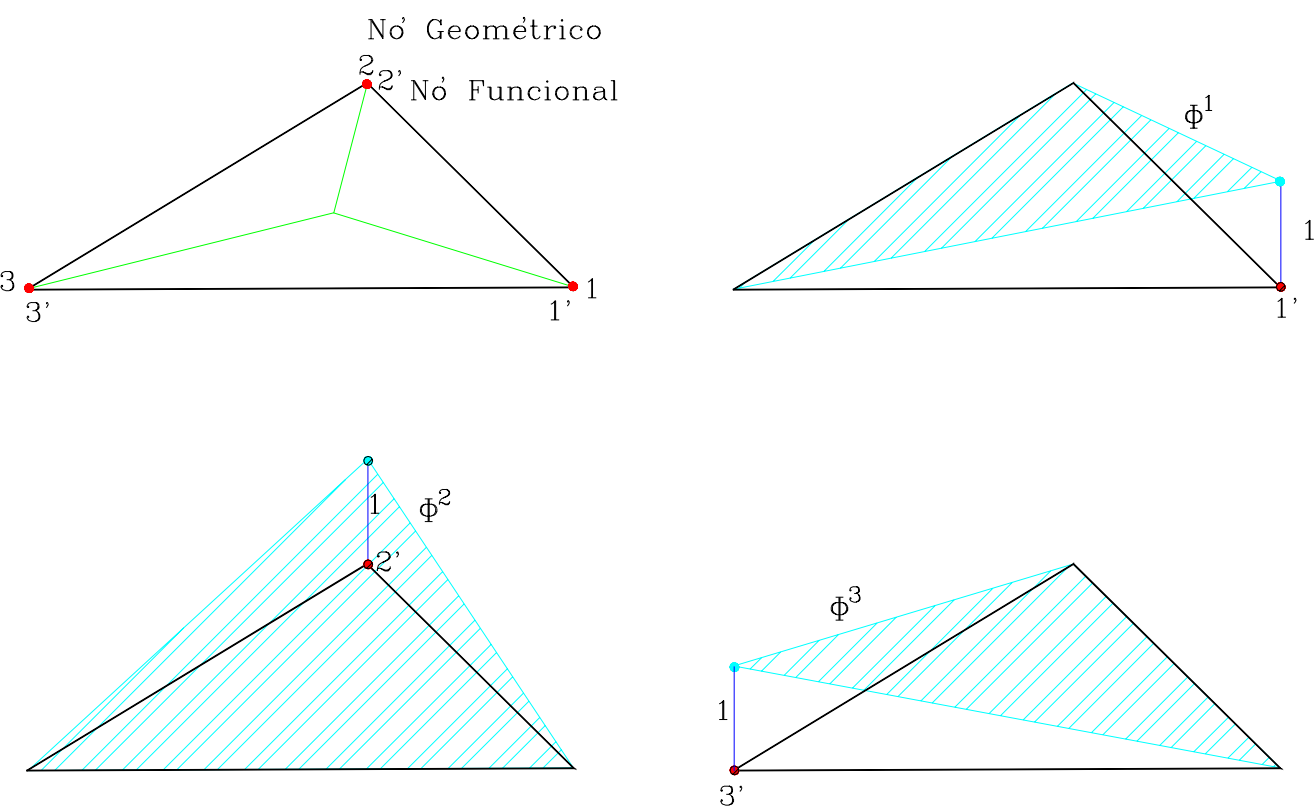

FIGURA 2.6.6 - Elemento triangular isoparamétrico linear.

As aproximações das variáveis do problema são expressas matricialmente por: 
$\left\{\begin{array}{l}\mathrm{u}_{1} \\ \mathrm{u}_{2} \\ \mathrm{u}_{3}\end{array}\right\}=\left[\begin{array}{ccccccccc}\xi_{1} & 0 & 0 & \xi_{2} & 0 & 0 & \xi_{3} & 0 & 0 \\ 0 & \xi_{1} & 0 & 0 & \xi_{2} & 0 & 0 & \xi_{3} & 0 \\ 0 & 0 & \xi_{1} & 0 & 0 & \xi_{2} & 0 & 0 & \xi_{3}\end{array}\right]\left\{\begin{array}{c}\mathrm{U}_{1}^{1} \\ \mathrm{U}_{2}^{1} \\ \mathrm{U}_{3}^{1} \\ \mathrm{U}_{1}^{2} \\ \mathrm{U}_{2}^{2} \\ \mathrm{U}_{3}^{2} \\ \mathrm{U}_{1}^{3} \\ \mathrm{U}_{2}^{3} \\ \mathrm{U}_{3}^{3}\end{array}\right\}, \mathrm{e}$

$$
\left\{\begin{array}{l}
\mathrm{p}_{1} \\
\mathrm{p}_{2} \\
\mathrm{p}_{3}
\end{array}\right\}=\left[\begin{array}{ccccccccc}
\xi_{1} & 0 & 0 & \xi_{2} & 0 & 0 & \xi_{3} & 0 & 0 \\
0 & \xi_{1} & 0 & 0 & \xi_{2} & 0 & 0 & \xi_{3} & 0 \\
0 & 0 & \xi_{1} & 0 & 0 & \xi_{2} & 0 & 0 & \xi_{3}
\end{array}\right]\left\{\begin{array}{l}
\mathrm{P}_{1}^{1} \\
\mathrm{P}_{2}^{1} \\
\mathrm{P}_{3}^{1} \\
\mathrm{P}_{1}^{2} \\
\mathrm{P}_{2}^{2} \\
\mathrm{P}_{3}^{2} \\
\mathrm{P}_{1}^{3} \\
\mathrm{P}_{2}^{3} \\
\mathrm{P}_{3}^{3}
\end{array}\right\}
$$

onde $u_{i}$ e $p_{i}$ representam as componentes de deslocamentos e forças de superfície, respectivamente, na direção i para um nó qualquer e $\mathrm{U}_{\mathrm{i}}^{\mathrm{n}}$ e $\mathrm{P}_{\mathrm{i}}^{\mathrm{n}}$ as componentes nodais na direção i de deslocamentos e forças de superfície, respectivamente.

No presente trabalho o elemento triangular linear contínuo só é utilizado para contornos suaves, sem angulosidades, o que significa que a matriz c da equação (2.6.5), tendo em vista os valores (2.5.9), tem os seguintes elementos:

$$
\mathbf{c}(\mathrm{S})=\left[\begin{array}{ccc}
\frac{1}{2} & 0 & 0 \\
0 & \frac{1}{2} & 0 \\
0 & 0 & \frac{1}{2}
\end{array}\right]
$$


B) Elemento Triangular Linear Descontínuo

O elemento descontínuo tem os três pontos de colocação deslizados para o seu interior, conforme figura 2.6.7, que define ainda as funções de interpolação $\phi$. Observando-se a localização de seus valores extremos, 0 e 1, nota-se que o nó funcional continua coincidente com o geométrico, havendo uma interpolação nos valores das variáveis (deslocamentos e forças de superfície), já que as equações de deslocamento são escritas para os pontos de colocação (internos).
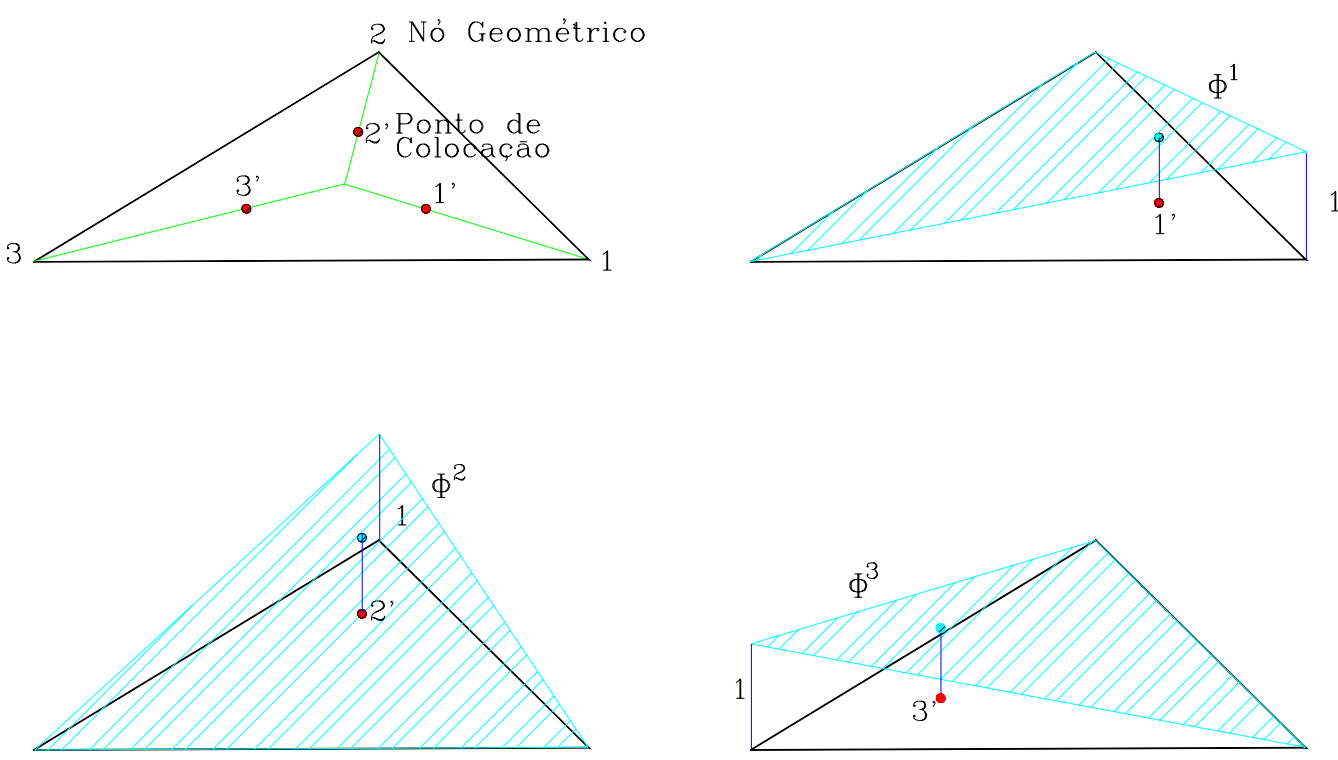

FIGURA 2.6.7 - Elemento triangular descontínuo.

As aproximações das variáveis do problema expressas por (2.6.15) e (2.6.16) são também válidas para o elemento descontínuo. A modificação se dá por interpolação, efetuada sobre a matriz $\overline{\mathbf{c}}$ em (2.6.6), ou seja,

$$
\overline{\mathbf{c}}(\mathrm{S})=\left[\begin{array}{ccc}
1 / 2 & 0 & 0 \\
0 & 1 / 2 & 0 \\
0 & 0 & 1 / 2
\end{array}\right]\left[\begin{array}{ccccccccc}
\bar{\xi}_{1} & 0 & 0 & \bar{\xi}_{2} & 0 & 0 & \bar{\xi}_{3} & 0 & 0 \\
0 & \bar{\xi}_{1} & 0 & 0 & \bar{\xi}_{2} & 0 & 0 & \bar{\xi}_{3} & 0 \\
0 & 0 & \bar{\xi}_{1} & 0 & 0 & \bar{\xi}_{2} & 0 & 0 & \bar{\xi}_{3}
\end{array}\right]
$$

onde as coordenadas homogêneas $\bar{\xi}_{\mathrm{i}}$ referem-se ao ponto de colocação S deslizado para o interior do elemento sobre a mediana do lado oposto ao vértice do triângulo 
relacionado ao ponto. Tomando-se a distância d entre o vértice do triângulo e o seu centróide, a posição do ponto de colocação fica definida utilizando-se

$$
0,35 \mathrm{~d} \text { ou } 0,475 \mathrm{~d}
$$

ressaltando-se que estes percentuais são utilizados a partir do centróide. O percentual 0,475 implica nos seguintes valores para as coordenadas homogêneas de um dado ponto S: $\bar{\xi}_{1}=\bar{\xi}_{2}=0,175$ e $\bar{\xi}_{3}=0,65$.

Alguns testes foram processados modificando-se a posição dos pontos de colocação. Os resultados foram mais precisos quando o ponto de colocação estava relacionado ao intervalo entre os valores definidos em (2.6.19).

\subsection{3 - Integrações Numéricas}

Considere-se a equação (2.6.5) sem o termo das forças de volume, por simplificação. Seus dois primeiros termos envolvem integrais sobre cada elemento de contorno. Definem-se, agora, duas matrizes $\mathbf{g}$ e $\mathbf{h}$ apresentando as seguintes expressões:

$$
\begin{aligned}
& \mathbf{g}=\int_{\Gamma_{\mathrm{j}}} \mathbf{u}^{*}(\mathrm{~S}, \mathrm{Q}) \phi^{\mathrm{T}}(\mathrm{Q}) \mathrm{d} \Gamma(\mathrm{Q}) \\
& \mathbf{h}=\int_{\Gamma_{\mathrm{j}}} \mathbf{p}^{*}(\mathrm{~S}, \mathrm{Q}) \phi^{\mathrm{T}}(\mathrm{Q}) \mathrm{d} \Gamma(\mathrm{Q})
\end{aligned}
$$

As soluções analíticas de (2.6.20a-b) são de difícil obtenção, dada à complexidade das funções a serem integradas. Portanto, justifica-se o emprego de esquemas numéricos de integração para viabilizar um procedimento padrão e eficiente de obtenção das matrizes $\mathbf{g}$ e $\mathbf{h}$.

As integrais (2.6.20a-b) são calculadas para duas situações distintas: quando o ponto de colocação S situa-se no elemento a ser integrado (integração singular ou 
semi-analítica) e quando posiciona-se fora (integração numérica). As duas integrações são definidas a seguir.

\subsubsection{1 - Integração Singular}

Na caso em que o ponto de colocação pertence ao elemento $\mathrm{j}$, a integração é chamada de singular, uma vez que as expressões de $\mathbf{u}^{*}$ e $\mathbf{p}^{*}$, escritas em função da coordenada esférica $\mathrm{r}$, apresentam singularidades nas vizinhanças do ponto $\mathrm{S}$.

Um procedimento bastante utilizado para determinação de uma integral singular, e que tem apresentado excelentes resultados, adota um sistema de eixos $(\bar{x}, \bar{y}$ e $\bar{z})$ de modo que o plano $\overline{x y}$ contenha o elemento. $O$ contorno $\Gamma_{j}$, que é o elemento, é substituído por uma relação entre as variáveis polares $\mathrm{r}$ e $\theta$, conforme mostra a figura 2.6.8. A integração singular é, então, efetuada em duas etapas: integração analítica em r e numérica em $\theta$.
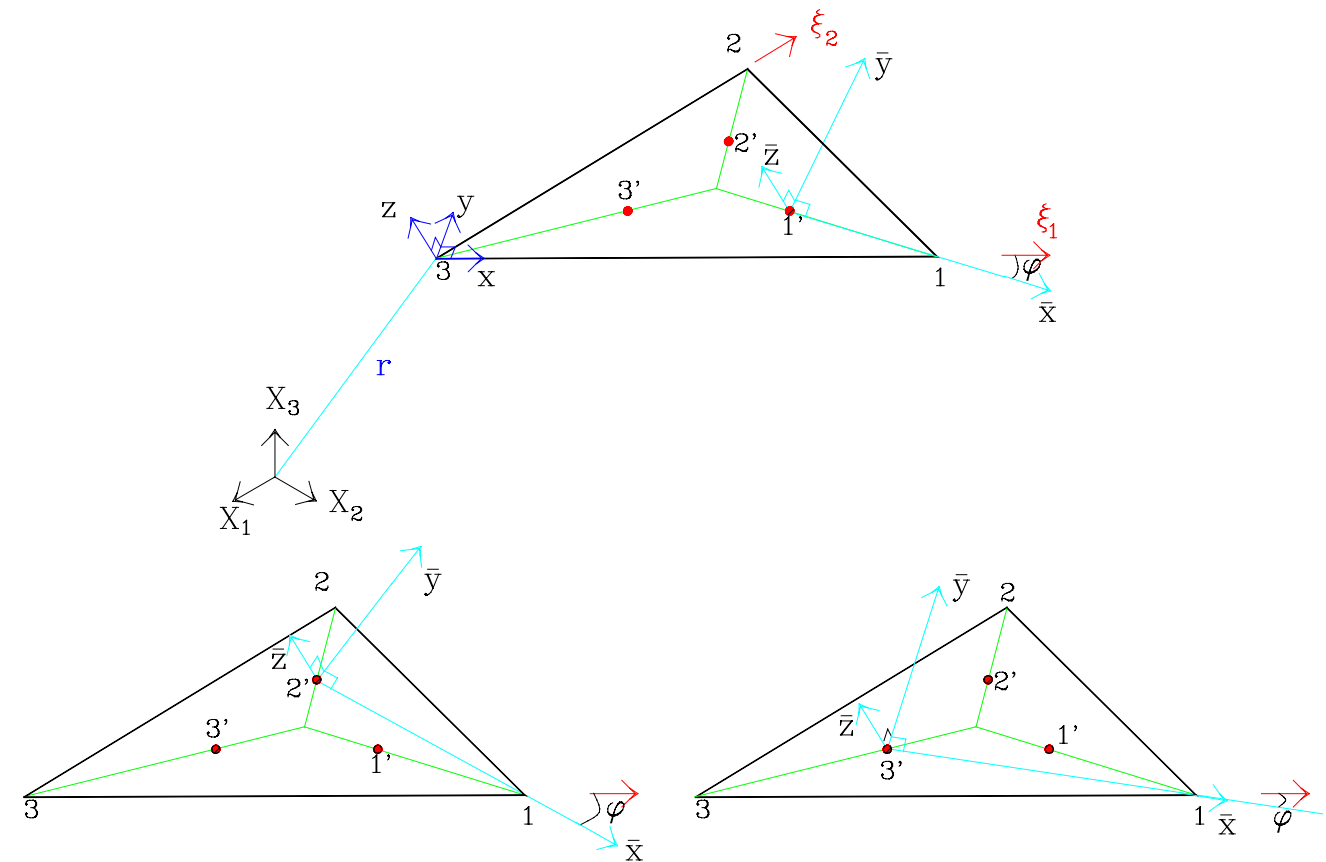

FIGURA 2.6.8 - Definição de parâmetros para a integração singular.

Para a solução fundamental de Kelvin (definida em (2.4.4) e (2.4.5)) a integração analítica em r pode ser resumida em três parcelas distintas: 


$$
\begin{aligned}
& \operatorname{parc1}(\ell)=\int_{\Gamma_{\mathrm{j}}} \frac{1}{\mathrm{r}} \xi_{\ell} \mathrm{d} \Gamma \\
& \operatorname{parc2}(\mathrm{i}, \mathrm{j}, \ell)=\int_{\Gamma_{\mathrm{j}}} \frac{\mathrm{r}_{\mathrm{i}} \mathrm{r}_{, j}}{\mathrm{r}} \xi_{\ell} \mathrm{d} \Gamma . \\
& \operatorname{parc3}(\mathrm{i}, \ell)=\int_{\Gamma_{\mathrm{j}}} \frac{\mathrm{r}_{, \mathrm{i}}}{\mathrm{r}^{2}} \xi_{\ell} \mathrm{d} \Gamma
\end{aligned}
$$

No plano xy, da figura 2.6.8, as coordenadas homogêneas podem ser obtidas a partir da expressão:

$$
\xi_{\ell}=\frac{1}{2 \mathrm{~A}}\left[\alpha_{\ell}+\beta_{\ell} \mathrm{x}+\gamma_{\ell} \mathrm{y}\right]
$$

onde

$$
\begin{aligned}
& \alpha_{\ell}=\mathrm{x}_{\mathrm{j}} \mathrm{y}_{\mathrm{k}}-\mathrm{x}_{\mathrm{k}} \mathrm{y}_{\mathrm{j}} \\
& \beta_{\ell}=\mathrm{y}_{\mathrm{j}}-\mathrm{y}_{\mathrm{k}} \\
& \gamma_{\ell}=\mathrm{x}_{\mathrm{k}}-\mathrm{x}_{\mathrm{j}} \\
& \begin{cases}\ell=1,2,3 \\
\mathrm{j}=2,3,1 \\
\mathrm{k}=3,1,2\end{cases}
\end{aligned}
$$

Sabe-se, ainda, que

$$
\begin{aligned}
& \bar{x}=r \cos \theta \\
& \bar{y}=r \operatorname{sen} \theta
\end{aligned}
$$

Portanto, para utilizar (2.6.22), tendo em vista (2.6.24), é necessária uma transformação de coordenadas, indicada da seguinte forma:

$$
\left\{\begin{array}{l}
x \\
y \\
z
\end{array}\right\}=\left[\begin{array}{ccc}
\cos \varphi & \operatorname{sen} \varphi & 0 \\
-\operatorname{sen} \varphi & \cos \varphi & 0 \\
0 & 0 & 1
\end{array}\right]\left\{\begin{array}{l}
\bar{x} \\
\bar{y} \\
\bar{z}
\end{array}\right\}+\left\{\begin{array}{l}
x_{o} \\
y_{o} \\
z_{o}
\end{array}\right\}
$$


Assim,

$$
\xi_{\ell}=\frac{1}{2 \mathrm{~A}}\left[\mathrm{~A}_{\ell}+\mathrm{B}_{\ell} \overline{\mathrm{x}}+\mathrm{C}_{\ell} \overline{\mathrm{y}}\right]
$$

onde

$$
\begin{aligned}
& \mathrm{A}_{\ell}=\alpha_{\ell}+\beta_{\ell} \mathrm{x}_{\mathrm{o}}+\gamma_{\ell} \mathrm{y}_{\mathrm{o}} \\
& \mathrm{B}_{\ell}=\beta_{\ell} \cos \varphi-\gamma_{\ell} \operatorname{sen} \varphi \\
& \mathrm{C}_{\ell}=\beta_{\ell} \operatorname{sen} \varphi+\gamma_{\ell} \cos \varphi
\end{aligned}
$$

Determinadas as coordenadas homogêneas, as integrais (2.6.21a-b) em r podem ser efetuadas, sendo $\mathrm{d} \Gamma=\operatorname{rdrd} \theta$, chegando-se em:

$$
\begin{aligned}
& \operatorname{parcl}(\ell)=\frac{1}{2 \mathrm{~A}} \int_{\theta}\left(\mathrm{A}_{\ell}+\frac{\mathrm{B}_{\ell}}{2} \mathrm{r} \cos \theta+\frac{\mathrm{C}_{\ell}}{2} \mathrm{r} \operatorname{sen} \theta\right) \mathrm{rd} \theta \\
& \operatorname{parc} 2(\mathrm{i}, \mathrm{j}, \ell)=\frac{1}{2 \mathrm{~A}} \int_{\theta} \mathrm{r}_{\mathrm{i}, \mathrm{r}} \mathrm{r}_{\mathrm{j}}\left(\mathrm{A}_{\ell}+\frac{\mathrm{B}_{\ell}}{2} \mathrm{r} \cos \theta+\frac{\mathrm{C}_{\ell}}{2} \mathrm{r} \operatorname{sen} \theta\right) \mathrm{rd} \theta .
\end{aligned}
$$

A parcela três, parc3(i, $\ell$ ), é singular para $r=0$ e, portanto, necessita de análise mais detalhada. A integração toma a seguinte forma:

$$
\begin{aligned}
\operatorname{parc} 3(\mathrm{i}, \ell) & =\frac{1}{2 \mathrm{~A}} \int_{\theta} \mathrm{r}_{\mathrm{i}}\left(\mathrm{A}_{\ell} \operatorname{Ln}(\mathrm{r})+\mathrm{B}_{\ell} \mathrm{r} \cos \theta+\mathrm{C}_{\ell} \mathrm{r} \operatorname{sen} \theta\right) \mathrm{d} \theta+ \\
& -\operatorname{Lim}_{\varepsilon \rightarrow 0} \int_{\theta} \mathrm{A}_{\ell} \operatorname{Ln}(\varepsilon) \mathrm{r}_{, \mathrm{i}} \mathrm{d} \theta
\end{aligned}
$$

Analisando-se o integrando da parcela do limite indicado em (2.6.29), verifica-se que a derivada da variável $\mathrm{r}$ em relação à direção cartesiana i pode ser representada por:

$$
\mathrm{r}_{, \mathrm{i}}=\mathrm{m}_{\mathrm{i} 1} \cos \theta+\mathrm{m}_{\mathrm{i} 2} \operatorname{sen} \theta \text {, }
$$


onde $\mathrm{m}_{\mathrm{ij}}$ são os cosenos diretores no ponto em análise em relação a $\mathrm{x}_{\mathrm{i}}$. Portanto, se a variação de $\theta$ obedece ao intervalo $0 \leq \theta \leq 2 \pi$, a integral tem valor nulo e o limite é igual a zero, ou seja,

$$
\operatorname{Lim}_{\varepsilon \rightarrow 0} \int_{0}^{2 \pi} A_{\ell} \operatorname{Ln}(\varepsilon)\left(m_{\mathrm{i} 1} \cos \theta+m_{\mathrm{i} 2} \operatorname{sen} \theta\right) \mathrm{d} \theta=0 .
$$

Logo, a parcela três passa a ser:

$$
\operatorname{parc3}(\mathrm{i}, \ell)=\frac{1}{2 \mathrm{~A}} \int_{\theta} \mathrm{r}_{\mathrm{i}}\left(\mathrm{A}_{\ell} \operatorname{Ln}(\mathrm{r})+\mathrm{B}_{\ell} \mathrm{r} \cos \theta+\mathrm{C}_{\ell} \mathrm{r} \operatorname{sen} \theta\right) \mathrm{d} \theta
$$

Cabe aqui uma consideração importante: a expressão (2.6.32), que representa a parc3, após a realização do limite (2.6.31), só é verdadeira para os extremos inferior e superior iguais a 0 e $2 \pi$, respectivamente, ou seja, se o ponto de colocação estiver deslocado para dentro do elemento (elemento descontínuo). Em elementos misto e contínuo isto só se verifica se a soma dos intervalos para a variável angular $\theta$, de cada elemento concorrente ao nó, atingir o extremo superior igual a $2 \pi$. Em cada elemento não há condição de limite, mas, quando são somadas as contribuições de cada elemento, chega-se no valor dado pela expressão (2.6.32).

$\mathrm{Na}$ determinação das expressões para as parcelas (2.6.21a-c), utiliza-se ainda um procedimento numérico para a integração em $\theta$ (2.6.28a-b) e (2.6.32). Pode-se efetuar a integração numérica diretamente em $\theta$, ou transformar cada elemento triangular plano em um domínio cujo contorno $\hat{\Gamma}$ tem três elementos unidimensionais retos (figura 2.6.9). 


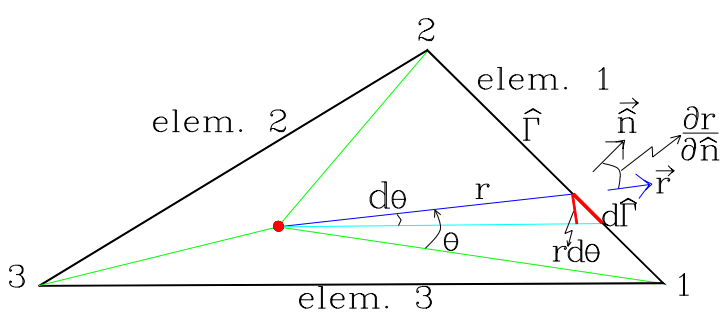

(a)

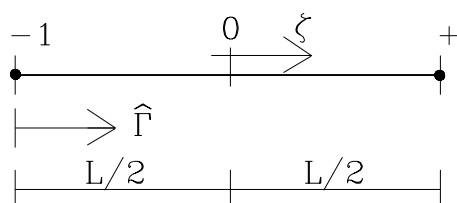

$d \widehat{\Gamma}=\frac{1}{2} d \zeta$

FIGURA 2.6.9 - (a) integração no contorno fictício do elemento triangular; (b) elemento unidimensional utilizado.

Para representar o elemento de superfície através de três elementos lineares de contorno, a diferencial em $\theta$ deve ser substituída por:

$$
\mathrm{d} \theta=\frac{1}{\mathrm{r}} \frac{\partial \mathrm{r}}{\partial \hat{\mathrm{n}}} \mathrm{d} \hat{\Gamma}
$$

Assim,

$$
\begin{aligned}
& \operatorname{parc1}(\ell)=\frac{1}{2 \mathrm{~A}} \int_{\hat{\Gamma}}\left(\mathrm{A}_{\ell}+\frac{\mathrm{B}_{\ell}}{2} \mathrm{r} \cos \theta+\frac{\mathrm{C}_{\ell}}{2} \mathrm{r} \operatorname{sen} \theta\right) \frac{\partial \mathrm{r}}{\partial \hat{\mathrm{n}}} \mathrm{d} \hat{\Gamma} \\
& \operatorname{parc2}(\mathrm{i}, \mathrm{j}, \ell)=\frac{1}{2 \mathrm{~A}} \int_{\hat{\Gamma}} \mathrm{r}_{, \mathrm{i}} \mathrm{r}_{, \mathrm{j}}\left(\mathrm{A}_{\ell}+\frac{\mathrm{B}_{\ell}}{2} \mathrm{r} \cos \theta+\frac{\mathrm{C}_{\ell}}{2} \mathrm{r} \operatorname{sen} \theta\right) \frac{\partial \mathrm{r}}{\partial \hat{\mathrm{n}}} \mathrm{d} \hat{\Gamma} \\
& \operatorname{parc3}(\mathrm{i}, \ell)=\frac{1}{2 \mathrm{~A}} \int_{\hat{\Gamma}} \mathrm{r}_{, \mathrm{i}}\left(\mathrm{A}_{\ell} \mathrm{Ln}(\mathrm{r})+\mathrm{B}_{\ell} \mathrm{r} \cos \theta+\mathrm{C}_{\ell} \mathrm{r} \operatorname{sen} \theta\right) \frac{1}{\mathrm{r}} \frac{\partial \mathrm{r}}{\partial \hat{\mathrm{n}}} \mathrm{d} \hat{\Gamma}
\end{aligned}
$$

Substituindo-se ainda as funções $\cos \theta$ e $\operatorname{sen} \theta$ em $(2.6 .34 \mathrm{a}-\mathrm{c})$, tendo em vista os valores dados em (2.6.24) e (2.6.25) mais os parâmetros (2.6.23), tem-se: 


$$
\begin{aligned}
& \operatorname{parc1}(\ell)=\frac{1}{4 \mathrm{~A}} \int_{\hat{\Gamma}}\left(2 \alpha_{\ell}+\beta_{\ell}\left(\mathrm{x}+\mathrm{x}_{\mathrm{o}}\right)+\gamma_{\ell}\left(\mathrm{x}+\mathrm{x}_{\mathrm{o}}\right)\right) \frac{\partial \mathrm{r}}{\partial \hat{\mathrm{n}}} \mathrm{d} \hat{\Gamma} \\
& \operatorname{parc2}(\mathrm{i}, \mathrm{j}, \ell)=\frac{1}{4 \mathrm{~A}} \int_{\hat{\Gamma}} \mathrm{r}_{, \mathrm{i}} \mathrm{r}_{\mathrm{j}}\left(2 \alpha_{\ell}+\beta_{\ell}\left(\mathrm{x}+\mathrm{x}_{\mathrm{o}}\right)+\gamma_{\ell}\left(\mathrm{x}+\mathrm{x}_{\mathrm{o}}\right)\right) \frac{\partial \mathrm{r}}{\partial \hat{\mathrm{n}}} \mathrm{d} \hat{\Gamma} \\
& \operatorname{parc3}(\mathrm{i}, \ell)=\frac{1}{2 \mathrm{~A}} \int_{\hat{\Gamma}}^{\mathrm{r}_{\mathrm{i}}} \frac{\mathrm{r}_{\mathrm{r}}}{\mathrm{r}}\left\{\alpha_{\ell} \operatorname{Ln}(\mathrm{r})+\beta_{\ell}\left[\mathrm{x}+\mathrm{x}_{\mathrm{o}}(\operatorname{Ln}(\mathrm{r})-1)\right]+\right. \\
& \left.+\gamma_{\ell}\left[\mathrm{y}+\mathrm{y}_{\mathrm{o}}(\operatorname{Ln}(\mathrm{r})-1)\right]\right\} \frac{\partial \mathrm{r}}{\partial \hat{\mathrm{n}}} \mathrm{d} \hat{\Gamma}
\end{aligned}
$$

As integrais (2.6.35a-c) são, então, calculadas numericamente através da quadratura Gaussiana (KANE, 1994). A figura 2.6.9b define o elemento linear utilizado no contorno fictício do elemento triangular, bem como seus parâmetros mais importantes. Assim, as matrizes $\mathbf{g}$ e h (2.6.20a-b) ficam determinadas por:

$$
\begin{aligned}
& \mathbf{g}=|\mathrm{J}| \sum_{\ell=1}^{\mathrm{N}_{\mathrm{PG}}} \overline{\mathbf{f}}_{\mathrm{g}}(\zeta) \mathrm{w}_{\ell} \\
& \mathbf{h}=|\mathrm{J}| \sum_{\ell=1}^{\mathrm{N}_{\mathrm{PG}}} \overline{\mathbf{f}}_{\mathrm{h}}(\zeta) \mathrm{w}_{\ell},
\end{aligned}
$$

onde $|\mathbf{J}|$ é o jacobiano de transformação de coordenadas para o elemento unidimensional reto, e vale $\frac{\mathrm{L}}{2}$ (metade de seu comprimento); $\mathrm{w}_{\ell}$ é o peso no ponto $\ell$, para a quadratura Gaussiana; $\overline{\mathbf{f}}_{\mathrm{g}}$ e $\overline{\mathbf{f}}_{\mathrm{h}}$ representam os integrandos (2.6.20a-b) já devidamente transformados utilizando-se convenientemente as parcelas apresentadas em (2.6.35a-c); e $\mathrm{N}_{\mathrm{PG}}$ representa o número de pontos de Gauss.

As expressões (2.6.35a-c) foram deduzidas a partir da solução fundamental de Kelvin. Para a solução fundamental de Mindlin, que pode ser entendida como uma parcela de Kelvin e outra complementar (BREBBIA et al., 1984), a aplicação dessas expressões é direta. Para a parcela complementar de Mindlin, tendo em vista que a imagem S' do ponto fonte, conforme a figura 2.4.4, não pertence ao elemento que está sendo integrado, o procedimento usado não é o de integração singular apresentado até aqui, e sim o próximo procedimento para integração numérica utilizando Hammer. 


\subsubsection{2 - Integração Numérica}

$\mathrm{Na}$ integração numérica, o ponto de colocação não pertence ao elemento a ser integrado. Para este caso, as tabelas de integração numérica de Hammer dá bons resultados.

A integração numérica é feita com relação às coordenadas homogêneas, utilizando-se o Jacobiano de transformação $|\mathrm{G}|$, (2.6.13). As integrais em (2.6.20a-b) para o caso onde o ponto de colocação não pertence ao elemento a ser integrado, passam a ser:

$$
\begin{aligned}
& \mathbf{g}=|\mathrm{G}| \int_{0}^{1}\left[\int_{0}^{1-\xi_{2}} \mathbf{f}_{\mathrm{g}}\left(\xi_{1}, \xi_{2}\right) \mathrm{d} \xi_{1}\right] \mathrm{d} \xi_{2} \\
& \mathbf{h}=|\mathrm{G}| \int_{0}^{1}\left[\int_{0}^{1-\xi_{2}} \mathbf{f}_{\mathrm{h}}\left(\xi_{1}, \xi_{2}\right) \mathrm{d} \xi_{1}\right] \mathrm{d} \xi_{2}
\end{aligned}
$$

onde as funções $\mathbf{f}_{\mathrm{g}}$ e $\mathbf{f}_{\mathrm{h}}$ são os integrandos de (2.6.20a-b).

Aplicando-se o procedimento de integração numérica de Hammer à expressão anterior, tem-se:

$$
\begin{aligned}
& \mathbf{g}=|\mathrm{G}| \sum_{\ell=1}^{\mathrm{N}_{\mathrm{PH}}} \mathbf{f}_{\mathrm{g}}\left(\xi_{1}^{\ell}, \xi_{2}^{\ell}\right) \mathrm{w}_{\ell} \\
& \mathbf{h}=|\mathrm{G}| \sum_{\ell=1}^{\mathrm{N}_{\mathrm{PH}}} \mathbf{f}_{\mathrm{h}}\left(\xi_{1}^{\ell}, \xi_{2}^{\ell}\right) \mathrm{w}_{\ell},
\end{aligned}
$$

onde $\mathrm{N}_{\mathrm{PH}}$ representa o número de pontos de integração de Hammer e $\mathrm{w}_{\ell} \mathrm{o}$ valor do peso no ponto $\ell$.

É importante salientar que os pesos do procedimento de Hammer apresentados nas diversas tabelas (como em BREBBIA et al., 1984), leva ao dobro do valor das integrais. Portanto, é necessário dividi-los por 2 (dois) a fim de equacionar os procedimentos de integração singular e numérico.

O procedimento numérico de integração de Hammer no presente trabalho, tem sua precisão afetada pela distância (r) entre os pontos fonte $\mathrm{S}$ e de campo Q, já que as funções envolvidas são singulares. A figura 2.6.10 mostra dois casos distintos 
que aparecem no procedimento numérico. Em (a) vê-se que a distância entre os pontos S e Q é relativamente grande quando comparada com o tamanho dos lados dos elementos triangulares. Portanto, o procedimento de Hammer fornece resultados bem satisfatórios. Em (b) o ponto $\mathrm{S}$ está muito próximo do elemento a ser integrado. Neste caso, por causa das funções envolvidas, a precisão da integração numérica diminui, comprometendo substancialmente os resultados.

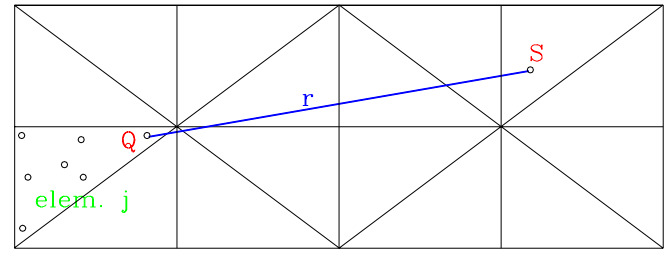

(a)

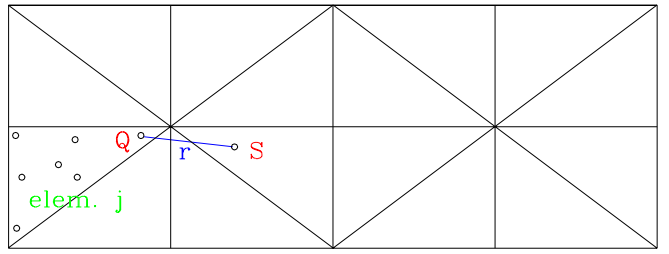

(b)

FIGURA 2.6.10 - Proximidade dos pontos fonte $\mathrm{S}$ e de campo Q.

Um procedimento utilizado para aumentar a precisão das integrações realizadas numericamente é aquele em que se divide o elemento j em sub-elementos e, em seguida, aplica-se o procedimento de Hammer a cada um deles (como em KANE, 1994). A divisão em sub-elementos pode ser feita de forma escalonada e não regular, em função da distância $r$ entre $S$ e Q. Neste trabalho, optou-se pela divisão única e regular, através de 25 sub-elementos, conforme mostra a figura 2.6.11.

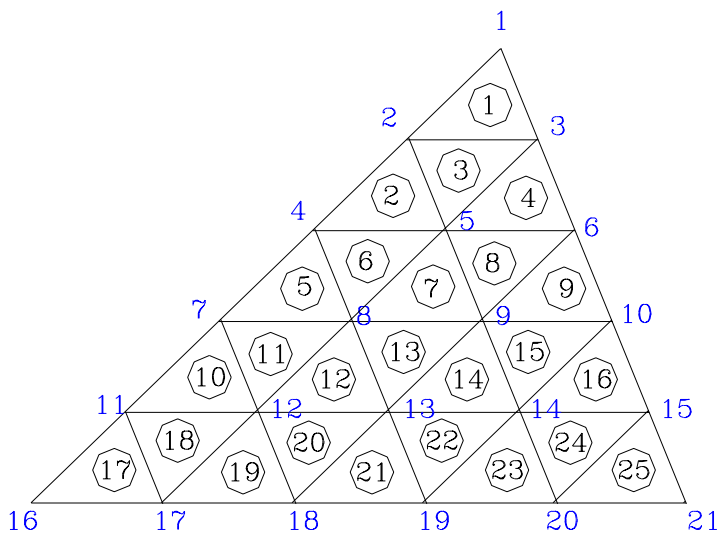

FIGURA 2.6.11 - Divisão do elemento triangular em 25 sub-elementos. 


\subsection{4 - Deslocamentos e Tensões em Pontos do Domínio}

A representação integral (2.5.1) fornece os valores de deslocamentos em pontos do domínio, conhecidos os valores de deslocamentos u e forças de superfície p no contorno. A discretização discutida no item 2.6.1 é também utilizada aqui, definindo (2.5.1) de forma semelhante à (2.6.5), ou seja:

$$
\begin{aligned}
\mathbf{u}(\mathrm{s}) & =-\sum_{\mathrm{j}=1}^{\mathrm{J}}\left[\int_{\Gamma_{\mathrm{j}}} \mathbf{p}^{*}(\mathrm{~s}, \mathrm{Q}) \phi^{\mathrm{T}}(\mathrm{Q}) \mathrm{d} \Gamma(\mathrm{Q})\right] \mathrm{U}^{\mathrm{n}}+ \\
& +\sum_{\mathrm{j}=1}^{\mathrm{J}}\left[\int_{\Gamma_{\mathrm{j}}} \mathbf{u}^{*}(\mathrm{~s}, \mathrm{Q}) \phi^{\mathrm{T}}(\mathrm{Q}) \mathrm{d} \Gamma(\mathrm{Q})\right] \mathrm{P}^{\mathrm{n}}+ \\
& +\sum_{\mathrm{m}=1}^{\mathrm{M}}\left[\int_{\Omega_{\mathrm{m}}} \mathbf{u}^{*}(\mathrm{~s}, \mathrm{q}) \phi_{\mathrm{c}}^{\mathrm{T}}(\mathrm{q}) \mathrm{d} \Omega(\mathrm{q})\right] \mathrm{B}^{\mathrm{n}},
\end{aligned}
$$

onde as respostas de deslocamentos são

$$
\mathbf{u}(\mathrm{s})=\left\{\begin{array}{l}
\mathrm{u}_{1} \\
\mathrm{u}_{2} \\
\mathrm{u}_{3}
\end{array}\right\} .
$$

As parcelas integrais de (2.6.39) são calculadas através do procedimento de integração numérica, semelhante àquele usado no caso onde $\mathrm{S}$ e $\mathrm{Q}$ pertencem a elementos diferentes. Também aqui adota-se a divisão do elemento triangular em sub-elementos, a fim de melhorar a precisão dos resultados.

Representando (2.6.39) na forma matricial, tem-se:

$$
\mathbf{u}=-\mathbf{H U}+\mathbf{G P}+\mathbf{D B}
$$

Para o cálculo das tensões em pontos do domínio, utiliza-se o mesmo tratamento dispensado à equação de deslocamentos. Assim, a representação integral (2.5.2) passa a ter a seguinte forma: 


$$
\begin{aligned}
\sigma(\mathrm{s}) & =-\sum_{\mathrm{j}=1}^{\mathrm{J}}\left[\int_{\Gamma_{\mathrm{j}}} \mathbf{S}^{*}(\mathrm{~s}, \mathrm{Q}) \phi^{\mathrm{T}}(\mathrm{Q}) \mathrm{d} \Gamma(\mathrm{Q})\right] \mathrm{U}^{\mathrm{n}}+ \\
& +\sum_{\mathrm{j}=1}^{\mathrm{J}}\left[\int_{\Gamma_{\mathrm{j}}} \mathbf{D}^{*}(\mathrm{~s}, \mathrm{Q}) \phi^{\mathrm{T}}(\mathrm{Q}) \mathrm{d} \Gamma(\mathrm{Q})\right] \mathrm{P}^{\mathrm{n}}+ \\
& +\sum_{\mathrm{m}=1}^{\mathrm{M}}\left[\int_{\Omega_{\mathrm{m}}} \mathbf{D}^{*}(\mathrm{~s}, \mathrm{q}) \phi_{\mathrm{c}}^{\mathrm{T}}(\mathrm{q}) \mathrm{d} \Omega(\mathrm{q})\right] \mathrm{B}^{\mathrm{n}},
\end{aligned}
$$

onde

$$
\sigma(s)=\left[\begin{array}{lll}
\sigma_{11} & \sigma_{12} & \sigma_{13} \\
\sigma_{21} & \sigma_{22} & \sigma_{23} \\
\sigma_{31} & \sigma_{32} & \sigma_{33}
\end{array}\right] \quad \text { ou } \quad \sigma(s)=\left\{\begin{array}{l}
\sigma_{11} \\
\sigma_{12} \\
\sigma_{13} \\
\sigma_{22} \\
\sigma_{23} \\
\sigma_{33}
\end{array}\right\}
$$

As integrais em (2.6.42) são resolvidas numericamente através do procedimento de Hammer, semelhante ao que já foi usado para os deslocamentos, observando-se que os tensores envolvidos, agora, são $\mathbf{S}^{*}$ e $\mathbf{D}^{*}-(2.5 .3)$, (2.5.4), (2.5.5) e (2.5.6).

Representando-se (2.6.42) na forma matricial, tem-se:

$$
\sigma=-\mathbf{H}^{\prime} \mathbf{U}+\mathbf{G}^{\prime} \mathbf{P}+\mathbf{D}^{\prime} \mathbf{B}
$$

\subsection{5 - Tensões em Pontos do Contorno}

As tensões em pontos do contorno podem ser obtidas através da equação (2.6.41) com alguma manipulação algébrica. Entretanto, os núcleos das integrais apresentam singularidades do tipo $\mathrm{r}^{-\mathrm{n}}$, motivo pelo qual este procedimento é evitado. 
Uma maneira mais simples e eficiente de se determinar tensões em pontos do contorno consiste em utilizar uma aproximação das deformações a partir dos valores nodais de deslocamentos de cada elemento. Esse procedimento é indicado em LACHAT (1975) e BREBBIA et al. (1984).

Considere-se a figura 2.6.12, que traz um elemento posicionado no contorno, com seus sistemas de coordenadas cartesianas global e local, para a análise de tensões. Um elemento deste ponto foi extraído, apresentando-se as suas componentes de tensão.

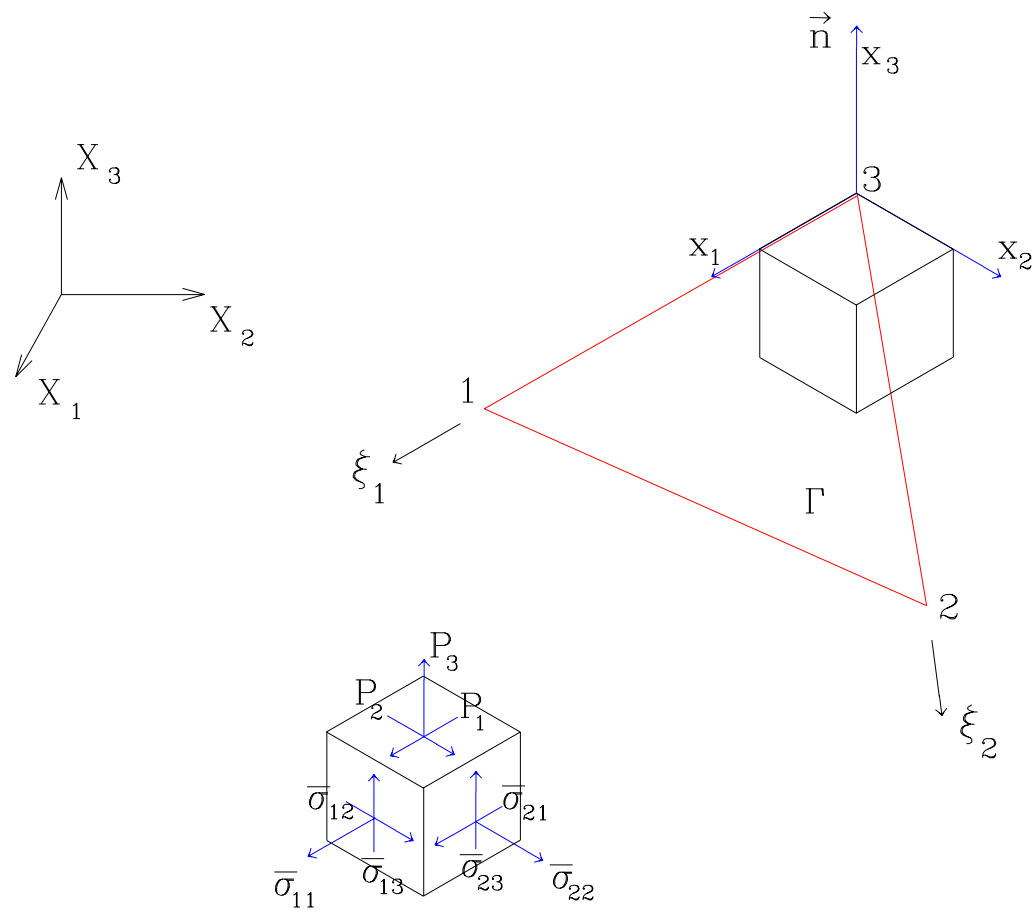

FIGURA. 2.6.12 - Ponto do contorno para análise de tensões.

O sistema local $\mathrm{x}_{\mathrm{i}}$ é definido tendo um de seus eixos perpendicular ao plano do elemento em questão, e os outros dois posicionados no plano do elemento. Através de (2.3.3) e (2.3.5), pode-se dizer que: 


$$
\begin{aligned}
& \bar{\sigma}_{11}=(2 \mathrm{G}+\lambda) \varepsilon_{11}+\lambda\left(\varepsilon_{22}+\varepsilon_{33}\right) \\
& \bar{\sigma}_{12}=2 \mathrm{G} \varepsilon_{12} \\
& \bar{\sigma}_{22}=(2 \mathrm{G}+\lambda) \varepsilon_{22}+\lambda\left(\varepsilon_{11}+\varepsilon_{33}\right) \\
& \bar{\sigma}_{31}=\mathrm{P}_{1} \\
& \bar{\sigma}_{32}=\mathrm{P}_{2} \\
& \bar{\sigma}_{33}=\mathrm{P}_{3}
\end{aligned}
$$

Neste caso, é possível eliminar a deformação na direção 3 já que se conhece o valor da tensão nesta direção. Assim,

$$
\bar{\sigma}_{33}=\mathrm{P}_{3}=(2 \mathrm{G}+\lambda) \varepsilon_{33}+\lambda\left(\varepsilon_{11}+\varepsilon_{22}\right),
$$

o que resulta em

$$
\varepsilon_{33}=\frac{1}{(2 \mathrm{G}+\lambda)}\left[\mathrm{P}_{3}-\lambda\left(\varepsilon_{11}+\varepsilon_{22}\right)\right]
$$

O valor encontrado (2.6.47) deve ser substituído em (2.6.45a-c).

Por outro lado, as deformações (2.3.4) estão escritas em função dos deslocamentos, aqui aproximados pelos valores nodais $\mathbf{U}_{\mathrm{i}}$ e a função aproximadora $\phi^{\mathrm{T}}$, já utilizada nos itens anteriores. Assim,

$$
\mathrm{u}_{\mathrm{i}}\left(\xi_{1}, \xi_{2}\right)=\phi_{\mathrm{k}} \mathrm{U}_{\mathrm{i}}^{\mathrm{k}}=\xi_{1}\left(\mathrm{U}_{\mathrm{i}}^{1}-\mathrm{U}_{\mathrm{i}}^{3}\right)+\xi_{2}\left(\mathrm{U}_{\mathrm{i}}^{2}-\mathrm{U}_{\mathrm{i}}^{3}\right)+\mathrm{U}_{\mathrm{i}}^{3}
$$

cujas derivadas são:

$$
\begin{aligned}
& \frac{\partial \mathrm{u}_{\mathrm{i}}}{\partial \xi_{1}}=\mathrm{U}_{\mathrm{i}}^{1}-\mathrm{U}_{\mathrm{i}}^{3} \\
& \frac{\partial \mathrm{u}_{\mathrm{i}}}{\partial \xi_{2}}=\mathrm{U}_{\mathrm{i}}^{2}-\mathrm{U}_{\mathrm{i}}^{3}
\end{aligned}
$$


Mas as derivadas necessárias para se utilizar (2.3.4) são relativas às coordenadas cartesianas. Assim, utilizando-se a regra da cadeia, tem-se:

$$
\frac{\partial \mathrm{u}_{\mathrm{i}}}{\partial \xi_{\mathrm{k}}}=\frac{\partial \mathrm{x}_{\mathrm{j}}}{\partial \xi_{\mathrm{k}}} \frac{\partial \mathrm{u}_{\mathrm{i}}}{\partial \mathrm{x}_{\mathrm{j}}}
$$

As coordenadas cartesianas locais também são escritas em função de seus valores nodais, da seguinte forma:

$$
\mathrm{x}_{\mathrm{j}}\left(\xi_{1}, \xi_{2}\right)=\phi_{\mathrm{k}} \mathrm{x}_{\mathrm{j}}^{\mathrm{k}}=\xi_{1}\left(\mathrm{x}_{\mathrm{j}}^{1}-\mathrm{x}_{\mathrm{j}}^{3}\right)+\xi_{2}\left(\mathrm{x}_{\mathrm{j}}^{2}-\mathrm{x}_{\mathrm{j}}^{3}\right)+\mathrm{x}_{\mathrm{j}}^{3}
$$

cujas derivadas são:

$$
\begin{aligned}
& \frac{\partial x_{1}}{\partial \xi_{1}}=x_{1}^{1}-x_{1}^{3} \\
& \frac{\partial x_{1}}{\partial \xi_{2}}=x_{1}^{2}-x_{1}^{3} \\
& \frac{\partial x_{2}}{\partial \xi_{1}}=x_{2}^{1}-x_{2}^{3} \\
& \frac{\partial x_{2}}{\partial \xi_{2}}=x_{2}^{2}-x_{2}^{3}
\end{aligned}
$$

O Jacobiano de transformação entre o sistema cartesiano local e o de coordenadas homogênea é $|\mathrm{G}|=2 \mathrm{~A}$, definido em (2.6.13). Com todos os parâmetros estabelecidos, a partir de (2.6.50), pode-se obter os valores aproximados para as deformações, dados a seguir. 


$$
\begin{aligned}
& \frac{\partial \mathrm{u}_{1}}{\partial \mathrm{x}_{1}}=\frac{1}{2 \mathrm{~A}}\left[\left(\mathrm{x}_{2}^{2}-\mathrm{x}_{2}^{3}\right)\left(\mathrm{U}_{1}^{1}-\mathrm{U}_{1}^{3}\right)-\left(\mathrm{x}_{2}^{1}-\mathrm{x}_{2}^{3}\right)\left(\mathrm{U}_{1}^{2}-\mathrm{U}_{1}^{3}\right)\right] \\
& \frac{\partial \mathrm{u}_{1}}{\partial \mathrm{x}_{2}}=\frac{1}{2 \mathrm{~A}}\left[\left(\mathrm{x}_{1}^{1}-\mathrm{x}_{1}^{3}\right)\left(\mathrm{U}_{1}^{2}-\mathrm{U}_{1}^{3}\right)-\left(\mathrm{x}_{1}^{2}-\mathrm{x}_{1}^{3}\right)\left(\mathrm{U}_{1}^{1}-\mathrm{U}_{1}^{3}\right)\right] \\
& \frac{\partial \mathrm{u}_{2}}{\partial \mathrm{x}_{1}}=\frac{1}{2 \mathrm{~A}}\left[\left(\mathrm{x}_{2}^{2}-\mathrm{x}_{2}^{3}\right)\left(\mathrm{U}_{2}^{1}-\mathrm{U}_{2}^{3}\right)-\left(\mathrm{x}_{2}^{1}-\mathrm{x}_{2}^{3}\right)\left(\mathrm{U}_{2}^{2}-\mathrm{U}_{2}^{3}\right)\right] \\
& \frac{\partial \mathrm{u}_{2}}{\partial \mathrm{x}_{2}}=\frac{1}{2 \mathrm{~A}}\left[\left(\mathrm{x}_{1}^{1}-\mathrm{x}_{1}^{3}\right)\left(\mathrm{U}_{2}^{2}-\mathrm{U}_{2}^{3}\right)-\left(\mathrm{x}_{1}^{2}-\mathrm{x}_{1}^{3}\right)\left(\mathrm{U}_{2}^{1}-\mathrm{U}_{2}^{3}\right)\right]
\end{aligned}
$$

Portanto, os valores das tensões em pontos do contorno ficam estabelecidos, de forma aproximada, pelas expressões:

$$
\begin{aligned}
& \bar{\sigma}_{11}=\frac{1}{1-v}\left[2 G\left(\frac{\partial u_{1}}{\partial x_{1}}+v \frac{\partial u_{2}}{\partial x_{2}}\right)+v P_{3}\right] \\
& \bar{\sigma}_{12}=G\left(\frac{\partial u_{1}}{\partial x_{2}}+v \frac{\partial u_{2}}{\partial x_{1}}\right) \\
& \bar{\sigma}_{22}=\frac{1}{1-v}\left[2 G\left(\frac{\partial u_{2}}{\partial x_{2}}+v \frac{\partial u_{1}}{\partial x_{1}}\right)+v P_{3}\right] \\
& \bar{\sigma}_{31}=P_{1} \\
& \bar{\sigma}_{32}=P_{2} \\
& \bar{\sigma}_{33}=P_{3}
\end{aligned}
$$

\section{7 - APLICAÇÕES}

2.7.1 - Aplicação 1: Sólido elástico paralelepipédico submetido a uma força estática.

O primeiro caso a ser processado é um sólido elástico paralelepipédico solicitado por uma força estática de tração, vinculado na base para evitar o deslocamento axial nestes pontos e totalmente contido nas laterais, conforme 
apresentado na figura 2.7.1. Além dos parâmetros geométricos definidos nesta figura, são considerados: $\mathrm{E}=100.000 \mathrm{~Pa} ; \mathrm{v}=0,25$.
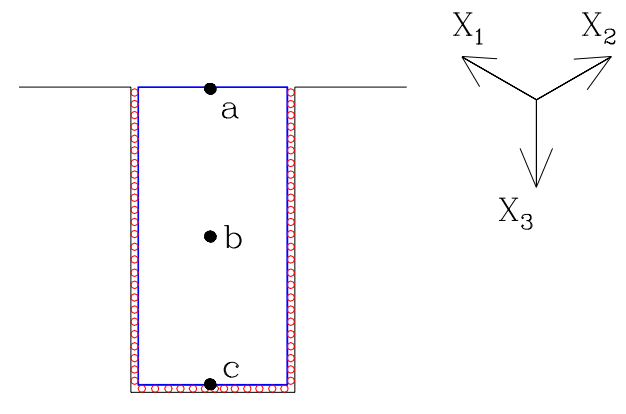

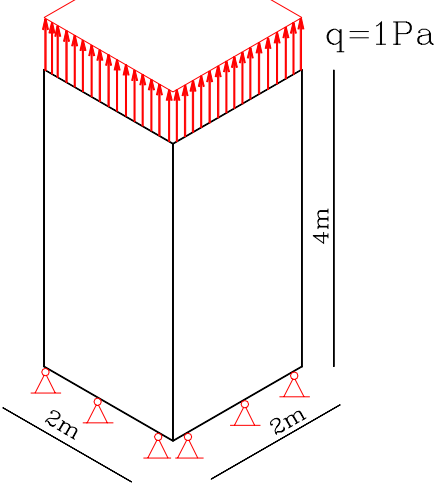

(b)
$\mathrm{E}=100.000 \mathrm{~Pa}$ $\nu=0,25$

FIGURA 2.7.1 - Definição do sólido e suas condições de contorno: (a) contorno contido lateralmente e pontos para avaliação; e (b) sólido, carga e condições de contorno parciais.

A formulação do método dos elementos de contorno apresentada neste capítulo foi testada utilizando-se duas discretizações: com 12 elementos triangulares descontínuos (36 pontos de colocação) e com 40 elementos (120 pontos de colocação), conforme mostra a figura 2.7.2. Os nós de cantos são avaliados através do nó deslocado dos elementos descontínuos.

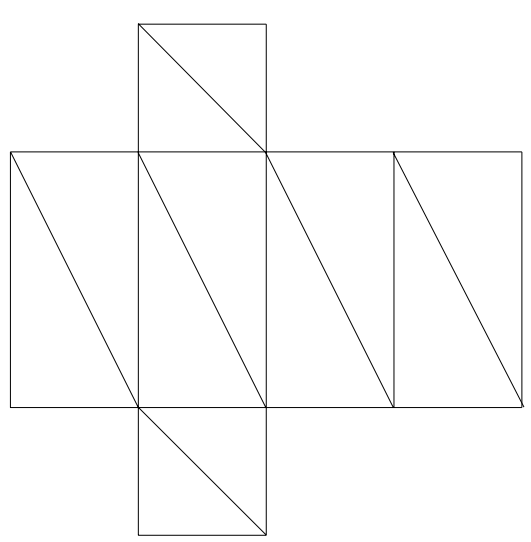

(a)

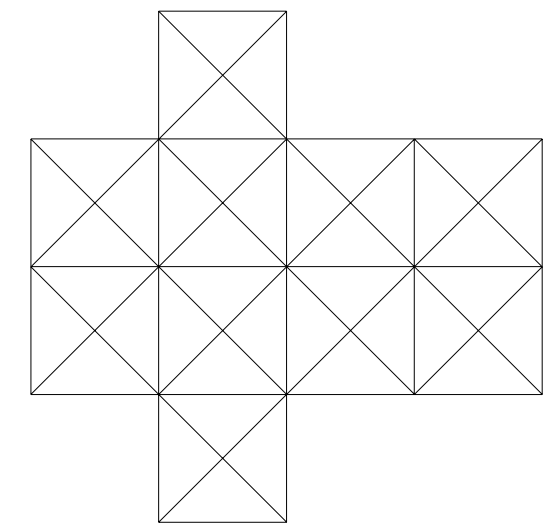

(b)

FIGURA 2.7.2 - Discretizações do contorno por elementos triangulares planos descontínuos: (a) 12 elementos e (b) 40 elementos. 
Os programas computacionais utilizados no processamento deste exemplo implementam as soluções fundamentais de Kelvin e Mindlin. Os resultados são apresentados na tabela 2.7.1 e evidencia a adequação da formulação apresentada, uma vez que os valores teóricos são alcançados pela simulação numérica. Deve-se lembrar que, para a solução de Mindlin, é necessário fazer com que o parâmetro "c" seja grande o suficiente para que esta coincida com a solução de Kelvin. Entretanto, por serem as exatas as funções aproximadoras, os resultados obtidos para "c" pequenos coincidem com os anteriores (neste caso).

TABELA 2.7.1 - Deslocamentos na direção axial, $\mathrm{X}_{3},\left(\times 10^{-5} \mathrm{~m}\right)$.

\begin{tabular}{|c|c|c|c|c|c|}
\hline \multirow{2}{*}{$\begin{array}{c}\text { Ponto } \\
\text { Observado }\end{array}$} & Valor & \multicolumn{2}{|c|}{ Sol. Fund. Kelvin } & \multicolumn{2}{c|}{ Sol. Fund. Mindlin } \\
\cline { 3 - 6 } & Teórico & 12 elem. & 40 elem. & 12 elem. & 40 elem. \\
\hline $\mathbf{a}$ & $-3,333 \ldots$ & $-3,332$ & $-3,333$ & $-3,332$ & $-3,333$ \\
\hline $\mathbf{b}$ & $-1,666 \ldots$ & $-1,667$ & $-1,667$ & $-1,667$ & $-1,667$ \\
\hline $\mathbf{c}$ & 0 & 0 & 0 & 0 & 0 \\
\hline
\end{tabular}

TABELA 2.7.2 - Tensões na direção axial, $\mathrm{X}_{3},(\mathrm{~Pa})$.

\begin{tabular}{|c|c|c|c|c|c|}
\hline \multirow{2}{*}{$\begin{array}{c}\text { Ponto } \\
\text { Observado }\end{array}$} & \multirow{2}{*}{$\begin{array}{c}\text { Valor } \\
\text { Teórico }\end{array}$} & \multicolumn{2}{|c|}{ Sol. Fund. Kelvin } & \multicolumn{2}{c|}{ Sol. Fund. Mindlin } \\
\cline { 3 - 6 } & 12 elem. & 40 elem. & 12 elem. & 40 elem. \\
\hline $\mathbf{a}$ & 1 & 1 & 1 & 1 & 1 \\
\hline $\mathbf{b}$ & 1 & 1,0002 & 1,0002 & 1,0002 & 1,0003 \\
\hline $\mathbf{c}$ & 1 & 1,0013 & 1,0013 & 1,0013 & 1,0013 \\
\hline
\end{tabular}

As tensões transversais encontradas com o emprego da formulação apresentada correspondem também aos valores teóricos obtidos através das relações da elasticidade; a saber:

$\sigma_{11}^{\mathrm{MEC}}=\sigma_{22}^{\mathrm{MEC}}=0,333$, enquanto que os valores teóricos são $: \sigma_{11}=\sigma_{22}=0,333 \ldots$.

Embora o exemplo processado seja extremamente simples, os resultados obtidos mostram que a formulação apresentada neste capítulo é adequada para a 
análise de sólidos elásticos tridimensionais. $\mathrm{O}$ elemento triangular plano com aproximação linear, de fácil implementação, possibilita uma discretização coerente.

A aproximação linear descontínua adotada elimina os problemas surgidos na análise dos nós de canto. As integrações utilizadas, tanto a numérica quanto a semianalítica, mostram-se eficientes junto à formulação. Por fim, a relação entre as soluções fundamentais de Kelvin e Mindlin apresentada neste capítulo se verifica à medida que os valores obtidos por ambas são coincidentes.

2.7.2 - Aplicação 2: Viga elástica solicitada à flexão.

Neste exemplo, é analisada uma viga engastada em uma extremidade e livre na outra, solicitada por uma força perpendicular ao seu eixo aplicada na extremidade livre. A figura 2.7.3 apresenta a viga em questão cujos parâmetros elásticos são: $\mathrm{E}=2.100 \mathrm{kN} / \mathrm{cm}^{2}$ e $v=0,3$.
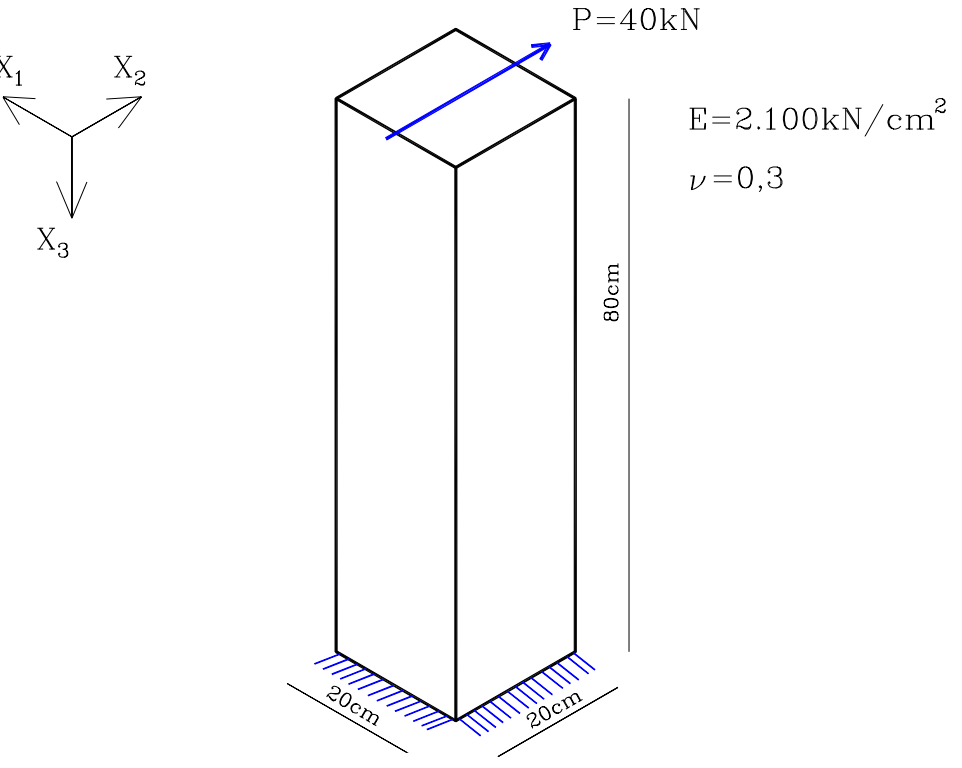

FIGURA 2.7.3 - Viga engastada com carregamento transversal na extremidade livre.

Duas discretizações são utilizadas para representar a superfície de contorno da viga, conforme mostrado na figura 2.7.4. A primeira utiliza 40 elementos 
triangulares planos com aproximação linear (120 pontos de colocação) e a segunda 72 elementos (216 pontos de colocação).

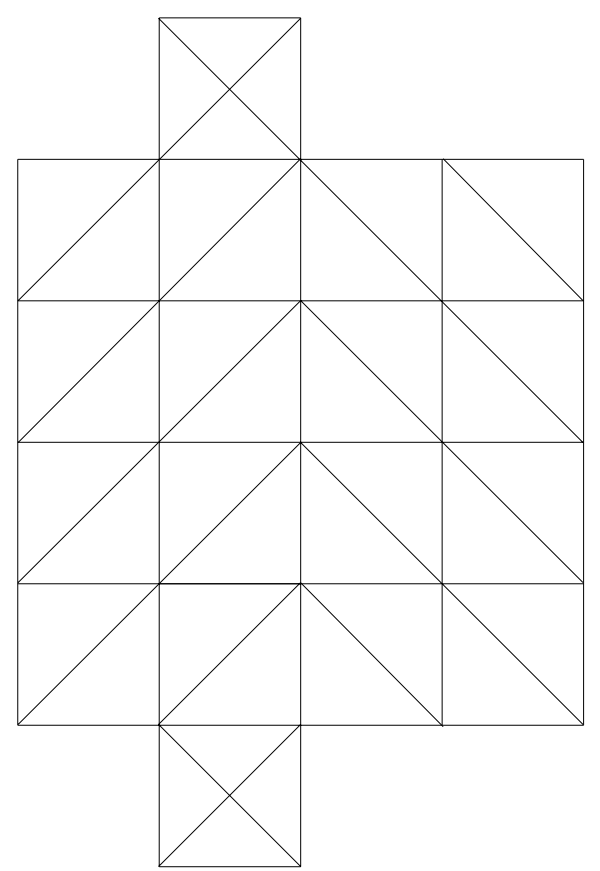

(a)

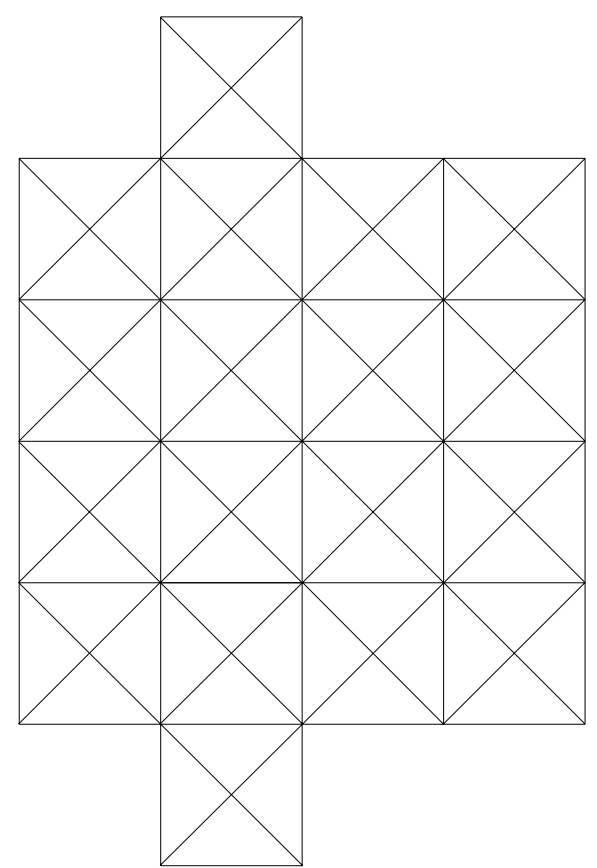

(b)

FIGURA 2.7.4 - Discretizações utilizadas: (a) 40 elementos triangulares planos; e (b) 72 elementos.

Após o processamento dos dois casos, foram encontrados os valores apresentados na tabela 2.7.3 e na figura 2.7.5. Foi utilizado o código computacional obtido utilizando-se a formulação do MEC com a solução fundamental de Kelvin.

TABELA 2.7.3 - Linha elástica da viga analisada (valores em $\mathrm{cm}$ ).

\begin{tabular}{|c|c|c|c|}
\hline Coord. $\mathrm{X}_{3}$ & Teoria de Vigas & $\begin{array}{c}\text { Discretização (a) } \\
40 \text { elementos }\end{array}$ & $\begin{array}{c}\text { Discretização (b) } \\
72 \text { elementos }\end{array}$ \\
\hline 0 & 0,2438 & 0,232 & 0,235 \\
\hline 20 & 0,1540 & 0,146 & 0,149 \\
\hline 40 & 0,0762 & 0,071 & 0,074 \\
\hline 60 & 0,0209 & 0,019 & 0,021 \\
\hline 80 & 0 & 0 & 0 \\
\hline
\end{tabular}


Os resultados obtidos, representados graficamente na figura 2.7.5, permite dizer que a formulação do Método dos Elementos de Contorno apresentada é coerente com o problema analisado. Note-se que, à medida que a discretização envolve mais elementos, os valores obtidos na análise se aproximam daqueles utilizando-se a teoria clássica das vigas.

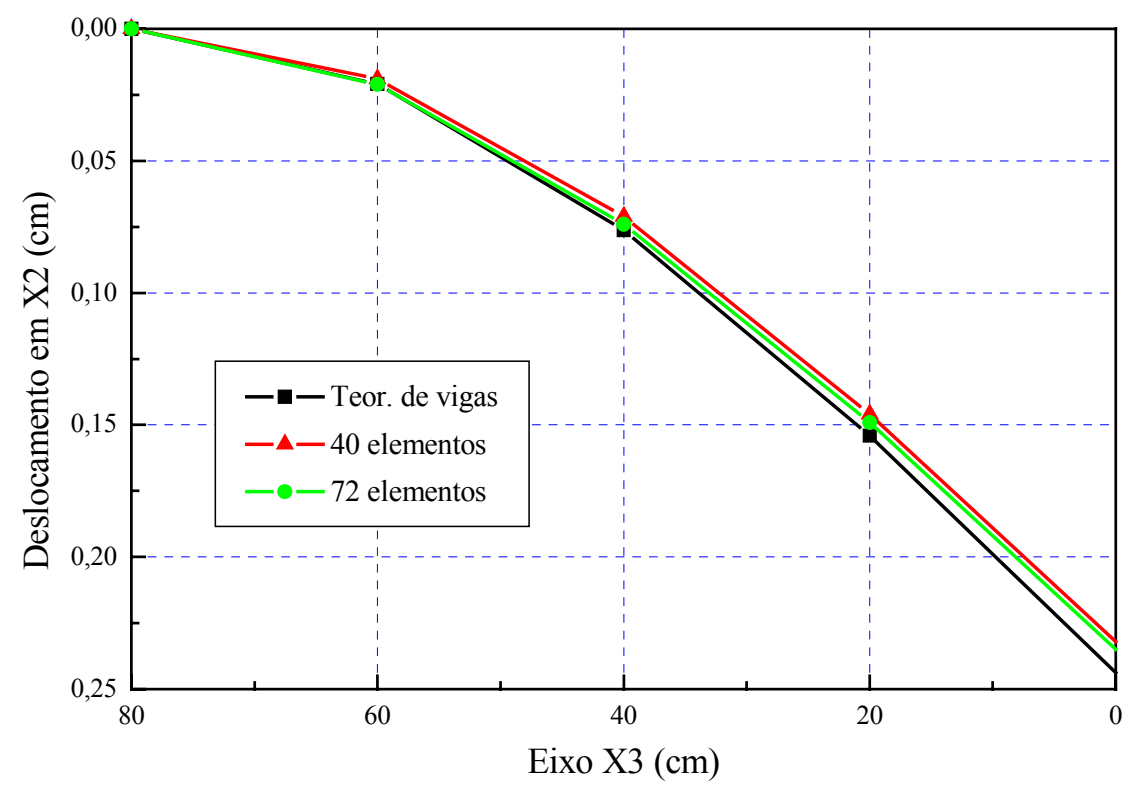

FIGURA 2.7.5 - Linha elástica da viga obtida das três formas.

2.7.3 - Aplicação 3: Sólido cruciforme.

O exemplo apresentado consiste de um sólido elástico em forma de cruz, carregado com forças auto-equilibrantes $t_{2}$ e $t_{3}$ de tração, conforme mostra a figura 2.7.6. O sistema global de coordenadas é adotado com a origem no centro do sólido, para manter a simetria apenas para facilitar a análise dos deslocamentos e tensões, uma vez que não é necessária para a exatidão dos resultados. 


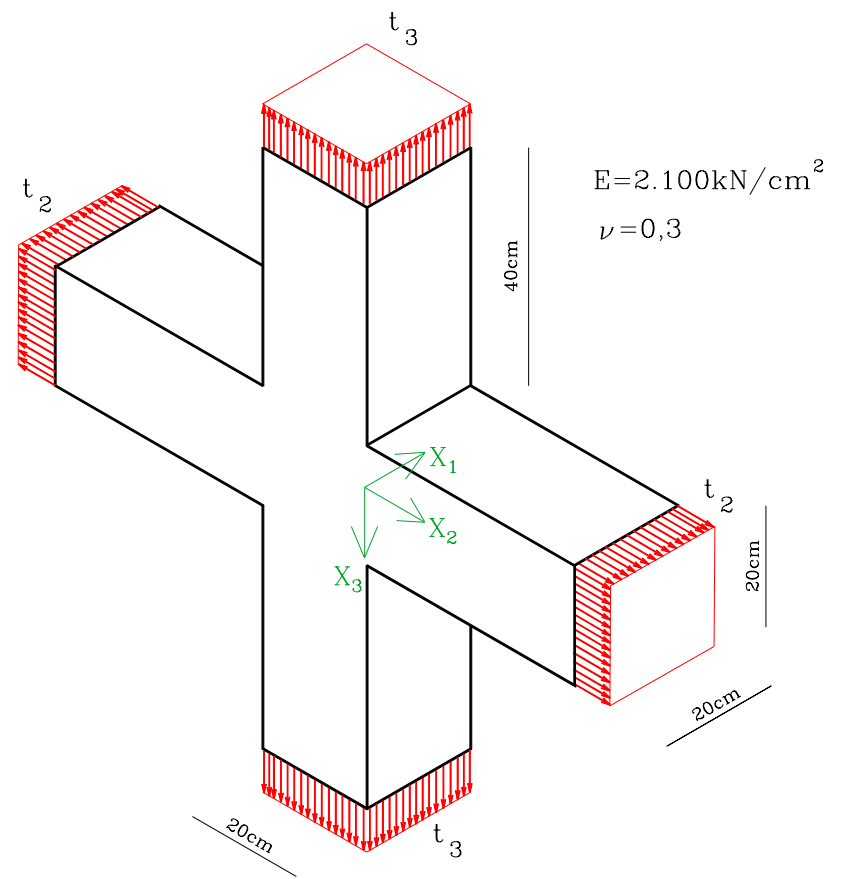

FIGURA 2.7.6 - Sólido elástico em forma de cruz.

A discretização do contorno do sólido é feita utilizando-se elementos triangulares planos com aproximação linear. Os problemas relacionados aos nós de canto estão solucionados à medida que o elemento descontínuo é utilizado. A figura 2.7.7 mostra o arranjo de elementos utilizado na discretização, formado por 152 elementos rebatidos em um único plano, de forma que a normal ao elemento está sempre para fora deste plano.
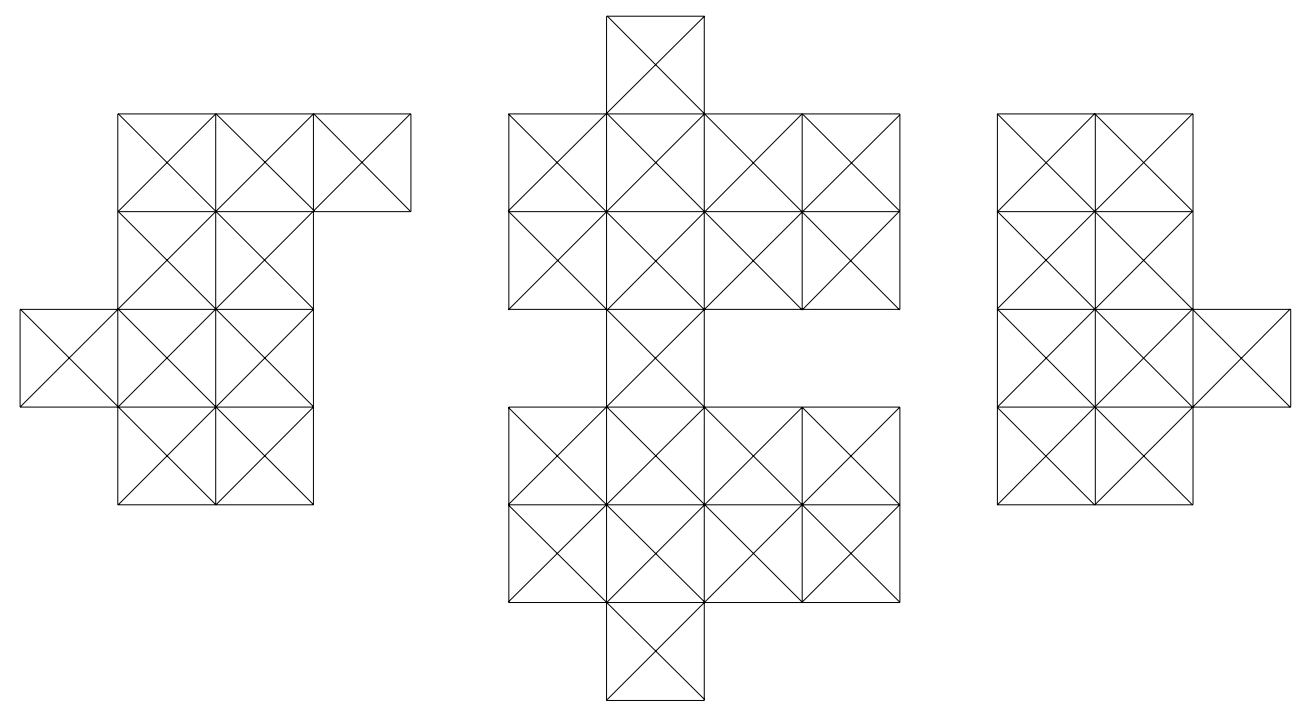

FIGURA 2.7.7 - Discretização em 152 elementos triangulares planos com aproximação linear (456 pontos de colocação). 
Adota-se, como primeira investigação, o par de forças $t_{2}$ e $t_{3}$ de valores iguais a $0,10 \mathrm{kN} / \mathrm{cm}^{2}$. Os valores de deslocamentos nas extremidades do sólido são obtidos na análise dos respectivos valores em pontos pertencentes aos elementos do contorno. A tabela 2.7.4 mostra esses valores nas duas direções investigadas.

TABELA 2.7.4 - Deslocamentos axiais máximos nas direções $\mathrm{X}_{2}$ e $\mathrm{X}_{3}$.

\begin{tabular}{|c|c|}
\hline Eixo & Desloc. Máximo $(\mathrm{cm})$ \\
\hline $\mathrm{X}_{2}$ & 0,00216 (along.) \\
\hline $\mathrm{X}_{3}$ & 0,00216 (along.) \\
\hline
\end{tabular}

Adota-se, ainda, uma malha de pontos internos localizada na região de interseção das duas direções do sólido, a fim de analisar o campo de tensões provocado pelas forças solicitantes. A figura 2.7.8 mostra a região central do sólido onde estão localizados os pontos internos (apenas em um quarto da região, por razões de simetria), e as direções principais das tensões, obtidas da investigação.

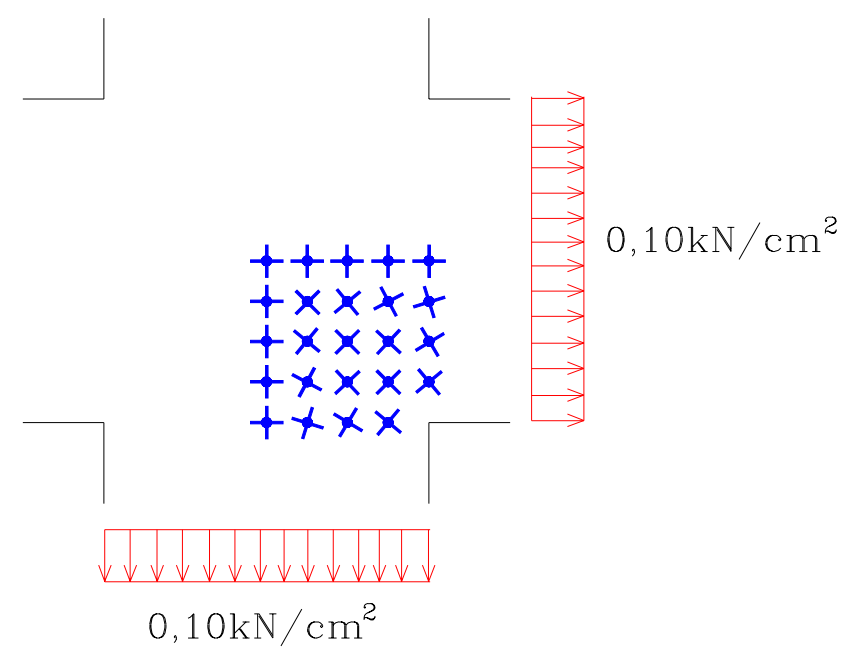

FIGURA 2.7.8 - Direções principais das tensões em pontos internos.

Outra investigação adotada para este sólido utiliza valores diferentes para o par de forças solicitantes, ou seja, $\mathrm{t}_{2}=0,10 \mathrm{kN} / \mathrm{cm}^{2}$ e $\mathrm{t}_{3}=0,20 \mathrm{kN} / \mathrm{cm}^{2}\left(\mathrm{t}_{3}=2 \mathrm{t}_{2}\right)$. Os valores obtidos dos deslocamentos nas extremidades estão mostrados na tabela 2.7.5. 
TABELA 2.7.5 - Deslocamentos axiais máximos nas direções $X_{2}$ e $X_{3}$.

\begin{tabular}{|c|c|}
\hline Eixo & Desloc. Máximo (cm) \\
\hline $\mathrm{X}_{2}$ & 0,00200 (along.) \\
\hline $\mathrm{X}_{3}$ & 0,00449 (along.) \\
\hline
\end{tabular}

Feita a análise das tensões nos pontos internos definidos na investigação anterior, os resultados qualitativos obtidos apresentam-se na figura 2.7.9.

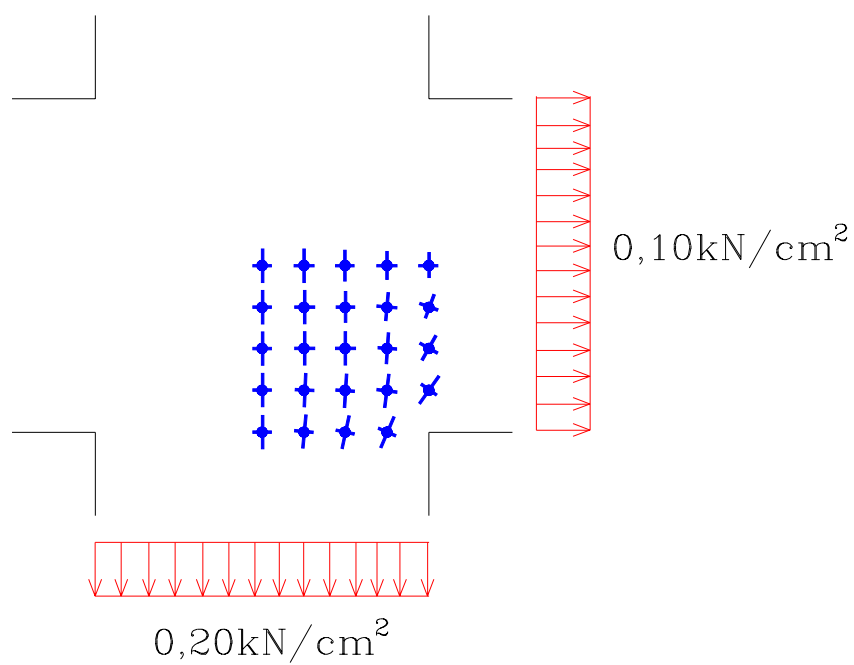

FIGURA 2.7.9 - Direções principais das tensões em pontos internos.

A formulação do Método dos Elementos de Contorno, apresentada neste capítulo, mostra-se adequada para a análise tanto das variáveis de contorno, deslocamentos e forças de superfície em pontos de contorno, quanto das de domínio, deslocamentos e tensões em pontos internos. Permite, por exemplo, que sejam traçadas as direções principais de tensões, identificados os pontos de concentração de tensões e possíveis superfícies de ruptura.

2.7.4 - Aplicação 4: Carregamento uniformemente distribuído sobre a superfície livre do semi-infinito.

Neste exemplo, uma carga uniformemente distribuída age sobre uma área retangular localizada na superfície do semi-infinito. A figura 2.7.10 mostra o 
retângulo de lados 9,15m e 18,3m, com a carga de $0,0956 \mathrm{kN} / \mathrm{m}^{2}$, módulo de elasticidade longitudinal igual a $44,42 \mathrm{kN} / \mathrm{m}^{2}$ e coeficiente de Poisson 0,3.
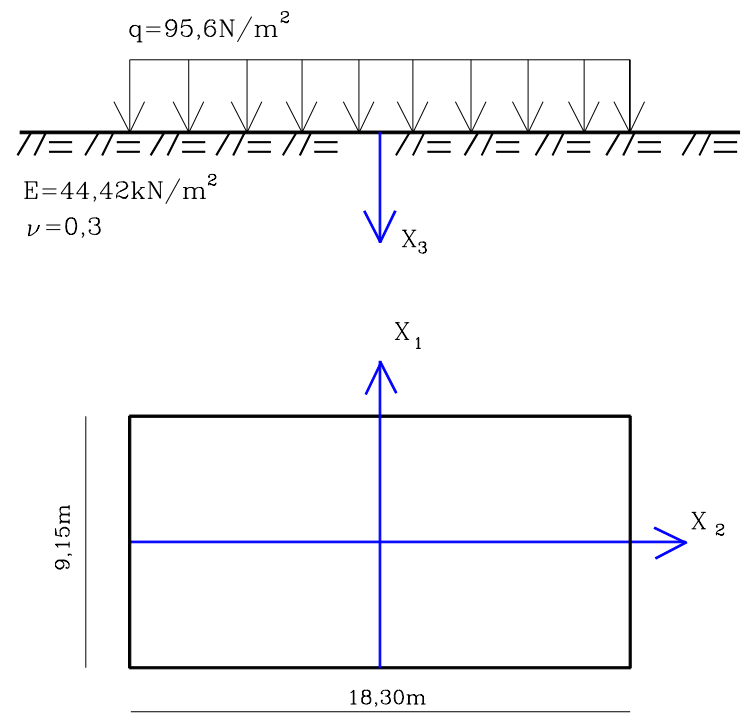

FIGURA 2.7.10 - Área retangular na superfície livre do semi-infinito, uniformemente carregada.

Este problema encontra-se resolvido em SÁ (1986), com valores apresentados para eixos diferentes aos utilizados na presente aplicação. Portanto, os resultados apresentados aqui são obtidos através do processamento utilizando as formulações apresentadas neste capítulo, com as soluções fundamentais de Kelvin e Mindlin.

As discretizações da superfície de contorno são apresentadas na figura 2.7.11. Novamente, utiliza-se o elemento triangular plano com aproximação linear.

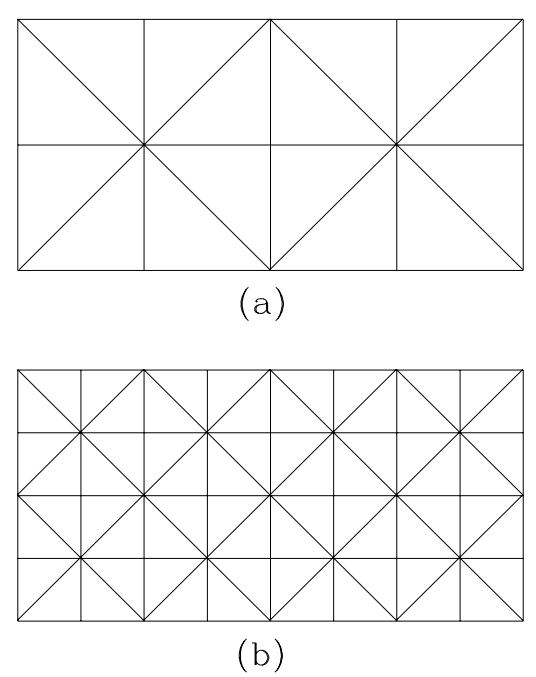

FIGURA 2.7.11 - Discretizações utilizadas: (a) 16 elementos; e (b) 64 elementos. 
Como primeira análise, faz-se a comparação entre os resultados obtidos utilizando-se a discretização (a), da figura 2.7.11, tanto para a solução fundamental de Kelvin quanto para a de Mindlin. A solução fundamental de Mindlin é adequada para este tipo de problemas, ou seja, relacionados ao semi-infinito com carregamentos ou escavações próximos à superfície livre. Com esta formulação, apenas as superfícies escavadas ou carregadas precisam ser discretizadas, como é o caso do presente exemplo e das discretizações apresentadas. Já para a solução fundamental de Kelvin, adequada para o espaço infinito, é necessário discretizar, além das superfícies escavadas e carregadas, a superfície livre de trações do semiinfinito. A análise apresentada utiliza a mesma discretização para ambas as soluções fundamentais. Os resultados encontram-se nas tabelas e gráficos dados a seguir.

TABELA 2.7.6 - Deslocamentos em $\mathrm{X}_{3}$ ao longo de $\mathrm{X}_{1}(\mathrm{em} \mathrm{cm})$.

\begin{tabular}{|c|c|c|}
\hline $\mathrm{X}_{1}$ & S.F. de Mindlin (a) & S.F. de Kelvin (a) \\
\hline 0 & 2,829 & 2,742 \\
\hline 4,575 & 2,416 & 2,248 \\
\hline 8 & 0,551 & 0,556 \\
\hline 15 & 0,311 & 0,310 \\
\hline
\end{tabular}

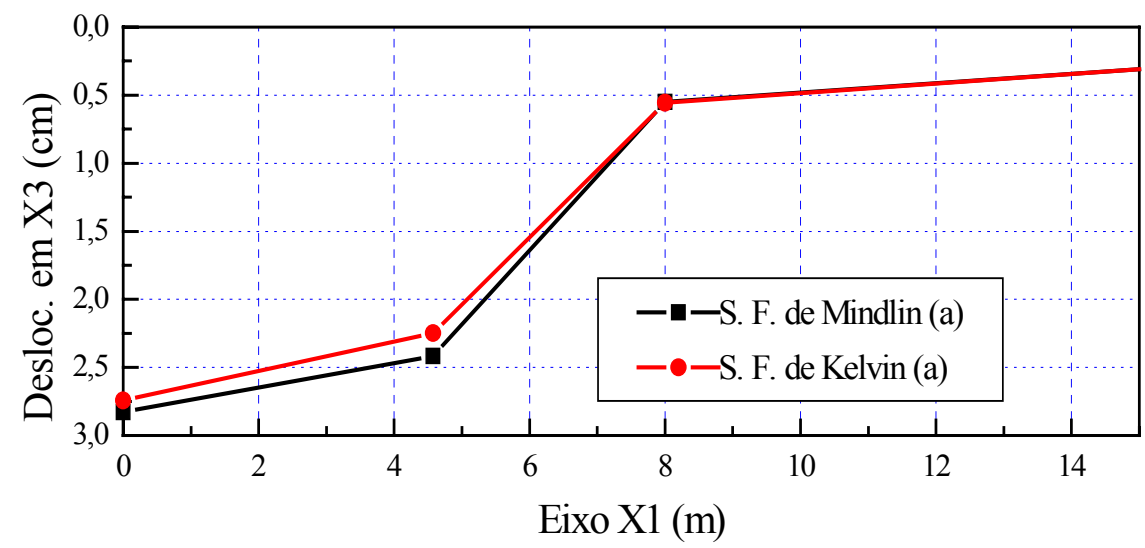

FIGURA 2.7.12 - Deslocamentos em $\mathrm{X}_{3}$ ao longo de $\mathrm{X}_{1}(\mathrm{em} \mathrm{cm})$ - comparação entre as soluções fundamentais de Mindlin e Kelvin (discretização (a)). 
TABELA 2.7.7 - Deslocamentos em $\mathrm{X}_{3}$ ao longo de $\mathrm{X}_{2}(\mathrm{em} \mathrm{cm})$.

\begin{tabular}{|c|c|c|}
\hline $\mathrm{X}_{2}$ & S.F. de Mindlin (a) & S.F. de Kelvin (a) \\
\hline 0 & 2,829 & 2,742 \\
\hline 4,575 & 2,780 & 2,748 \\
\hline 9,15 & 2,020 & 1,827 \\
\hline 15 & 0,364 & 0,359 \\
\hline
\end{tabular}

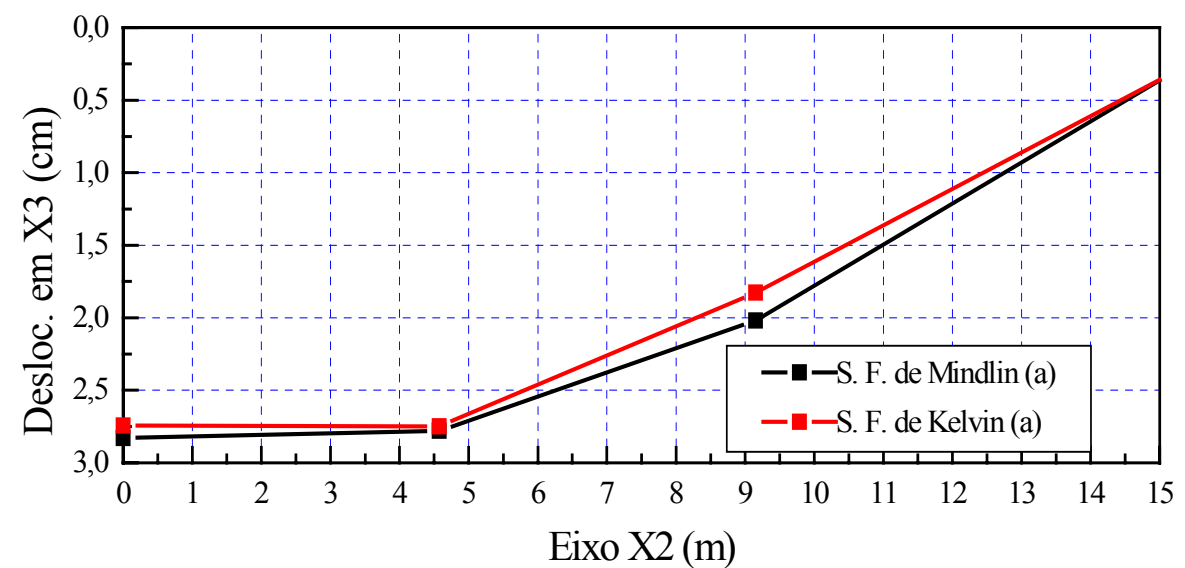

FIGURA 2.7.13 - Deslocamentos em $X_{3}$ ao longo de $X_{2}$ (em cm) - comparação entre as soluções fundamentais de Mindlin e Kelvin (discretização (a)).

Os valores obtidos e apresentados nos gráficos acima mostram uma pequena diferença entre as formulações com a solução fundamental de Mindlin, adotada como referência, e a de Kelvin, prejudicada pela discretização apenas da região carregada, não interpretando de maneira correta a existência da superfície livre de trações.

Os resultados mostrados na seqüência referem-se a comparações entre a mesma solução fundamental, de Mindlin, variando-se as discretizações da superfície de contorno, apresentadas na figura 2.7.11.

Pode-se verificar que os resultados são bastante próximos, mostrando o grau de influência das discretizações utilizadas. É interessante ressaltar que a discretização feita com mais elementos consegue captar melhor a influência das variáveis de contorno sobre as de domínio, o que resulta em valores para pontos internos mais precisos. 
TABELA 2.7.8 - Deslocamentos em $\mathrm{X}_{3}$ ao longo de $\mathrm{X}_{1}(\mathrm{em} \mathrm{cm})$.

\begin{tabular}{|c|c|c|}
\hline $\mathrm{X}_{1}$ & S.F. de Mindlin (a) & S.F. de Mindlin (b) \\
\hline 0 & 2,829 & 2,790 \\
\hline 4,575 & 2,416 & 2,221 \\
\hline 8 & 0,551 & 0,552 \\
\hline 15 & 0,311 & 0,311 \\
\hline
\end{tabular}

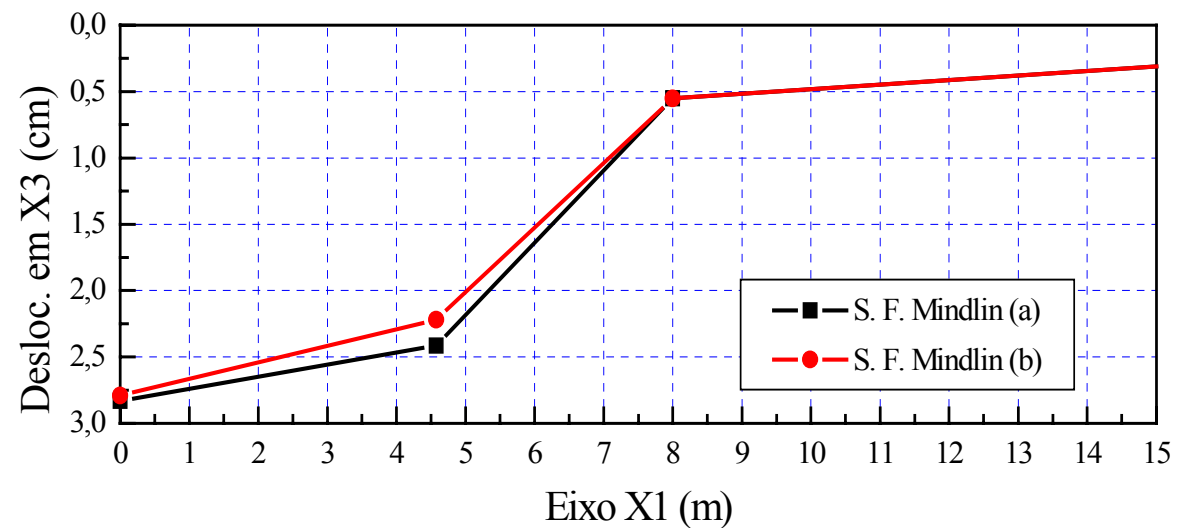

FIGURA 2.7.14 - Deslocamentos em $X_{3}$ ao longo de $X_{1}(e m$ cm) - comparação entre as discretizações (a) e (b) para a solução fundamental de Mindlin.

TABELA 2.7.9 - Deslocamentos em $X_{3}$ ao longo de $X_{2}$ (em cm).

\begin{tabular}{|c|c|c|}
\hline $\mathrm{X}_{2}$ & S.F. de Mindlin (a) & S.F. de Mindlin (b) \\
\hline 0 & 2,829 & 2,790 \\
\hline 4,575 & 2,780 & 2,653 \\
\hline 9,15 & 2,020 & 1,970 \\
\hline 15 & 0,364 & 0,363 \\
\hline 20 & 0,257 & 0,257 \\
\hline
\end{tabular}




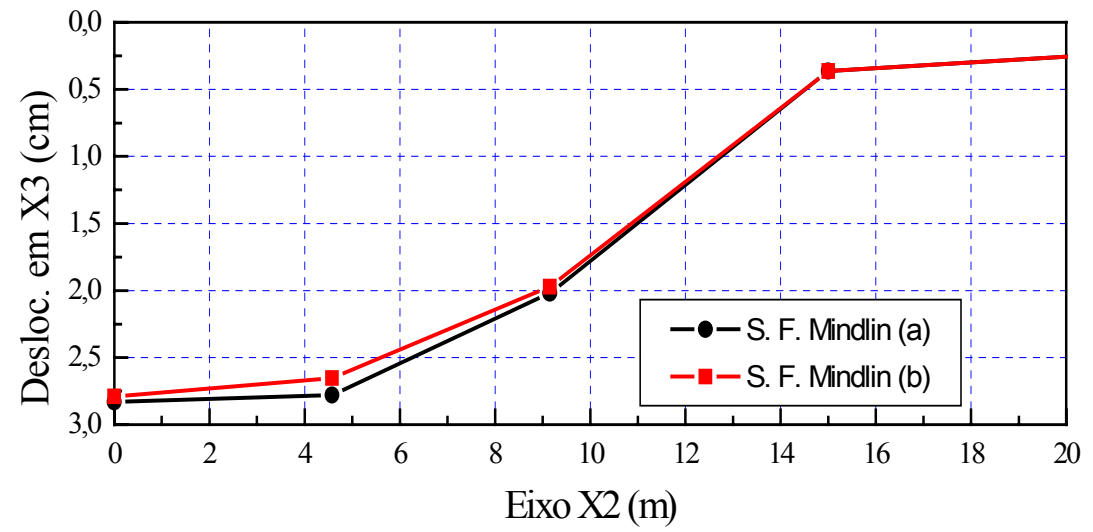

FIGURA 2.7.15 - Deslocamentos em $X_{3}$ ao longo de $X_{2}(\mathrm{em} \mathrm{cm})$ - comparação entre as discretizações (a) e (b) para a solução fundamental de Mindlin.

TABELA 2.7.10 -Tensões $\sigma_{33}$ ao longo do eixo $\mathrm{X}_{3}(\mathrm{~Pa})$.

\begin{tabular}{|c|c|c|}
\hline $\mathrm{X}_{3}$ & S.F. de Mindlin (a) & S.F. de Mindlin (b) \\
\hline 0 & $-0,0956$ & $-0,0956$ \\
\hline 1 & $-0,0911$ & $-0,0911$ \\
\hline 2 & $-0,1000$ & $-0,1012$ \\
\hline 4 & $-0,0889$ & $-0,0881$ \\
\hline 8 & $-0,0521$ & $-0,0516$ \\
\hline 15 & $-0,0204$ & $-0,0203$ \\
\hline
\end{tabular}

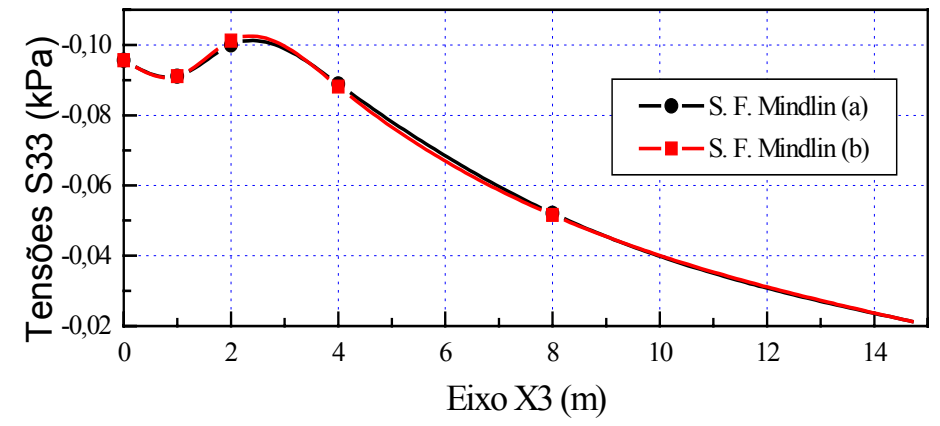

FIGURA 2.7.16 - Tensões $\sigma_{33}$ ao longo do eixo $\mathrm{X}_{3}$ (em Pa) - comparação entre as discretizações (a) e (b) para a solução fundamental de Mindlin.

A formulação do MEC com a solução fundamental de Mindlin mostra-se adequada para a análise de problemas onde há carregamentos próximos à superfície livre do espaço semi-infinito. 
2.7.5 - Aplicação 5: Cavidade próxima à superfície do semi-infinito.

Neste exemplo é analisado um problema de escavação próxima à superfície do sólido semi-infinito - o solo. Trata-se de uma cavidade na superfície do solo com uma carga distribuída em uma região da superfície próxima à escavação, conforme mostra a figura 2.7.17.

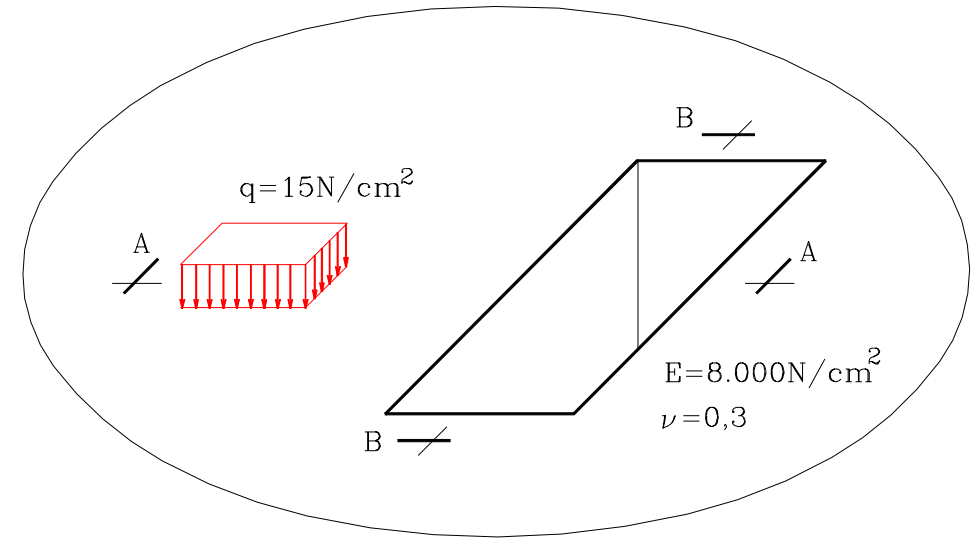

(a) CAVIDADE E CARGA

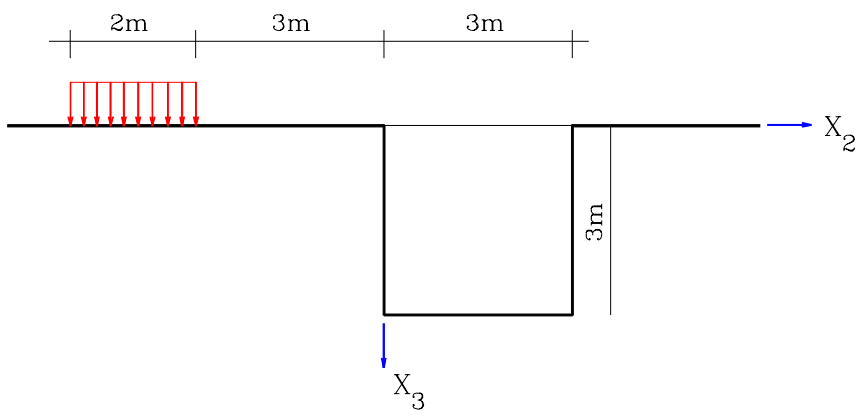

(b) CORTE AA

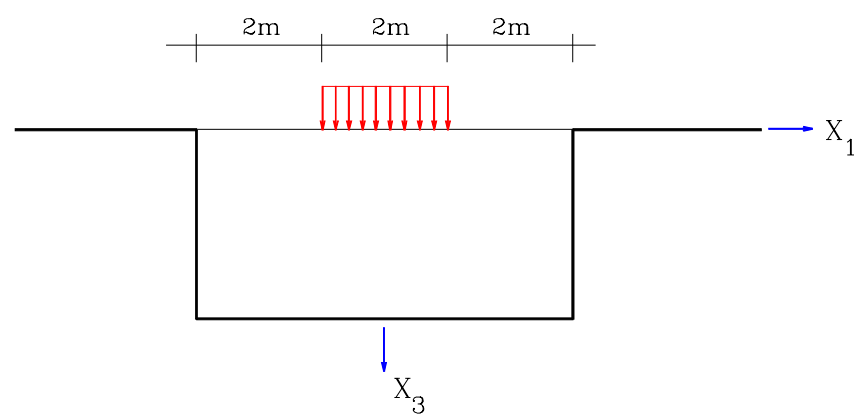

(c) CORTE BB

FIGURA 2.7.17 - Definição do problema: cavidade no meio semi-infinito, próxima da superfície, com carga distribuída agindo na superfície. 
Com o emprego da formulação do Método dos Elementos de Contorno usando a solução fundamental de Mindlin, o problema é resolvido de maneira mais adequada, pois a discretização utilizada apenas precisa ser feita no contorno da escavação e na região da carga.

A discretização do contorno utilizada é formada por 152 elementos triangulares planos com aproximação linear, conforme mostra a figura 2.7.18.

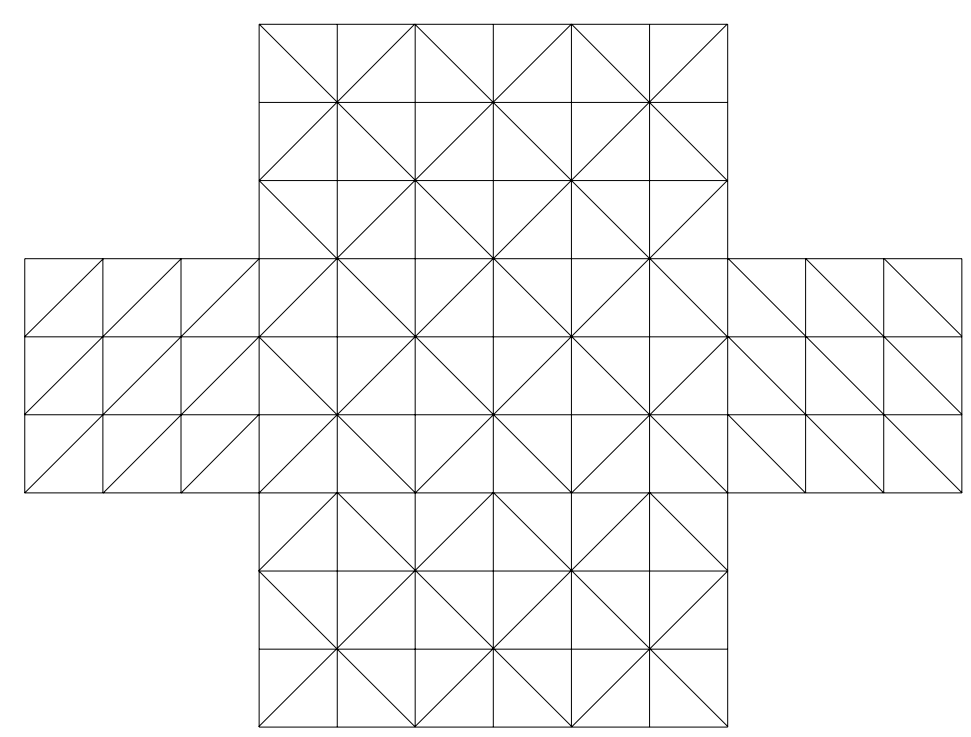

FIGURA 2.7.18 - Discretização utilizada: 152 elementos triangulares planos.

As investigações são efetuadas no lado da escavação mais próximo da área carregada (plano $\mathrm{X}_{1} \mathrm{X}_{3}$, com $\mathrm{X}_{2}=0$ ), onde são medidos os deslocamentos em vários níveis, conforme mostram as figuras 2.7.19 e 2.7.20. 


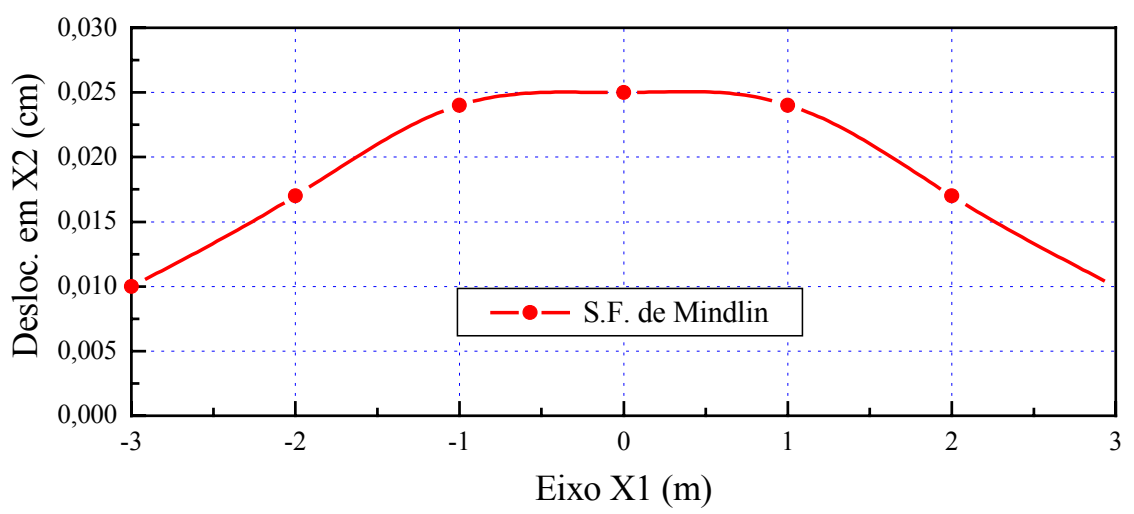

FIGURA 2.7.19 - Deslocamentos horizontais na face mais próxima da carga, em $\mathrm{X}_{3}=2 \mathrm{~m}$.

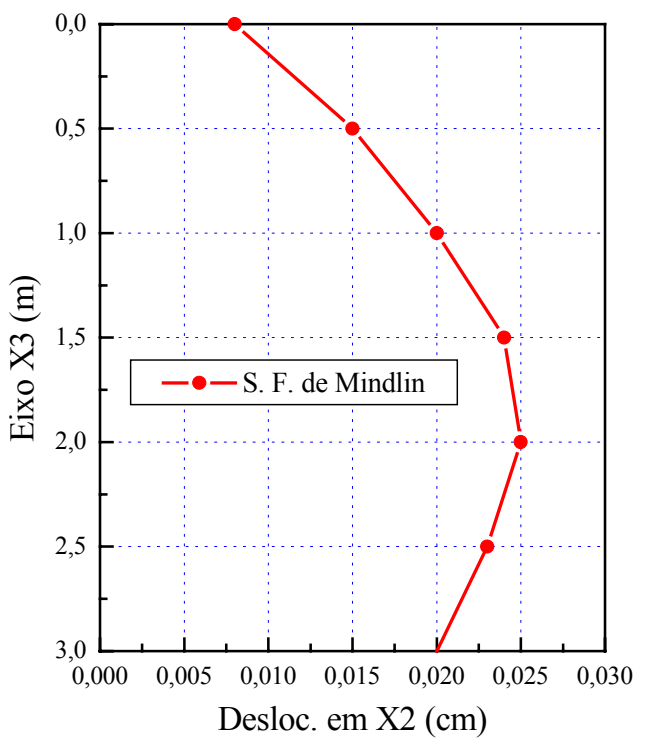

FIGURA 2.7.20 - Deslocamentos horizontais na face mais próxima da carga, no eixo $\mathrm{X}_{3}$.

Os valores dos deslocamentos obtidos descrevem uma superfície, onde os gráficos mostrados nas figuras 2.7.19 e 2.7.20 são traços desta, relacionada ao que se chama bulbo de pressão na área de Mecânica dos Solos. A superfície de deslocamento da face vertical mais próxima da carga está representada na figura 2.7.31, apresentada na forma de curvas de nível. 


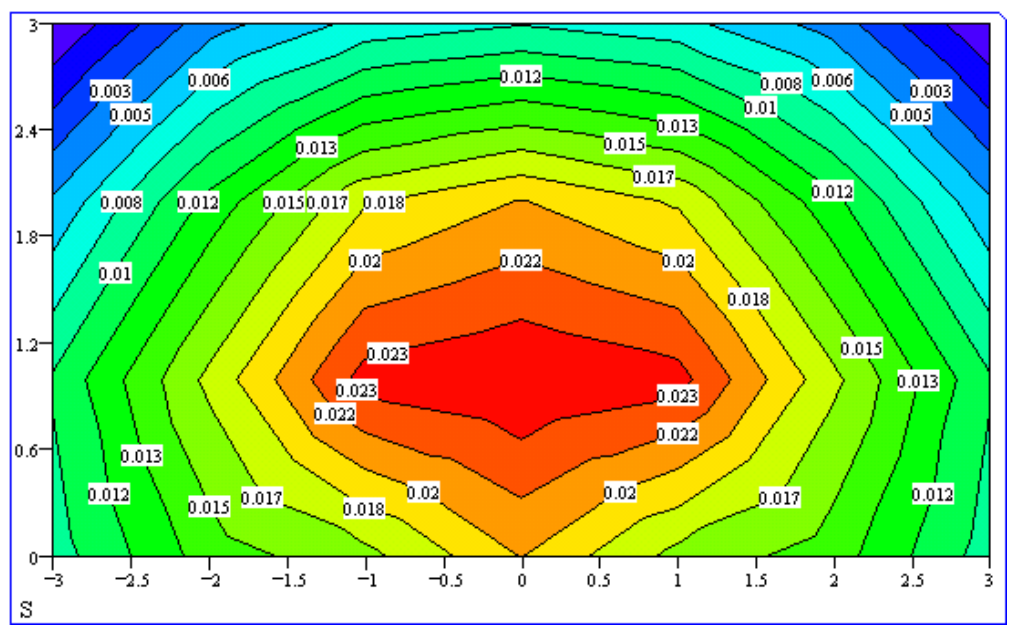

FIGURA 2.7.21 - Superfície de deslocamento da face vertical da escavação mais próxima da carga: curvas de nível (valores em $\mathrm{cm}$ ). A graduação do eixo vertical do gráfico estabelece seu zero em $X_{3}=3 \mathrm{~m}$. 


\section{CAPÍTULO 3}

\section{FORMULAÇÃO ELASTODINÂMICA TRIDIMEN- SIONAL DO MEC - MATRIZ DE MASSA}

\section{1- INTRODUÇÃO}

A mudança com o tempo da posição relativa de um corpo no espaço é chamada de movimento. Para se falar de movimento, é necessário estabelecer uma forma para descrevê-lo e um referencial para observá-lo. Estabelecido isto, é possível escrever a expressão denominada "equação de movimento", que rege o problema dinâmico.

Neste capítulo é apresentada uma formulação dinâmica do Método dos Elementos de Contorno para sólidos elásticos tridimensionais. A formulação é desenvolvida utilizando-se o conceito de "matriz de massa".

Inicialmente, apresentam-se as relações mais importantes da elastodinâmica, de forma sucinta, referenciando o que já foi desenvolvido para a estática no capítulo anterior. Indicam-se as representações integrais tanto para pontos do domínio quanto do contorno. Para transformar os termos de domínio para o contorno, relativos às forças de inércia que surgem nas representações integrais, são apresentados o Método da Reciprocidade Dual (MRD) e a Técnica da Integração Direta. Para os dois procedimentos são mostradas as equações básicas e discretizadas através do emprego de elementos triangulares planos (apresentados no item 2.6.2). Indicam-se, ainda, os algoritmos de Newmark e Houbolt para a integração no tempo. Por fim, 
apresentam-se alguns exemplos para mostrar a eficiência do método, comparando-se os resultados com os dois procedimentos indicados para transformar o termo de domínio para o contorno.

\section{2 - RELAÇÕES DA ELASTODINÂMICA}

Considere-se um sólido elástico, homogêneo, isotrópico e com distribuição contínua de matéria em seu domínio $\Omega$, cujo contorno é $\Gamma$, conforme mostra a figura 3.2.1.

FIGURA 3.2.1 - Definição do objeto de estudo: sólido tridimensional de domínio $\Omega$ e contorno $\Gamma$ (nos instantes t e $\Delta \mathrm{t})$.

Um corpo qualquer, durante o seu movimento e sob a ação de um sistema de forças, deve obedecer aos princípios de conservação da quantidade de movimento linear e da conservação da quantidade de movimento angular, de forma a se ter garantidas as condições de equilíbrio dinâmico. Assim, a equação de equilíbrio para o problema elastodinâmico é:

$$
\sigma_{\mathrm{ij}, \mathrm{j}}+\rho \mathrm{b}_{\mathrm{i}}-\rho \ddot{u}_{\mathrm{i}}=0,
$$


onde:

$\sigma_{\mathrm{ij}, \mathrm{j}}(\chi, \mathrm{t})=$ a derivada no espaço do tensor das tensões;

$\mathrm{b}_{\mathrm{i}}(\chi, \mathrm{t})=$ as forças volumétricas por densidade;

$\rho(\chi)=$ a densidade de massa $\mathrm{e}$

$\ddot{\mathrm{u}}_{\mathrm{i}}=\mathrm{a}$ aceleração do ponto na direção $\mathrm{X}_{\mathrm{i}},\left(\frac{\partial^{2} \mathrm{u}_{\mathrm{i}}}{\partial \mathrm{t}^{2}}\right)$.

A equação de equilíbrio (3.2.1) é semelhante à (2.3.1), diferenciando-se substancialmente à medida que depende de uma variável no espaço (como na estática) e de uma variável no tempo. As demais relações do equilíbrio estático também são válidas para o problema dinâmico, incluindo-se a variável tempo.

A simetria do tensor das tensões apresenta-se da seguinte forma:

$$
\sigma_{\mathrm{ij}}(\chi, \mathrm{t})=\sigma_{\mathrm{ji}}(\chi, \mathrm{t})
$$

Adapta-se, também, a expressão das forças de superfície,

$$
\mathrm{p}_{\mathrm{i}}(\chi, \mathrm{t})=\sigma_{\mathrm{ji}}(\chi, \mathrm{t}) \mathrm{n}_{\mathrm{j}}(\chi)
$$

e a relação cinemática

$$
\varepsilon_{\mathrm{ij}}(\chi, \mathrm{t})=\frac{1}{2}\left(\mathrm{u}_{\mathrm{i}, \mathrm{j}}(\chi, \mathrm{t})+\mathrm{u}_{\mathrm{j}, \mathrm{i}}(\chi, \mathrm{t})\right),
$$

A equação constitutiva do material é semelhante à já apresentada em (2.3.5). A relação linear entre as componentes de tensão e deformação, conhecida como lei de Hooke, equação (2.3.5), também se aplica para o problema dinâmico. Observa-se, entretanto, que as componentes de tensão e deformação estão expressas em função das variáveis no espaço $\chi$ e no tempo t. Assim, 


$$
\sigma_{\mathrm{ij}}(\chi, \mathrm{t})=\frac{E}{(1+v)}\left[\frac{v}{(1-2 v))} \delta_{\mathrm{ij}} \varepsilon_{\mathrm{kk}}(\chi, \mathrm{t})+\varepsilon_{\mathrm{ij}}(\chi, \mathrm{t})\right] .
$$

As constantes elásticas da lei de Hooke são apresentadas em (2.3.7a-c). Com as equações aqui apresentadas, que juntas formam um sistema completo de equações que governam o movimento de sólidos elásticos tridimensionais, pode-se obter a equação diferencial do movimento em termos de deslocamentos:

$$
u_{i, j j}(\chi, t)+\frac{1}{(1-2 v)} u_{j, i j}(\chi, t)+\frac{1}{G} \rho b_{i}(\chi, t)-\frac{1}{G} \rho \ddot{u}_{i}(\chi, t)=0
$$

Para a análise completa do problema elastodinâmico, faz-se necessário estabelecer as condições de contorno. Aqui, também, do mesmo modo que o problema elastostático, tem-se as definições das condições essenciais e naturais, (2.3.10a-b), relativas ao espaço. São necessárias, ainda, as condições iniciais para qualquer ponto $\chi$ do sólido $\Omega$ no tempo $\mathrm{t}=0$, que se traduz em:

$$
\begin{aligned}
& \mathrm{u}_{\mathrm{i}}(\chi, 0)=\mathrm{u}_{\mathrm{oi}}(\chi) \\
& \dot{\mathrm{u}}_{\mathrm{i}}(\chi, 0)=\mathrm{v}_{\mathrm{oi}}(\chi)
\end{aligned}
$$

\section{3 - REPRESENTAÇÕES INTEGRAIS}

\subsection{1 - Equações Básicas}

Analogamente ao que foi feito para o caso estático, pretende-se aplicar o teorema da reciprocidade de Maxwell-Betti a dois estados independentes de deslocamento: um elastodinâmico e outro elastostático. Para este último foi escolhido o fundamental. Tendo em vista a equação de equilíbrio dinâmico (3.2.1), estes estados têm as seguintes características: 
- estado elastodinâmico:

$$
\begin{aligned}
& \sigma_{\mathrm{ij}, \mathrm{j}}(\chi, \mathrm{t}), \quad \mathrm{u}_{\mathrm{i}}(\chi, \mathrm{t}), \mathrm{p}_{\mathrm{i}}(\chi, \mathrm{t}), \ddot{\mathrm{u}}_{\mathrm{i}}(\chi, \mathrm{t}) \text { e } \mathrm{b}_{\mathrm{i}}(\chi, \mathrm{t}) \\
& \sigma_{\mathrm{ij}, \mathrm{j}}(\chi, \mathrm{t})=\rho\left(\ddot{\mathrm{u}}_{\mathrm{i}}(\chi, \mathrm{t})+\mathrm{b}_{\mathrm{i}}(\chi, \mathrm{t})\right)
\end{aligned}
$$

- estado elastostático (problema fundamental):

$$
\begin{aligned}
& \sigma_{\mathrm{ij}, \mathrm{j}}^{*}(\chi), \quad u_{\mathrm{i}}^{*}(\chi), \quad \mathrm{p}_{\mathrm{i}}^{*}(\chi) \text { e } \mathrm{b}_{\mathrm{i}}^{*}(\chi) \\
& \sigma_{\mathrm{ij}, \mathrm{j}}^{*}(\chi)=\mathrm{b}_{\mathrm{i}}^{*}(\chi)=\delta(\chi, \mathrm{q}) \delta_{\mathrm{ki}}
\end{aligned}
$$

Aplicando-se o teorema da reciprocidade aos dois estados escolhidos, tem-se:

$$
\int_{\Omega}\left(u_{\mathrm{i}}^{*} \sigma_{\mathrm{ij}, \mathrm{j}}-\mathrm{u}_{\mathrm{i}} \sigma_{\mathrm{ij}, \mathrm{j}}^{*}\right) \mathrm{d} \Omega=\int_{\Gamma}\left(\mathrm{u}_{\mathrm{i}}^{*} \mathrm{p}_{\mathrm{i}}-\mathrm{u}_{\mathrm{i}} \mathrm{p}_{\mathrm{i}}^{*}\right) \mathrm{d} \Gamma
$$

Sabe-se que:

$$
\begin{aligned}
& \mathrm{u}_{\mathrm{j}}^{*}=\mathrm{u}_{\mathrm{kj}}^{*} \delta_{\mathrm{ik}}=\mathrm{u}_{\mathrm{ij}}^{*}, \mathrm{e} \\
& \mathrm{p}_{\mathrm{j}}^{*}=\mathrm{p}_{\mathrm{kj}}^{*} \delta_{\mathrm{ik}}=\mathrm{p}_{\mathrm{ij}}^{*} .
\end{aligned}
$$

Após alguns desenvolvimentos matemáticos, chega-se na versão para a elastodinâmica da representação integral de Somigliana (2.5.1) que é:

$$
\begin{aligned}
\mathrm{u}_{\mathrm{i}}(\mathrm{s}, \mathrm{t}) & =-\int_{\Gamma} \mathrm{p}_{\mathrm{ij}}^{*}(\mathrm{~s}, \mathrm{Q}) \mathrm{u}_{\mathrm{j}}(\mathrm{Q}, \mathrm{t}) \mathrm{d} \Gamma+\int_{\Gamma} \mathrm{u}_{\mathrm{ij}}^{*}(\mathrm{~s}, \mathrm{Q}) \mathrm{p}_{\mathrm{j}}(\mathrm{Q}, \mathrm{t}) \mathrm{d} \Gamma+ \\
& +\int_{\Omega} \rho \mathrm{u}_{\mathrm{ij}}^{*}(\mathrm{~s}, \mathrm{q}) \mathrm{b}_{\mathrm{j}}(\mathrm{q}, \mathrm{t}) \mathrm{d} \Omega-\int_{\Omega} \rho \mathrm{u}_{\mathrm{ij}}^{*}(\mathrm{~s}, \mathrm{q}) \ddot{\mathrm{u}}_{\mathrm{j}}(\mathrm{q}, \mathrm{t}) \mathrm{d} \Omega,
\end{aligned}
$$

lembrando-se que $\mathrm{Q}$ representa um ponto no contorno $\Gamma$; q e s são pontos no domínio $\Omega$; e t a variável tempo.

Estabelecida a equação integral de deslocamentos para o problema elastodinâmico, com o auxílio das equações (3.2.4) e (3.2.5), pode-se determinar a equação integral para as tensões em pontos do domínio $\Omega$ : 


$$
\begin{aligned}
\sigma_{\mathrm{ij}}(\mathrm{s}, \mathrm{t}) & =-\int_{\Gamma} \mathrm{S}_{\mathrm{ijk}}^{*}(\mathrm{~s}, \mathrm{Q}) \mathrm{u}_{\mathrm{k}}(\mathrm{Q}, \mathrm{t}) \mathrm{d} \Gamma+\int_{\Gamma} \mathrm{D}_{\mathrm{ijk}}^{*}(\mathrm{~s}, \mathrm{Q}) \mathrm{p}_{\mathrm{k}}(\mathrm{Q}, \mathrm{t}) \mathrm{d} \Gamma+ \\
& +\int_{\Omega} \rho \mathrm{D}_{\mathrm{ijk}}^{*}(\mathrm{~s}, \mathrm{q}) \mathrm{b}_{\mathrm{k}}(\mathrm{q}, \mathrm{t}) \mathrm{d} \Omega-\int_{\Omega} \rho \mathrm{D}_{\mathrm{ijk}}^{*}(\mathrm{~s}, \mathrm{q}) \ddot{\mathrm{u}}_{\mathrm{k}}(\mathrm{q}, \mathrm{t}) \mathrm{d} \Omega .
\end{aligned}
$$

Os tensores $\mathrm{S}_{\mathrm{ijk}}^{*} \mathrm{e} \mathrm{D}_{\mathrm{ijk}}^{*}$, relativos à solução fundamental empregada são os mesmos já apresentados para a elastostática, ou seja, as expressões (2.5.3), (2.5.4), (2.5.5) e (2.5.6).

\subsection{2 - Para Pontos do Contorno}

A obtenção da representação integral de deslocamentos para pontos do contorno, a partir de (3.3.5), segue o mesmo procedimento utilizado para a elastostática, mostrado no item 2.5.2. Sendo assim, a representação integral em deslocamentos para pontos do contorno é:

$$
\begin{aligned}
\mathrm{c}_{\mathrm{ij}} \mathrm{u}_{\mathrm{j}}(\mathrm{S}, \mathrm{t})= & -\int_{\Gamma} \mathrm{p}_{\mathrm{ij}}^{*}(\mathrm{~S}, \mathrm{Q}) \mathrm{u}_{\mathrm{j}}(\mathrm{Q}, \mathrm{t}) \mathrm{d} \Gamma+\int_{\Gamma} \mathrm{u}_{\mathrm{ij}}^{*}(\mathrm{~S}, \mathrm{Q}) \mathrm{p}_{\mathrm{j}}(\mathrm{Q}, \mathrm{t}) \mathrm{d} \Gamma+ \\
& +\int_{\Omega} \rho \mathrm{u}_{\mathrm{ij}}^{*}(\mathrm{~S}, \mathrm{q}) \mathrm{b}_{\mathrm{j}}(\mathrm{q}, \mathrm{t}) \mathrm{d} \Omega-\int_{\Omega} \rho \mathrm{u}_{\mathrm{ij}}^{*}(\mathrm{~S}, \mathrm{q}) \ddot{\mathrm{u}}_{\mathrm{j}}(\mathrm{q}, \mathrm{t}) \mathrm{d} \Omega,
\end{aligned}
$$

lembrando-se que $c_{i j}$ possui os mesmos valores dados em (2.5.9).

As tensões em pontos do contorno podem ser obtidas utilizando-se o procedimento apresentado no item 2.6.5. As tensões, então, são obtidas a partir dos valores aproximados das deformações, escritas em função dos valores nodais de deslocamentos. Na elastodinâmica, esse procedimento é utilizado para cada instante de tempo.

Os valores das tensões em pontos do contorno são calculados de forma aproximada, utilizando-se as expressões: 


$$
\begin{aligned}
& \bar{\sigma}_{11}(\chi, \mathrm{t})=\frac{1}{1-v}\left[2 \mathrm{G}\left(\frac{\partial \mathrm{u}_{1}(\chi, \mathrm{t})}{\partial \mathrm{x}_{1}}+v \frac{\partial \mathrm{u}_{2}(\chi, \mathrm{t})}{\partial \mathrm{x}_{2}}\right)+v \mathrm{P}_{3}(\chi, \mathrm{t})\right] \\
& \bar{\sigma}_{12}(\chi, \mathrm{t})=\mathrm{G}\left(\frac{\partial \mathrm{u}_{1}(\chi, \mathrm{t})}{\partial \mathrm{x}_{2}}+v \frac{\partial \mathrm{u}_{2}(\chi, \mathrm{t})}{\partial \mathrm{x}_{1}}\right) \\
& \bar{\sigma}_{22}(\chi, \mathrm{t})=\frac{1}{1-v}\left[2 \mathrm{G}\left(\frac{\partial \mathrm{u}_{2}(\chi, \mathrm{t})}{\partial \mathrm{x}_{2}}+v \frac{\partial \mathrm{u}_{1}(\chi, \mathrm{t})}{\partial \mathrm{x}_{1}}\right)+v \mathrm{P}_{3}(\chi, \mathrm{t})\right] \\
& \bar{\sigma}_{31}(\chi, \mathrm{t})=\mathrm{P}_{1}(\chi, \mathrm{t}) \\
& \bar{\sigma}_{32}(\chi, \mathrm{t})=\mathrm{P}_{2}(\chi, \mathrm{t}) \\
& \bar{\sigma}_{33}(\chi, \mathrm{t})=\mathrm{P}_{3}(\chi, \mathrm{t})
\end{aligned}
$$

onde:

$$
\begin{aligned}
& \frac{\partial \mathrm{u}_{1}(\chi, \mathrm{t})}{\partial \mathrm{x}_{1}}=\frac{1}{2 \mathrm{~A}}\left[\left(\mathrm{x}_{2}^{2}-\mathrm{x}_{2}^{3}\right)\left(\mathrm{U}_{1}^{1}(\mathrm{t})-\mathrm{U}_{1}^{3}(\mathrm{t})\right)-\left(\mathrm{x}_{2}^{1}-\mathrm{x}_{2}^{3}\right)\left(\mathrm{U}_{1}^{2}(\mathrm{t})-\mathrm{U}_{1}^{3}(\mathrm{t})\right)\right] \\
& \frac{\partial \mathrm{u}_{1}(\chi, \mathrm{t})}{\partial \mathrm{x}_{2}}=\frac{1}{2 \mathrm{~A}}\left[\left(\mathrm{x}_{1}^{1}-\mathrm{x}_{1}^{3}\right)\left(\mathrm{U}_{1}^{2}(\mathrm{t})-\mathrm{U}_{1}^{3}(\mathrm{t})\right)-\left(\mathrm{x}_{1}^{2}-\mathrm{x}_{1}^{3}\right)\left(\mathrm{U}_{1}^{1}(\mathrm{t})-\mathrm{U}_{1}^{3}(\mathrm{t})\right)\right] \\
& \frac{\partial \mathrm{u}_{2}(\chi, \mathrm{t})}{\partial \mathrm{x}_{1}}=\frac{1}{2 \mathrm{~A}}\left[\left(\mathrm{x}_{2}^{2}-\mathrm{x}_{2}^{3}\right)\left(\mathrm{U}_{2}^{1}(\mathrm{t})-\mathrm{U}_{2}^{3}(\mathrm{t})\right)-\left(\mathrm{x}_{2}^{1}-\mathrm{x}_{2}^{3}\right)\left(\mathrm{U}_{2}^{2}(\mathrm{t})-\mathrm{U}_{2}^{3}(\mathrm{t})\right)\right] \\
& \frac{\partial \mathrm{u}_{2}(\chi, \mathrm{t})}{\partial \mathrm{x}_{2}}=\frac{1}{2 \mathrm{~A}}\left[\left(\mathrm{x}_{1}^{1}-\mathrm{x}_{1}^{3}\right)\left(\mathrm{U}_{2}^{2}(\mathrm{t})-\mathrm{U}_{2}^{3}(\mathrm{t})\right)-\left(\mathrm{x}_{1}^{2}-\mathrm{x}_{1}^{3}\right)\left(\mathrm{U}_{2}^{1}(\mathrm{t})-\mathrm{U}_{2}^{3}(\mathrm{t})\right)\right]
\end{aligned}
$$

\section{4 - MÉTODO DA RECIPROCIDADE DUAL}

\subsection{1 - Formulação Integral}

Neste trabalho, o Método da Reciprocidade Dual (MRD) é utilizado para transformar o último termo de domínio de (3.3.7), considerando as forças de inércia como forças de volume e tratando o problema dinâmico com as soluções fundamentais do problema estático. A partir disso, aplicando-se o Teorema da Reciprocidade na referida parcela, pela segunda vez na formulação do Método dos Elementos de Contorno (MEC) (daí a denominação “dual”), chega-se a uma representação somente com termos de contorno. 
Embora não se utilize a discretização do domínio em células tridimensionais, o MDR pode exigir a consideração de pontos internos, chamados de "pólos", para dar mais precisão aos resultados.

O Método da Reciprocidade Dual (MRD) foi originalmente apresentado em NARDINI \& BREBBIA (1982), aplicando-o na solução de problemas elastodinâmicos.

Considere-se, então, o termo integral de forças de inércia isoladamente,

$$
\int_{\Omega} \rho u_{\mathrm{ij}}^{*}(\mathrm{~S}, \mathrm{q}) \ddot{\mathrm{u}}_{\mathrm{j}}(\mathrm{q}, \mathrm{t}) \mathrm{d} \Omega \text {. }
$$

$\mathrm{Na}$ transformação do termo de domínio (3.4.1) para o contorno, faz-se necessário definir uma aproximação para a densidade $\ddot{u}_{i}$, incógnita do problema. $\mathrm{O}$ MDR pode ser interpretado como sendo uma superposição de soluções particulares localizadas, e sugere uma série de funções, tais que:

$$
\begin{aligned}
& \mathrm{u}_{j}(\mathrm{q}, \mathrm{t})=\alpha_{\mathrm{j}}^{\mathrm{m}}(\mathrm{t}) \mathrm{f}_{\mathrm{m}}(\chi, \mathrm{q}) \mathrm{e} \\
& \ddot{\mathrm{u}}_{\mathrm{j}}(\mathrm{q}, \mathrm{t})=\ddot{\alpha}_{\mathrm{j}}^{\mathrm{m}}(\mathrm{t}) \mathrm{f}_{\mathrm{m}}(\chi, q),
\end{aligned}
$$

onde $\mathrm{f}_{\mathrm{m}}$ são funções linearmente independentes, definidas sobre todo o domínio (escritas para pontos do domínio e do contorno), $\ddot{\alpha}_{j}^{m}$ coeficientes a serem determinados, e $\mathrm{m}$ a somatória $(\mathrm{m}=1,2, \cdots, \mathrm{M})$.

Considerando-se a aproximação (3.4.2), o termo de domínio (3.4.1) passa a ser:

$$
\begin{aligned}
\int_{\Omega} \rho u_{i j}^{*}(S, q) \ddot{u}_{j}(q, t) d \Omega & =\left(\int_{\Omega} \rho u_{i j}^{*}(S, q) f_{m}(\chi, q) d \Omega\right) \ddot{\alpha}_{j}^{m}(t) \\
& =\left(\int_{\Omega} \rho u_{i j}^{*}(S, q) \delta_{j k} f_{m}(\chi, q) d \Omega\right) \ddot{\alpha}_{k}^{m}(t) .
\end{aligned}
$$


Adota-se um estado elastostático no domínio infinito devido à aplicação de forças de volume $f_{m}(q)$ na direção $k$, com as seguintes características:

$$
\hat{\mathrm{u}}_{\mathrm{jk}}^{\mathrm{m}}(\chi, \mathrm{q}) ; \quad \rho b_{\mathrm{j}}^{\mathrm{m}}(\chi, \mathrm{q})=-\mathrm{f}_{\mathrm{m}}(\chi, q) \delta_{\mathrm{jk}} ; \quad \text { e } \quad \hat{\mathrm{p}}_{\mathrm{jk}}^{\mathrm{m}}(\chi, \mathrm{q})
$$

onde $\hat{\mathrm{u}}_{\mathrm{jk}}^{\mathrm{m}}$ e $\hat{\mathrm{p}}_{\mathrm{jk}}^{\mathrm{m}}$ são soluções particulares do problema adotado, governado pela equação:

$$
\hat{\sigma}_{\mathrm{j} \ell \ell}+\delta_{\mathrm{jk}} \mathrm{f}_{\mathrm{m}}=0
$$

Por outro lado, o problema fundamental elastostático fica caracterizado por:

$$
u_{\mathrm{ij}}^{*}(\mathrm{~s}, \mathrm{q}) ; \quad \rho b_{\mathrm{j}}^{*}(\mathrm{~s}, \mathrm{q})=\delta(\mathrm{s}, \mathrm{q}) \delta_{\mathrm{ij}} ; \quad \mathrm{e} \quad \mathrm{p}_{\mathrm{ij}}^{*}(\mathrm{~s}, \mathrm{q}) \text {. }
$$

Aplicando-se o teorema da Reciprocidade de Betti aos dois estados apresentados, (3.4.4a-c) e (3.4.6a-c), chega-se em:

$$
\begin{aligned}
c_{\mathrm{ij}}(\chi) \hat{\mathrm{u}}_{\mathrm{jk}}^{\mathrm{m}}(\chi) & =-\int_{\Gamma} \mathrm{p}_{\mathrm{ij}}^{*}(\mathrm{~s}, \mathrm{q}) \hat{\mathrm{u}}_{\mathrm{jk}}^{\mathrm{m}}(\chi, \mathrm{q}) \mathrm{d} \Gamma+\int_{\Gamma} \mathrm{u}_{\mathrm{ij}}^{*}(\mathrm{~s}, \mathrm{q}) \hat{\mathrm{p}}_{\mathrm{jk}}^{\mathrm{m}}(\chi, \mathrm{q}) \mathrm{d} \Gamma+ \\
& -\int_{\Omega} \mathrm{u}_{\mathrm{ij}}^{*}(\mathrm{~s}, \mathrm{q}) \mathrm{f}_{\mathrm{m}}(\chi, \mathrm{q}) \delta_{\mathrm{jk}} \mathrm{d} \Omega
\end{aligned}
$$

ou, ainda,

$$
\begin{aligned}
\int_{\Omega} \mathrm{u}_{\mathrm{ij}}^{*}(\mathrm{~s}, \mathrm{q}) \mathrm{f}_{\mathrm{m}}(\chi, \mathrm{q}) \delta_{\mathrm{jk}} \mathrm{d} \Omega & =-\mathrm{c}_{\mathrm{ij}}(\chi) \hat{\mathrm{u}}_{\mathrm{jk}}^{\mathrm{m}}(\chi)-\int_{\Gamma} \mathrm{p}_{\mathrm{ij}}^{*}(\mathrm{~s}, \mathrm{q}) \hat{\mathrm{u}}_{\mathrm{jk}}^{\mathrm{m}}(\chi, \mathrm{q}) \mathrm{d} \Gamma+ \\
& +\int_{\Gamma} \mathrm{u}_{\mathrm{ij}}^{*}(\mathrm{~s}, \mathrm{q}) \hat{\mathrm{p}}_{\mathrm{jk}}^{\mathrm{m}}(\chi, \mathrm{q}) \mathrm{d} \Gamma
\end{aligned}
$$

Substituindo-se (3.4.7) em (3.4.3), chega-se na representação transformada para o contorno do termo de domínio, ou seja: 


$$
\begin{aligned}
\rho \int_{\Omega} u_{i j}^{*}(s, q) \ddot{u}_{j}(q, t) d \Omega & =\rho\left(-c_{i j}(\chi) \hat{u}_{j k}^{m}(\chi)-\int_{\Gamma} p_{i j}^{*}(s, q) \hat{u}_{j k}^{m}(\chi, q) d \Gamma+\right. \\
& \left.+\int_{\Gamma} u_{i j}^{*}(s, q) \hat{p}_{j k}^{m}(\chi, q) d \Gamma\right) \ddot{\alpha}_{k}^{m}(t) .
\end{aligned}
$$

Portanto, a representação integral de deslocamento (3.3.7), desconsiderandose a parcela referente às forças de volume, passa a ser escrita somente com termos do contorno, tendo em vista (3.4.9). Assim,

$$
\begin{aligned}
\mathrm{c}_{\mathrm{ij}}(\mathrm{S}) \mathrm{u}_{\mathrm{j}}(\mathrm{S}, \mathrm{t}) & =-\int_{\Gamma} \mathrm{p}_{\mathrm{ij}}^{*}(\mathrm{~S}, \mathrm{Q}) \mathrm{u}_{\mathrm{j}}(\mathrm{Q}, \mathrm{t}) \mathrm{d} \Gamma+\int_{\Gamma} \mathrm{u}_{\mathrm{ij}}^{*}(\mathrm{~S}, \mathrm{Q}) \mathrm{p}_{\mathrm{j}}(\mathrm{Q}, \mathrm{t}) \mathrm{d} \Gamma+ \\
& +\rho\left(\mathrm{c}_{\mathrm{ij}}(\chi) \hat{\mathrm{u}}_{\mathrm{jk}}^{\mathrm{m}}(\chi)+\int_{\Gamma} \mathrm{p}_{\mathrm{ij}}^{*}(\mathrm{~s}, \mathrm{q}) \hat{\mathrm{u}}_{\mathrm{jk}}^{\mathrm{m}}(\chi, \mathrm{q}) \mathrm{d} \Gamma+\right. \\
& \left.-\int_{\Gamma} \mathrm{u}_{\mathrm{ij}}^{*}(\mathrm{~s}, \mathrm{q}) \hat{\mathrm{p}}_{\mathrm{jk}}^{\mathrm{m}}(\chi, \mathrm{q}) \mathrm{d} \Gamma\right) \ddot{\alpha}_{\mathrm{k}}^{\mathrm{m}}(\mathrm{t})
\end{aligned}
$$

onde $\mathrm{u}_{\mathrm{ij}}^{*}$ e $\mathrm{p}_{\mathrm{ij}}^{*}$ são os tensores já apresentados em (2.4.4) e (2.4.5) para Kelvin, e (2.4.8) e (2.4.9) para Mindlin; $\chi$ pode ser um ponto do domínio ou do contorno, e os coeficientes $\mathrm{c}_{\mathrm{ij}}$ são aqueles já definidos em (2.5.9). Os novos tensores que surgem em (3.4.10) são as respostas, deslocamentos e forças de superfície referentes ao problema particular (3.4.5), que dependem da função $\mathrm{f}_{\mathrm{m}}$ adotada.

Nos trabalhos PARTRIDGE et al. (1992), VENTURINI (1994a), podem ser encontradas famílias de funções adequadas para os diversos tipos de problemas da engenharia, além de discutirem as vantagens e desvantagens de utilizar-se de cada uma. Neste trabalho adota-se a seguinte função:

$$
\mathrm{f}_{\mathrm{m}}=1+\mathrm{r} \text {. }
$$

Portanto, resolvendo-se (3.4.5) para a função adotada, chega-se às expressões para os tensores de deslocamentos: 


$$
\hat{\mathrm{u}}_{\mathrm{j}}^{\mathrm{m}}=\frac{(1-2 v)}{(6-4 v) \mathrm{G}} \mathrm{r}_{\mathrm{rm}} \mathrm{r}_{, \mathrm{j}} \mathrm{r}^{2}+\frac{1}{48(1-v) \mathrm{G}}\left[\left(\frac{11}{3}-4 v\right) \delta_{\mathrm{mj}}-\mathrm{r}_{, \mathrm{m}} \mathrm{r}_{, \mathrm{j}}\right] \mathrm{r}^{3},
$$

e de forças de superfície:

$$
\begin{aligned}
\hat{\mathrm{p}}_{\mathrm{j}}^{\mathrm{m}} & =\frac{(1-2 v)}{(3-2 v)}\left[\frac{(1+2 v)}{(1-2 v)} \mathrm{r}_{\mathrm{m}} \mathrm{n}_{\mathrm{j}}+\frac{1}{2} \mathrm{r}_{, \mathrm{j}} \mathrm{n}_{\mathrm{m}}+\frac{1}{2} \delta_{\mathrm{mj}} \frac{\partial \mathrm{r}}{\partial \mathrm{n}}\right] \mathrm{r}+ \\
& +\frac{1}{24(1-v)}\left[(5-6 v) \mathrm{r}_{, \mathrm{j}} \mathrm{n}_{\mathrm{m}}-(1-6 v) \mathrm{r}_{\mathrm{,m}} \mathrm{n}_{\mathrm{j}}+\left((5-6 v) \delta_{\mathrm{mj}}-\mathrm{r}_{, \mathrm{m}} \mathrm{r}_{\mathrm{j}}\right) \frac{\partial \mathrm{r}}{\partial \mathrm{n}}\right] \mathrm{r}^{2} .
\end{aligned}
$$

A representação integral para pontos localizados no domínio é obtida utilizando-se o procedimento clássico, baseado na lei de Hooke (2.3.5), substituindose convenientemente as derivadas da expressão (3.4.10) de deslocamentos. Assim,

$$
\begin{aligned}
\sigma_{\mathrm{i} \ell}(\mathrm{s}, \mathrm{t}) & =-\int_{\Gamma} \mathrm{S}_{\mathrm{ij} \ell}^{*}(\mathrm{~s}, \mathrm{Q}) \mathrm{u}_{\mathrm{j}}(\mathrm{Q}, \mathrm{t}) \mathrm{d} \Gamma+\int_{\Gamma} \mathrm{D}_{\mathrm{ij} \ell}^{*}(\mathrm{~s}, \mathrm{Q}) \mathrm{p}_{\mathrm{j}}(\mathrm{Q}, \mathrm{t}) \mathrm{d} \Gamma+ \\
& +\rho\left\{\left[\lambda \delta_{\mathrm{i} \ell} \frac{\partial \hat{\mathrm{u}}_{\mathrm{wk}}^{\mathrm{m}}(\mathrm{s}, \mathrm{q})}{\partial \mathrm{X}_{\mathrm{w}}}+\mathrm{G}\left(\frac{\partial \hat{\mathrm{u}}_{\mathrm{jk}}^{\mathrm{m}}(\mathrm{s}, \mathrm{q})}{\partial \mathrm{X}_{\ell}}+\frac{\partial \hat{\mathrm{u}}_{\ell \mathrm{k}}^{\mathrm{m}}(\mathrm{s}, \mathrm{q})}{\partial \mathrm{X}_{\mathrm{j}}}\right)\right]+\right. \\
& \left.+\int_{\Gamma} \mathrm{S}_{\mathrm{ij} \ell}^{*}(\mathrm{~s}, \mathrm{q}) \hat{\mathrm{u}}_{\mathrm{jk}}^{\mathrm{m}}(\mathrm{s}, \mathrm{q}) \mathrm{d} \Gamma-\int_{\Gamma} \mathrm{D}_{\mathrm{ij} \ell}^{*}(\mathrm{~s}, \mathrm{q}) \hat{\mathrm{p}}_{\mathrm{jk}}^{\mathrm{m}}(\mathrm{s}, \mathrm{q}) \mathrm{d} \Gamma\right\} \ddot{\alpha}_{\mathrm{k}}^{\mathrm{m}}(\mathrm{t}),
\end{aligned}
$$

onde os tensores $\mathrm{S}_{\mathrm{ijk}}^{*}$ e $\mathrm{D}_{\mathrm{ijk}}^{*}$ são os mesmos apresentados nas expressões (2.5.3), (2.5.4), (2.5.5) e (2.5.6). Efetuando-se as operações algébricas do termo entre colchetes, relativo ao termo independente $\hat{\mathrm{u}}_{\mathrm{j} \ell}^{\mathrm{m}}$, chega-se à expressão de um novo tensor, definido por $\hat{\mathrm{D}}_{\mathrm{ijk}}$ :

$$
\begin{aligned}
\hat{D}_{\mathrm{ijk}} & =\frac{(1-2 v)}{(3-2 v)}\left[\frac{(1+2 v)}{(1-2 v)} \mathrm{r}_{, \mathrm{k}} \delta_{\mathrm{ij}}+\frac{1}{2} \delta_{\mathrm{jk}} \mathrm{r}_{, \mathrm{i}}+\frac{1}{2} \delta_{\mathrm{ik}} \mathrm{r}_{, \mathrm{j}}\right] \mathrm{r}+ \\
& +\frac{1}{24(1-v)}\left[(5-6 v)\left(\mathrm{r}_{, \mathrm{i}} \delta_{\mathrm{kj}}+\mathrm{r}_{, \mathrm{j}} \delta_{\mathrm{ik}}\right)-(1-6 v) \mathrm{r}_{, \mathrm{k}} \delta_{\mathrm{ij}}-\mathrm{r}_{, \mathrm{i}} \mathrm{r}_{\mathrm{j}} \mathrm{r}_{, \mathrm{k}}\right] \mathrm{r}^{2}
\end{aligned}
$$

A dedução do tensor (3.4.15) é apresentada no anexo C. 
Portanto, as tensões em pontos do domínio são obtidas através seguinte representação integral:

$$
\begin{aligned}
\sigma_{\mathrm{ij}}(\mathrm{s}, \mathrm{t}) & =-\int_{\Gamma} \mathrm{S}_{\mathrm{ij} \ell}^{*}(\mathrm{~s}, \mathrm{Q}) \mathrm{u}_{\mathrm{j}}(\mathrm{Q}, \mathrm{t}) \mathrm{d} \Gamma+\int_{\Gamma} \mathrm{D}_{\mathrm{ij} \ell}^{*}(\mathrm{~s}, \mathrm{Q}) \mathrm{p}_{\mathrm{j}}(\mathrm{Q}, \mathrm{t}) \mathrm{d} \Gamma+ \\
& +\rho\left\{\hat{\mathrm{D}}_{\mathrm{ij} \ell}^{\mathrm{k}}(\mathrm{s}, \mathrm{q})+\int_{\Gamma} \mathrm{S}_{\mathrm{ij} \ell}^{*}(\mathrm{~s}, \mathrm{q}) \hat{\mathrm{u}}_{\mathrm{jk}}^{\mathrm{m}}(\mathrm{s}, \mathrm{q}) \mathrm{d} \Gamma-\int_{\Gamma} \mathrm{D}_{\mathrm{ij} \ell}^{*}(\mathrm{~s}, \mathrm{q}) \hat{\mathrm{p}}_{\mathrm{jk}}^{\mathrm{m}}(\mathrm{s}, \mathrm{q}) \mathrm{d} \Gamma\right\} \ddot{\alpha}_{\mathrm{k}}^{\mathrm{m}}(\mathrm{t})
\end{aligned}
$$

\subsection{2 - Discretizações}

As representações integrais aqui apresentadas são discretizadas utilizando-se os elementos e as aproximações definidos no item 2.6.

Além dos “J" elementos de contorno, dos "N" pontos nodais (nós funcionais), deve-se considerar o número de pólos, denotado por "Np".

Considere-se a expressão integral para deslocamentos (3.4.10). Substitui-se os deslocamentos e forças de superfície ( $u$ e p) pelo produto entre as funções aproximadoras e seus respectivos valores nodais, expressões (2.6.2a-b). O mesmo deve ser feito para os tensores dos deslocamentos e forças de superfície do problema particular $\left(\hat{\mathrm{u}}_{\mathrm{jk}}^{\mathrm{m}} \mathrm{e} \hat{\mathrm{p}}_{\mathrm{jk}}^{\mathrm{m}}\right)$. Adotando-se as mesmas funções aproximadoras de (2.6.2a-b) chega-se às mesmas matrizes $\mathbf{H}$ e $\mathbf{G}$ do contorno, também para o termo de domínio transformado para o contorno em (3.4.10). Portanto, adotam-se:

$$
\begin{aligned}
& \hat{\mathbf{u}}_{\mathrm{k}}=\phi^{\mathrm{T}} \hat{\mathbf{U}}_{\mathrm{m}}^{\mathrm{n}} \mathrm{e} \\
& \hat{\mathbf{p}}_{\mathrm{k}}=\phi^{\mathrm{T}} \hat{\mathbf{P}}_{\mathrm{m}}^{\mathrm{n}} .
\end{aligned}
$$

A expressão (3.4.10), na sua forma discretizada, passa a ser: 


$$
\begin{aligned}
\mathbf{c}(\mathrm{S}, \mathrm{t}) \mathbf{u}(\mathrm{S}, \mathrm{t})= & -\sum_{\mathrm{j}=1}^{\mathrm{J}}\left[\int_{\Gamma_{\mathrm{j}}} \mathbf{p}^{*}(\mathrm{~S}, \mathrm{Q}) \phi^{\mathrm{T}}(\mathrm{Q}) \mathrm{d} \Gamma\right] \mathbf{U}^{\mathrm{n}}(\mathrm{Q}, \mathrm{t})+ \\
& +\sum_{\mathrm{j}=1}^{\mathrm{J}}\left[\int_{\Gamma_{\mathrm{j}}} \mathbf{u}^{*}(\mathrm{~S}, \mathrm{Q}) \phi^{\mathrm{T}}(\mathrm{Q}) \mathrm{d} \Gamma\right] \mathbf{P}^{\mathrm{n}}(\mathrm{Q}, \mathrm{t})+ \\
& +\rho\left\{\mathbf{c}(\mathrm{s}) \hat{\mathbf{u}}^{\mathrm{m}}(\mathrm{s}, \mathrm{t})+\sum_{\mathrm{j}=1}^{\mathrm{J}}\left[\int_{\Gamma_{\mathrm{j}}} \mathbf{p}^{*}(\mathrm{~s}, \mathrm{q}) \phi^{\mathrm{T}}(\mathrm{q}) \mathrm{d} \Gamma\right] \mathbf{U}_{\mathrm{m}}^{\mathrm{n}}(\mathrm{s}, \mathrm{q})+\right. \\
& \left.-\sum_{\mathrm{j}=1}^{\mathrm{J}}\left[\int_{\Gamma_{\mathrm{j}}} \mathbf{u}^{*}(\mathrm{~s}, \mathrm{q}) \phi^{\mathrm{T}}(\mathrm{q}) \mathrm{d} \Gamma\right] \mathbf{P}_{\mathrm{m}}^{\mathrm{n}}(\mathrm{s}, \mathrm{q})\right\} \ddot{\alpha}^{\mathrm{m}}(\mathrm{t}) .
\end{aligned}
$$

Calculadas as integrais de (3.4.18) para todos os pontos de colocação, chegase em:

$$
\overline{\mathbf{c}} \mathbf{U}+\hat{\mathbf{H}} \mathbf{U}=\mathbf{G P}+\rho(\overline{\mathbf{c}} \hat{\mathbf{U}}+\hat{\mathbf{H}} \hat{\mathbf{U}}-\mathbf{G} \hat{\mathbf{P}}) \ddot{\alpha}
$$

ou

$$
\mathbf{H U}=\mathbf{G P}+\rho(\mathbf{H} \hat{\mathbf{U}}-\mathbf{G} \hat{\mathbf{P}}) \ddot{\alpha}
$$

Os coeficientes $\ddot{\alpha}$ são determinados tomando-se a forma inversa de (3.4.2b), uma vez que as funções $f_{m}$ são conhecidas. Assim,

$$
\ddot{\alpha}=\mathbf{F}^{-1} \ddot{\mathbf{U}} \text {. }
$$

Substituindo-se o valor desses coeficientes em (3.4.20), determina-se a equação matricial representativa do problema dinâmico em questão:

$$
\mathbf{H U}=\mathbf{G P}+\rho(\mathbf{H} \hat{\mathbf{U}}-\mathbf{G} \hat{\mathbf{P}}) \mathbf{F}^{-1} \ddot{\mathbf{U}}
$$

Finalmente, utilizando-se o conceito de matriz de massa, obtém-se a equação do movimento para um sistema dinâmico forçado sem amortecimento: 


$$
\mathbf{M U ̈}+\mathbf{H U}=\mathbf{G P},
$$

onde a matriz de massa $\mathbf{M}$ é definida por

$$
\mathbf{M}=-\rho(\mathbf{H} \hat{\mathbf{U}}-\mathbf{G} \hat{\mathbf{P}}) \mathbf{F}^{-1}
$$

As matrizes aqui apresentadas possuem características próprias que devem ser conhecidas para sua perfeita montagem. Iniciando-se pelas matrizes de deslocamentos e forças de superfície do problema particular, dadas em (3.4.22), apresentam-se suas submatrizes e as respectivas ordens. Fica estabelecido que $\mathrm{N}$ é o número de pontos funcionais no contorno; $\mathrm{N}_{\mathrm{p}} \mathrm{o}$ número de pontos funcionais no domínio, ou pólos; e $\mathrm{N}_{\mathrm{n}}$ é o número total de pontos funcionais. Os símbolos que representam o domínio $\Omega$ e o contorno $\Gamma$ são utilizados para indicar a localização dos pontos envolvidos. Assim,

$$
\hat{\mathbf{U}}=\left[\begin{array}{ll}
\hat{\mathbf{U}}_{\mathrm{N} \times \mathrm{N}}^{\Gamma \Gamma} & \hat{\mathbf{U}}_{\mathrm{N} \times \mathrm{Np}}^{\Gamma \Omega} \\
\hat{\mathbf{U}}_{\mathrm{Np} \times \mathrm{N}}^{\Omega \Gamma} & \hat{\mathbf{U}}_{\mathrm{Np} \times \mathrm{Np}}^{\Omega \Omega}
\end{array}\right]_{\mathrm{Nn} \times \mathrm{Nn}} \quad \text { e } \quad \hat{\mathbf{P}}=\left[\begin{array}{ll}
\mathbf{P}_{\mathrm{N} \times \mathrm{N}}^{\Gamma \Gamma} & \mathbf{P}_{\mathrm{N} \times \mathrm{Np}}^{\Gamma \Omega} \\
\mathbf{P}_{\mathrm{Np} \times \mathrm{N}}^{\Omega \Gamma} & \mathbf{P}_{\mathrm{Np} \times \mathrm{Np}}^{\Omega \Omega}
\end{array}\right]_{\mathrm{Nn} \times \mathrm{Nn}} ;
$$

$\mathrm{e}$

$$
\mathbf{F}=\left[\begin{array}{ll}
\mathbf{F}_{\mathrm{N} \times N}^{\Gamma \Gamma} & \mathbf{F}_{N \times N p}^{\Gamma \Omega} \\
\mathbf{F}_{N p \times N}^{\Omega \Gamma} & \mathbf{F}_{N p \times N p}^{\Omega \Omega}
\end{array}\right]_{N n \times N n}=\left[\begin{array}{ccccccc}
f_{\mathrm{n}} & 0 & 0 & \mathrm{f}_{\mathrm{n}} & 0 & 0 & \cdots \\
0 & \mathrm{f}_{\mathrm{n}} & 0 & 0 & \mathrm{f}_{\mathrm{n}} & 0 & \cdots \\
0 & 0 & \mathrm{f}_{\mathrm{n}} & 0 & 0 & \mathrm{f}_{\mathrm{n}} & \cdots \\
\mathrm{f}_{\mathrm{n}} & 0 & 0 & \mathrm{f}_{\mathrm{n}} & 0 & 0 & \cdots \\
0 & \mathrm{f}_{\mathrm{n}} & 0 & 0 & \mathrm{f}_{\mathrm{n}} & 0 & \cdots \\
0 & 0 & \mathrm{f}_{\mathrm{n}} & 0 & 0 & \mathrm{f}_{\mathrm{n}} & \cdots \\
\vdots & \vdots & \vdots & \vdots & \vdots & \vdots & \ddots
\end{array}\right] .
$$

As matrizes em (3.4.23) têm a seguinte configuração: 


$$
\begin{aligned}
& \mathbf{M}=\left[\begin{array}{ll}
\mathbf{M}_{\mathrm{N} \times \mathrm{N}}^{\Gamma \Gamma} & \mathbf{M}_{\mathrm{N} \times \mathrm{Np}}^{\Gamma \Omega} \\
\mathbf{M}_{\mathrm{Np} \times \mathrm{N}}^{\Omega \Gamma} & \mathbf{M}_{\mathrm{Np} \times \mathrm{Np}}^{\Omega \Omega}
\end{array}\right]_{\mathrm{Nn} \times \mathrm{Nn}} \quad \text { e } \quad \ddot{\mathbf{U}}=\left\{\begin{array}{c}
\ddot{\mathbf{U}}_{\mathrm{N}}^{\Gamma} \\
\ddot{\mathbf{U}}_{\mathrm{Np}}^{\Omega}
\end{array}\right\}_{\mathrm{Nn}} ; \\
& \mathbf{H}=\left[\begin{array}{ll}
\mathbf{H}_{\mathrm{N} \times \mathrm{N}}^{\Gamma \Gamma} & \mathbf{0}_{\mathrm{N} \times \mathrm{Np}}^{\Gamma \Omega} \\
\mathbf{H}_{\mathrm{Np} \times \mathrm{N}}^{\Omega \Gamma} & \mathbf{I}_{\mathrm{Np} \times \mathrm{Np}}^{\Omega \Omega}
\end{array}\right]_{\mathrm{Nn \times Nn}} \quad \text { e } \quad \mathbf{U}=\left\{\begin{array}{c}
\mathbf{U}_{\mathrm{N}}^{\Gamma} \\
\mathbf{U}_{\mathrm{Np}}^{\Omega}
\end{array}\right\}_{\mathrm{Nn}} ; \mathrm{e} \\
& \mathbf{G}=\left[\begin{array}{ll}
\mathbf{G}_{\mathrm{N} \times \mathrm{N}}^{\Gamma \Gamma} & \mathbf{0}_{\mathrm{N} \times \mathrm{Np}}^{\Gamma \Omega} \\
\mathbf{G}_{\mathrm{Np} \times \mathrm{N}}^{\Omega \Gamma} & \mathbf{0}_{\mathrm{Np} \times \mathrm{Np}}^{\Omega \Omega}
\end{array}\right]_{\mathrm{Nn} \times \mathrm{Nn}} \quad \text { e } \quad \mathbf{P}=\left\{\begin{array}{l}
\mathbf{P}_{\mathrm{N}}^{\Gamma} \\
\mathbf{0}_{\mathrm{Np}}^{\Omega}
\end{array}\right\}_{\mathrm{Nn}} ;
\end{aligned}
$$

onde $\mathbf{0}$ representa a matriz nula e I a matriz identidade.

Pode-se, ainda, determinar os deslocamentos em pontos do domínio utilizando-se os valores nodais de deslocamentos, forças de superfície e acelerações determinados a partir da resolução do sistema dado por (3.4.23). Assim, partindo-se de (3.4.18) para pontos do domínio, chega-se à

$$
\mathbf{u}=-\mathbf{H U}+\mathbf{G P}-\mathbf{M} \ddot{\mathbf{U}} .
$$

Para o cálculo das tensões em pontos do domínio, utiliza-se o mesmo procedimento de discretização executado para a equação de deslocamentos (3.4.18). Assim, sua representação integral tem a seguinte forma: 


$$
\begin{aligned}
\sigma(\mathrm{s}, \mathrm{t}) & =-\sum_{\mathrm{j}=1}^{\mathrm{J}}\left[\int_{\Gamma_{\mathrm{j}}} \mathbf{S}^{*}(\mathrm{~s}, \mathrm{Q}) \phi^{\mathrm{T}}(\mathrm{Q}) \mathrm{d} \Gamma\right] \mathbf{U}^{\mathrm{n}}(\mathrm{Q}, \mathrm{t})+ \\
& +\sum_{\mathrm{j}=1}^{\mathrm{J}}\left[\int_{\Gamma_{\mathrm{j}}} \mathbf{D}^{*}(\mathrm{~s}, \mathrm{Q}) \phi^{\mathrm{T}}(\mathrm{Q}) \mathrm{d} \Gamma\right] \mathbf{P}^{\mathrm{n}}(\mathrm{Q}, \mathrm{t})+ \\
& +\rho\left\{\hat{\mathbf{D}}^{\mathrm{k}}(\mathrm{s}, \mathrm{q})+\sum_{\mathrm{j}=1}^{\mathrm{J}}\left[\int_{\Gamma_{\mathrm{j}}} \mathbf{S}^{*}(\mathrm{~s}, \mathrm{Q}) \phi^{\mathrm{T}}(\mathrm{Q}) \mathrm{d} \Gamma\right] \hat{\mathbf{U}}^{\mathrm{k}}(\mathrm{s}, \mathrm{q})+\right. \\
& \left.-\sum_{\mathrm{j}=1}^{\mathrm{J}}\left[\int_{\Gamma_{\mathrm{j}}} \mathbf{D}^{*}(\mathrm{~s}, \mathrm{Q}) \phi^{\mathrm{T}}(\mathrm{Q}) \mathrm{d} \Gamma\right] \hat{\mathbf{P}}^{\mathrm{k}}(\mathrm{s}, \mathrm{q})\right\} \mathbf{F}^{-1}(\mathrm{~s}, \mathrm{q}) \ddot{\mathbf{U}}^{\mathrm{k}}(\mathrm{q}, \mathrm{t}),
\end{aligned}
$$

ou

$$
\sigma=-\mathbf{H}^{\prime} \mathbf{U}+\mathbf{G}^{\prime} \mathbf{P}-\mathbf{M}^{\prime} \ddot{\mathbf{U}}
$$

onde

$$
\mathbf{M}^{\prime}=-\rho\left(\hat{\mathbf{D}}+\mathbf{H}^{\prime} \hat{\mathbf{U}}-\mathbf{G}^{\prime} \hat{\mathbf{P}}\right) \mathbf{F}^{-1}
$$

\section{5 - TÉCNICA DA INTEGRAÇÃO DIRETA}

3.5.1 - Transformação do Termo de Domínio para o Contorno - Forças de Inércia

Deseja-se transformar o termo definido sobre o domínio para o contorno, referente às forças de inércia na expressão integral (3.3.7), desconsiderando-se o termo de forças de volume. Isto pode ser feito discretizando-se $\Omega$ em células tridimensionais, procedimento bastante utilizado no Método dos Elementos Finitos (como em BREBBIA \& FERRANTE, 1975) e incorporado ao Método dos Elementos de Contorno (MEC) em diversos trabalhos, como em NAKAGUMA, 1979. Esta solução, porém, não corresponde, a princípio, à idéia do Método dos 
Elementos de Contorno de resolver os problemas aplicando somente integrais de contorno para problemas lineares. Uma alternativa viável para se contornar este problema, mas ainda utilizando células, é aquela que transforma a parcela integral de domínio para o contorno das células. Como conseqüência, na expressão (3.3.7) só restam termos sobre o contorno, e as células podem ter forma qualquer, não mais obedecendo às formas preestabelecidas. Na realidade, a razão mais forte pela qual se optou por este procedimento é a eliminação da singularidade que ele proporciona. Assim, este último procedimento é utilizado pelo presente trabalho, cuja formulação é apresentada a seguir.

Destaque-se o termo de domínio referente às forças de inércia da expressão (3.3.7):

$$
\int_{\Omega} \rho u_{\mathrm{ij}}^{*}(\mathrm{~S}, \mathrm{q}) \ddot{\mathrm{u}}_{\mathrm{j}}(\mathrm{q}, \mathrm{t}) \mathrm{d} \Omega \text {. }
$$

Observa-se (3.5.1) que é necessário utilizar uma forma aproximada para as componentes da aceleração utilizando-se seus valores nodais e funções interpoladoras. Define-se, portanto,

$$
\ddot{\mathrm{u}}_{\mathrm{j}}(\mathrm{q}, \mathrm{t})=\phi_{\mathrm{jm}}(\mathrm{q}) \ddot{\mathrm{U}}_{\mathrm{m}}^{\mathrm{N}}(\mathrm{q}, \mathrm{t})
$$

Utilizando-se a aproximação (3.5.2) para as acelerações, o termo de domínio (3.5.1) passa a ser:

$$
\int_{\Omega} \rho u_{\mathrm{ij}}^{*}(\mathrm{~S}, \mathrm{q}) \ddot{\mathrm{u}}_{\mathrm{j}}(\mathrm{q}, \mathrm{t}) \mathrm{d} \Omega=\left(\int_{\Omega} \rho \mathrm{u}_{\mathrm{ij}}^{*}(\mathrm{~S}, \mathrm{q}) \phi_{\mathrm{jm}}(\mathrm{q}) \mathrm{d} \Omega\right) \ddot{\mathrm{U}}_{\mathrm{m}}^{\mathrm{n}}(\mathrm{q}, \mathrm{t}) .
$$

Em seguida, adota-se um tensor $\mathrm{T}_{\mathrm{ij}}^{*}$ que satisfaz a seguinte condição:

$$
\nabla^{2} \mathrm{~T}_{\mathrm{ij}}^{*}=\mathrm{u}_{\mathrm{ij}}^{*}
$$

A primitiva $\mathrm{T}_{\mathrm{ij}}^{*}$ pode ser determinada a partir dos procedimentos mostrados por CRUSE (1975) e DANSON (1981), entre outros, uma vez que o tensor 
fundamental de deslocamentos é conhecido. Os trabalhos citados utilizam o tensor de Galerkin para chegar na primitiva. Outro procedimento, consiste em efetuar a integração direta em relação à coordenada radial de um sistema de coordenadas polares associado. Neste trabalho, utiliza-se este último procedimento, cujas deduções encontram-se no anexo D.

Admitindo-se a existência de uma primitiva para (3.5.4), a expressão (3.5.3) pode ser rescrita, tornando-se

$$
\int_{\Omega} \rho u_{i j}^{*}(S, q) \phi_{j m}(q) d \Omega=\int_{\Omega} \rho \nabla^{2} T_{i j}^{*}(S, q) \phi_{j m}(q) d \Omega
$$

Para transformar o termo de domínio de (3.5.5), é preciso integrá-lo por partes, duas vezes, resultando na seqüência:

$$
\rho \int_{\Omega} \mathrm{u}_{\mathrm{ij}}^{*}(\mathrm{~S}, \mathrm{q}) \phi_{\mathrm{jm}}(\mathrm{q}) \mathrm{d} \Omega=\rho\left(\int_{\Gamma} \mathrm{T}_{\mathrm{ij}, \ell}^{*}(\mathrm{~S}, \mathrm{q}) \phi_{\mathrm{jm}}(\mathrm{q}) \mathrm{n}_{\ell}(\mathrm{q}) \mathrm{d} \Gamma-\int_{\Omega} \mathrm{T}_{\mathrm{ij}, \ell}^{*}(\mathrm{~S}, \mathrm{q}) \phi_{\mathrm{jm}, \ell}(\mathrm{q}) \mathrm{d} \Omega\right)
$$

$$
\begin{aligned}
\rho \int_{\Omega} \mathrm{u}_{\mathrm{ij}}^{*}(\mathrm{~S}, \mathrm{q}) \phi_{\mathrm{jm}}(\mathrm{q}) \mathrm{d} \Omega & =\rho\left(\int_{\Gamma} \mathrm{T}_{\mathrm{ij}, \ell}^{*}(\mathrm{~S}, \mathrm{q}) \phi_{\mathrm{jm}}(\mathrm{q}) \mathrm{n}_{\ell}(\mathrm{q}) \mathrm{d} \Gamma\right. \\
& \left.-\int_{\Gamma} \mathrm{T}_{\mathrm{ij}}^{*}(\mathrm{~S}, \mathrm{q}) \phi_{\mathrm{jm}, \ell}(\mathrm{q}) \mathrm{n}_{\ell}(\mathrm{q}) \mathrm{d} \Gamma+\int_{\Omega} \mathrm{T}_{\mathrm{ij}}^{*}(\mathrm{~S}, \mathrm{q}) \phi_{\mathrm{jm}, \ell \ell}(\mathrm{q}) \mathrm{d} \Gamma\right)
\end{aligned}
$$

A função de interpolação $\phi$ aqui adotada é a linear, já definida no item 2.6. Portanto, o último termo de (3.5.7) tem valor nulo, uma vez que a derivada segunda da função de interpolação é zero. Assim, substitui-se (3.5.7) por (3.5.1), resultando em

$$
\rho \int_{\Omega} \mathrm{u}_{\mathrm{ij}}^{*}(\mathrm{~S}, \mathrm{q}) \ddot{\mathrm{u}}_{\mathrm{j}}(\mathrm{q}, \mathrm{t}) \mathrm{d} \Omega=\rho \int_{\Gamma}\left(\mathrm{T}_{\mathrm{ij}, \ell}^{*}(\mathrm{~S}, \mathrm{q}) \phi_{\mathrm{jm}}(\mathrm{q})-\mathrm{T}_{\mathrm{ij}}^{*}(\mathrm{~S}, \mathrm{q}) \phi_{\mathrm{jm}, \ell}\right) \mathrm{n}_{\ell}(\mathrm{q}) \mathrm{d} \Gamma \ddot{\mathbf{U}}_{\mathrm{m}}^{\mathrm{n}}(\mathrm{q}, \mathrm{t})
$$


A expressão integral (3.5.8) representa a transformação do termo de domínio para o contorno. Ressalte-se que o domínio deve ser discretizado por células tridimensionais, de forma qualquer, cujo contorno é, por sua vez, discretizado por elementos bidimensionais.

\subsection{2 - Discretizações}

As discretizações das representações integrais aqui apresentadas são efetuadas utilizando-se os elementos e as aproximações definidos no item 2.6 do capítulo anterior.

A forma discretizada da representação de deslocamentos equação (3.3.7), desconsiderando-se o termo de forças de volume e observando-se o que foi encontrado em (3.5.8), é

$$
\begin{aligned}
\mathbf{c}(\mathrm{S}) \mathbf{u}(\mathrm{S}, \mathrm{t}) & =-\sum_{\mathrm{j}=1}^{\mathrm{J}}\left[\int_{\Gamma_{\mathrm{j}}} \mathbf{p}^{*}(\mathrm{~S}, \mathrm{Q}) \phi^{\mathrm{T}}(\mathrm{Q}) \mathrm{d} \Gamma\right] \mathbf{U}^{\mathrm{n}}(\mathrm{Q}, \mathrm{t})+ \\
& +\sum_{\mathrm{j}=1}^{\mathrm{J}}\left[\int_{\Gamma_{\mathrm{j}}} \mathbf{u}^{*}(\mathrm{~S}, \mathrm{Q}) \phi^{\mathrm{T}}(\mathrm{Q}) \mathrm{d} \Gamma\right] \mathbf{P}^{\mathrm{n}}(\mathrm{Q}, \mathrm{t})+ \\
& -\sum_{\mathrm{c}=1}^{\mathrm{Nc}} \rho\left[\int_{\Gamma_{\mathrm{c}}}\left(\mathbf{T}_{\ell}^{*}(\mathrm{~S}, \mathrm{Q}) \phi^{\mathrm{T}}(\mathrm{Q}) \mathrm{d} \Gamma-\mathbf{T}^{*}(\mathrm{~S}, \mathrm{Q}) \phi_{, \ell}^{\mathrm{T}}(\mathrm{Q}) \mathrm{d} \Gamma\right) \mathrm{n}_{\ell} \mathrm{d} \Gamma\right] \ddot{\mathbf{U}}^{\mathrm{n}}(\mathrm{q}, \mathrm{t}) .
\end{aligned}
$$

Escrevendo-se (3.5.9) na forma matricial e efetuando-se as integrais sobre todos os elementos para todos os pontos de colocação, tem-se

$$
\overline{\mathbf{c}} \mathbf{U}+\hat{\mathbf{H}} \mathbf{U}=\mathbf{G P}-\rho\left(\mathbf{T}_{, \mathrm{n}} \phi-\mathbf{T} \phi_{, \mathrm{n}}\right) \ddot{\mathbf{U}} .
$$

Obtém-se, agora, a expressão matricial do problema dinâmico, com o conceito da matriz de massa, semelhante a que foi obtida para o Método da Reciprocidade Dual: 


$$
\mathbf{M U ̈}+\mathbf{H U}=\mathbf{G P}
$$

onde a matriz de massa $\mathbf{M}$ é definida por

$$
\mathbf{M}=\rho\left(\mathbf{T}_{, \mathrm{n}} \phi-\mathbf{T} \phi_{, \mathrm{n}}\right)
$$

As divisões em submatrizes apresentadas em (3.4.27a-b), (3.4.28a-b) e (3.4.29a-b) são também aqui consideradas.

Pode-se, ainda, determinar os deslocamentos em pontos do domínio utilizando-se os valores nodais de deslocamentos, forças de superfície e acelerações determinados a partir da resolução do sistema dado por (3.4.23). Assim, partindo-se de (3.5.9), para pontos do domínio, chega-se à

$$
\mathbf{u}=-\mathbf{H U}+\mathbf{G P}-\mathbf{M} \ddot{\mathbf{U}} .
$$

Para a análise das tensões em pontos do domínio, basta diferenciar a expressão de deslocamento, equação (3.5.9), e substituir convenientemente os resultados na equação (3.2.5). Assim, resulta em:

$$
\begin{aligned}
\sigma(\mathrm{S}, \mathrm{t}) & =-\sum_{\mathrm{j}=1}^{\mathrm{J}}\left[\int_{\Gamma_{\mathrm{j}}} \mathbf{S}^{*}(\mathrm{~S}, \mathrm{Q}) \phi^{\mathrm{T}}(\mathrm{Q}) \mathrm{d} \Gamma\right] \mathbf{U}^{\mathrm{n}}(\mathrm{Q}, \mathrm{t})+ \\
& +\sum_{\mathrm{j}=1}^{\mathrm{J}}\left[\int_{\Gamma_{\mathrm{j}}} \mathbf{D}^{*}(\mathrm{~S}, \mathrm{Q}) \phi^{\mathrm{T}}(\mathrm{Q}) \mathrm{d} \Gamma\right] \mathbf{P}^{\mathrm{n}}(\mathrm{Q}, \mathrm{t})+ \\
& -\sum_{\mathrm{c}=1}^{\mathrm{Nc}} \rho\left[\int_{\Gamma_{\mathrm{c}}}\left(\overline{\mathbf{T}}_{, \ell}^{*}(\mathrm{~S}, \mathrm{Q}) \phi^{\mathrm{T}}(\mathrm{Q}) \mathrm{d} \Gamma-\overline{\mathbf{T}}^{*}(\mathrm{~S}, \mathrm{Q}) \phi_{, \ell}^{\mathrm{T}}(\mathrm{Q}) \mathrm{d} \Gamma\right) \mathrm{n}_{\ell} \mathrm{d} \Gamma\right] \ddot{\mathbf{U}}^{\mathrm{n}}(\mathrm{q}, \mathrm{t}),
\end{aligned}
$$

ou:

$$
\sigma=-\mathbf{H}^{\prime} \mathbf{U}+\mathbf{G}^{\prime} \mathbf{P}-\mathbf{M}^{\prime} \ddot{\mathbf{U}}
$$


onde

$\mathbf{M}^{\prime}=\rho\left(\mathbf{T}_{, \mathrm{n}}^{\prime} \phi-\mathbf{T}^{\prime} \phi_{, \mathrm{n}}\right)$.

A dedução dos tensores em (3.5.13) encontra-se no anexo E.

\section{6 - ALGORITMOS PARA INTEGRAÇÃO NO TEMPO}

Até aqui, foram determinadas as equações que permitem a análise de tensões e deslocamentos para sólidos tridimensionais no regime dinâmico. Diferentemente do caso estático, a solução dessas equações requer o uso de procedimentos numéricos específicos. COOK et al. (1989) e DOMINGUEZ (1993), entre outros, apontam dois algoritmos próprios para as equações dinâmicas apresentadas de Houbolt e de Newmark. Neste trabalho, optou-se por utilizar o algoritmo de Newmark sem amortecimento para resolver as integrais no tempo, dado o seu extenso uso em diversos casos. Sua formulação, portanto, garante segurança com relação à convergência e precisão. Também, em KATONA \& ZIENKIEWICZ (1985) pode-se encontrar as formulações para os algoritmos de Newmark e Houbolt.

\subsection{1 - Algoritmo de Newmark}

Em WARBURTON (1976), pode-se encontrar a apresentação detalhada da formulação do algoritmo de Newmark que, resumidamente, possui duas equações básicas: uma usada na partida do processo numérico de resolução e outra para os demais passos de tempo. A equação para a partida do processos sem amortecimento é

$$
\left[\mathbf{M}+\beta \Delta \mathrm{t}^{2} \mathbf{H}\right] \mathbf{U}_{1}=\Delta \mathrm{t}^{2} \beta \mathbf{F}_{1}+\Delta \mathrm{t}^{2}\left[\left(\frac{1}{2}-\beta\right) \mathbf{I}\right] \mathbf{F}_{0},
$$


onde $\beta$ é o parâmetro que permite a calibragem do algoritmo; $\Delta \mathrm{t}$ representa o intervalo de tempo entre dois instantes consecutivos; $\mathbf{F}_{\mathrm{s}}$ o vetor das forças atuantes no instante de tempo "s"; e $\mathbf{U}_{\mathrm{s}}$ o vetor de deslocamentos no instante de tempo "s".

Vê-se em (3.6.1), que o algoritmo necessita dos valores das forças atuantes nos instantes de tempo 0 (zero - de partida) e 1 (um - o de partida, mais o intervalo $\Delta \mathrm{t})$ para que se determine os valores dos deslocamentos no instante 1 , lembrando que o sólido está em repouso no instante 0 (prescrição deste trabalho).

Para os demais instantes de tempo s, o algoritmo Newmark fornece a seguinte equação:

$$
\begin{aligned}
{\left[\mathbf{M}+\beta \Delta \mathrm{t}^{2} \mathbf{H}\right] \mathbf{U}_{\mathrm{s}+1} } & =\Delta \mathrm{t}^{2}\left[\beta \mathbf{F}_{\mathrm{s}+1}+(1-2 \beta) \mathbf{F}_{\mathrm{s}}+\beta \mathbf{F}_{\mathrm{s}-1}\right]+ \\
& +\left[2 \mathbf{M}-(1-2 \beta) \Delta \mathrm{t}^{2} \mathbf{H}\right] \mathbf{U}_{\mathrm{s}}-\left[\mathbf{M}+\beta \Delta \mathrm{t}^{2} \mathbf{H}\right] \mathbf{U}_{\mathrm{s}-1},
\end{aligned}
$$

utilizando-se os valores dos deslocamentos nos dois instantes de tempo subsequentes, e os valores das forças em três instantes consecutivos: o atual e dois anteriores.

A aplicação imediata de Newmark à equação (3.4.23) é inadequada pois as suas incógnitas, deslocamentos e forças de superfície, podem estar dos dois lados da igualdade. Portanto, é necessário fazer um arranjo nas matrizes desta equação de modo que as incógnitas sejam apenas deslocamentos e acelerações. Assim, considerando-se a divisão em: parcela onde as forças de superfície são prescritas, representada pelo índice 1; e deslocamentos prescritos (nulos), índice 2, tem-se

$$
\left[\begin{array}{ll}
\mathbf{M}^{11} & \mathbf{M}^{12} \\
\mathbf{M}^{21} & \mathbf{M}^{22}
\end{array}\right]\left\{\begin{array}{c}
\ddot{\mathbf{U}}^{1} \\
\ddot{\mathbf{U}}^{2}
\end{array}\right\}+\left[\begin{array}{cc}
\mathbf{H}^{11} & \mathbf{H}^{12} \\
\mathbf{H}^{21} & \mathbf{H}^{22}
\end{array}\right]\left\{\begin{array}{c}
\mathbf{U}^{1} \\
\mathbf{U}^{2}
\end{array}\right\}=\left[\begin{array}{cc}
\mathbf{G}^{11} & \mathbf{G}^{12} \\
\mathbf{G}^{21} & \mathbf{G}^{22}
\end{array}\right]\left\{\begin{array}{l}
\mathbf{P}^{1} \\
\mathbf{P}^{2}
\end{array}\right\} .
$$

Desenvolvendo a equação (3.6.3), determina-se a equação do movimento resolvida pelo algoritmo Newmark. Surge, então,

$$
\mathbf{M}^{1} \ddot{\mathbf{U}}^{1}+\mathbf{H}^{1} \mathbf{U}^{1}=\mathbf{F}^{1}
$$

onde 


$$
\begin{aligned}
& \mathbf{M}^{1}=\mathbf{M}^{11}-\mathbf{G}^{12}\left(\mathbf{G}^{22}\right)^{-1} \mathbf{M}^{21} \\
& \mathbf{H}^{1}=\mathbf{H}^{11}-\mathbf{G}^{12}\left(\mathbf{G}^{22}\right)^{-1} \mathbf{H}^{21} \\
& \mathbf{F}^{1}=\left[\mathbf{G}^{11}-\mathbf{G}^{12}\left(\mathbf{G}^{22}\right)^{-1} \mathbf{G}^{21}\right] \mathbf{P}^{1}
\end{aligned}
$$

Determina-se, ainda, a equação das forças incógnitas:

$$
\mathbf{P}^{2}=\left(\mathbf{G}^{22}\right)^{-1}\left(\mathbf{M}^{21} \ddot{\mathbf{U}}^{1}+\mathbf{H}^{21} \mathbf{U}^{1}-\mathbf{G}^{21} \mathbf{P}^{1}\right)
$$

É conveniente que se monte as matrizes já na forma particionada, bastando utilizar um vetor índice para ordenar a colocação de seus elementos. Este assunto será abordado mais detalhadamente no capítulo 7 .

\subsection{2 - Algoritmo de Houbolt}

Em DOMINGUEZ (1993), pode-se encontrar as expressões sucintas do algoritmo de Houbolt.

Considere-se a equação do movimento definida no item 3.4.2, explicitando-se o instante de tempo genérico.

$$
\mathbf{M} \ddot{\mathbf{U}}_{\mathrm{t}+\Delta \mathrm{t}}+\mathbf{H} \mathbf{U}_{\mathrm{t}+\Delta \mathrm{t}}=\mathbf{G P}_{\mathrm{t}+\Delta \mathrm{t}}
$$

Adote-se a seguinte aproximação, em diferenças, para a aceleração.

$$
\ddot{\mathbf{U}}_{\mathrm{t}+\Delta \mathrm{t}}=\frac{1}{\Delta \mathrm{t}^{2}}\left(2 \mathbf{U}_{\mathrm{t}+\Delta \mathrm{t}}-5 \mathbf{U}_{\mathrm{t}}+4 \mathbf{U}_{\mathrm{t}-\Delta \mathrm{t}}-\mathbf{U}_{\mathrm{t}-2 \Delta \mathrm{t}}\right)
$$

São necessárias informações, deslocamentos, de três instantes de tempo anteriores, $\mathrm{t}-2 \Delta \mathrm{t}, \mathrm{t}-\Delta \mathrm{t}$ e $\mathrm{t}$, para se obter a expressão da aceleração em $\mathrm{t}+\Delta \mathrm{t}$.

Substituindo-se (3.6.7) em (3.4.23)rep., encontra-se: 


$$
\mathbf{M}\left[\frac{1}{\Delta \mathrm{t}^{2}}\left(2 \mathbf{U}_{\mathrm{t}+\Delta \mathrm{t}}-5 \mathbf{U}_{\mathrm{t}}+4 \mathbf{U}_{\mathrm{t}-\Delta \mathrm{t}}-\mathbf{U}_{\mathrm{t}-2 \Delta \mathrm{t}}\right)\right]+\mathbf{H} \mathbf{U}_{\mathrm{t}+\Delta \mathrm{t}}=\mathbf{G} \mathbf{P}_{\mathrm{t}+\Delta \mathrm{t}}
$$

ou

$$
\left[\frac{2}{\Delta \mathrm{t}^{2}} \mathbf{M}+\mathbf{H}\right] \mathbf{U}_{\mathrm{t}+\Delta \mathrm{t}}=\mathbf{G} \mathbf{P}_{\mathrm{t}+\Delta \mathrm{t}}+\mathbf{M}\left[\frac{1}{\Delta \mathrm{t}^{2}}\left(5 \mathbf{U}_{\mathrm{t}}-4 \mathbf{U}_{\mathrm{t}-\Delta \mathrm{t}}+\mathbf{U}_{\mathrm{t}-2 \Delta \mathrm{t}}\right)\right]
$$

Pode-se escrever (3.6.9) de forma resumida, resultando:

$$
\overline{\mathbf{H}} \mathbf{U}_{\mathrm{t}+\Delta \mathrm{t}}=\mathbf{G} \mathbf{P}_{\mathrm{t}+\Delta \mathrm{t}}+\overline{\mathbf{F}}_{\mathrm{t}+\Delta \mathrm{t}}
$$

onde

$$
\begin{aligned}
& \overline{\mathbf{H}}=\left[\frac{2}{\Delta \mathrm{t}^{2}} \mathbf{M}+\mathbf{H}\right] \\
& \overline{\mathbf{F}}_{\mathrm{t}+\Delta \mathrm{t}}=\mathbf{M}\left[\frac{1}{\Delta \mathrm{t}^{2}}\left(5 \mathbf{U}_{\mathrm{t}}-4 \mathbf{U}_{\mathrm{t}-\Delta \mathrm{t}}+\mathbf{U}_{\mathrm{t}-2 \Delta \mathrm{t}}\right)\right]
\end{aligned}
$$

É possível, agora, trocar as colunas de $\overline{\mathbf{H}}$ e $\mathbf{G}$ para deixar as incógnitas convenientemente em um único vetor do lado esquerdo da igualdade. Assim, a equação (3.6.10) passa a ser:

$$
\mathbf{A X}_{\mathrm{t}+\Delta \mathrm{t}}=\mathbf{F}_{\mathrm{t}+\Delta \mathrm{t}}
$$

cuja solução é imediata.

As matrizes envolvidas nessa formulação possuem elementos relacionados com forças e deslocamentos, o que pode gerar algum distúrbio nas respostas; além do fato das matrizes não serem simétricas.

Pode-se trabalhar com as matrizes particionadas, semelhantemente ao que foi utilizado no algoritmo de Newmark, homogeneizando-as (apenas deslocamentos incógnitos). 


\section{7 - APLICAÇÕES}

3.7.1 - Aplicação 1: Sólido contido lateralmente.

Como primeira aplicação da formulação elastodinâmica abordada neste capítulo, apresenta-se um sólido contido lateralmente com uma extremidade livre (axialmente livre), conforme mostra a figura 3.7.1. A força é aplicada bruscamente na extremidade, mantendo-se constante ao longo do tempo. São adotados os seguintes valores para os parâmetros elastodinâmicos: $E=1 \times 10^{5} \mathrm{~Pa}, \mathrm{v}=0,25$, $\rho=1 \mathrm{~kg} / \mathrm{m}^{3}$ e $\Delta \mathrm{t}=0,004 \mathrm{~s}$.

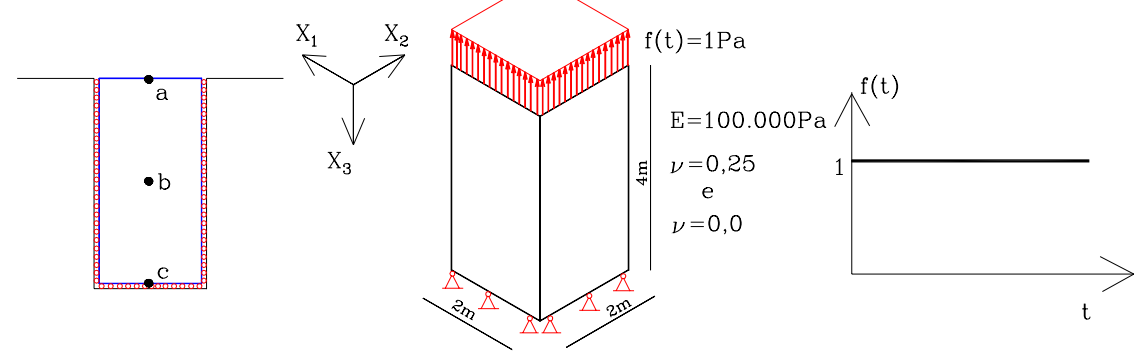

FIGURA 3.7.1 - Características geométricas do sólido, condições de contorno e comportamento temporal da força $\mathrm{f}(\mathrm{t})$.

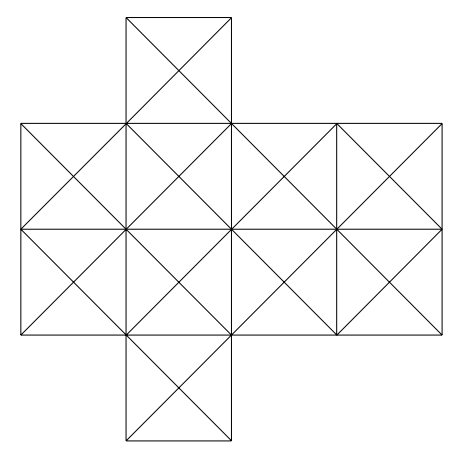

(a)

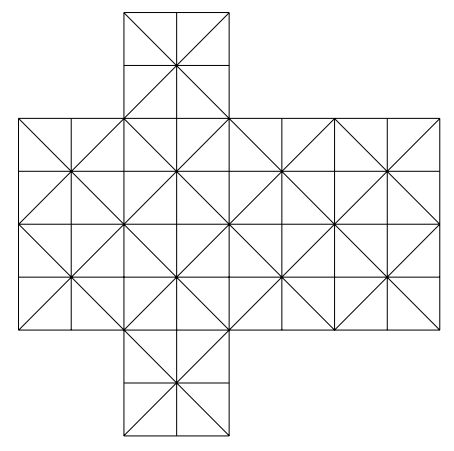

(b)

FIGURA 3.7.2 - Discretizações do contorno por elementos triangulares planos: (a) 40 elementos e (b) 80 elementos. 
TABELA 3.7.1 - Casos processados.

\begin{tabular}{|c|c|c|c|c|}
\hline Caso & Formulação & Discretização & "Pólos" Internos & Células \\
\hline RD1 & Reciprocidade. Dual & a & 0 & - \\
RD2 & & a & 15 & \\
RD3 & & b & 15 & \\
\hline CT1 & Células & a & - & 2 \\
CT2 & Tridimensionais & b & & 2 \\
\hline
\end{tabular}

A análise é feita tomando-se as duas discretizações do contorno apresentadas na figura 3.7.2, seguindo o roteiro de casos da tabela 3.7.1, utilizando-se o algoritmo de Newmark para integração no tempo. Os resultados para $v=0,25$ são mostrados nas figuras 3.7.3 e 3.7.4, para as discretizações (a) e (b), respectivamente. Os sentidos das reações de apoio e deslocamentos estão relacionados ao sistema de eixos adotado.
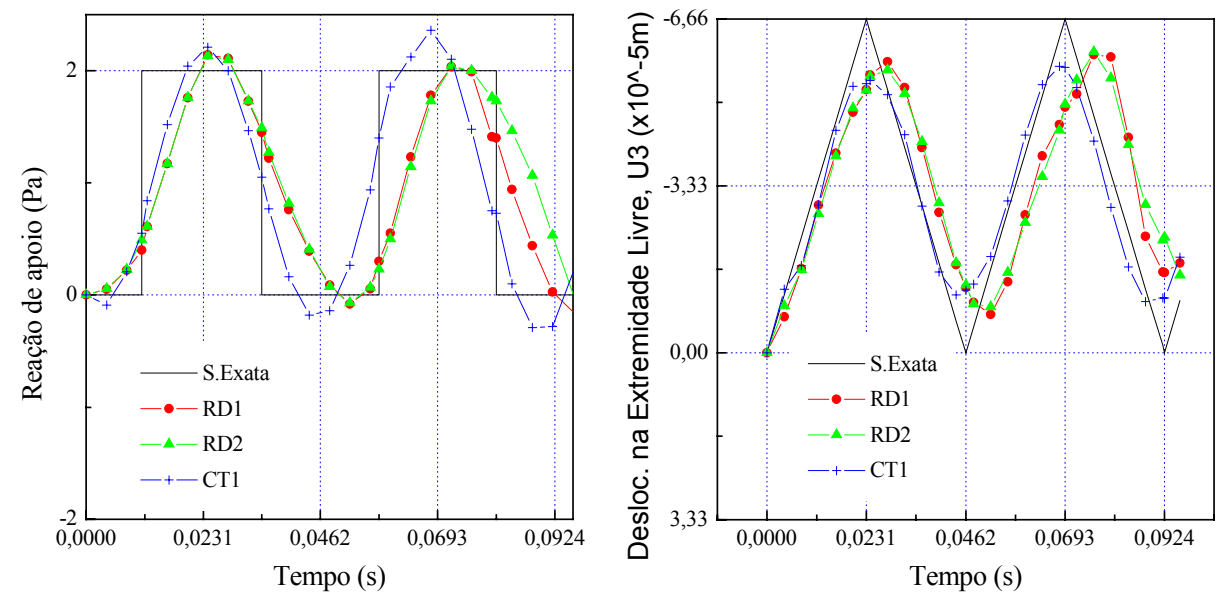

FIGURA 3.7.3 - Resultados para a discretização (a).
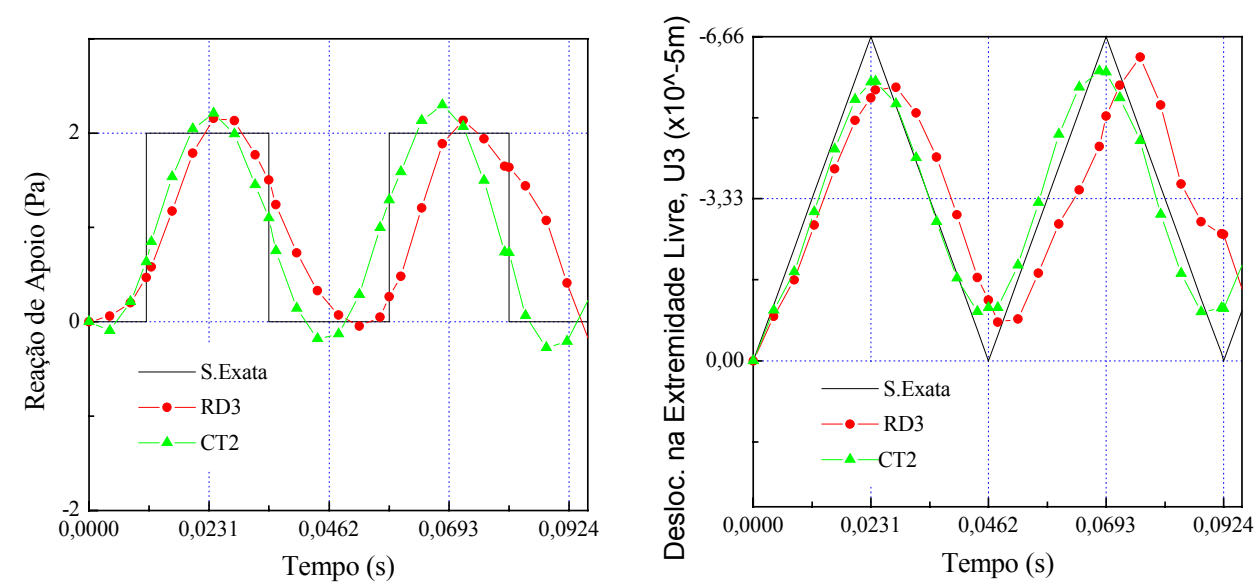

FIGURA 3.7.4 - Resultados para a discretização (b). 
Realiza-se, ainda, um teste comparativo entre os algoritmos de integração no tempo, Newmark e Houbolt. Os resultados são mostrados na figura 3.7 .5 deslocamentos em um ponto da extremidade livre do sólido analisado e força de vinculação.
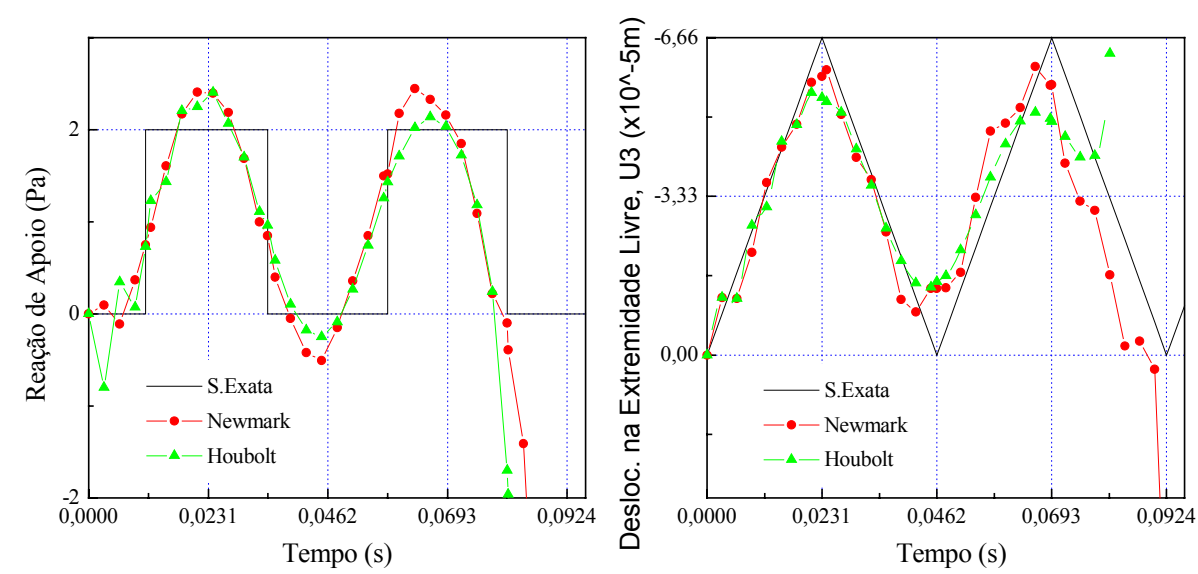

FIGURA 3.7.5 - Resultados de comparação entre os algoritmos de Newmark e Houbolt.

Considere-se o coeficiente de Poisson nulo, $v=0,0$. Os resultados do processamento deste exemplo para tal valor encontra-se nos gráficos da figura 3.7.6, mostrados a seguir.
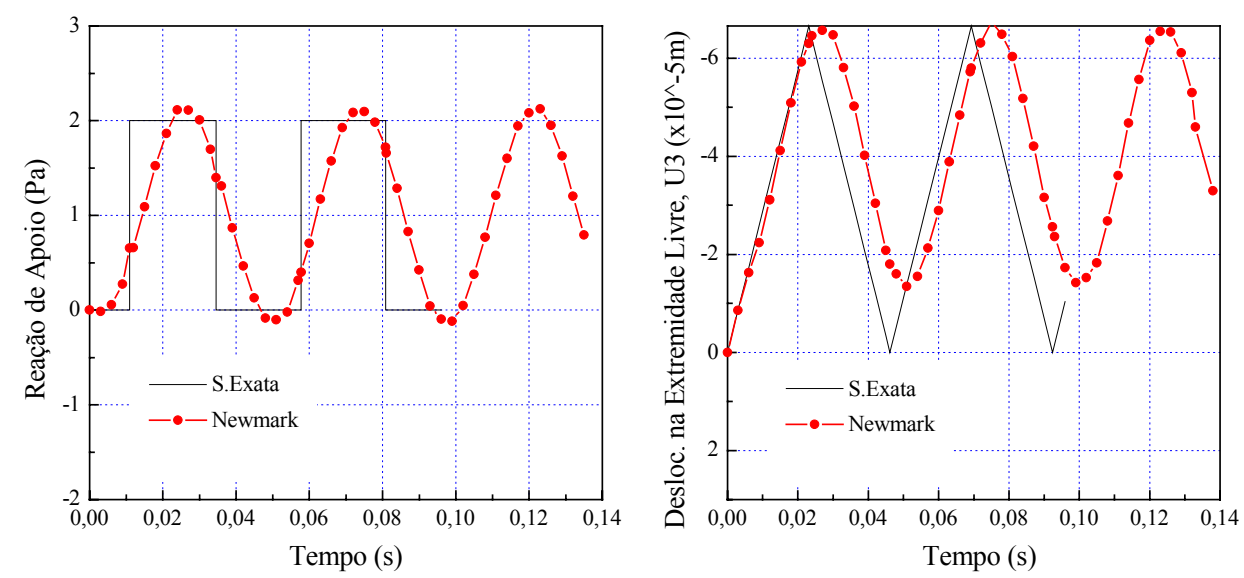

FIGURA 3.7.6 - Resultados do processamento utilizando-se Newmark para $v=0,0$.

Como conclusão genérica, pode-se dizer que o Método dos Elementos de Contorno se mostra adequado à análise transiente de sólidos tridimensionais, 
formulado através da Reciprocidade Dual e da utilização de células, conforme os valores apresentados nas figuras 3.7.3 e 3.7.4.

$\mathrm{O}$ uso do elemento triangular com aproximação linear, de fácil implementação, exige que a discretização seja mais refinada, na busca de melhores resultados. A integração temporal através do algoritmo de Newmark permite os resultados apresentados.

Deve-se ter especial atenção com o passo de tempo $\Delta$ t adotado, uma vez que pode haver suavização da resposta ou divergência, de acordo com a escolha de valores maiores e menores, respectivamente.

Os resultados obtidos através do MEC com a Reciprocidade Dual tendem para os valores da solução exata, observando-se o mesmo para os obtidos com a utilização de células, estes últimos um pouco mais precisos. Já o tempo computacional gasto no processamento pelo MRD é bem maior, uma vez que a sua formulação exige a inversão da matriz das funções $\mathrm{f}_{\mathrm{n}}$. Entretanto, tal observação é irrelevante quando se analisa a evolução tecnológica dos computadores, cada vez mais velozes e disponíveis.

O teste comparativo entre os algoritmos de integração no tempo, mostra uma pequena vantagem para o algoritmo de Newmark frente ao de Houbolt. Este último apresenta variação dos resultados nos passos iniciais, como mostra a figura 3.7.5. A expectativa é que essa variação possa ser bastante diminuída com a redução do passo de tempo $\Delta \mathrm{t}$, ou ainda, formulando o método com diferenças que não a central, para melhorar as respostas dos passos iniciais. $O$ teste realizado não tem a pretensão de responder de forma definitiva a questão formulada. Trata-se apenas de um teste comparativo das formulações apresentadas dentro do contexto desta aplicação.

Quando se altera o coeficiente de Poisson para o valor nulo, resultados na figura 3.7.6, verifica-se que o processamento apresenta comportamento estável ao longo do tempo, diferentemente do que se nota nas figuras anteriores a esta.

\subsection{2 - Aplicação 2: Sólido à flexão.}

Esta aplicação consiste em analisar o comportamento do sólido elástico tridimensional solicitado por uma distribuição transversal ao seu eixo, caracterizando 
um impulso cisalhante. O sólido, engastado na base e livre na extremidade, é definido na figura 3.7.7. Na mesma figura, é definida a força aplicada bruscamente na extremidade, que desaparece algumas frações de segundo depois. São adotados os seguintes valores para os parâmetros elastodinâmicos: $E=1 \times 10^{5} \mathrm{~Pa}, v=0,25$, $\rho=1 \mathrm{~kg} / \mathrm{m}^{3}$ e $\Delta \mathrm{t}=0,015 \mathrm{~s}$.
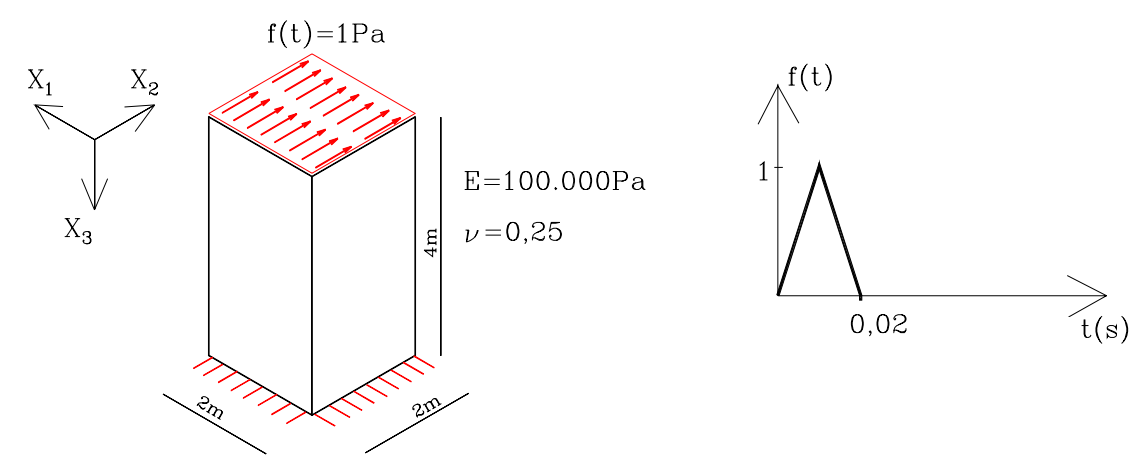

FIGURA 3.7.7 - Características geométricas do sólido, condições de contorno e comportamento temporal da força $f(t)$.

Pretende-se calcular os deslocamentos vertical e horizontal do ponto A definido na figura anterior.

A discretização do contorno é feita através de elementos triangulares planos com aproximação linear. São utilizados 80 elementos, conforme figura 3.7.8.

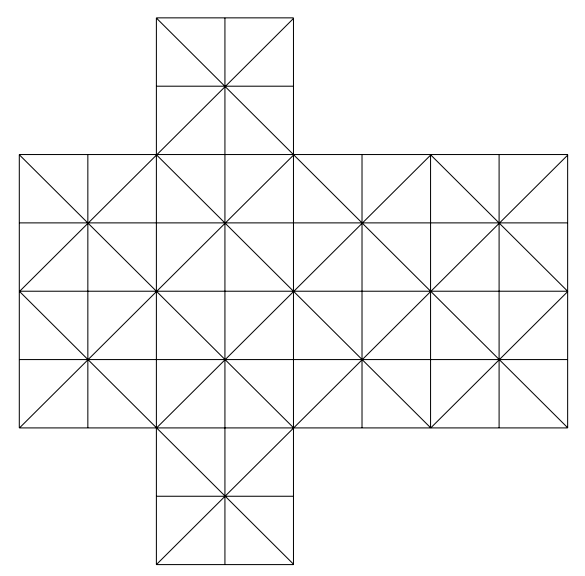

FIGURA 3.7.8 - Discretização do contorno do sólido - 80 elementos triangulares planos.

Juntamente com a discretização do contorno são definidos pólos, pontos internos, constituindo três casos para processamento numérico, intencionando a 
utilização do Método dos Elementos de Contorno com a Reciprocidade Dual. Os casos são:

- 80 elementos no contorno e 1 pólo interno;

- 80 elementos no contorno e 3 pólos internos; e

- 80 elementos no contorno e 28 pólos internos.

As figuras 3.7.9 e 3.7.10 trazem os gráficos dos deslocamentos vertical e horizontal do ponto A para os três casos definidos.

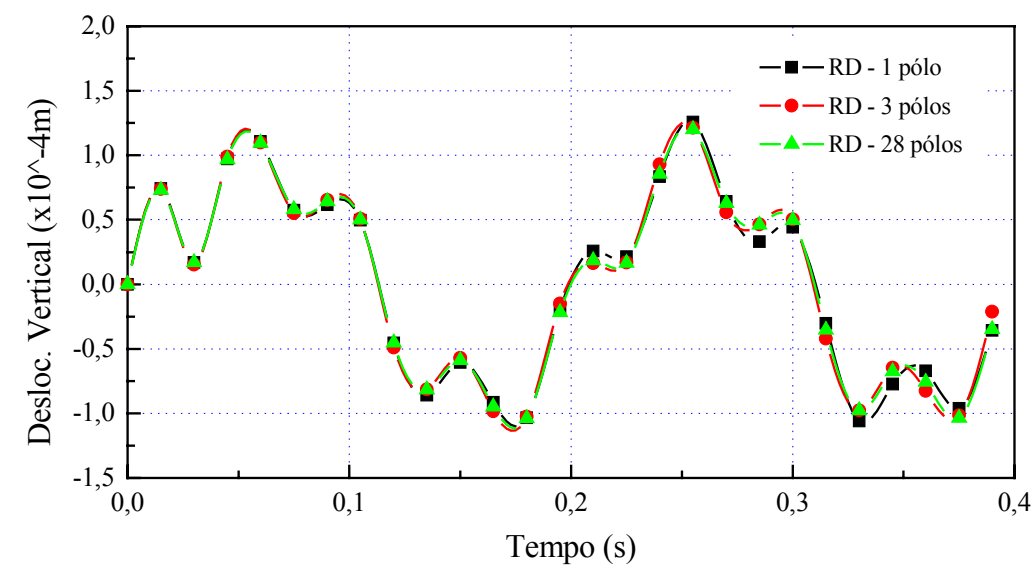

FIGURA 3.7.9 - Deslocamentos verticais no ponto A ao longo do tempo.

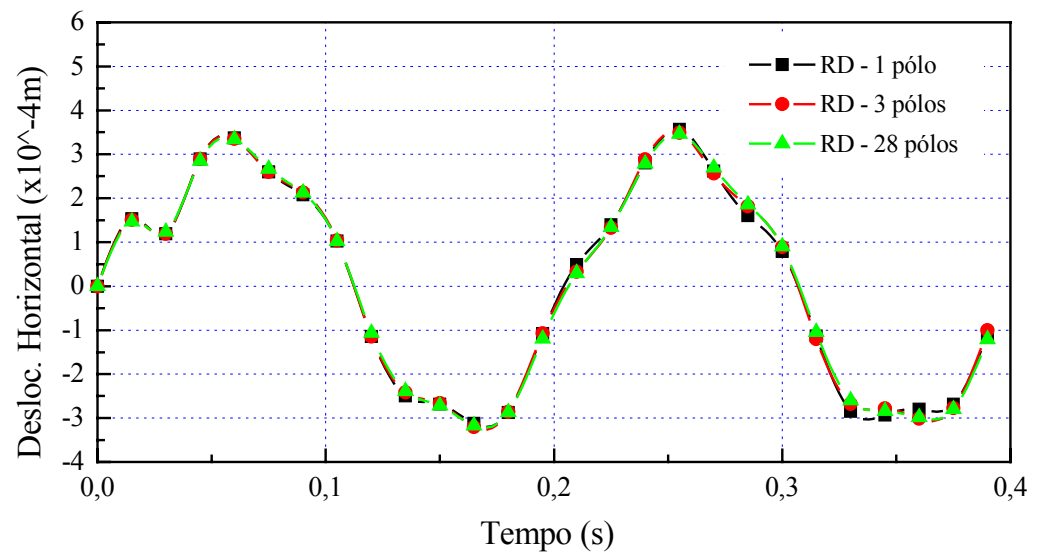

FIGURA 3.7.10 - Deslocamentos horizontais no ponto A ao longo do tempo.

A inclusão de mais pontos internos (pólos) não representa, neste caso, melhoria nos resultados obtidos; os valores encontrados para os três casos simulados são bastante coincidentes. As curvas apresentadas nas figuras 3.7 .9 e 3.7 .10 são semelhantes àquelas mostradas por PARTRIDGE et al. (1992) para uma aplicação bidimensional. 
$\mathrm{O}$ aumento do valor do passo de tempo $\Delta \mathrm{t}$ pode trazer como conseqüência a suavização da curva, como mostra a figura 3.7.11. Valores menores de $\Delta$ t permitem a inclusão de modos secundários de freqüência que interferem na resposta procurada. Os modos secundários alteram os valores oscilando sobre a resposta principal do problema (figura 3.7.12).

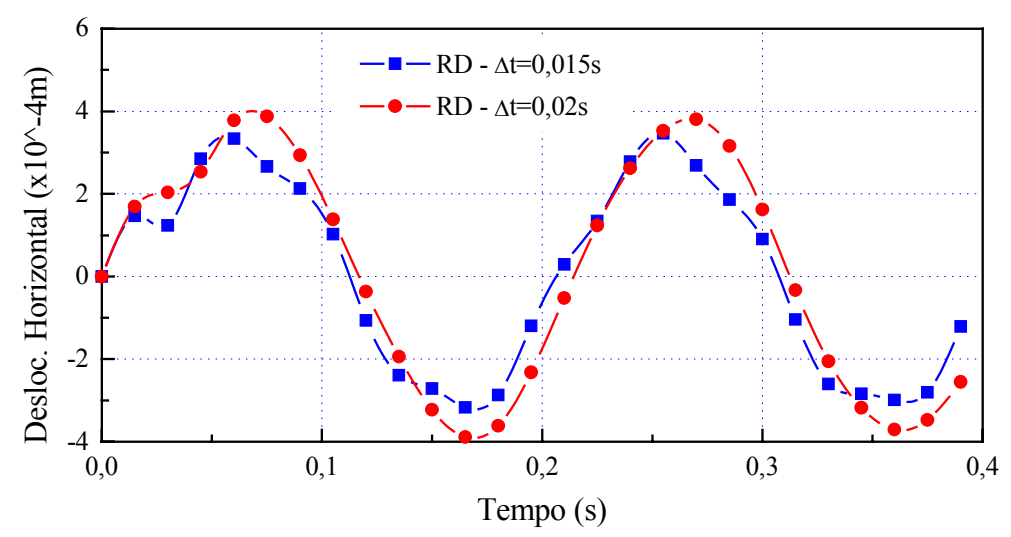

FIGURA 3.7.11 - Suavização da resposta à medida que se aumenta o valor de $\Delta \mathrm{t}$.

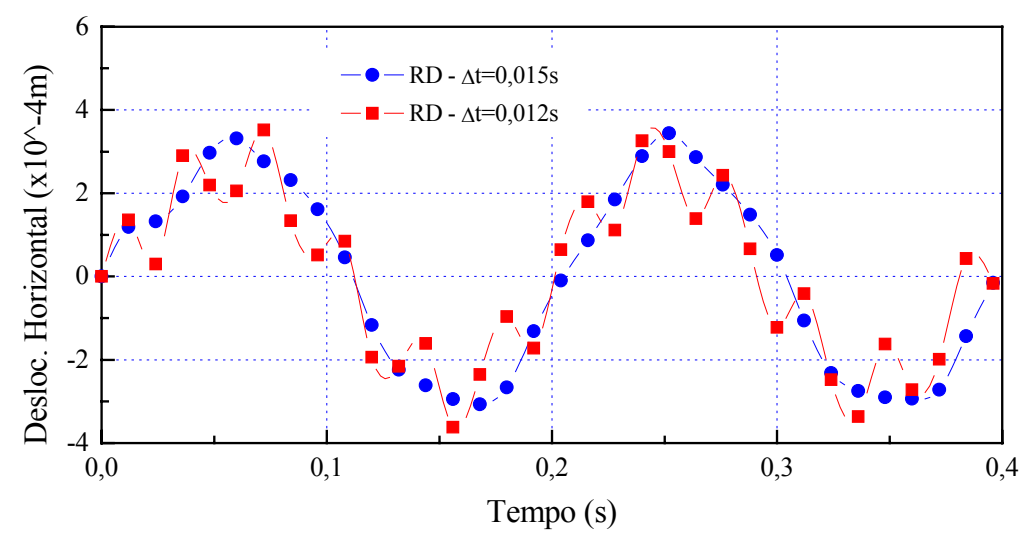

FIGURA 3.7.12 - Oscilação da resposta à medida que se diminui o valor de $\Delta \mathrm{t}$. 


\section{CAPÍTULO 4}

\section{MECÂNICA DO FRATURAMENTO - MODELO COESIVO}

\section{1 - INTRODUÇÃO}

Neste capítulo é apresentada uma introdução à Mecânica do Fraturamento. Enfoca-se a parte inicial de seu desenvolvimento com os trabalhos clássicos dos precursores, chegando-se nas expressões dos fatores de intensidade de tensão para o caso tridimensional. Apresenta-se, ainda, o modelo de fratura coesiva utilizado neste trabalho.

\section{2 - MECÂNICA DA FRATURA ELÁTICA E LINEAR}

\subsection{1 - Generalidades}

Segundo TIMOSHENKO (1953), os primeiros relatos sobre a observação do fenômeno de fraturamento devem-se à Leonardo da Vinci, a partir de um estudo sobre a variação da resistência de materiais em corpos de prova de diferentes comprimentos e mesmo diâmetro. O efeito do tamanho, hoje denominado "efeito 
escala", é também estudado para o aço por Lloyd and Hodkinson ${ }^{1}$, assim como Anderegg ${ }^{2}$, apud OWEN \& FAWKES (1983), para lamelas finas de vidro e fibras.

Mas é em 1913 que se apresenta uma aproximação matemática para representar o efeito do fraturamento, em INGLIS (1913), que serve de base para a mecânica da fratura. INGLIS (1913) ensaia experimentalmente uma chapa solicitada por tensão constante na presença de um furo elíptico com o raio maior na direção perpendicular à da aplicação do carregamento, conforme figura 4.2.1. O furo age como um concentrador de tensões, provocando o colapso do material assim que a tensão na ponta do entalhe (região próxima ao eixo $\mathrm{x}$ ) ultrapassa a resistência do material.

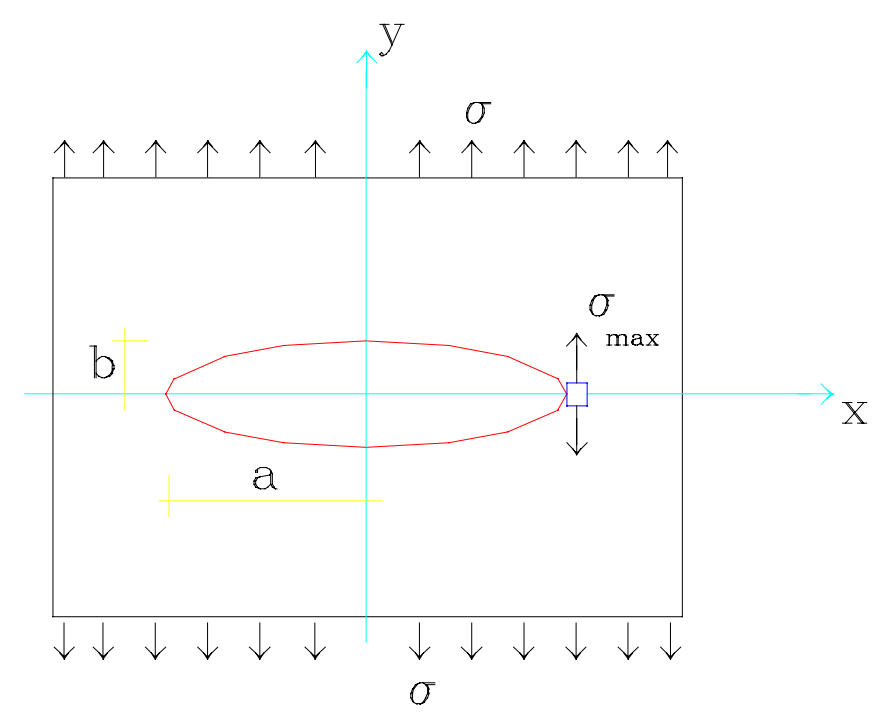

FIGURA 4.2.1 - Modelo experimentado por Inglis - furo elíptico em uma placa solicitada por tensões constantes.

Como resultado, é determinado que a máxima tensão ocorre no ponto extremo do eixo maior da elipse onde o raio de curvatura é mínimo $\left(\rho=b^{2} / a\right)$, cujo valor é

$$
\sigma_{\mathrm{y}}^{\max }=\sigma(1+2 \mathrm{a} / \mathrm{b})
$$

\footnotetext{
${ }^{1}$ TODHUNTER, I. \& PEARSON, K. (1886). History of the theory of elasticity and of the strength of materials from Galileo to the present time, Cambridge University Press.

${ }^{2}$ ANDEREGG, F.O. (1939) Industr. Eng. Chem., 31, 290.
} 
No limite quando o eixo $b=0$, situação real de uma trinca, o valor da tensão máxima próxima da ponta da mesma tem o valor infinitamente grande, dado por

$$
\sigma_{\mathrm{y}}^{\max }=2 \sigma \sqrt{\frac{\mathrm{a}}{\rho}} .
$$

Como conseqüência, poder-se-ia dizer que o elemento estrutural que apresentasse alguma trinca não suportaria carregamento algum. Estes resultados são reavaliados em trabalhos posteriores, como em GRIFFITHS (1924).

GRIFFITHS (1924) realiza experimentos com placas de vidro, material de ruptura frágil, na presença de uma trinca centralizada, semelhante à figura 4.2.1. Ele propõe que uma trinca pode tornar-se instável somente quando a taxa de decréscimo de energia de deformação elástica, devido ao aumento da trinca, excede a taxa de acréscimo da energia de superfície associada à nova forma da superfície da trinca. A taxa de energia de deformação na formação da trinca de comprimento a, em um corpo inicialmente homogêneo é definida por:

$$
U= \begin{cases}\frac{\sigma^{2}}{2 \mathrm{E}} \pi \mathrm{a}^{2}, & \text { para o estado plano de tensão; } \\ \frac{\sigma^{2}}{2 \mathrm{E}} \pi \mathrm{a}^{2}\left(1-v^{2}\right), & \text { para o estado plano de deformação, }\end{cases}
$$

onde E representa o módulo de elasticidade; $v$ o coeficiente de Poisson; $\sigma$ a tensão aplicada perpendicularmente à trinca e $2 \mathrm{a}$ o comprimento da trinca.

Como conclusão, GRIFFITHS (1924) determina que a taxa de energia de deformação crítica liberada no fenômeno de fraturamento é uma propriedade intrínseca do material, já que está associada ao rompimento das forças atômicas de coesão na ponta da trinca. Isto permite determinar a resistência de uma peça, composta de material frágil, relacionando a sua tenacidade (resistência ao fraturamento) ao tamanho da trinca $\mathrm{a}_{\mathrm{c}}$. 
As investigações científicas para os materiais dúcteis, por exemplo os metais, são iniciadas por IRWIN (1948) e OROWAN (1955), baseando-se no balanço energético devido a GRIFFITHS (1924). A extensão para os materiais dúcteis, cujo fraturamento é acompanhado de uma deformação permanente, originou a seguinte expressão:

$$
\mathrm{G} \geq 2 \Gamma+\Delta
$$

onde

G representa a taxa de energia de deformação liberada;

$\Gamma$ representa o trabalho necessário para a formação de uma nova trinca; e

$\Delta$ representa o trabalho não recuperado, relativo à deformação permanente na ponta da trinca.

Para os materiais dúcteis, os metais por exemplo, o valor de $\Delta$ é bem maior do que o de $\Gamma$, ao ponto de se considerar apenas

$$
\mathrm{G} \geq \Delta
$$

Isso explica porque os materiais dúcteis necessitam de maior trabalho para ser fraturado em comparação àqueles considerados frágeis ou quase-frágeis.

O postulado fundamental da Mecânica da Fratura Elástica Linear (MFEL) diz que o comportamento das trincas é determinado somente pelo valor dos fatores de intensidade de tensão. Existem três modos básicos de deformação para um corpo fraturado: modo I, abertura; modo II, de deslizamento; e modo III, de rasgamento. Esses modos são caracterizados pelos movimentos relativos das duas superfícies da trinca, conforme mostra a figura 4.2.2. 

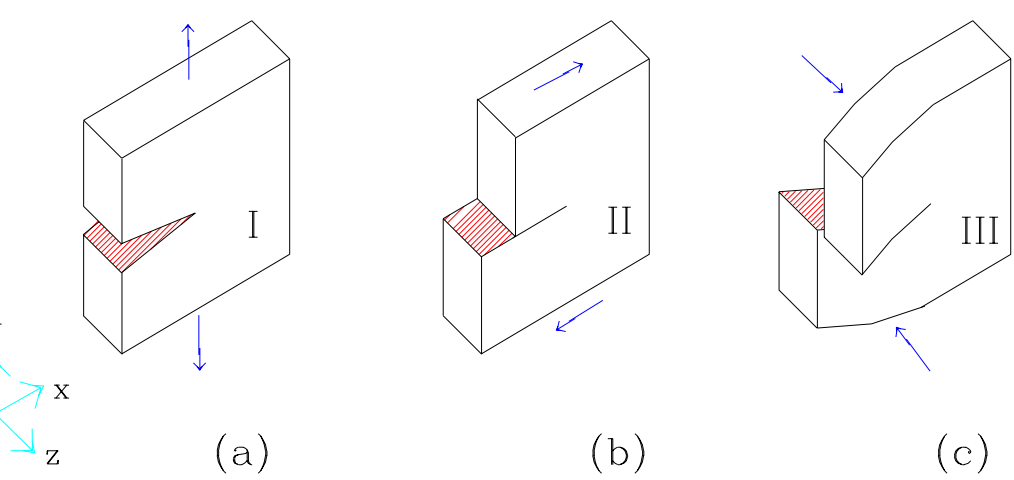

FIGURA 4.2.2 - Modos de Fraturamento: (a) modo I - abertura; (b) modo II, de deslizamento; (c) modo III, de rasgamento.

A figura 4.2.2 (a) mostra o modo fundamental de fratura I, cuja conseqüência é a abertura das superfícies da trinca, uma vez solicitada por tensões de tração. A figura 4.2.2 (b) mostra o modo II, com deslizamento relativo das superfícies da trinta, provocado por tensões cisalhantes. A figura 4.2 .2 (c) mostra o modo III, com rasgamento entre as duas superfícies, provocado por tensões cisalhantes.

A partir dos valores dos fatores de intensidade de tensão dos modos de fraturamento $\mathrm{K}_{\mathrm{I}}, \mathrm{K}_{\mathrm{II}}$ e $\mathrm{K}_{\mathrm{III}}$, são determinadas expressões para o cálculo das tensões em um ponto "p" nas proximidades da frente da fratura (ponta da trinca), definido pelas coordenadas esféricas ( $r, \theta$ e $\phi)$ - como em HARTRANFT \& SIH (1977) conforme mostra a figura 4.2.3.

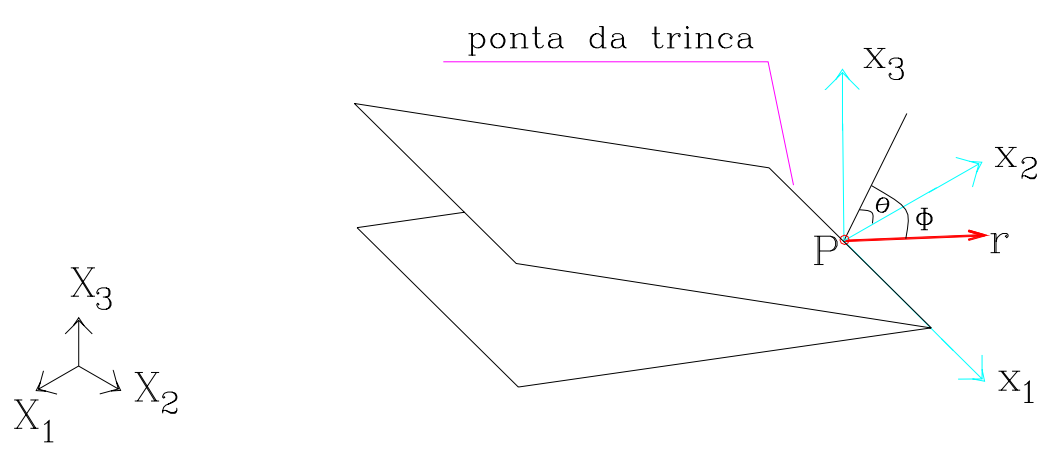

FIGURA 4.2.3 - Frente da fratura - sistemas de coordenadas. 
HARTRANFT \& SIH (1977) determinam as expressões para tensões e deslocamentos nas direções normal e tangenciais, trabalhando em um plano perpendicular à frente da fratura. Estendendo-as para um ponto qualquer sobre uma esfera centrada no ponto "p" (pertencente à frente da fratura), tem-se as seguintes expressões:

$$
\begin{aligned}
& \sigma_{11}=\frac{1}{\sqrt{2 \mathrm{r} \cos \phi}}\left\{2 \nu \mathrm{K}_{\mathrm{I}} \cos \frac{\theta}{2}+2 \nu \mathrm{K}_{\mathrm{II}} \operatorname{sen} \frac{\theta}{2}\right\} \\
& \sigma_{12}=\frac{1}{\sqrt{2 \mathrm{r} \cos \phi}}\left\{-\mathrm{K}_{\mathrm{III}} \operatorname{sen} \frac{\theta}{2}\right\} \\
& \sigma_{13}=\frac{1}{\sqrt{2 \mathrm{r} \cos \phi}}\left\{\mathrm{K}_{\mathrm{III}} \cos \frac{\theta}{2}\right\} \\
& \sigma_{22}=\frac{1}{\sqrt{2 \mathrm{r} \cos \phi}}\left\{\mathrm{K}_{\mathrm{I}} \cos \frac{\theta}{2}\left(1-\operatorname{sen} \frac{\theta}{2} \operatorname{sen} \frac{3 \theta}{2}\right)-\mathrm{K}_{\mathrm{II}} \operatorname{sen} \frac{\theta}{2}\left(2+\cos \frac{\theta}{2} \cos \frac{3 \theta}{2}\right)\right\} \\
& \sigma_{23}=\frac{1}{\sqrt{2 \mathrm{r} \cos \phi}}\left\{\mathrm{K}_{\mathrm{I}} \operatorname{sen} \frac{\theta}{2} \cos \frac{\theta}{2} \operatorname{sen} \frac{3 \theta}{2}+\mathrm{K}_{\mathrm{II}} \cos \frac{\theta}{2}\left(1-\operatorname{sen} \frac{\theta}{2} \operatorname{sen} \frac{3 \theta}{2}\right)\right\} \\
& \sigma_{33}=\frac{1}{\sqrt{2 \mathrm{r} \cos \phi}}\left\{\mathrm{K}_{\mathrm{I}} \cos \frac{\theta}{2}\left(1+\operatorname{sen} \frac{\theta}{2} \operatorname{sen} \frac{3 \theta}{2}\right)+\mathrm{K}_{\mathrm{II}} \operatorname{sen} \frac{\theta}{2} \cos \frac{\theta}{2} \cos \frac{3 \theta}{2}\right\}
\end{aligned}
$$

\subsection{2 - Fatores de Intensidade de Tensão}

Através de experimentos de vários problemas elástico-lineares bidimensionais de fratura, IRWIN (1957) verifica que há uma regularidade de forma no campo de tensões nas proximidades da ponta da trinca. Propõe, então, uma representação matemática para o campo de tensões, tomando-se por base um ponto da ponta da trinca, isto é,

$$
\sigma(\mathrm{r}, \theta)=\frac{\mathrm{K}}{\sqrt{2 \pi \mathrm{r}}} \mathbf{f}+\ldots
$$


onde $r$ e $\theta$ são coordenadas polares. Os demais termos da série apresentada em (4.2.7) são constantes ou tendem a zero. Mas o primeiro, observando-se a coordenada $\mathrm{r}$ bem próximo da origem (ponta da trinca), eleva consideravelmente $\mathrm{o}$ valor das tensões, fazendo-o dominante aos demais. A constante $\mathrm{K}$ é batizada de Fator de Intensidade de Tensão - FIT (SIF em inglês). Portanto, o FIT caracteriza o campo de tensões nos pontos bem próximos à ponta da trinca.

IRWIN (1957) determina, ainda, uma relação entre o fator de intensidade de tensão e a taxa de energia de deformação liberada,

$$
\mathrm{K}^{2} \propto \mathrm{G}
$$

A constante de proporcionalidade de (4.2.8) é função das constantes elásticas do material. Essa relação origina-se de uma ligação entre o campo de tensões na ponta da trinca e a energia requerida para propagação da mesma. Isto significa que o critério de balanço de energia para a propagação de uma trinca pode ser interpretado em termos dos valores críticos de $\mathrm{K}$ para o início da trinca.

Partindo-se da consideração básica da Mecânica da Fratura, a de que o controle da propagação da trinca (estável ou instável) é feito através do campo de tensões na ponta da mesma, pode-se dizer que a propagação é caracterizada através do parâmetro K. Isso significa que duas trincas diferentes, de valores iguais a K, têm o mesmo comportamento. Assim, para se prever a propagação de uma trinca em um componente estrutural, é necessário avaliar os fatores de intensidade de tensão do mesmo. De um modo geral, $\mathrm{K}$ é função dos comprimento e forma da trinca, do tipo de carregamento e configuração geométrica da estrutura. $\mathrm{O}$ fator de intensidade de tensão pode ser escrito da seguinte forma:

$$
\mathrm{K}=\mathrm{Y} \sigma \sqrt{\pi \mathrm{a}}
$$

onde $\sigma$ representa uma tensão, a é a medida do comprimento da trinca e Y é uma função adimensional da geometria. 
Desde o trabalho pioneiro de IRWIN (1957) muitos outros têm se seguido, estabelecendo-se vários procedimentos para a obtenção dos fatores de intensidade de tensão. Os procedimentos mais comuns, divididos em três grandes categorias, são:

- métodos teóricos simples: superposição, concentração de tensões, distribuição de tensões, funções de Green e funções peso;

- métodos teóricos avançados (numéricos): colocação (mapeamento), transformações integrais, técnicas alternativas, Método dos Elementos de Contorno, Método dos Elementos Finitos; e

- métodos experimentais.

A utilização dos fatores de intensidade de tensão, uma vez determinados, pode-se verificar em três áreas diferentes:

- na determinação da resistência estática de uma estrutura fraturada (obtenção da resistência residual);

- na determinação da taxa de propagação de uma trinca em uma estrutura submetida a cargas variáveis (fadiga); e

- na determinação da taxa de propagação de uma trinca em uma estrutura carregada em um ambiente corrosivo (tensões de corrosão).

\section{3 - MODELO COESIVO}

O modelo de fratura coesiva tem sido empregado com eficiência para representar o comportamento do material do tipo "quasi-brittle" frente ao processo de fraturamento. Em HILLERBORG et al. (1976), o modelo é testado e modificado utilizando-se corpo de prova homogêneo e de área constante, solicitado até a ruptura, em ensaios laboratoriais. É definida uma zona de fratura (ou zona de processo) com largura limitada na direção da tensão, formada em algum lugar do modelo. Esta zona de fratura perde gradualmente suas propriedades mecânicas à medida que o dano causado pelo surgimento de microfissuras aumenta e, portanto, tem-se um comportamento de material coesivo. Da mesma forma, em CARPINTERI (1989) são 
encontrados novos experimentos e análise através de modelagem numérica, constituindo-se em uma referência importante sobre o assunto.

O modelo de fratura coesiva apresentado por HILLERBORG et al. (1976) é considerado neste trabalho. O modelo é recomendado para os casos onde a zona plástica (ou zona de processo) é suficientemente fina ao ponto de se poder representá-la por uma superfície sem que isso ocasione perda de precisão. A idealização do modelo é apresentada na figura 4.3.1.

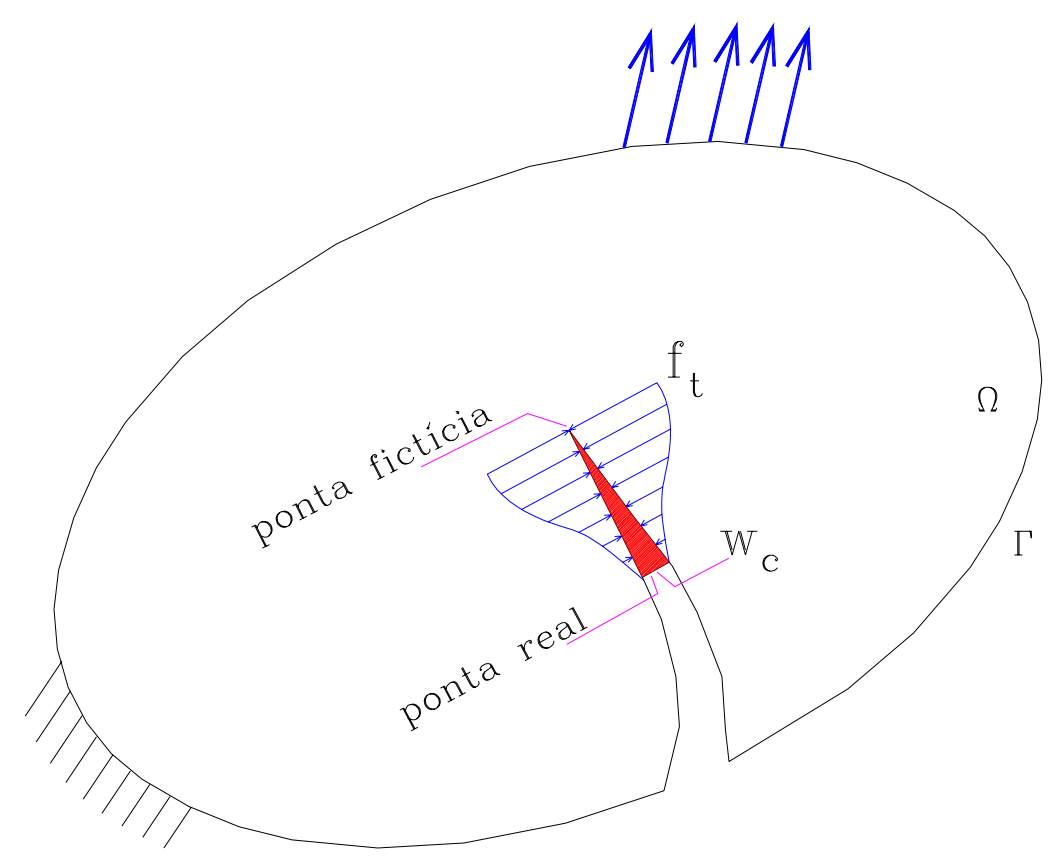

FIGURA 4.3.1 - Definição do modelo idealizado - distribuição de tensões na zona coesiva.

É definida, na figura 4.3.1, a existência de duas extremidades para a trinca: a real, localizada no ponto onde o valor crítico da abertura da trinca $\mathrm{w}_{\mathrm{c}}$ é atingido, onde a tensão normal à superfície da trinca é nula; e a fíctícia, definida no ponto onde a tensão normal atinge o seu valor máximo $\mathrm{f}_{\mathrm{t}}$, onde a abertura da fratura é nula. 
O modelo de fratura coesiva baseia-se nas seguintes considerações:

- a linearidade geométrica (pequenas deformações) é considerada;

- o material é considerado homogêneo e elástico linear, exceto na superfície de fraturamento;

- a zona de fratura coesiva ou zona de processo (fig. 4.3.1) inicia o seu desenvolvimento quando a tensão principal máxima atinge a tensão $f_{t}$; e

- o material na zona de processo está parcialmente danificado, ou seja, ainda consegue transmitir tensões. Quando a abertura da trinca atingir o deslocamento $\mathrm{w}_{\mathrm{c}}$ (fig. 4.3.1), o material não mais transmite tensões.

Portanto, as trincas coesivas surgem quando a máxima tensão principal de tração atinge seu valor limite, $\mathrm{f}_{\mathrm{t}}$, conforme mostra a relação constitutiva apresentada na figura 4.3.2a. Admite-se que a resistência do material sofre uma contínua redução até o valor zero, quando o comprimento da abertura da trinca chegar no seu valor limite $\mathrm{w}_{\mathrm{c}}$, figuras $4.3 .2 \mathrm{~b}, \mathrm{c}$.

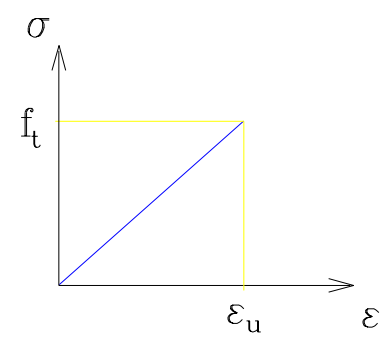

(a)

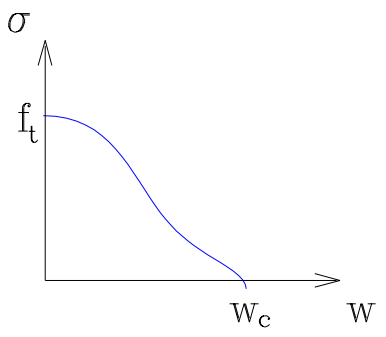

(b)

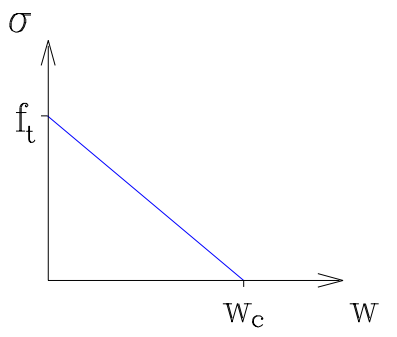

(c)

FIGURA 4.3.2 - Leis constitutivas do material: (a) tensão $\times$ deformação; (b) tensão $\times$ abertura da trinca - modelo ideal; (c) tensão $\times$ abertura da trinca - modelo simplificado.

O modelo simplificado, figura 4.3.2c, conduz a resultados satisfatórios para materiais classificados como "quasi-brittle" (concreto, cerâmicos, por exemplo). Este modelo é definido apenas por dois parâmetros, $\mathrm{f}_{\mathrm{t}}$ e $\mathrm{w}_{\mathrm{c}}$, os quais podem ser obtidos experimentalmente em laboratório. A relação constitutiva do modelo coesivo pode ser escrita através da forma clássica dos critérios de plasticidade, conforme mostrado por CEN \& MAIER (1992). Essa representação é usada no presente trabalho e tem a seguinte forma: 


$$
\Phi(\sigma)=\sigma-\mathrm{f}_{\mathrm{t}}\left(1-\frac{\mathrm{w}}{\mathrm{w}_{\mathrm{c}}}\right)
$$

onde $\sigma$ é a tensão de tração na direção perpendicular à superfície da trinca, e w representa o deslocamento normal entre as superfícies da trinca (comprimento da abertura da trinca), desprezando-se as tensões tangenciais coesivas. As seguintes condições devem ser levadas em consideração, uma vez que regulamenta o funcionamento do critério (4.3.2):

$$
\begin{aligned}
& \dot{\mathrm{W}} \geq 0 \\
& \Phi \dot{\mathrm{W}}=0 .
\end{aligned}
$$




\section{CAPÍTULO 5}

\section{FORMULAÇÃO DO MEC PARA PROBLEMAS DE FRATURAMENTO - TEORIA DAS TENSÕES INICIAIS}

\section{1 - INTRODUÇÃO}

Uma das características positivas do Método dos Elementos de Contorno é a representação adequada de problemas com gradientes altos em corpos elásticos com precisão, como em fraturamento, onde há pontos com alta concentração de tensões.

No presente trabalho utiliza-se uma formulação do Método dos Elementos de Contorno para a análise de fraturas coesivas, utilizando os conceitos apresentados em VENTURINI (1994a, 1995) e LOPES Jr. (1996), estendidos para problemas tridimensionais. O modelo coesivo idealizado por HILLERBORG et al. (1976) é incorporado à formulação.

Neste capítulo é apresentada uma formulação do Método dos Elementos de Contorno (MEC) para análise de fratura mecânica em sólidos tridimensionais, utilizando o modelo coesivo. A partir da imposição de campos de tensões iniciais, formulados através da definição de dipolos, são modelados os efeitos de separação entre as superfícies da fratura. São calculados deslocamentos e tensões para o corpo sem fraturamento, da maneira usual do MEC, e, em seguida, corrigidos através dos efeitos da distribuição dos dipolos, representando o corpo fraturado. Para a discretização do contorno do sólido são utilizados os elementos triangulares planos 
com aproximação linear - contínuos e descontínuos. São inseridos elementos descontínuos à medida que a trinca se propaga. Apresentam-se, ainda, exemplos processados para mostrar a precisão da formulação.

\section{2 - EQUAÇÕES GOVERNANTES}

Considere-se que um sólido elástico isotrópico esteja submetido a um estado de tensão, igual ao que foi apresentado no capítulo 2, e, ainda, submetido a um campo de tensões iniciais. Admite-se que este campo de tensões tenha provocado deformações, denotadas por deformações iniciais.

No intuito de tentar visualizar o comportamento do sólido na presença de deformações iniciais, toma-se o exemplo da Teoria da Plasticidade, mostrado na figura 5.2.1.

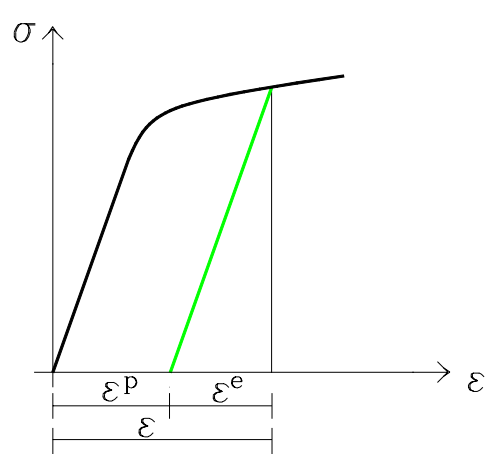

FIGURA 5.2.1 - Modelo Elastoplástico - parcelas de deformação.

A teoria estabelece que a deformação total $\varepsilon$ pode ser entendida como a soma das parcelas elástica $\varepsilon^{\mathrm{e}}$ e plástica $\varepsilon^{\mathrm{p}}$, ou seja,

$$
\varepsilon=\varepsilon^{\mathrm{e}}+\varepsilon^{\mathrm{p}}
$$

Como a natureza da deformação plástica, ou permanente, é a mesma da deformação inicial, pode-se assumir que 


$$
\varepsilon_{\mathrm{ij}}=\varepsilon_{\mathrm{ij}}^{\mathrm{e}}+\varepsilon_{\mathrm{ij}}^{0}
$$

onde $\varepsilon_{\mathrm{ij}}$ representa o tensor da deformação total,

$\varepsilon_{\mathrm{ij}}^{\mathrm{e}}$ representa o tensor da deformação elástica e

$\varepsilon_{\mathrm{ij}}^{0}$ representa o tensor da deformação inicial.

A relação entre tensão e deformação (2.3.5), a lei de Hooke, sofre modificações uma vez que utilizam-se as deformações elásticas obtidas a partir da diferença entre as deformações total e inicial. Assim, para um material elástico, temse

$$
\sigma_{\mathrm{ij}}=\lambda \delta_{\mathrm{ij}}\left(\varepsilon_{\mathrm{kk}}-\varepsilon_{\mathrm{kk}}^{0}\right)+2 \mathrm{G}\left(\varepsilon_{\mathrm{ij}}-\varepsilon_{\mathrm{ij}}^{0}\right)
$$

ou

$$
\sigma_{\mathrm{ij}}=\lambda \delta_{\mathrm{ij}} \varepsilon_{\mathrm{kk}}+2 \mathrm{G} \varepsilon_{\mathrm{ij}}-\sigma_{\mathrm{ij}}^{0}
$$

onde

$$
\sigma_{\mathrm{ij}}^{0}=\lambda \delta_{\mathrm{ij}} \varepsilon_{\mathrm{kk}}^{0}+2 \mathrm{G} \varepsilon_{\mathrm{ij}}^{0} .
$$

A lei de Hooke (5.2.4) em termos de deslocamentos, considerando as tensões iniciais, equivale a

$$
\sigma_{i j}=\lambda \delta_{i j} u_{k, k}+2 G\left(u_{i, j}+u_{j, i}\right)-\sigma_{i j}^{0} .
$$

A equação de equilíbrio de Navier, em termos de deslocamentos, envolvendo tensões iniciais, é 


$$
u_{i, j j}+\frac{1}{1-2 v} u_{j, i j}+\frac{1}{G} b_{i}-\frac{1}{G} \sigma_{i j, j}^{0}=0 .
$$

Estabelecidas as equações constituintes do material, Lei de Hooke e equação de Navier, a formulação fica definida no tocante às equações governantes

\section{3 - EQUAÇÕES INTEGRAIS}

A formulação empregada baseia-se na equação Somigliana (2.5.1), acrescentando-se um novo termo correspondente a um campo de tensões iniciais aplicado no domínio, o que se traduz em:

$$
\mathrm{u}_{\mathrm{i}}+\int_{\Gamma} \mathrm{p}_{\mathrm{ik}}^{*} \mathrm{u}_{\mathrm{k}} \mathrm{d} \Gamma=\int_{\Gamma} \mathrm{u}_{\mathrm{ik}}^{*} \mathrm{p}_{\mathrm{k}} \mathrm{d} \Gamma+\int_{\Omega} \mathrm{u}_{\mathrm{ik}}^{*} \mathrm{~b}_{\mathrm{k}} \mathrm{d} \Omega+\int_{\Omega_{\mathrm{c}}} \varepsilon_{\mathrm{ijk}}^{*} \sigma_{\mathrm{jk}}^{\mathrm{o}} \mathrm{d} \Omega
$$

A inclusão de um termo é válida uma vez que a representação integral é sempre escrita para um meio infinito, ficando os limites de determinado sólido definidos pelas condições de contorno a ele impostas. Portanto, vale a superposição de efeitos.

De outra forma, a equação integral (5.3.1), válida também para o contorno, sem as forças volumétricas, apresenta-se como

$$
\mathrm{c}_{\mathrm{ik}} \mathrm{u}_{\mathrm{k}}+\int_{\Gamma} \mathrm{p}_{\mathrm{ik}}^{*} \mathrm{u}_{\mathrm{k}} \mathrm{d} \Gamma=\int_{\Gamma} \mathrm{u}_{\mathrm{ik}}^{*} \mathrm{p}_{\mathrm{k}} \mathrm{d} \Gamma+\int_{\Omega_{\mathrm{c}}} \varepsilon_{\mathrm{ijk}}^{*} \sigma_{\mathrm{jk}}^{\mathrm{o}} \mathrm{d} \Omega
$$

Excetuando-se a parcela correspondente às tensões iniciais em (5.3.2), as integrais já foram devidamente resolvidas nos capítulos anteriores. Portanto, todos os procedimentos apresentados daqui por diante serão referentes à nova parcela.

Passando-se a trabalhar com deslocamentos e não com deformações, tem-se:

$$
\varepsilon_{\mathrm{ijk}}^{*} \sigma_{\mathrm{jk}}^{\mathrm{o}}=\mathrm{u}_{\mathrm{ij}, \mathrm{k}}^{*} \sigma_{\mathrm{jk}}^{\mathrm{o}} \text {. }
$$


Considere-se uma sub-região estreita $\Omega_{\mathrm{c}}$ (figura 5.3.1), representando uma área onde são aplicadas as tensões iniciais, sendo transformada em superfícies de descontinuidade. Note-se que o contorno $\Gamma_{\mathrm{c}}$ dessa região de tensões iniciais ou de descontinuidade pode ser dividida em duas partes, $\Gamma_{c}{ }^{1}$ e $\Gamma_{c}{ }^{2}$, desconsiderando-se as extremidades da mesma. Assume-se que a espessura 2a é muito pequena em comparação com o comprimento dessa faixa estreita. Assim, a integral sobre $\Gamma_{\mathrm{c}}$, que aparece na equação 5.3.2, pode ser aproximada levando-se em conta somente as integrais sobre as superfícies independentes $\Gamma_{\mathrm{c}}{ }^{1} \mathrm{e} \Gamma_{\mathrm{c}}{ }^{2}$.

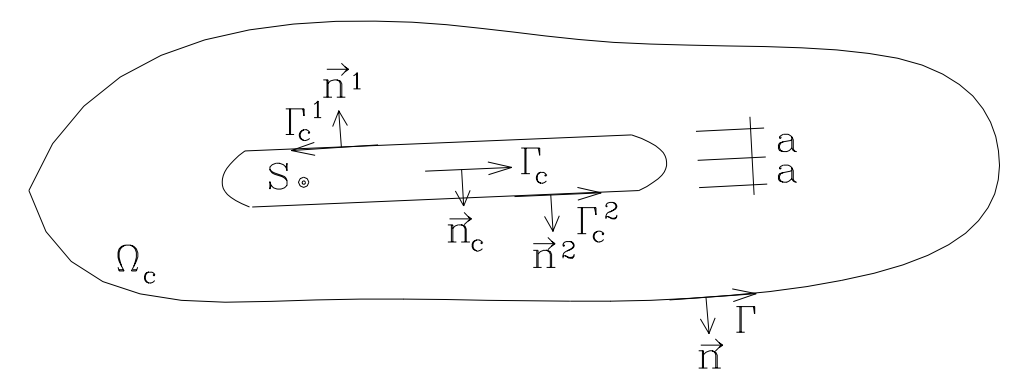

FIGURA 5.3.1 - Definição da descontinuidade no meio (fratura).

Para o desenvolvimento algébrico que será aqui utilizado, consideram-se os sistemas de coordenadas global e local apresentados na figura 5.3.2:

FIGURA 5.3.2 - Coordenadas globais e locais para a superfície de fraturamento. 
A parcela integral de (5.3.1) correspondente às tensões iniciais passa a ser escrita utilizando-se a derivada do tensor de deslocamentos, (5.3.3), ou seja,

$$
\int_{\Omega_{\mathrm{c}}} \varepsilon_{\mathrm{ijk}}^{*} \sigma_{\mathrm{jk}}^{\mathrm{o}} \mathrm{d} \Omega=\int_{\Omega_{\mathrm{c}}} \frac{\partial \mathrm{u}_{\mathrm{ij}}^{*}}{\partial \mathrm{x}_{\mathrm{k}}} \sigma_{\mathrm{jk}}^{\mathrm{o}} \mathrm{d} \Omega .
$$

Para transformar esta parcela integral de domínio para o contorno, reduzindo sua singularidade, utiliza-se a técnica da integração por partes que, aplicada a (5.3.4), resulta em:

$$
\int_{\Omega_{\mathrm{c}}} \frac{\partial \mathrm{u}_{\mathrm{ij}}^{*}}{\partial \mathrm{x}_{\mathrm{k}}} \sigma_{\mathrm{jk}}^{\mathrm{o}} \mathrm{d} \Omega=\int_{\Gamma_{\mathrm{c}}} \mathrm{u}_{\mathrm{ij}}^{*} \sigma_{\mathrm{jk}}^{\mathrm{o}} \mathrm{n}_{\mathrm{k}} \mathrm{d} \Gamma-\int_{\Omega_{\mathrm{c}}} \mathrm{u}_{\mathrm{ij}}^{*} \sigma_{\mathrm{jk}, \mathrm{k}}^{\mathrm{o}} \mathrm{d} \Omega .
$$

Considere-se a variável $\mathrm{p}_{\mathrm{j}}^{\mathrm{o}}$ que representa as forças de superfície relacionadas às tensões iniciais atuantes na faixa estreita $\Omega_{\mathrm{c}}$. Pode-se substituir o valor destas forças em (5.3.5), sabendo-se que $p_{j}^{o}=\sigma_{j k}^{o} n_{k}$, resultando em

$$
\int_{\Omega_{\mathrm{c}}} \frac{\partial \mathrm{u}_{\mathrm{ij}}^{*}}{\partial \mathrm{x}_{\mathrm{k}}} \sigma_{\mathrm{jk}}^{\mathrm{o}} \mathrm{d} \Omega=\int_{\Gamma_{\mathrm{c}}} \mathrm{u}_{\mathrm{ij}}^{*} \mathrm{p}_{\mathrm{j}}^{\mathrm{o}} \mathrm{d} \Gamma-\int_{\Omega_{\mathrm{c}}} \mathrm{u}_{\mathrm{ij}}^{*} \sigma_{\mathrm{jk}, \mathrm{k}}^{\mathrm{o}} \mathrm{d} \Omega .
$$

Passa-se a tratar cada uma das parcelas integrais dadas em (5.3.6) isoladamente. Primeiramente, tendo em vista a figura 5.3.1, desenvolve-se a parcela integral sobre o contorno (no caso, contorno da fratura).

Define-se por "a" a semi-largura da faixa estreita que representa a fratura, cujo valor é muito menor que a de seu comprimento, e as superfícies da sub-região por $\mathrm{S}+\mathrm{a}$ e $\mathrm{S}-\mathrm{a}$, sendo $\mathrm{S}$ um ponto deste contorno particular.

Pode-se, agora, escrever a primeira integral de (5.3.6) - parcela sobre o contorno $\Gamma_{\mathrm{c}}$ - admitindo-se a existência das duas superfícies que definem a fratura. Assim, 


$$
\int_{\Gamma_{\mathrm{c}}} \mathrm{u}_{\mathrm{ij}}^{*} \mathrm{p}_{\mathrm{j}}^{\mathrm{o}} \mathrm{d} \Gamma=\int_{\Gamma_{\mathrm{c}}^{1}} \mathrm{u}_{\mathrm{ij}}^{*}(\mathrm{p}, \mathrm{S}-\mathrm{a}) \mathrm{p}_{\mathrm{j}}^{\mathrm{o}}(\mathrm{S}-\mathrm{a}) \mathrm{d} \Gamma+\int_{\Gamma_{\mathrm{c}}^{2}} \mathrm{u}_{\mathrm{ij}}^{*}(\mathrm{p}, \mathrm{S}+\mathrm{a}) \mathrm{p}_{\mathrm{j}}^{\mathrm{o}}(\mathrm{S}+\mathrm{a}) \mathrm{d} \Gamma
$$

Adota-se a direção do eixo coordenado local $\mathrm{x}_{3}$ (figura 5.3.2) normal à fratura. Assumindo-se que as superfícies da fratura $\Gamma_{\mathrm{c}}^{1}$ e $\Gamma_{\mathrm{c}}^{2}$ estão bem próximas, pode-se escrever as seguintes expressões:

$$
\begin{aligned}
& u_{i j}^{*}(p, S+a)=u_{i j}^{*}(p, S)+\frac{\partial u_{i j}^{*}}{\partial x_{3}}(p, S) a \\
& u_{i j}^{*}(p, S-a)=u_{i j}^{*}(p, S)-\frac{\partial u_{i j}^{*}}{\partial x_{3}}(p, S) a
\end{aligned}
$$

Levando-se (5.3.8) para (5.3.7), chega-se a:

$$
\begin{aligned}
\int_{\Gamma_{\mathrm{c}}} \mathrm{u}_{\mathrm{ij}}^{*} \mathrm{p}_{\mathrm{j}}^{\mathrm{o}} \mathrm{d} \Gamma & =\int_{-\Gamma_{\mathrm{c}}}\left[\mathrm{u}_{\mathrm{ij}}^{*}(\mathrm{p}, \mathrm{S})-\frac{\partial \mathrm{u}_{\mathrm{ij}}^{*}}{\partial \mathrm{x}_{3}}(\mathrm{p}, \mathrm{S}) \mathrm{a}\right] \mathrm{p}_{\mathrm{j}}^{\mathrm{o} 3}(\mathrm{~S}) \mathrm{d} \Gamma+ \\
& +\int_{\Gamma_{\mathrm{c}}}\left[\mathrm{u}_{\mathrm{ij}}^{*}(\mathrm{p}, \mathrm{S})+\frac{\partial \mathrm{u}_{\mathrm{ij}}^{*}}{\partial \mathrm{x}_{3}}(\mathrm{p}, \mathrm{S}) \mathrm{a}\right] \mathrm{p}_{\mathrm{j}}^{\mathrm{o} 3}(\mathrm{~S}) \mathrm{d} \Gamma
\end{aligned}
$$

ou, resumidamente,

$$
\int_{\Gamma_{\mathrm{c}}} \mathrm{u}_{\mathrm{ij}}^{*} \mathrm{p}_{\mathrm{j}}^{\mathrm{o}} \mathrm{d} \Gamma=\int_{\Gamma_{\mathrm{c}}} 2 \mathrm{a} \frac{\partial \mathrm{u}_{\mathrm{ij}}^{*}}{\partial \mathrm{x}_{3}} \mathrm{p}_{\mathrm{j}}^{\mathrm{o} 3} \mathrm{~d} \Gamma .
$$

Portanto, com a integral (5.3.10) chega-se na influência da componente normal de tensão sobre a fratura.

A parcela de domínio em (5.3.6) tem, ainda, que ser desenvolvida. Explicitando-se esta parcela e já substituindo o valor da diferencial de volume, temse: 


$$
\int_{\Omega_{\mathrm{c}}} \mathrm{u}_{\mathrm{ij}}^{*} \sigma_{\mathrm{jk}, \mathrm{k}}^{\mathrm{o}} \mathrm{d} \Omega=\int_{\Omega_{\mathrm{c}}} \mathrm{u}_{\mathrm{ij}}^{*} \sigma_{\mathrm{jk}, \mathrm{k}}^{\mathrm{o}} \mathrm{dx} \mathrm{x}_{1} \mathrm{dx} \mathrm{dx}_{3}
$$

O termo das derivadas das tensões iniciais deve ser desenvolvido, pois a sua definição utiliza sistema de coordenadas globais e (5.3.11) o sistema local, ou seja,

$$
\sigma_{\mathrm{jk}, \mathrm{k}}^{\mathrm{o}}=\frac{\partial \sigma_{\mathrm{jk}}^{\mathrm{o}}}{\partial \mathrm{X}_{\mathrm{k}}}=\frac{\partial \sigma_{\mathrm{j} 1}^{\mathrm{o}}}{\partial \mathrm{X}_{1}}+\frac{\partial \sigma_{\mathrm{j} 2}^{\mathrm{o}}}{\partial \mathrm{X}_{2}}+\frac{\partial \sigma_{\mathrm{j} 3}^{\mathrm{o}}}{\partial \mathrm{X}_{3}} .
$$

Utilizando-se a regra da cadeia e levando-se em conta que as derivadas na direção normal à fratura $\left(\mathrm{x}_{3}\right)$ são nulas, pela consideração de que $\sigma_{\mathrm{jk}}^{\mathrm{o}}$ é constante, (5.3.12) passa a ser:

$$
\begin{aligned}
\sigma_{\mathrm{jk}, \mathrm{k}}^{\mathrm{o}}= & \frac{\partial \sigma_{\mathrm{j} 1}^{\mathrm{o}}}{\partial \mathrm{x}_{1}} \frac{\partial \mathrm{x}_{1}}{\partial \mathrm{X}_{1}}+\frac{\partial \sigma_{\mathrm{j} 1}^{\mathrm{o}}}{\partial \mathrm{x}_{2}} \frac{\partial \mathrm{x}_{2}}{\partial \mathrm{X}_{1}}+ \\
& \frac{\partial \sigma_{\mathrm{j} 2}^{\mathrm{o}}}{\partial \mathrm{x}_{1}} \frac{\partial \mathrm{x}_{1}}{\partial \mathrm{X}_{2}}+\frac{\partial \sigma_{\mathrm{j} 2}^{\mathrm{o}}}{\partial \mathrm{x}_{2}} \frac{\partial \mathrm{x}_{2}}{\partial \mathrm{X}_{2}}+ \\
& \frac{\partial \sigma_{\mathrm{j} 3}^{\mathrm{o}}}{\partial \mathrm{x}_{1}} \frac{\partial \mathrm{x}_{1}}{\partial \mathrm{X}_{3}}+\frac{\partial \sigma_{\mathrm{j} 3}^{\mathrm{o}}}{\partial \mathrm{x}_{2}} \frac{\partial \mathrm{x}_{2}}{\partial \mathrm{X}_{3}}
\end{aligned}
$$

Utilizando-se os co-senos diretores definidos na figura 5.3.1, tem-se

$$
\begin{aligned}
\sigma_{\mathrm{jk}, \mathrm{k}}^{\mathrm{o}}= & \frac{\partial \sigma_{\mathrm{j} 1}^{\mathrm{o}}}{\partial \mathrm{x}_{1}} \mathrm{~m}_{1}+\frac{\partial \sigma_{\mathrm{j} 1}^{\mathrm{o}}}{\partial \mathrm{x}_{2}} \mathrm{~s}_{1}+ \\
& \frac{\partial \sigma_{\mathrm{j} 2}^{\mathrm{o}}}{\partial \mathrm{x}_{1}} \mathrm{~m}_{2}+\frac{\partial \sigma_{\mathrm{j} 2}^{\mathrm{o}}}{\partial \mathrm{x}_{2}} \mathrm{~s}_{2}+ \\
& \frac{\partial \sigma_{\mathrm{j} 3}^{\mathrm{o}}}{\partial \mathrm{x}_{1}} \mathrm{~m}_{3}+\frac{\partial \sigma_{\mathrm{j} 3}^{\mathrm{o}}}{\partial \mathrm{x}_{2}} \mathrm{~s}_{3}
\end{aligned}
$$

ou, resumidamente,

$$
\sigma_{j k, k}^{o}=\frac{\partial}{\partial x_{1}}\left(\sigma_{j k}^{o} m_{k}\right)+\frac{\partial}{\partial x_{2}}\left(\sigma_{j k}^{o} s_{k}\right)
$$


onde as componentes das tensões iniciais já encontram-se escritas em função das coordenadas locais.

Substituindo-se o resultado de (5.3.15) na expressão (5.3.11), esta passa a se apresentar da seguinte forma:

$$
\int_{\Omega_{\mathrm{c}}} \mathrm{u}_{\mathrm{ij}}^{*} \sigma_{\mathrm{jk}, \mathrm{k}}^{\mathrm{o}} \mathrm{d} \Omega=\int_{\Omega_{\mathrm{c}}} \mathrm{u}_{\mathrm{ij}}^{*}\left[\frac{\partial}{\partial \mathrm{x}_{1}}\left(\sigma_{\mathrm{jk}}^{\mathrm{o}} \mathrm{m}_{\mathrm{k}}\right)+\frac{\partial}{\partial \mathrm{x}_{2}}\left(\sigma_{\mathrm{jk}}^{\mathrm{o}} \mathrm{s}_{\mathrm{k}}\right)\right] \mathrm{dx_{1 }} \mathrm{d} \mathrm{x}_{2} \mathrm{dx} \mathrm{x}_{3} .
$$

Admitindo-se que não há variações na distância entre as superfícies da fratura, na sua direção normal, e que a integral correspondente a diferencial $\mathrm{dx}_{3}$ é igual a 2a, a integral (5.3.16) passa a ser:

$$
\int_{\Omega_{\mathrm{c}}} \mathrm{u}_{\mathrm{ij}}^{*} \sigma_{\mathrm{jk}, \mathrm{k}}^{\mathrm{o}} \mathrm{d} \Omega=\iint_{\Gamma_{\mathrm{c}}} \mathrm{u}_{\mathrm{ij}}^{*} \frac{\partial}{\partial \mathrm{x}_{1}}\left(\sigma_{\mathrm{jk}}^{\mathrm{o}} \mathrm{m}_{\mathrm{k}}\right) 2 \mathrm{adx}_{1} \mathrm{dx} \mathrm{x}_{2}+\iint_{\Gamma_{\mathrm{c}}} \mathrm{u}_{\mathrm{ij}}^{*} \frac{\partial}{\partial \mathrm{x}_{2}}\left(\sigma_{\mathrm{jk}}^{\mathrm{o}} \mathrm{s}_{\mathrm{k}}\right) 2 \mathrm{adx}_{1} \mathrm{dx} \mathrm{x}_{2} .
$$

Obtém-se, neste ponto, a transformação da parcela de domínio para o contorno.

Integrando-se por partes os dois termos integrais do lado direito da igualdade em (5.3.17), tem-se

$$
\begin{aligned}
& \iint_{\Gamma_{\mathrm{c}}} \mathrm{u}_{\mathrm{ij}}^{*} \frac{\partial}{\partial \mathrm{x}_{1}}\left(\sigma_{\mathrm{jk}}^{\mathrm{o}} \mathrm{m}_{\mathrm{k}}\right) 2 \mathrm{adx}_{1} \mathrm{dx} \mathrm{x}_{2}=\mathrm{u}_{\mathrm{ij}}^{*} \sigma_{\mathrm{jk}}^{\mathrm{o}} \mathrm{m}_{\mathrm{k}} 2 \mathrm{a}\left(\mathrm{x}_{1}^{2}-\mathrm{x}_{1}^{1}\right)-\int_{\Gamma_{\mathrm{c}}} \frac{\partial \mathrm{u}_{\mathrm{ij}}^{*}}{\partial \mathrm{x}_{1}} \sigma_{\mathrm{jk}}^{\mathrm{o}} \mathrm{m}_{\mathrm{k}} 2 \mathrm{ad} \Gamma \\
& \iint_{\Gamma_{\mathrm{c}}} \mathrm{u}_{\mathrm{ij}}^{*} \frac{\partial}{\partial \mathrm{x}_{2}}\left(\sigma_{\mathrm{jk}}^{\mathrm{o}} \mathrm{s}_{\mathrm{k}}\right) 2 \mathrm{adx}_{1} \mathrm{dx} \mathrm{x}_{2}=\mathrm{u}_{\mathrm{ij}}^{*} \sigma_{\mathrm{jk}}^{\mathrm{o}} \mathrm{m}_{\mathrm{k}} 2 \mathrm{a}\left(\mathrm{x}_{2}^{2}-\mathrm{x}_{2}^{1}\right)-\int_{\Gamma_{\mathrm{c}}} \frac{\partial \mathrm{u}_{\mathrm{ij}}^{*}}{\partial \mathrm{x}_{2}} \sigma_{\mathrm{jk}}^{\mathrm{o}} \mathrm{s}_{\mathrm{k}} 2 \mathrm{ad} \Gamma .
\end{aligned}
$$

Os primeiros termos de (5.3.18) e (5.3.19) após a igualdade tendem a zero quando a abertura da fratura, $2 \mathrm{a}$, tende a zero. Assim, (5.3.17) passa a ser

$$
\int_{\Omega_{\mathrm{c}}} \mathrm{u}_{\mathrm{ij}}^{*} \sigma_{\mathrm{jk}, \mathrm{k}}^{\mathrm{o}} \mathrm{d} \Omega=-\int_{\Gamma_{\mathrm{c}}} \frac{\partial \mathrm{u}_{\mathrm{ij}}^{*}}{\partial \mathrm{x}_{1}} \sigma_{\mathrm{jk}}^{\mathrm{o}} \mathrm{m}_{\mathrm{k}} 2 \mathrm{ad} \Gamma-\int_{\Gamma_{\mathrm{c}}} \frac{\partial \mathrm{u}_{\mathrm{ij}}^{*}}{\partial \mathrm{x}_{2}} \sigma_{\mathrm{jk}}^{\mathrm{o}} \mathrm{s}_{\mathrm{k}} 2 \mathrm{ad} \Gamma .
$$


Utilizando-se, novamente, as componentes das forças de superfície na fratura, agora para as direções dos eixos $x_{1}$ e $x_{2}\left(p_{j}^{o 1}=\sigma_{j k}^{o} m_{k}\right.$ e $\left.p_{j}^{o 2}=\sigma_{j k}^{o} s_{k}\right)$, a integral (5.3.20) torna-se

$$
\int_{\Omega_{\mathrm{c}}} \mathrm{u}_{\mathrm{ij}}^{*} \sigma_{\mathrm{jk}, \mathrm{k}}^{\mathrm{o}} \mathrm{d} \Omega=-\int_{\Gamma_{\mathrm{c}}} 2 \mathrm{a} \frac{\partial \mathrm{u}_{\mathrm{ij}}^{*}}{\partial \mathrm{x}_{1}} \mathrm{p}_{\mathrm{j}}^{\mathrm{ol}} \mathrm{d} \Gamma-\int_{\Gamma_{\mathrm{c}}} 2 \mathrm{a} \frac{\partial \mathrm{u}_{\mathrm{ij}}^{*}}{\partial \mathrm{x}_{2}} \mathrm{p}_{\mathrm{j}}^{\mathrm{o} 2} \mathrm{~d} \Gamma,
$$

e representa a influência das componentes tangenciais de tensão sobre a fratura.

As duas parcelas integrais de (5.3.6) encontram-se determinadas em (5.3.10) e (5.3.21). Portanto, a parcela relativa às tensões iniciais em (5.3.2) fica devidamente resolvida, apresentando como resultado

$$
\int_{\Omega_{\mathrm{c}}} \varepsilon_{\mathrm{ijk}}^{*} \sigma_{\mathrm{jk}}^{\mathrm{o}} \mathrm{d} \Omega=\int_{\Gamma_{\mathrm{c}}} 2 \mathrm{a} \frac{\partial \mathrm{u}_{\mathrm{ij}}^{*}}{\partial \mathrm{x}_{1}} \mathrm{p}_{\mathrm{j}}^{\mathrm{o1}} \mathrm{d} \Gamma+\int_{\Gamma_{\mathrm{c}}} 2 \mathrm{a} \frac{\partial \mathrm{u}_{\mathrm{ij}}^{*}}{\partial \mathrm{x}_{2}} \mathrm{p}_{\mathrm{j}}^{\mathrm{o} 2} \mathrm{~d} \Gamma+\int_{\Gamma_{\mathrm{c}}} 2 \mathrm{a} \frac{\partial \mathrm{u}_{\mathrm{ij}}^{*}}{\partial \mathrm{x}_{3}} \mathrm{p}_{\mathrm{j}}^{\mathrm{o} 3} \mathrm{~d} \Gamma
$$

ou, resumidamente,

$$
\int_{\Omega_{\mathrm{c}}} \varepsilon_{\mathrm{ijk}}^{*} \sigma_{\mathrm{jk}}^{\mathrm{o}} \mathrm{d} \Omega=\int_{\Gamma_{\mathrm{c}}} 2 \mathrm{a} \frac{\partial \mathrm{u}_{\mathrm{ij}}^{*}}{\partial \mathrm{x}_{\ell}} \mathrm{p}_{\mathrm{j}}^{\mathrm{o} \ell} \mathrm{d} \Gamma
$$

As manipulações algébricas realizadas até chegar a (5.3.23), são efetuadas tendo como sistema de referência os eixos $\mathrm{x}_{1}, \mathrm{x}_{2}$ e $\mathrm{x}_{3}$, ou seja, o sistema local de coordenadas (relativo à superfície da fratura). Esse pode variar em relação ao referencial global à medida que a trinca vai se propagando. É necessário, portanto, obter a resposta algébrica em função das coordenadas globais $X_{1}, X_{2}$ e $X_{3}$. A transformação é feita como se segue: 


$$
\begin{aligned}
\frac{\partial \mathrm{u}_{\mathrm{ij}}^{*}}{\partial \mathrm{x}_{\ell}} \mathrm{p}_{\mathrm{j}}^{\mathrm{\ell}} & =\frac{\partial \mathrm{u}_{\mathrm{ij}}^{*}}{\partial \mathrm{X}_{\mathrm{m}}} \frac{\partial \mathrm{X}_{\mathrm{m}}}{\partial \mathrm{x}_{\ell}} \mathrm{p}_{\mathrm{j}}^{\mathrm{o} \ell} \\
& =\frac{\partial \mathrm{u}_{\mathrm{ij}}^{*}}{\partial \mathrm{X}_{1}} \frac{\partial \mathrm{X}_{1}}{\partial \mathrm{x}_{\ell}} \mathrm{p}_{\mathrm{j}}^{\mathrm{o} \ell}+\frac{\partial \mathrm{u}_{\mathrm{ij}}^{*}}{\partial \mathrm{X}_{2}} \frac{\partial \mathrm{X}_{2}}{\partial \mathrm{x}_{\ell}} \mathrm{p}_{\mathrm{j}}^{\mathrm{o} \ell}+\frac{\partial \mathrm{u}_{\mathrm{ij}}^{*}}{\partial \mathrm{X}_{3}} \frac{\partial \mathrm{X}_{3}}{\partial \mathrm{x}_{\ell}} \mathrm{p}_{\mathrm{j}}^{\mathrm{o} \ell},
\end{aligned}
$$

onde aparecem os novos co-senos diretores que relacionam os sistemas global e local de coordenadas, representados por:

$$
\begin{gathered}
\overline{\mathrm{m}}_{\ell}=\frac{\partial \mathrm{X}_{1}}{\partial \mathrm{x}_{\ell}} \\
\overline{\mathrm{s}}_{\ell}=\frac{\partial \mathrm{X}_{2}}{\partial \mathrm{x}_{\ell}} \\
\overline{\mathrm{n}}_{\ell}=\frac{\partial \mathrm{X}_{3}}{\partial \mathrm{x}_{\ell}}
\end{gathered}
$$

Portanto, pode-se determinar um tensor das forças de superfície aplicadas na fratura, escrito nas coordenadas globais, utilizando-se uma matriz de transformação, ou seja,

$$
\overline{\mathrm{p}}_{\mathrm{j}}^{\mathrm{om}}=\mathrm{T}_{\mathrm{m} \ell} \mathrm{p}_{\mathrm{j}}^{\mathrm{o} \ell}
$$

onde

$$
\mathrm{T}_{\mathrm{m} \ell}=\left[\begin{array}{ccc}
\overline{\mathrm{m}}_{1} & \overline{\mathrm{m}}_{2} & \overline{\mathrm{m}}_{3} \\
\overline{\mathrm{s}}_{1} & \overline{\mathrm{s}}_{2} & \overline{\mathrm{s}}_{3} \\
\overline{\mathrm{n}}_{1} & \overline{\mathrm{n}}_{2} & \overline{\mathrm{n}}_{3}
\end{array}\right] \quad \text { e } \quad \mathrm{p}_{\mathrm{j}}^{\mathrm{o} \ell}=\left\{\begin{array}{l}
\mathrm{p}_{\mathrm{j}}^{\mathrm{o} 1} \\
\mathrm{p}_{\mathrm{j}}^{\mathrm{o} 2} \\
\mathrm{p}_{\mathrm{j}}^{\mathrm{o} 3}
\end{array}\right\}
$$

A expressão (5.3.24) passa a ser escrita na forma:

$$
\frac{\partial \mathrm{u}_{\mathrm{ij}}^{*}}{\partial \mathrm{x}_{\ell}} \mathrm{p}_{\mathrm{j}}^{\mathrm{o} \ell}=\frac{\partial \mathrm{u}_{\mathrm{ij}}^{*}}{\partial \mathrm{X}_{\mathrm{m}}} \overline{\mathrm{p}}_{\mathrm{j}}^{\mathrm{om}} .
$$


Pode-se, então, escrever (5.3.23) em função das coordenadas globais:

$$
\int_{\Omega} \varepsilon_{\mathrm{ijk}}^{*} \sigma_{\mathrm{jk}}^{\mathrm{o}} \mathrm{d} \Omega=\int_{\Gamma_{\mathrm{c}}} 2 \mathrm{a} \frac{\partial \mathrm{u}_{\mathrm{ij}}^{*}}{\partial \mathrm{X}_{\ell}^{\mathrm{p}}} \overline{\mathrm{p}}_{\mathrm{j}}^{\mathrm{\ell}} \mathrm{d} \Gamma .
$$

As deduções realizadas até aqui, baseiam-se na definição da faixa estreita de inclusão da descontinuidade (fratura), mostrada na figura 5.3.1. Quando a sua espessura tende para zero, são necessárias tensões iniciais de valores infinitos devido à natureza do problema abordado. Assim, para se escrever o termo integral correspondente às tensões iniciais, no qual é possível assumir que a espessura da faixa é igual a zero, é necessário definir um novo tensor, denominado "dipolo" de forças, representado por:

$$
\mathrm{q}_{\mathrm{j}}^{\ell}=2 \mathrm{a} \overline{\mathrm{p}}_{\mathrm{j}}^{\mathrm{\ell}}
$$

Essas forças recebem este nome por analogia ao caso do dipolo elétrico. Uma de suas características é permitir a inclusão de um campo de tensões no meio contínuo sem apresentar uma resultante de forças.

De (5.3.29) desenvolve-se, ainda, a derivada do tensor dos deslocamentos fundamentais, representada por

$$
\mathrm{G}_{\mathrm{ij}}^{\ell}=\frac{\partial \mathrm{u}_{\mathrm{ij}}^{*}}{\partial \mathrm{X}_{\ell}}
$$

Portanto, (5.3.29) passa a ser:

$$
\int_{\Omega_{\mathrm{c}}} \varepsilon_{\mathrm{ijk}}^{*} \sigma_{\mathrm{jk}}^{\mathrm{o}} \mathrm{d} \Omega=\int_{\Gamma_{\mathrm{c}}} \mathrm{G}_{\mathrm{ij}}^{\ell} \mathrm{q}_{\mathrm{j}}^{\ell} \mathrm{d} \Gamma
$$

que é a parcela adicional na expressão Somigliana devido à presença da fratura, cuja influência é considerada através dos dipolos. Portanto, a representação dos 
deslocamentos, já levando-se em conta a contribuição das tensões iniciais concentradas em uma superfície, é

$$
\mathrm{c}_{\mathrm{ik}} \mathrm{u}_{\mathrm{i}}+\int_{\Gamma} \mathrm{p}_{\mathrm{ik}}^{*} \mathrm{u}_{\mathrm{k}} \mathrm{d} \Gamma=\int_{\Gamma} \mathrm{u}_{\mathrm{ik}}^{*} \mathrm{p}_{\mathrm{k}} \mathrm{d} \Gamma+\int_{\Gamma_{\mathrm{c}}} \mathrm{G}_{\mathrm{ij}}^{\ell} \mathrm{q}_{\mathrm{j}}^{\ell} \mathrm{d} \Gamma
$$

\section{4 - SOBRE O INTEGRANDO CORRESPONDENTE AOS DIPOLOS}

Com os índices i, j e $\ell$ variando de 1 a 3 (no espaço tridimensional), a última parcela de (5.3.33) tem suas componentes explicitadas conforme (5.4.1):

$$
\begin{aligned}
G_{i j}^{\ell} q_{j}^{\ell}= & G_{i j}^{1} q_{j}^{1}+G_{i j}^{2} q_{j}^{2}+G_{i j}^{3} q_{j}^{3} \\
G_{i j}^{\ell} q_{j}^{\ell}= & G_{i 1}^{1} q_{1}^{1}+G_{i 1}^{2} q_{1}^{2}+G_{i 1}^{3} q_{1}^{3} \\
& G_{i 2}^{1} q_{2}^{1}+G_{i 2}^{2} q_{2}^{2}+G_{i 2}^{3} q_{2}^{3} \\
& G_{i 3}^{1} q_{3}^{1}+G_{i 3}^{2} q_{3}^{2}+G_{i 3}^{3} q_{3}^{3}
\end{aligned}
$$

$\mathrm{Na}$ forma matricial, tem-se

$$
\left[\begin{array}{lllllllll}
G_{i 1}^{1} & G_{i 2}^{1} & G_{i 3}^{1} & G_{i 1}^{2} & G_{i 2}^{2} & G_{i 3}^{2} & G_{i 1}^{3} & G_{i 2}^{3} & G_{i 3}^{3}
\end{array}\right]\left\{\begin{array}{l}
q_{1}^{1} \\
q_{2}^{1} \\
q_{3}^{1} \\
q_{1}^{2} \\
q_{2}^{2} \\
q_{3}^{2} \\
q_{1}^{3} \\
q_{2}^{3} \\
q_{3}^{3}
\end{array}\right\}
$$

A definição das componentes dos dipolos é obtida considerando-se a superfície da fratura, ou seja, determinando-se as tensões neste contorno particular.

Considere-se a figura 5.4.1 mostrada a seguir: 

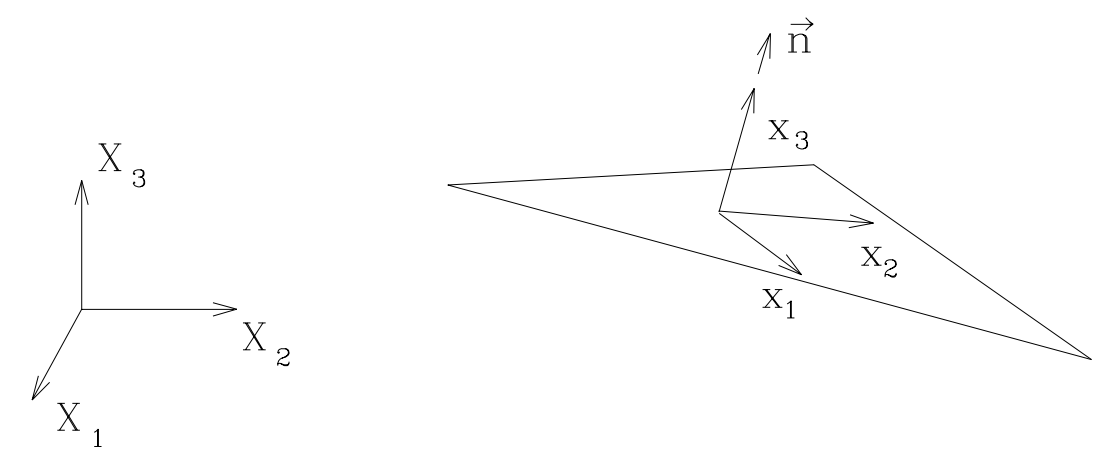

FIGURA 5.4.1 - Elemento do contorno da fratura com o seu sistema local de coordenadas e vetor normal.

$\mathrm{Na}$ forma clássica, as tensões no contorno apresentam as seguintes expressões, já apresentadas em (2.6.45a-f):

$$
\begin{aligned}
\sigma_{11} & =\frac{1}{1-v}\left[v \sigma_{33}+2 \mathrm{G}\left(\varepsilon_{11}+v \varepsilon_{22}\right)\right] \\
\sigma_{12} & =2 \mathrm{G} \varepsilon_{12} \\
\sigma_{22} & =\frac{1}{1-v}\left[v \sigma_{33}+2 \mathrm{G}\left(\varepsilon_{22}+v \varepsilon_{11}\right)\right] \\
\sigma_{13} & =p_{1} \\
\sigma_{23} & =p_{2} \\
\sigma_{33} & =p_{3}
\end{aligned}
$$

Admitindo-se que as deformações $\varepsilon_{11}$ e $\varepsilon_{22}$ são nulas no plano da fratura, chega-se em

$$
\begin{aligned}
& \sigma_{11}=\frac{v}{1-v} \sigma_{33} \\
& \sigma_{22}=\frac{v}{1-v} \sigma_{33}
\end{aligned}
$$

Considere-se a figura 5.4.2 mostrada a seguir: 


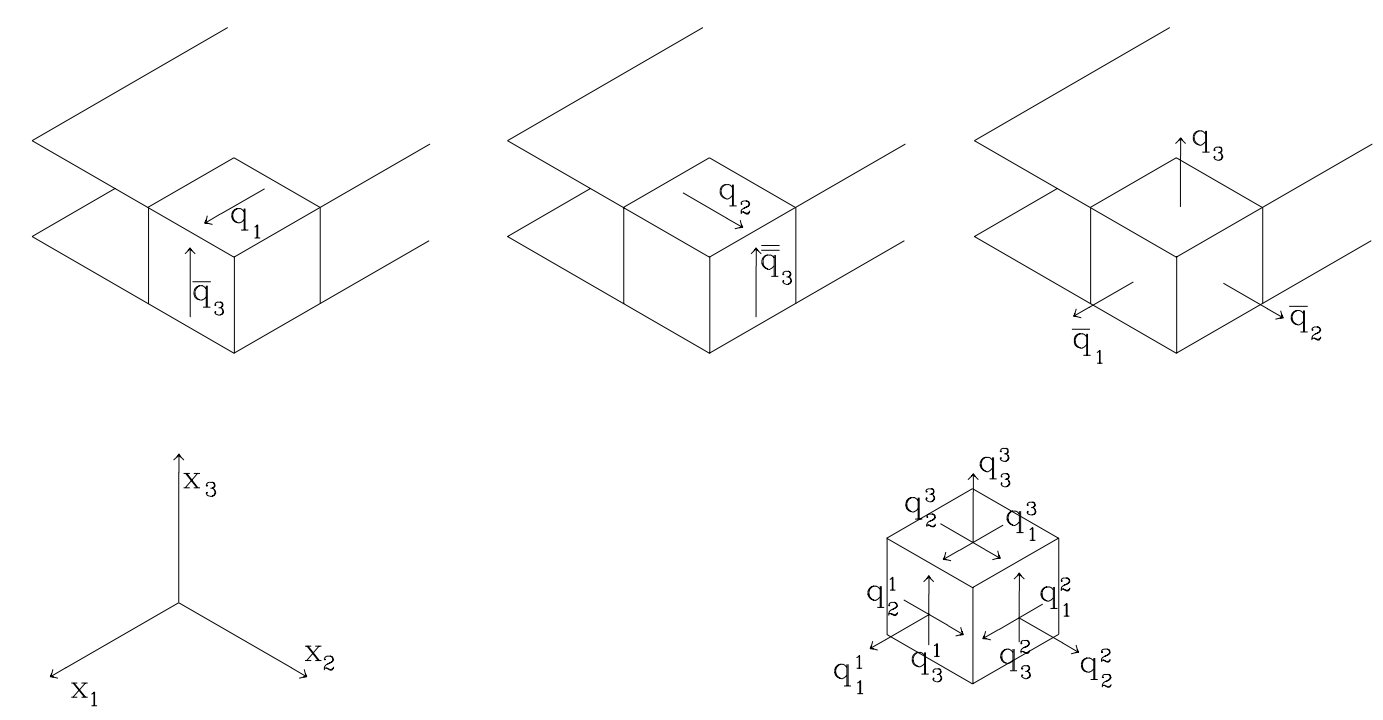

FIGURA 5.4.2 - Elemento infinitesimal no contorno da fratura com as componentes dos dipolos.

A aplicação de $\mathrm{q}_{3}$ produz "empuxo" entre os pontos de aplicação das forças, causando perturbações na distribuição de tensão, devido ao coeficiente de Poisson. Para eliminar este empuxo devido a $\mathrm{q}_{3}$, deve-se aplicar dois dipolos $\overline{\mathrm{q}}_{1}$ e $\overline{\mathrm{q}}_{2}$ tais que impeçam a deformação na direção transversal.

Relacionando-se os dipolos necessários para o equilíbrio na região das tensões iniciais com as componentes de tensão de um elemento infinitesimal, chegase aos seguintes valores:

$$
\begin{aligned}
& \mathrm{q}_{1}^{1}=\overline{\mathrm{q}}_{1} \\
& \mathrm{q}_{2}^{1}=0 \\
& \mathrm{q}_{3}^{1}=\overline{\mathrm{q}}_{3} \\
& \mathrm{q}_{1}^{2}=0 \\
& \mathrm{q}_{2}^{2}=\overline{\mathrm{q}}_{2} \\
& \mathrm{q}_{3}^{2}=\overline{\overline{\mathrm{q}}}_{3} \\
& \mathrm{q}_{1}^{3}=\mathrm{q}_{1} \\
& \mathrm{q}_{2}^{3}=\mathrm{q}_{2} \\
& \mathrm{q}_{3}^{3}=\mathrm{q}_{3}
\end{aligned}
$$


Utilizando-se os resultados de (5.4.3), (5.4.4) e (5.4.5), obtêm-se as componentes dos dipolos necessárias à análise do efeito da fratura em meio contínuo devidamente relacionadas entre si.

$$
\begin{aligned}
& \mathrm{q}_{3}^{1}=\mathrm{q}_{1}^{3} \\
& \mathrm{q}_{3}^{2}=\mathrm{q}_{2}^{3} \\
& \mathrm{q}_{1}^{1}=\frac{\mathrm{v}}{1-\mathrm{v}} \mathrm{q}_{3}^{3} \\
& \mathrm{q}_{2}^{2}=\frac{\mathrm{v}}{1-v} \mathrm{q}_{3}^{3}
\end{aligned}
$$

É possível reduzir as matrizes apresentadas em (5.4.2) utilizando-se as relações (5.4.6) encontradas para as componentes dos dipolos escritas em função das componentes independentes. Assim,

$$
\left[\mathrm{G}_{\mathrm{i} 3}^{1}+\mathrm{G}_{\mathrm{i} 1}^{3} \quad \mathrm{G}_{\mathrm{i} 3}^{2}+\mathrm{G}_{\mathrm{i} 2}^{3} \frac{v}{1-v}\left(\mathrm{G}_{\mathrm{i} 1}^{1}+\mathrm{G}_{\mathrm{i} 2}^{2}\right)+\mathrm{G}_{\mathrm{i} 3}^{3}\right]\left\{\begin{array}{l}
\mathrm{q}_{1}^{3} \\
\mathrm{q}_{2}^{3} \\
\mathrm{q}_{3}^{3}
\end{array}\right\} .
$$

Pode-se escrever (5.4.7) de forma concisa, bastando definir uma matriz de contribuição dos dipolos $[\mathrm{K}]$, o que resulta em

$$
[\mathrm{K}]\{\mathrm{q}\} \text {. }
$$

As componentes da matriz $[\mathrm{K}]$ são:

$$
\begin{aligned}
& \mathrm{K}_{\mathrm{i} 1}=\mathrm{G}_{\mathrm{i} 3}^{1}+\mathrm{G}_{\mathrm{i} 1}^{3} \\
& \mathrm{~K}_{\mathrm{i} 2}=\mathrm{G}_{\mathrm{i} 3}^{2}+\mathrm{G}_{\mathrm{i} 2}^{3} \\
& \mathrm{~K}_{\mathrm{i} 3}=\frac{\mathrm{v}}{1-\mathrm{v}}\left(\mathrm{G}_{\mathrm{i} 1}^{1}+\mathrm{G}_{\mathrm{i} 2}^{2}\right)+\mathrm{G}_{\mathrm{i} 3}^{3}
\end{aligned}
$$


Portanto, para um ponto qualquer da superfície de fratura, o integrando da última parcela integral de (5.3.33), escrito de forma matricial, é

$$
\left[\begin{array}{lll}
\mathrm{K}_{11} & \mathrm{~K}_{12} & \mathrm{~K}_{13} \\
\mathrm{~K}_{21} & \mathrm{~K}_{22} & \mathrm{~K}_{23} \\
\mathrm{~K}_{31} & \mathrm{~K}_{32} & \mathrm{~K}_{33}
\end{array}\right]\left\{\begin{array}{l}
\mathrm{q}_{1}^{3} \\
\mathrm{q}_{2}^{3} \\
\mathrm{q}_{3}^{3}
\end{array}\right\}
$$

Os valores das componentes da matriz de influência dos dipolos estão relacionados à solução fundamental utilizada, posto que suas expressões decorrem da derivação do tensor de deslocamento fundamental, conforme (5.3.31).

Escolhendo-se a solução fundamental de Kelvin, apresentada na seção 2.4.1 expressão (2.4.4) - chega-se a:

$$
\mathrm{G}_{\mathrm{ij}}^{\ell}=\frac{\partial \mathrm{u}_{\mathrm{ij}}^{*}}{\partial \mathrm{X}_{\ell}}=\frac{\partial}{\partial \mathrm{X}_{\ell}}\left\{\frac{1}{16 \pi(1-v) \mathrm{G}}\left[\frac{(3-4 v)}{\mathrm{r}} \delta_{\mathrm{ij}}+\frac{1}{\mathrm{r}} \mathrm{r}_{\mathrm{i}, \mathrm{r}} \mathrm{r}_{\mathrm{j}}\right]\right\},
$$

obtendo-se o núcleo singular

$$
\mathrm{G}_{\mathrm{ij}}^{\ell}=\frac{1}{16 \pi(1-v) \mathrm{Gr}^{2}}\left\{-(3-4 v) \mathrm{r}_{, \ell} \delta_{\mathrm{ij}}+\mathrm{r}_{, \mathrm{j}} \delta_{\ell \mathrm{i}}+\mathrm{r}_{, \mathrm{i}} \delta_{\ell \mathrm{j}}-3 \mathrm{r}_{, \mathrm{i}} \mathrm{r}_{\mathrm{j}, ~} \mathrm{r}_{, \ell}\right\}
$$

cujas deduções encontram-se no anexo F.

Por outro lado, escolhendo-se a solução fundamental de Mindlin, apresentada na seção 2.4.2 - expressão (2.4.8), tem-se mais de trabalho algébrico. Entendendo-se a solução fundamental de Mindlin como sendo formada por duas partes: a parcela de Kelvin e outra complementar, conforme apresentado em (2.4.11a), divide-se o núcleo $\mathrm{G}_{\mathrm{ij}}^{\ell}$ da seguinte forma:

$$
\left(\mathrm{G}_{\mathrm{ij}}^{\ell}\right)^{\mathrm{M}}=\left(\mathrm{G}_{\mathrm{ij}}^{\ell}\right)^{\mathrm{K}}+\left(\mathrm{G}_{\mathrm{ij}}^{\ell}\right)^{\mathrm{C}} .
$$


Uma vez que já se obteve a expressão do núcleo para Kelvin, procura-se efetuar as derivadas parciais indicadas em (5.3.31) apenas para a parte complementar. Assim,

$$
\begin{aligned}
& \left(\mathrm{G}_{11}^{1}\right)^{\mathrm{C}}=\mathrm{K}_{\mathrm{d}} \mathrm{r}_{1}\left\{\frac{\left(2 \mathrm{C}_{3}-1\right)}{\mathrm{R}^{3}}-\frac{3 \mathrm{C}_{3} \mathrm{r}_{1}^{2}}{\mathrm{R}^{5}}-\frac{6 \mathrm{cz}}{\mathrm{R}^{5}}\left(1-\frac{3 \mathrm{r}_{1}^{2}}{\mathrm{R}^{2}}\right)+\frac{12 \mathrm{cz}}{\mathrm{R}^{5}}\left(\frac{\mathrm{r}_{1}^{2}}{\mathrm{R}^{2}}-1\right)+\right. \\
& -\frac{4 \mathrm{C}_{1} \mathrm{C}_{2}}{\mathrm{R}\left(\mathrm{R}+\mathrm{R}_{3}\right)^{2}}-\frac{4 \mathrm{C}_{1} \mathrm{C}_{2} \mathrm{r}_{1}}{\left[\mathrm{R}\left(\mathrm{R}+\mathrm{R}_{3}\right)^{2}\right]^{2}}\left(3 \mathrm{r}_{1} \mathrm{R}+4 \mathrm{r}_{1} \mathrm{R}_{3}+\frac{\mathrm{r}_{1} \mathrm{R}_{3}^{2}}{\mathrm{R}}\right)+ \\
& \left.-\frac{8 \mathrm{C}_{1} \mathrm{C}_{2}}{\left[\mathrm{R}\left(\mathrm{R}+\mathrm{R}_{3}\right)^{2}\right]}\right\} \\
& \left(\mathrm{G}_{11}^{2}\right)^{\mathrm{C}}=\mathrm{K}_{\mathrm{d}} \mathrm{r}_{2}\left\{-\frac{1}{\mathrm{R}^{3}}-\frac{3 \mathrm{C}_{3} \mathrm{r}_{1}^{2}}{\mathrm{R}^{5}}-\frac{6 \mathrm{cz}}{\mathrm{R}^{5}}\left(1-\frac{3 \mathrm{r}_{1}^{2}}{\mathrm{R}^{2}}\right)+\frac{12 \mathrm{czr}_{1}^{2}}{\mathrm{R}^{7}}-\frac{4 \mathrm{C}_{1} \mathrm{C}_{2}}{\mathrm{R}\left(\mathrm{R}+\mathrm{R}_{3}\right)^{2}}+\right. \\
& \left.+\frac{4 \mathrm{C}_{1} \mathrm{C}_{2} \mathrm{r}_{1}^{2}}{\left[\mathrm{R}\left(\mathrm{R}+\mathrm{R}_{3}\right)^{2}\right]^{2}}\left(3 \mathrm{R}+4 \mathrm{R}_{3}+\frac{\mathrm{R}_{3}^{2}}{\mathrm{R}}\right)\right\} \\
& \left(\mathrm{G}_{11}^{3}\right)^{\mathrm{C}}=\mathrm{K}_{\mathrm{d}}\left\{-\frac{\mathrm{R}_{3}}{\mathrm{R}^{3}}+\frac{2 \mathrm{c}}{\mathrm{R}^{3}}\left(1-\frac{3 \mathrm{r}_{1}^{2}}{\mathrm{R}^{2}}\right)-\frac{3 \mathrm{C}_{3} \mathrm{r}_{1}^{2} \mathrm{R}_{3}}{\mathrm{R}^{5}}-\frac{6 \mathrm{czR} \mathrm{R}_{3}}{\mathrm{R}^{5}}\left(1-\frac{3 \mathrm{r}_{1}^{2}}{\mathrm{R}^{2}}\right)+\right. \\
& +\frac{12 \operatorname{czr}_{1}^{2} \mathrm{R}_{3}}{\mathrm{R}^{7}}-\frac{4 \mathrm{C}_{1} \mathrm{C}_{2}}{\left(\mathrm{R}+\mathrm{R}_{3}\right)^{2}}\left(1+\frac{\mathrm{R}_{3}}{\mathrm{R}}\right)+ \\
& \left.+\frac{4 \mathrm{C}_{1} \mathrm{C}_{2} \mathrm{r}_{1}^{2}}{\left[\mathrm{R}\left(\mathrm{R}+\mathrm{R}_{3}\right)^{2}\right]^{2}}\left(2 \mathrm{R}^{2}+5 \mathrm{RR}_{3}+4 \mathrm{R}_{3}^{2}+\frac{\mathrm{R}_{3}^{3}}{\mathrm{R}}\right)\right\} \\
& \left(G_{12}^{1}\right)^{C}=K_{d} r_{2}\left\{\frac{C_{3} r_{2}}{R^{3}}-\frac{3 C_{3} r_{1}^{2}}{R^{5}}-\frac{6 c z}{R^{5}}+\frac{30 c_{1}^{2}}{R^{7}}-\frac{4 C_{1} C_{2}}{R\left(R+R_{3}\right)^{2}}+\right. \\
& \left.+\frac{4 \mathrm{C}_{1} \mathrm{C}_{2} \mathrm{r}_{1}^{2}}{\mathrm{R}^{3}\left(\mathrm{R}+\mathrm{R}_{3}\right)^{2}}+\frac{8 \mathrm{C}_{1} \mathrm{C}_{2} \mathrm{r}_{1}^{2}}{\mathrm{R}^{2}\left(\mathrm{R}+\mathrm{R}_{3}\right)^{3}}\right\} \\
& \left(\mathrm{G}_{12}^{2}\right)^{\mathrm{C}}=\mathrm{K}_{\mathrm{d}} \mathrm{r}_{1}\left\{\frac{\mathrm{C}_{3} \mathrm{r}_{1}}{\mathrm{R}^{3}}-\frac{3 \mathrm{C}_{3} \mathrm{r}_{2}^{2}}{\mathrm{R}^{5}}-\frac{6 \mathrm{cz}}{\mathrm{R}^{5}}+\frac{30 \mathrm{czr}_{2}^{2}}{\mathrm{R}^{7}}-\frac{4 \mathrm{C}_{1} \mathrm{C}_{2}}{\mathrm{R}\left(\mathrm{R}+\mathrm{R}_{3}\right)^{2}}+\right. \\
& \left.+\frac{4 \mathrm{C}_{1} \mathrm{C}_{2} \mathrm{r}_{2}^{2}}{\mathrm{R}^{3}\left(\mathrm{R}+\mathrm{R}_{3}\right)^{2}}+\frac{8 \mathrm{C}_{1} \mathrm{C}_{2} \mathrm{r}_{2}^{2}}{\mathrm{R}^{2}\left(\mathrm{R}+\mathrm{R}_{3}\right)^{3}}\right\}
\end{aligned}
$$




$$
\begin{aligned}
& \left(\mathrm{G}_{12}^{3}\right)^{\mathrm{C}}=\mathrm{K}_{\mathrm{d}} \mathrm{r}_{1} \mathrm{r}_{2}\left\{-\frac{3 \mathrm{C}_{3} \mathrm{R}_{3}}{\mathrm{R}^{5}}-\frac{6 \mathrm{c}}{\mathrm{R}^{5}}+\frac{30 \mathrm{czR} \mathrm{R}_{3}}{\mathrm{R}^{7}}+\frac{4 \mathrm{C}_{1} \mathrm{C}_{2} \mathrm{R}_{3}}{\mathrm{R}^{3}\left(\mathrm{R}+\mathrm{R}_{3}\right)^{2}}+\right. \\
& \left.+\frac{8 \mathrm{C}_{1} \mathrm{C}_{2}}{\mathrm{R}\left(\mathrm{R}+\mathrm{R}_{3}\right)^{3}}\left(1+\frac{\mathrm{R}_{3}}{\mathrm{R}}\right)\right\} \\
& \left(G_{13}^{1}\right)^{C}=K_{d}\left\{\frac{C_{3} r_{3}}{R^{3}}-\frac{3 C_{3} r_{1}^{2} r_{3}}{R^{5}}-\frac{6 c z R_{3}}{R^{5}}+\frac{30 \operatorname{czr}_{1}^{2} R_{3}}{R^{7}}+\frac{4 C_{1} C_{2}}{R\left(R+R_{3}\right)}+\right. \\
& \left.-\frac{4 \mathrm{C}_{1} \mathrm{C}_{2} \mathrm{r}_{1}^{2}}{\mathrm{R}^{2}\left(\mathrm{R}+\mathrm{R}_{3}\right)^{2}}-\frac{4 \mathrm{C}_{1} \mathrm{C}_{2} \mathrm{r}_{1}^{2}}{\mathrm{R}^{3}\left(\mathrm{R}+\mathrm{R}_{3}\right)}\right\} \\
& \left(\mathrm{G}_{13}^{2}\right)^{\mathrm{C}}=\mathrm{K}_{\mathrm{d}} \mathrm{r}_{1} \mathrm{r}_{2}\left\{-\frac{3 \mathrm{C}_{3} \mathrm{r}_{3}}{\mathrm{R}^{5}}+\frac{30 \mathrm{czR} \mathrm{R}_{3}}{\mathrm{R}^{7}}-\frac{4 \mathrm{C}_{1} \mathrm{C}_{2}}{\mathrm{R}^{2}\left(\mathrm{R}+\mathrm{R}_{3}\right)^{2}}-\frac{4 \mathrm{C}_{1} \mathrm{C}_{2}}{\mathrm{R}^{3}\left(\mathrm{R}+\mathrm{R}_{3}\right)}\right\} \\
& \left(\mathrm{G}_{13}^{3}\right)^{\mathrm{C}}=\mathrm{K}_{\mathrm{d}} \mathrm{r}_{1}\left(\frac{\mathrm{C}_{3}}{\mathrm{R}^{3}}-\frac{3 \mathrm{C}_{3} \mathrm{r}_{3} \mathrm{R}_{3}}{\mathrm{R}^{5}}-\frac{6 \mathrm{c}}{\mathrm{R}^{5}}\left(\mathrm{z}+\mathrm{R}_{3}\right)+\frac{30 \mathrm{czR} \mathrm{R}_{3}^{2}}{\mathrm{R}^{7}}+\right. \\
& \left.-\frac{4 \mathrm{C}_{1} \mathrm{C}_{2}}{\mathrm{R}\left(\mathrm{R}+\mathrm{R}_{3}\right)^{2}}\left(1+\frac{\mathrm{R}_{3}}{\mathrm{R}}\right)-\frac{4 \mathrm{C}_{1} \mathrm{C}_{2} \mathrm{R}_{3}}{\mathrm{R}^{3}\left(\mathrm{R}+\mathrm{R}_{3}\right)}\right\} \\
& \left(\mathrm{G}_{21}^{\ell}\right)^{\mathrm{C}}=\left(\mathrm{G}_{12}^{\ell}\right)^{\mathrm{C}} \\
& \left(G_{22}^{1}\right)^{C}=K_{d} r_{1}\left\{-\frac{1}{R^{3}}-\frac{3 C_{3} r_{2}^{2}}{R^{5}}-\frac{6 c z}{R^{5}}\left(1-\frac{3 r_{2}^{2}}{R^{2}}\right)+\frac{12 c r_{2}^{2}}{R^{7}}-\frac{4 C_{1} C_{2}}{R\left(R+R_{3}\right)^{2}}\right. \text {. } \\
& \left.+\frac{4 \mathrm{C}_{1} \mathrm{C}_{2} \mathrm{r}_{2}^{2}}{\left[\mathrm{R}\left(\mathrm{R}+\mathrm{R}_{3}\right)^{2}\right]^{2}}\left(3 \mathrm{R}+4 \mathrm{R}_{3}+\frac{\mathrm{R}_{3}^{2}}{\mathrm{R}}\right)\right\} \\
& \left(\mathrm{G}_{22}^{2}\right)^{\mathrm{C}}=\mathrm{K}_{\mathrm{d}} \mathrm{r}_{2}\left\{\frac{\left(2 \mathrm{C}_{3}-1\right)}{\mathrm{R}^{3}}-\frac{3 \mathrm{C}_{3} \mathrm{r}_{2}^{2}}{\mathrm{R}^{5}}-\frac{6 \mathrm{cz}}{\mathrm{R}^{5}}\left(1-\frac{3 \mathrm{r}_{2}^{2}}{\mathrm{R}^{2}}\right)+\frac{12 \mathrm{cz}}{\mathrm{R}^{5}}\left(\frac{\mathrm{r}_{2}^{2}}{\mathrm{R}^{2}}-1\right)+\right. \\
& -\frac{4 C_{1} C_{2}}{R\left(R+R_{3}\right)^{2}}-\frac{4 C_{1} C_{2} r_{2}}{\left[R\left(R+R_{3}\right)^{2}\right]^{2}}\left(3 r_{2} R+4 r_{2} R_{3}+\frac{r_{2} R_{3}^{2}}{R}\right)+ \\
& \left.-\frac{8 \mathrm{C}_{1} \mathrm{C}_{2}}{\left[\mathrm{R}\left(\mathrm{R}+\mathrm{R}_{3}\right)^{2}\right]}\right\}
\end{aligned}
$$




$$
\begin{aligned}
& \left(\mathrm{G}_{22}^{3}\right)^{\mathrm{C}}=\mathrm{K}_{\mathrm{d}}\left\{-\frac{\mathrm{R}_{3}}{\mathrm{R}^{3}}+\frac{2 \mathrm{c}}{\mathrm{R}^{3}}\left(1-\frac{3 \mathrm{r}_{2}^{2}}{\mathrm{R}^{2}}\right)-\frac{3 \mathrm{C}_{3} \mathrm{r}_{2}^{2} \mathrm{R}_{3}}{\mathrm{R}^{5}}-\frac{6 \mathrm{czR} \mathrm{R}_{3}}{\mathrm{R}^{5}}\left(1-\frac{3 \mathrm{r}_{2}^{2}}{\mathrm{R}^{2}}\right)+\right. \\
& +\frac{12 \operatorname{czr}_{2}^{2} \mathrm{R}_{3}}{\mathrm{R}^{7}}-\frac{4 \mathrm{C}_{1} \mathrm{C}_{2}}{\left(\mathrm{R}+\mathrm{R}_{3}\right)^{2}}\left(1+\frac{\mathrm{R}_{3}}{\mathrm{R}}\right)+ \\
& \left.+\frac{4 \mathrm{C}_{1} \mathrm{C}_{2} \mathrm{r}_{2}^{2}}{\left[\mathrm{R}\left(\mathrm{R}+\mathrm{R}_{3}\right)^{2}\right]^{2}}\left(2 \mathrm{R}^{2}+5 \mathrm{RR}_{3}+4 \mathrm{R}_{3}^{2}+\frac{\mathrm{R}_{3}^{3}}{\mathrm{R}}\right)\right\} \\
& \left(\mathrm{G}_{23}^{1}\right)^{\mathrm{C}}=\frac{\mathrm{r}_{2}}{\mathrm{r}_{1}}\left(\mathrm{G}_{13}^{1}\right)^{\mathrm{C}} \\
& \left(\mathrm{G}_{31}^{1}\right)^{\mathrm{C}}=\mathrm{K}_{\mathrm{d}}\left\{\frac{\mathrm{C}_{3} \mathrm{r}_{3}}{\mathrm{R}^{3}}-\frac{3 \mathrm{C}_{3} \mathrm{r}_{1}^{2} \mathrm{r}_{3}}{\mathrm{R}^{5}}+\frac{6 \mathrm{czR} \mathrm{R}_{3}}{\mathrm{R}^{5}}-\frac{30 \mathrm{czr}_{1}^{2} \mathrm{R}_{3}}{\mathrm{R}^{7}}-\frac{4 \mathrm{C}_{1} \mathrm{C}_{2}}{\mathrm{R}\left(\mathrm{R}+\mathrm{R}_{3}\right)}+\right. \\
& \left.+\frac{4 \mathrm{C}_{1} \mathrm{C}_{2} \mathrm{r}_{1}^{2}}{\mathrm{R}^{2}\left(\mathrm{R}+\mathrm{R}_{3}\right)^{2}}+\frac{4 \mathrm{C}_{1} \mathrm{C}_{2} \mathrm{r}_{1}^{2}}{\mathrm{R}^{3}\left(\mathrm{R}+\mathrm{R}_{3}\right)}\right\} \\
& \left(\mathrm{G}_{31}^{2}\right)^{\mathrm{C}}=\mathrm{K}_{\mathrm{d}} \mathrm{r}_{1} \mathrm{r}_{2}\left\{-\frac{3 \mathrm{C}_{3} \mathrm{r}_{3}}{\mathrm{R}^{5}}-\frac{30 \mathrm{czR} \mathrm{R}_{3}}{\mathrm{R}^{7}}+\frac{4 \mathrm{C}_{1} \mathrm{C}_{2}}{\mathrm{R}^{2}\left(\mathrm{R}+\mathrm{R}_{3}\right)^{2}}+\frac{4 \mathrm{C}_{1} \mathrm{C}_{2}}{\mathrm{R}^{3}\left(\mathrm{R}+\mathrm{R}_{3}\right)}\right\} \\
& \left(\mathrm{G}_{31}^{3}\right)^{\mathrm{C}}=\mathrm{K}_{\mathrm{d}} \mathrm{r}_{1}\left\{\frac{\mathrm{C}_{3}}{\mathrm{R}^{3}}-\frac{3 \mathrm{C}_{3} \mathrm{r}_{3} \mathrm{R}_{3}}{\mathrm{R}^{5}}+\frac{6 \mathrm{c}}{\mathrm{R}^{5}}\left(\mathrm{z}+\mathrm{R}_{3}\right)-\frac{30 \mathrm{czR} \mathrm{R}_{3}^{2}}{\mathrm{R}^{7}}+\right. \\
& \left.+\frac{4 \mathrm{C}_{1} \mathrm{C}_{2}}{\mathrm{R}\left(\mathrm{R}+\mathrm{R}_{3}\right)^{2}}\left(1+\frac{\mathrm{R}_{3}}{\mathrm{R}}\right)+\frac{4 \mathrm{C}_{1} \mathrm{C}_{2} \mathrm{R}_{3}}{\mathrm{R}^{3}\left(\mathrm{R}+\mathrm{R}_{3}\right)}\right\} \\
& \left(\mathrm{G}_{32}^{1}\right)^{\mathrm{C}}=\frac{\mathrm{r}_{2}}{\mathrm{r}_{1}}\left(\mathrm{G}_{31}^{1}\right)^{\mathrm{C}} \\
& \left(\mathrm{G}_{33}^{1}\right)^{\mathrm{C}}=\mathrm{K}_{\mathrm{d}} \mathrm{r}_{1}\left\{-\frac{\left(8 \mathrm{C}_{1}^{2}-\mathrm{C}_{3}\right)}{\mathrm{R}^{3}}-\frac{3}{\mathrm{R}^{5}}\left(-2 \mathrm{cz}+\mathrm{C}_{3} \mathrm{R}_{3}^{2}\right)-\frac{30 \mathrm{czR} \mathrm{R}_{3}^{2}}{\mathrm{R}^{7}}\right\} \\
& \left(G_{33}^{2}\right)^{C}=K_{d} r_{2}\left\{-\frac{\left(8 C_{1}^{2}-C_{3}\right)}{R^{3}}-\frac{3}{R^{5}}\left(-2 c z+C_{3} R_{3}^{2}\right)-\frac{30 c z R_{3}^{2}}{R^{7}}\right\} \\
& \left(G_{33}^{3}\right)^{C}=K_{d}\left\{-\frac{\left(8 C_{1}^{2}-C_{3}\right) R_{3}}{R^{3}}-\frac{2 c+2 C_{3} R_{3}}{R^{3}}-\frac{3 R_{3}}{R^{5}}\left(-2 c z+C_{3} R_{3}^{2}\right)+\right. \\
& \left.+\frac{12 \mathrm{czR}_{3}}{\mathrm{R}^{5}}+\frac{6 \mathrm{cR} \mathrm{R}_{3}^{2}}{\mathrm{R}^{5}}-\frac{30 \mathrm{czR} \mathrm{R}_{3}^{3}}{\mathrm{R}^{7}}\right\}
\end{aligned}
$$




\section{5 - CONTRIBUIÇÃO DOS DIPOLOS PARA A ABERTURA DA} TRINCA

A abertura da trinca é dada pelo deslocamento relativo entre as suas superfícies em um determinado ponto. Tal valor pode ser determinado através da análise dos deslocamentos obtidos pela aplicação da equação integral (5.3.33) às imagens do ponto escolhido sobre as superfícies da fratura. A figura 5.5.1 mostra a representação de uma das faces da fratura com a definição do ponto a ser estudado, sua imagem e os parâmetros envolvidos na análise.

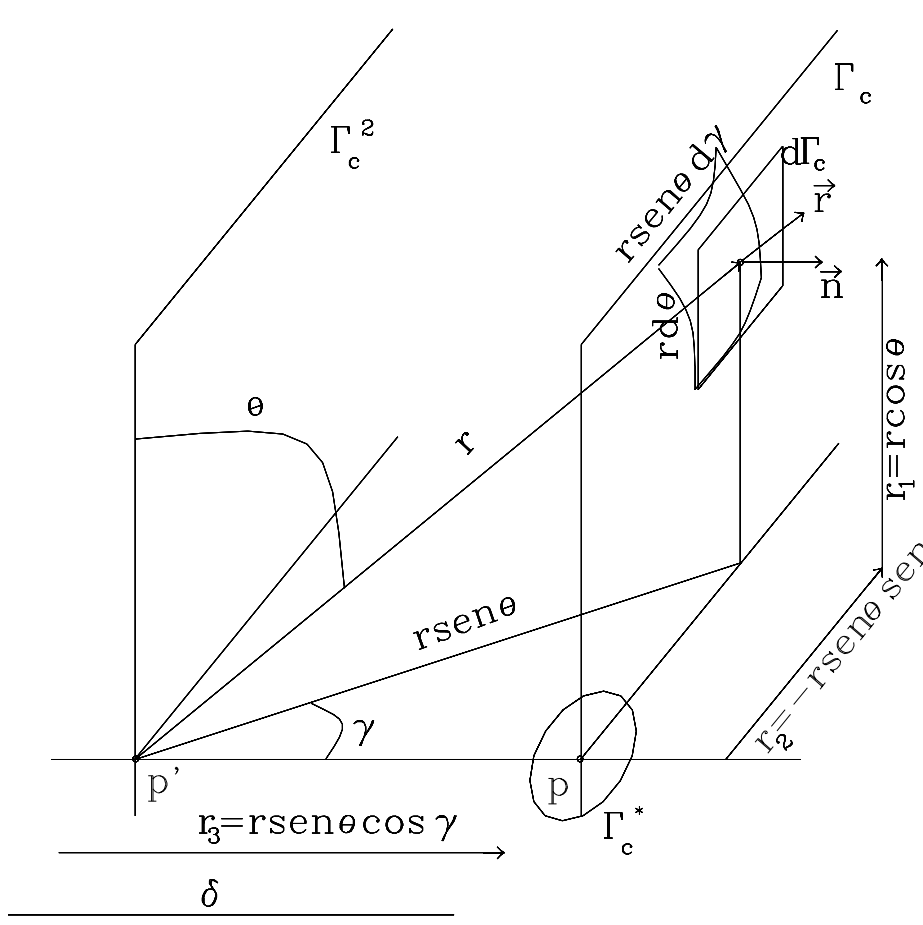

FIGURA 5.5.1 - Esquema de dois pontos próximos da fratura - representado o da esquerda; o da direita é de forma similar.

Da figura 5.5.1 obtém-se os seguintes parâmetros: 


$$
\begin{aligned}
& \mathrm{r}_{1}=\mathrm{r} \cos \theta \rightarrow \mathrm{r}_{, 1}=\cos \theta \\
& \mathrm{r}_{2}=-\mathrm{r} \operatorname{sen} \theta \operatorname{sen} \gamma \rightarrow \mathrm{r}_{, 2}=-\operatorname{sen} \theta \operatorname{sen} \gamma \\
& \mathrm{r}_{3}=\mathrm{r} \operatorname{sen} \theta \cos \gamma \rightarrow \mathrm{r}_{, 3}=\operatorname{sen} \theta \cos \gamma \\
& \mathrm{dS}=\mathrm{r}^{2} \operatorname{sen} \theta \mathrm{d} \theta \mathrm{d} \gamma \\
& \frac{\partial \mathrm{r}}{\partial \mathrm{n}}=\frac{1}{\mathrm{r}}\left(\mathrm{r}_{1} \mathrm{n}_{1}+\mathrm{r}_{2} \mathrm{n}_{2}+\mathrm{r}_{3} \mathrm{n}_{3}\right)=\mathrm{r}_{, 3} \\
& \mathrm{~d} \Gamma \frac{\partial \mathrm{r}}{\partial \mathrm{n}}=\mathrm{dS} \rightarrow \mathrm{d} \Gamma=\frac{\mathrm{r}^{2}}{\cos \gamma} \mathrm{d} \theta \mathrm{d} \gamma
\end{aligned}
$$

A última parcela integral de (5.3.33), escrita na forma matricial, é aqui analisada uma vez que através dela é introduzida a descontinuidade no meio contínuo. Assim, toma-se somente

$$
\int_{\Gamma_{\mathrm{c}}}[\mathrm{K}]\{\mathrm{q}\} \mathrm{d} \Gamma
$$

Considere-se a figura 5.5.1, onde é mostrada a imagem do ponto "P" à esquerda da superfície média da fratura. Quando a imagem representada por p' tende para o plano médio da trinca (o mesmo ocorre para o ponto p" à direita), onde serão dispostos os elementos especiais de contorno para simular a trinca, chega-se no seguinte limite:

$$
\operatorname{Lim}_{\mathrm{p}^{\prime} \rightarrow \mathrm{p}}\{\mathrm{u}\}^{\mathrm{p}^{\prime}}=\operatorname{Lim}_{\substack{\delta \rightarrow 0 \\ \Gamma^{*} \rightarrow 0}} \int_{\Gamma_{\mathrm{c}}-\Gamma_{\mathrm{c}}}[\mathrm{K}]\{\mathrm{q}\} \mathrm{d} \Gamma+\operatorname{Lim}_{\substack{\delta \rightarrow 0 \\ \Gamma^{*} \rightarrow 0}} \int_{\Gamma_{\mathrm{c}}^{*}}[\mathrm{~K}]\{\mathrm{q}\} \mathrm{d} \Gamma
$$

ou, ainda,

$$
\operatorname{Lim}_{\mathrm{p}^{\prime} \rightarrow \mathrm{p}}\{\mathrm{u}\}^{\mathrm{p}^{\prime}}=\operatorname{Lim}_{\substack{\delta \rightarrow 0 \\ \Gamma^{*} \rightarrow 0}} \int_{\Gamma_{\mathrm{c}}-\Gamma_{\mathrm{c}}^{*}}[\mathrm{~K}]\{\mathrm{q}\} \mathrm{d} \Gamma+\underset{\substack{\delta \rightarrow 0 \\ \Gamma^{* \rightarrow 0} \Gamma_{\mathrm{c}}^{*}}}{\operatorname{Lim}_{\substack{\Gamma^{*} \\ \text { termo independente }}}[\mathrm{K}] \mathrm{d} \Gamma\{\mathrm{q}\}^{\mathrm{p}^{\prime}}}
$$


onde $\Gamma_{\mathrm{c}}^{*}$ é a parte do contorno da trinca com centro no ponto $\mathrm{p}$ e $\{\mathrm{q}\}^{\mathrm{P}^{\prime}}$ são valores nodais dos dipolos, por isso retirados da integral.

Procurando-se efetuar o limite para o termo independente de (5.5.4), genericamente, tem-se:

$$
\overline{\mathrm{K}}_{\mathrm{ij}} \mathrm{q}_{\mathrm{j}}^{3}=\int_{\Gamma_{\mathrm{c}}^{*}} \mathrm{~K}_{\mathrm{ij}} \mathrm{q}_{\mathrm{j}}^{3} \mathrm{~d} \Gamma=\iint_{\Gamma_{\mathrm{c}}^{*}} \mathrm{~K}_{\mathrm{ij}} \frac{\mathrm{r}^{2}}{\cos \gamma} \mathrm{d} \theta \mathrm{d} \gamma \mathrm{q}_{\mathrm{j}}^{3}
$$

Para o ponto p' situado à esquerda do plano médio da trinca valem os seguintes limites para os ângulos (mostrados na figura 5.5.1):

$$
\begin{aligned}
& -\frac{\pi}{2} \leq \gamma \leq \frac{\pi}{2} \\
& 0 \leq \theta \leq \pi
\end{aligned}
$$

O resultado para a análise da imagem do ponto à esquerda, tendo em vista (5.5.5) e (5.5.6), é

$$
\begin{aligned}
& \overline{\mathrm{K}}^{\mathrm{e}}{ }_{11}=-\frac{1}{2 \mathrm{G}} \\
& \overline{\mathrm{K}}_{12}^{\mathrm{e}}=0 \\
& \overline{\mathrm{K}}^{\mathrm{e}}{ }_{13}=0 \\
& \overline{\mathrm{K}}^{\mathrm{e}}{ }_{21}=0 \\
& \overline{\mathrm{K}}^{\mathrm{e}}{ }_{22}=-\frac{1}{2 \mathrm{G}} \\
& \overline{\mathrm{K}}_{23}^{\mathrm{e}}=0 \\
& \overline{\mathrm{K}}_{31}^{\mathrm{e}}=0 \\
& \overline{\mathrm{K}}_{32}^{\mathrm{e}}=0 \\
& \overline{\mathrm{K}}^{\mathrm{e}}{ }_{33}=-\frac{(1-2 v)}{4(1-v) \mathrm{G}}
\end{aligned}
$$

Para o ponto p', imagem do ponto $\mathrm{P}$, situado à direita do plano médio da trinca, modificam-se os limites para os ângulos envolvidos na análise, passando a ser 


$$
\begin{aligned}
& \frac{\pi}{2} \leq \gamma \leq \frac{3 \pi}{2} \\
& \pi \leq \theta \leq 2 \pi
\end{aligned}
$$

permanecendo igual o procedimento utilizado para o ponto à esquerda. Isto resulta em:

$$
\begin{aligned}
\overline{\mathrm{K}}_{11}^{\mathrm{d}} & =\frac{1}{2 \mathrm{G}} \\
\overline{\mathrm{K}}_{12}^{\mathrm{d}} & =0 \\
\overline{\mathrm{K}}_{13}^{\mathrm{d}} & =0 \\
\overline{\mathrm{K}}_{21}^{\mathrm{d}} & =0 \\
\overline{\mathrm{K}}_{22}^{\mathrm{d}} & =\frac{1}{2 \mathrm{G}} \\
\overline{\mathrm{K}}_{23}^{\mathrm{d}} & =0 \\
\overline{\mathrm{K}}_{31}^{\mathrm{d}} & =0 \\
\overline{\mathrm{K}}_{32}^{\mathrm{d}} & =0 \\
\overline{\mathrm{K}}_{33}^{\mathrm{d}} & =\frac{(1-2 \mathrm{v})}{4(1-v) \mathrm{G}}
\end{aligned}
$$

Fazendo-se a diferença entre os deslocamentos à esquerda e à direita, tomando-se o valor absoluto, chega-se aos deslocamentos relativos entre as faces da trinca (que é a abertura da trinca). Assim,

$$
\{\Delta \mathrm{w}\}=\left\{\begin{array}{l}
\Delta \mathrm{w}_{1} \\
\Delta \mathrm{w}_{2} \\
\Delta \mathrm{w}_{3}
\end{array}\right\}=\{\mathrm{u}\}^{\mathrm{p}^{\prime}}-\{\mathrm{u}\}^{\mathrm{p}^{\prime \prime}} \mid=\left[\begin{array}{ccc}
\frac{1}{\mathrm{G}} & 0 & 0 \\
0 & \frac{1}{\mathrm{G}} & 0 \\
0 & 0 & \frac{(1-2 v)}{2(1-v) \mathrm{G}}
\end{array}\right]\left\{\begin{array}{l}
\mathrm{q}_{1}^{3} \\
\mathrm{q}_{2}^{3} \\
\mathrm{q}_{3}^{3}
\end{array}\right\}^{\mathrm{p}}
$$

Portanto, a abertura da trinca ou modo I de fraturamento, é dada por:

$$
\Delta \mathrm{w}_{3}=\frac{(1-2 v)}{2(1-v) \mathrm{G}} \mathrm{q}_{3}^{3}
$$


Já os deslizamentos no plano da fratura, modos II e III, são dados por:

$$
\begin{aligned}
& \Delta \mathrm{w}_{1}=\frac{1}{\mathrm{G}} \mathrm{q}_{1}^{3} \\
& \Delta \mathrm{w}_{2}=\frac{1}{\mathrm{G}} \mathrm{q}_{2}^{3}
\end{aligned}
$$

\section{6 - CONTRIBUIÇÃO DOS DIPOLOS NAS TENSÕES EM PONTOS INTERNOS}

Pode-se obter a representação integral das tensões para pontos internos utilizando-se o procedimento clássico baseado na lei de Hooke, ou seja,

$$
\begin{aligned}
& \sigma_{\mathrm{ij}}=\lambda \delta_{\mathrm{ij}} \varepsilon_{\mathrm{ww}}+2 \mathrm{G} \varepsilon_{\mathrm{ij}} \\
& \varepsilon_{\mathrm{ij}}=\frac{1}{2}\left(\frac{\partial \mathrm{u}_{\mathrm{i}}}{\partial \mathrm{X}_{\mathrm{j}}}+\frac{\partial \mathrm{u}_{\mathrm{j}}}{\partial \mathrm{X}_{\mathrm{i}}}\right)
\end{aligned}
$$

Portanto,

$$
\sigma_{\mathrm{im}}=\lambda \delta_{\mathrm{im}} \frac{\partial \mathrm{u}_{\mathrm{w}}}{\partial \mathrm{X}_{\mathrm{w}}}+\mathrm{G}\left(\frac{\partial \mathrm{u}_{\mathrm{i}}}{\partial \mathrm{X}_{\mathrm{m}}}+\frac{\partial \mathrm{u}_{\mathrm{m}}}{\partial \mathrm{X}_{\mathrm{i}}}\right)
$$

Utilizando-se a representação integral para deslocamentos, (5.3.33), e efetuando as suas derivadas convenientemente à (5.6.2), chega-se a:

$$
\begin{aligned}
\sigma_{\text {im }} & =-\int_{\Gamma} \mathrm{S}_{\mathrm{imk}} \mathrm{u}_{\mathrm{k}} \mathrm{d} \Gamma+\int_{\Gamma} \mathrm{D}_{\mathrm{imk}} \mathrm{p}_{\mathrm{k}} \mathrm{d} \Gamma+ \\
& +\left\{\lambda \delta_{\text {im }} \frac{\partial}{\partial \mathrm{X}_{\mathrm{w}}} \int_{\Gamma_{\mathrm{c}}} \mathrm{G}_{\mathrm{wj}}^{\ell} \mathrm{q}_{\mathrm{j}}^{\ell} \mathrm{d} \Gamma+\mathrm{G} \frac{\partial}{\partial \mathrm{X}_{\mathrm{m}}} \int_{\Gamma_{\mathrm{c}}} \mathrm{G}_{\mathrm{ij}}^{\ell} \mathrm{q}_{\mathrm{j}}^{\ell} \mathrm{d} \Gamma+\frac{\partial}{\partial \mathrm{X}_{\mathrm{i}}} \int_{\Gamma_{\mathrm{c}}} \mathrm{G}_{\mathrm{mj}}^{\ell} \mathrm{q}_{\mathrm{j}}^{\ell} \mathrm{d} \Gamma\right\}
\end{aligned}
$$

Os tensores S e D em (5.6.3) são os já conhecidos da formulação clássica do MEC. Os termos que encontram-se entre parênteses em (5.6.3) referem-se à fratura e, devido à presença de singularidade, devem ser analisados com cuidado. 
Considere-se isoladamente o termo de (5.6.3) conforme a seguir:

$$
\mathrm{I}=\frac{\partial}{\partial \mathrm{X}_{\mathrm{m}}} \int_{\Gamma_{\mathrm{c}}} \mathrm{G}_{\mathrm{ij}}^{\ell} \mathrm{q}_{\mathrm{j}}^{\ell} \mathrm{d} \Gamma
$$

Utilizando-se o conceito de integral singular devido a Miklin, conforme VENTURINI (1982), pode-se obter a derivada parcial antes de realizar a integração, assumindo que as densidades q satisfazem à condição de Lipschitz com expoente positivo. Assim, (5.6.4) equivale a:

$$
\mathrm{I}=\int_{\Gamma_{\mathrm{c}}} \frac{\partial}{\partial \mathrm{X}_{\mathrm{m}}}\left[\mathrm{G}_{\mathrm{ij}}^{\ell} \mathrm{g}_{\mathrm{j}}^{\ell} \mathrm{d} \Gamma\right]-\mathrm{q}_{\mathrm{j}}^{\ell} \int_{0}^{2 \pi} \mathrm{rG}_{\mathrm{ij}}^{\ell} \mathrm{r}_{\mathrm{m}} \mathrm{d} \phi
$$

Pode-se, agora, escrever (5.6.3) da seguinte forma:

$$
\sigma_{i m}=-\int_{\Gamma} S_{i m k} u_{k} d \Gamma+\int_{\Gamma} D_{i m k} p_{k} d \Gamma+\int_{\Gamma_{\mathrm{c}}} G_{i j}^{m \ell} q_{j}^{\ell} d \Gamma+g_{i j}^{m \ell}\left[\sigma_{j \ell}(p)\right]
$$

onde:

$$
\mathrm{g}_{\mathrm{ij}}^{\mathrm{m} \ell}(\mathrm{p})=\left\{\begin{array}{c}
0 \\
\sigma_{\mathrm{im}}^{\mathrm{o}}
\end{array},\right.
$$

para o cálculo da tensão plástica ou elástica, respectivamente, e

$$
\mathrm{G}_{\mathrm{ij}}^{\mathrm{m} \ell}=\lambda \delta_{\mathrm{ij}} \frac{\partial \mathrm{G}_{\mathrm{wj}}^{\ell}}{\partial \mathrm{X}_{\mathrm{w}}}+\mathrm{G}\left(\frac{\partial \mathrm{G}_{\mathrm{ij}}^{\ell}}{\partial \mathrm{X}_{\mathrm{m}}}+\frac{\partial \mathrm{G}_{\mathrm{mj}}^{\ell}}{\partial \mathrm{X}_{\mathrm{i}}}\right)
$$

Efetuando-se individualmente as derivadas parciais de (5.6.8), observando-se que as mesmas têm sinais negativos, já que a derivação é feita para o ponto $\mathrm{p}$ e não para o ponto s, chega-se na expressão: 


$$
\begin{array}{r}
\frac{\partial \mathrm{G}_{\mathrm{ij}}^{\ell}}{\partial \mathrm{X}_{\mathrm{m}}}=\frac{1}{16 \pi(1-v) \mathrm{G}}\left\{-(3-4 v) \frac{\partial}{\partial \mathrm{X}_{\mathrm{m}}}\left(\frac{\mathrm{r}_{, \ell}}{\mathrm{r}^{2}}\right) \delta_{\mathrm{ij}}+\frac{\partial}{\partial \mathrm{X}_{\mathrm{m}}}\left(\frac{\mathrm{r}_{, \mathrm{j}}}{\mathrm{r}^{2}}\right) \delta_{\mathrm{i} \ell}+\right. \\
+\frac{\partial}{\partial \mathrm{X}_{\mathrm{m}}}\left(\frac{\mathrm{r}_{\mathrm{i}}}{\mathrm{r}^{2}}\right) \delta_{\ell \mathrm{j}}-3 \frac{\partial}{\partial \mathrm{X}_{\mathrm{m}}}\left(\frac{1}{\mathrm{r}^{2}}\right) \mathrm{r}_{, \mathrm{i}} \mathrm{r}_{\mathrm{j},} \mathrm{r}_{, \ell}-\frac{3}{\mathrm{r}^{2}} \frac{\partial \mathrm{r}_{, \mathrm{i}}}{\partial \mathrm{X}_{\mathrm{m}}} \mathrm{r}_{, \mathrm{j}} \mathrm{r}_{, \ell}+ \\
\left.-\frac{3}{\mathrm{r}^{2}} \frac{\partial \mathrm{r}_{, \mathrm{j}}}{\partial \mathrm{X}_{\mathrm{m}}} \mathrm{r}_{, \mathrm{r}} \mathrm{r}_{, \ell}-\frac{3}{\mathrm{r}^{2}} \frac{\partial \mathrm{r}_{, \ell}}{\partial \mathrm{X}_{\mathrm{m}}} \mathrm{r}_{, \mathrm{i}} \mathrm{r}_{\mathrm{j}}\right\}
\end{array}
$$

Os valores das derivadas auxiliares são dados pelas expressões abaixo.

$$
\begin{aligned}
& \frac{\partial}{\partial \mathrm{X}_{\mathrm{m}}}\left(\frac{\mathrm{r}_{, \ell}}{\mathrm{r}^{2}}\right)=\frac{\partial}{\partial \mathrm{X}_{\mathrm{m}}}\left(\frac{1}{\mathrm{r}^{2}}\right)+\frac{1}{\mathrm{r}^{2}} \frac{\partial \mathrm{r}_{, \ell}}{\partial \mathrm{X}_{\mathrm{m}}} \\
&=-\left(-\frac{2 \mathrm{r}_{\mathrm{m}} \mathrm{r}_{, \ell}}{\mathrm{r}^{3}}+\frac{1}{\mathrm{r}^{2}}\left[\frac{1}{\mathrm{r}}\left(\delta_{\mathrm{ml}}-\mathrm{r}_{, \mathrm{m}} \mathrm{r}_{, \ell}\right)\right]\right) \\
&=\frac{1}{\mathrm{r}^{3}}\left(3 \mathrm{r}_{, \mathrm{m}} \mathrm{r}_{, \ell}-\delta_{\mathrm{m} \ell}\right) \\
& \frac{\partial}{\partial \mathrm{X}_{\mathrm{m}}}\left(\frac{\mathrm{r}_{, \mathrm{j}}}{\mathrm{r}^{2}}\right)=\frac{1}{\mathrm{r}^{3}}\left(3 \mathrm{r}_{, \mathrm{m}} \mathrm{r}_{\mathrm{j}}-\delta_{\mathrm{mj}}\right) \\
& \frac{\partial}{\partial \mathrm{X}_{\mathrm{m}}}\left(\frac{\mathrm{r}_{\mathrm{i}}}{\mathrm{r}^{2}}\right)=\frac{1}{\mathrm{r}^{3}}\left(3 \mathrm{r}_{, \mathrm{m}} \mathrm{r}_{\mathrm{i}}-\delta_{\mathrm{mi}}\right) \\
& \frac{\partial}{\partial \mathrm{X}_{\mathrm{m}}}\left(\frac{1}{\mathrm{r}^{2}}\right)=\frac{2 \mathrm{r}_{\mathrm{m}}}{\mathrm{r}^{3}} \\
& \frac{\partial \mathrm{r}_{, \mathrm{i}}}{\partial \mathrm{X}_{\mathrm{m}}}=\frac{1}{\mathrm{r}}\left(\mathrm{r}_{, \mathrm{m}} \mathrm{r}_{\mathrm{i}}-\delta_{\mathrm{mi}}\right) \\
& \frac{\partial \mathrm{r}_{, \mathrm{j}}}{\partial \mathrm{X}_{\mathrm{m}}}=\frac{1}{\mathrm{r}}\left(\mathrm{r}_{, \mathrm{m}} \mathrm{r}_{, \mathrm{j}}-\delta_{\mathrm{mj}}\right) \\
& \frac{\partial \mathrm{r}_{, \ell}}{\partial \mathrm{X}_{\mathrm{m}}}=\frac{1}{\mathrm{r}}\left(\mathrm{r}_{, \mathrm{m}} \mathrm{r}_{, \ell}-\delta_{\mathrm{m} \ell}\right)
\end{aligned}
$$


Substituindo-se (5.6.10) em (5.6.9), encontra-se

$$
\begin{aligned}
& \frac{\partial \mathrm{G}_{\mathrm{ij}}^{\ell}}{\partial \mathrm{X}_{\mathrm{m}}}=\frac{1}{16 \pi(1-v) \mathrm{Gr}^{3}}\left\{-(3-4 v)\left(\delta_{\mathrm{ij}} \delta_{\mathrm{m} \ell}-3 \delta_{\mathrm{ij}, \mathrm{r}_{\mathrm{m}}, \ell} \mathrm{r}_{\ell}\right)-\left(\delta_{\mathrm{mj}} \delta_{\ell \mathrm{i}}+\delta_{\mathrm{mi}} \delta_{\ell \mathrm{j}}\right)+\right. \\
& +3\left(\delta_{\ell \mathrm{j}, \mathrm{m}} \mathrm{r}_{, \ell} \mathrm{r}_{\mathrm{ij}, \mathrm{m}, \mathrm{i}}+\delta_{\mathrm{i} \ell} \mathrm{r}_{\mathrm{m}, \mathrm{m}} \mathrm{r}_{\mathrm{j}}+\delta_{\mathrm{mj}, \mathrm{i}, \ell} \mathrm{r}_{,} \mathrm{r}_{\mathrm{mi}}, \delta_{\mathrm{j}, \ell} \mathrm{r}_{,}\right)+ \\
& \left.-15 \mathrm{r}_{, \mathrm{i}} \mathrm{r}_{\mathrm{j}, \ell} \mathrm{r}_{,} \mathrm{r}_{\mathrm{r}}\right\}
\end{aligned}
$$

Aproveitando-se o resultado de (5.6.11) para as outras derivada em (5.6.8), encontra-se:

$$
\begin{aligned}
& \frac{\partial \mathrm{G}_{\mathrm{mj}}^{\ell}}{\partial \mathrm{X}_{\mathrm{i}}}=\frac{1}{16 \pi(1-v) \mathrm{Gr}^{3}}\left\{-(3-4 v)\left(\delta_{\mathrm{mj}} \delta_{\mathrm{i} \ell}-3 \delta_{\mathrm{mj}, \mathrm{r}_{\mathrm{i}}, \ell} \mathrm{r}_{\ell}\right)-\left(\delta_{\mathrm{ij}} \delta_{\ell \mathrm{m}}+\delta_{\mathrm{mi}} \delta_{\ell \mathrm{j}}\right)+\right. \\
& +3\left(\delta_{\ell \mathrm{j}, \mathrm{i}} \mathrm{r}_{, \ell} \mathrm{r}_{1}+\delta_{\mathrm{mj}, \mathrm{m}, \mathrm{i}} \mathrm{r}_{\mathrm{i}}+\delta_{\mathrm{m} \ell} \mathrm{r}_{\mathrm{i}} \mathrm{r}_{\mathrm{j}}+\delta_{\mathrm{ij}} \mathrm{r}_{\mathrm{m}} \mathrm{r}_{, \ell}+\delta_{\mathrm{mi}, \mathrm{j}} \mathrm{r}_{, \ell} \mathrm{r}_{\ell}\right)+ \\
& \left.-15 \mathrm{r}_{, \mathrm{i}, \mathrm{j}, \ell} \mathrm{r}_{,} \mathrm{r}_{, \mathrm{m}}\right\}
\end{aligned}
$$

e

$$
\frac{\partial \mathrm{G}_{\mathrm{wj}}^{\ell}}{\partial \mathrm{X}_{\mathrm{w}}}=\frac{(1-2 v)}{16 \pi(1-v) \mathrm{Gr}^{3}}\left(2 \delta_{\ell \mathrm{j}}-6 \mathrm{r}_{, \mathrm{j}, \ell} \mathrm{r}_{\mathrm{r}}\right)
$$

Levando-se as expressões obtidas, (5.6.11), (5.6.12) e (5.6.13) para (5.6.8), chega-se no tensor:

$$
\begin{aligned}
\mathrm{G}_{\mathrm{ij}}^{\mathrm{m} \ell}= & \frac{1}{8 \pi(1-v) \mathrm{r}^{3}}\left\{(1-2 v)\left(\delta_{\mathrm{mj}} \delta_{\mathrm{i} \ell}+\delta_{\mathrm{ij}} \delta_{\ell \mathrm{m}}-\delta_{\mathrm{mi}} \delta_{\ell \mathrm{j}}\right)+3(1-2 v)\left(\delta_{\mathrm{mj}} \mathrm{r}_{\mathrm{i}} \mathrm{r}_{, \ell}+\right.\right. \\
& \left.\left.+\delta_{\mathrm{ij}} \mathrm{r}_{\mathrm{m}} \mathrm{r}_{, \ell}-\delta_{\mathrm{im}} \mathrm{r}_{, \mathrm{j}} \mathrm{r}_{, \ell}\right)-3\left(\delta_{\ell \mathrm{j}} \mathrm{r}_{\mathrm{i}} \mathrm{r}_{, \mathrm{m}}+\delta_{\mathrm{m} \ell} \mathrm{r}_{, \mathrm{j}} \mathrm{r}_{\mathrm{i}}+\delta_{\ell \mathrm{i}} \mathrm{r}_{\mathrm{r}, \mathrm{m}} \mathrm{r}_{\mathrm{j}}\right)-15 \mathrm{r}_{\mathrm{i}} \mathrm{r}_{\mathrm{j}, \mathrm{j}, \ell} \mathrm{r}_{, \mathrm{m}} \mathrm{r}_{\mathrm{m}}\right\}
\end{aligned}
$$

Uma vez definido o tensor (5.6.14) hipersingular dos coeficientes de influência dos dipolos para as tensões em pontos internos, pode-se organizar a penúltima parcela de (5.6.6), resultando em 


$$
\begin{aligned}
\mathrm{G}_{\mathrm{ij}}^{\mathrm{m} \ell} \mathrm{q}_{\mathrm{j}}^{\ell}= & \mathrm{G}_{\mathrm{ij}}^{\mathrm{m} 1} \mathrm{q}_{\mathrm{j}}^{1}+\mathrm{G}_{\mathrm{ij}}^{\mathrm{m} 2} \mathrm{q}_{\mathrm{j}}^{2}+\mathrm{G}_{\mathrm{ij}}^{\mathrm{m} 3} \mathrm{q}_{\mathrm{j}}^{3} \\
\mathrm{G}_{\mathrm{ij}}^{\mathrm{m} \ell} \mathrm{q}_{\mathrm{j}}^{\ell}= & \mathrm{G}_{\mathrm{i} 1}^{\mathrm{m} 1} \mathrm{q}_{1}^{1}+\mathrm{G}_{\mathrm{i} 1}^{\mathrm{m} 2} \mathrm{q}_{1}^{2}+\mathrm{G}_{\mathrm{i} 1}^{\mathrm{m} 3} \mathrm{q}_{1}^{3}+ \\
& +\mathrm{G}_{\mathrm{i} 2}^{\mathrm{m} 1} \mathrm{q}_{2}^{1}+\mathrm{G}_{\mathrm{i} 2}^{\mathrm{m} 2} \mathrm{q}_{2}^{2}+\mathrm{G}_{\mathrm{i} 2}^{\mathrm{m} 3} \mathrm{q}_{2}^{3}+ \\
& +\mathrm{G}_{\mathrm{i} 3}^{\mathrm{m} 1} \mathrm{q}_{3}^{1}+\mathrm{G}_{\mathrm{i} 3}^{\mathrm{m} 2} \mathrm{q}_{3}^{2}+\mathrm{G}_{\mathrm{i} 3}^{\mathrm{m} 3} \mathrm{q}_{3}^{3}
\end{aligned}
$$

É conveniente escrever (5.6.15) na forma matricial, ou seja,

$$
\left[\begin{array}{lllllllll}
\mathrm{G}_{\mathrm{i} 1}^{\mathrm{m} 1} & \mathrm{G}_{\mathrm{i} 2}^{\mathrm{m} 1} & \mathrm{G}_{\mathrm{i} 3}^{\mathrm{m} 1} & \mathrm{G}_{\mathrm{i} 1}^{\mathrm{m} 2} & \mathrm{G}_{\mathrm{i} 2}^{\mathrm{m} 2} & \mathrm{G}_{\mathrm{i} 3}^{\mathrm{m} 2} & \mathrm{G}_{\mathrm{i} 1}^{\mathrm{m} 3} & \mathrm{G}_{\mathrm{i} 2}^{\mathrm{m} 3} & \mathrm{G}_{\mathrm{i} 3}^{\mathrm{m} 3}
\end{array}\right]\left[\begin{array}{c}
\mathrm{q}_{1}^{1} \\
\mathrm{q}_{2}^{1} \\
\mathrm{q}_{3}^{1} \\
\mathrm{q}_{1}^{2} \\
\mathrm{q}_{2}^{2} \\
\mathrm{q}_{3}^{2} \\
\mathrm{q}_{1}^{3} \\
\mathrm{q}_{2}^{3} \\
\mathrm{q}_{3}^{3}
\end{array}\right\}
$$

Tendo em vista o mesmo procedimento utilizado para condensar as componentes tensor $\mathrm{G}_{\mathrm{ij}}^{\ell}$, que resultou em (5.4.7), pode-se rescrever a expressão (5.6.16), obtendo-se:

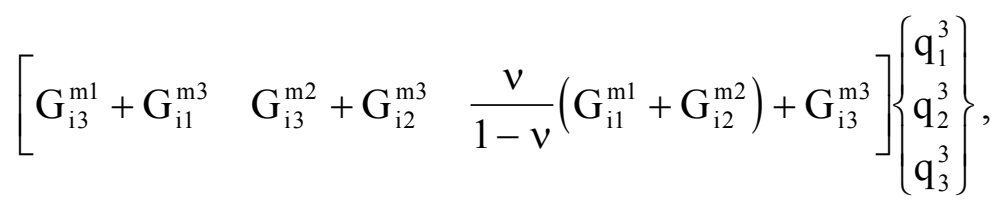

ou, ainda, na forma concisa

$$
[\mathrm{KS}]\{\mathrm{q}\} .
$$

Portanto, para um ponto qualquer da superfície de fratura, o integrando da penúltima parcela integral de (5.6.6), escrito de forma matricial, é 


$$
\left[\begin{array}{ccc}
\mathrm{KS}_{11}^{\mathrm{m}} & \mathrm{KS}_{12}^{\mathrm{m}} & \mathrm{KS}_{13}^{\mathrm{m}} \\
\mathrm{KS}_{21}^{\mathrm{m}} & \mathrm{KS}_{22}^{\mathrm{m}} & \mathrm{KS}_{23}^{\mathrm{m}} \\
\mathrm{KS}_{31}^{\mathrm{m}} & \mathrm{KS}_{32}^{\mathrm{m}} & \mathrm{KS}_{33}^{\mathrm{m}}
\end{array}\right]\left\{\begin{array}{l}
\mathrm{q}_{1}^{3} \\
\mathrm{q}_{2}^{3} \\
\mathrm{q}_{3}^{3}
\end{array}\right\}
$$

As componentes da matriz [KS] são:

$$
\begin{aligned}
\mathrm{KS}_{\mathrm{i} 1}^{\mathrm{m}} & =\mathrm{G}_{\mathrm{i} 3}^{\mathrm{m} 1}+\mathrm{G}_{\mathrm{i} 1}^{\mathrm{m} 3} \\
\mathrm{KS}_{\mathrm{i} 2}^{\mathrm{m}} & =\mathrm{G}_{\mathrm{i} 3}^{\mathrm{m} 2}+\mathrm{G}_{\mathrm{i} 2}^{\mathrm{m} 3} \\
\mathrm{KS}_{\mathrm{i} 3}^{\mathrm{m}} & =\frac{v}{1-v}\left(\mathrm{G}_{\mathrm{i} 1}^{\mathrm{m} 1}+\mathrm{G}_{\mathrm{i} 2}^{\mathrm{m} 2}\right)+\mathrm{G}_{\mathrm{i} 3}^{\mathrm{m} 3}
\end{aligned}
$$

As expressões das componentes da matriz $[\mathrm{KS}]$ encontram-se no anexo G.

\section{7 - DISCRETIZAÇÕES}

Mostradas as expressões matemáticas da formulação, necessárias à análise do problema proposto, necessita-se, agora, transformá-las para uso em um algoritmo computacional para a solução automática do problema. Assim, são utilizados elementos planos triangulares descontínuos com aproximação linear (com três pontos de colocação) para a discretização do contorno do corpo. Para a fratura são utilizados os mesmos elementos com aproximação constante (um ponto de colocação). Elementos com outras aproximações poderiam ser utilizados sem nenhum prejuízo à formulação, lembrando-se que as fraturas impõem descontinuidades ao contorno (quando o atinge), e são somente representadas por elementos descontínuos.

Escrevendo-se (5.3.33) para os pontos de colocação definidos no contorno do corpo e nos elementos da fratura, obtém-se:

$$
\mathbf{H U}=\mathbf{G P}+\mathbf{K Q}
$$


onde as matrizes $\mathbf{H}$ e $\mathbf{G}$ são as já conhecidas do MEC. A matriz $\mathbf{K}$ é obtida a partir dos núcleos dados em (5.4.12), observando-se convenientemente as componentes necessárias e a relação entre elas (conforme figura 5.4.2). Fazendo a troca de colunas entre as matrizes $\mathbf{H}$ e $\mathbf{G}$, do modo usual do MEC, a fim de estabelecer um vetor de incógnitas (deslocamentos e forças de superfície) e um vetor de valores prescritos, obtém-se o seguinte sistema de equações algébricas:

$$
\mathbf{X}=\mathbf{M}+\mathbf{R} \mathbf{Q}
$$

onde

$$
\begin{aligned}
& \mathbf{M}=\mathbf{A}^{-1} \mathbf{F} \\
& \mathbf{R}=\mathbf{A}^{-1} \mathbf{K}
\end{aligned}
$$

A equação (5.7.2) pode ser usada na forma incremental, para calcular os efeitos dos incrementos da carga dados por $\mathbf{M}$ ou $\Delta \mathbf{M}$, ou incrementos dos dipolos, $\Delta \mathbf{Q}$, requeridos para corrigir o nível de tensão sobre a superfície da fratura.

A expressão integral para as tensões (5.6.6), é transformada de forma semelhante à de deslocamentos, com as matrizes usuais do MEC $\mathbf{H}^{\prime}$ e $\mathbf{G}^{\prime}$ ( tensores $\mathbf{S}^{*}$ e $\mathbf{D}^{*}$ - (2.5.3), (2.5.4), (2.5.5) e (2.5.6)), mais a matriz $\mathbf{K}^{\prime}$ obtida com os núcleos dados por (5.6.14). O resultado é:

$$
\sigma=-\mathbf{H}^{\prime} \mathbf{U}+\mathbf{G}^{\prime} \mathbf{P}+\mathbf{K}^{\prime} \mathbf{Q}
$$

Da mesma forma que a expressão para deslocamentos, a de tensões pode ser escrita separando-se em duas parcelas, uma elástica e a outra que corrige a primeira pelo emprego dos dipolos. Assim,

$$
\sigma=\mathbf{N}+\mathbf{S Q}
$$

onde 


$$
\begin{aligned}
& \mathbf{N}=\mathbf{F}^{\prime}-\mathbf{A}^{\prime} \mathbf{M} \\
& \mathbf{S}=\mathbf{K}^{\prime}-\mathbf{A}^{\prime} \mathbf{R}
\end{aligned}
$$

\section{8 - ASPECTOS COMPUTACIONAIS}

O algoritmo empregado na solução das equações apresentadas até aqui, é do tipo incremental iterativo. Calculam-se as incógnitas, deslocamentos e forças de superfície e, eventualmente, tensões internas com as parcelas elásticas das equações (5.7.2) e (5.7.5) para cada passo incremental. Verifica-se, então, se o critério de resistência é atingido e, em caso afirmativo, entra-se em um processo iterativo. Os valores verdadeiros de deslocamentos, forças de superfície e tensões são obtidos corrigindo-se os valores elásticos através das parcelas relacionadas com os dipolos. Os valores dos dipolos devem ser estimados a partir do valor local das tensões de cada elemento de fratura ou nó, como se fossem campos independentes, já que o processo é não-linear. Portanto, com os valores residuais das tensões e das submatrizes de $\mathbf{S}$, obtém-se de modo simplificado os valores parciais dos dipolos, que são posteriormente acumulados em $\mathbf{Q}$. Obedecendo-se ao critério do modelo coesivo apresentado em (4.3.1), verifica-se o surgimento da abertura de fratura limite, $\mathrm{w}_{\mathrm{c}}$, condição necessária para se afirmar que neste nó houve fraturamento.

É importante destacar que o emprego de elementos descontínuos na discretização do contorno é necessário para permitir o surgimento de fraturas.

O procedimento descrito é similar a outros já empregados em conjunto com o MEC; e pode ser utilizado no estudo de sólidos quaisquer sujeitos ao surgimento de uma linha de fratura ou a multifraturamento.

Nesta formulação apresentada, as matrizes $\mathbf{G}$ e $\mathbf{H}$ usuais do MEC sofrem inclusões de linhas e colunas à medida que novos elementos são exigidos para representar o crescimento da fratura. Portanto, não se trata de redefinir uma nova rede de elementos, e sim de aproveitar os valores das componentes dessas matrizes, já calculados, aumentando a economia de tempo computacional. 
Sendo hipersingulares os núcleos envolvidos nesta formulação, deve-se ter cuidado especial com os procedimentos de integração, principalmente se o ponto de colocação pertencer ao elemento integrado (utilizando-se o conceito de parte finita de Hadamard, conforme PORTELA, 1993).

\section{9 - APLICAÇÕES}

A seguir, são apresentados alguns exemplos de aplicação da formulação apresentada.

5.9.1 - Aplicação 1: Sólido paralelepipédico elástico com deslocamento uniforme aplicado.

É escolhido um caso simples da mecânica da fratura, um sólido paralelepipédico, definido conforme a figura 5.9.1, que é analisado assumindo-se deslocamentos prescritos sobre suas duas extremidades. São adotados os seguintes valores que definem as propriedades físicas do sólido bem como os parâmetros do modelo coesivo: $\mathrm{E}=1 \times 10^{5}, \mathrm{v}=0,0, \mathrm{f}_{\mathrm{t}}=1,0$ e $\mathrm{w}_{\mathrm{c}}=0,00004$.

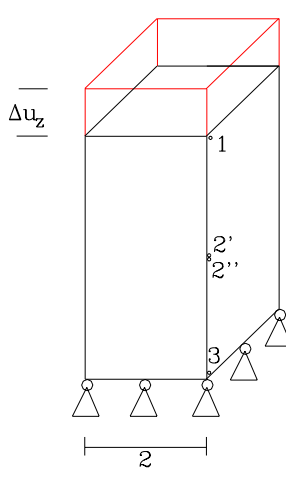

(a)

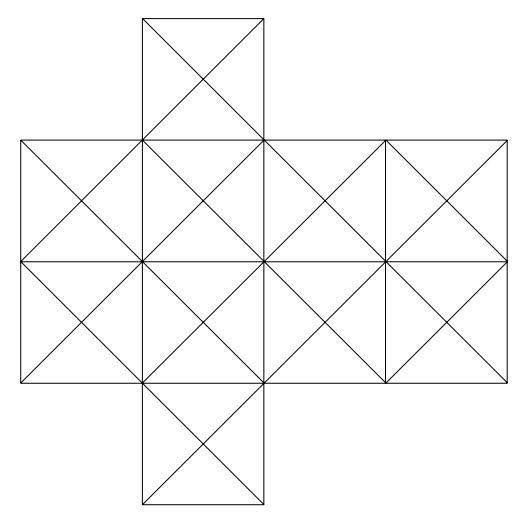

(b)

FIGURA 5.9.1 - Sólido para análise. (a) geometria e deslocamentos prescritos; (b) discretização do contorno; (c) discretização da superfície da trinca. 
Na figura 5.9.1a são definidos quatro pontos: um na extremidade livre, outro próximo aos apoios e dois na metade do comprimento (um imediatamente acima da metade e outro abaixo). Para esses pontos são apresentados valores de deslocamentos para alguns passos de cálculo do algoritmo apresentado.

A discretização do contorno do corpo é feita através de elementos triangulares planos com aproximação linear, conforme figura 5.9.1b, e são descontínuos para permitir o surgimento de trincas ao longo do comprimento do corpo. A superfície fictícia de fraturamento é discretizada por elementos triangulares planos com aproximação constante (figura 5.9.1c) e divide o corpo hipoteticamente em dois cubos.

Os resultados obtidos do processamento são apresentados a seguir. A figura 5.9.2a mostra que, ao ser atingida a tensão limite, inicia-se o processo de fraturamento, obedecendo-se ao critério adotado em (4.3.1) e verificando-se o deslocamento relativo entre os pontos $2^{\prime}$ e $2 "$ (abertura da fratura). Na figura 5.9.2b observa-se bem o trecho de deslocamentos elásticos, que são corrigidos no segundo trecho, moldando-se à nova situação de fraturamento.

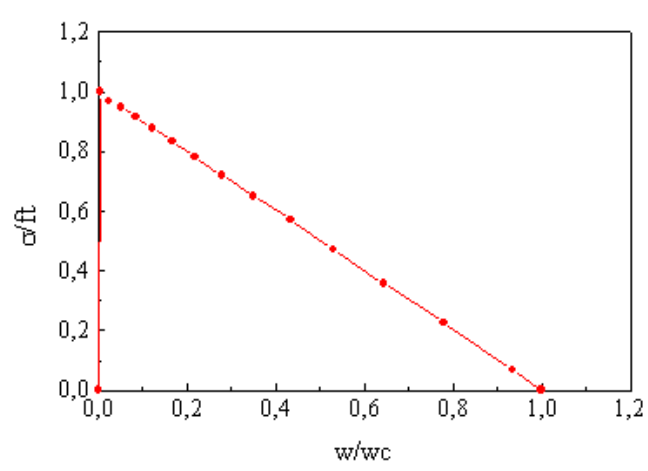

(a)

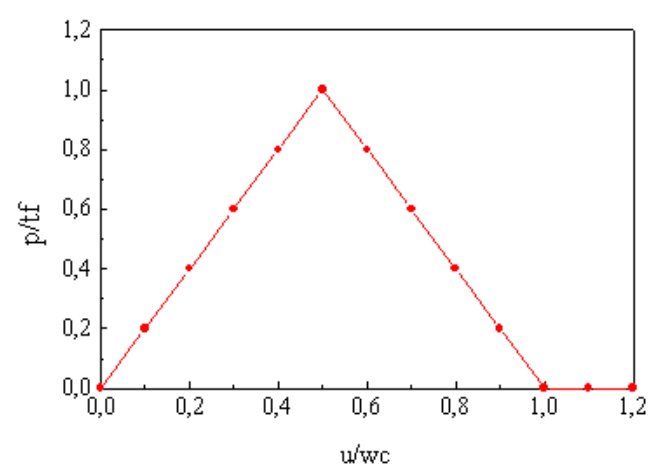

(b)

FIGURA. 5.9.2 - Resultados: (a) tensão em um nó da fratura $\times$ abertura da fratura;

(b) força de superfície na extremidade do sólido $\times$ deslocamento no nó 2'.

Os valores de deslocamentos obtidos para os quatro pontos definidos na figura (5.9.1a) estão resumidos na tabela 5.9.1: 
TABELA 5.9.1 - Deslocamento durante o processo de carregamento.

\begin{tabular}{|c|c|c|c|c|c|c|c|}
\hline & \multicolumn{5}{|c|}{ Fase Elástica } & \multicolumn{2}{c|}{ Fase Fraturada } \\
\hline Nó & $\alpha=0$ & $\alpha=0,25$ & $\alpha=0,50$ & $\alpha=0,75$ & $\alpha=1,00$ & $\alpha=1,00$ & $\alpha=1,25$ \\
\hline 1 & 0 & 0,000010 & 0,000020 & 0,000030 & 0,000040 & 0,000040 & 0,000050 \\
\hline $2^{\prime}$ & 0 & 0,000005 & 0,000010 & 0,000015 & 0,000020 & 0,000040 & 0,000050 \\
\hline $2^{\prime \prime}$ & 0 & 0,000005 & 0,000010 & 0,000015 & 0,000020 & 0,000000 & 0,000000 \\
\hline 3 & 0 & 0 & 0 & 0 & 0 & 0 & 0 \\
\hline
\end{tabular}

Os dados da tabela 5.9.1 revelam duas fases distintas do comportamento do corpo em questão. Na primeira, o corpo íntegro deforma-se elasticamente até que a tensão limite é atingida. A partir daí, já no processo iterativo, são determinados os dipólos que fazem a correção dos valores elásticos neste incremento, para os valores verdadeiros, já com o corpo fraturado. A segunda fase caracteriza-se pelo descolamento das duas partes do domínio, uma permanecendo imóvel e a outra tendo deslocamento de corpo livre. A configuração final do corpo, após o fraturamento, está apresentada na figura 5.9.3.

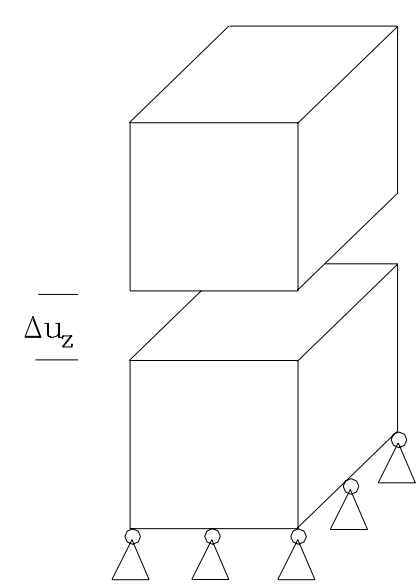

FIGURA 5.9.3 - Configuração final do corpo fraturado.

A formulação apresentada neste capítulo mostra-se adequada para solucionar o caso simples de problema tridimensional de fratura coesiva. O algoritmo é convergente e bastante versátil. Após discretizado o contorno do corpo e encontradas as matrizes usuais do MEC, basta que se acrescente os elementos na superfície fictícia da fratura, gerando novas linhas e novas colunas, mantendo-se a rede de 
elementos do contorno. Isso traz uma economia de tempo computacional e dá ao Método dos Elementos de Contorno uma formulação elegante para a análise do processo de fraturamento. Vale destacar que esta formulação é válida para quaisquer outros modelos que não o coesivo, inclusive o elástico.

5.9.2 - Aplicação 2: Sólido paralelepipédico elástico com deslocamento linear aplicado.

Apresenta-se um sólido paralelepipédico engastado na base e livre na outra extremidade, conforme mostra a figura 5.9.4. É imposto um deslocamento escalonado de forma triangular na extremidade livre. São adotados os seguintes valores que definem as propriedades físicas do sólido bem como os parâmetros do modelo coesivo: $\mathrm{E}=1 \times 10^{5}, \mathrm{v}=0,0, \mathrm{f}_{\mathrm{t}}=1,0$ e $\mathrm{w}_{\mathrm{c}}=0,00004$.

Na figura 5.9.4 estão definidos 3 pontos ao longo do comprimento do sólido: ponto 1, na extremidade livre, e 2'e 2", imediatamente acima e abaixo da sua metade, respectivamente.
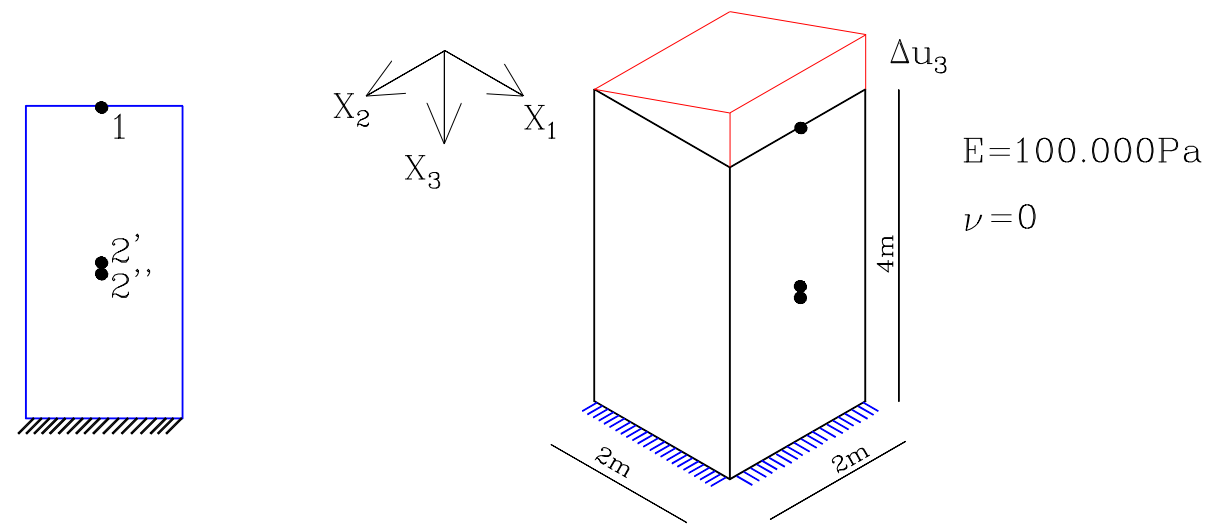

FIGURA 5.9.4 - Geometria e deslocamentos prescritos.

A discretização do contorno é feita utilizando-se o elemento triangular plano descontínuo, com uma só distribuição, conforme mostra a figura 5.9.5. 


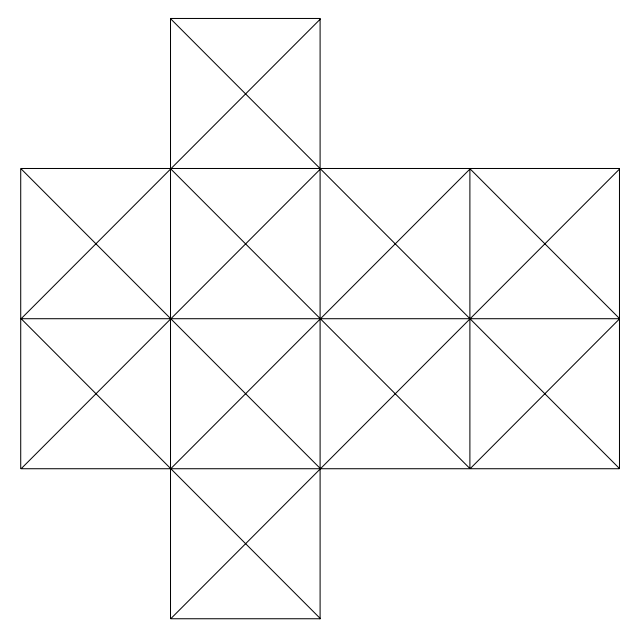

FIGURA 5.9.5 - Discretização do contorno do sólido: 40 elementos triangulares planos.

A superfície da fratura é discretizada por elementos triangulares planos com aproximação constante (figura 5.9.6), e está posicionada no plano $X_{3}=2 \mathrm{~m}$. Três diferentes distribuições são utilizadas para analisar deslocamentos e tensões em pontos da fratura: discretização (a) com 32 elementos, (b) com 64 elementos e (c) com 64 elementos.

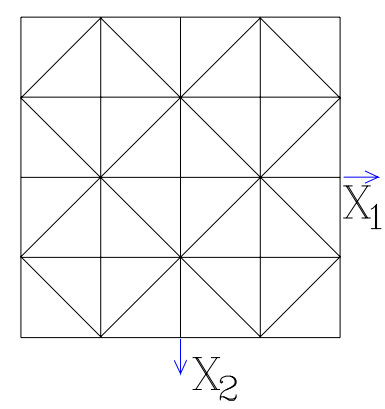

(a)

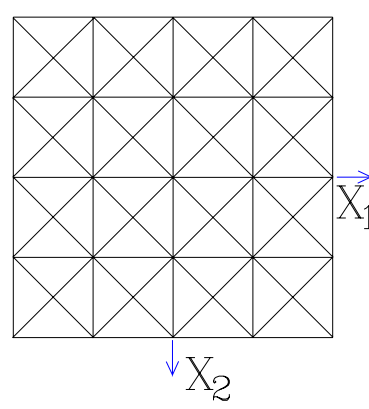

(b)

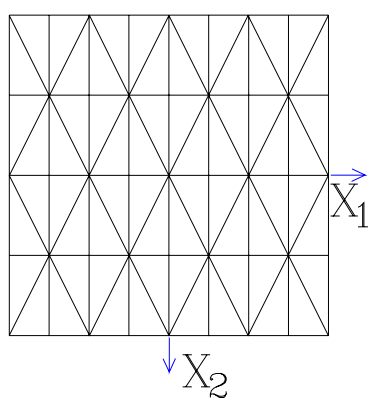

(c)

FIGURA 5.9.6 - Discretizações da superfície fictícia da fratura: (a) 32 elementos constantes, (b) 64 elementos e (c) 64 elementos.

Através de um processamento incremental dos deslocamentos prescritos, e outro interno de caráter iterativo, chega-se na análise do sólido quando uma trinca vai se propagando. A tabela 5.9.2 apresenta os valores da abertura de fratura obtidos 
para as três discretizações consideradas. A resposta é mais precisa à medida que a rede de elementos é melhor definida (melhor caso: discretização da figura 5.9.6c).

TABELA 5.9.2 - Valores de $\mathrm{w}_{\mathrm{c}}$ (abertura da fratura) ao longo de $\mathrm{X}_{1}$, para

$$
\Delta \mathrm{u}_{3}=0,00008 \text {. }
$$

\begin{tabular}{|c|c|c|c|}
\hline \multirow{2}{*}{$\mathrm{X}_{1}(\mathrm{~m})$} & \multicolumn{3}{|c|}{ Valores da abertura da fratura $\left(\times 10^{-5} \mathrm{~m}\right)$} \\
\cline { 2 - 4 } & Discretização (a) & Discretização (b) & Discretização (c) \\
\hline 0 & 0 & 0 & 0 \\
\hline 0,1 & 2,156 & 2,407 & 2,386 \\
\hline 0,2 & 3,546 & 3,167 & 3,213 \\
\hline 0,3 & 4,045 & 3,839 & 3,823 \\
\hline 0,4 & 4,444 & 4,424 & 4,459 \\
\hline 0,5 & 4,906 & 4,930 & 4,989 \\
\hline 0,6 & 5,435 & 5,418 & 5,507 \\
\hline 0,7 & 5,894 & 5,898 & 6,042 \\
\hline 0,8 & 6,256 & 6,355 & 6,503 \\
\hline 0,9 & 6,609 & 6,790 & 7,041 \\
\hline 1 & 6,99 & 7,225 & 7,615 \\
\hline
\end{tabular}

$\mathrm{Na}$ figura 5.9.7 é mostrado o gráfico dos valores apresentados na tabela 5.9.2, comparando a abertura de fratura determinada utilizando-se as discretizações adotadas. Considera-se a discretização 5.9.6(c) a que melhor representa o comportamento do sólido no fraturamento.

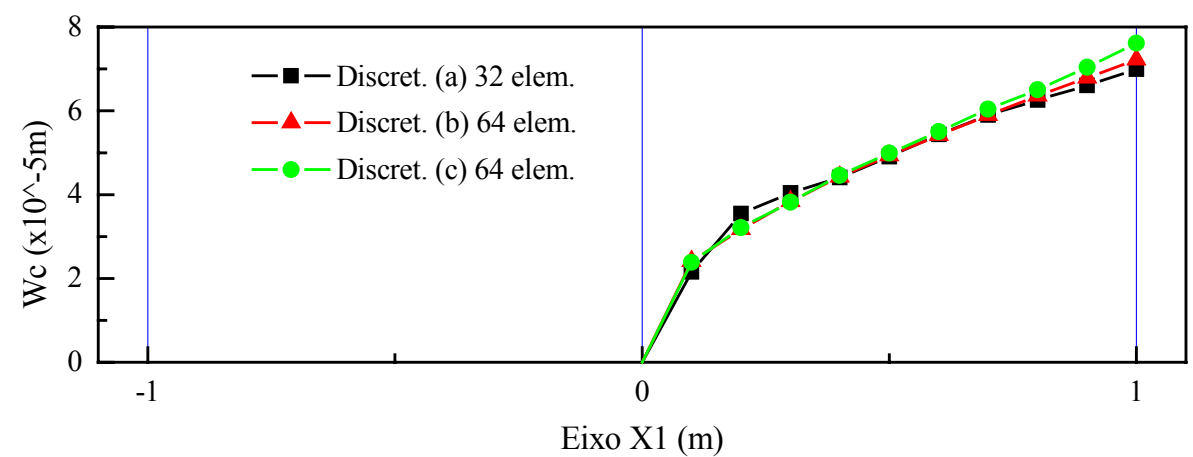

FIGURA 5.9.7 - Abertura da fratura para as discretizações adotadas. 
A figura 5.9.8 mostra os deslocamentos na direção do eixo $X_{3}$ para os três pontos definidos previamente. No ponto 1 , o deslocamento $\mathrm{u}_{3}$ é imposto externamente. $\mathrm{O}$ ponto $2^{\prime}$ tem deslocamento $\mathrm{u}_{3}$ igual à metade do prescrito, até atingir o limite de tensão estabelecido, passando a ter um novo coeficiente angular, tendendo ao valor prescrito. Já o ponto 2", localizado na metade inferior do sólido, tem aproximadamente o mesmo valor do 2' até atingir o limite de tensão, tendendo para o valor zero.

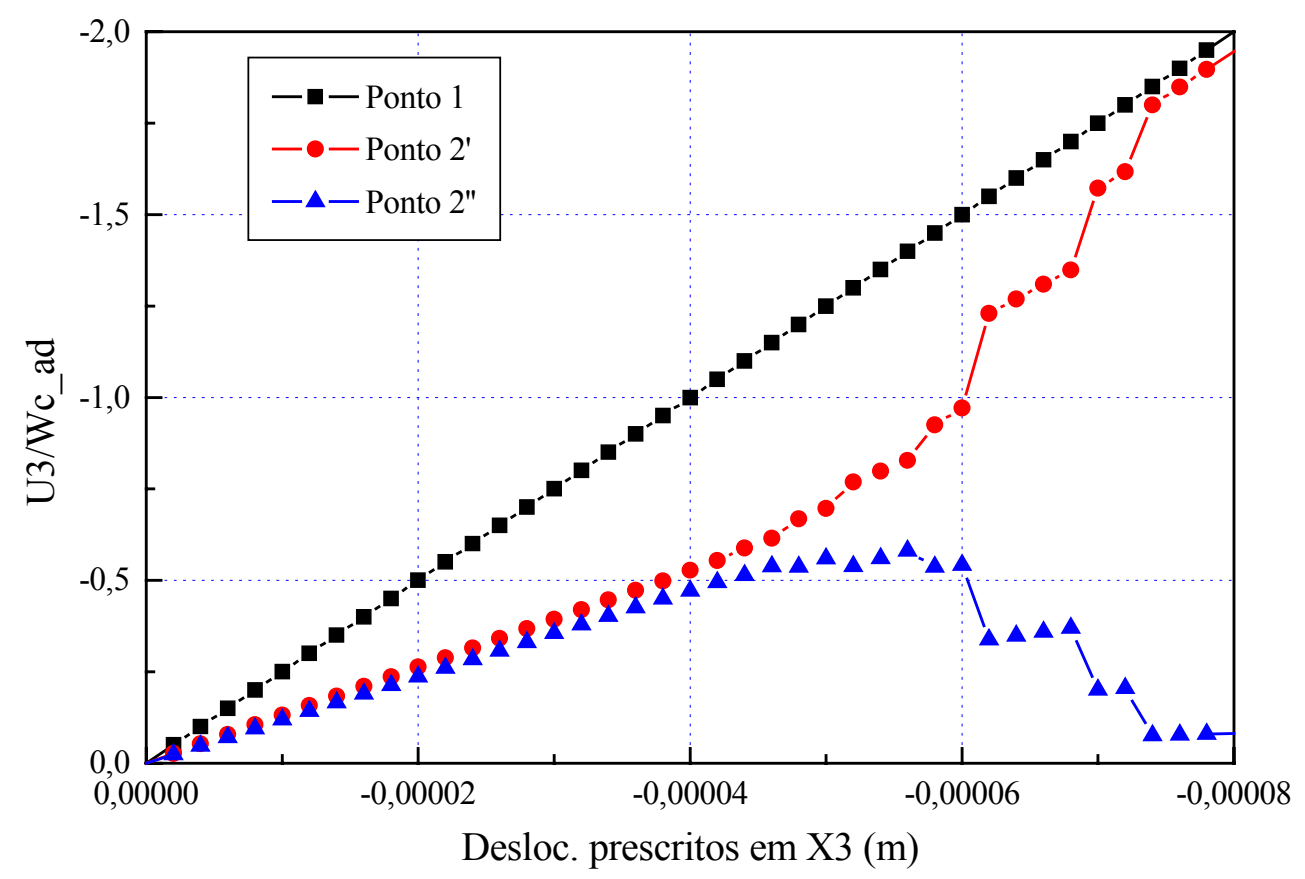

FIGURA 5.9.8 - Deslocamentos na direção do eixo $X_{3}$ para os três pontos previamente definidos.

O critério adotado do modelo de fratura coesiva é verificado, conforme mostra a figura 5.9.9. 


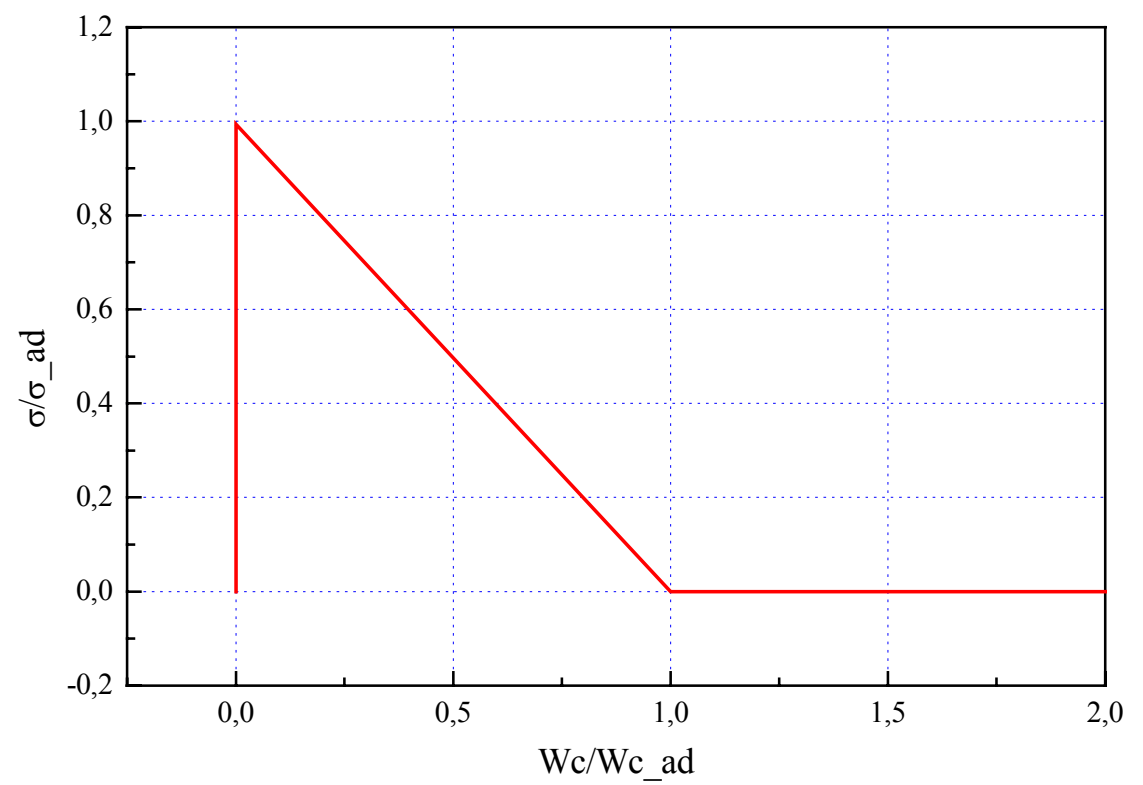

FIGURA 5.9.9 - Redução gradativa da capacidade de resistência da zona de fraturamento - modelo coesivo.

A formulação do Método dos Elementos de Contorno para análise de fraturas mecânicas, apresentada neste capítulo, mostra-se adequada para avaliar sólidos onde há propagação de fratura. O exemplo apresentado mostra a importância da discretização da superfície fictícia da fratura para se chegar a valores mais próximos dos reais. 


\section{CAPÍTULO 6}

\section{FRATURAMENTO DINÂMICO - TEORIA DE TENSÕES INICIAIS E MATRIZ DE MASSA}

\section{1 - INTRODUÇÃO}

Duas formulações para o Método dos Elementos de Contorno foram até aqui desenvolvidas: uma para a análise dinâmica (com células ou reciprocidade dual) e outra para a análise estática de fraturamento. Agora, a intenção é unir as duas formulações a fim de trabalhar com a análise dinâmica de fraturas.

Neste capítulo é apresentado o problema de fratura dinâmica e suas equações básicas. Aplicam-se os procedimentos numéricos para resolução do problema utilizando-se o Método dos Elementos de Contorno. São apresentadas as equações integrais discretizadas, utilizando-se o elemento triangular plano com aproximação linear para o contorno e constante para a superfície fictícia de fratura, além dos pontos internos, quando necessários, adequados ao uso do Método da Reciprocidade Dual. São analisadas aplicações através de um código computacional, a fim de mostrar que o método é adequado na resolução desse tipo de problema. 


\section{2 - EQUAÇÕES GOVERNANTES}

Considere-se um sólido elástico, homogêneo, isotrópico e com distribuição contínua de matéria em seu domínio $\Omega$, submetido a um campo de tensões iniciais, conforme mostra a figura 6.2.1.

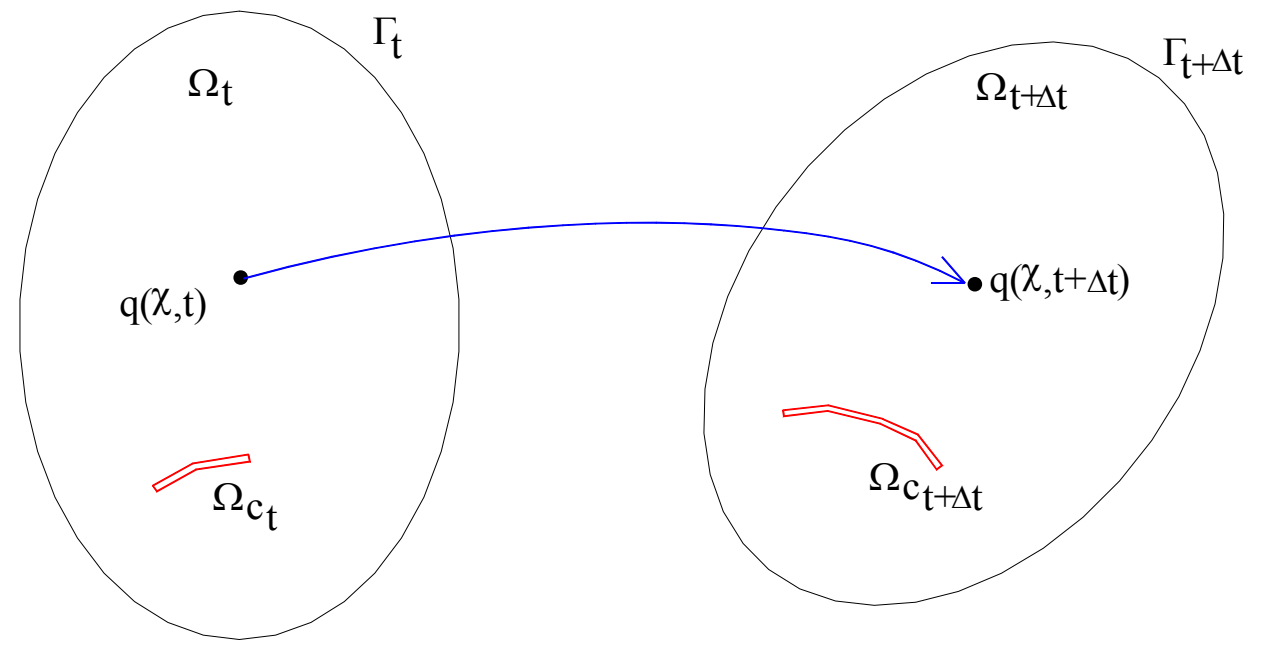

FIGURA 6.2.1 - Sólido em movimento com uma região de tensões iniciais $\Omega_{\mathrm{c}}$.

Admite-se que esse campo de tensões provoque deformações, denominadas de deformações iniciais, caracterizando uma região de descontinuidade representando a fratura.

Considerando-se, ainda, as equações básicas apresentadas nos capítulos 3 e 5 , chega-se à lei de Hooke adaptada para o problema,

$$
\sigma_{\mathrm{ij}}(\chi, \mathrm{t})=\frac{\mathrm{E}}{(1+v)}\left[\frac{v}{(1-2 v))} \delta_{\mathrm{ij}} \varepsilon_{\mathrm{kk}}(\chi, \mathrm{t})+\varepsilon_{\mathrm{ij}}(\chi, \mathrm{t})\right]-\sigma_{\mathrm{ij}}^{0}
$$

onde:

$\sigma_{\mathrm{ij}}^{0}=\lambda \delta_{\mathrm{ij}} \varepsilon_{\mathrm{kk}}^{0}+2 \mathrm{G} \varepsilon_{\mathrm{ij}}^{0}$.

A equação constitutiva (6.2.1) em termos de deslocamentos equivale a: 


$$
\sigma_{i j}(\chi, t)=\lambda \delta_{i j} u_{k, k}(\chi, t)+2 G\left(u_{i, j}(\chi, t)+u_{j, i}(\chi, t)\right)-\sigma_{i j}^{0}
$$

Pode-se obter a equação diferencial do movimento para deslocamentos equação de Navier - admitindo-se a presença de uma região submetida à tensões iniciais:

$$
u_{i, j j}(\chi, t)+\frac{1}{(1-2 v)} u_{j, i j}(\chi, t)+\frac{1}{G} \rho b_{i}(\chi, t)-\frac{1}{G} \rho \ddot{u}_{i}(\chi, t)-\frac{1}{G} \sigma_{i j, j}^{0}=0 .
$$

Para a análise completa do problema elastodinâmico, faz-se necessário estabelecer as condições de contorno. Aqui também, do mesmo modo que no problema elastostático, tem-se as definições das condições essenciais e naturais (2.3.10a-b), relativas ao espaço. São necessárias, ainda, as condições iniciais para qualquer ponto $\chi$ do sólido $\Omega$ no tempo $\mathrm{t}=0$, definidas em (3.2.7a-b).

\section{3 - EQUAÇÕES INTEGRAIS}

A formulação empregada baseia-se na equação Somigliana (2.5.1), acrescentando-se um novo termo correspondente a um campo de tensões iniciais aplicado no domínio, além da influência dinâmica provocada por forças externas atuantes. Portanto, considerando-se as parcelas de forças de massa e de tensões iniciais, a seguinte representação integral de deslocamentos pode ser escrita para pontos do domínio:

$$
\mathrm{u}_{\mathrm{i}}+\int_{\Gamma} \mathrm{p}_{\mathrm{ik}}^{*} \mathrm{u}_{\mathrm{k}} \mathrm{d} \Gamma=\int_{\Gamma} \mathrm{u}_{\mathrm{ik}}^{*} \mathrm{p}_{\mathrm{k}} \mathrm{d} \Gamma-\rho \int_{\Omega} \mathrm{u}_{\mathrm{ik}}^{*} \ddot{\mathrm{u}}_{\mathrm{k}} \mathrm{d} \Omega+\int_{\Omega_{\mathrm{c}}} \varepsilon_{\mathrm{ijk}}^{*} \sigma_{\mathrm{jk}}^{\mathrm{o}} \mathrm{d} \Omega
$$

As operações algébricas realizadas nos capítulos anteriores, tanto para a formulação dinâmica quanto para a formulação de fratura, são também utilizadas para a obtenção de (6.3.1). Assim, considerando-se o conceito de dipolo definido em 
(5.3.30) e as transformações pertinentes para que (6.3.1) também tenha validade para pontos do contorno, tem-se:

$$
\mathrm{c}_{\mathrm{ik}} \mathrm{u}_{\mathrm{k}}+\int_{\Gamma} \mathrm{p}_{\mathrm{ik}}^{*} \mathrm{u}_{\mathrm{k}} \mathrm{d} \Gamma=\int_{\Gamma} \mathrm{u}_{\mathrm{ik}}^{*} \mathrm{p}_{\mathrm{k}} \mathrm{d} \Gamma-\rho \int_{\Omega} \mathrm{u}_{\mathrm{ik}}^{*} \ddot{\mathrm{u}}_{\mathrm{k}} \mathrm{d} \Omega+\int_{\Gamma_{\mathrm{c}}} \mathrm{G}_{\mathrm{ij}}^{\ell} \mathrm{q}_{\mathrm{j}}^{\ell} \mathrm{d} \Gamma
$$

Os tensores $\mathrm{u}_{\mathrm{ik}}^{*}$ e $\mathrm{p}_{\mathrm{ik}}^{*}$ são os utilizados na elastostática, expressões (2.4.4), (2.4.5), (2.4.8) e (2.4.9). O tensor correspondente ao dipolo é o mesmo apresentado em (5.4.12) e (5.4.13). Os coeficientes $c_{i k}$ são aqueles já definidos em (2.5.9) e permitem o emprego da equação integral de deslocamentos (6.3.2) para pontos do contorno, domínio ou fora dele.

A representação integral para as tensões é obtida derivando-se a equação de deslocamentos (6.3.2), semelhante ao que foi feito para as equações apresentadas em (3.3.6) e (5.6.6). Assim,

$$
\begin{aligned}
\sigma_{\mathrm{ijj}}(\mathrm{s}, \mathrm{t}) & =-\int_{\Gamma} \mathrm{S}_{\mathrm{ijk}}^{*}(\mathrm{~s}, \mathrm{Q}) \mathrm{u}_{\mathrm{k}}(\mathrm{Q}, \mathrm{t}) \mathrm{d} \Gamma+\int_{\Gamma} \mathrm{D}_{\mathrm{ijk}}^{*}(\mathrm{~s}, \mathrm{Q}) \mathrm{p}_{\mathrm{k}}(\mathrm{Q}, \mathrm{t}) \mathrm{d} \Gamma+ \\
& +\int_{\Omega} \rho \mathrm{D}_{\mathrm{ijk}}^{*}(\mathrm{~s}, \mathrm{q}) \mathrm{b}_{\mathrm{k}}(\mathrm{q}, \mathrm{t}) \mathrm{d} \Omega-\int_{\Omega} \rho D_{\mathrm{ijk}}^{*}(\mathrm{~s}, \mathrm{q}) \ddot{\mathrm{u}}_{\mathrm{k}}(\mathrm{q}, \mathrm{t}) \mathrm{d} \Omega+ \\
& +\int_{\Gamma_{\mathrm{c}}} \mathrm{G}_{\mathrm{ik}}^{\mathrm{j} \ell}(\mathrm{s}, \mathrm{q}) \mathrm{q}_{\mathrm{k}}^{\ell}(\mathrm{q}, \mathrm{t}) \mathrm{d} \Gamma+\mathrm{g}_{\mathrm{ik}}^{\mathrm{j} \ell}\left[\sigma_{\mathrm{k} \ell}(\mathrm{p})\right] .
\end{aligned}
$$

onde:

$$
g_{i k}^{j \ell}(p)=\left\{\begin{array}{c}
0 \\
\sigma_{i j}^{o}
\end{array},\right.
$$

para o cálculo da tensão plástica ou elástica, respectivamente.

Os tensores $\mathrm{S}_{\mathrm{ijk}}^{*}$ e $\mathrm{D}_{\mathrm{ijk}}^{*}$ em (6.3.3), relativos à solução fundamental empregada, são os mesmos já apresentados para a eletrostática, ou seja, as expressões (2.5.3), (2.5.4), (2.5.5) e (2.5.6). O tensor $\mathrm{G}_{\mathrm{ik}}^{\mathrm{j} \ell}$ é o mesmo definido em (5.6.14). 


\section{4 - EMPREGO DO MÉTODO DA RECIPROCIDADE DUAL}

Na transformação do termo de domínio em (6.3.2), excetuando-se o termo referente às forças de volume, é utilizado o Método da Reciprocidade Dual (MRD), apresentado em detalhes no capítulo 3. Assim, a representação integral de deslocamentos passa a ser:

$$
\begin{aligned}
\mathrm{c}_{\mathrm{ij}}(\mathrm{S}) \mathrm{u}_{\mathrm{j}}(\mathrm{S}, \mathrm{t}) & =-\int_{\Gamma} \mathrm{p}_{\mathrm{ij}}^{*}(\mathrm{~S}, \mathrm{Q}) \mathrm{u}_{\mathrm{j}}(\mathrm{Q}, \mathrm{t}) \mathrm{d} \Gamma+\int_{\Gamma} \mathrm{u}_{\mathrm{ij}}^{*}(\mathrm{~S}, \mathrm{Q}) \mathrm{p}_{\mathrm{j}}(\mathrm{Q}, \mathrm{t}) \mathrm{d} \Gamma+ \\
& +\rho\left(\mathrm{c}_{\mathrm{ij}}(\chi) \hat{\mathrm{u}}_{\mathrm{jk}}^{\mathrm{m}}(\chi)+\int_{\Gamma} \mathrm{p}_{\mathrm{ij}}^{*}(\mathrm{~s}, \mathrm{q}) \hat{\mathrm{u}}_{\mathrm{jk}}^{\mathrm{m}}(\chi, \mathrm{q}) \mathrm{d} \Gamma+\right. \\
& \left.-\int_{\Gamma} \mathrm{u}_{\mathrm{ij}}^{*}(\mathrm{~s}, \mathrm{q}) \hat{\mathrm{p}}_{\mathrm{jk}}^{\mathrm{m}}(\chi, \mathrm{q}) \mathrm{d} \Gamma\right) \ddot{\alpha}_{\mathrm{k}}^{\mathrm{m}}(\mathrm{t})+\int_{\Gamma_{\mathrm{c}}} \mathrm{G}_{\mathrm{ij}}^{\ell} \mathrm{q}_{\mathrm{j}}^{\ell} \mathrm{d} \Gamma .
\end{aligned}
$$

A representação integral para pontos localizados no domínio é obtida utilizando-se o procedimento clássico, baseado na lei de Hooke (6.2.3), substituindose convenientemente as derivadas da expressão (6.3.2) de deslocamentos. Assim,

$$
\begin{aligned}
\sigma_{\mathrm{ij}}(\mathrm{s}, \mathrm{t}) & =-\int_{\Gamma} \mathrm{S}_{\mathrm{ij} \ell}^{*}(\mathrm{~s}, \mathrm{Q}) \mathrm{u}_{\mathrm{j}}(\mathrm{Q}, \mathrm{t}) \mathrm{d} \Gamma+\int_{\Gamma} D_{\mathrm{ij} \ell}^{*}(\mathrm{~s}, \mathrm{Q}) \mathrm{p}_{\mathrm{j}}(\mathrm{Q}, \mathrm{t}) \mathrm{d} \Gamma+ \\
& +\rho\left\{\hat{\mathrm{D}}_{\mathrm{ij} \ell}^{\mathrm{k}}(\mathrm{s}, \mathrm{q})+\int_{\Gamma} \mathrm{S}_{\mathrm{ij} \ell}^{*}(\mathrm{~s}, \mathrm{q}) \hat{\mathrm{u}}_{\mathrm{jk}}^{\mathrm{m}}(\mathrm{s}, \mathrm{q}) \mathrm{d} \Gamma-\int_{\Gamma} D_{\mathrm{ij} \ell}^{*}(\mathrm{~s}, \mathrm{q}) \hat{\mathrm{p}}_{\mathrm{jk}}^{\mathrm{m}}(\mathrm{s}, \mathrm{q}) \mathrm{d} \Gamma\right\} \ddot{\alpha}_{\mathrm{k}}^{\mathrm{m}}(\mathrm{t})+ \\
& +\int_{\Gamma_{\mathrm{c}}} \mathrm{G}_{\mathrm{ik}}^{\mathrm{j} \ell}(\mathrm{s}, \mathrm{q}) \mathrm{q}_{\mathrm{k}}^{\ell}(\mathrm{q}, \mathrm{t}) \mathrm{d} \Gamma+\mathrm{g}_{\mathrm{ik}}^{\mathrm{j} \ell}\left[\sigma_{\mathrm{k} \ell}(\mathrm{p})\right],
\end{aligned}
$$

onde os tensores $\mathrm{S}_{\mathrm{ij \textrm {k }}}^{*}$ e $\mathrm{D}_{\mathrm{ij \textrm {k }}}^{*}$ são os mesmos apresentados nas expressões (2.5.3), (2.5.4), (2.5.5) e (2.5.6) e $\hat{\mathrm{D}}_{\mathrm{ijk}}$ é o tensor definido em (3.4.15). 


\section{5 - DISCRETIZAÇÕES}

As representações integrais (6.4.1) e (6.4.2) são discretizadas utilizando-se os elementos e as aproximações definidos no item 2.6.

Além dos "J" elementos de contorno e dos " $\mathrm{N}$ " pontos nodais (nós funcionais), deve-se considerar o número de pólos, denotado por " $\mathrm{Np}$ ", e os “ $\mathrm{J}_{\mathrm{c}}$ " elementos constantes utilizados na superfície fictícia da fratura.

Considere-se a expressão integral para deslocamentos (6.4.1). Substituem-se os deslocamentos e forças de superfície $\left(u_{j}\right.$ e $\left.p_{j}\right)$ pelo produto entre as funções aproximadoras e seus respectivos valores nodais, expressões (2.6.2a-b). O mesmo deve ser feito para os tensores dos deslocamentos e forças de superfície do problema particular $\left(\hat{\mathrm{u}}_{\mathrm{jk}}^{\mathrm{m}} \mathrm{e} \hat{\mathrm{p}}_{\mathrm{jk}}^{\mathrm{m}}\right)$. Adotando-se as mesmas funções aproximadoras de (2.6.2a-b), chega-se às mesmas matrizes $\mathbf{H}$ e $\mathbf{G}$ do contorno, também para o termo de domínio transformado para o contorno em (6.4.1). Portanto, adotam-se:

$$
\begin{aligned}
& \hat{\mathbf{u}}_{\mathrm{k}}=\phi^{\mathrm{T}} \hat{\mathbf{U}}_{\mathrm{m}}^{\mathrm{n}} \mathrm{e} \\
& \hat{\mathbf{p}}_{\mathrm{k}}=\phi^{\mathrm{T}} \hat{\mathbf{P}}_{\mathrm{m}}^{\mathrm{n}} .
\end{aligned}
$$

A expressão (6.4.1), na sua forma discretizada, passa a ser:

$$
\begin{aligned}
\mathbf{c}(\mathrm{S}, \mathrm{t}) \mathbf{u}(\mathrm{S}, \mathrm{t})= & -\sum_{\mathrm{j}=1}^{\mathrm{J}+\mathrm{J}_{\mathrm{c}}}\left[\int_{\Gamma_{\mathrm{j}}} \mathbf{p}^{*}(\mathrm{~S}, \mathrm{Q}) \phi^{\mathrm{T}}(\mathrm{Q}) \mathrm{d} \Gamma\right] \mathbf{U}^{\mathrm{n}}(\mathrm{Q}, \mathrm{t})+ \\
& +\sum_{\mathrm{j}=1}^{\mathrm{J}+\mathrm{J}_{\mathrm{c}}}\left[\int_{\Gamma_{\mathrm{j}}} \mathbf{u}^{*}(\mathrm{~S}, \mathrm{Q}) \phi^{\mathrm{T}}(\mathrm{Q}) \mathrm{d} \Gamma\right] \mathbf{P}^{\mathrm{n}}(\mathrm{Q}, \mathrm{t})+ \\
& +\rho\left\{\mathbf{c}(\mathrm{s}) \hat{\mathbf{u}}^{\mathrm{m}}(\mathrm{s}, \mathrm{t})+\sum_{\mathrm{j}=1}^{\mathrm{J}+\mathrm{J}_{\mathrm{c}}}\left[\int_{\Gamma_{\mathrm{j}}} \mathbf{p}^{*}(\mathrm{~s}, \mathrm{q}) \phi^{\mathrm{T}}(\mathrm{q}) \mathrm{d} \Gamma\right] \mathbf{U}_{\mathrm{m}}^{\mathrm{n}}(\mathrm{s}, \mathrm{q})+\right. \\
& \left.-\sum_{\mathrm{j}=1}^{\mathrm{J}+\mathrm{J}_{\mathrm{c}}}\left[\int_{\Gamma_{\mathrm{j}}} \mathbf{u}^{*}(\mathrm{~s}, \mathrm{q}) \phi^{\mathrm{T}}(\mathrm{q}) \mathrm{d} \Gamma\right] \mathbf{P}_{\mathrm{m}}^{\mathrm{n}}(\mathrm{s}, \mathrm{q})\right\} \ddot{\alpha}^{\mathrm{m}}(\mathrm{t})+ \\
& +\sum_{\mathrm{j}=1}^{\mathrm{J}_{\mathrm{c}}}\left[\int_{\Gamma_{\mathrm{j}}} \mathbf{G}^{*}(\mathrm{~s}, \mathrm{q}) \mathrm{d} \Gamma\right] \mathbf{Q}(\mathrm{q}, \mathrm{t}) .
\end{aligned}
$$


Efetuadas as integrais de (6.5.1) para todos os pontos de colocação, representadas na forma matricial, tem-se:

$$
\overline{\mathbf{c}} \mathbf{U}+\hat{\mathbf{H}} \mathbf{U}=\mathbf{G P}+\rho(\overline{\mathbf{c}} \hat{\mathbf{U}}+\hat{\mathbf{H}} \hat{\mathbf{U}}-\mathbf{G} \hat{\mathbf{P}}) \ddot{\alpha}+\mathbf{K Q},
$$

ou, ainda,

$$
\mathbf{H U}=\mathbf{G P}-\mathbf{M} \ddot{\mathbf{U}}+\mathbf{K Q},
$$

onde $\mathbf{M}$ é a matriz de massa definida por

$$
\mathbf{M}=-\rho(\mathbf{H} \hat{\mathbf{U}}-\mathbf{G} \hat{\mathbf{P}}) \mathbf{F}^{-1}
$$

A equação de deslocamentos para pontos do domínio está também definida em (6.5.2), ou seja,

$$
\mathbf{u}=-\mathbf{H U}+\mathbf{G P}-\mathbf{M} \ddot{\mathbf{U}}+\mathbf{K Q}
$$

Para o cálculo das tensões em pontos do domínio, utiliza-se o mesmo procedimento de discretização efetuado para a equação de deslocamentos que aplicado à equação (6.4.2), resulta em:

$$
\begin{aligned}
\sigma(\mathrm{s}, \mathrm{t}) & =-\sum_{\mathrm{j}=1}^{\mathrm{J}+\mathrm{J}_{\mathrm{c}}}\left[\int_{\Gamma_{\mathrm{j}}} \mathbf{S}^{*}(\mathrm{~s}, \mathrm{Q}) \phi^{\mathrm{T}}(\mathrm{Q}) \mathrm{d} \Gamma\right] \mathbf{U}^{\mathrm{n}}(\mathrm{Q}, \mathrm{t})+ \\
& +\sum_{\mathrm{j}=1}^{\mathrm{J}+\mathrm{J}_{\mathrm{c}}}\left[\int_{\Gamma_{\mathrm{j}}} \mathbf{D}^{*}(\mathrm{~s}, \mathrm{Q}) \phi^{\mathrm{T}}(\mathrm{Q}) \mathrm{d} \Gamma\right] \mathbf{P}^{\mathrm{n}}(\mathrm{Q}, \mathrm{t})+ \\
& +\rho\left\{\hat{\mathbf{D}}^{\mathrm{k}}(\mathrm{s}, \mathrm{q})+\sum_{\mathrm{j}=1}^{\mathrm{J}+\mathrm{J}_{\mathrm{c}}}\left[\int_{\Gamma_{\mathrm{j}}} \mathbf{S}^{*}(\mathrm{~s}, \mathrm{Q}) \phi^{\mathrm{T}}(\mathrm{Q}) \mathrm{d} \Gamma\right] \hat{\mathbf{U}}^{\mathrm{k}}(\mathrm{s}, \mathrm{q})+\right. \\
& \left.-\sum_{\mathrm{j}=1}^{\mathrm{J}+\mathrm{J}_{\mathrm{c}}}\left[\int_{\Gamma_{\mathrm{j}}} \mathbf{D}^{*}(\mathrm{~s}, \mathrm{Q}) \phi^{\mathrm{T}}(\mathrm{Q}) \mathrm{d} \Gamma\right] \hat{\mathbf{P}}^{\mathrm{k}}(\mathrm{s}, \mathrm{q})\right\} \mathbf{F}^{-1}(\mathrm{~s}, \mathrm{q}) \ddot{\mathbf{U}}^{\mathrm{k}}(\mathrm{q}, \mathrm{t})+ \\
& +\sum_{\mathrm{j}=1}^{\mathrm{J}_{\mathrm{c}}}\left[\int_{\Gamma_{\mathrm{j}}}\left[\mathbf{G}_{\sigma}^{*}(\mathrm{~s}, \mathrm{q}) \mathrm{d} \Gamma\right] \mathbf{Q}(\mathbf{q}, \mathbf{t}),\right.
\end{aligned}
$$


Escrevendo-se (6.5.5) de forma matricial, tem-se

$$
\sigma=-\mathbf{H}^{\prime} \mathbf{U}+\mathbf{G}^{\prime} \mathbf{P}-\mathbf{M}^{\prime} \ddot{\mathbf{U}}+\mathbf{K}^{\prime} \mathbf{Q}
$$

onde,

$$
\mathbf{M}^{\prime}=-\rho\left(\hat{\mathbf{D}}+\mathbf{H}^{\prime} \hat{\mathbf{U}}-\mathbf{G}^{\prime} \hat{\mathbf{P}}\right) \mathbf{F}^{-1}
$$

\section{6 - SOLUÇÃO DAS EQUAÇÕES}

No item 3.6.6 são apresentados os algoritmos de Newmark e Houbolt para a integração no tempo, destacando-se as equações básicas e o conveniente particionamento das matrizes $\mathbf{H}, \mathbf{G}$ e M. Já no item 5.7 é apresentado um algoritmo incremental iterativo para resolução das equações de deslocamentos e tensões, levando-se em conta a contribuição do dipolos (representando a existência da fratura). A intenção, agora, é unir os dois algoritmos (integração no tempo e iterativo) para a solução do problema de fratura dinâmica.

\subsection{1 - Algoritmo de Newmark}

Admitindo-se a separação das matrizes em sub-matrizes convenientemente, conforme 3.6.1, a equação de deslocamentos (6.5.3) passa a ser:

$$
\mathbf{M U ̈}+\mathbf{H U}=\mathbf{G P}+\mathbf{K Q}
$$

Empregando-se a equação de Newmark para a partida do processo (3.6.1) à equação do movimento (6.6.1), obtém-se o sistema algébrico:

$$
\mathbf{A} \mathbf{U}_{1}=\mathbf{F}_{1}+\mathbf{K} \mathbf{Q}_{1}
$$


onde:

$$
\begin{aligned}
& \mathbf{A}=\left[\mathbf{M}+\beta(\Delta \mathrm{t})^{2} \mathbf{H}\right] \\
& \mathbf{F}_{1}=(\Delta \mathrm{t})^{2} \beta \overline{\mathbf{F}}_{1}+(\Delta \mathrm{t})^{2}\left[\left(\frac{1}{2}-\beta\right) \mathbf{I}\right] \overline{\mathbf{F}}_{\mathrm{o}}
\end{aligned}
$$

A solução do sistema (6.6.2) é obtida por:

$$
\mathbf{U}_{1}=\overline{\mathbf{M}}_{1}+\mathbf{R} \mathbf{Q}_{1},
$$

onde:

$\overline{\mathbf{M}}_{1}=\mathbf{A}^{-1} \mathbf{F}_{1}$, representa a parcela elástica dos deslocamentos;

$\mathbf{R}=\mathbf{A}^{-1} \mathbf{K}$, representa a parcela não-linear, relacionada à presença da fratura.

Para os demais termos e adotando-se Newmark, considera-se a expressão (3.6.2) que aplicada à (6.6.1) resulta em:

$$
\mathbf{A} \mathbf{U}_{\mathrm{t}+1}=\mathbf{F}_{\mathrm{t}+1}+\mathbf{K} \mathbf{Q}_{\mathrm{t}+1}
$$

onde:

$$
\begin{aligned}
& \mathbf{A}=\left[\mathbf{M}+\beta(\Delta t)^{2} \mathbf{H}\right] \\
& \mathbf{B}=\left[2 \mathbf{M}-(\Delta \mathrm{t})^{2}(1-2 \beta) \mathbf{H}\right] \\
& \mathbf{F}_{\mathrm{t}+1}=(\Delta \mathrm{t})^{2}\left[\beta \overline{\mathbf{F}}_{\mathrm{t}+1}+(1-2 \beta) \overline{\mathbf{F}}_{\mathrm{t}}+\beta \overline{\mathbf{F}}_{\mathrm{t}-1}\right]+\mathbf{B} \mathbf{U}_{\mathrm{t}}-\mathbf{A} \mathbf{U}_{\mathrm{t}-1}
\end{aligned}
$$

A solução do sistema (6.6.6) é:

$$
\mathbf{U}_{\mathrm{t}+1}=\overline{\mathbf{M}}_{\mathrm{t}+1}+\mathbf{R} \mathbf{Q}_{\mathrm{t}+1}
$$


onde:

$$
\begin{aligned}
& \overline{\mathbf{M}}_{\mathrm{t}+1}=\mathbf{A}^{-1} \mathbf{F}_{\mathrm{t}+1} \\
& \mathbf{R}=\mathbf{A}^{-1} \mathbf{K}
\end{aligned}
$$

De maneira semelhante ao que foi feito para a representação de deslocamentos, pode-se trabalhar a expressão de tensões (6.5.6). Tem-se, portanto,

$$
\sigma+\mathbf{M}^{\prime} \ddot{\mathbf{U}}+\mathbf{H}^{\prime} \mathbf{U}=\mathbf{G}^{\prime} \mathbf{P}+\mathbf{K}^{\prime} \mathbf{Q}
$$

Aplicando-se a expressão do algoritmo para o termo de partida, tem-se:

$$
\sigma_{1}+\mathbf{A}_{1}^{\prime} \mathbf{U}=\mathbf{F}_{1}^{\prime}+\mathbf{K}^{\prime} \mathbf{Q}_{1}
$$

onde:

$$
\begin{aligned}
& \mathbf{A}^{\prime}=\left\lfloor\mathbf{M}^{\prime}+\beta(\Delta \mathrm{t})^{2} \mathbf{H}^{\prime}\right\rfloor \\
& \mathbf{F}_{1}^{\prime}=(\Delta \mathrm{t})^{2} \beta \overline{\mathbf{F}}_{1}+(\Delta \mathrm{t})^{2}\left[\left(\frac{1}{2}-\beta\right) \mathbf{I}\right] \overline{\mathbf{F}}_{\mathrm{o}} .
\end{aligned}
$$

Utilizando-se os deslocamentos calculados em (6.6.4), para o primeiro termo, pode-se escrever (6.6.11) da forma:

$$
\sigma_{1}+\mathbf{A}_{1}^{\prime}\left(\overline{\mathbf{M}}_{1}+\mathbf{R} \mathbf{Q}_{1}\right)=\mathbf{F}_{1}^{\prime}+\mathbf{K}^{\prime} \mathbf{Q}_{1}
$$

ou

$$
\sigma_{1}=\mathbf{N}_{1}+\mathbf{S Q}_{1}
$$

onde

$$
\begin{aligned}
& \mathbf{N}_{1}=\mathbf{F}_{1}^{\prime}-\mathbf{A}^{\prime} \overline{\mathbf{M}}_{1} \\
& \mathbf{S}=\mathbf{K}^{\prime}-\mathbf{A}^{\prime} \mathbf{R} .
\end{aligned}
$$

Aplicando-se o algoritmo de Newmark para os demais termos, tem-se: 


$$
\sigma_{\mathrm{t}+1}+\mathbf{A}^{\prime} \mathbf{U}_{\mathrm{t}+1}=\mathbf{F}_{\mathrm{t}+1}^{\prime}+\mathbf{K}^{\prime} \mathbf{Q}_{\mathrm{t}+1}
$$

onde:

$$
\begin{aligned}
& \mathbf{A}^{\prime}=\left[\mathbf{M}^{\prime}+\beta(\Delta t)^{2} \mathbf{H}^{\prime}\right] \\
& \mathbf{B}^{\prime}=\left[2 \mathbf{M}^{\prime}-(\Delta t)^{2}(1-2 \beta) \mathbf{H}^{\prime}\right] \\
& \mathbf{F}_{t+1}^{\prime}=(\Delta t)^{2}\left[\beta \overline{\mathbf{F}}_{\mathrm{t}+1}+(1-2 \beta) \overline{\mathbf{F}}_{\mathrm{t}}+\beta \overline{\mathbf{F}}_{\mathrm{t}-1}\right]+\mathbf{B}^{\prime} \mathbf{U}_{\mathrm{t}}-\mathbf{A}^{\prime} \mathbf{U}_{\mathrm{t}-1} .
\end{aligned}
$$

Utilizando-se os deslocamentos calculados em (6.6.8), a expressão (6.6.16) passa a ser:

$$
\sigma_{t+1}+\mathbf{A}^{\prime}\left(\overline{\mathbf{M}}_{\mathrm{t}+1}+\mathbf{R} \mathbf{Q}_{\mathrm{t}+1}\right)=\mathbf{F}_{\mathrm{t}+1}^{\prime}+\mathbf{K}^{\prime} \mathbf{Q}_{\mathrm{t}+1}
$$

Portanto, as tensões no instante de tempo $\mathrm{t}+1$ são calculados utilizando-se

$$
\sigma_{\mathrm{t}+1}=\mathbf{N}_{\mathrm{t}+1}+\mathbf{S} \mathbf{Q}_{\mathrm{t}+1},
$$

onde:

$$
\begin{aligned}
& \mathbf{N}_{\mathrm{t}+1}=\mathbf{F}_{\mathrm{t}+1}^{\prime}-\mathbf{A}^{\prime} \overline{\mathbf{M}}_{\mathrm{t}+1} \\
& \mathbf{S}=\mathbf{K}^{\prime}-\mathbf{A}^{\prime} \mathbf{R} .
\end{aligned}
$$

\subsection{2 - Algoritmo de Houbolt}

Considere-se a equação do movimento definida em (6.5.3). Utilizando-se a formulação do algoritmo de Houbolt apresentada no item 3.6.2, tem-se, para um instante de tempo genérico,

$$
\mathbf{M} \ddot{\mathbf{U}}_{\mathrm{t}+\Delta \mathrm{t}}+\mathbf{H} \mathbf{U}_{\mathrm{t}+\Delta \mathrm{t}}=\mathbf{G} \mathbf{P}_{\mathrm{t}+\Delta \mathrm{t}}+\mathbf{K} \mathbf{Q}_{\mathrm{t}+\Delta \mathrm{t}}
$$


ou

$$
\overline{\mathbf{H}} \mathbf{U}_{\mathrm{t}+\Delta \mathrm{t}}=\mathbf{G} \mathbf{P}_{\mathrm{t}+\Delta \mathrm{t}}+\overline{\mathbf{F}}_{\mathrm{t}+\Delta \mathrm{t}}+\mathbf{K} \mathbf{Q}_{\mathrm{t}+\Delta \mathrm{t}}
$$

onde

$$
\begin{aligned}
& \overline{\mathbf{H}}=\left[\frac{2}{\Delta \mathrm{t}^{2}} \mathbf{M}+\mathbf{H}\right] \\
& \overline{\mathbf{F}}_{\mathrm{t}+\Delta \mathrm{t}}=\mathbf{M}\left[\frac{1}{\Delta \mathrm{t}^{2}}\left(5 \mathbf{U}_{\mathrm{t}}-4 \mathbf{U}_{\mathrm{t}-\Delta \mathrm{t}}+\mathbf{U}_{\mathrm{t}-2 \Delta \mathrm{t}}\right)\right] .
\end{aligned}
$$

Fazendo-se a troca de colunas entre as matrizes $\mathbf{H}$ e $\mathbf{G}$, do modo usual do MEC, a fim de estabelecer um vetor de incógnitas (deslocamentos e forças de superfície) e um vetor de valores prescritos, obtém-se a seguinte solução:

$$
\mathbf{X}_{\mathrm{t}+\Delta \mathrm{t}}=\overline{\mathbf{M}}_{\mathrm{t}+\Delta \mathrm{t}}+\mathbf{R} \mathbf{Q}_{\mathrm{t}+\Delta \mathrm{t}}
$$

onde:

$$
\begin{aligned}
& \mathbf{X}_{\mathrm{t}+\Delta \mathrm{t}}=\text { vetor misto de incógnitas } \\
& \overline{\mathbf{M}}_{\mathrm{t}+\Delta \mathrm{t}}=\mathbf{A}^{-1}\left(\mathbf{G P}_{\mathrm{t}+\Delta \mathrm{t}}+\overline{\mathbf{F}}_{\mathrm{t}+\Delta \mathrm{t}}\right) \\
& \mathbf{R}=\mathbf{A}^{-1} \mathbf{K}
\end{aligned}
$$

De maneira semelhante, manipula-se algebricamente a equação das tensões (6.5.6) resultando em:

$$
\sigma_{\mathrm{t}+\Delta \mathrm{t}}+\overline{\mathbf{H}}^{\prime} \mathbf{U}_{\mathrm{t}+\Delta \mathrm{t}}=\mathbf{G}^{\prime} \mathbf{P}_{\mathrm{t}+\Delta \mathrm{t}}+\overline{\mathbf{F}}_{\mathrm{t}+\Delta \mathrm{t}}^{\prime}+\mathbf{K}^{\prime} \mathbf{Q}_{\mathrm{t}+\Delta \mathrm{t}}
$$

onde: 


$$
\begin{aligned}
& \overline{\mathbf{H}}^{\prime}=\left[\frac{2}{\Delta \mathrm{t}^{2}} \mathbf{M}^{\prime}+\mathbf{H}^{\prime}\right] \\
& \overline{\mathbf{F}}_{\mathrm{t}+\Delta \mathrm{t}}^{\prime}=\mathbf{M}^{\prime}\left[\frac{1}{\Delta \mathrm{t}^{2}}\left(5 \mathbf{U}_{\mathrm{t}}-4 \mathbf{U}_{\mathrm{t}-\Delta \mathrm{t}}+\mathbf{U}_{\mathrm{t}-2 \Delta \mathrm{t}}\right)\right] .
\end{aligned}
$$

A expressão (6.6.26) pode ser ainda escrita na forma:

$$
\sigma_{\mathrm{t}+\Delta \mathrm{t}}+\mathbf{A}^{\prime} \mathbf{X}_{\mathrm{t}+\Delta \mathrm{t}}=\mathbf{F}_{\mathrm{t}+\Delta \mathrm{t}}^{\prime}+\mathbf{K}^{\prime} \mathbf{Q}_{\mathrm{t}+\Delta \mathrm{t}}
$$

onde:

$\mathbf{X}_{\mathrm{t}+\Delta \mathrm{t}}=$ vetor misto de incógnitas

$\mathbf{F}_{\mathrm{t}+\Delta \mathrm{t}}^{\prime}=\mathbf{G}^{\prime} \mathbf{P}_{\mathrm{t}+\Delta \mathrm{t}}+\overline{\mathbf{F}}_{\mathrm{t}+\Delta \mathrm{t}}^{\prime}$

Utilizando-se o vetor de incógnitas definido em (6.6.24), tem-se:

$$
\sigma_{\mathrm{t}+\Delta t}=\mathbf{N}_{\mathrm{t}+\Delta \mathrm{t}}+\mathbf{S} \mathbf{Q}_{\mathrm{t}+\Delta \mathrm{t}},
$$

onde:

$$
\begin{aligned}
& \mathbf{N}_{\mathrm{t}+\Delta \mathrm{t}}=\mathbf{F}_{\mathrm{t}+\Delta \mathrm{t}}^{\prime}-\mathbf{A}^{\prime} \overline{\mathbf{M}}_{\mathrm{t}+\Delta \mathrm{t}} \\
& \mathbf{S}=\mathbf{K}^{\prime}-\mathbf{A}^{\prime} \mathbf{R} .
\end{aligned}
$$




\section{7 - APLICAÇÕES}

6.7.1 - Aplicação 1: Problema de Chen.

O problema de uma chapa com uma fratura central e solicitada por tensões dinâmicas nas extremidades opostas foi resolvido em CHEN (1975) utilizando diferenças finitas. Constitui-se em um trabalho de referência para a avaliação de outras formulações sobre fratura dinâmica. Neste exemplo, para testar a formulação apresentada, tem-se a resolução desse problema (na forma tridimensional).

Considere-se o problema de um sólido paralelepipédico solicitado por tensões de tração nas extremidades opostas, conforme mostra a figura 6.7.1. Define-se, ainda, uma descontinuidade em sua seção transversal média. Os parâmetros elastodinâmicos adotados são: $\quad E=2 \times 10^{5} \mathrm{GPa}, \quad v=0,3, \quad \rho=500 \mathrm{~kg} / \mathrm{m}^{3}$, $\Delta \mathrm{t}=0,0004 \mathrm{~s}, \mathrm{f}_{\mathrm{t}}=0,0 \mathrm{~Pa}$ e a largura da fratura $2 \mathrm{a}=4,8 \mathrm{~mm}$.
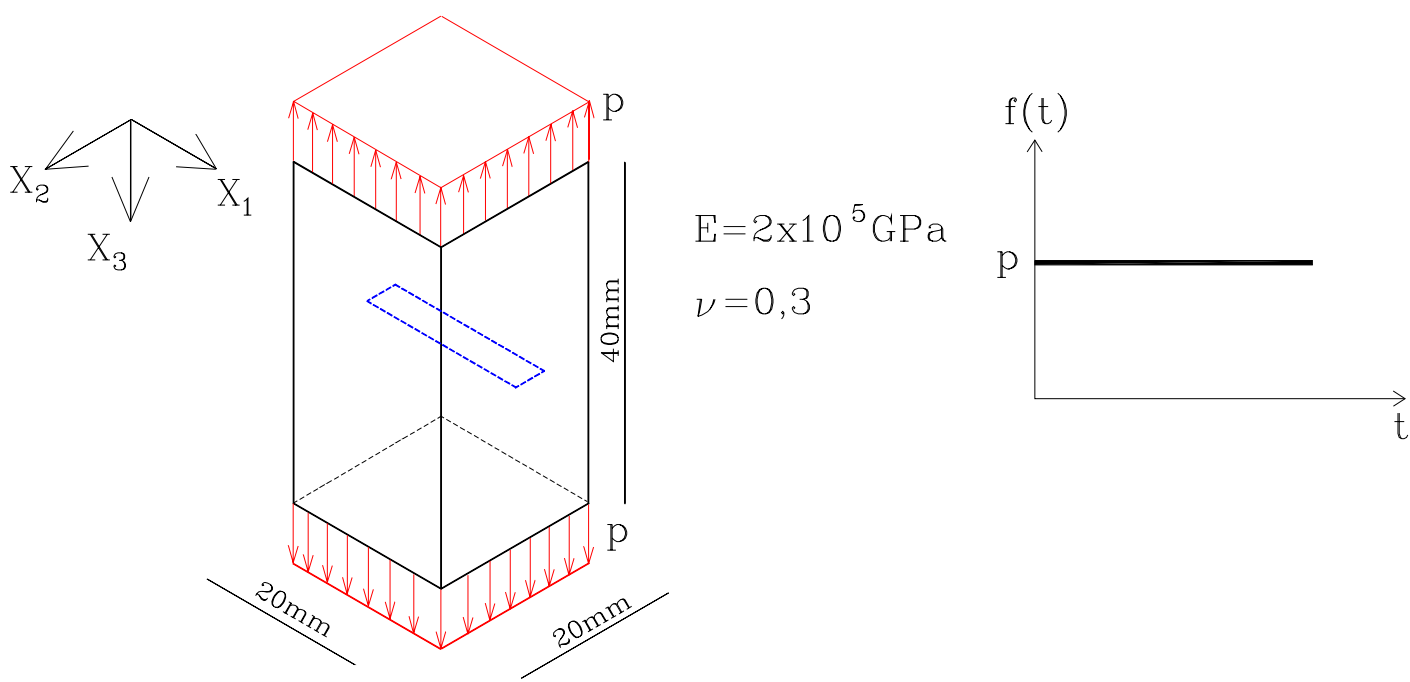

FIGURA 6.7.1 - Definição do objeto de estudo: geometria do sólido, condições de contorno e comportamento temporal da força. 
O contorno do sólido é discretizado utilizando-se 80 elementos triangulares planos, conforme mostra a figura 6.7.2.

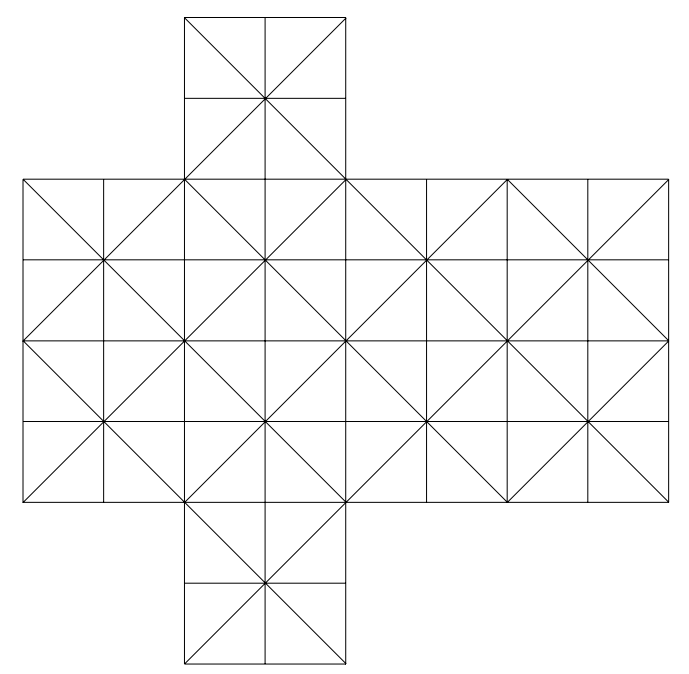

FIGURA 6.7.2 - Discretização do contorno do sólido por elementos lineares.

A descontinuidade na seção média do sólido é discretizada por elementos triangulares com aproximação constante (figura 6.7.3).

FIGURA 6.7.3 - Discretização da superfície da trinca na seção central do sólido.

O exemplo é resolvido utilizando-se um algoritmo computacional incremental (no tempo) e iterativo, cuja formulação encontra-se no item 6.2.2 deste capítulo. São medidas as tensões em pontos próximos da linha "extremidade da trinca" e apresentadas na forma de fatores de intensidade de tensão (relativos ao módulo I de fraturamento), a fim de comparar com a curva apresentada em CHEN (1975). Os resultados são mostrados na figura 6.7.4. 


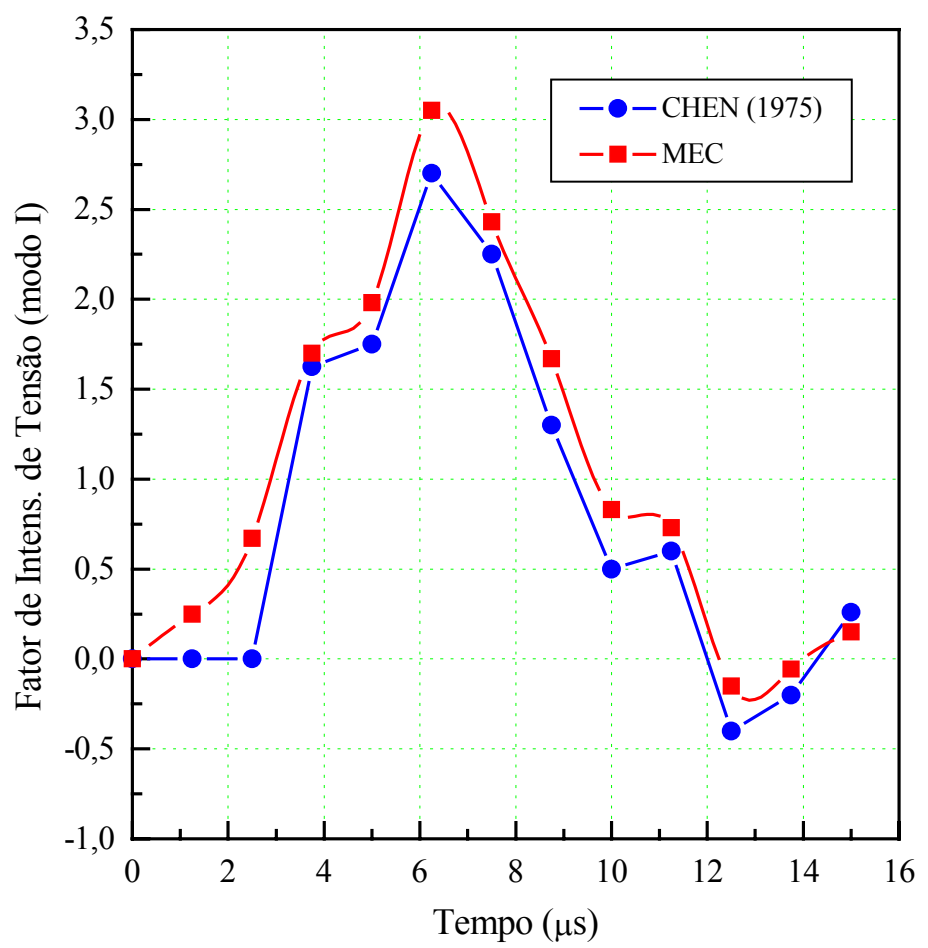

FIGURA 6.7.4 - Valores de $\mathrm{K}_{\mathrm{I}}$ (normalizado por $\sigma \sqrt{\pi \mathrm{a}}$ ) ao longo do tempo.

De acordo com a figura 6.7.4, comprova-se que a formulação apresentada fornece bons resultados, estando em conformidade com valores apresentados por outras soluções.

6.7.2 - Aplicação 2: Sólido paralelepipédico elástico solicitado por tensões dinâmicas.

Como outro teste da formulação apresentada, considera-se o exemplo de um sólido paralelepipédico engastado na base e livre na outra extremidade, solicitado bruscamente por uma força na extremidade livre, conforme mostra a figura 6.7.5. Os parâmetros elastodinâmicos adotados são: $E=1 \times 10^{5} \mathrm{~Pa}, \quad v=0,0, \quad \rho=1 \mathrm{~kg} / \mathrm{m}^{3}$, $\Delta \mathrm{t}=0,003 \mathrm{~s}, \mathrm{f}_{\mathrm{t}}=1,0 \mathrm{~Pa}$ e $\mathrm{w}_{\mathrm{c} \_ \text {ad }}=0,00004 \mathrm{~m}$. 

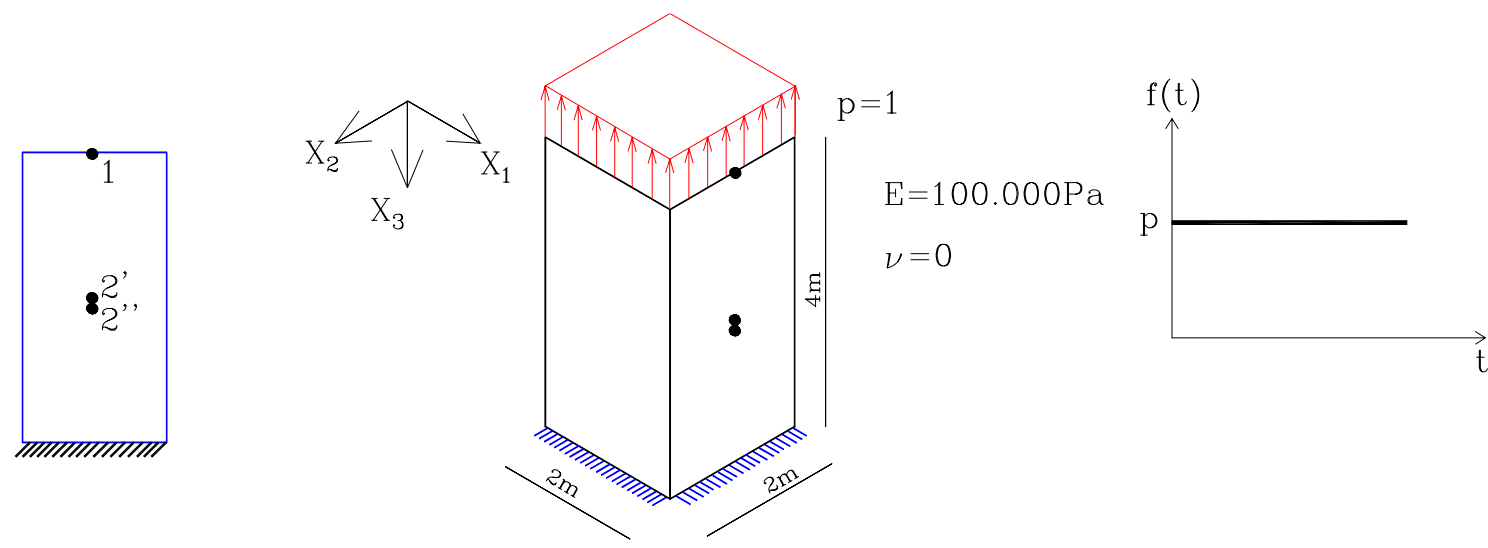

FIGURA 6.7.5 - Definição do objeto de estudo: geometria do sólido, condições de contorno e comportamento temporal da força.

$\mathrm{Na}$ figura 6.7.5 estão definidos 3 pontos ao longo do comprimento do sólido: ponto 1 na extremidade livre, e 2'e 2', imediatamente acima e abaixo da sua metade, respectivamente.

$\mathrm{Na}$ fase transiente da resposta do sólido à solicitação, verifica-se o surgimento de uma fratura, definida na sua seção transversal central através da discretização de uma superfície fíctícia por elementos triangulares planos com aproximação constante. O contorno é discretizado por elementos triangulares descontínuos. As discretizações são mostras na figura 6.7.6.
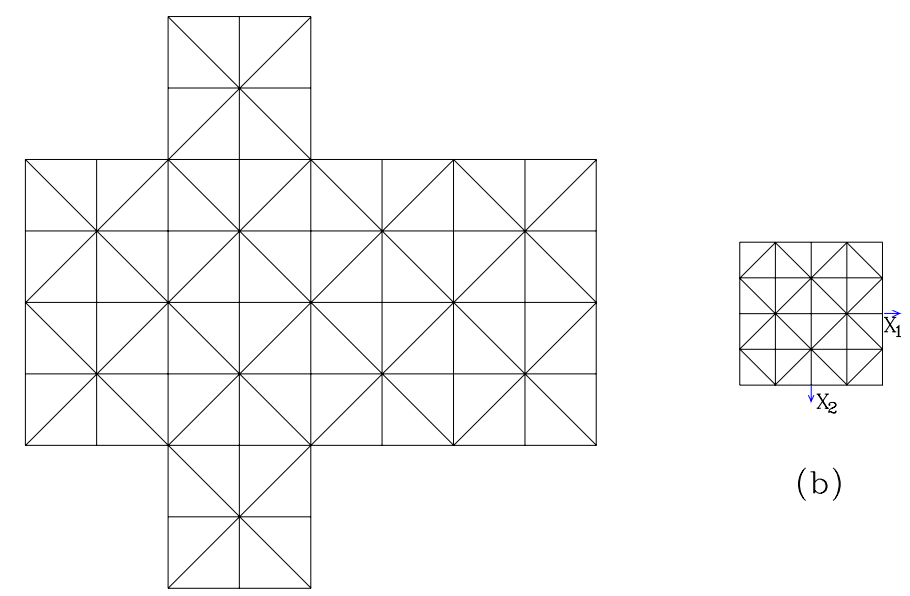

(a)

FIGURA 6.7.6 - Discretizações: (a) contorno do sólido e (b) superfície da fratura. 
O exemplo é resolvido em um algoritmo computacional incremental (no tempo) e iterativo. Ao atingir a tensão limite $\mathrm{f}_{t}$, o sólido começa a se separar em duas partes, tendo a seção central como a fratura propriamente dita. Os resultados encontrados são apresentados nas figuras 6.7.7, 6.7.8 e 6.7.9.

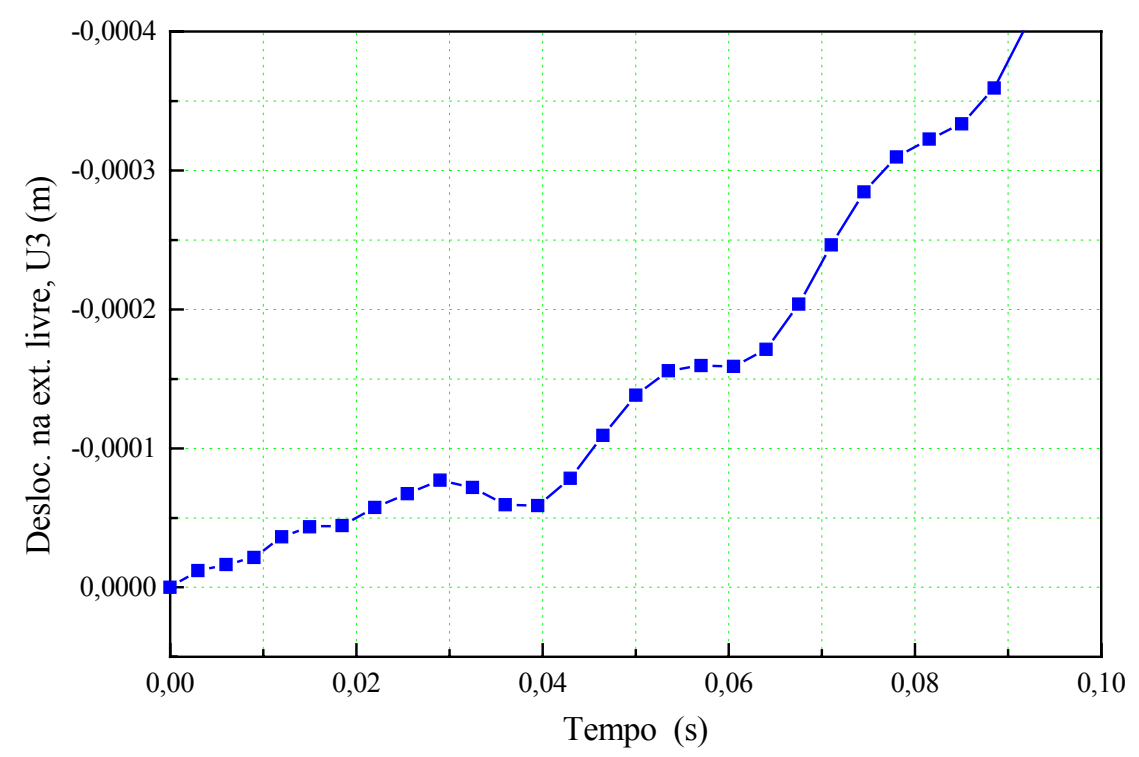

FIGURA 6.7.7 - Deslocamentos no ponto 1 na direção $X_{3}$, ao longo do tempo.

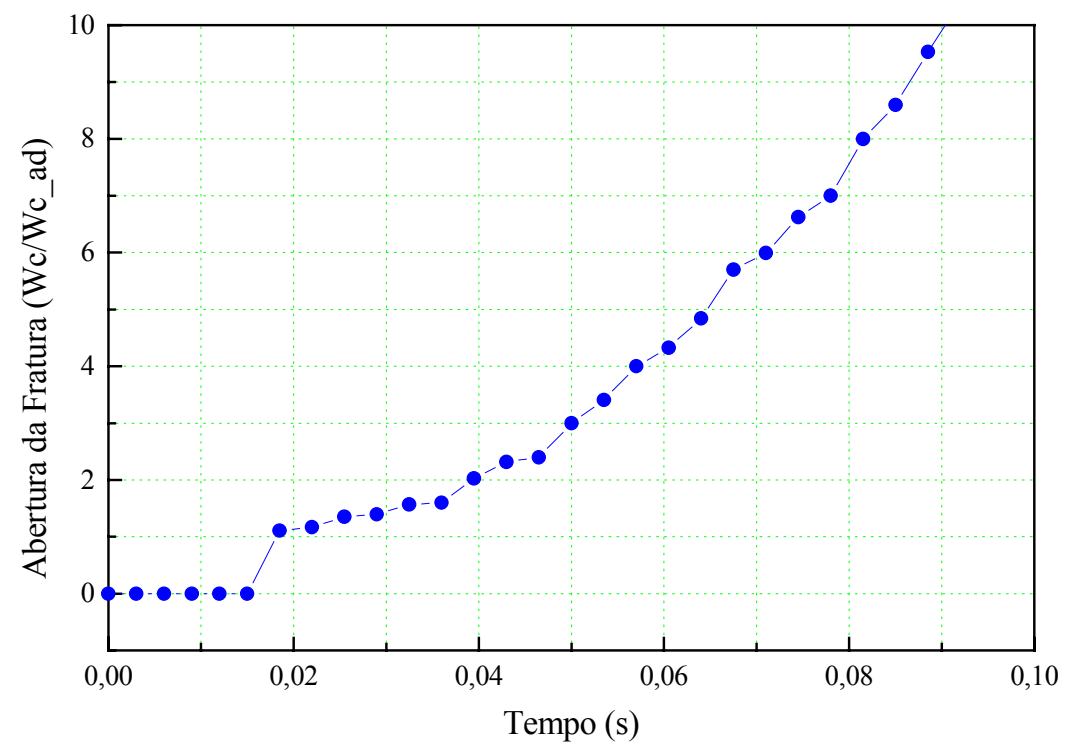

FIGURA 6.7.8 - Abertura da fratura $\left(\mathrm{w}_{\mathrm{c}} / \mathrm{w}_{\mathrm{c}_{-} \text {ad }}\right)$ ao longo do tempo. 


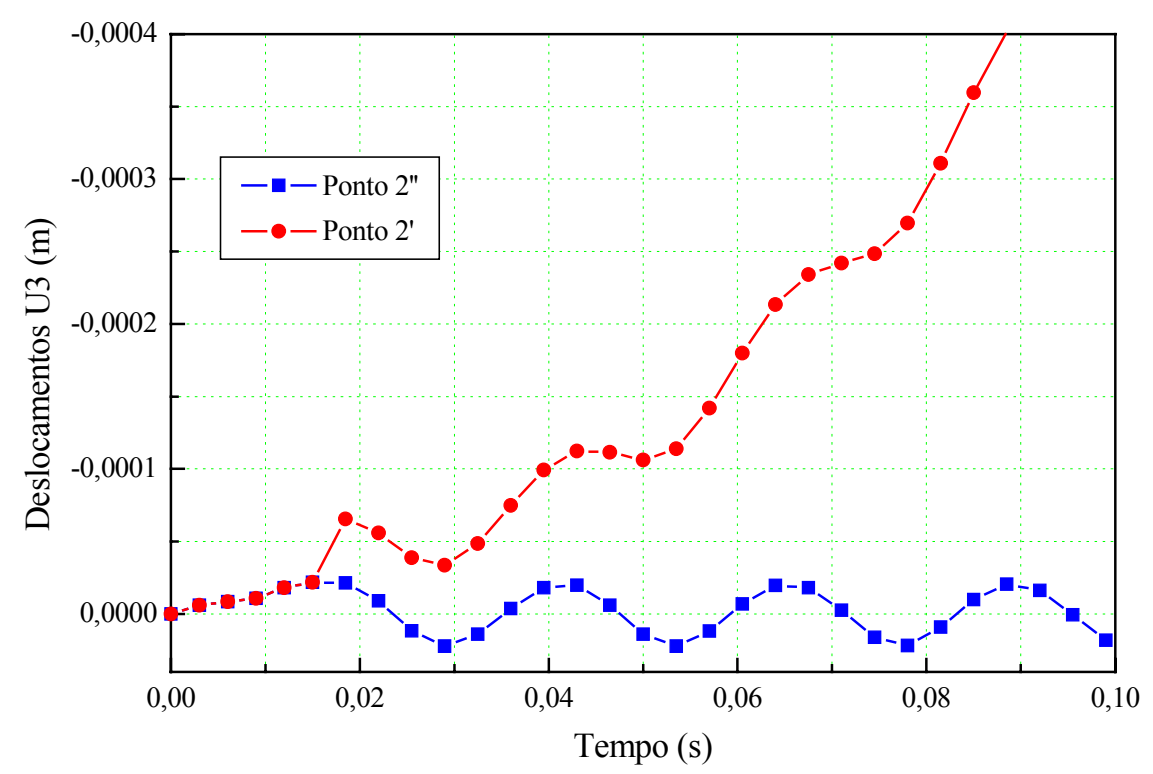

FIGURA 6.7.9 - Deslocamentos (corrigidos) dos pontos 2' e 2',

Os parâmetros utilizados no código computacional impõem que a abertura de fratura se observa apenas quando o deslocamento relativo das superfícies atinge o valor $\mathrm{w}_{\mathrm{c}-\mathrm{ad}}=0,00004$. A formulação deve ser capaz de permitir a separação das duas partes, apresentando, portanto, a fratura. Para tanto, foi necessário adotar células diferentes para as partes a fim de possibilitar a separação também em termos da matriz de massa.

O código computacional elaborado, tendo em vista a formulação apresentada, consegue fornecer respostas adequadas para o problema de fratura com solicitações dinâmicas. 


\section{CAPÍTULO 7}

\section{IMPLEMENTAÇÕES COMPUTACIONAIS}

\section{1 - INTRODUÇÃO}

Os códigos computacionais para a implementação das formulações apresentadas foram elaborados utilizando-se a linguagem de programação $\mathrm{C}++$, para uso em microcomputadores (Pentium ${ }^{1}$ MMX $200 \mathrm{MHz}$ ou mais atual) com $64 \mathrm{Mb}$ de memória central.

A estrutura principal dos códigos computacionais é formada por módulos ligados a arquivos de informações armazenados em memória de massa. A idéia é permitir o uso racional da memória central e avaliar várias alternativas sem a necessidade de processar toda a formulação.

Neste capítulo são apresentados os fluxogramas principais e de algumas rotinas mais importantes dos códigos computacionais elaborados para as diversas formulações deste trabalho.

\section{2 - ALGORITMO ELASTOSTÁTICO}

O algoritmo elastostático implementa computacionalmente a formulação apresentada no item 2.6 deste trabalho.

\footnotetext{
${ }^{1}$ Processador Intel, marca registrada
} 
O desenvolvimento do algoritmo é feito a partir de quatro módulos, na tentativa de dividir tarefas para não repeti-las, conforme figura 7.2.1.

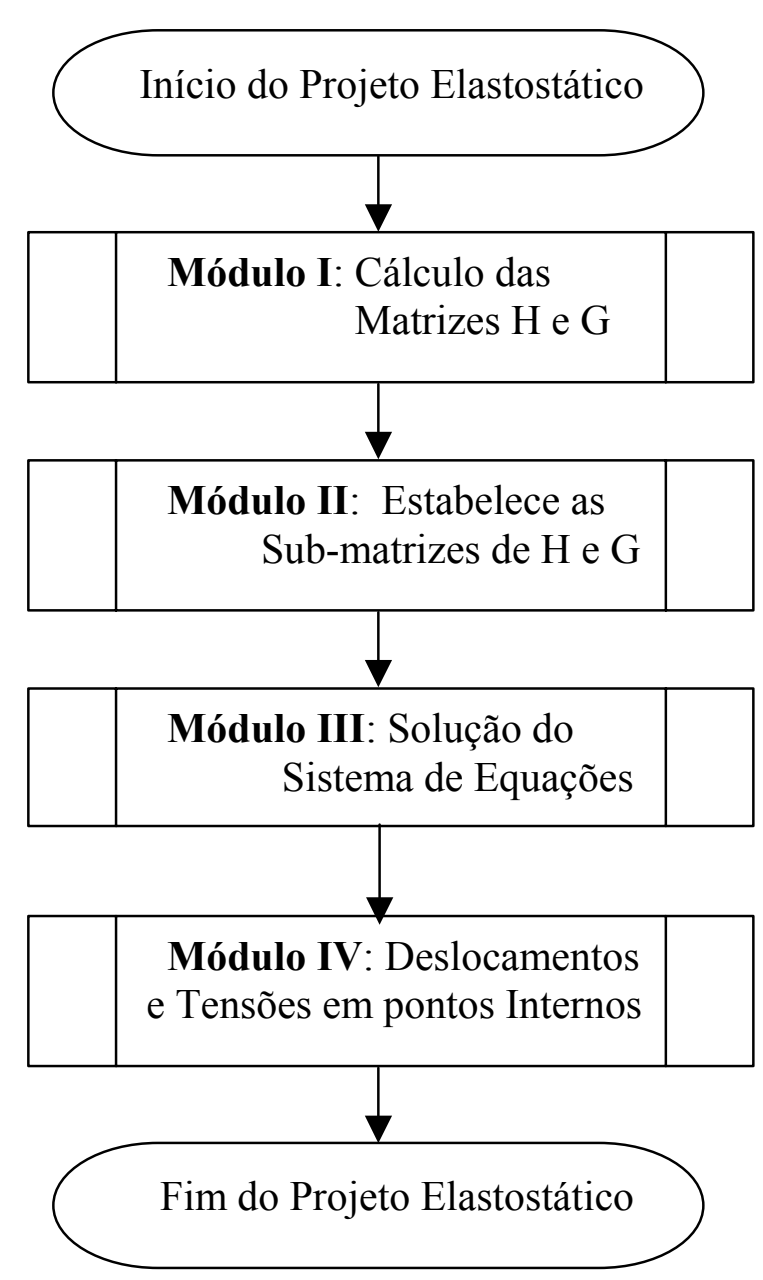

FIGURA 7.2.1 - Desenvolvimento em módulos do algoritmo elastostático.

A) Módulo I:

O módulo I tem a finalidade de calcular as componentes das matrizes $\mathrm{H}$ e G. A seqüência das rotinas envolvidas é mostradas na figura 7.2.2. 


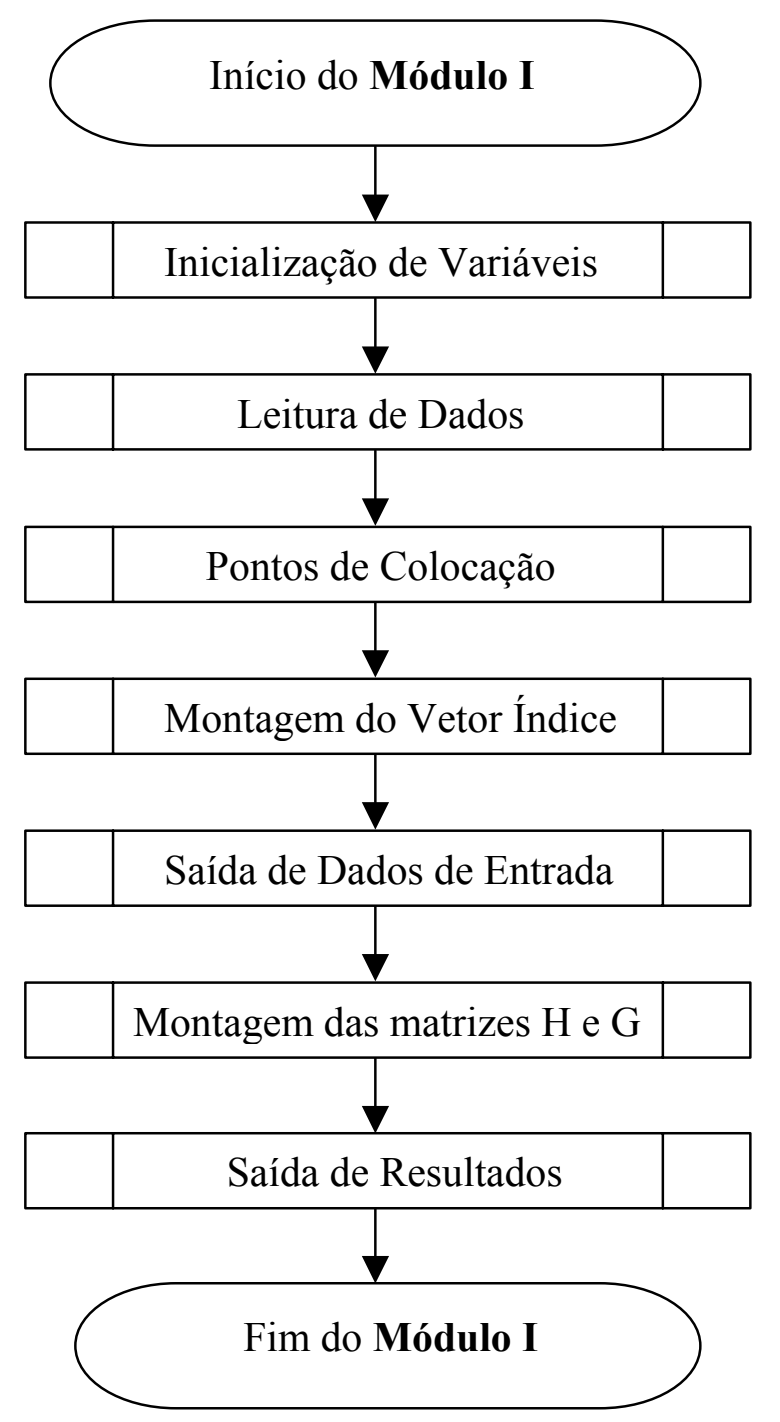

FIGURA 7.2.2 - Rotina principal do Módulo I do programa elastostático.

A sub-rotina "Inicialização de variáveis" define as matrizes do tipo ponteiro obedecendo o limite estabelecido para número máximo de elementos e de nós, nas primeiras linhas do programa e define, ainda, as variáveis simples do programa.

"...

define Nmaxnos 500 // ... número máximo de nós ...

define NmaxElem $350 / / .$. número máximo de elementos ... ..."

A sub-rotina "Leitura de dados" permite a entrada dos parâmetros elásticos e geométricos do problema. $\mathrm{O}$ arquivo de entrada é mostrado a seguir: 


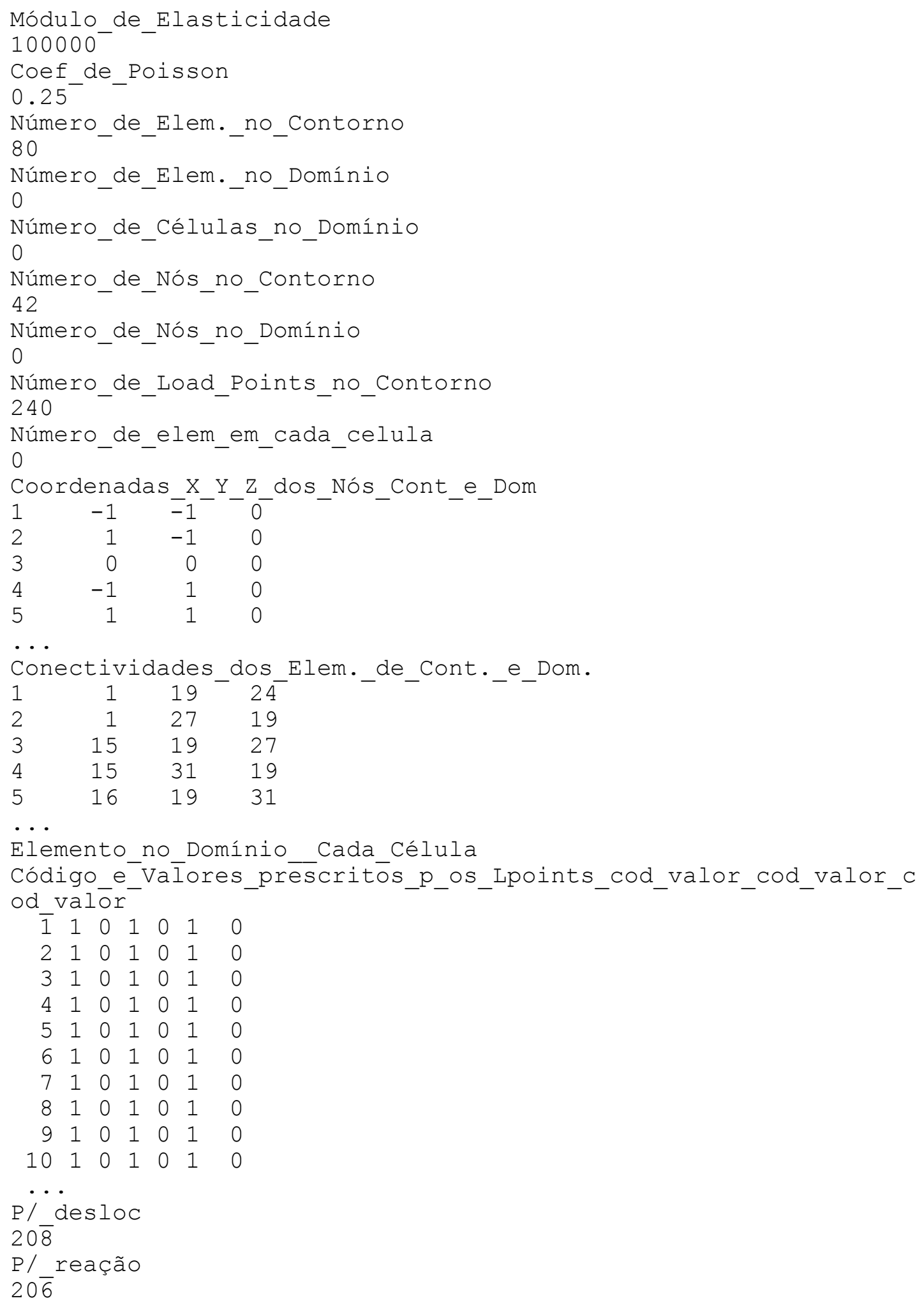


A sub-rotina "Pontos de colocação" define as coordenadas dos pontos para os quais são escritas as equações integrais de deslocamento. É definida, ainda, a conectividade de cada elemento frente aos novos pontos. A sub-rotina utiliza os valores estabelecidos no intervalo (2.6.19) para deslocar os pontos dos vértices do elemento triangular para seu interior (elemento descontínuo).

$\mathrm{Na}$ forma clássica de montagem das matrizes $\mathrm{H}$ e $\mathrm{G}$ utiliza-se a ordem de definição das coordenadas, já que todos os valores prescritos são levados para um único vetor misto (contendo deslocamentos e forças de superfície) e, portanto, encontrando-se respostas também mistas. Este procedimento dá bons resultados e é empregado tradicionalmente nos algoritmos elastostáticos.

Outro procedimento que pode ser utilizado, faz a montagem das matrizes $\mathrm{H} \mathrm{e}$ $\mathrm{G}$ de forma particionada, estabelecendo os blocos de coordenadas com valores prescritos para forças de superfície e deslocamentos, conforme mostra o item 3.6.1. Assim, o vetor das incógnitas é definido apenas para deslocamentos e as forças de superfície, calculados em uma etapa posterior. Trata-se de um artifício visando o algoritmo elastodinâmico, como por exemplo, Newmark.

A sub-rotina "Montagem do vetor índice" estabelece a ordem em que as componentes de $\mathrm{H}$ e $\mathrm{G}$ devem ser arranjadas para que o particionamento das matrizes aconteça de forma direta. É utilizado o vetor "código" que define a característica dos graus de liberdade: se 1 (um), a força de superfície é prescrita; se 0 (zero), o deslocamento é prescrito.

Na sub-rotina "Saída de dados de entrada" armazena-se um relatório dos dados de entrada e mais alguns gerados (coordenadas dos pontos de colocação, conectividade dos elementos para estes pontos, etc.) em um arquivo tipo texto: "EX1.SAI", por exemplo. O arquivo recebe mais dados a medida que as variáveis do contorno são calculadas (Módulo III).

A sub-rotina "Montagem das matrizes $\mathrm{H}$ e $\mathrm{G}$ " efetua as integrações estabelecidas no item 2.6.3, que resulta nas componentes das matrizes citadas. $\mathrm{O}$ fluxograma desta sub-rotina é mostrado na figura 7.2.3. 


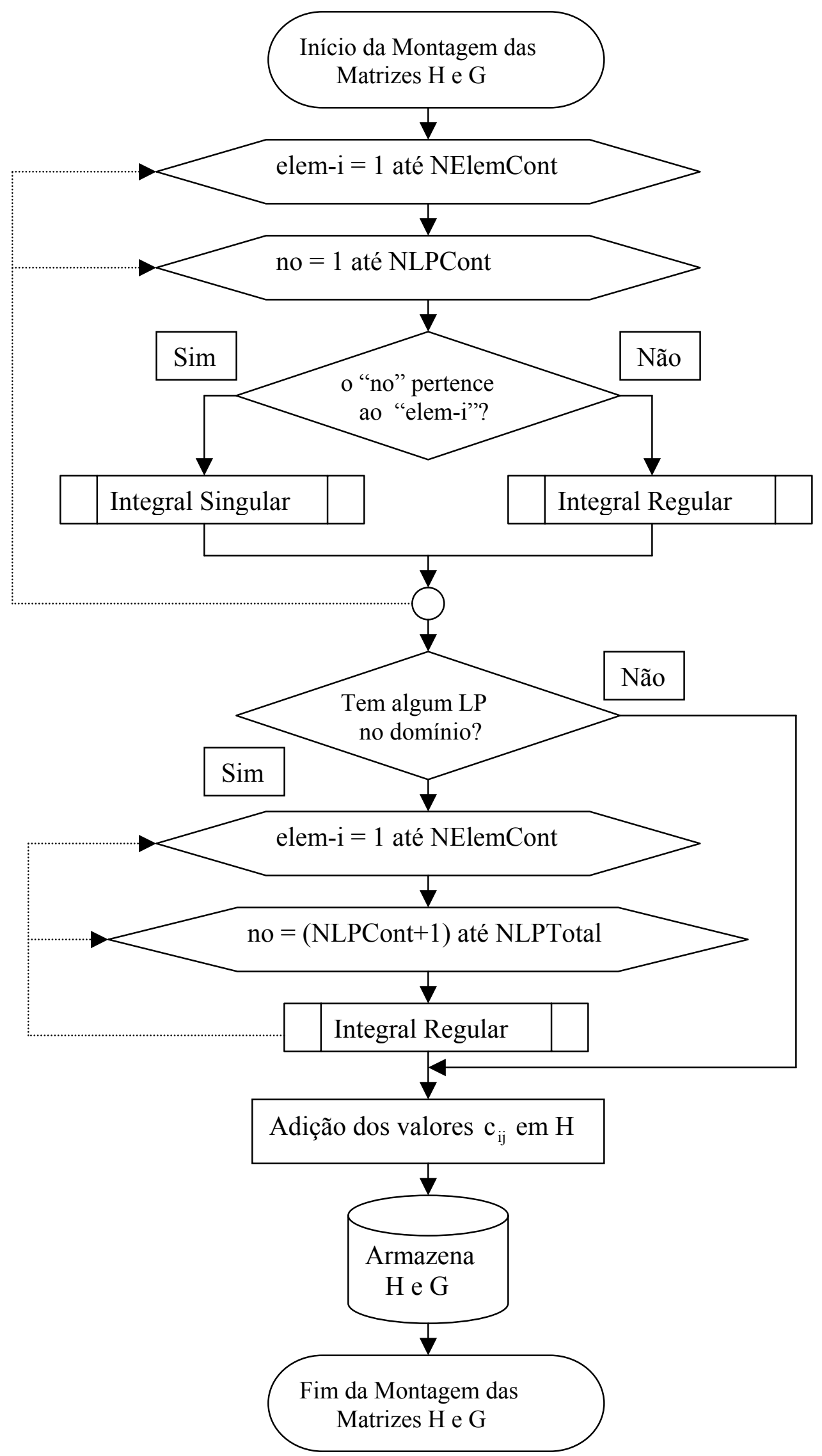

FIGURA 7.2.3 - Fluxograma da sub-rotina “montagem de H e G”. 
Para efetuar a sub-rotina "integral singular" são utilizados os passos apresentados no item 2.6.3.1.

$\mathrm{Na}$ sub-rotina “integral regular", os procedimentos são os do item 2.6.3.2, lembrando-se que a divisão do elemento em sub-elementos, utilizada neste trabalho, propicia resultados mais precisos quando o ponto de colocação está próximo do elemento a ser integrado.

$\mathrm{Na}$ sub-rotina "Saída de resultados" são armazenadas no arquivo tipo texto "EX1.SAI", informações, por exemplo, sobre a matriz $\mathrm{H}$ calculada, e valores que medem seu nível de precisão.

B) Módulo II:

O módulo II é destinado às operações de particionamento das matrizes H e G.

$\mathrm{Na}$ forma tradicional do algoritmo do MEC, o módulo II pode ser dispensado, passando-se diretamente para a resolução do sistema (módulo III), envolvendo um vetor de incógnitas misto, ou seja, com deslocamentos e forças de superfícies desconhecidos.

Pensando-se em utilizar a seqüência da figura 7.2.1 também para o algoritmo elastodinâmico, busca-se o particionamento das matrizes $\mathrm{H}$ e $\mathrm{G}$ e as operações matriciais necessárias, definidas no item 3.6.1, expressões (3.6.5a-c) e (3.6.6).

Os valores necessários para as operações são lidos dos arquivos "MATH.MEC", "MATG.MEC", "DAD.MEC" e "IND.MEC", armazenados na memória de massa.

C) Módulo III:

O módulo III é dedicado à resolução do sistema de equações algébricas obtido na formulação.

No modo clássico, basta utilizar o algoritmo de Gauss para resolver o sistema de equações simultâneas, onde 


$$
\mathbf{V}_{\mathrm{DF}}=\mathbf{A}^{-1} \mathbf{F}
$$

Com a opção pelo particionamento das matrizes $\mathrm{H}$ e $\mathrm{G}$, pode-se utilizar o primeiro termo de Newmark para obter os valores das variáveis de contorno, obtendo-se as mesmas respostas que (2.6.9).

Para o módulo III, são requeridas as informações armazenadas nos arquivos "DAD.MEC", "IND.MEC", MATG.MEC" e "MATH.MEC". As matrizes do tipo ponteiro são inicializadas com as ordens passadas do arquivo "DAD.MEC", utilizando-se a memória central requerida especificamente para aquele problema.

O arquivo de saída “*.SAI” acumula informações do módulo I e se completa no módulo III com os valores das variáveis do contorno, conforme mostra a listagem a seguir:

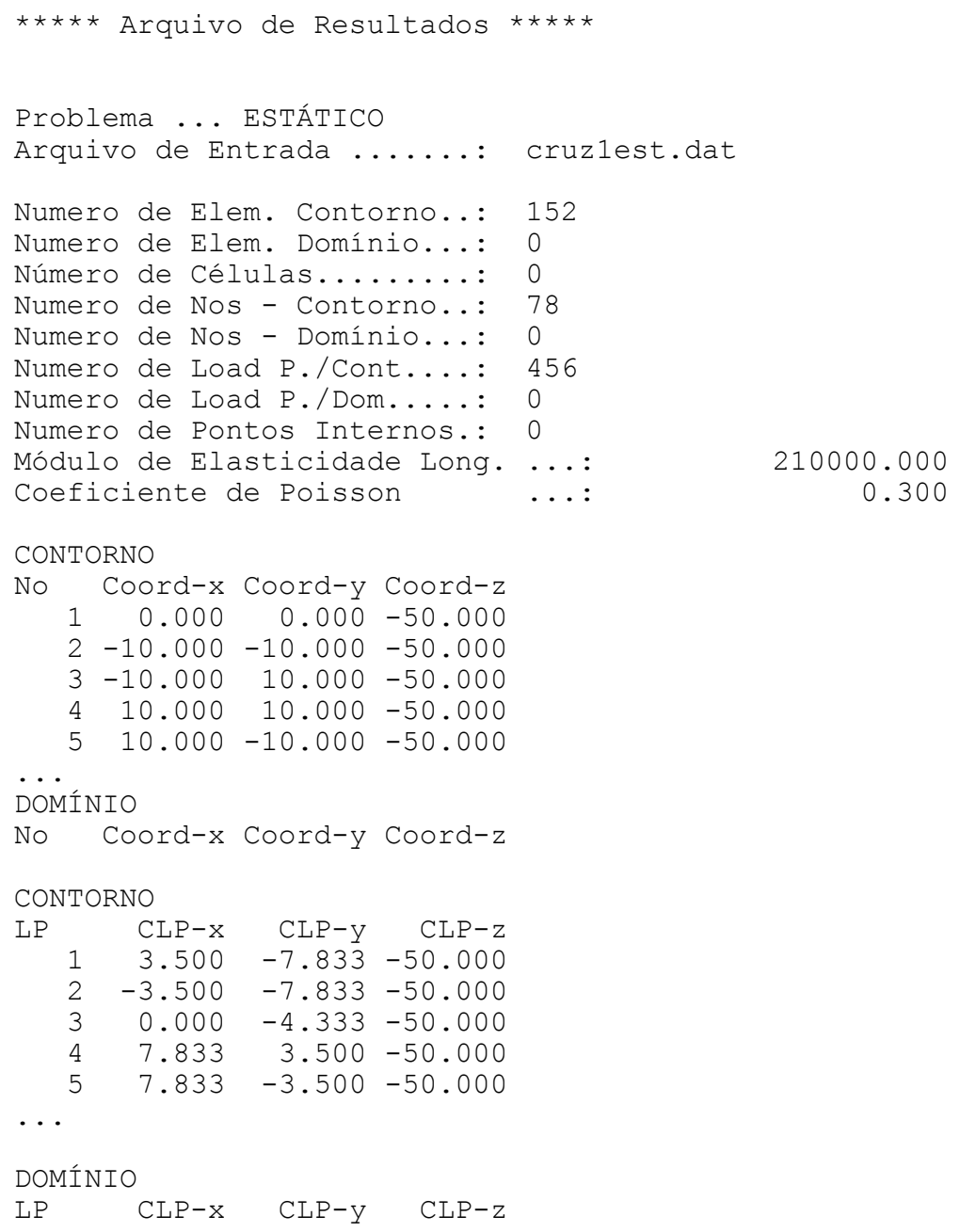




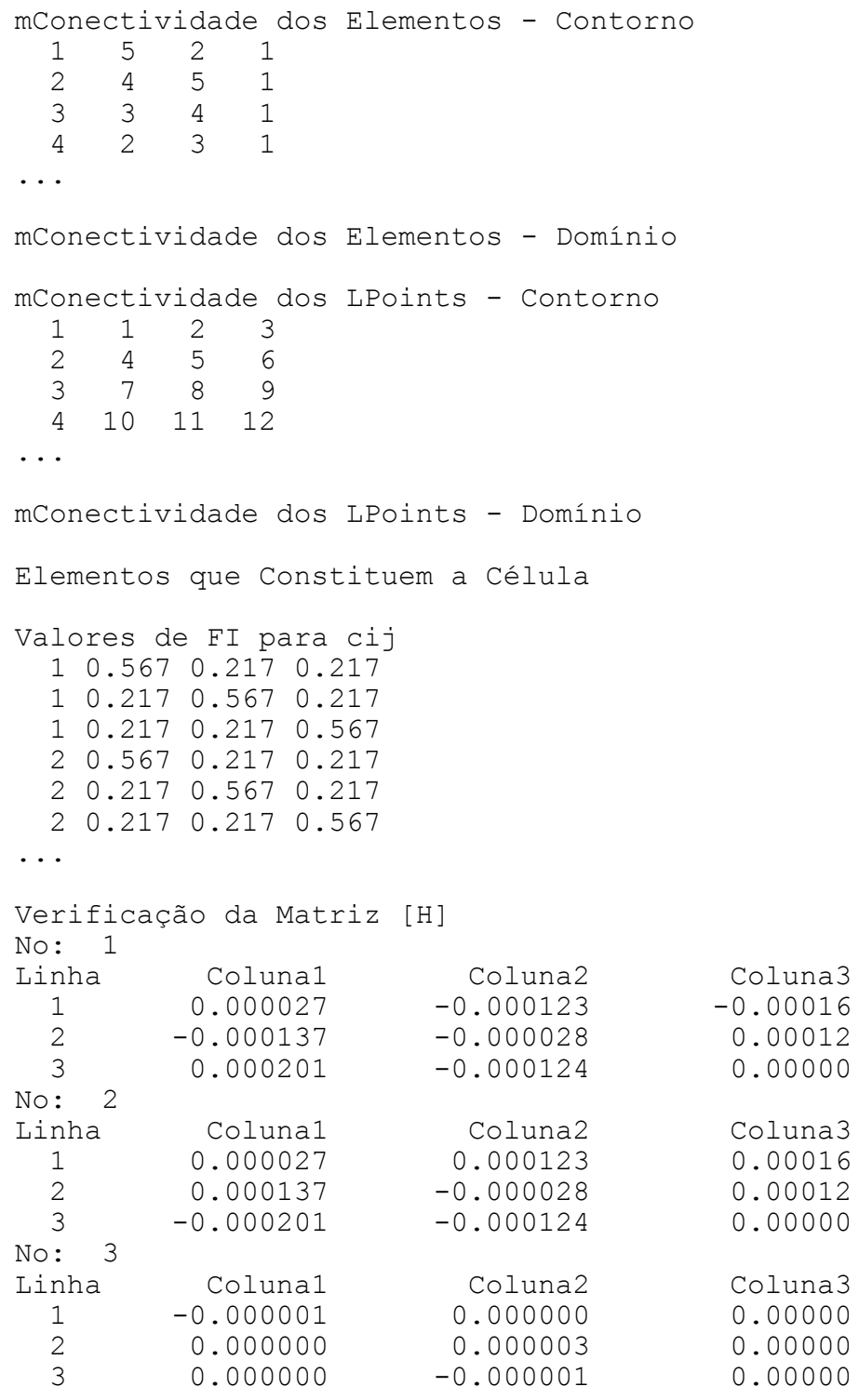

\footnotetext{
$\star \star \star \star *$ VALORES ENCONTRADOS $\star * \star \star \star *$
}

\begin{tabular}{|c|c|c|c|c|c|c|}
\hline pas & sSO & & & & & \\
\hline $\begin{array}{l}\text { Lpt } \\
1\end{array}$ & $\begin{array}{r}\text { Desl } x \\
-0.00014523\end{array}$ & $\begin{array}{c}\text { Desl } \\
0.000143 \overline{6} 1\end{array}$ & $\begin{array}{r}\text { Desl } z \\
-0.002163 \overline{5} 9\end{array}$ & $\begin{array}{r}\text { Forc } x \\
-0.000001 \overline{8} 3\end{array}$ & $\begin{array}{r}\text { Forc }-\frac{y}{2} \\
-0.00000302\end{array}$ & $\begin{array}{r}\text { Forc } z \\
-0.000312 \overline{4} 9\end{array}$ \\
\hline 2 & 0.00014523 & 0.00014361 & -0.00216359 & 0.00000183 & -0.00000302 & -0.0003124 \\
\hline 3 & 0.00000000 & -0.00000055 & -0.00216289 & 0.00000000 & -0.00000170 & -0.00037017 \\
\hline 4 & -0.00014395 & -0.00014462 & -0.00216384 & -0.00000408 & 0.00000132 & -0.00031263 \\
\hline 5 & -0.00014 & 0.00014462 & -0.00216384 & -0.00000408 & -0.00000132 & -0.00031263 \\
\hline 6 & 0.00 & 0.00000000 & -0.00216290 & -0.00000229 & 0.00000000 & -0.0003702 \\
\hline
\end{tabular}

D) Módulo IV:

Este módulo é destinado ao cálculo das variáveis em pontos do domínio, conhecidos os valores das variáveis do contorno e implementadas as expressões do item 2.6.4. 
São requeridas para o módulo IV as informações dos arquivos "EX1.DAT", "EX1.INT" e os demais auxiliares. O arquivo "EX1.DAT" informa os dados de entrada (módulo I). O arquivo "EX1.INT" fornece o número de pontos internos e suas coordenadas cartesianas, conforme mostra a seqüência a seguir:

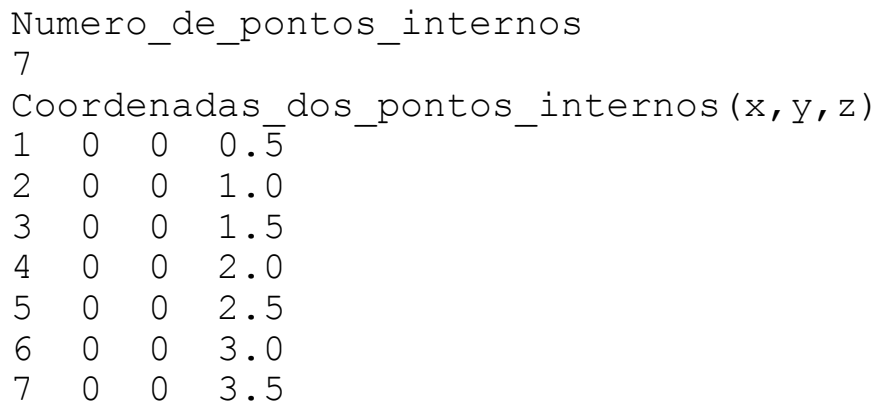

Se o usuário não se satisfizer com a análise para os pontos estabelecidos, pode-se definir um novo arquivo "EX1a.INT" e processá-lo sem envolver os outros módulos, o que traz versatilidade ao algoritmo.

O módulo IV fornece os valores de deslocamentos e tensões calculados para pontos internos através do arquivo “*.DT”. Sua forma apresenta-se conforme trecho do arquivo a seguir:

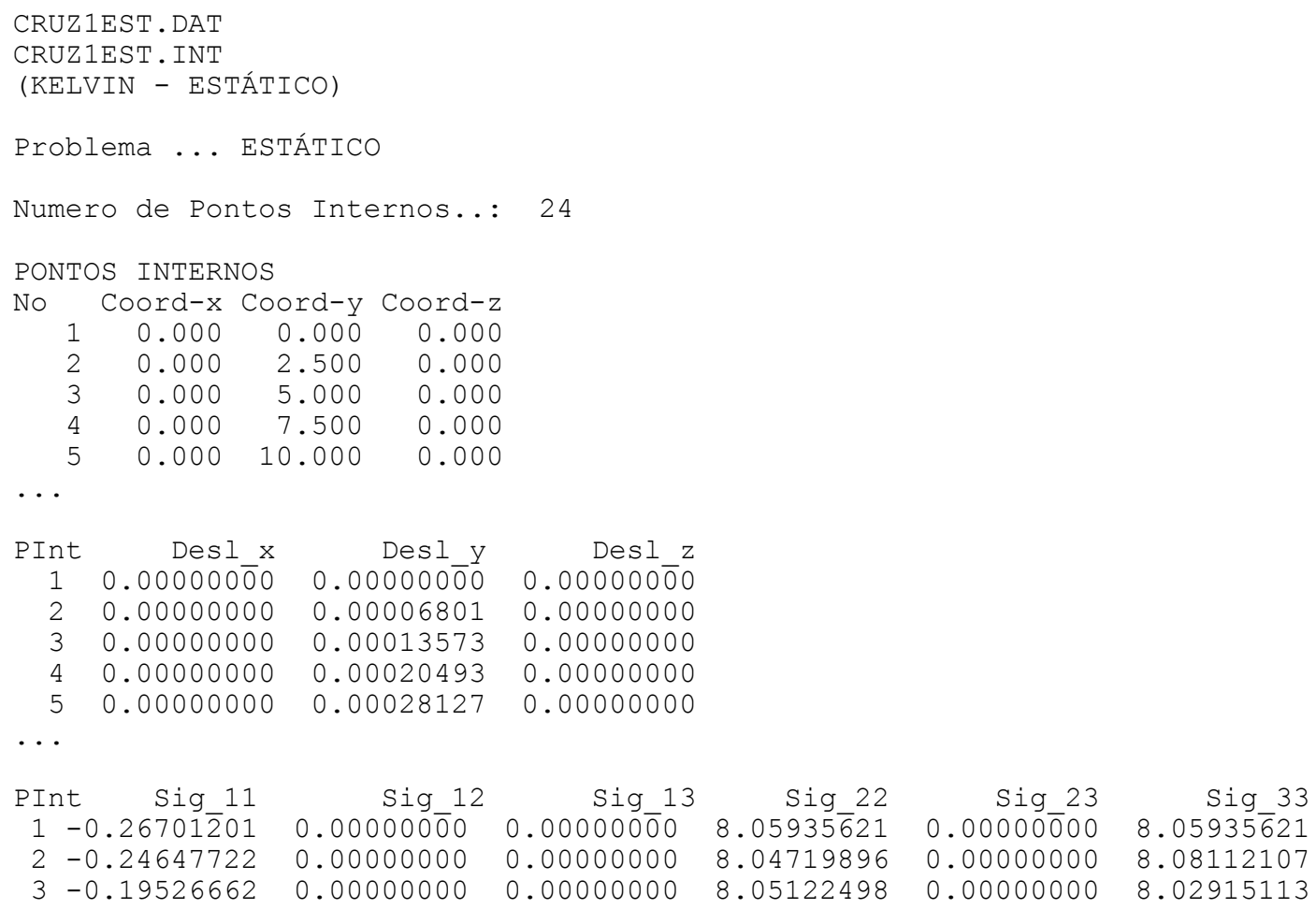


É importante enfatizar que as sub-rotinas apresentadas até aqui servem para qualquer solução fundamental. A particularização pode ser feita modificando-se as sub-rotinas de integração regular e singular do módulo I e a regular do módulo IV.

\subsection{1 - Solução Fundamental de Kelvin}

Considerando-se a solução fundamental de Kelvin, item 2.4.1, a sub-rotina "Integral_Singular" segue os passos mostrados a seguir:

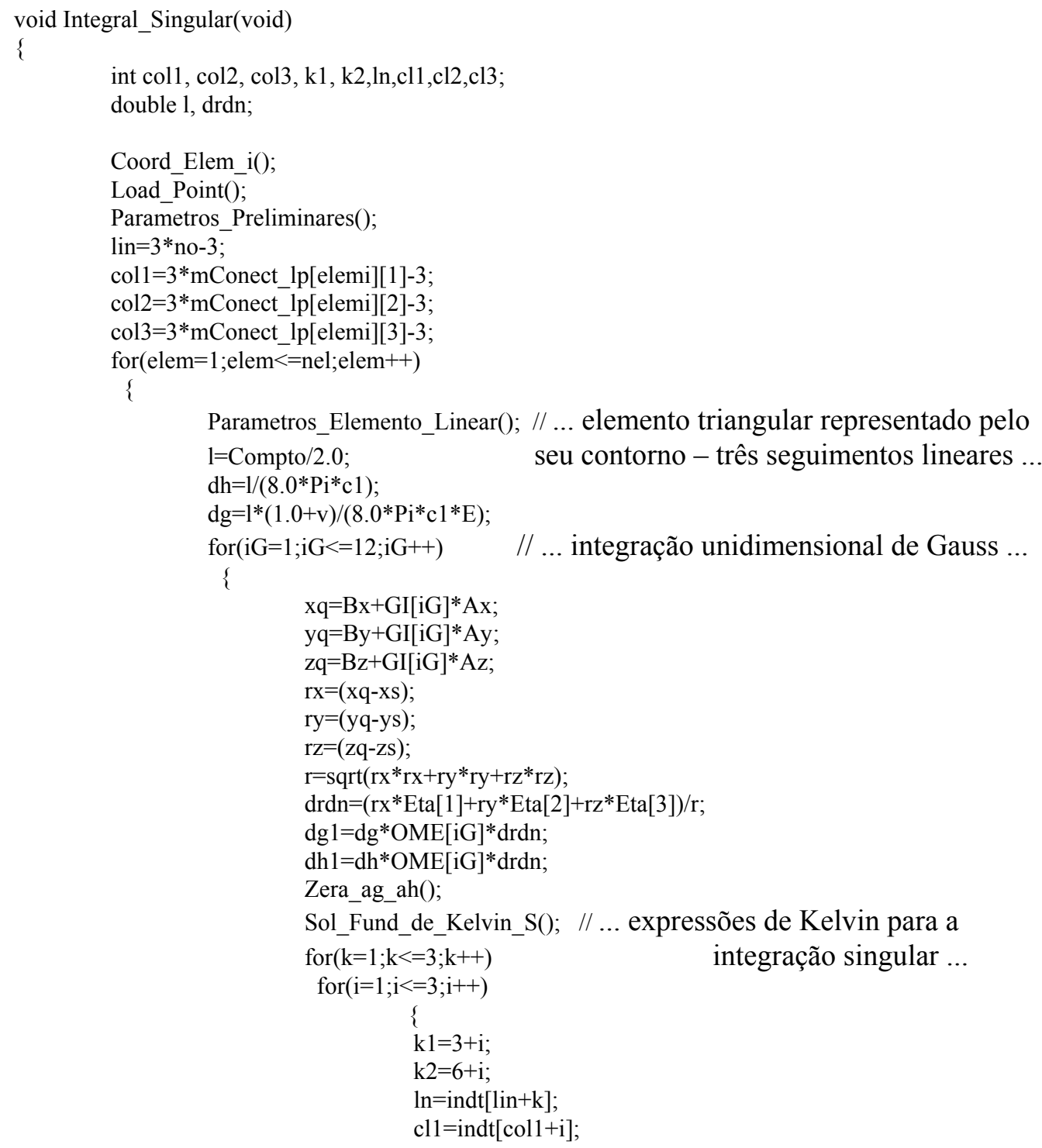




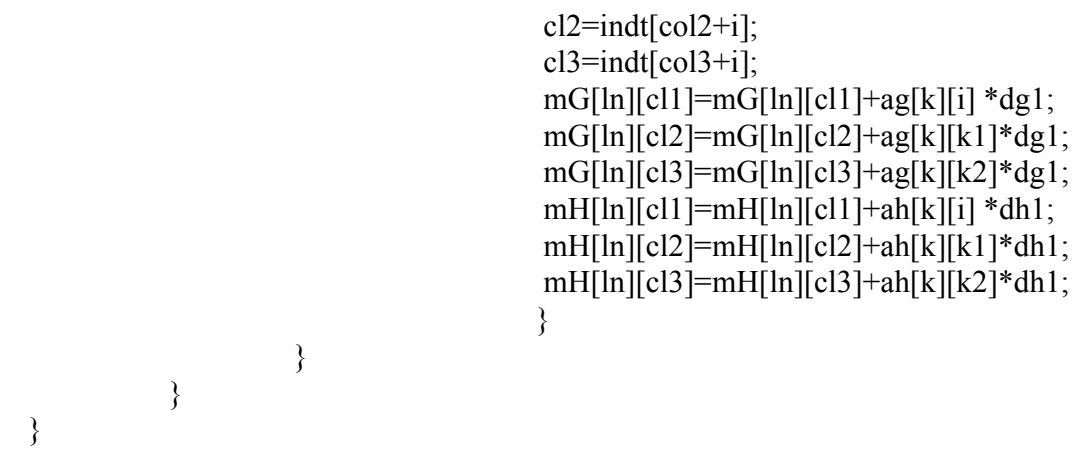

A sub-rotina “Integral_Regular” faz uma verificação preliminar para avaliar a distância entre o ponto funcional e o elemento integrado, e empregar ou não, a subdivisão do elemento. Assim, tem-se:

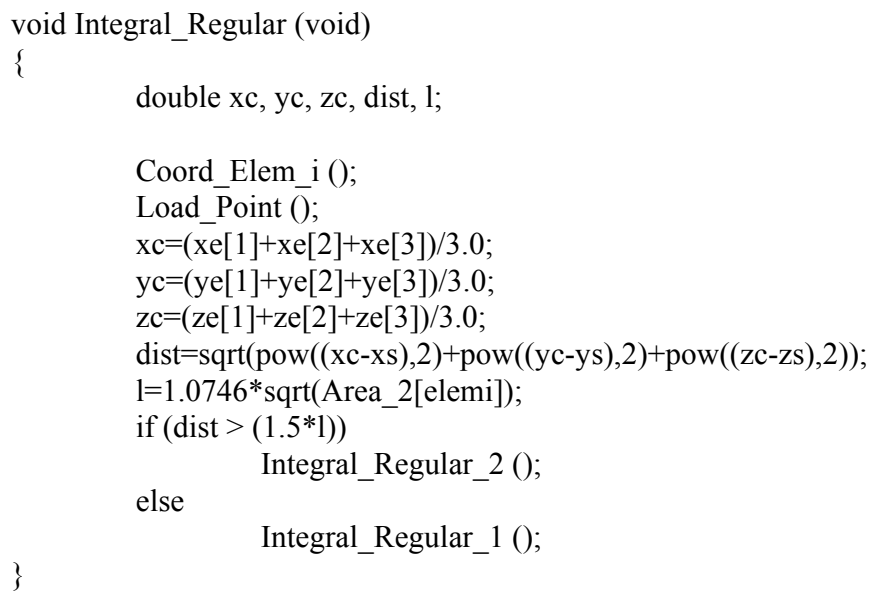

A sub-rotina "Integral_Regular_1" tem os seguintes passos:

void Integral_Regular_1(void)

\{

int col1, $\operatorname{col} 2, \operatorname{col} 3, \ln , \mathrm{cl} 1, \mathrm{cl} 2, \mathrm{cl} 3$;

lin $=3 *$ no- 3 ;

coll $=3 *$ mConect_lp[elemi] $[1]-3$;

col2 $=3 *$ mConect_lp[elemi][2]-3;

col3 $=3 *$ mConect_lp[elemi][3]-3;

for $($ sElem $=1 ;$ sElem $<=$ nst;sElem ++ )

\{

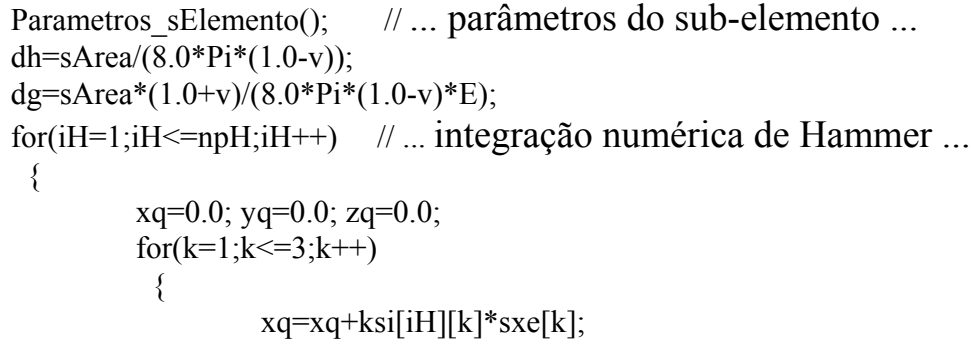




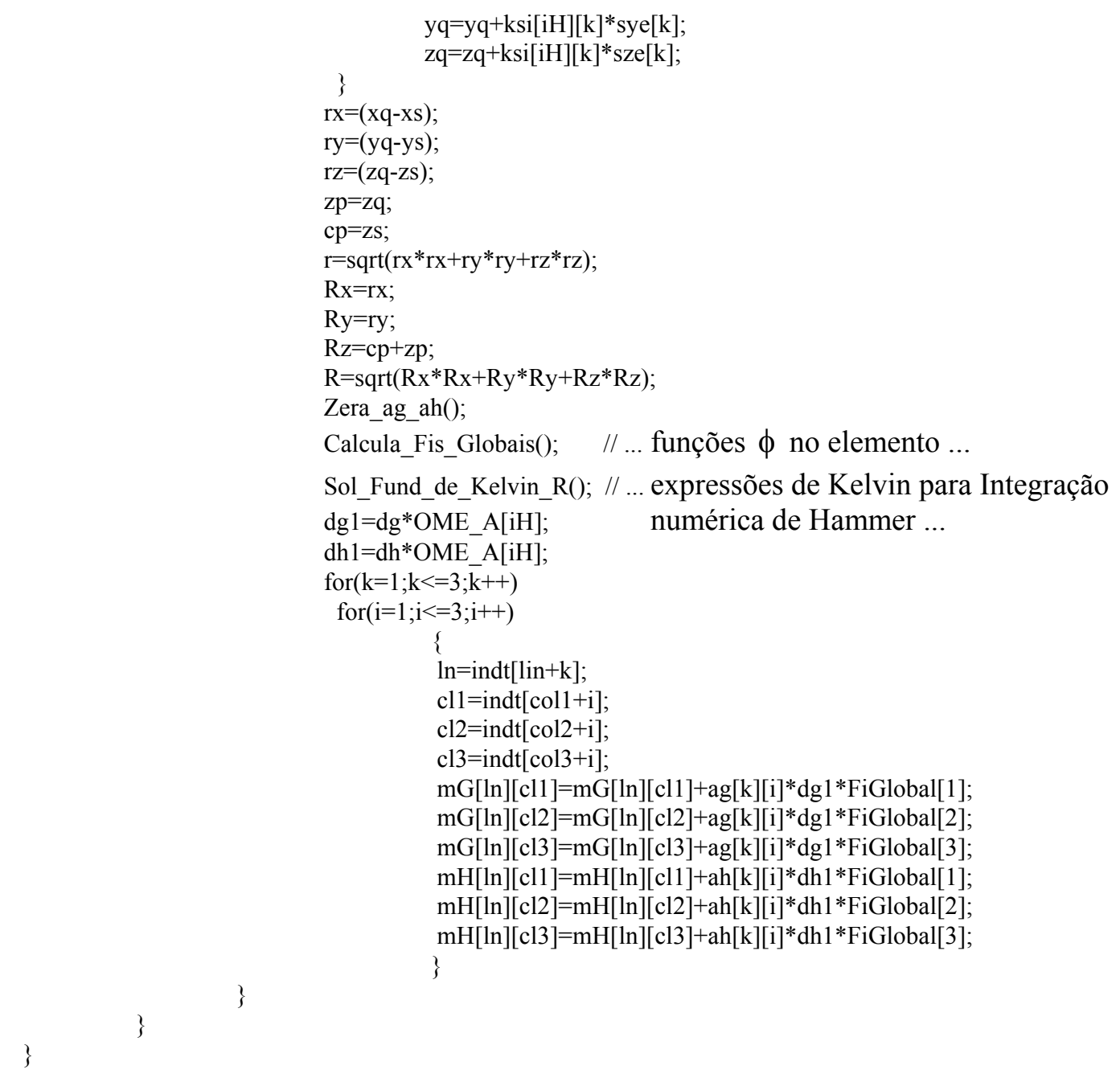

7.2.2 - Solução Fundamental de Mindlin

Para a solução fundamental de Mindlin, os trechos do programa computacional apresentados são válidos modificando-se apenas as chamadas das sub-rotinas das expressões de Kelvin para Mindlin. Utiliza-se a solução de Mindlin como sendo formada pelas parcelas de Kelvin e complementar, conforme (2.4.11ab). Outra opção consiste em usar as expressões de Boussinesq-Cerruti, quando o ponto funcional encontra-se na superfície livre de trações. Assim, a seqüência de códigos para a sub-rotina "Integral Singular" é como se apresenta a seguir: 


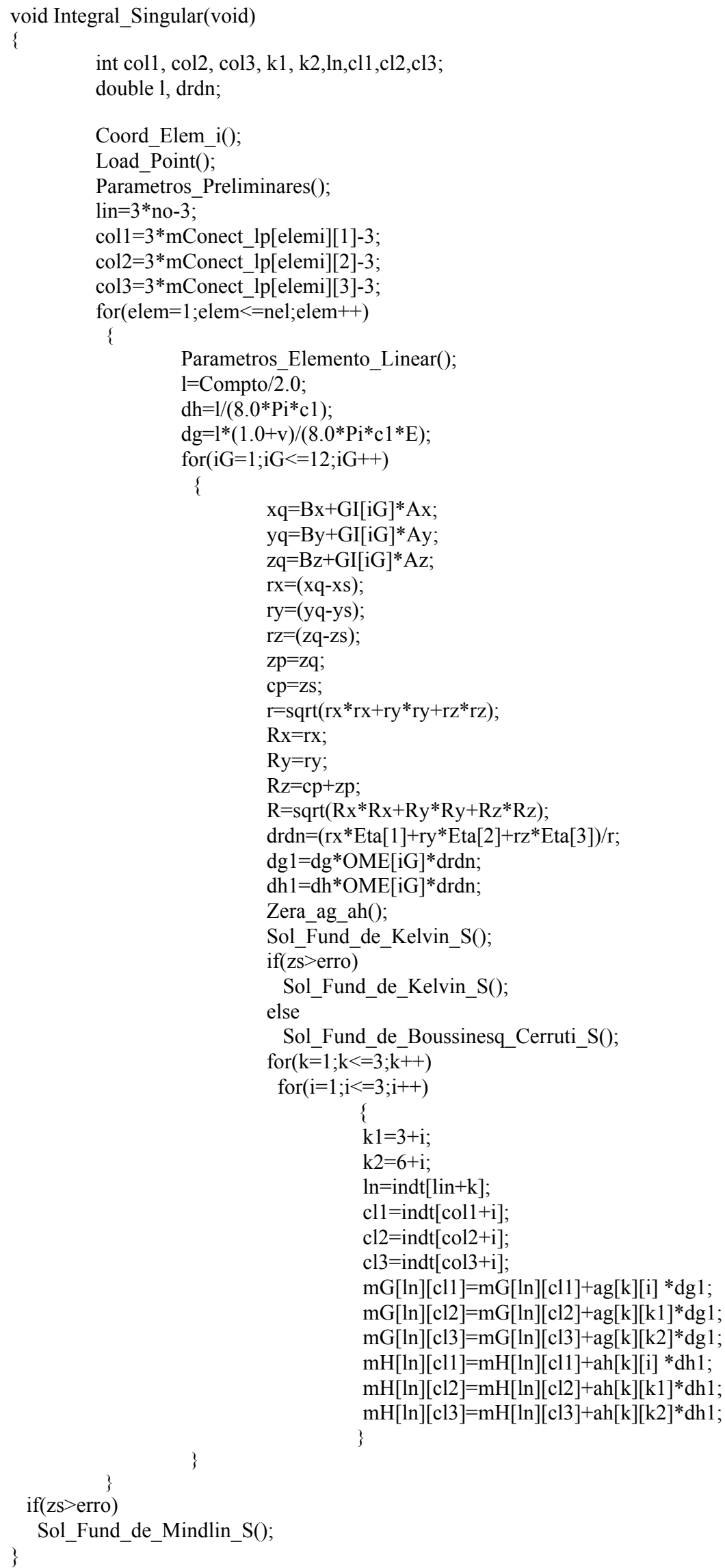


As demais sub-rotinas, definidas para a solução fundamental de Kelvin, são modificadas da mesma maneira descrita acima.

\section{3 - ALGORITMO ELASTODINÂMICO}

O algoritmo elastodinâmico é semelhante ao elastostático mostrado no item anterior. A única modificação consiste na inclusão da matriz de massa, calculada no módulo I. Portanto, o esquema mostrado na figura 7.2.1 é também representativo deste algoritmo.

A figura 7.3.1 mostra a seqüência principal de rotinas do módulo I para o algoritmo elastodinâmico.

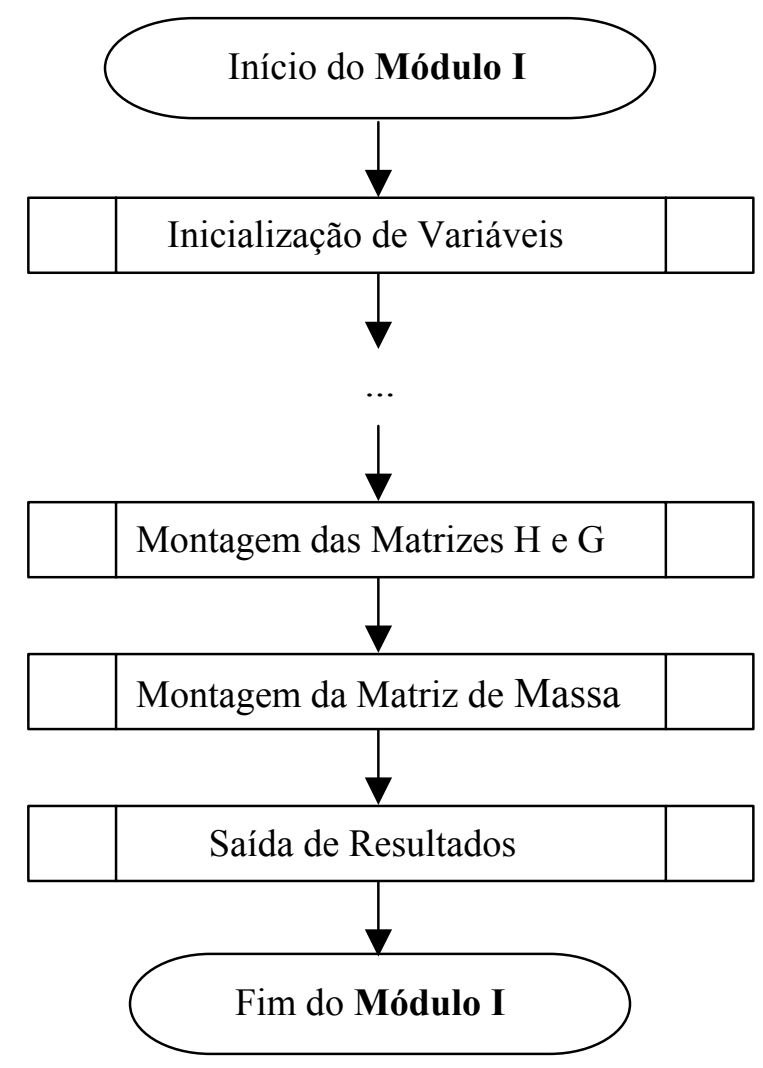

FIGURA 7.3.1 - Rotina principal do algoritmo elastodinâmico.

A sub-rotina "Montagem da matriz de massa" é particular para cada técnica usada para obtenção da matriz de influência dinâmica. Neste trabalho utiliza-se o Método da Reciprocidade Dual e a Técnica da Integração Direta, cujos algoritmos são descritos a seguir. 
7.3.1 - Método da Reciprocidade Dual

O módulo I correspondente ao algoritmo elastodinâmico com a Reciprocidade Dual sofre algumas alterações em relação ao original, iniciando-se pelo arquivo de dados de entrada (“*.DAT”) que passa a prever a definição de pontos no domínio (pólos), conforme mostra a listagem a seguir:

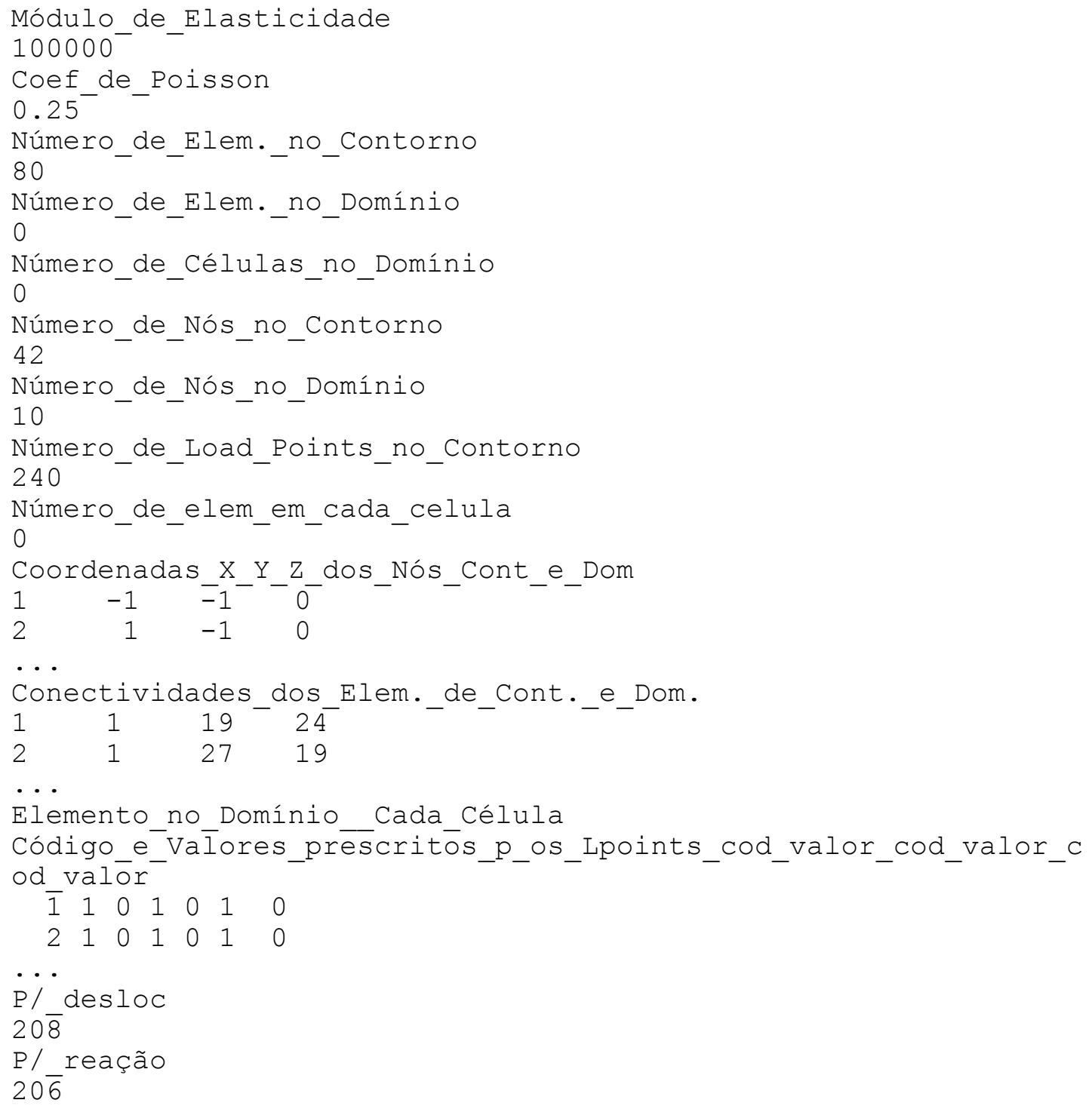

Nas últimas linhas do arquivo de entrada são informados dois nós, escolhidos para dar saída em seus valores de deslocamentos e forças para um arquivo 
“GRF.DAT” que pode ser lido por um aplicativo gráfico (Origin ${ }^{2}$, por exemplo) e traçado seus gráficos na variável tempo.

Outra modificação necessária no módulo I, para o regime elastodinâmico, é a inclusão da sub-rotina "Montagem da matriz de massa" que, para a Reciprocidade Dual apresentada no item 3.4, tem o fluxograma mostrado conforme a figura 7.3.2

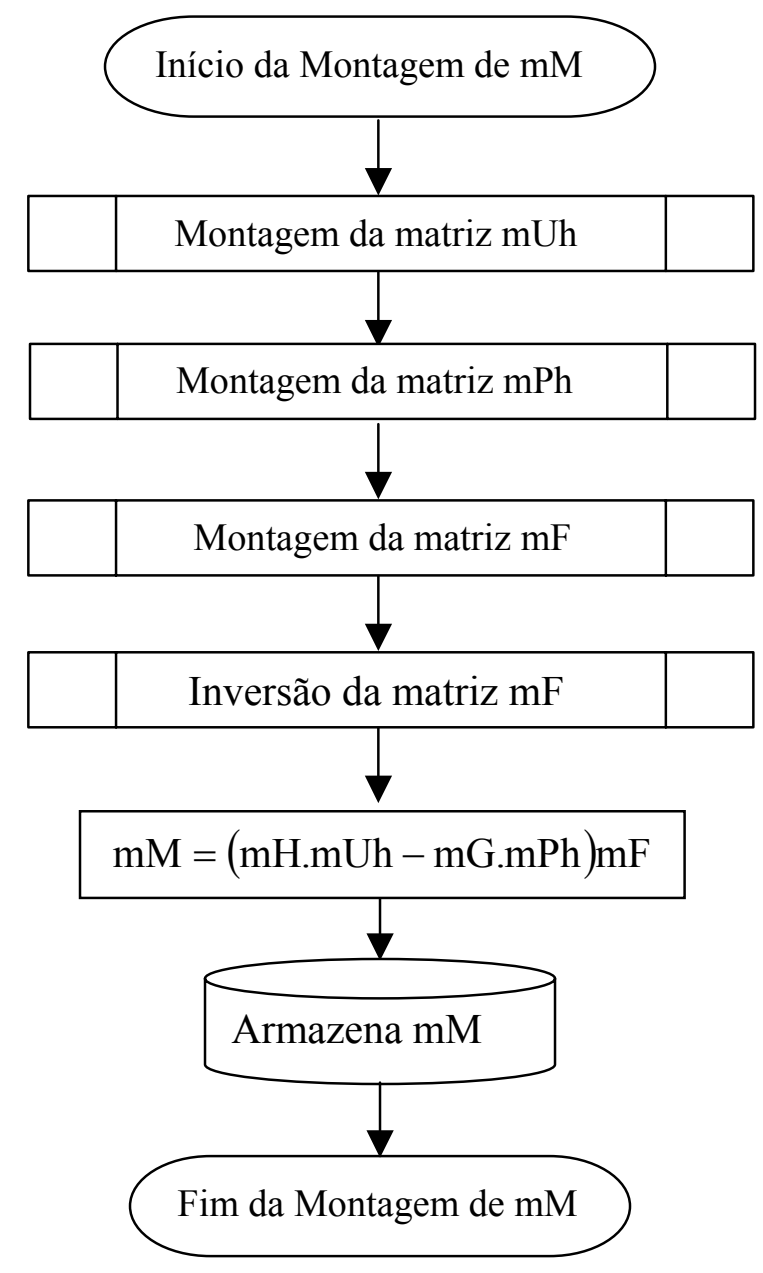

FIGURA 7.3.2 - Fluxograma da sub-rotina de montagem da matriz de massa.

As demais sub-rotinas são as mesmas apresentadas para o algoritmo elastostático.

\footnotetext{
${ }^{2}$ Microcal Origin, da Microcal Software Inc.
} 


\subsection{2 - Técnica da Integração Direta}

O módulo I do algoritmo elastodinâmico, onde a matriz de massa é obtida a partir da discretização do domínio em células não convencionais, necessita de dados de entrada referentes aos elementos do contorno de cada célula e sua quantidade. Portanto, o arquivo de entrada (“*.DAT") passa a ter novos campos, conforme mostra a listagem a seguir:

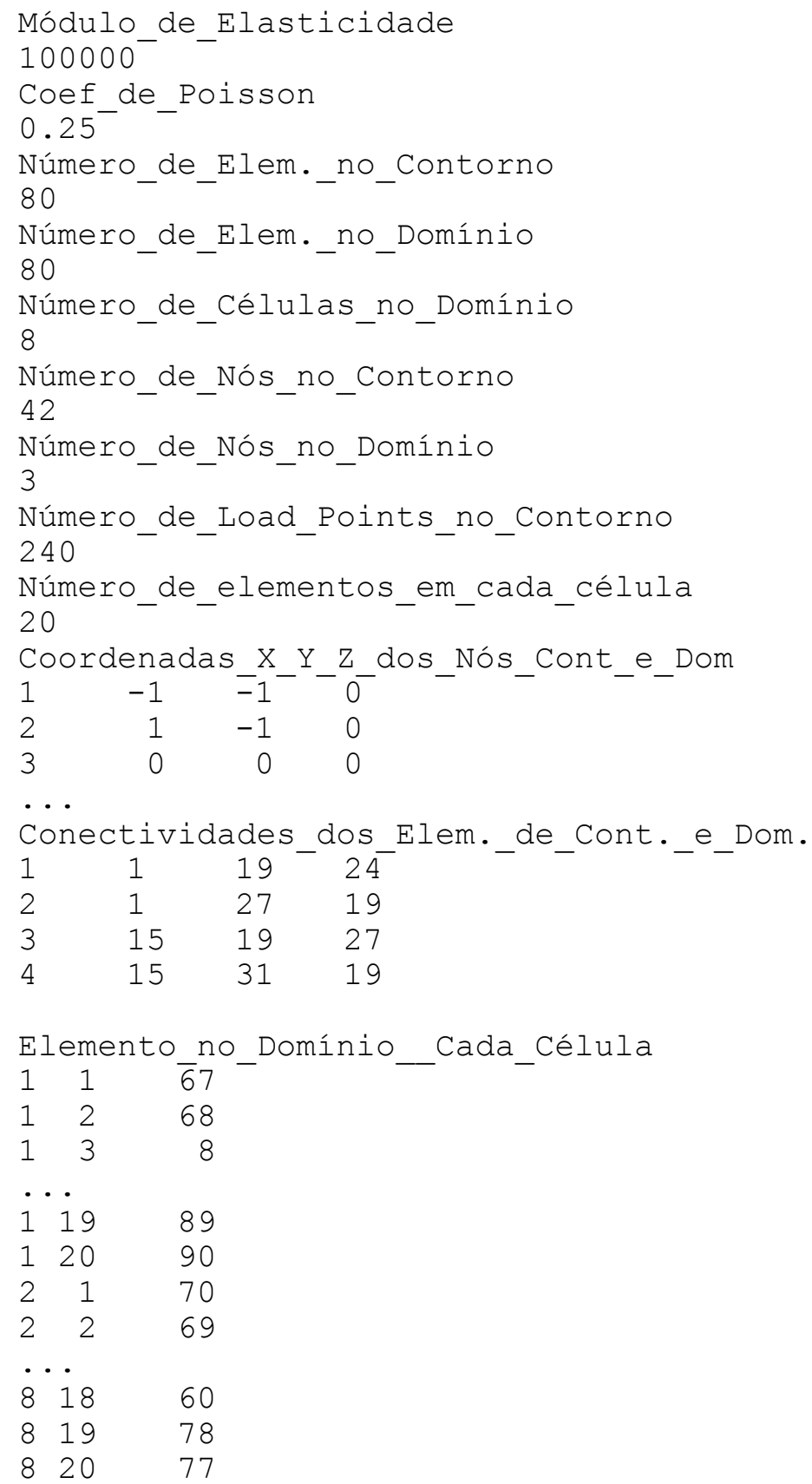




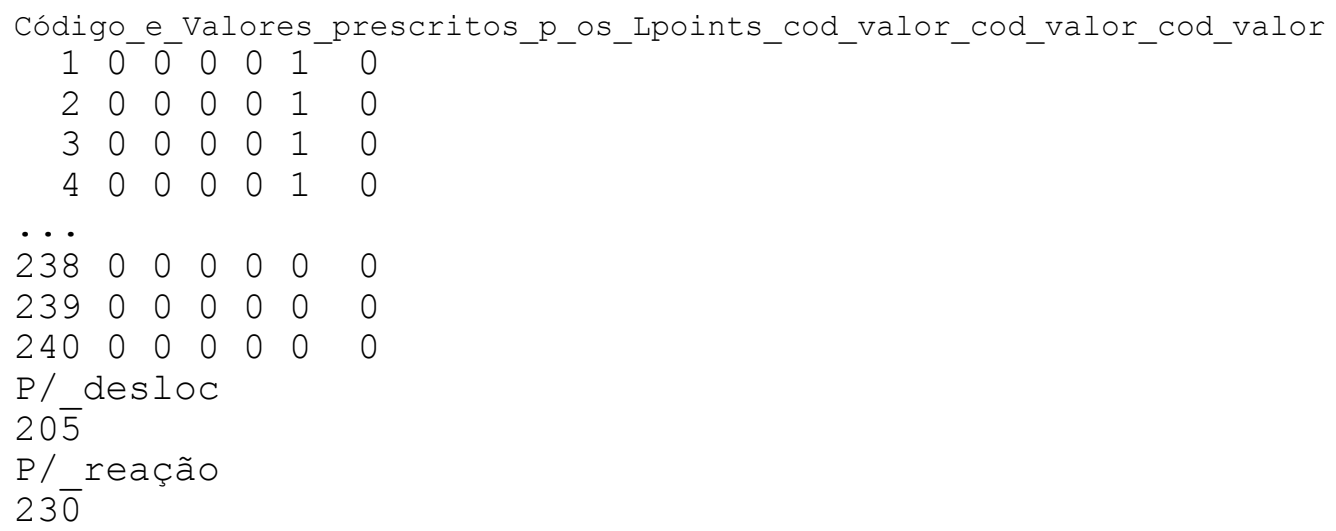

$\mathrm{Na}$ listagem acima, surgem informações adicionais quanto ao número de células (Ncel), número de elementos no domínio (nas faces internas do contorno das células - NelemDom), número de nós no domínio (NNosDom), número de elementos em cada célula (nelemcel) e quais os elementos que constituem cada célula (mElemDom[i][j]).

Nas últimas linhas do arquivo de entrada, semelhante ao apresentado no item 7.3.2, são informados dois nós, escolhidos para dar saída em seus valores de deslocamentos e forças para um arquivo "GRF.DAT" que pode ser lido por um programa gráfico (Origin, por exemplo) e traçado seus gráficos ao longo do tempo.

Outra modificação exigida é apresentada na sub-rotina "Montagem da matriz mM", onde as expressões envolvidas não possuem problemas de singularidade e, portanto, o algoritmo segue a forma da sub-rotina "Integral regular" da elastostática, implementando-se (3.5.11), e tendo-se em vista o que é estabelecido no anexo D.

\section{4 - ALGORITMO PARA FRATURA MECÂNICA}

O algoritmo para análise de fratura mecânica é desenvolvido considerando-se três módulos, como mostra a figura 7.4.1. 


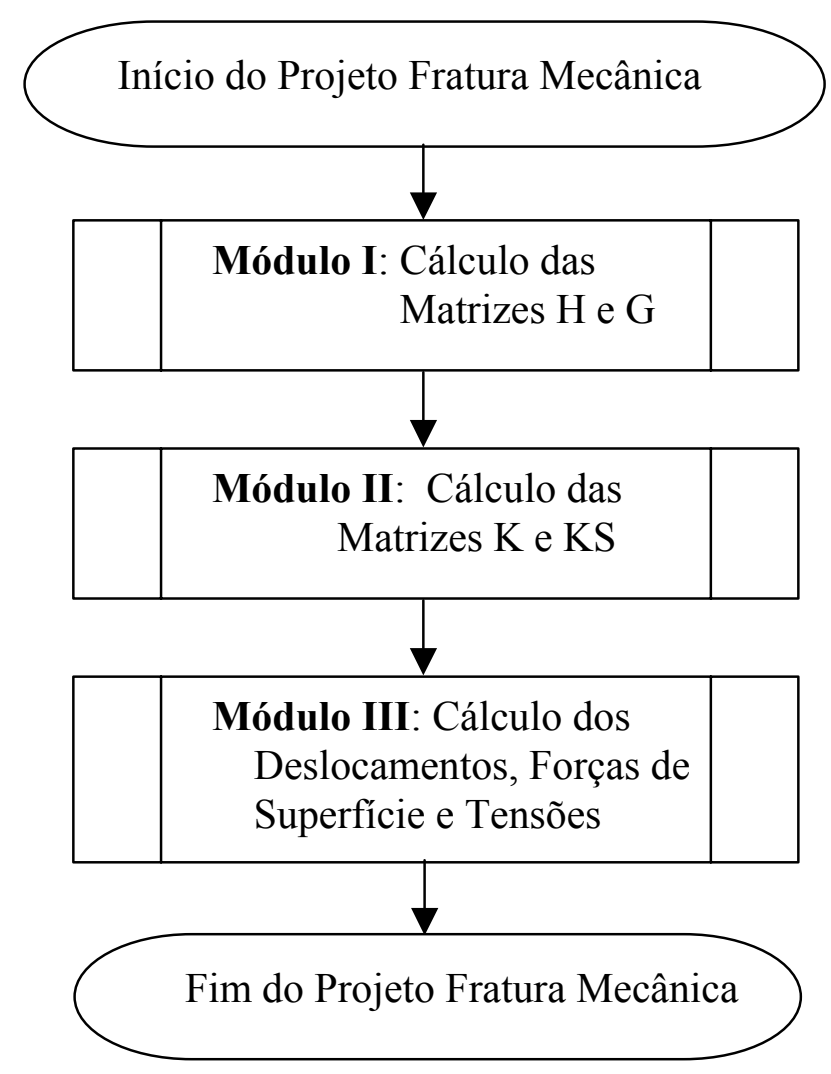

FIGURA 7.4.1 - Desenvolvimento em módulos do algoritmo para análise de fratura mecânica.

A) Módulo I:

O módulo I deste item segue a mesma seqüência apresentada para o algoritmo elastostático, figura 7.2.2, apenas excluindo-se a sub-rotina do vetor índice. $\mathrm{O}$ arquivo de entrada é o mesmo, assim como o fluxograma da sub-rotina de montagem das matrizes $\mathrm{H}$ e $\mathrm{G}$, figura 7.2.3.

B) Módulo II:

O módulo II do algoritmo da fratura mecânica é destinado ao cálculo das matrizes K e KS, que contêm os coeficientes de influência dos dipolos relacionados a 
deslocamentos e tensões, respectivamente. Implementa computacionalmente a formulação apresentada nos itens 5.4 e 5.6.

A sub-rotina usada para determinar a matriz $\mathrm{K}(\mathrm{mK})$ é mostrada na figura 7.4.2.

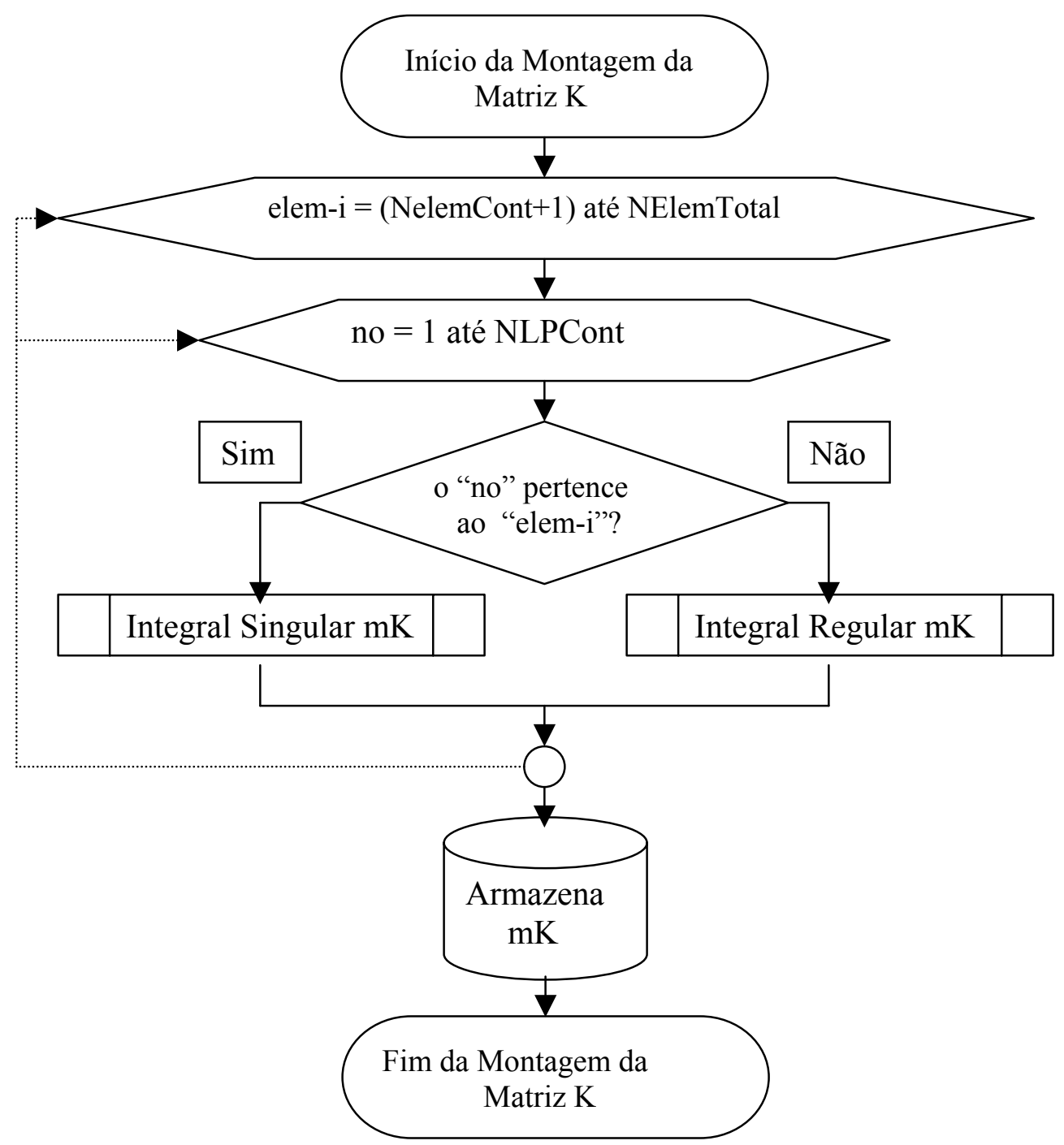

FIGURA 7.4.2 - Fluxograma da sub-rotina que calcula a matriz K dos dipolos.

As sub-rotinas "Integral singular mK" e "Integral regular mK" são elaboradas da mesma forma que as "Integral singular" e "Integral regular" do algoritmo elastostático. A expressão utilizada na determinação dos coeficientes de $\mathrm{K}$, exp. (5.4.12), tem singularidade forte igualmente encontrada nas expressões (2.4.4) e (2.4.5) da elastostática. 
A sub-rotina que determina a matriz $\mathrm{KS}(\mathrm{mKS})$ é mostrada na figura 7.4.3.

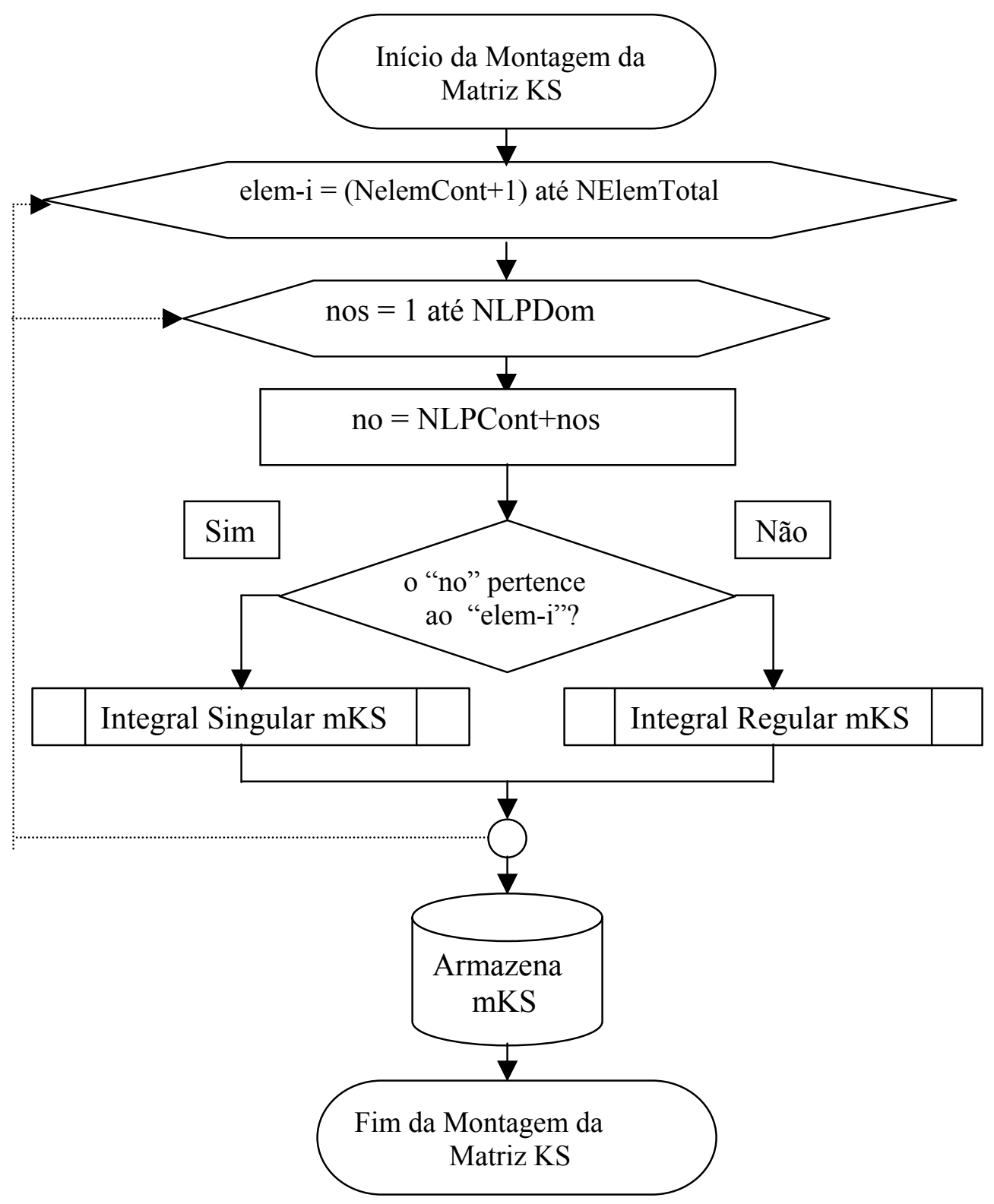

FIGURA 7.4.3 - Fluxograma da sub-rotina que calcula a matriz KS dos dipolos.

A sub-rotina "Integral regular mKS" na figura 7.4.3, é elaborada seguindo-se os moldes da "Integral regular" da elastostática, item 7.2, utilizando-se a integração numérica de Hammer e a subdivisão do elemento.

A sub-rotina "Integral singular mKS", referenciada na figura 7.4.3, segue a forma da "Integral singular" da elastostática. É utilizado o procedimento de transformar a integral sobre o elemento triangular em uma integral em seu contorno, 
efetuando-se a integração analítica na variável $r$ e numérica (Gauss) na variável $\theta$. Sendo hipersingular a expressão que determina os coeficientes de mKS, exp. (5.6.14), a integração em $r$ deve ser efetuada considerando-se a parte finita de Hadamard (PORTELA, 1993).

C) Módulo III:

O módulo III do algoritmo de fratura mecânica é responsável pelos cálculos dos deslocamentos e forças de superfície em pontos do contorno, bem como as tensões em pontos internos para as diversas etapas de um processo incremental (de forças ou deslocamentos). Portanto, os cálculos são efetuados para o sólido íntegro e na presença da fratura.

São implementadas as equações matriciais apresentadas no item 5.7.

O fluxograma principal do módulo III é mostrado na figura 7.4.4.

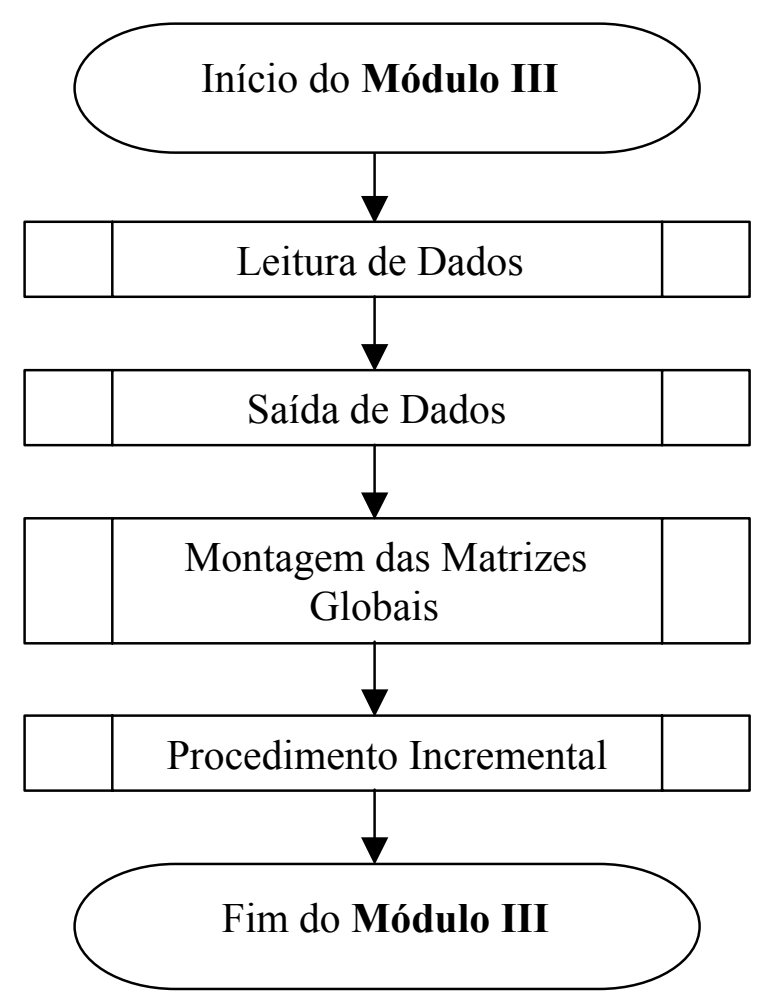

FIGURA 7.4.4 - Rotina principal do Módulo III do programa de fratura mecânica. 
A sub-rotina "Leitura de dados" recebe informações dos arquivos “*.DAT", “DAD.MEC", "MATK.MEC”, "MATKS.MEC", "MATH.MEC" e "MATG.MEC", arquivos de dados iniciais e os gerados nos módulos I e II.

A sub-rotina "Saída de dados" prepara os cabeçalhos dos arquivos de relatório (“*.SAI”, continuação) e de gráficos (“GRF.DAT”), que são alimentados com os dados calculados no processo incremental.

A sub-rotina "Montagem das matrizes globais" tem a finalidade de calcular as matrizes $\mathbf{M}$ e $\mathbf{R}$, exp. (5.7.3a-b), e $\mathbf{N}$ e $\mathbf{S}$, exp. (5.7.6a-b), para deslocamentos e tensões, respectivamente.

A sub-rotina "Procedimento incremental" calcula, para cada passo incremental (de força ou deslocamento), deslocamentos e forças de superfície com o sólido no regime elástico e verifica se o limite de resistência estabelecido foi atingido. Nesse procedimento, existe uma varredura sobre os pontos funcionais dos elementos de fratura para determinar quantos e quais excederam o limite estabelecido. A partir daí, é chamada uma sub-rotina iterativa que calcula os valores dos dipolos correspondentes à fratura (figura 7.4.5).

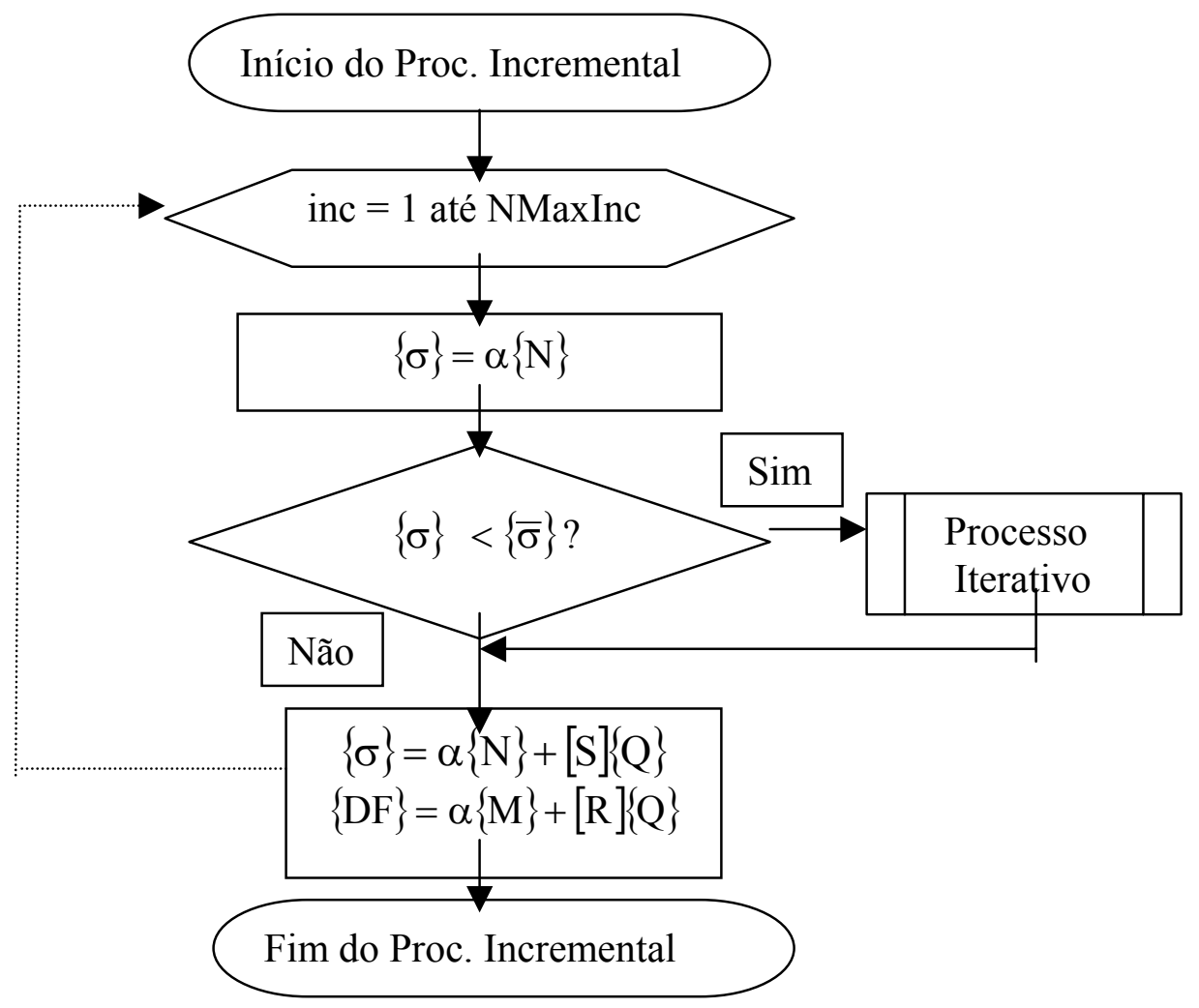

FIGURA 7.4.5 - Fluxograma do procedimento incremental. 
O fluxograma da sub-rotina iterativa é mostrado na figura 7.4.6.

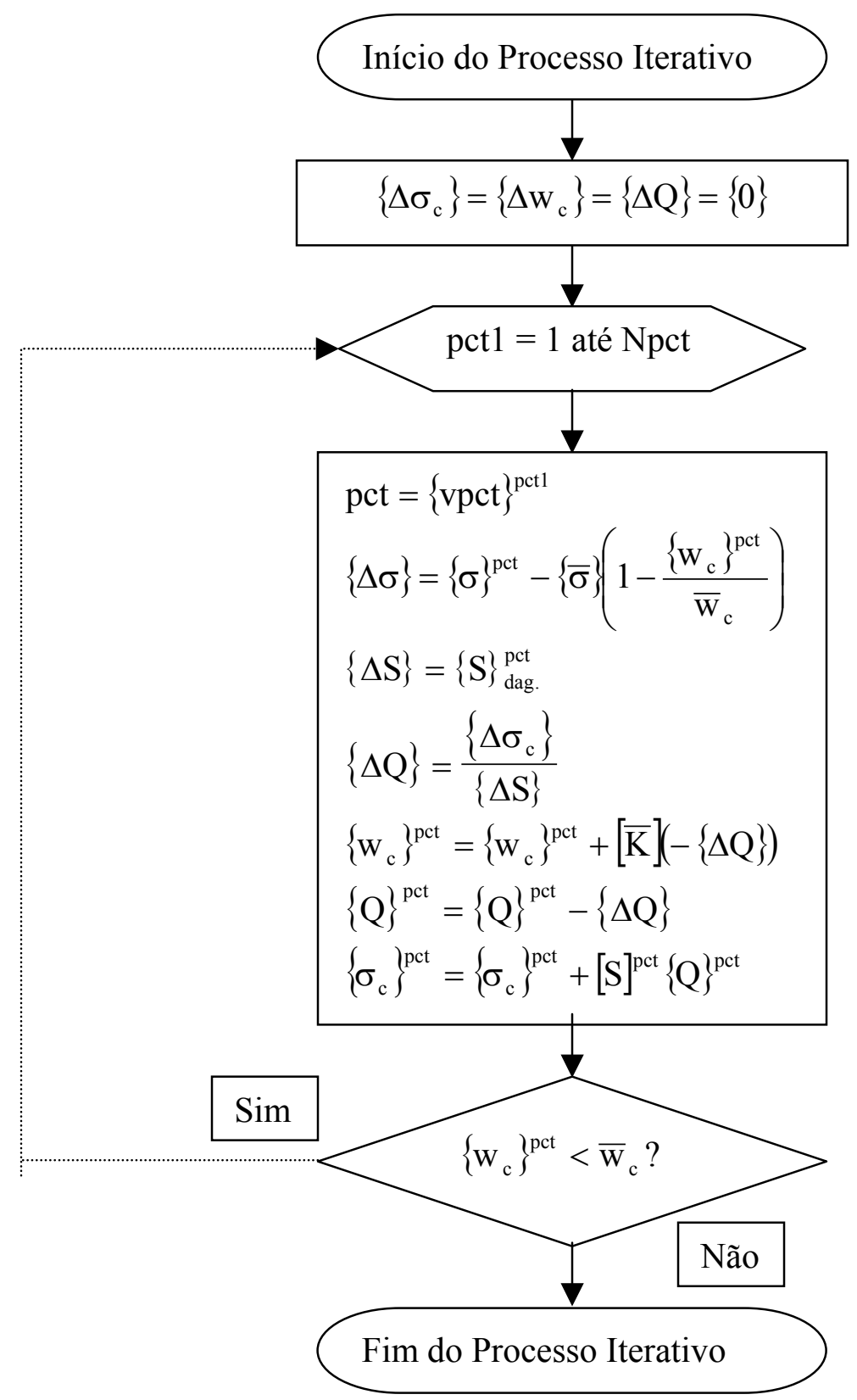

FIGURA 7.4.6 - Fluxograma do procedimento iterativo de fratura mecânica.

O fluxograma do processo iterativo mostra as principais operações efetuadas para se determinar os valores dos dipolos, responsáveis pela correção dos valores obtidos na análise elástica do corpo. Calculam-se os acréscimos de tensão, levandose em conta o critério adotado do modelo de fratura coesiva (item 4.3) e, em seguida, encontram-se os valores dos dipolos correspondentes. Calculam-se, então, os valores da variável abertura da fratura, que retroalimenta a equação do modelo coesivo em 
uma nova iteração. Uma vez atingida a abertura de fratura limite, o procedimento é finalizado, estabelecendo-se antes os novos valores para deslocamentos e forças de superfície, considerando-se agora o sólido fraturado.

Algumas informações sobre as matrizes envolvidas neste modulo III do algoritmo de fratura mecânica, quanto à ordem e componentes, estão mostradas a seguir. Inicialmente, considere-se que:

$$
\begin{aligned}
& \mathrm{nt}=3 * \text { NLPTotal } \\
& \mathrm{nd}=6 * \text { NLPDom } \\
& \mathrm{nd} 1=3 * \text { NLPDom }
\end{aligned}
$$

Assim,

$$
\begin{aligned}
& \sigma_{\text {im }} \Rightarrow\{\sigma\}^{\text {pct }}=\left\{\begin{array}{l}
\sigma_{11} \\
\sigma_{12} \\
\sigma_{13} \\
\sigma_{22} \\
\sigma_{23} \\
\sigma_{33}
\end{array}\right\} \Rightarrow\{\sigma\}=\left\{\begin{array}{l}
\{\sigma\}^{1} \\
\{\sigma\}^{2} \\
\{\sigma\}^{\text {NLPDom }}
\end{array}\right\} \Rightarrow\left\{\begin{array}{c}
\text { nd } \\
\text { nd }
\end{array}\right. \\
& \mathrm{SS}_{\mathrm{imk}} \Rightarrow\left[\mathrm{SS}^{\mathrm{pct}}=\left[\begin{array}{lllll}
\mathrm{SS}_{111} & \cdots & \mathrm{SS}_{11 \mathrm{k}} & \cdots & \mathrm{SS}_{11 \mathrm{nt}} \\
\mathrm{SS}_{121} & \cdots & \mathrm{SS}_{12 \mathrm{k}} & \cdots & \mathrm{SS}_{12 \mathrm{nt}} \\
\mathrm{SS}_{131} & \cdots & \mathrm{SS}_{13 \mathrm{k}} & \cdots & \mathrm{SS}_{13 \mathrm{nt}} \\
\mathrm{SS}_{221} & \cdots & \mathrm{SS}_{22 \mathrm{k}} & \cdots & \mathrm{SS}_{22 \mathrm{nt}} \\
\mathrm{SS}_{231} & \cdots & \mathrm{SS}_{23 \mathrm{k}} & \cdots & \mathrm{SS}_{23 \mathrm{nt}} \\
\mathrm{SS}_{331} & \cdots & \mathrm{SS}_{33 \mathrm{k}} & \cdots & \mathrm{SS}_{33 \mathrm{nt}}
\end{array}\right] \Rightarrow[\mathrm{SS}]=\left[\begin{array}{l}
{[\mathrm{SS}]^{1}} \\
{[\mathrm{SS}]^{2}} \\
\vdots \\
{[\mathrm{SS}]^{\mathrm{NLPDom}}}
\end{array}\right] \Rightarrow \underset{\text { nd Xnt }}{[\mathrm{SS}},\right. \\
& \mathrm{KS}_{\text {imk }} \Rightarrow[\mathrm{KS}]^{\mathrm{pct}}=\left[\begin{array}{lllll}
\mathrm{KS}_{111} & \cdots & \mathrm{KS}_{11 \mathrm{k}} & \cdots & \mathrm{KS}_{11 \text { nd } 1} \\
\mathrm{KS}_{121} & \cdots & \mathrm{KS}_{12 \mathrm{k}} & \cdots & \mathrm{KS}_{12 \text { nd }} \\
\mathrm{KS}_{131} & \cdots & \mathrm{KS}_{13 \mathrm{k}} & \cdots & \mathrm{KS}_{13 \text { nd }} \\
\mathrm{KS}_{221} & \cdots & \mathrm{KS}_{22 \mathrm{k}} & \cdots & \mathrm{KS}_{22 \text { nd }} \\
\mathrm{KS}_{231} & \cdots & \mathrm{KS}_{23 \mathrm{k}} & \cdots & \mathrm{KS}_{23 \mathrm{nd} 1} \\
\mathrm{KS}_{331} & \cdots & \mathrm{KS}_{33 \mathrm{k}} & \cdots & \mathrm{KS}_{33 \mathrm{nd} 1}
\end{array}\right] \Rightarrow[\mathrm{KS}]=\left[\begin{array}{l}
{[\mathrm{KS}]^{1}} \\
{[\mathrm{KS}]^{2}} \\
\vdots \\
{[\mathrm{KS}]^{\mathrm{NLPDom}}}
\end{array}\right] \Rightarrow[\mathrm{KS}]_{\text {nd nd1 }}, \mathrm{e} \\
& \mathrm{S}_{\text {imk }} \Rightarrow[\mathrm{S}]^{\text {pct }}=\left[\begin{array}{lllll}
\mathrm{S}_{111} & \cdots & \mathrm{S}_{11 \mathrm{k}} & \cdots & \mathrm{S}_{11 \text { nd } 1} \\
\mathrm{~S}_{121} & \cdots & \mathrm{S}_{12 \mathrm{k}} & \cdots & \mathrm{S}_{12 \text { nd } 1} \\
\mathrm{~S}_{131} & \cdots & \mathrm{S}_{13 \mathrm{k}} & \cdots & \mathrm{S}_{13 \mathrm{nd} 1} \\
\mathrm{~S}_{221} & \cdots & \mathrm{S}_{22 \mathrm{k}} & \cdots & \mathrm{S}_{22 \text { nd } 1} \\
\mathrm{~S}_{231} & \cdots & \mathrm{S}_{23 \mathrm{k}} & \cdots & \mathrm{S}_{23 \mathrm{nd} 1} \\
\mathrm{~S}_{331} & \cdots & \mathrm{S}_{33 \mathrm{k}} & \cdots & \mathrm{S}_{33 \mathrm{nd} 1}
\end{array}\right] \Rightarrow[\mathrm{S}]=\left[\begin{array}{l}
{[\mathrm{S}]^{1}} \\
{[\mathrm{~S}]^{2}} \\
\vdots \\
{[\mathrm{S}]^{\text {NLPDom }}}
\end{array}\right] \Rightarrow[\mathrm{S}] \text { nd x nd1 }
\end{aligned}
$$




\section{5 - ALGORITMO PARA FRATURA DINÂMICA}

O algoritmo para análise de fratura dinâmica está dividido em três módulos usados para implementar as equações apresentadas nos itens 6.5 e 6.6, como mostra a figura 7.5.1. Estes módulos reúnem partes dos algoritmos referenciados neste capítulo.

O módulo I deste item é o mesmo abordado no item 7.3.

O módulo II do algoritmo para fratura dinâmica é o mesmo apresentado no item 7.4.

O módulo III corresponde aproximadamente aos módulos IV do algoritmo elastodinâmico e III do fratura mecânica. Em relação ao módulo III, o processo incremental dá lugar ao processo dinâmico. O procedimento iterativo permanece praticamente o mesmo, exceto por uma nova instrução para avaliar os efeitos da força não mais monotônica.

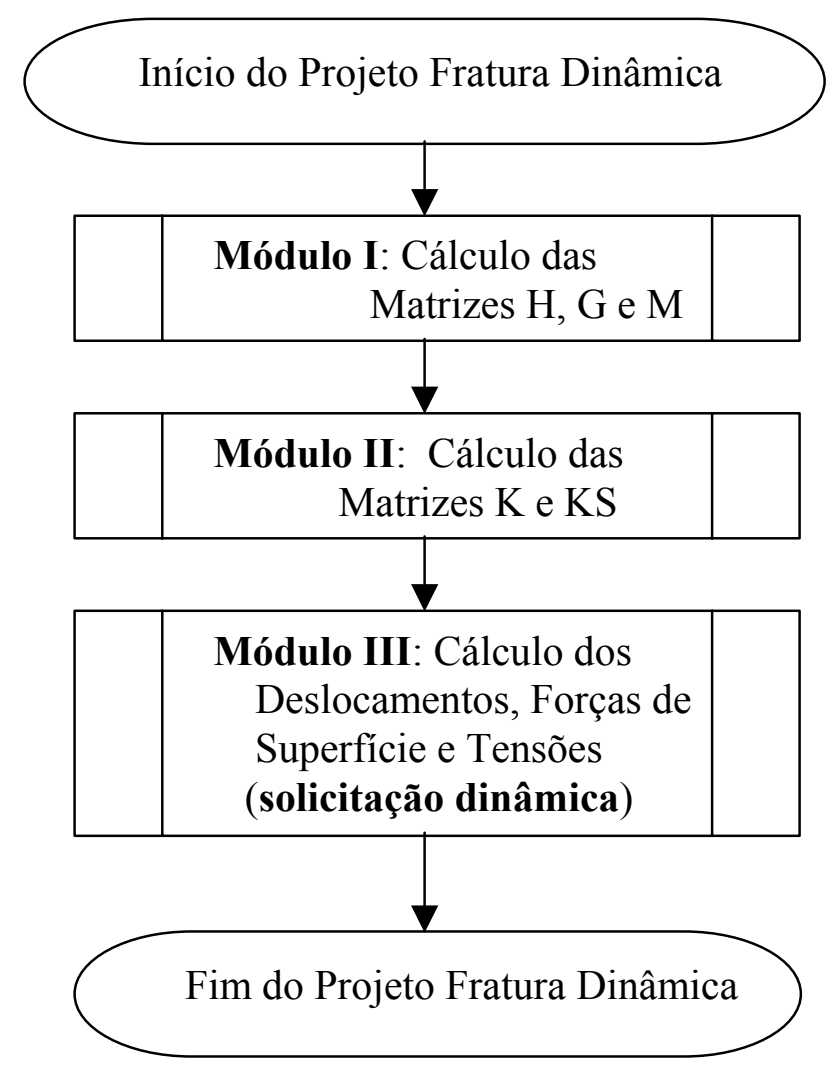

FIGURA 7.5.1 - Desenvolvimento em módulos do algoritmo para análise de fratura dinâmica. 


\section{CAPÍTULO 8}

\section{CONCLUSÕES}

Neste trabalho desenvolveu-se uma formulação do Método dos Elementos de Contorno (MEC) para análise de fratura dinâmica, a partir de formulações independentes da elastostática, elastodinâmica e fratura mecânica.

$\mathrm{Na}$ elastostática tridimensional, confirmou-se que o MEC é uma alternativa viável com alguma vantagem frente a outros métodos numéricos - com discretização apenas do contorno do sólido. O elemento triangular plano com aproximação linear, utilizado nas discretizações, mostrou-se adequado à forma do sólido e aos valores das variáveis envolvidas.

É necessário, porém, atenção às integrações numéricas efetuadas quando o ponto de colocação está muito próximo do elemento em operação. Uma alternativa, utilizada neste trabalho, é a subdivisão do elemento triangular empregado, que mostrou dar bons resultados.

As soluções fundamentais adotadas dão versatilidade ao algoritmo computacional elaborado. A solução de Kelvin é utilizada para sólidos de domínio finito, resultando em respostas com satisfatório grau de precisão. Já para problemas envolvendo escavações próximas à superfície do solo (problema no espaço semiinfinito), as soluções fundamentais de Mindlin e Boussinesq-Cerruti mostram-se mais adequadas, pois a discretização necessária se dá apenas na região do carregamento.

O algoritmo computacional, elaborado em quatro módulos, permite melhor uso da memória central do computador, além de efetuar os cálculos das variáveis de 
contorno isoladamente. Com isso, várias investigações em pontos do domínio podem ser feitas, bastando informar o novo arquivo de dados dos pontos de domínio.

$\mathrm{Na}$ elastodinâmica tridimensional, o MEC é testado utilizando-se as soluções fundamentais da elastostática, através do emprego do Método da Reciprocidade Dual (MRD) e da discretização em células. As formulações, empregadas na análise de problemas transientes, apresentam bons resultados, em consonância com trabalhos publicados. Sabe-se, entretanto, que discretizações com maior número de elementos (com relação ao que foi utilizado neste trabalho) são recomendadas para o problema dinâmico.

O MRD mostra-se inserido com o propósito principal do MEC que é o de analisar problemas com equações escritas para pontos no contorno, embora necessite da inclusão de pontos internos (pólos) em alguns casos. Na formulação em células, é aplicado um procedimento que permite parametrizá-las através de seus contornos, permitindo a existência de células de forma qualquer. Os resultados desta são um pouco melhores do que os apresentados com o emprego do MRD, além de necessitar de menor tempo computacional. Os algoritmos de integração no tempo escolhidos (Newnark e Houbolt) apresentam bons resultados, com valores bem próximos entre eles.

A formulação do MEC para fraturas mecânicas apresentada mostra-se eficiente e versátil, já que o uso de dipolos de forças permite a representação das superfícies de fratura apenas introduzindo uma rede de elementos na superfície média da mesma. Seus valores corrigem as respostas provenientes do cálculo elástico das variáveis, efetuado na forma clássica do método, com economia de tempo computacional, uma vez que as matrizes envolvidas na discretização do contorno permanecem as mesmas, apenas acrescentando-se as componentes relativas aos pontos internos (na superfície fictícia da fratura), à medida que estes são definidos.

Os exemplos processados ao longo do trabalho demostram a eficiência das formulações. As expressões das contribuições dos dipolos para deslocamentos e tensões, representadas pelas componentes dos tensores deduzidos e apresentados, são contribuições efetivas para o desenvolvimento desta área de conhecimento. $\mathrm{O}$ algoritmo computacional elaborado mantém a característica básica de módulos, acionados para tarefas específicas, racionalizando o uso da memória central e 
permitindo investigações diferentes sem a necessidade de processar toda a formulação.

A discretização do contorno do sólido, feita através de elementos triangulares planos com aproximação linear descontínua, permite a abordagem de descontinuidade entre dois elementos, o que efetivamente representa a possibilidade de fraturamento. A superfície fictícia de fratura é discretizada pelo mesmo elemento com aproximação constante, e os resultados são bem satisfatórios, demostrando a validade de seu uso.

A formulação do MEC apresentada para fratura dinâmica utiliza os conceitos apresentados para as formulações elastostática, elastodinâmica e da fratura mecânica. Os resultados obtidos comprovam o bom condicionamento da formulação para a análise deste problema, demostrando ser mais uma ferramenta alternativa entre várias outras de resolução. A formulação é precisa e permite que a rede de elementos seja acrescida de outros na superfície fíctícia da fratura, não necessitando de uma redefinição total da rede, com diminuição de tempo computacional.

O presente trabalho envolve três grandes áreas de conhecimento: elastostática, elastodinâmica e mecânica da fratura, e limitou-se, entre as muitas alternativas possíveis de abordagem, ao desenvolvimento de formulações do Método dos Elementos de Contorno de uma parte da mecânica da fratura. Portanto, algumas possíveis extensões da presente pesquisa podem se constituir em temas para investigações futuras, sugerindo-se, entre elas:

- trabalhos de iteração solo-estrutura, utilizando-se a solução fundamental de Kelvin para o sólido (estrutura) e a solução de Mindlin para o solo (meio semi-infinito);

- trabalhos de iteração solo estrutura, utilizando-se formulações do Método dos Elementos Finitos (MEF) para a estrutura acopladas à formulação do MEC com a solução fundamental de Mindlin;

- utilização, a partir dos trabalhos citados nos itens anteriores, de elementos curvos ou planos com aproximação de ordem superior à utilizada;

- trabalhos no âmbito da elastodinâmica com combinação do MEF com o MEC; 
- avaliação de outras famílias de funções para uso do Método da Reciprocidade Dual;

- avaliação dos algoritmos de integração no tempo, Newmark e Houbolt por exemplo, de forma mais sistemática, partindo-se da característica de não simetria das matrizes envolvidas;

- avaliação de problemas de multifraturamento concorrentes;

- utilização de elementos descontínuos com aproximação de ordem superior à que foi utilizada na discretização da superfície fictícia de fratura;

- avaliação de algoritmos eficientes de propagação da superfície fictícia da fratura;

- trabalhos para elaboração de pré e pós-processadores gráficos para os diversos algoritmos computacionais elaborados, permitindo uma apresentação mais adequada. 


\section{ANEXO A}

\section{DELTA DE DIRAC}

Neste anexo é mostrado o importante conceito da distribuição Delta de Dirac, utilizada na formulação do Método dos Elementos de Contorno.

A distribuição Delta de Dirac, denotada por $\delta(s, q)$ ou $\delta(s, q)=\Delta^{s}$, corresponde a um pulso retangular unitário, como mostra a figura A.1.
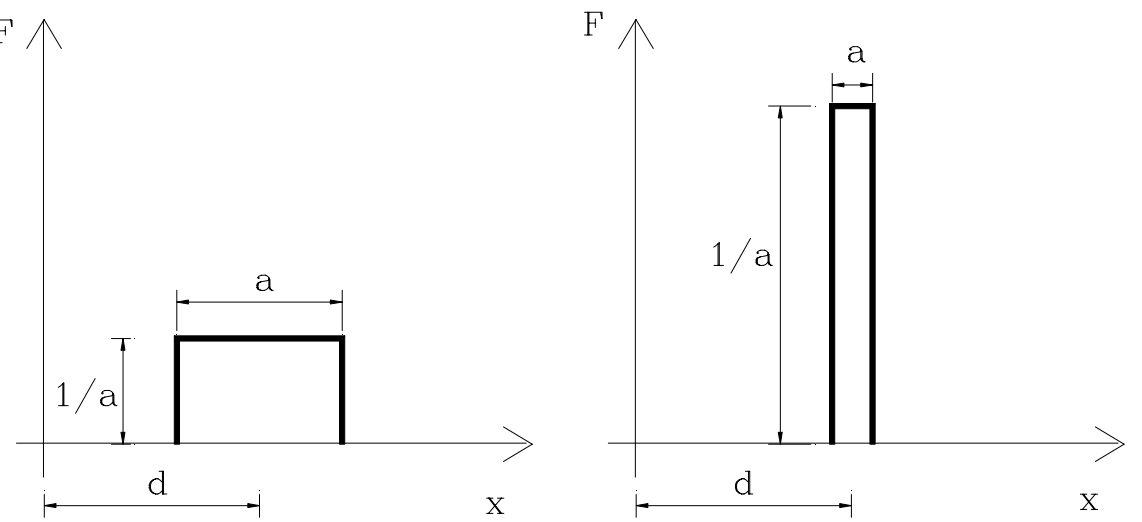

FIGURA A.1 - Pulso retangular unitário.

A função $\mathrm{F}(\mathrm{x}, \mathrm{d}, \mathrm{a})$ representada na figura $\mathrm{A} .1$, tem como característica o valor unitário de sua integral qualquer que seja o domínio. É definida da seguinte forma:

$$
\mathrm{F}(\mathrm{x}, \mathrm{d}, \mathrm{a})= \begin{cases}0 ; & \mathrm{x}<\mathrm{d}-\frac{\mathrm{a}}{2} \\ \frac{1}{\mathrm{a}} ; & \mathrm{d}-\frac{\mathrm{a}}{2} \leq \mathrm{x} \leq \mathrm{d}+\frac{\mathrm{a}}{2} \\ 0 ; & \mathrm{x}>\mathrm{d}+\frac{\mathrm{a}}{2}\end{cases}
$$


Denomina-se de distribuição Delta de Dirac o limite da função pulso unitário quando a largura "a" do retângulo tende para zero, ou seja,

$$
\delta(x-d)=\underset{a \rightarrow 0}{\operatorname{Lim}} F(x, d, a)
$$

Para domínios n-dimensionais, a distribuição Delta de Dirac apresenta-se com as seguintes propriedades:

$$
\begin{aligned}
& \delta(s, q)=0, \text { se } q \neq s ; \\
& \delta(s, q)=\infty, \text { se } q=s ; e \\
& \int_{\Omega} \rho(q) \delta(s, q) d \Omega=\rho(s)
\end{aligned}
$$

onde s e q são pontos do domínio $\Omega$, e $\rho(q)$ uma função qualquer.

Pode-se obter maiores informações sobre o assunto em KANE (1994). 


\section{ANEXO B}

\section{DERIVADAS DO TENSOR DAS TENSÕES PARA A SOLUÇÃO FUNDAMENTAL DE MINDLIN}

Neste anexo são mostradas as derivadas do tensor de terceira ordem das tensões utilizadas para a determinação de $S_{i j k}^{*}$ e $D_{i j k}^{*}$ da solução fundamental de Mindlin.

As derivadas foram transcritas do trabalho de LAETHEM et al. (1984).

Os parâmetros $\mathrm{K}_{\mathrm{s}}, \mathrm{r}_{\mathrm{i}}, \mathrm{R}_{\mathrm{i}}$, c e z são definidos no item 2.4 .2 do capítulo 2. A seguir, são definidas as expressões auxiliares:
$\mathrm{A}_{1}=(1-2 v)$
$A_{2}=(3-4 v)$
$\mathrm{A}_{3}=(1-v)$
$\mathrm{D}=2 \delta_{3 \mathrm{i}}-1$

As expressões indiciais a seguir são denominadas de (B.2a-j).

$$
\begin{aligned}
& \mathrm{C}_{1}(\mathrm{i}, \mathrm{j})=\frac{6 \mathrm{r}_{\mathrm{j}} \delta_{\mathrm{ij}}}{\mathrm{r}^{5}}-\frac{15 \mathrm{r}_{\mathrm{j}}^{2} \mathrm{r}_{\mathrm{i}}}{\mathrm{r}^{7}} \\
& \mathrm{C}_{2}(\mathrm{i}, \mathrm{j})=\frac{6 \mathrm{~A}_{2} \mathrm{r}_{\mathrm{j}} \delta_{\mathrm{ij}}}{\mathrm{R}^{5}}-\frac{15 \mathrm{~A}_{2} \mathrm{r}_{\mathrm{j}}^{2} \mathrm{R}_{\mathrm{i}} \mathrm{D}}{\mathrm{R}^{7}}
\end{aligned}
$$




$$
\begin{aligned}
& C_{3}(i, j, k)=\frac{4 A_{3} A_{1}}{R^{2}\left(R+R_{3}\right)^{4}}\left[k-\frac{r_{j}^{2}\left(3 R+R_{3}\right)}{R^{2}\left(R+R_{3}\right)}\right]\left[\frac{R_{i} D}{R}\left(R+R_{3}\right)^{2}+2 R\left(R+R_{3}\right)\left(\frac{R_{i} D}{R}+\delta_{3 i}\right)\right]+ \\
& -\frac{4 A_{3} A_{1}}{R\left(R+R_{3}\right)^{2}}\left[\frac{2 r_{j}\left(3 R+R_{3}\right) \delta_{i j}}{R^{2}\left(R+R_{3}\right)}-\frac{r_{j}^{2}\left(\frac{3 R_{i} D}{R}+\delta_{3 i}\right)}{R^{2}\left(R+R_{3}\right)}+\frac{r_{j}^{2}\left(3 R+R_{3}\right)}{R^{4}\left(R+R_{3}\right)^{2}}\left[2 R_{i} D\left(R+R_{3}\right)+R^{2}\left(\frac{R_{i} D}{R}+\delta_{3 i}\right)\right]\right] \\
& \mathrm{C}_{4}(\mathrm{i}, \mathrm{j}, \mathrm{k}, \ell, \mathrm{m})=\left[\frac{6 \delta_{3 \mathrm{i}}}{\mathrm{R}^{5}}-\frac{30 \mathrm{cR} \mathrm{i}_{\mathrm{i}} \mathrm{D}}{\mathrm{R}^{7}}\right]\left[\mathrm{mc}-(\mathrm{k}-1 v) \mathrm{R}_{3}+\frac{5 \mathrm{r}_{\mathrm{j}}^{2} \mathrm{z}}{\mathrm{R}^{2}}\right]+ \\
& +\frac{6 \mathrm{c}}{\mathrm{R}^{5}}\left[\mathrm{~m} \delta_{3 \mathrm{i}}-(\mathrm{k}-1 v) \delta_{3 \mathrm{i}}-\frac{10 \mathrm{r}_{\mathrm{j}} \mathrm{z} \delta_{\mathrm{ij}}}{\mathrm{R}^{2}}-\frac{10 \mathrm{r}_{\mathrm{j}} \mathrm{zR} \mathrm{R}_{\mathrm{i}} \mathrm{D}}{\mathrm{R}^{4}}\right] \\
& \mathrm{C}_{5}(\mathrm{i}, \mathrm{j})=\left[-\frac{6 \mathrm{z} \delta_{3 \mathrm{i}}}{\mathrm{R}^{5}}+\frac{30 \mathrm{czR} \mathrm{R}_{\mathrm{i}} \mathrm{D}}{\mathrm{R}^{7}}\right]\left[1-\frac{5 \mathrm{r}_{\mathrm{j}}^{2}}{\mathrm{R}^{2}}\right]-\frac{6 \mathrm{cz}}{\mathrm{R}^{5}}\left[\frac{10 \mathrm{r}_{\mathrm{j}} \delta_{\mathrm{ij}}}{\mathrm{R}^{2}}+\frac{10 \mathrm{r}_{\mathrm{j}}^{2} \mathrm{R}_{\mathrm{i}} \mathrm{D}}{\mathrm{R}^{4}}\right] \\
& \mathrm{C}_{6}(\mathrm{i}, \mathrm{j})=\left[-\frac{6 \delta_{3 i}}{\mathrm{R}^{5}}+\frac{30 \mathrm{cR} \mathrm{i}_{\mathrm{i}} \mathrm{D}}{\mathrm{R}^{7}}\right]\left[\mathrm{zR}_{3}-\mathrm{A}_{1} \mathrm{r}_{\mathrm{j}}{ }^{2}-\frac{5 \mathrm{r}_{2}^{\mathrm{j}} \mathrm{ZR}_{3}}{\mathrm{R}^{2}}\right]+ \\
& -\frac{6 c}{R^{5}}\left[z \delta_{3 i}+2 A_{1} r_{j} \delta_{i j}+\frac{10 r_{j} z R_{3} \delta_{i j}}{R^{2}}-\frac{5 r_{j}^{2} z \delta_{3 i}}{R^{2}}+\frac{10 r_{j}^{2} z R_{3} R_{i} D}{R^{4}}\right] \\
& \mathrm{C}_{7}(\mathrm{i}, \mathrm{j})=\frac{6 \mathrm{r}_{\mathrm{j}} \mathrm{r}_{3} \delta_{\mathrm{ij}}}{\mathrm{r}^{5}}+\frac{3 \mathrm{r}_{\mathrm{j}}^{2} \delta_{3 \mathrm{i}}}{\mathrm{r}^{5}}-\frac{15 \mathrm{r}_{\mathrm{j}}^{2} \mathrm{r}_{3} \mathrm{r}_{\mathrm{i}}}{\mathrm{r}^{7}} \\
& \mathrm{C}_{8}(\mathrm{i}, \mathrm{j})=\frac{6 \mathrm{~A}_{2} \mathrm{r}_{\mathrm{i}} \mathrm{r}_{3} \delta_{\mathrm{ij}}+3 \mathrm{~A}_{2} \mathrm{r}_{\mathrm{j}}^{2} \delta_{3 \mathrm{i}}}{\mathrm{R}^{5}}+\frac{6\left(\mathrm{R}_{3}+\mathrm{z}\right) \delta_{3 \mathrm{i}}\left(\mathrm{A}_{1} \mathrm{z}-2 v \mathrm{c}\right)}{\mathrm{R}^{5}}-\frac{12 \mathrm{cR} \mathrm{R}_{3} v \delta_{3 \mathrm{i}}}{\mathrm{R}^{5}}+ \\
& +\frac{5\left(3 \mathrm{~A}_{2} \mathrm{r}_{j}^{2} \mathrm{r}_{3}-6 \mathrm{cR} \mathrm{R}_{3}\left(\mathrm{~A}_{1} \mathrm{z}-2 v \mathrm{c}\right)\right) \mathrm{R}_{\mathrm{i}} \mathrm{D}}{\mathrm{R}^{7}} \\
& \mathrm{C}_{9}(\mathrm{i}, \mathrm{j})=\frac{-30 \delta_{3 \mathrm{i}} \mathrm{r}_{\mathrm{j}}^{2} \mathrm{zR} \mathrm{R}_{3}-30 \mathrm{cr}_{\mathrm{j}}^{2} z \delta_{3 \mathrm{i}}+60 \mathrm{cr}_{\mathrm{j}} \mathrm{zR} \mathrm{R}_{3} \delta_{\mathrm{ij}}}{\mathrm{R}^{7}}+\frac{\left(210 \mathrm{cr}_{\mathrm{j}}^{2} z \mathrm{R}_{3}\right) \mathrm{R}_{\mathrm{i}} \mathrm{D}}{\mathrm{R}^{9}} \\
& \mathrm{C}_{10}(\mathrm{i}, \mathrm{j})=\frac{4 \mathrm{~A}_{3} \mathrm{~A}_{1}}{\mathrm{R}^{2}\left(\mathrm{R}+\mathrm{R}_{3}\right)^{2}}\left[1-\frac{\mathrm{r}_{\mathrm{j}}^{2}}{\mathrm{R}\left(\mathrm{R}+\mathrm{R}_{3}\right)}-\frac{\mathrm{r}_{\mathrm{j}}^{2}}{\mathrm{R}^{2}}\right]\left[\frac{\mathrm{R}_{\mathrm{i}} \mathrm{D}}{\mathrm{R}}\left(\mathrm{R}+\mathrm{R}_{3}\right)+\mathrm{R}\left(\frac{\mathrm{R}_{\mathrm{i}} \mathrm{D}}{\mathrm{R}}+\delta_{3 \mathrm{i}}\right)\right]-\frac{4 \mathrm{~A}_{3} \mathrm{~A}_{1}}{\mathrm{R}\left(\mathrm{R}+\mathrm{R}_{3}\right)} \\
& {\left[\frac{2 r_{j} \delta_{i j}}{R\left(R+R_{3}\right)}+\frac{r_{j}^{2}}{R^{2}\left(R+R_{3}\right)^{2}}\left[\frac{R_{i} D}{R}\left(R+R_{3}\right)+R\left(\frac{R_{i} D}{R}+\delta_{3 i}\right)\right]+\frac{2 r_{j} \delta_{i j}}{R^{2}}+\frac{2 r_{j}^{2} R_{i} D}{R^{4}}\right]}
\end{aligned}
$$


Expressões das derivadas do tensor de terceira ordem, denominadas de (B.3a-r):

$$
\begin{aligned}
& \sigma_{11, \mathrm{i}}^{* 1}=-\frac{\delta_{1 i}}{\mathrm{r}_{1}} \sigma_{11}^{* 1}+\mathrm{K}_{\mathrm{s}} \mathrm{r}_{1}\left[\begin{array}{l}
-\frac{3 \mathrm{~A}_{1} \mathrm{r}_{\mathrm{j}}}{\mathrm{r}^{5}}-\frac{3 \mathrm{~A}_{1}(5-4 v) \mathrm{R}_{\mathrm{i}} \mathrm{D}}{\mathrm{R}^{5}}+ \\
+\mathrm{C}_{1}(\mathrm{i}, 1)+\mathrm{C}_{2}(\mathrm{i}, 1)+\mathrm{C}_{3}(\mathrm{i}, 1,3)+\mathrm{C}_{4}(\mathrm{i}, 1,3,2,3)
\end{array}\right] \\
& \sigma_{12, \mathrm{i}}^{* 1}=-\frac{\delta_{2 \mathrm{i}}}{\mathrm{r}^{2}} \sigma_{12}^{* 1}+\mathrm{K}_{\mathrm{s}} \mathrm{r}_{2}\left[-\frac{3 \mathrm{~A}_{1} \mathrm{r}_{\mathrm{i}}}{\mathrm{r}^{5}}-\frac{3 \mathrm{~A}_{1} \mathrm{R}_{\mathrm{i}} \mathrm{D}}{\mathrm{R}^{5}}+\mathrm{C}_{1}(\mathrm{i}, 1)+\mathrm{C}_{2}(\mathrm{i}, 1)+\mathrm{C}_{3}(\mathrm{i}, 1,1)+\mathrm{C}_{5}(\mathrm{i}, 1)\right] \\
& \sigma_{13, i}^{* 1}=K_{s}\left[\begin{array}{l}
\frac{A_{1} \delta_{3 i}}{r^{3}}-\frac{3 A_{1} r_{3} r_{i}}{r^{5}}-\frac{A_{1} \delta_{3 i}}{R^{3}}-\frac{3 A_{1} r_{3} R_{i} D}{R^{5}}+\frac{3 r_{1}^{2} \delta_{3 i}}{r^{5}}+r_{3} C_{1}(i, 1)-\frac{3 A_{2} r_{1}^{2} \delta_{3 i}}{R^{5}}+ \\
+R_{3} C_{2}(i, 1)+C_{6}(i, 1)
\end{array}\right] \\
& \sigma_{22, \mathrm{i}}^{* 1}=-\frac{\delta_{1 \mathrm{i}}}{\mathrm{r}_{1}} \sigma_{22}^{* 1}+\mathrm{K}_{\mathrm{s}} \mathrm{r}_{1}\left[\begin{array}{l}
-\frac{3 \mathrm{~A}_{1} \mathrm{r}_{\mathrm{i}}}{\mathrm{r}^{5}}-\frac{3 \mathrm{~A}_{1} \mathrm{~A}_{2} \mathrm{R}_{\mathrm{i}} \mathrm{D}}{\mathrm{R}^{5}}+ \\
+\mathrm{C}_{1}(\mathrm{i}, 2)+\mathrm{C}_{2}(\mathrm{i}, 2)+\mathrm{C}_{3}(\mathrm{i}, 2,1)+\mathrm{C}_{4}(\mathrm{i}, 2,1,2,1)
\end{array}\right]
\end{aligned}
$$

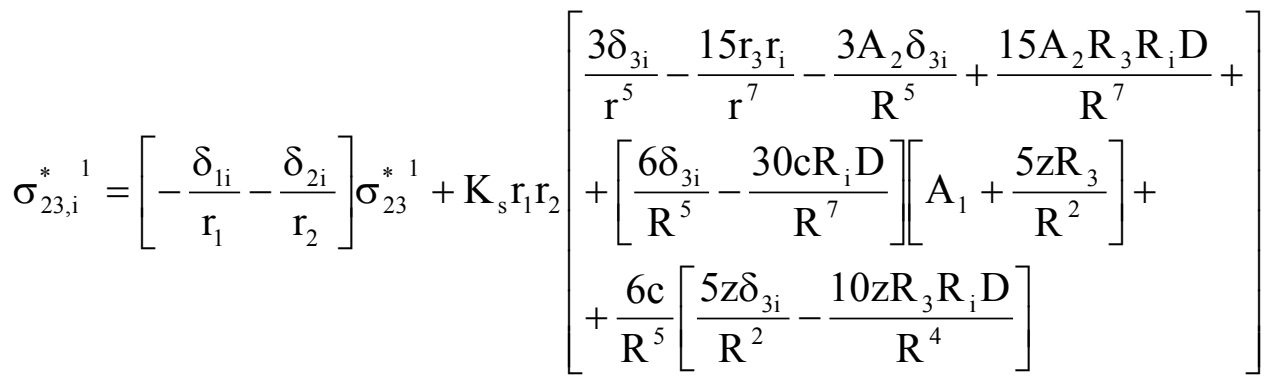

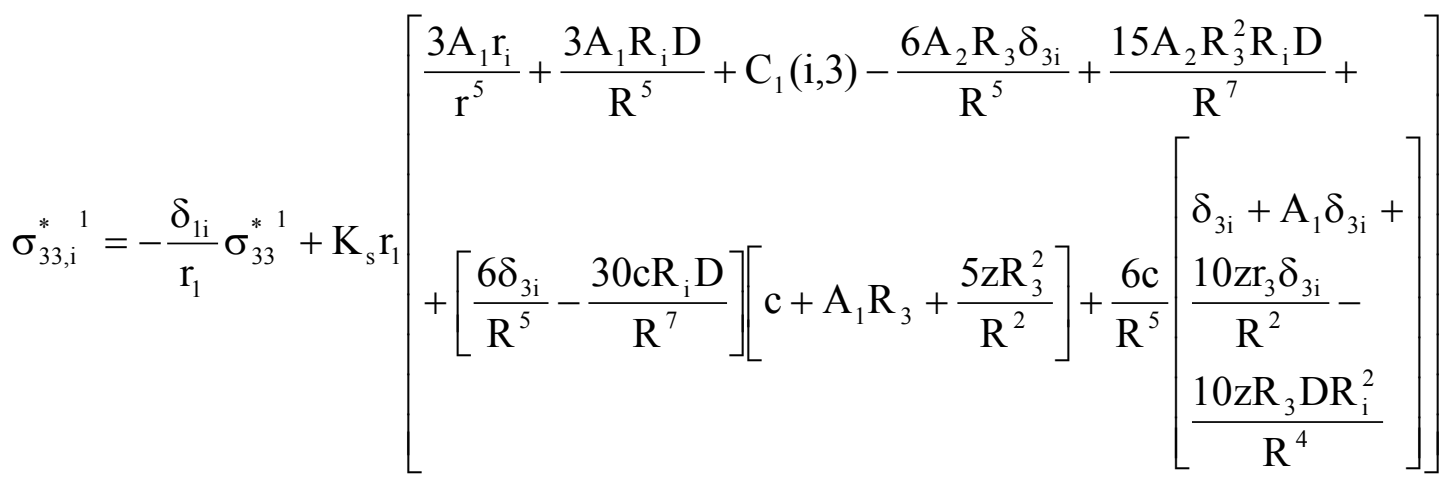

$$
\begin{aligned}
& \sigma_{11, \mathrm{i}}^{* 2}=-\frac{\delta_{2 \mathrm{i}}}{\mathrm{r}_{2}} \sigma_{11}^{* 2}+\mathrm{K}_{\mathrm{s}} \mathrm{r}_{2}\left[\frac{3 \mathrm{~A}_{1} \mathrm{r}_{\mathrm{i}}}{\mathrm{r}^{5}}-\frac{3 \mathrm{~A}_{1} \mathrm{~A}_{2} \mathrm{R}_{\mathrm{i}} \mathrm{D}}{\mathrm{R}^{5}}+\mathrm{C}_{1}(\mathrm{i}, 1)+\mathrm{C}_{2}(\mathrm{i}, 1)+\mathrm{C}_{3}(\mathrm{i}, 1,1)+\mathrm{C} 3(\mathrm{i}, 1,2,1)\right] \\
& \sigma_{12, \mathrm{i}}^{*}{ }^{2}=-\frac{\delta_{1 \mathrm{i}}}{\mathrm{r}_{1}}{\sigma_{12}^{*}}^{2}+\mathrm{K}_{\mathrm{s}} \mathrm{r}_{1}\left[-\frac{3 \mathrm{~A}_{1} \mathrm{r}_{\mathrm{i}}}{\mathrm{r}^{5}}-\frac{3 \mathrm{~A}_{1} \mathrm{R}_{\mathrm{i}} \mathrm{D}}{\mathrm{R}^{5}}+\mathrm{C}_{1}(\mathrm{i}, 2)+\mathrm{C}_{2}(\mathrm{i}, 2)+\mathrm{C}_{3}(\mathrm{i}, 2,1)+\mathrm{C}_{5}(\mathrm{i}, 2)\right]
\end{aligned}
$$




$$
\begin{aligned}
& \sigma_{13, \mathrm{i}}^{*}{ }^{2}=\sigma_{23, \mathrm{i}}^{*} \\
& \sigma_{22, \mathrm{i}}^{* 2}=-\frac{\delta_{2 \mathrm{i}}}{\mathrm{r}_{2}} \sigma_{22}^{* 2}+\mathrm{K}_{\mathrm{s}} \mathrm{r}_{2}\left[\begin{array}{l}
\left.-\frac{3 \mathrm{~A}_{1} \mathrm{r}_{\mathrm{i}}}{\mathrm{r}^{5}}-\frac{3 \mathrm{~A}_{1}(5-4 v) \mathrm{R}_{\mathrm{i}} \mathrm{D}}{\mathrm{R}^{5}}+\mathrm{C}_{1}(\mathrm{i}, 2)+\mathrm{C}_{2}(\mathrm{i}, 2)+\mathrm{C}_{3}(\mathrm{i}, 2,3)+\right] \\
+\mathrm{C}_{4}(\mathrm{i}, 2,3,2,3)
\end{array}\right] \\
& \sigma_{23, i}^{* 2}=K_{s}\left[\begin{array}{l}
\frac{A_{1} \delta_{3 i}}{r^{3}}-\frac{3 A_{1} r_{3} r_{i}}{r^{5}}-\frac{A_{1} \delta_{3 i}}{R^{3}}-\frac{3 A_{1} r_{3} R_{i} D}{R^{5}}+C_{7}(i, 2)+R_{3} C_{2}(i, 2) \\
-\frac{3 A_{2} r_{2}^{2} \delta_{3 i}}{R^{5}}+C_{6}(i, 2)
\end{array}\right] \\
& \sigma_{33, \mathrm{i}}^{* 2}=\frac{\mathrm{r}_{2}}{\mathrm{r}_{1}} \sigma_{33, \mathrm{i}}^{* 1}+\sigma_{33}^{* 1}\left(\delta_{1 \mathrm{i}} \frac{\mathrm{r}_{2}}{\mathrm{r}_{1}^{2}}-\frac{\delta_{2 \mathrm{i}}}{\mathrm{r}_{1}}\right) \\
& \sigma_{11, \mathrm{i}}^{* 3}=\mathrm{K}_{\mathrm{s}}\left[\begin{array}{l}
-\frac{\mathrm{A}_{1} \delta_{3 \mathrm{i}}}{\mathrm{r}^{3}}+\frac{3 \mathrm{~A}_{1} \mathrm{r}_{3} \mathrm{r}_{\mathrm{i}}}{\mathrm{r}^{5}}-\frac{\mathrm{A}_{1}(3+4 v) \delta_{3 \mathrm{i}}}{\mathrm{R}^{3}}+\mathrm{C}_{7}(\mathrm{i}, 1)-\frac{3 \mathrm{~A}_{1}\left(3 \mathrm{r}_{3}-4 v \mathrm{R}_{3}\right) \mathrm{R}_{\mathrm{i}} \mathrm{D}}{\mathrm{R}^{5}}+ \\
+\mathrm{C}_{8}(\mathrm{i}, 1)+\mathrm{C}_{9}(\mathrm{i}, 1)+\mathrm{C}_{10}(\mathrm{i}, 1)
\end{array}\right] \\
& \sigma_{12, \mathrm{i}}^{* 3}=\left[-\frac{\delta_{1 \mathrm{i}}}{\mathrm{r}_{1}}-\frac{\delta_{2 \mathrm{i}}}{\mathrm{r}_{2}}\right] \sigma_{12, \mathrm{i}}^{*^{3}}+\mathrm{K}_{\mathrm{s}} \mathrm{r}_{1} \mathrm{r}_{2}\left[\begin{array}{l}
\frac{3 \delta_{3 \mathrm{i}}}{\mathrm{r}^{5}}-\frac{15 \mathrm{r}_{3} \mathrm{r}_{\mathrm{i}}}{\mathrm{r}^{7}}+\frac{3 \mathrm{~A}_{2} \delta_{3 \mathrm{i}}}{\mathrm{R}^{5}}+\frac{15 \mathrm{~A}_{2} \mathrm{R}_{3} \mathrm{R}_{\mathrm{i}} \mathrm{D}}{\mathrm{R}^{7}}+ \\
-\frac{30 \mathrm{z} \delta_{3 \mathrm{i}}\left(\mathrm{R}_{3}+\mathrm{c}\right)}{\mathrm{R}^{7}}+\frac{210 \mathrm{czR} \mathrm{R}_{3} \mathrm{D}}{\mathrm{R}^{9}}+\frac{4 \mathrm{~A}_{3} \mathrm{~A}_{1}}{\mathrm{R}^{2}\left(\mathrm{R}+\mathrm{R}_{3}\right)} \\
{\left[\begin{array}{l}
\frac{\left(\mathrm{R}+\delta_{3 \mathrm{i}} \mathrm{D}\right) / \mathrm{R}}{\left(\mathrm{R}+\mathrm{R}_{3}\right)^{2} \mathrm{D}} \\
\mathrm{R}^{3}
\end{array}\right]-\frac{4 \mathrm{~A}_{3} \mathrm{~A}_{1}}{\mathrm{R}^{4}\left(\mathrm{R}+\mathrm{R}_{3}\right)^{2}}\left[\frac{1}{\mathrm{R}+\mathrm{R}_{3}}+\frac{1}{\mathrm{R}}\right]} \\
{\left[2 \mathrm{R}_{\mathrm{i}} \mathrm{D}\left(\mathrm{R}+\mathrm{R}_{3}\right)+\mathrm{R}^{2}\left(\frac{\mathrm{R}_{\mathrm{i}} \mathrm{D}}{\mathrm{R}}+\delta_{3 \mathrm{i}}\right)\right]}
\end{array}\right] \\
& \sigma_{13, \mathrm{i}}^{* 3}=-\frac{\delta_{1 \mathrm{i}}}{\mathrm{r}_{1}} \sigma_{13}^{* 3}+\mathrm{K}_{\mathrm{s}} \mathrm{r}_{1}\left[\begin{array}{l}
-\frac{3 \mathrm{~A}_{1} \mathrm{r}_{\mathrm{i}}}{\mathrm{r}^{5}}-\frac{3 \mathrm{~A}_{1} \mathrm{R}_{\mathrm{i}} \mathrm{D}}{\mathrm{R}^{5}}+\mathrm{C}_{1}(\mathrm{i}, 3)-\frac{3 \mathrm{~A}_{2} \mathrm{z}_{3 \mathrm{i}}-3 \delta_{3 \mathrm{i}}(3 \mathrm{z}+2 \mathrm{c})}{\mathrm{R}^{5}} \\
+\frac{5\left(3 \mathrm{~A}_{2} \mathrm{zR}_{3}-3 \mathrm{c}(3 \mathrm{z}+\mathrm{c})\right) \mathrm{R}_{\mathrm{i}} \mathrm{D}}{\mathrm{R}^{7}}+\frac{210 \mathrm{czR} \mathrm{R}_{3}^{2} \mathrm{R}_{\mathrm{i}} \mathrm{D}}{\mathrm{R}}- \\
-\frac{30 \delta_{3 \mathrm{i}} \mathrm{zR}_{3}^{2}+60 \mathrm{czR} \delta_{3 \mathrm{i}}}{\mathrm{R}^{7}}
\end{array}\right] \\
& \sigma_{22, \mathrm{i}}^{*}=\mathrm{K}_{\mathrm{s}}\left[\begin{array}{l}
-\frac{\mathrm{A}_{1} \delta_{3 \mathrm{i}}}{\mathrm{r}^{3}}+\frac{3 \mathrm{~A}_{1} \mathrm{r}_{3} \mathrm{r}_{\mathrm{i}}}{\mathrm{r}^{5}}+\mathrm{C}_{7}(\mathrm{i}, 2)-\frac{\mathrm{A}_{1}(3-4 v) \delta_{3 \mathrm{i}}}{\mathrm{R}^{3}}-\frac{3 \mathrm{~A}_{1}\left(3 \mathrm{r}_{3}-4 v \mathrm{R}_{3}\right) \mathrm{R}_{\mathrm{i}} \mathrm{D}}{\mathrm{R}^{5}}+ \\
\mathrm{C}_{8}(\mathrm{i}, 2)+\mathrm{C}_{9}(\mathrm{i}, 2)+\mathrm{C}_{10}(\mathrm{i}, 2)
\end{array}\right]
\end{aligned}
$$




$$
\begin{aligned}
& \sigma_{23, \mathrm{i}}^{*}{ }^{3}=\frac{\mathrm{r}_{2}}{\mathrm{r}_{1}} \sigma_{13, \mathrm{i}}^{*}+\sigma_{13, \mathrm{i}}^{*^{3}}\left[\delta_{1 \mathrm{i}} \frac{\mathrm{r}_{2}}{\mathrm{r}_{1}^{2}}-\frac{\delta_{2 \mathrm{i}}}{\mathrm{r}_{1}}\right] \\
& \sigma_{23, \mathrm{i}}^{*}{ }^{3}=\mathrm{K}_{\mathrm{s}}\left[\begin{array}{l}
\frac{\mathrm{A}_{1} \delta_{3 \mathrm{i}}}{\mathrm{r}^{3}}-\frac{3 \mathrm{~A}_{1} \mathrm{r}_{3} \mathrm{r}_{\mathrm{i}}}{\mathrm{r}^{5}}-\frac{\mathrm{A}_{1} \delta_{3 \mathrm{i}}}{\mathrm{R}^{3}}-\frac{3 \mathrm{~A}_{1} \mathrm{r}_{3} \mathrm{R}_{\mathrm{i}} \mathrm{D}}{\mathrm{R}^{5}}+\frac{9 \mathrm{r}_{3}^{2} \delta_{3 \mathrm{i}}}{\mathrm{r}^{5}}-\frac{15 \mathrm{r}_{3}^{3} \mathrm{r}_{\mathrm{i}}}{\mathrm{r}^{7}}+\frac{210 \mathrm{czR}{ }_{3}^{3} \mathrm{R}_{\mathrm{i}} \mathrm{D}}{\mathrm{R}^{9}}+ \\
-\frac{6 \mathrm{~A}_{2} \mathrm{zcR}_{3} \delta_{3 \mathrm{i}}-3\left(\mathrm{R}_{3}+\mathrm{c}\right) \delta_{3 \mathrm{i}}(5 \mathrm{z}-\mathrm{c})}{\mathrm{R}^{5}}+\frac{3 \mathrm{cR} \mathrm{R}_{3} \delta_{3 \mathrm{i}}}{\mathrm{R}^{5}}+ \\
+\frac{5\left(3 \mathrm{~A}_{2} \mathrm{zR}_{3}^{2}-3 \mathrm{cR} \mathrm{R}_{3}(5 \mathrm{z}-\mathrm{c})\right) \mathrm{R}_{\mathrm{i}} \mathrm{D}}{\mathrm{R}^{7}}-\frac{30 \mathrm{zR}_{3}^{3} \delta_{3 \mathrm{i}}+90 \mathrm{czR}_{3}^{2} \delta_{3 \mathrm{i}}}{\mathrm{R}^{7}}
\end{array}\right]
\end{aligned}
$$




\section{ANEXO C}

\section{DEDUÇÃO DO TENSOR D̂}

No capítulo 3, a equação (3.4.15) apresenta um tensor utilizado na equação de tensões do Método da Reciprocidade Dual. Aqui, são apresentados alguns passos para a dedução deste tensor.

Considere-se o tensor de deslocamentos (3.4.12),

$$
\hat{\mathrm{u}}_{\mathrm{ij}}=\mathrm{a}_{1} \mathrm{r}_{, \mathrm{i}} \mathrm{r}_{\mathrm{j}} \mathrm{r}^{2}+\mathrm{a}_{2}\left(\mathrm{a}_{3} \delta_{\mathrm{ij}}-\mathrm{r}_{, \mathrm{i}} \mathrm{r}_{\mathrm{j}}\right) \mathrm{r}^{3}
$$

onde:

$$
\begin{aligned}
& \mathrm{a}_{1}=\frac{1-2 v}{(6-4 v) G} \\
& \mathrm{a}_{2}=\frac{1}{48(1-v) \mathrm{G}} \\
& \mathrm{a}_{3}=\left(\frac{11}{3}-4 v\right)
\end{aligned}
$$

Considerando-se a lei de Hooke para o tensor $\hat{u}$, estabelece-se que:

$$
\hat{\mathrm{D}}_{\mathrm{ijk}}=\lambda \delta_{\mathrm{ik}} \frac{\partial \hat{\mathrm{u}}_{\mathrm{wj}}}{\partial \mathrm{X}_{\mathrm{w}}}+\mathrm{G}\left(\frac{\partial \hat{\mathrm{u}}_{\mathrm{ij}}}{\partial \mathrm{X}_{\mathrm{k}}}+\frac{\partial \hat{\mathrm{u}}_{\mathrm{kj}}}{\partial \mathrm{X}_{\mathrm{i}}}\right)
$$

Sabe-se que: 


$$
\begin{aligned}
& r_{, i w}=\frac{1}{r}\left(\delta_{i w}-r_{, i} r_{, w}\right) \\
& r_{, j w}=\frac{1}{r}\left(\delta_{j w}-r_{, j} r_{, w}\right) \\
& r_{, w}^{2}=2 r_{, w} \\
& r_{, w}^{3}=3 r^{2} r_{, w}
\end{aligned}
$$

Efetuando-se as derivadas de (C.3), utilizando-se (C.4a-d), chega-se a:

$$
\begin{gathered}
\frac{\partial \hat{\mathrm{u}}_{\mathrm{wj}}}{\partial \mathrm{X}_{\mathrm{w}}}=\left(4 \mathrm{a}_{1} \mathrm{r}_{, \mathrm{j}}\right) \mathrm{r}+\mathrm{a}_{2} \mathrm{r}^{2}\left(3 \mathrm{a}_{3} \mathrm{r}_{, \mathrm{j}}-5 \mathrm{r}_{, \mathrm{j}}\right), \\
\frac{\partial \hat{\mathrm{u}}_{\mathrm{ij}}}{\partial \mathrm{X}_{\mathrm{k}}}=\mathrm{a}_{1} \mathrm{r}\left(\delta_{\mathrm{ik}} \mathrm{r}_{\mathrm{j}}+\delta_{\mathrm{jk}} \mathrm{r}_{, \mathrm{i}}\right)+\mathrm{a}_{2} \mathrm{r}^{2}\left(3 \mathrm{a}_{3} \delta_{\mathrm{ij}} \mathrm{r}_{, \mathrm{k}}-\delta_{\mathrm{ik}} \mathrm{r}_{, \mathrm{j}}-\delta_{\mathrm{jk}} \mathrm{r}_{\mathrm{i}}-\mathrm{r}_{, \mathrm{i}} \mathrm{r}_{, j} \mathrm{r}_{, \mathrm{k}}\right), \mathrm{e} \\
\frac{\partial \hat{\mathrm{u}}_{\mathrm{kj}}}{\partial \mathrm{X}_{\mathrm{i}}}=\mathrm{a}_{1} \mathrm{r}\left(\delta_{\mathrm{ki}} \mathrm{r}_{, \mathrm{j}}+\delta_{\mathrm{ji}} \mathrm{r}_{, \mathrm{k}}\right)+\mathrm{a}_{2} \mathrm{r}^{2}\left(3 \mathrm{a}_{3} \delta_{\mathrm{kj}} \mathrm{r}_{\mathrm{i}}-\delta_{\mathrm{ki}} \mathrm{r}_{, \mathrm{j}}-\delta_{\mathrm{ji}} \mathrm{r}_{\mathrm{k}}-\mathrm{r}_{, \mathrm{k}} \mathrm{r}_{, \mathrm{j}} \mathrm{r}_{\mathrm{i}}\right) .
\end{gathered}
$$

Levando-se as derivadas (C.5), (C.6) e (C.7) para (C.3), tem-se:

$$
\begin{aligned}
& \hat{D}_{\mathrm{ijk}}=\frac{(1-2 v)}{(3-2 v)} \mathrm{r}\left[\frac{(1+2 v)}{(1-2 v)} \delta_{\mathrm{ik}} \mathrm{r}_{, \mathrm{j}}+\frac{1}{2}\left(\delta_{\mathrm{jk}} \mathrm{r}_{, \mathrm{i}}+\delta_{\mathrm{ij}} \mathrm{r}_{, \mathrm{k}}\right)\right]+ \\
& +\frac{\mathrm{r}^{2}}{24(1-v)}\left[(5-6 v)\left(\delta_{\mathrm{ij}} \mathrm{r}_{, \mathrm{k}}+\delta_{\mathrm{kj}} \mathrm{r}_{\mathrm{i}}\right)-(1-6 v) \delta_{\mathrm{ik}} \mathrm{r}_{, \mathrm{j}}-\mathrm{r}_{, \mathrm{i}} \mathrm{r}_{, \mathrm{j}} \mathrm{r}_{, \mathrm{k}}\right]
\end{aligned}
$$




\section{DETERMINAÇÃO DE UMA PRIMITIVA}

A formulação empregada no item 3.5 exige a determinação de uma primitiva relativa à expressão do tensor de deslocamentos fundamentais.

A expressão (3.5.4) estabelece a relação entre dois tensores:

$$
\nabla^{2} \mathrm{~T}_{\mathrm{ij}}^{*}=\mathrm{u}_{\mathrm{ij}}^{*}
$$

Portanto, o objetivo é encontrar $\mathrm{T}_{\mathrm{ij}}^{*}$. Para tanto, pode-se utilizar o procedimento de integrar em relação à coordenada radial de um sistema de coordenadas polares.

O tensor dos deslocamentos é:

$$
\mathrm{u}_{\mathrm{ij}}^{*}(\mathrm{~s}, \mathrm{q})=\frac{1}{16 \pi(1-v) \mathrm{Gr}}\left\{(3-4 v) \delta_{\mathrm{ij}}+\mathrm{r}_{\mathrm{i}} \mathrm{r}_{\mathrm{j}}\right\}
$$

Uma primitiva possível para (3.5.4), tendo em vista (2.4.4), é:

$$
\mathrm{T}_{\mathrm{ij}}^{*}=\frac{1}{4 \times 16 \pi(1-v)}\left\{(7-8 v) \delta_{\mathrm{ij}}-\mathrm{r}_{, \mathrm{i}} \mathrm{r}_{\mathrm{j}}\right\}
$$




\section{DETERMINAÇÃO DE TENSORES PARA M'}

Considere-se a expressão de uma primitiva possível para a relação (3.5.4),

$$
\mathrm{T}_{\mathrm{ij}}^{*}=\frac{1}{4 \times 16 \pi(1-v)}\left\{(7-8 v) \delta_{\mathrm{ij}}-\mathrm{r}_{\mathrm{i}, \mathrm{i}} \mathrm{r}_{\mathrm{j}}\right\}
$$

e a sua derivada

$$
\mathrm{T}_{\mathrm{ij}, \ell}^{*}=\frac{\mathrm{r}}{4 \times 16 \pi(1-v) \mathrm{G}}\left\{(7-8 v) \delta_{\mathrm{ij}, \ell} \mathrm{r}_{,}-\delta_{\mathrm{i} \ell} \mathrm{r}_{\mathrm{ij}}-\delta_{\mathrm{j} \ell} \mathrm{r}_{, \mathrm{i}} \mathrm{r}_{, \ell} \mathrm{r}_{, j}\right\} .
$$

Na expressão das tensões (3.5.12) surgem dois novos tensores $\overline{\mathrm{T}}_{\mathrm{ij}}^{*} \mathrm{e} \overline{\mathrm{T}}_{\mathrm{ij}, \ell}^{*} \equiv \overline{\mathrm{T}}_{\mathrm{ij}}^{* \ell}$ utilizados na composição de $\mathbf{M}^{\prime}$. A determinação de ambos é como se segue.

Sabe-se que:

$$
\overline{\mathrm{T}}_{\mathrm{ik}}^{*}=\lambda \delta_{\mathrm{ik}} \frac{\partial \mathrm{T}^{*} \mathrm{wj}}{\partial \mathrm{X}_{\mathrm{w}}}+\mathrm{G}\left(\frac{\partial \mathrm{T}_{\mathrm{ij}}^{*}}{\partial \mathrm{X}_{\mathrm{k}}}+\frac{\partial \mathrm{T}^{*} \mathrm{kj}_{\mathrm{i}}}{\partial \mathrm{X}_{\mathrm{i}}}\right) .
$$

Efetuando-se as derivadas em (E.3), tem-se: 


$$
\frac{\partial \mathrm{T}_{\mathrm{wj}}^{*}}{\partial \mathrm{X}_{\mathrm{w}}}=\frac{\partial}{\partial \mathrm{X}_{\mathrm{w}}}\left[\frac{1}{4 \times 16 \pi(1-v) \mathrm{G}}\left((7-8 v) \mathrm{r}-\mathrm{rr}_{, \mathrm{w}} \mathrm{r}_{\mathrm{j}}\right)\right] .
$$

Determinam-se as derivadas auxiliares:

$$
\begin{aligned}
& \frac{\partial \mathrm{r}}{\partial \mathrm{X}_{\mathrm{w}}}=-\mathrm{r}_{, \mathrm{w}} \\
& \frac{\partial\left(\mathrm{r}_{\mathrm{r}, \mathrm{w}}\right)}{\partial \mathrm{X}_{\mathrm{w}}}=\frac{1}{\mathrm{r}}\left(\delta_{\mathrm{ww}}-\mathrm{r}_{, \mathrm{w}} \mathrm{r}_{\mathrm{w}}\right) \\
& \frac{\partial\left(\mathrm{r}_{, \mathrm{j}}\right)}{\partial \mathrm{X}_{\mathrm{w}}}=\frac{1}{\mathrm{r}}\left(\delta_{\mathrm{jw}}-\mathrm{r}_{, \mathrm{j}} \mathrm{r}_{\mathrm{w}}\right)
\end{aligned}
$$

Levando-se (E.5a-c) para (E.4), tem-se:

$$
\frac{\partial \mathrm{T}_{\mathrm{wj}}^{*}}{\partial \mathrm{X}_{\mathrm{w}}}=\frac{1}{4 \times 16 \pi(1-v) \mathrm{G}}\left\{-(7-8 v) \delta_{\mathrm{wj}} \mathrm{r}_{\mathrm{w}}-3 \mathrm{r}_{, \mathrm{j}}-\mathrm{r}_{, \mathrm{j}}+3 \mathrm{r}_{, \mathrm{j}}\right\}
$$

ou, ainda,

$$
\frac{\partial \mathrm{T}_{\mathrm{wj}}^{*}}{\partial \mathrm{X}_{\mathrm{w}}}=\frac{1}{4 \times 16 \pi(1-v) \mathrm{G}}\left\{-8(1-v) \mathrm{r}_{, \mathrm{j}}\right\}
$$

Portanto, a primeira parcela de (E.3) é:

$$
\lambda \delta_{\mathrm{ik}} \frac{\partial \mathrm{T}_{\mathrm{wj}}^{*}}{\partial \mathrm{X}_{\mathrm{w}}}=-\frac{1}{4 \times 16 \pi(1-v)} \times \frac{16 v \mathrm{r}_{, \mathrm{j}} \delta_{\mathrm{ik}}}{(1-2 v)} .
$$

Para a outra derivada de (E.3), tem-se

$$
\frac{\partial \mathrm{T}_{\mathrm{ij}}^{*}}{\partial \mathrm{X}_{\mathrm{k}}}=\frac{\partial}{\partial \mathrm{X}_{\mathrm{k}}}\left[\frac{1}{4 \times 16 \pi(1-v) \mathrm{G}}\left((7-8 v) \mathrm{r}-\mathrm{rr}_{, \mathrm{i}, \mathrm{j}} \mathrm{r}_{\mathrm{j}}\right)\right] .
$$


Efetuando-se as derivadas de (E.9), encontra-se a segunda parcela de (E.3):

$$
\mathrm{G} \frac{\partial \mathrm{T}_{\mathrm{ij}}^{*}}{\partial \mathrm{X}_{\mathrm{k}}}=\frac{\mathrm{G}}{4 \times 16 \pi(1-v) \mathrm{G}}\left\{-(7-8 v) \delta_{\mathrm{ij}, \mathrm{r}} \mathrm{r}-\delta_{\mathrm{ik}} \mathrm{r}_{, \mathrm{j}}-\delta_{\mathrm{jk}} \mathrm{r}_{, \mathrm{i}}+3 \mathrm{r}_{\mathrm{i}, \mathrm{r}} \mathrm{r}_{\mathrm{j}, \mathrm{r}}\right\} .
$$

Para a última derivada de (E.3), tem-se

$$
\frac{\partial \mathrm{T}_{\mathrm{kj}}^{*}}{\partial \mathrm{X}_{\mathrm{i}}}=\frac{\partial}{\partial \mathrm{X}_{\mathrm{i}}}\left[\frac{1}{4 \times 16 \pi(1-v) \mathrm{G}}\left((7-8 v) \mathrm{r}-\mathrm{rr}_{, \mathrm{k}} \mathrm{r}_{, \mathrm{j}}\right)\right]
$$

Efetuando-se as derivadas de (E.11), encontra-se a última parcela de (E.3):

$$
\mathrm{G} \frac{\partial \mathrm{T}_{\mathrm{kj}}^{*}}{\partial \mathrm{X}_{\mathrm{i}}}=\frac{\mathrm{G}}{4 \times 16 \pi(1-v) \mathrm{G}}\left\{-(7-8 v) \delta_{\mathrm{kj}} \mathrm{r}_{, \mathrm{i}}-\delta_{\mathrm{ik}} \mathrm{r}_{, \mathrm{j}}-\delta_{\mathrm{ji}} \mathrm{r}_{, \mathrm{k}}+3 \mathrm{r}_{, \mathrm{i}} \mathrm{r}_{, \mathrm{j}} \mathrm{r}_{\mathrm{k}}\right\} .
$$

Levando-se os resultados (E.8), (E.10) e (E.12) para (E.3), chega-se à expressão do tensor procurado, (E.13):

$$
\begin{aligned}
\overline{\mathrm{T}}_{\mathrm{ik}} & =\frac{1}{4 \times 16 \pi(1-v)}\left\{(7-8 v)\left(-\delta_{\mathrm{ij}, \mathrm{r}}-\delta_{\mathrm{kj}} \mathrm{r}_{, \mathrm{i}}\right)-\right. \\
& \left.-2\left(1+\frac{8 v}{(1-2 v)}\right) \delta_{\mathrm{ik}} \mathrm{r}_{, \mathrm{j}}-\delta_{\mathrm{jk}} \mathrm{r}_{, \mathrm{i}}-\delta_{\mathrm{ij}, \mathrm{k}} \mathrm{r}+6 \mathrm{r}_{, \mathrm{i}} \mathrm{r}_{, \mathrm{j}} \mathrm{r}_{, \mathrm{k}}\right\}
\end{aligned}
$$

Repete-se o procedimento utilizado acima, agora para $\overline{\mathrm{T}}_{\mathrm{ij}}^{* \ell}$.

Sabe-se que:

$$
\overline{\mathrm{T}}_{\mathrm{ik}}^{* \ell}=\lambda \delta_{\mathrm{ik}} \frac{\partial \mathrm{T}_{\mathrm{wj}}^{\ell}}{\partial \mathrm{X}_{\mathrm{w}}}+\mathrm{G}\left(\frac{\partial \mathrm{T}_{\mathrm{ij}}^{\ell}}{\partial \mathrm{X}_{\mathrm{k}}}+\frac{\partial \mathrm{T}_{\mathrm{kj}}^{\ell}}{\partial \mathrm{X}_{\mathrm{i}}}\right)
$$

Efetua-se individualmente as derivadas definidas em (E.14), como se segue: 


$$
\begin{aligned}
\frac{\partial \mathrm{T}_{\mathrm{ij}}^{\ell}}{\partial \mathrm{X}_{\mathrm{k}}}= & \mathrm{k}\left\{(7-8 \mathrm{v}) \delta_{\mathrm{ij}} \frac{\partial \mathrm{r}_{, \ell}}{\partial \mathrm{X}_{\mathrm{k}}}-\delta_{\mathrm{i} \ell} \frac{\partial \mathrm{r}_{, \mathrm{j}}}{\partial \mathrm{X}_{\mathrm{k}}}-\delta_{\mathrm{j} \ell} \frac{\partial \mathrm{r}_{\mathrm{i}}}{\partial \mathrm{X}_{\mathrm{k}}}+\frac{\partial \mathrm{r}_{, \mathrm{j}}}{\partial \mathrm{X}_{\mathrm{k}}} \mathrm{r}_{, \mathrm{i}, \ell} \mathrm{r}_{,}+\right. \\
& \left.+\frac{\partial \mathrm{r}_{, \mathrm{i}}}{\partial \mathrm{X}_{\mathrm{k}}} \mathrm{r}_{, \mathrm{j}} \mathrm{r}_{\ell}+\frac{\partial \mathrm{r}_{, \ell}}{\partial \mathrm{X}_{\mathrm{k}}} \mathrm{r}_{\mathrm{i}} \mathrm{r}_{\mathrm{j}}\right\}
\end{aligned}
$$

Determinam-se as derivadas auxiliares:

$$
\begin{gathered}
\frac{\partial \mathrm{r}_{, \ell}}{\partial \mathrm{X}_{\mathrm{k}}}=\frac{1}{\mathrm{r}}\left(\delta_{\ell \mathrm{k}}-\mathrm{r}_{, \ell} \mathrm{r}_{\mathrm{k}}\right) \\
\frac{\partial \mathrm{r}_{, \mathrm{j}}}{\partial \mathrm{X}_{\mathrm{k}}}=\frac{1}{\mathrm{r}}\left(\delta_{\mathrm{jk}}-\mathrm{r}_{, \mathrm{j}, \mathrm{k}} \mathrm{r}_{\mathrm{k}}\right) \\
\frac{\partial \mathrm{r}_{, \mathrm{i}}}{\partial \mathrm{X}_{\mathrm{k}}}=\frac{1}{\mathrm{r}}\left(\delta_{\mathrm{ik}}-\mathrm{r}_{, \mathrm{i}, \mathrm{k}}\right)
\end{gathered}
$$

Levando-se as derivadas auxiliares (E.16) para (E.15), encontra-se:

$$
\frac{\partial \mathrm{T}_{\mathrm{wj}}^{\ell}}{\partial \mathrm{X}_{\mathrm{w}}}=\frac{v \delta_{\mathrm{ik}}}{8 \pi(1-v) \mathrm{r}}\left\{\delta_{\mathrm{j} \ell}-\mathrm{r}_{, \mathrm{j}, \ell} \mathrm{r}_{,}\right\}
$$

As demais derivadas de (E.14) resultam em:

$$
\begin{aligned}
\frac{\partial \mathrm{T}_{\mathrm{ij}}^{\ell}}{\partial \mathrm{X}_{\mathrm{k}}}= & \frac{1}{16 \pi(1-v)} \frac{1}{\mathrm{r}}\left\{(7-8 v) \delta_{\mathrm{ij}}\left(\delta_{\ell \mathrm{k}}-\mathrm{r}_{, \ell} \mathrm{r}_{, \mathrm{k}}\right)-\delta_{\mathrm{i} \ell} \delta_{\mathrm{jk}}-\delta_{\mathrm{j} \ell} \delta_{\mathrm{ik}}+\right. \\
& \left.+\delta_{\mathrm{i} \ell} \mathrm{r}_{, j} \mathrm{r}_{\mathrm{k}}+\delta_{\mathrm{j} \ell} \mathrm{r}_{\mathrm{i}, \mathrm{r}} \mathrm{r}_{\mathrm{k}}+\delta_{\mathrm{ik}} \mathrm{r}_{, \mathrm{j}} \mathrm{r}_{, \ell}+\delta_{\mathrm{jk}} \mathrm{r}_{, \mathrm{i}} \mathrm{r}_{\ell}+\delta_{\ell \mathrm{k}} \mathrm{r}_{, \mathrm{i}} \mathrm{r}_{\mathrm{j}}-3 \mathrm{r}_{\mathrm{i}} \mathrm{r}_{\mathrm{j}, \mathrm{j}, \mathrm{k}} \mathrm{r}_{, \ell}\right\}
\end{aligned}
$$

e

$$
\begin{aligned}
& \frac{\partial \mathrm{T}_{\mathrm{kj}}^{\ell}}{\partial \mathrm{X}_{\mathrm{i}}}=\frac{1}{16 \pi(1-v)} \frac{1}{\mathrm{r}}\left\{(7-8 v) \delta_{\mathrm{kj}}\left(\delta_{\ell \mathrm{i}}-\mathrm{r}_{, \ell} \mathrm{r}_{\mathrm{i}}\right)-\delta_{\mathrm{k} \ell} \delta_{\mathrm{ji}}-\delta_{\mathrm{j} \ell} \delta_{\mathrm{ki}}+\right.
\end{aligned}
$$

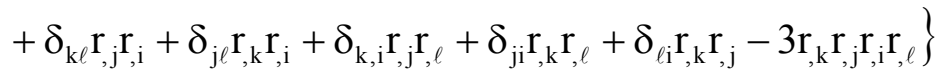


Levando-se os resultados (E.17), (E.18) e (E.19) para (E.14), chega-se à expressão do segundo tensor procurado, (E.20):

$$
\begin{aligned}
& \overline{\mathrm{T}}_{\mathrm{ik}}^{* \ell}=\frac{1}{2 \times 16 \pi(1-v) \mathrm{r}}\left\{(3-4 v)\left(\delta_{\mathrm{ij}} \delta_{\ell \mathrm{k}}+\delta_{\mathrm{kj}} \delta_{\mathrm{i} \ell}-\delta_{\mathrm{ij}} \mathrm{r}_{, \ell} \mathrm{r}_{\mathrm{k}}-\delta_{\mathrm{kj}, \ell} \mathrm{r}_{,} \mathrm{r}_{\mathrm{i}}\right)+\right. \\
& \left.+(1-4 v)\left(-\delta_{\mathrm{ik}} \delta_{\mathrm{j} \ell}+\delta_{\mathrm{ik}} \mathrm{r}_{, j} \mathrm{r}_{\ell}\right)+\delta_{\mathrm{i} \ell} \mathrm{r}_{\mathrm{j}, \mathrm{r}} \mathrm{r}_{\mathrm{k}}+\delta_{\ell \mathrm{k}} \mathrm{r}_{\mathrm{i}, \mathrm{r}} \mathrm{r}_{\mathrm{j}}-3 \mathrm{r}_{\mathrm{i}, \mathrm{r}, \mathrm{j}, \ell} \mathrm{r}_{,} \mathrm{r}_{\mathrm{k}}\right\}
\end{aligned}
$$




\section{DESENVOLVIMENTO DAS DERIVADAS PARA SE} OBTER O TENSOR $\mathrm{G}_{\mathrm{ij}}^{\ell}$, SUAS COMPONENTES E A MATRIZ K

O tensor da equação de deslocamentos associado aos dipolos está definido em (5.4.11), considerando-se a solução fundamental de Kelvin. Assim,

$$
\mathrm{G}_{\mathrm{ij}}^{\ell}=\frac{\partial \mathrm{u}_{\mathrm{ij}}^{*}}{\partial \mathrm{X}_{\ell}}=\frac{\partial}{\partial \mathrm{X}_{\ell}}\left\{\frac{1}{16 \pi(1-v) \mathrm{G}}\left[\frac{(3-4 v)}{\mathrm{r}} \delta_{\mathrm{ij}}+\frac{1}{\mathrm{r}} \mathrm{r}_{\mathrm{i}, \mathrm{r}}\right]\right\} .
$$

A derivada da segunda parcela de (5.4.11) é

$$
\begin{aligned}
\frac{\partial}{\partial X_{\ell}}\left(\frac{1}{r} r_{, i} r_{, j}\right) & =\frac{\partial}{\partial X_{\ell}}\left(\frac{1}{\mathrm{r}}\right) \mathrm{r}_{, \mathrm{i}, \mathrm{j}}+\frac{1}{\mathrm{r}} \frac{\partial^{2} \mathrm{r}}{\partial \mathrm{X}_{\ell} \partial \mathrm{X}_{\mathrm{i}}} \mathrm{r}_{, \mathrm{j}}+\frac{1}{\mathrm{r}} \frac{\partial^{2} \mathrm{r}}{\partial \mathrm{X}_{\ell} \partial \mathrm{X}_{\mathrm{j}}} \mathrm{r}_{\mathrm{i}} \\
& =\left(-\frac{1}{\mathrm{r}^{2}} \mathrm{r}_{, \ell}\right) \mathrm{r}_{, \mathrm{i}} \mathrm{r}_{\mathrm{j}}+\frac{1}{\mathrm{r}}\left[\frac{1}{\mathrm{r}}\left(\delta_{\ell \mathrm{i}}-\mathrm{r}_{, \ell} \mathrm{r}_{\mathrm{i}}\right)\right] \mathrm{r}_{, \mathrm{j}}+\frac{1}{\mathrm{r}}\left[\frac{1}{\mathrm{r}}\left(\delta_{\ell \mathrm{j}}-\mathrm{r}_{, \ell} \mathrm{r}_{\mathrm{i}}\right)\right] \mathrm{r}_{, \mathrm{i}}
\end{aligned}
$$

Levando-se o resultado (F.1) para (5.4.11), e efetuando-se a derivada da primeira parcela, tem-se: 


$$
\begin{aligned}
\mathrm{G}_{\mathrm{ij}}^{\ell} & =\frac{1}{16 \pi(1-v) \mathrm{G}}\left\{-\frac{1}{\mathrm{r}^{2}} \mathrm{r}_{, \ell}(3-4 v) \delta_{\mathrm{ij}}-\frac{1}{\mathrm{r}^{2}} \mathrm{r}_{\mathrm{i}, \mathrm{j}} \mathrm{r}_{, \ell}+\right. \\
& \left.+\frac{1}{\mathrm{r}^{2}}\left(\delta_{\ell \mathrm{i}}-\mathrm{r}_{, \ell} \mathrm{r}_{\mathrm{i}}\right) \mathrm{r}_{, \mathrm{j}}+\frac{1}{\mathrm{r}^{2}}\left(\delta_{\ell \mathrm{j}}-\mathrm{r}_{, \ell} \mathrm{r}_{\mathrm{j}}\right) \mathrm{r}_{, \mathrm{i}}\right\}
\end{aligned}
$$

ou

$$
\mathrm{G}_{\mathrm{ij}}^{\ell}=\frac{1}{16 \pi(1-v) \mathrm{Gr}^{2}}\left\{-(3-4 v) \mathrm{r}_{, \ell} \delta_{\mathrm{ij}}+\mathrm{r}_{, \mathrm{j}} \delta_{\ell \mathrm{i}}+\mathrm{r}_{, \mathrm{i}} \delta_{\ell \mathrm{j}}-3 \mathrm{r}_{, \mathrm{i}} \mathrm{r}_{\mathrm{j},} \mathrm{r}_{,}\right\} .
$$

As componentes do tensor $\mathrm{G}_{\mathrm{ij}}^{\ell}$ são:

$$
\begin{aligned}
& \mathrm{G}_{\mathrm{i} 1}^{1}=\frac{1}{16 \pi(1-v) \mathrm{Gr}^{2}}\left\{-(3-4 v) \mathrm{r}_{, 1} \delta_{\mathrm{i} 1}+\mathrm{r}_{, 1} \delta_{1 \mathrm{i}}+\mathrm{r}_{, \mathrm{i}}-3 \mathrm{r}_{, 1}^{2} \mathrm{r}_{\mathrm{i}}\right\} \\
& \mathrm{G}_{\mathrm{i} 2}^{1}=\frac{1}{16 \pi(1-v) \mathrm{Gr}^{2}}\left\{-(3-4 v) \mathrm{r}_{, 1} \delta_{\mathrm{i} 2}+\mathrm{r}_{, 2} \delta_{1 \mathrm{i}}-3 \mathrm{r}_{, 1} \mathrm{r}_{, 2} \mathrm{r}_{, \mathrm{i}}\right\} \\
& \mathrm{G}_{\mathrm{i} 3}^{1}=\frac{1}{16 \pi(1-v) \mathrm{Gr}^{2}}\left\{-(3-4 v) \mathrm{r}_{, 1} \delta_{\mathrm{i} 3}+\mathrm{r}_{, 3} \delta_{1 \mathrm{i}}-3 \mathrm{r}_{, 1} \mathrm{r}_{, 3} \mathrm{r}_{, \mathrm{i}}\right\} \\
& \mathrm{G}_{\mathrm{i} 1}^{2}=\frac{1}{16 \pi(1-v) \mathrm{Gr}^{2}}\left\{-(3-4 v) \mathrm{r}_{, 2} \delta_{\mathrm{i} 1}+\mathrm{r}_{, 1} \delta_{2 \mathrm{i}}-3 \mathrm{r}_{, 2} \mathrm{r}_{, \mathrm{i}} \mathrm{r}_{, 1}\right\} \\
& \mathrm{G}_{\mathrm{i} 2}^{2}=\frac{1}{16 \pi(1-v) \mathrm{Gr}^{2}}\left\{-(3-4 v) \mathrm{r}_{, 2} \delta_{\mathrm{i} 2}+\mathrm{r}_{, 2} \delta_{2 \mathrm{i}}+\mathrm{r}_{, \mathrm{i}}-3 \mathrm{r}_{, 2}^{2} \mathrm{r}_{, \mathrm{i}}\right\} \\
& \mathrm{G}_{\mathrm{i} 3}^{2}=\frac{1}{16 \pi(1-v) \mathrm{Gr}^{2}}\left\{-(3-4 v) \mathrm{r}_{, 2} \delta_{\mathrm{i} 3}+\mathrm{r}_{, 3} \delta_{2 \mathrm{i}}-3 \mathrm{r}_{, 2} \mathrm{r}_{\mathrm{i}} \mathrm{r}_{, 3}\right\} \\
& \mathrm{G}_{\mathrm{i} 1}^{3}=\frac{1}{16 \pi(1-v) \mathrm{Gr}^{2}}\left\{-(3-4 v) \mathrm{r}_{, 3} \delta_{\mathrm{i} 1}+\mathrm{r}_{, 1} \delta_{3 \mathrm{i}}-3 \mathrm{r}_{, 3} \mathrm{r}_{, \mathrm{i}} \mathrm{r}_{, 1}\right\} \\
& \mathrm{G}_{\mathrm{i} 2}^{3}=\frac{1}{16 \pi(1-v) \mathrm{Gr}^{2}}\left\{-(3-4 v) \mathrm{r}_{, 3} \delta_{\mathrm{i} 2}+\mathrm{r}_{, 2} \delta_{3 \mathrm{i}}-3 \mathrm{r}_{, 3} \mathrm{r}_{\mathrm{i}} \mathrm{r}_{, 2}\right\} \\
& \mathrm{G}_{\mathrm{i} 3}^{3}=\frac{1}{16 \pi(1-v) \mathrm{Gr}^{2}}\left\{-(3-4 v) \mathrm{r}_{, 3} \delta_{\mathrm{i} 3}+\mathrm{r}_{, 3} \delta_{3 \mathrm{i}}+\mathrm{r}_{, \mathrm{i}}-3 \mathrm{r}_{, 3}^{2} \mathrm{r}_{\mathrm{i}}\right\}
\end{aligned}
$$

A formulação desenvolvida neste trabalho utiliza as componentes do tensor $\mathrm{G}_{\mathrm{ij}}^{\ell}$ de forma condensada, definida em (5.4.9a-c), ou seja: 


$$
\begin{aligned}
& \mathrm{K}_{\mathrm{i} 1}=\mathrm{G}_{\mathrm{i} 3}^{1}+\mathrm{G}_{\mathrm{i} 1}^{3} \\
& \mathrm{~K}_{\mathrm{i} 2}=\mathrm{G}_{\mathrm{i} 3}^{2}+\mathrm{G}_{\mathrm{i} 2}^{3} \\
& \mathrm{~K}_{\mathrm{i} 3}=\frac{v}{1-v}\left(\mathrm{G}_{\mathrm{i} 1}^{1}+\mathrm{G}_{\mathrm{i} 2}^{2}\right)+\mathrm{G}_{\mathrm{i} 3}^{3}
\end{aligned}
$$

Operando-se algebricamente as expressões (F.3), tendo em vista (5.4.9a-c), chega-se às componentes da matriz de coeficientes de influência dos dipolos, matriz $\mathrm{K}$ :

$$
\begin{aligned}
& \mathrm{K}_{11}=\frac{1}{16 \pi(1-v) \mathrm{Gr}^{2}} 2 \mathrm{r}_{, 3}\left[-(1-2 v)-3 \mathrm{r}_{, 1}^{2}\right] \\
& \mathrm{K}_{12}=\frac{1}{16 \pi(1-v) \mathrm{Gr}^{2}}\left(-6 \mathrm{r}_{, 1} \mathrm{r}_{, 2} \mathrm{r}_{, 3}\right) \\
& \mathrm{K}_{13}=\frac{1}{16 \pi(1-v) \mathrm{Gr}^{2}} \mathrm{r}_{, 1} \frac{\mathrm{r}_{, 1}}{(1-v)}\left[1-(1-4 v) v-3 v\left(\mathrm{r}_{, 1}^{2}+\mathrm{r}_{, 2}^{2}-\mathrm{r}_{, 3}^{2}\right)-3 \mathrm{r}_{, 3}^{2}\right] \\
& \mathrm{K}_{21}=\frac{1}{16 \pi(1-v) \mathrm{Gr}^{2}}\left(-6 \mathrm{r}_{, 1} \mathrm{r}_{2} \mathrm{r}_{, 3}\right) \\
& \mathrm{K}_{22}=\frac{1}{16 \pi(1-v) \mathrm{Gr}^{2}} 2 \mathrm{r}_{, 3}\left[-(1-2 v)-3 \mathrm{r}_{, 2}^{2}\right] \\
& \mathrm{K}_{23}=\frac{1}{16 \pi(1-v) \mathrm{Gr}^{2}} \frac{\mathrm{r}_{, 2}}{(1-v)}\left[1-(1-4 v) v-3 v\left(\mathrm{r}_{, 1}^{2}+\mathrm{r}_{, 2}^{2}-\mathrm{r}_{, 3}^{2}\right)-3 \mathrm{r}_{, 3}^{2}\right] \\
& \mathrm{K}_{31}=\frac{1}{16 \pi(1-v) \mathrm{Gr}^{2}} 2 \mathrm{r}_{, 1}\left[-(1-2 v)-3 \mathrm{r}_{3}^{2}\right] \\
& \mathrm{K}_{32}=\frac{1}{16 \pi(1-v) \mathrm{Gr}^{2}} 2 \mathrm{r}_{, 2}\left[-(1-2 v)-3 \mathrm{r}_{3}^{2}\right] \\
& \mathrm{K}_{33}=\frac{1}{16 \pi(1-v) \mathrm{Gr}^{2}} \frac{\mathrm{r}_{, 3}}{(1-v)}\left[-1+(7-4 v) v-3 v\left(\mathrm{r}_{, 1}^{2}+\mathrm{r}_{, 2}^{2}-\mathrm{r}_{, 3}^{2}\right)-3 \mathrm{r}_{, 3}^{2}\right]
\end{aligned}
$$




\section{COMPONENTES DA MATRIZ DE CONTRIBUIÇÃO DOS DIPOLOS Às TENSÕES EM PONTOS INTERNOS}

As componentes da matriz de contribuição dos dipolos para as tensões em pontos internos KS são definidas por (5.6.20a-c), ou seja,

$$
\begin{aligned}
& \mathrm{KS}_{\mathrm{i} 1}^{\mathrm{m}}=\mathrm{G}_{\mathrm{i} 3}^{\mathrm{m} 1}+\mathrm{G}_{\mathrm{i} 1}^{\mathrm{m} 3} \\
& \mathrm{KS}_{\mathrm{i} 2}^{\mathrm{m}}=\mathrm{G}_{\mathrm{i} 3}^{\mathrm{m} 2}+\mathrm{G}_{\mathrm{i} 2}^{\mathrm{m} 3} \\
& \mathrm{KS}_{\mathrm{i} 3}^{\mathrm{m}}=\frac{v}{1-v}\left(\mathrm{G}_{\mathrm{i} 1}^{\mathrm{m} 1}+\mathrm{G}_{\mathrm{i} 2}^{\mathrm{m} 2}\right)+\mathrm{G}_{\mathrm{i} 3}^{\mathrm{m} 3}
\end{aligned}
$$

De forma explícita, pode-se escrever:

$$
\begin{aligned}
\mathrm{KS}_{11}^{1} & =\frac{1}{8 \pi(1-v) \mathrm{r}^{3}} 6 \mathrm{r}_{, 1} \mathrm{r}_{, 3}\left[1-5 \mathrm{r}_{, 1}^{2}\right] \\
\mathrm{KS}_{12}^{1} & =\frac{1}{8 \pi(1-v) \mathrm{r}^{3}} 6 \mathrm{r}_{, 2} \mathrm{r}_{, 3}\left[(1-2 v)-5 \mathrm{r}_{, 1}^{2}\right] \\
\mathrm{KS}_{13}^{1} & =\frac{1}{8 \pi(1-v) \mathrm{r}^{3}} \frac{1}{(1-v)}\left[-\left(1-3 v+2 v^{2}\right)+3\left(r_{, 1}^{2}+r_{, 3}^{2}-5 \mathrm{r}_{, 1}^{2} \mathrm{r}_{, 3}^{2}\right)+\right. \\
& \left.-3 v\left(-2 \mathrm{r}_{, 1}^{2}+5 \mathrm{r}_{, 1}^{4}-\mathrm{r}_{, 2}^{2}+5 \mathrm{r}_{, 1}^{2} \mathrm{r}_{, 2}^{2}+3 \mathrm{r}_{, 3}^{2}-5 \mathrm{r}_{, 1}^{2} \mathrm{r}_{, 3}^{2}\right)-6 v^{2}\left(-\mathrm{r}_{, 1}^{2}+\mathrm{r}_{, 2}^{2}-\mathrm{r}_{, 3}^{2}\right)\right]
\end{aligned}
$$




$$
\begin{aligned}
& \mathrm{KS}_{21}^{1}=\frac{1}{8 \pi(1-v) \mathrm{r}^{3}} 6 \mathrm{r}_{, 2} \mathrm{r}_{, 3}\left[v-5 \mathrm{r}_{, 1}^{2}\right] \\
& \mathrm{KS}_{22}^{1}=\frac{1}{8 \pi(1-v) \mathrm{r}^{3}} 6 \mathrm{r}_{, 1} \mathrm{r}_{, 3}\left[v-5 \mathrm{r}_{, 2}^{2}\right] \\
& \mathrm{KS}_{23}^{1}=\frac{1}{8 \pi(1-v) \mathrm{r}^{3}} \frac{3 \mathrm{r}_{, 1} \mathrm{r}_{, 2}}{(1-v)}\left[\left(1+v+4 v^{2}\right)-5\left(\mathrm{r}_{, 3}^{2}+\mathrm{r}_{, 1}^{2} v+\mathrm{r}_{, 2}^{2} v-\mathrm{r}_{, 3}^{2} v\right)\right] \\
& \mathrm{KS}_{31}^{1}=\frac{1}{8 \pi(1-v) \mathrm{r}^{3}}\left[2(1-2 v)+6\left(-5 \mathrm{r}_{, 1}^{2} \mathrm{r}_{, 3}^{2}+\mathrm{r}_{, 1}^{2} v+\mathrm{r}_{, 3}^{2} v\right)\right] \\
& \mathrm{KS}_{32}^{1}=\frac{1}{8 \pi(1-v) \mathrm{r}^{3}} 6 \mathrm{r}_{, 1} \mathrm{r}_{, 2}\left[v-5 \mathrm{r}_{, 3}^{2}\right] \\
& \mathrm{KS}_{33}^{1}=\frac{1}{8 \pi(1-v) \mathrm{r}^{3}} \frac{3 \mathrm{r}_{1} \mathrm{r}_{, 3}}{(1-v)}\left[(1+3 v)-5\left(\mathrm{r}_{, 3}^{2}+\mathrm{r}_{, 1}^{2} v+\mathrm{r}_{, 2}^{2} v-\mathrm{r}_{, 3}^{2} v\right)\right] \\
& \mathrm{KS}_{11}^{2}=\frac{1}{8 \pi(1-v) \mathrm{r}^{3}} 6 \mathrm{r}_{, 2} \mathrm{r}_{, 3}\left[v-5 \mathrm{r}_{, 1}^{2}\right] \\
& \mathrm{KS}_{12}^{2}=\frac{1}{8 \pi(1-v) \mathrm{r}^{3}} 6 \mathrm{r}_{, 1} \mathrm{r}_{, 3}\left[v-5 \mathrm{r}_{, 2}^{2}\right] \\
& \mathrm{KS}_{13}^{2}=\frac{1}{8 \pi(1-v) \mathrm{r}^{3}} \frac{3 \mathrm{r}_{, 1} \mathrm{r}_{, 2}}{(1-v)}\left[\left(1+v+4 v^{2}\right)-5\left(\mathrm{r}_{, 3}^{2}+\mathrm{r}_{, 1}^{2} v+\mathrm{r}_{, 2}^{2} v-\mathrm{r}_{, 3}^{2} v\right)\right] \\
& \mathrm{KS}_{21}^{2}=\frac{1}{8 \pi(1-v) \mathrm{r}^{3}} 6 \mathrm{r}_{, 1} \mathrm{r}_{, 3}\left[(1-2 v)-5 \mathrm{r}_{, 2}^{2}\right] \\
& \mathrm{KS}_{22}^{2}=\frac{1}{8 \pi(1-v) \mathrm{r}^{3}} 6 \mathrm{r}_{, 2} \mathrm{r}_{, 3}\left[1-5 \mathrm{r}_{, 2}^{2}\right] \\
& \mathrm{KS}_{23}^{2}=\frac{1}{8 \pi(1-v) \mathrm{r}^{3}} \frac{1}{(1-v)}\left[-\left(1-3 v+2 v^{2}\right)+3\left(\mathrm{r}_{, 2}^{2}+\mathrm{r}_{, 3}^{2}-5 \mathrm{r}_{, 2}^{2} \mathrm{r}_{, 3}^{2}\right)+\right. \\
& \left.-3 v\left(-2 r_{, 2}^{2}+5 r_{, 2}^{4}-r_{, 1}^{2}+5 r_{, 1}^{2} r_{, 2}^{2}+3 r_{, 3}^{2}-5 r_{, 2}^{2} r_{, 3}^{2}\right)-6 v^{2}\left(r_{, 1}^{2}-r_{, 2}^{2}-r_{, 3}^{2}\right)\right] \\
& \mathrm{KS}_{31}^{2}=\frac{1}{8 \pi(1-v) \mathrm{r}^{3}} 6 \mathrm{r}_{, 1} \mathrm{r}_{, 2}\left[v-5 \mathrm{r}_{, 3}^{2}\right] \\
& \mathrm{KS}_{32}^{2}=\frac{1}{8 \pi(1-v) \mathrm{r}^{3}}\left[2(1-2 v)+6\left(-5 \mathrm{r}_{, 2}^{2} \mathrm{r}_{, 3}^{2}+\mathrm{r}_{, 2}^{2} v+\mathrm{r}_{, 3}^{2} v\right)\right] \\
& \mathrm{KS}_{33}^{2}=\frac{1}{8 \pi(1-v) \mathrm{r}^{3}} \frac{3 \mathrm{r}_{, 2} \mathrm{r}_{, 3}}{(1-v)}\left[(1+3 v)-5\left(\mathrm{r}_{, 3}^{2}+\mathrm{r}_{, 1}^{2} v+\mathrm{r}_{, 2}^{2} v-\mathrm{r}_{, 3}^{2} v\right)\right] \\
& \mathrm{KS}_{31}^{3}=\frac{1}{8 \pi(1-v) \mathrm{r}^{3}}\left[2(1-2 v)+6\left(-5 \mathrm{r}_{, 1}^{2} \mathrm{r}_{, 3}^{2}+\mathrm{r}_{, 1}^{2} v+\mathrm{r}_{, 3}^{2} v\right)\right] \\
& \mathrm{KS}_{32}^{3}=\frac{1}{8 \pi(1-v) \mathrm{r}^{3}} 6 \mathrm{r}_{, 1} \mathrm{r}_{, 2}\left[v-5 \mathrm{r}_{, 3}^{2}\right] \\
& \mathrm{KS}_{33}^{3}=\frac{1}{8 \pi(1-v) \mathrm{r}^{3}} \frac{3 \mathrm{r}_{, 1} \mathrm{r}_{, 3}}{(1-v)}\left[(1+3 v)-5\left(\mathrm{r}_{, 3}^{2}+\mathrm{r}_{, 1}^{2} v+\mathrm{r}_{, 2}^{2} v-\mathrm{r}_{, 3}^{2} v\right)\right]
\end{aligned}
$$




$$
\begin{aligned}
\mathrm{KS}_{21}^{3} & =\frac{1}{8 \pi(1-v) \mathrm{r}^{3}} 6 \mathrm{r}_{, 1} \mathrm{r}_{, 2}\left[v-5 \mathrm{r}_{, 3}^{2}\right] \\
\mathrm{KS}_{22}^{2} & =\frac{1}{8 \pi(1-v) \mathrm{r}^{3}}\left[(2-4 v)+6\left(-5 \mathrm{r}_{, 2}^{2} \mathrm{r}_{, 3}^{2}+\mathrm{r}_{, 2}^{2} v+\mathrm{r}_{, 3}^{2} v\right)\right] \\
\mathrm{KS}_{23}^{2} & =\frac{1}{8 \pi(1-v) \mathrm{r}^{3}} \frac{3 \mathrm{r}_{, 2} \mathrm{r}_{, 3}}{(1-v)}\left[(1+3 v)-5\left(\mathrm{r}_{, 3}^{2}+\mathrm{r}_{, 1}^{2} v+\mathrm{r}_{, 2}^{2} v-\mathrm{r}_{, 3}^{2} v\right)\right] \\
\mathrm{KS}_{31}^{2} & =\frac{1}{8 \pi(1-v) \mathrm{r}^{3}} 6 \mathrm{r}_{, 1} \mathrm{r}_{, 3}\left[1-5 \mathrm{r}_{, 3}^{2}\right] \\
\mathrm{KS}_{32}^{2} & =\frac{1}{8 \pi(1-v) \mathrm{r}^{3}} 6 \mathrm{r}_{, 2} \mathrm{r}_{, 3}\left[1-5 \mathrm{r}_{, 3}^{2}\right] \\
\mathrm{KS}_{33}^{2} & =\frac{1}{8 \pi(1-v) \mathrm{r}^{3}} \frac{1}{(1-v)}\left[-\left(-1+5 v-6 v^{2}\right)+3\left(2 r_{, 3}^{2}-5 \mathrm{r}_{, 3}^{4}\right)+\right. \\
& \left.-3 v\left(-\mathrm{r}_{, 1}^{2}-\mathrm{r}_{, 2}^{2}+5 \mathrm{r}_{, 1}^{2} \mathrm{r}_{, 3}^{2}-2 \mathrm{r}_{, 3}^{2}-5 \mathrm{r}_{, 3}^{4}+5 \mathrm{r}_{, 2}^{2} \mathrm{r}_{, 3}^{2}\right)-6 v^{2}\left(\mathrm{r}_{, 1}^{2}+\mathrm{r}_{, 2}^{2}+\mathrm{r}_{, 3}^{2}\right)\right]
\end{aligned}
$$




\section{BIBLIOGRAFIA}

ADEY, R.A and BREBBIA, C.A. (1983). Basic computational techniques for engineers. London, Pentech Press.

ALIABADI, M.H. et al. (1987). Weighted gaussian methods for three-dimensional boundary element kernel integration. Comm. Appl. Numer. Methods, 3, 89-96.

ALIABADI, M.H. \& BREBBIA, C.A. (1993). Advances in boundary element methods for fracture mechanics. London. Computational Mechanics Publications.

ALIABADI, M.H., BREBBIA, C.A., \& PARTON, V.Z. (1994). Static and dynamic fracture mechanics. Southampton, CMP, UK.

ALIABADI, M.H. \& ROOKE, D.P. (1991). Numerical Fracture Mechanics. Computational Mechanics Publications. Southampton and Kluwer Academic Publishers, Dordrecht.

ALIABADI, M.H., ROOKE, D.P. and CARTWRIGHT, D.J. (1987). An improved boundary element formulation for calculating stress intensity factors: application to aerospace structures. J. Strain Anal., 22 (4), pp. 1-5.

ANDERSEN,R.S. et al. (1980). The application and numerical solution of integral equations. Alphen aan den Rijn, The Netherlands, Sijthoff \& Noordhoff.

ARAÚJO, F.C. (1994). Formulação acoplada FEM/BEM no domínio do tempo para a resolução de problemas elastodinâmicos 3D. In: XV CILAMCE, v. 1, pp. 621630. Belo Horizonte, BR.

ARAÚJO, T.D.P.; CAVALCANTI, NETO, J.B.; CARVALHO, M.T.M. (1997). Simulação adaptativa de processos de fraturamento baseada em técnicas de enumeração espacial recursiva. In: XVIII CILAMCE, v. 3, pp. 1185-1192. Brasília, BR.

ARAÚJO, T.D.P.; ROEHL, D.; BITTENCOURT, T.N.; MARTHA, L.F. (1998). Adaptative simulation of elastic-plastic fracture processes. In: IV WCCM, CDROM Proceedings. Buenos Aires, AR. 
BAINS, R.; ALIABADI, M.H. and ROOKE, D.P. (1992). Int. Jour. for Numerical Meth. in Eng., v. 35, n. 1, pp. 179-202.

BAINS, R.; ALIABADI, M.H. and ROOKE, D.P. (1993). Stress intensity factor weight functions for cracks in 3-d finite geometries. In: ALIABADI, M.H.; BREBBIA, C.A. (eds) Advances in boundary element methods for fracture mechanics, CMP, Southampton.

BANERJEE, P.K. and BUTTERFIELD, R. (1977). Boundary Element Method in Geomechanics. In: Finite Elements in Geomechanics, G. Gudehus (ed.), pp. 529570, J. Wiley, London.

BARBIRATO, J.C.C. (1991) Formulação do método dos elementos de contorno para sólidos elásticos tridimensionais, baseada na solução fundamental de Mindlin. São Carlos, Dissertação (Mestrado) - Escola de Engenharia de São Carlos, Universidade de São Paulo.

BARBIRATO, J.C.C. and VENTURINI, W.S. (1991). Formulação do método dos elementos de contorno, baseada na solução fundamental de Mindlin, para problemas elásticos tridimensionais. In: $X X V$ Jornadas Sul-Americanas de Engenharia Estrutural, v. 1, pp. 431-442. Porto Alegre, BR.

BARBIRATO, J.C.C. \& VENTURINI, W.S. (1997). Análise transiente de sólidos tridimensionais através do método dos elementos de contorno. In: XVIII CILAMCE, v. 1, pp. 397-404. Brasília, BR.

BARBIRATO, J.C.C. \& VENTURINI, W.S. (1998). Boundary element method for three-dimensional cohesive crack problems. In: IV WCCM, CD-ROM Proceedings. Buenos Aires, AR.

BARRA, L.P.S. \& TELLES, J.C.F. (1999). A hyper-singular numerical Green's function generation for BEM applied to dynamic SIF problems. Engineering Analysis with Boundary Elements, 23, pp. 77-87.

BARRA, L.P.S.; TELLES, J.C.F.; GUIMARÃES, S. (1997). Aplicação do MEC à mecânica da fratura elastodinâmica com funções de Green numéricas via transformada de Laplace. In: XVIII CILAMCE, v. 1, pp. 325-332. Brasília, BR.

BATCHELOR, G.K., WUNSCH, C. \& RICE, J. (1990). Cambridge monografhs on mechanics and applied mathematics - dynamic fracture mechanics. Cambridge University Press, New York.

BECKER, A.A. (1992). The boundary element method in engineering. London, MacGraw-Hill.

BESKOS, D.E. (1987). Boundary element methods in dynamic analysis, Appl. Mech. Reviews, Vol. 40, pp. 1-23. 
BRADY, B.H. \& BRAY, J.W. (1978). The boundary element method for elastic analysis of tabular orebody extraction, assuming complete plane strain. Int. J. Rock. Mech. Min. Sci. \& Geomech. Abstr., v. 15, pp. 29-37.

BREBBIA, C.A. (1978) The boundary element method for engineers, London, Pentech Press.

BREBBIA, C.A. \& DOMÍNGUEZ, J. (1977). Boundary element methods for potential problems. Appl. Math. Modelling, v. 1, pp. 372-378.

BREBBIA, C.A. \& DOMÍNGUEZ, J. (1989). Boundary elements - an introductory course. London. Computational Mechanics Publications.

BREBBIA, C.A. \& FERRANTE, A. J. (1975). The finite element technique - an introduction for engineers. Editora da URGS, Porto Alegre

BREBBIA, C.A. \& NARDINI, D. (1983). Dynamic analysis in solid mechanics by and alternative boundary element procedure. Int. Jour. of Soil Dynamics and Earthquake Engineering, v. 2, n. 4, pp. 228-233.

BREBBIA, C.A. \& NARDINI, D. (1986). Solution of parabolic and hyperbolic time dependent problems using elements. Comp. \& Math. with Appls., 12B, n. 5/6: 1061-1072.

BREBBIA, C.A.; TELLES, J.C.F.; WROBEL, L.C. (1984). Boundary element techniques. Spring-Verlag, Berlin.

BROEK, D. (1989). The pratical use of fracture mechanics. London, Kluwer Academic Publishers.

CALDERÓN, E.T. and VENTURINI, W.S. (1991). Um processo alternativo para o tratamento de integrais de domínio no método dos elementos de contorno: aplicação em placa sobre fundação elástica. In: XXV Jornadas Sul-Americanas de Engenharia Estrutural, v. 1, pp. 409-419. Porto Alegre, BR.

CARPINTERI, A. (1989). Softening and snap-back instability in cohesive solids. International Journal for numerical methods in engineering, v. 28, pp. 15211537.

CARRER, J.A.M. \& MANSUR, W.J. (1995). Análise elastodinâmica transiente pelo método dos elementos de contorno com o emprego de soluções fundamentais dependentes do tempo. In: XVI CILAMCE, pp. 109-119, Rio de Janeiro.

CARRER, J.A.M. \& MANSUR, W.J. (1999). Stress and velocity in 2D transiente elastodynamic analysis by the boundary element method. Engineering Analysis with Boundary Elements, 23, pp. 233-245. 
CARTWRIGHT, D.J. and ROOKE, D.P. (1985). An efficient boundary element method for calculating Green's functions in fracture mechanics. Int. J. of Fract., 27, R43-R-50.

CASTOR, G.S.; TELLES, J.C.F. (1997). Aplicação da função de Green numérica a problemas tridimensionais da mecânica da fratura. In: XVIII CILAMCE, v. 1, pp. 113-120. Brasília, BR.

CEN, Z. and MAIER, G. (1992). Bifurcations and instabilities in fracture of cohesive-softening structures: a boundary element analysis. Fatigue Fract. Engng. Mater., Vol. 15(9), 911-928.

CHEN, Y.M. (1975). Numerical computation of dynamic stress intensity factor by lagrangian finite-difference method. Engng. Fracture Mech., vol. 7, pp. 653-660.

CHEN, T.C. \& CHEN, W.H. (1998). Frictional contact analysis of multiple cracks by incremental displacement and resultant traction boundary integral equations. Engineering Analysis with Boundary Elements, 21, pp. 339-348.

CHIRINO, F. et al. (1994). Comparative study of three boundary element approaches to transient dynamics crack problems. Engineering Analysis with Boundary Elements, v. 13, n. 1, pp. 11-19.

CISILINO, A. P. \& ALIABADI, M.H. (1998). Three-dimensional elastoplastic analysis of cracked bodies using the dual boundary element method. In: IV WCCM, CD-ROM Proceedings. Buenos Aires, AR.

CODA, H.B. (1990). Análise da vibração livre de meios bidimensionais pelo método dos elementos de contorno. São Carlos. Dissertação (Mestrado) - Escola de Engenharia de São Carlos, Universidade de São Paulo.

CODA, H.B. (1993). Análise tridimensional transiente de estruturas pela combinação entre o método dos elementos de contorno e o método dos elementos finitos. São Carlos. Tese (Doutorado) - Escola de Engenharia de São Carlos, Universidade de São Paulo.

CODA, H.B. \& VENTURINI, W.S. (1990a). Alternative boundary element formulation for elastodynamics. In: International Conference on Boundary Elements in Engineering, 12. Sapporo, Japan. CMP, v. 1, pp. 517-534.

CODA, H.B. \& VENTURINI, W.S. (1990b). Vibração livre de meios elásticos bidimensionais pelo método dos elementos de contorno. In: Congresso Ibero Latino Americano sobre Métodos Computacionais para Engenharia, 11. Rio de Janeiro. Anais, v. 2, pp. 781-790.

CODA, H.B. \& VENTURINI, W.S. (1995a). Three-dimensional transient BEM analysis. Computers \& Structures, v. 56, n. 5, pp. 751-768. 
CODA, H.B. \& VENTURINI, W.S. (1995b). Non-singular time-stepping BEM for transient elastodynamic analysis. Eng. Analysis with Boundary Elements, 15, pp. $11-18$.

CODA, H.B. \& VENTURINI, W.S. (1996). A simple comparison between two 3D time domain elastodynamic boundary element formulations. Eng. Analysis with Boundary Elements, 17, pp. 33-44.

COOK, R.D., MALKUS, D.S. and PLESHA, M.E. (1989). Concepts and applications of finite element analysis. 3a. ed. John Wiley \& Sons Ed., New York.

CROUCH, S.L. \& STARFIELD, A.M. (1983). Boundary element methods in solid mechanics. Lodon. George Allen \& Unwin.

CRUSE, T.A. (1968). A direct formulation and numerical solution of the general transient elastodynamic problem II. Journal Math. Anal. Appl., v. 22.

CRUSE, T.A.(1969). Numerical solutions in three dimensional elastostatics. Int. Journal of Solids and Structures. v. 5, pp. 1259-1274.

CRUSE, T.A (1973). Application of the boundary-integral equation method to three dimensional stress analysis. Computers and Structures, 3, pp. 509-527.

CRUSE, T.A (1974). An improved boundary-integral equation method for three dimensional elastic stress analysis. Computers and Structures, v. 4, pp. 741-754.

CRUSE, T.A., (1975). Boundary-integral equation method for three-dimensional elastic fracture mechanics analysis, AFOSR-TR-75-0813.

CRUSE, T.A. (1988). Boundary element analysis in computational fracture mechanics. London, Kluwer Academic Publishers.

CRUSE, T.A. (1995). BIE fracture mechanics analysis - 25 years of developments. In: $I A B E M / 95$. pp. 2848-2855.

CRUSE, T.A. \& MEYERS, G.J. (1977). Three dimensional fracture mechanics analysis. ASCE Journal of the Structural Division, n. 103, pp. 309-320.

CRUSE, T.A. \& RIZZO, F.J. (1968). A direct formulation and numerical solution of the general transient elastodynamic problem I. Journal Math. Anal. Appl., v. 22, pp. 224-259.

CRUSE, T.A. and Van BUREN (1971). Three dimensional elastic stress analysis of a fracture specimen with an edge crack. Int. J. Fract. Mech., v. 7, pp. 1-15.

CUROTTO, C.L. (1981). Método dos elementos de contorno para elasticidade tridimensional. Rio de Janeiro. Dissertação (Mestrado), COPPE-UFRJ. 
DANSON, D.J. (1981). A boundary element formulation of problems in linear isotropic elasticity with body forces. In: Boundary Element Methods, pp. 105122 , Springer-Verlag.

DOMINGUEZ, J. (1977). Computation of stresses near anchor plates: an application of the boundary element method. Ph. D. Thesis, Univ. of Seville, Spanish.

DOMINGUEZ, J. (1993). Boundary elements in dynamics. London, CMP.

DOMINGUEZ, J. \& GALLEGO, R. (1991). The time domain boundary element for elastodynamic problems. Journal of Math. and Computer Modelling, n. 15, pp. 119-129.

DOMINGUEZ, J. \& GALLEGO, R. (1992). Time domain boundary element method for dynamic stress intensity factor computations. Int. J. Numer. Methods Eng., v. 33, pp. 635-647.

DOMINGUEZ, J.; SÁEZ, A. (1998). Boundary element analysis of 3-D dynamic crack problems in isotropic and transversely isotropic solids. In: IV WCCM, CDROM Proceedings. Buenos Aires, AR.

DUMONT, N.A.; OLIVEIRA, R. de (1997). The exact dynamic formulation of the hybrid boundary element method. In: XVIII CILAMCE, v. 1, pp. 357-364. Brasília, BR.

FEDELINSKI, P.; ALIABADI, M.H.; ROOKE, D.P. (1993). The dual boundary element method in dynamic fracture mechanics. Eng. Analysis with Boundary Elements, 12, pp. 203-210.

FEDELINSKI, P.; ALIABADI, M.H.; ROOKE, D.P. (1996). Boundary element formulations for the dynamic analysis of cracked structures. Eng. Analysis with Boundary Elements, 17, pp. 45-56.

FERREIRA, W. G. (1990). Implementação de elemento de colocação não nodal para análise tridimensional pelo método dos elementos de contorno. Rio de Janeiro. Dissertação (Mestrado) - COPPE/UFRJ.

FLEURY Jr., P.; AZEVEDO, J.P.S.; MANSUR, W.J. (1997). Formulação hipersingular do método dos elementos de contorno aplicada à simulação da propagação de ondas gravitacionais de grande amplitude. In: XVIII CILAMCE, v. 1, pp. 301-308. Brasília, BR.

FREDHOLM, I. (1903). Sur une classe d'equations functionelles. Acta Math., v. 27, pp. 365-390.

GRIFFITHS, A.A. (1924). Proceedings of the $1^{\text {st }}$ International Congress for Applied Mechanics, Delft, pp. 55. 
GUIMARÃES, S. (1992). Sobre o método dos elementos de contorno aplicado à mecânica da fratura. Rio de Janeiro. Tese (Doutorado) - COPPE, Universidade Federal do Rio de Janeiro.

GUIMARÃES, S. \& TELLES, J.C.F. (1994). On the hyper-singular boundary element formulation for fracture mechanics applications. Eng. Analysis with Boundary Elements, 13, pp. 353-363.

GURTIN, M.E (1981). An introduction to continuum mechanics. Academic Press, New York.

HADAMARD, J. (1923). Lectures on Cauchy's problem in linear differential equations. New Haven, Yale University Press.

HARTRANFT, R.J. (1977). Stress singularity for a crack with an arbitrary curved crack front. Engng. Fracture Mech., 9, pp. 705-718.

HILLERBORG, A.; MODÉER, M. P.E. (1976). Analysis of crack formation and crack growth in concrete by means of fracture mechanics and finite elements. Cement and Concrete Res., pp. 773-782.

INGRAFFEA, A.R. and MANU, C.(1980). Stress-intensity factor computation in three dimensions with quarter-point elements. International Journal for Numerical Methods in Engineering, v. 15, pp. 1427-1445.

INGLIS, C.E. (1913). Stresses in a plate due to the presence of cracks and sharp corners. Proc. Inst. Naval Architects 60.

IRWIN, G.R. (1948). Fracture dynamics. In: Fracturing of Metals, ASM Cleveland.

IRWIN, G.R. (1957). Analysis of stresses and strains near the end of a crack traversing a plate". Trans. ASME, J. Appl. Mech..

JIA, Z.H. \& SHIPPY, D.J. (1989). Three-dimensional crack analysis using singular boundary elements. International Journal for Numerical Methods in Engineering, vol. 28, pp. 2257-2273.

JIANG, Y.; VENTURINI, W.S. (1998). Limit analysis of slope stability with boundary element methods. In: IV WCCM, CD-ROM Proceedings. Buenos Aires, AR.

KANE, J.H. (1994) Boundary element in engineering continuum mechanics. Prentice-Hall, Inc., New York.

KATONA, M.G. and ZIENKIEWICZ, O.C. (1985). A unified set of single step algorithms - part 3: the beta-m method, a generalisation of the Newmark scheme. 
International Journal for Numerical Methods in Engineering, vol. 21, pp. 13451359.

KUPRADZE, V.D. (1965). Potential methods in theory of elasticity. Jerusalem. Israel Program for Scientific Translations.

KUTT, H.R. (1975). Quadrature formulae for finite-part integrals Report WISK 178, National Research Institute for Mathematical Sciences, Pretoria, South Africa.

LACHAT, J.C. (1975). A further development of the boundary integral technique for elastostatics. Ph.D. Thesis, University of Southampton.

LACHAT, J.C. and WATSON, J.O. (1976). Effective treatment of boundary integral equations: a formulation for three-dimensional elastostatics. Int. J. Num. Meth. Engng., v. 10, pp. 991-1005.

LAETHEM, M. VAN et al. (1984). The use of boundary elements to represent the far field in soil-structure interaction. Nuclear Engineering and Design, vol. 78, pp 313-327, North-Holland Publishing Division.

LEITÃO, V.M.A. (1998). Applications of multi-region Trefftz-collocation to fracture mechanics. Engineering Analysis with Boundary Elements, 22, pp. 251256.

LOEFFLER, C.F. (1994). Comparações entre modelos elastodinâmicos estabelecidos com o método dos elementos de contorno. In: XV CILAMCE, vol. I, pp. 611-620.

LOPES JR., M.C. (1996). Modelagem numérica do crescimento de fraturas através do método dos elementos de contorno. Dissertação de Mestrado, Escola de Engenharia de São Carlos, USP, São Carlos.

LOVE, A.E.H. (1927). A treatise on the mathematical theory of elasticity. General Publishing Company Ltd, $4^{\mathrm{a}}$ edição.

LUCHI, M.L. and RIZZUTI, S. (1987). Boundary elements for three-dimensional elastic crack analysis. International Journal for Numerical Methods in Engineering, v. 24, pp. 2253-2271.

LUTZ, E.; INGRAFFEA, A.R. and GRAY, L. (1992). Use of "simple solutions" for boundary integral methods in elasticity and fracture analysis. Int. Journ. for Num. Meth. in Engng., v. 35, pp. 1737-1751.

MANOLIS, G.D. and BESKOS, D.E. (1981). Dynamic stress concentration studies by boundary integrals and Laplace transform. Int. J. Num. Meth. Engng., v. 17, pp. 573-599.

MANOLIS, G.D. and BESKOS, D.E. (1988). Boundary element methods in elastodynamics. Unwin Hyman, London-UK. 
MANSUR, W.J. and BREBBIA, C.A. (1982). Numerical implementation of the boundary element method for two-dimensional transient scalar wave propagation problems. Appl. Math. Modelling, v. 6, pp. 299-306.

MANSUR, W.J. and BREBBIA, C.A. (1985). Transient Elastodynamics. In: Topics in Boundary Element Research. v. 2, C.A.Brebbia (Ed.), pp. 124-155, SpringerVerlag.

MARTHA, L.F.; GRAY, L.J. and INGRAFFEA, A.R. (1992). Three-dimensional fracture simulation with a single-domain, direct boundary element formulation. Int. Journ. for Num. Meth. in Engng., v. 35, pp. 1907-1921.

MI, Y. and ALIABADI, M.H. (1992). Dual boundary element method for threedimensional fracture mechanics analysis. Engineering Analysis with Boundary Elements, v. 10, pp. 161-171.

MI, Y. and ALIABADI, M.H. (1994). Three-dimensional crack growth simulation using BEM. Computers \& Structures, v. 52, n. 5, pp. 871-878.

MIKHLIN, S.G. (1957). Integral equations. London. Pergamon Press (International series of monographs in pure and applied mathematics).

MINDLIN, R.D. (1936). Force at a point in the interior of a semi-infinite solid. $J$. Physics, v. 7, pp. 195-202.

MINGHAO, Z.; YUANJIE, L.; CHANGJUN, C. (1994). Boundary-integral equations and the boundary-element method for three-dimensional fracture mechanics. Engineering Analysis with Boundary Elements, v. 13, pp. 333-338.

MOAN et al. (1993). Structural dynamics - EURODYN'93. V. 1 e 2. Trondhein, Norway.

MUKHERJEE, Y.X.; SHAH, K. \& MUKHERJEE, S. (1999). Thermoelastic fracture mechanics with regularized hypersingular boundary integral equations. Engineering Analysis with Boundary Elements, 23, pp. 89-96.

MUSKHELISHVILI, N.I. (1953). Some basic problems of the mathematical theory of elasticity. Groningen Holland, Noordhoff.

NAKAGUMA, R.K. (1979). Three dimensional elastostatics using the boundary element method. Southampton. Tese (PhD) - University of Southampton.

NARDINI, D. and BREBBIA, C.A. (1982). A new approach to free vibration analysis using boundary elements. In: Boundary Element Methods in Engineering, CMP, Southampton.

NARDINI, D. and BREBBIA, C.A. (1983). A new approach to free vibration analysis using boundary elements. Appl. Math. Modelling, v. 7, pp. 157-162. 
NARDINI, D. and BREBBIA, C.A. (1985). Boundary integral formulation of mass matrices for dynamic analysis. In: Topics in Boundary Element Research, v. 2, C.A. Brebbia (Ed.), pp. 191-208, Springer-Verlag.

NEVES, A.C. \& BREBBIA, C.A. (1991). The multiple reciprocity boundary element method in elasticity: a new approach for transforming domain integrals to the boundary. International Journal for Numerical Methods in Engineering, vol. 31, pp. 709-727.

NISHIOKA, T. (1994). The state of the art in computational dynamic fracture mechanics. JSME Intern. Journ. - Series A, v. 37, n. 4, pp. 313-333.

NISITANI, H and CHEN, D.H. (1993). Body force method. In: ALIABADI, M.H.; BREBBIA, C.A. (eds.). Advances in boundary element methods for fracture mechanics, Southampton, CMP.

NOVAK, A.J. \& PARTRIDGE, P.W. (1992). Comparison of the dual reciprocity and the multiple reciprocity methods. Engineering Analysis with Boundary Elements, v. 10 , pp. 155-160.

OROWAN, E. (1955). Energy criteria of fracture. Weld J. Res. Suppl. 20, 1575.

OWEN, D.R.J. and FAWKES, A.J. (1983). Engineering fracture mechanics: numerical methods and applications. Pineridge Press Ltd, Swansea, UK.

PANASYUK, A.E.; ANDREJKIV, A.E.; STADNIK, M.M. (1981). Threedimensional static crack problems solution (a review). Engineering Analysis with Boundary Elements, v. 14, pp. 245-260.

PARTRIDGE, P.W. (1995). Reciprocidade dual no MEC: comparação de funções locais e globais de aproximação para problemas de convecção, de difusão e similares. Revista internacional de métodos numéricos para cálculo y diseño en ingeniería, v. 11, n.4, pp.529-541.

PARTRIDGE, P.W.; BREBBIA, C.A. and WROBEL, L.C. (1992). The dual reciprocity boundary element method. 2.ed., Southampton, CMP.

PORTELA, A. (1993). Topics in Engineering - Dual boundary analysis of crack growth. Computational Mechanics Publications, Southampton.

PORTELA, A.; ALIABADI, M.H. and ROOKE, D.P. (1993) Dual boundary element analysis of fatigue crack growth. In: ALIABADI, M.H.; BREBBIA, C.A. (eds.). Advances in boundary element methods for fracture mechanics, Southampton, CMP

RIGBY, R.H. \& ALIABADI, M.H. (1993). Mixed-mode J-integral method for analysis of 3D fracture problems using BEM. Engineering Analysis with Boundary Elements, v. 11, pp. 239-256. 
RIZZO, F.J. (1967). An integral approach to boundary value problems of classical elastostatics. Quartely of Applied Mathematics, v. 25 (1), pp. 83-92.

RIZZO, F.J. \& SHIPPY, D.J. (1968). A formulation and solution procedure for the general non-homogeneous elastic inclusion problem. Int. J. Solids Structures, v. 4, pp. 1161-1179.

ROCHA, F.S. (1988). Análise de descontinuidades pelo método dos elementos de contorno. São Carlos. Tese (Doutorado) - Escola de Engenharia de São Carlos, Universidade de São Paulo.

ROCHA, J.A. de L. \& VENTURINI, W.S. (1998). Griffith's generalised and thermodynamically consistent criterion for fracture initiation. In: $I V W C C M$, CRROM Proceedings. Buenos Aires, AR.

SÁ, P.A.C.O. and TELLES, J.C.F. (1986). Análise de problemas de elasticidade linear tridimensional pelo método dos elementos de contorno utilizando as soluções fundamentais de Kelvin e Mindlin. In: Congresso Latino-Americano sobre Métodos Computacionais para Engenharia, v. 7, pp. 43-60. São Carlos.

SALEH, A.L. and ALIABADI, M.H. (1994). Cracking of strain softening material. In: BREBBIA, C.A. ed., Boundary Element Method XVI, pp. 328-334. Southampton, UK.

SILVA, J.J.R. (1989). MEC3DE - Um programa para análise elástica tridimensional com o método dos elementos de contorno. Rio de Janeiro. Dissertação (Mestrado), COPPE-UFRJ.

SILVA, N.A. (1996). Aplicação do método dos elementos de contorno à placas com enrijecedores utilizando a teoria de Reissner. Tese de Doutorado, Escola de Engenharia de São Carlos, USP, São Carlos-SP.

SILVEIRA, N.P.P. \& TELLES, J.C.F. (1997). Implementação da formulação hipersingular do método dos elementos de contorno e função de Green numérica para trincas de geometria qualquer. . In: XVIII CILAMCE, v. 1, pp. 285-292. Brasília, BR.

SNYDER, M.D. and CRUSE, T.A. (1975). Boundary-integral equation analysis of cracked anisotropic plates. Int. J. Fracture, 11, pp. 315-28.

SOUZA, J.L.A.O; BITTENCOURT, T.N. and MARTHA, L.F. (1995). Simulação tridimensional de propagação de fissuras não planas em sólidos de geometria arbitrária. In: XVI CILAMCE, anais, v. 2, pp. 1092-1101. Curitiba, BR.

TANDON, S.; FABER, K.T.; BAZANT, Z.P.; LI, N.Y. (1995). Cohesive crack modeling of influence of sudden changes in loading rate on concrete fracture. Engineering Fracture Mechanics, v. 52, n. 6, pp. 987-997. 
TELLES, J.C.F.; CASTOR, G.S. and GUIMARÃES, S. (1994). A hipersingular Green's function generation for fracture mechanics problems. In: BREBBIA, C.A. ed., Boundary Element Method XVI, pp. 443-452. Southampton, UK.

TELLES, J.C.F.; GUIMARÃES, S. (1998). Green's function: a numerical generation for fracture mechanics problems via boundary elements. In: IV WCCM, CR-ROM Proceedings. Buenos Aires, AR.

TIMOSHENKO, S.P. (1953). History of strength of materials - with a brief account of the history of theory of elasticity and theory of structures. London. McGrawHill.

TREVELYAN, J. (1992). Use of discontinuous boundary elements for fracture mechanics analysis. Engineering Analysis with Boundary Elements, v. 10, 353358.

TUHKURI, J. (1997). Dual boundary element analysis of closed cracks. Int. Journ. of Numer. Meth. in Engin., v. 40, pp. 2995-3014.

VALENTIM, V.A.; SANTIAGO, J.A.F. \& TELLES, J.C.F. (1995). Uma técnica eficiente para implementação de subregiões no método dos elementos de contorno. In: XVI CILAMCE, vol. I, pp. 236-245, Belo Horizonte-BR.

VENTURINI, W.S. (1982). Application of the boundary element formulation to solve geomechanical problems. Ph.D. Thesis, University of Southampton.

VENTURINI, W.S. (1988). Um estudo sobre o método dos elementos de contorno e suas aplicações em problemas de engenharia. São Carlos. Tese (Livre-Docência) - Escola de Engenharia de São Carlos, Universidade de São Paulo.

VENTURINI, W.S. (1994a). Reciprocidade dual para análise não-linear através do método dos elementos de contorno. In: XV CILANCE, v. 1, pp. 576-590. Belo Horizonte, BR.

VENTURINI, W.S. (1994b). A new boundary element formulation for crack analysis. In: BREBBIA, C.A. ed., Boundary Element Method XVI, pp. 405-412. Southampton, UK.

VENTURINI, W.S. (1995). Análise de problemas de fratura coesiva usando o método dos elementos de contorno. In: XVI CILAMCE, anais, v. 2, pp. 10121021. Curitiba, BR.

VOLTERRA, V. (1956). Opere mathematiche. Acad. Naz. Lincei, 2: 216-275, Rome.

WANG, E.Z.; SHRIVE, N.G. (1995). Brittle fracture in compression: mechanisms, models and criteria. Engineering Fracture Mechanics, v. 52, n. 6, pp. 1107-1126. 
WARBURTON, G.B. (1976). The dynamical behaviour of structures. 2.ed., Oxford, Pergamon Press.

WEARING, J.L. \& AHMADI-BROOGHANI, S.Y. (1999). The evaluation of stress intensity factors in plate bending problems using the dual boundary element method. Engineering Analysis with Boundary Elements, 23, pp. 3-19.

WEN, P.H.; ALIABADI, M.H.; ROOKE, D.P. (1995). An indirect boundary element method for three-dimensional dynamic fracture mechanics. Engineering Analysis with Boundary Elements, 16, pp. 351-362.

WEN, P.H.; ALIABADI, M.H.; ROOKE, D.P. (1999). Three-dimensional dynamic fracture analysis with the dual reciprocity method in Laplace domain. Engineering Analysis with Boundary Elements, 23, pp. 51-58.

ZAHAO, M.H.; SHEN, Y.P.; LIU, Y.J. \& LIU, G.N. (1998). The method of analysis of cracks in three-dimensional transversely isotropic media: boundary integral equation approch. Engineering Analysis with Boundary Elements, 21, pp. 169178. 